Keywords: SCIX, DWPF,

Titanium, Glass, Durability

Retention: Permanent

\title{
Impacts of Small Column Ion Exchange Streams on DWPF Glass Formulation: KT01, KT02, KT03, and KT04-Series Glass Compositions
}

K. M. Fox

T. B. Edwards

November 2010

Savannah River National Laboratory Savannah River Nuclear Solutions, LLC Aiken, SC 29808

Prepared for the U.S. Department of Energy under contract number DE-AC09-08SR22470.

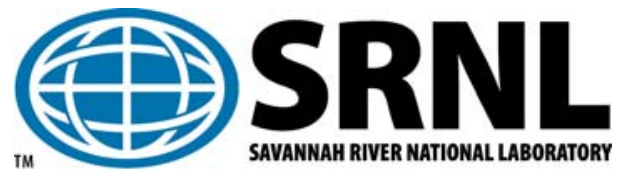


SRNL-STI-2010-00566

Revision 0

\section{DISCLAIMER}

This work was prepared under an agreement with and funded by the U.S. Government. Neither the U.S. Government or its employees, nor any of its contractors, subcontractors or their employees, makes any express or implied:

1. warranty or assumes any legal liability for the accuracy, completeness, or for the use or results of such use of any information, product, or process disclosed; or

2. representation that such use or results of such use would not infringe privately owned rights; or

3. endorsement or recommendation of any specifically identified commercial product, process, or service.

Any views and opinions of authors expressed in this work do not necessarily state or reflect those of the United States Government, or its contractors, or subcontractors.

\section{Printed in the United States of America \\ Prepared for \\ U.S. Department of Energy}




\section{REVIEWS AND APPROVALS}

AUTHORS:

K. M. Fox, Process Technology Programs

Date

T. B. Edwards, Applied Computational Engineering and Statistics

Date

TECHNICAL REVIEW:

D. K. Peeler, Process Technology Programs

Date

APPROVAL:

C. C. Herman, Manager

Date

Process Technology Programs

F. M. Pennebaker, Manager

Date

SRNL SCIX Project

S.L. Marra, Manager

Date

Environmental \& Chemical Process Technology Research Programs

J. E. Occhipinti, Manager

Date

SRR Engineering

T. H. Huff, Manager

Date

SCIX Engineering 


\section{EXECUTIVE SUMMARY}

Four series of glass compositions were selected, fabricated, and characterized as part of a study to determine the impacts of the addition of Crystalline Silicotitanate (CST) and Monosodium Titanate (MST) from the Small Column Ion Exchange (SCIX) process on the Defense Waste Processing Facility (DWPF) glass waste form and the applicability of the DWPF process control models. The KT01 and KT02-series of glasses were chosen to allow for the identification of the influence of the concentrations of major components of the glass on the retention of $\mathrm{TiO}_{2}$. The KT03 series of glasses was chosen to allow for the identification of these influences when higher $\mathrm{Nb}_{2} \mathrm{O}_{5}$ and $\mathrm{ZrO}_{2}$ concentrations are included along with $\mathrm{TiO}_{2}$. The KT04 series of glasses was chosen to investigate the properties and performance of glasses based on the best available projections of actual compositions to be processed at the DWPF (i.e., future sludge batches including the SCIX streams).

The X-ray Diffraction (XRD) results showed no titanium containing phases in the KT01-series glasses, regardless of whether they had been air quenched or slowly cooled. The target $\mathrm{TiO}_{2}$ concentration had to be increased to $12 \mathrm{wt} \%$ in glass in the KT02-series before any compositional impacts on $\mathrm{TiO}_{2}$ retention (e.g., the formation of titanium containing crystals) were apparent. Titanium containing crystalline phases formed in the slowly cooled versions of all of the KT02 compositions except for the low $\mathrm{Al}_{2} \mathrm{O}_{3}$ concentration glass, the low $\mathrm{Fe}_{2} \mathrm{O}_{3}$ concentration glass, the high $\mathrm{Na}_{2} \mathrm{O}$ concentration glass, and the high $\mathrm{K}_{2} \mathrm{O}$ concentration glass. The impacts of these components will need additional investigation before drawing further conclusions. However, one should keep in mind that the $12 \mathrm{wt} \% \mathrm{TiO}_{2}$ concentrations in these glasses are unrealistic for DWPF processing with the SCIX and Salt Waste Processing Facility (SWPF) streams. Further characterization of the KT02-series glasses was not performed because of their unusually high $\mathrm{TiO}_{2}$ concentrations. Spinels were identified in some of the KT03 glasses after slow cooling. The spinels did not adversely impact the durability of the glasses but have the potential to impact liquidus temperature determinations since spinel is the primary crystalline phase predicted by the current model. The KT04-series glasses were amorphous regardless of heat treatment.

All of the glasses studied were considerably more durable than the benchmark Environmental Assessment (EA) glass. The measured Product Consistency Test (PCT) responses were compared with the predicted values from the current DWPF durability model. One of the KT01series and two of the KT03-series glasses had measured PCT responses that were outside the lower bound of the durability model. All three of these glasses had intentionally high $\mathrm{K}_{2} \mathrm{O}$ concentrations (to evaluate the impact of $\mathrm{K}_{2} \mathrm{O}$ on $\mathrm{TiO}_{2}$ retention), which may indicate a lack of applicability for the durability model in this composition region. However, this is likely of little practical importance since $\mathrm{K}_{2} \mathrm{O}$ concentrations of this magnitude (more than 9 wt \%) are unrealistic for actual compositions to be processed at the DWPF. All of the KT04 glasses had durabilities that were predictable regardless of heat treatment or compositional view.

All but two of the KT01, KT03, and KT04-series glasses had measured viscosities that were predictable using the current DWPF viscosity model. The viscosities of two of the high $\mathrm{K}_{2} \mathrm{O}$ concentration glasses fell above the upper confidence intervals for the model prediction. These glasses have $\mathrm{K}_{2} \mathrm{O}$ concentrations that are above the DWPF viscosity model development range for $\mathrm{K}_{2} \mathrm{O}$. Another high $\mathrm{K}_{2} \mathrm{O}$ concentration glass had a measured viscosity that was well predicted by the current DWPF model. While these results point to lack of applicability for the DWPF viscosity model for increased $\mathrm{K}_{2} \mathrm{O}$ concentrations, it is again important to note that these $\mathrm{K}_{2} \mathrm{O}$ concentrations are unrealistic for actual DWPF processing. In general, the measured viscosity 
values of the KT01, KT03, and KT04-series glasses are well predicted by the current DWPF viscosity model.

The results of liquidus temperature $\left(\mathrm{T}_{\mathrm{L}}\right)$ measurements for the KT01-series glasses were mixed with regard to the predictability of the $T_{L}$ for each glass. All of the measured $T_{L}$ values were higher than the model predicted values, although most fell within the $95 \%$ confidence intervals. Four of the KT01 glasses had measured $\mathrm{T}_{\mathrm{L}}$ values that were above the upper $95 \%$ confidence bounds on the predicted values. The concentrations of some of the components in these glasses, particularly $\mathrm{TiO}_{2}$, fall outside the region of applicability of the current $\mathrm{T}_{\mathrm{L}}$ model. These results indicate that the model may need to be adjusted in order to more correctly predict the $T_{L}$ of glasses when the SCIX streams are incorporated, although further data are necessary for a more complete assessment. Liquidus temperature measurements for the KT03 and KT04-series glasses are underway.

Overall, the results presented in this report show a reasonable ability to incorporate the anticipated SCIX streams into the DWPF-type glass compositions studied, with $\mathrm{TiO}_{2}$ concentrations of $4-5 \mathrm{wt} \%$ in glass. The durability and viscosity models satisfactorily predicted the measured values for the study glasses with the exception of a small number of extreme compositions. The liquidus temperature model may need to be adjusted to correctly predict the liquidus temperatures of glasses including the SCIX streams based on the data measured. Additional experiments are currently underway. Once completed, all of the measured data will be reviewed and compared to model predictions to determine whether the validation range of the DWPF process control models can be confidently extended, or whether refitting of the models will be necessary. 


\section{TABLE OF CONTENTS}

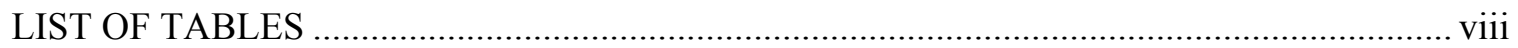

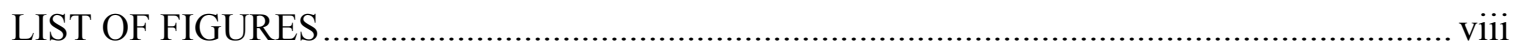

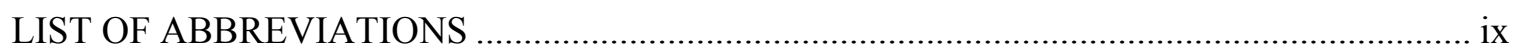

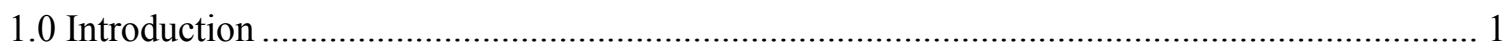

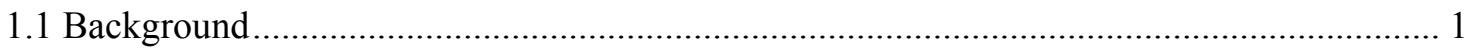

1.2 Potential Impacts of SCIX on DWPF Glass Formulation.................................................. 1

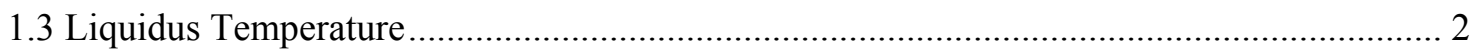

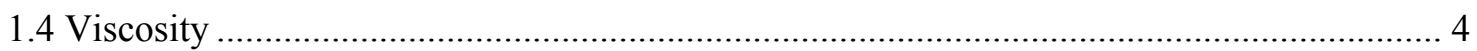

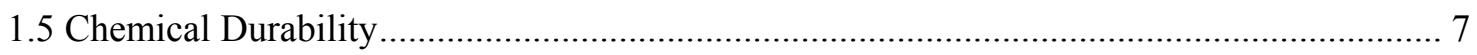

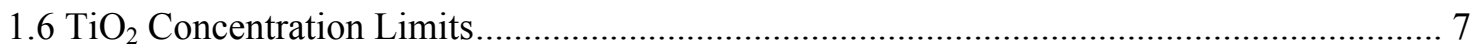

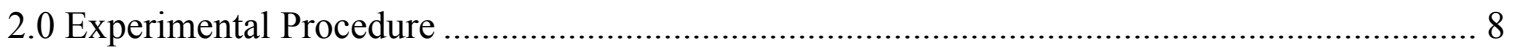

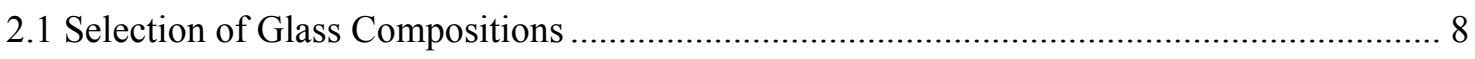

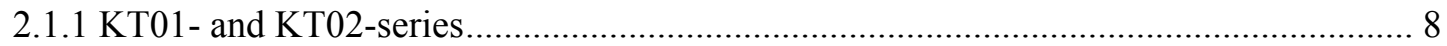

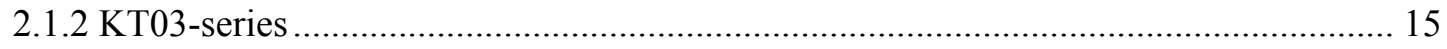

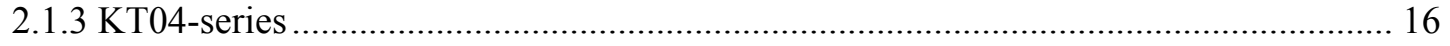

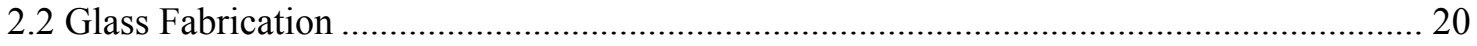

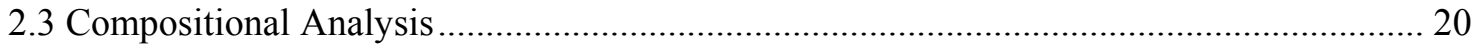

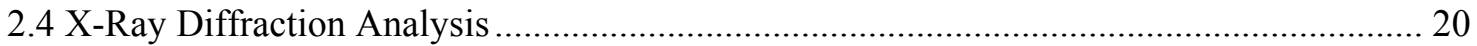

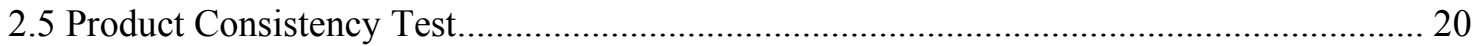

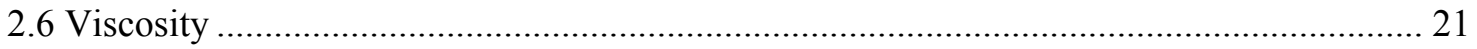

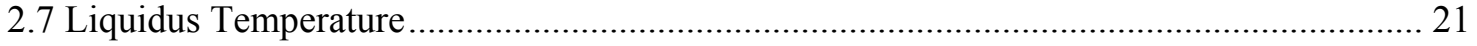

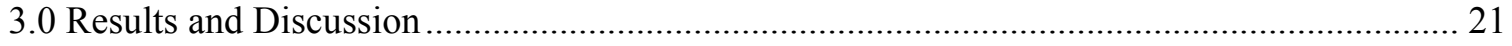

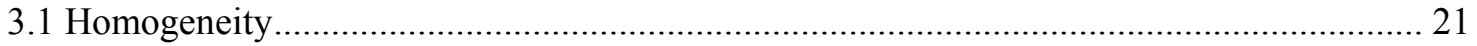

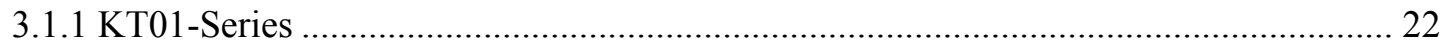

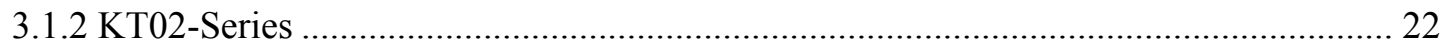

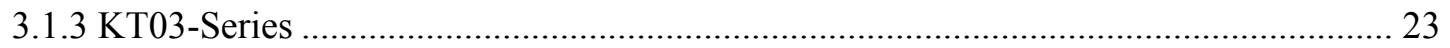

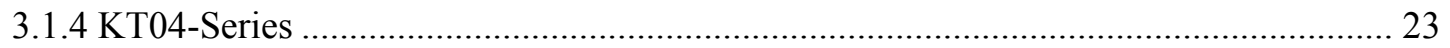

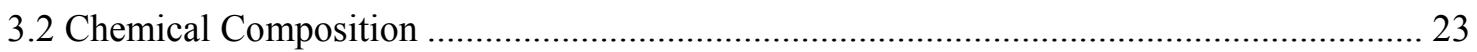

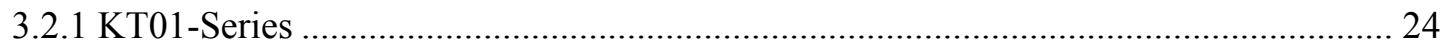

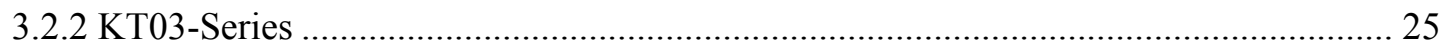

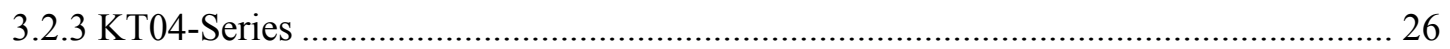

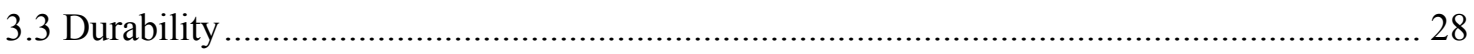

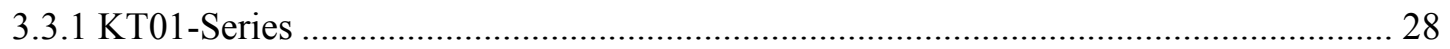


SRNL-STI-2010-00566

Revision 0

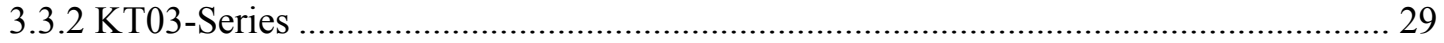

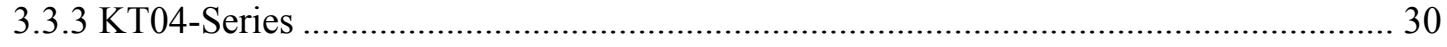

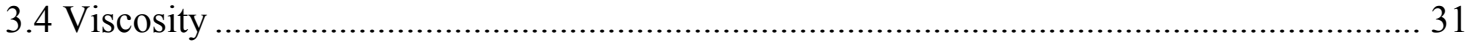

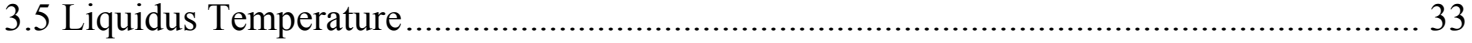

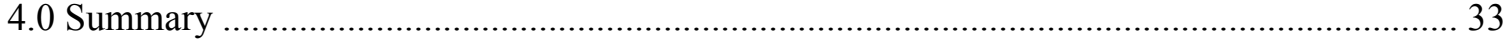

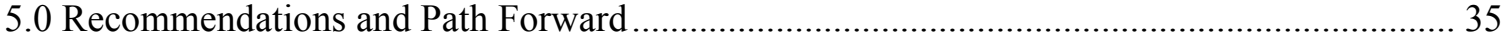

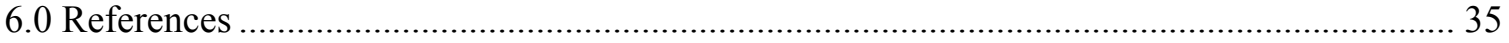




\section{LIST OF TABLES}

Table 1-1. Component Ranges (wt \%) Describing the Region of Applicability of the Liquidus Temperature Model. (from Brown et al. ${ }^{9}$ ). 3

Table 1-2. Component Ranges (wt \%) Describing the 155 Selected Non-Model Glasses Used in Evaluating the DWPF Liquidus Model. (from Brown et al. ${ }^{9}$ )............................................... 3

Table 1-3. Temperature, Viscosity, and Composition Ranges (wt \%) for the DWPF Viscosity Model. (from Jantzen ${ }^{14}$ ) 5

Table 1-4. Temperature, Viscosity, and Composition Ranges (wt \%) for Validation of the DWPF Viscosity Model. (from Jantzen ${ }^{14}$ )

Table 2-1. Projected Average, Minimum, and Maximum Concentrations (wt \%) of HLW Glass Components in Out-Year Processing at DWPF with SCIX and SWPF Streams.

Table 2-2. Target Compositions (wt \%) of the KT01-Series Glasses. ........................................ 11

Table 2-3. Target Compositions (wt \%) of the KT02-Series Glasses. ........................................ 13

Table 2-4. Target Compositions (wt \%) of the KT03-Series Glasses. ......................................... 15

Table 2-5. Projected Compositions (wt \%) of the Final SRAT Batches of Sludge Batches 8 through 17 Used to Develop the KT04-Series Glass Compositions. .................................... 17

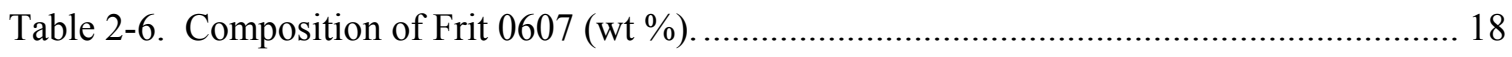

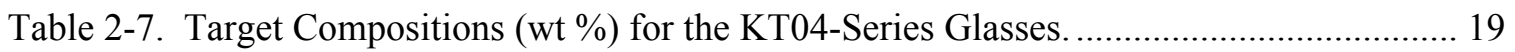

Table 3-1. Summary of XRD Results for the KT02-Series Glasses............................................ 23

\section{LIST OF FIGURES}

Figure 3-1. Measured Versus Predicted Viscosities at $1150{ }^{\circ} \mathrm{C}$ for the KT01, KT03, and KT04Series Glasses, Based on the Measured Compositions.

Figure 3-2. Predicted and Measured Liquidus Temperatures for the KT01-Series Glasses, Based on the Measured Compositions. 


\section{LIST OF ABBREVIATIONS}

\begin{tabular}{|c|c|}
\hline $\mathrm{AD}$ & Analytical Development \\
\hline ANOVA & ANalysis Of VAriance \\
\hline ARM & Approved Reference Material \\
\hline $\mathrm{CCC}$ & Canister Centerline Cooled \\
\hline CST & Crystalline Silicotitanate \\
\hline DWPF & Defense Waste Processing Facility \\
\hline EA & Environmental Assessment \\
\hline HLW & High Level Waste \\
\hline ICP-AES & Inductively Coupled Plasma - Atomic Emission Spectroscopy \\
\hline $\mathrm{LM}$ & Lithium-Metaborate Fusion \\
\hline M\&TE & Measurement and Test Equipment \\
\hline MAR & Measurement Acceptability Region \\
\hline MSP & Modular Salt Processing \\
\hline MST & Monosodium Titanate \\
\hline PCCS & Product Composition Control System \\
\hline PCT & Product Consistency Test \\
\hline $\mathrm{PF}$ & Sodium Peroxide Fusion \\
\hline PSAL & Process Science Analytical Laboratory \\
\hline $\mathrm{RF}$ & Resorcinol Formaldehyde \\
\hline RMF & Rotary Micro Filtration \\
\hline SCIX & Small Column Ion Exchange \\
\hline SME & Slurry Mix Evaporator \\
\hline SRAT & Sludge Receipt and Adjustment Tank \\
\hline SRNL & Savannah River National Laboratory \\
\hline SRS & Savannah River Site \\
\hline SWPF & Salt Waste Processing Facility \\
\hline THERMO & Thermodynamic Hydration Energy Reaction MOdel \\
\hline $\mathrm{T}_{\mathrm{L}}$ & Liquidus Temperature \\
\hline XRD & X-ray Diffraction \\
\hline
\end{tabular}




\subsection{Introduction}

\subsection{Background}

The Savannah River Site (SRS) Tank Farm will begin a process referred to as Small Column Ion Exchange (SCIX) to disposition salt solution in fiscal year 2014. In the first step of the process, salt solution retrieved from various waste tanks will be struck with Monosodium Titanate (MST) to remove key actinides. The salt solution will then be processed using Rotary Micro Filtration (RMF) to remove the MST and any insoluble solids. The MST and insoluble solids will accumulate on the bottom of Tank 41. The filtrate from RMF will be fed to ion exchange columns, also in Tank 41, to remove the ${ }^{137}$ Cs using Crystalline Silicotitanate (CST) resin. The decontaminated salt solution from SCIX will be sent to the Saltstone Facility for immobilization in grout. The ${ }^{137} \mathrm{Cs}$-laden CST resin will be sluiced and ground for particle size reduction, then sent to the Defense Waste Processing Facility (DWPF) for immobilization in glass. These processes mirror the current disposition paths for streams associated with the Salt Waste Processing Facility (SWPF), which is under construction.

The MST and insoluble solids from Tank 41 will periodically be transferred to a sludge batch preparation tank (e.g., Tank 42 or Tank 51) as part of the High Level Waste (HLW) sludge batch preparation process for DWPF. The ground, ${ }^{137} \mathrm{Cs}-\mathrm{laden}$ CST material (hereafter referred to simply as CST) from SCIX will be periodically transferred to Tank 40 prior to being processed at DWPF. Periodic additions of CST to Tank 40 would result in a changing composition of each sludge batch as it is processed since Tank 40 serves as the feed tank for the DWPF. Work is currently in progress to determine the feasibility of dropping the ground CST into Tank 41. If ground CST can be dropped into Tank 41 (depending on heat loading issues, among others), the CST would be sent to Tank 42 or Tank 51 using an existing transfer line. Therefore, the studies of SCIX impacts on DWPF glass formulation will encompass scenarios where the CST is sent to either Tank 40 or a sludge batch preparation tank.

This work was initiated by a DWPF Technical Task Request ${ }^{1}$ and is performed following a Task Technical and Quality Assurance Plan. ${ }^{2}$

\subsection{Potential Impacts of SCIX on DWPF Glass Formulation}

The MST and CST from the SCIX process will significantly increase the concentrations of $\mathrm{Nb}_{2} \mathrm{O}_{5}$, $\mathrm{TiO}_{2}$, and $\mathrm{ZrO}_{2}$ in the DWPF feed. Other constituents of MST and CST $-\mathrm{Na}_{2} \mathrm{O}$ and $\mathrm{SiO}_{2}-$ are already present in high concentrations in DWPF glass; thus their influences are well understood. The increased concentrations of $\mathrm{Nb}_{2} \mathrm{O}_{5}, \mathrm{TiO}_{2}$, and $\mathrm{ZrO}_{2}$ will likely have some impact on the properties and performance of the DWPF glass product. Properties such as the liquidus temperature, viscosity, and rate of melting of the glass may be impacted. The performance of the glass, particularly its chemical durability as it pertains to repository acceptance requirements, may also be impacted. The DWPF uses a set of semi-empirical and first-principles models referred to as the Product Composition Control System (PCCS) ${ }^{3}$ to predict the properties and performance of a glass based on its composition since it is not possible to measure these attributes during processing. The objective of this study is to evaluate the impacts of the SCIX streams on the properties and performance of the DWPF glass product and on the applicability of the current process control models.

Previous studies have provided data on the potential impacts of MST and CST specifically on DWPF-type glasses. Edwards and Harbour completed a series of studies on the coupling of CST and MST with Purex and HM sludges for DWPF processing in $1999 .^{4-8}$ A total of 25 glass 
compositions were fabricated and characterized for durability, viscosity, and liquidus temperature. All of the glasses had very good measured durability values, although there were minor issues with the predictions of the durability model and the majority of the glasses failed the homogeneity constraint. ${ }^{8}$ The glasses generally had estimated liquidus temperatures that would be acceptable for DWPF processing. The viscosities of the glasses produced with CST and the Purex sludge were acceptable for processing but not well predicted. The viscosities of the glasses fabricated with CST and HM sludge were too high for processing but were better predicted.

A later report by Peeler and Edwards provided paper study evaluations of the projected impact of a similar Modular Salt Processing (MSP) strategy using CST or eluant from Resorcinol Formaldehyde (RF) resin on DWPF processing. ${ }^{9}$ This study again noted that the compositions were outside the validation ranges for many of the PCCS models, although the current models continued to be used for the evaluation. Using updated sludge and salt processing composition projections, this study found that no options for producing an acceptable glass composition were available when CST or RF were sent to Tank 40. However, direct transfer of RF or CST to DWPF appeared to be viable options in terms of producing an acceptable glass. Transfer of the CST to Tank 51 also appeared to be a viable option, with the caveat that the PCCS models be updated to incorporate higher concentrations of the CST components.

Fox and Edwards recently performed a paper study evaluation using updated projections for sludge batch compositions and SCIX CST and MST addition rates. ${ }^{10}$ This study found that, as a result of the updated composition projections, several viable options were predicted to be available for incorporation of the SCIX streams into either Tank 40 or a sludge batch preparation tank. Transfer of the CST to a sludge batch preparation tank was the preferred option since it allowed more compositional flexibility for frit development while maintaining sufficient projected operating windows. The report again identified several assumptions and limitations associated with the current PCCS models, and recommended that these be further evaluated.

The following sections provide more detailed descriptions of the potential impacts of incorporation of the SCIX streams on the properties and performance of DWPF glass, and the models that are used to predict these attributes.

\subsection{Liquidus Temperature}

The liquidus temperature $\left(T_{\mathrm{L}}\right)$ of a glass is the maximum temperature at which the formation of glass liquid and the formation of crystals are at equilibrium. Liquidus temperature is an important parameter in operation of the DWPF melter since the bulk formation of crystals in the melt pool can cause non-recoverable process problems. If not controlled, crystallization in the melt could potentially occur very rapidly and throughout the entire volume of the melter. The crystalline phases formed can have a melting temperature much higher than the temperature of the melt pool and, thus, be impossible to dissolve back into the glass. This same crystalline material once formed in the melt pool can potentially clog the pour spout and interfere with Joule heating. Finally, crystals can also cause an undesirable increase in viscosity of the glass. To avoid detrimental crystallization in the DWPF melter, PCCS limits the predicted liquidus temperature to a maximum of $1050{ }^{\circ} \mathrm{C}$. This provides a $100{ }^{\circ} \mathrm{C}$ margin of safety below the nominal melt pool temperature of $1150{ }^{\circ} \mathrm{C}$ before crystallization of spinels is predicted to occur. ${ }^{\mathrm{a}}$

\footnotetext{
${ }^{a}$ Note that a reduction in the magnitude of the $\mathrm{T}_{\mathrm{L}}$ margin (or increase in the allowable predicted $\mathrm{T}_{\mathrm{L}}$ ) was proposed at the time the current model was developed, ${ }^{11}$ and has recently been proposed again, ${ }^{12}$ to allow DWPF access to higher waste loadings.
} 
While several models for predicting the liquidus temperature of a glass melt have been reported (a detailed review is provided by Jantzen and Brown ${ }^{13}$ ), a unique model was developed at the Savannah River National Laboratory (SRNL) relevant to the complex nature of DWPF-type glass compositions. $^{11,13,14}$ This semi-empirical model is currently incorporated in PCCS to predict the spinel liquidus temperature from glass composition. ${ }^{3}$ Spinels are expected to be the primary crystalline phase in DWPF glasses. The model assumes that pyroxene-like melt phase complexes or precursors control the heterogeneous volume crystallization of spinels. The basis of the model is mechanistic, based on known speciation of cations in the crystalline pyroxene and nepheline lattices, and including a mathematical representation of the freezing point depression equation. Coefficients within the model were determined through a least squares fit of a carefully reviewed and qualified data set. These data represent 105 individual glasses with compositions that are relevant to DWPF processing. A region of compositional applicability, shown in Table 1-1, was defined along with the model as a result of its semi-empirical nature. Measured values for compositions outside this range must be collected and evaluated against the model predicted values to determine further applicability.

Table 1-1. Component Ranges (wt \%) Describing the Region of Applicability of the Liquidus Temperature Model. (from Brown et al. ${ }^{11}$ )

\begin{tabular}{||c|c|c|c|c|c|c|c|c|c||}
\hline & $\mathbf{A l}_{2} \mathbf{O}_{3}$ & $\mathbf{B}_{\mathbf{2}} \mathbf{O}_{3}$ & $\mathbf{C a O}$ & $\mathbf{C r}_{\mathbf{2}} \mathbf{O}_{3}$ & $\mathbf{F e O}$ & $\mathbf{F e}_{\mathbf{2}} \mathbf{O}_{3}$ & $(\Sigma \mathbf{F e})_{\mathbf{2}} \mathbf{O}_{3}$ & $\mathbf{K}_{\mathbf{2}} \mathbf{O}$ & $\mathbf{L i}_{2} \mathbf{O}$ \\
\hline Maximum & 14.162 & 12.652 & 2.007 & 0.3008 & 6.901 & 16.977 & 17.60 & 3.8846 & 6.1576 \\
\hline Minimum & 0.99 & 4.893 & 0.3053 & 0 & 0.0161 & 3.427 & 3.452 & 0 & 2.4901 \\
\hline & & & & & & & & & \\
\hline & $\mathbf{M g O}$ & $\mathbf{M n O}$ & $\mathbf{N a}_{\mathbf{2}} \mathbf{O}$ & $\mathbf{N i O}$ & $\mathbf{S i O}_{2}$ & $\mathbf{T i O}_{2}$ & $\mathbf{U}_{3} \mathbf{O}_{\mathbf{8}}$ & $\mathbf{Z r O}_{2}$ & $\mathbf{T o t a l}^{\dagger}$ \\
\hline Maximum & 2.6502 & 3.25 & 14.901 & 3.045 & 58.230 & 1.8549 & 5.1387 & 0.97 & 94.5479 \\
\hline Minimum & 0.470 & 0.7392 & 5.989 & 0.0379 & 41.795 & 0 & 0 & 0 & 81.5746 \\
\hline
\end{tabular}

"The shaded components are included in the current liquidus temperature model. As indicated in the Total column, they constitute between approximately 81.6 and $94.5 \mathrm{wt} \%$ of the model glasses.

The DWPF liquidus temperature model was also examined with non-model data to provide insight into the applicability of the model to these systems. ${ }^{11}$ Predicted spinel liquidus temperatures for 155 non-model glasses, within the composition ranges shown in Table 1-2, were compared with their measured values. The results, detailed by Brown et al., showed significant prediction biases for many of the glasses, although the model did appear to describe the general trend of liquidus temperature as a function of composition. ${ }^{11}$ Brown et al. recommended that if compositions outside the composition space of the model glasses (Table 1-1) become likely for DWPF processing, the model may have to be adjusted considering such components as $\mathrm{CaO}$, $\mathrm{Na}_{2} \mathrm{O}, \mathrm{SiO}_{2}$, and $\mathrm{ZrO}_{2}{ }^{11}$ Note that an individual component limit of $0.3 \mathrm{wt} \%$ for $\mathrm{Cr}_{2} \mathrm{O}_{3}$ is used in PCCS, ${ }^{3}$ so it was excluded from this list.

Table 1-2. Component Ranges (wt \%) Describing the 155 Selected Non-Model Glasses Used in Evaluating the DWPF Liquidus Model. (from Brown et al. ${ }^{11}$ )

\begin{tabular}{|l|c|c|c|c|c|c|c|c||}
\hline & $\mathbf{A l}_{2} \mathbf{O}_{3}$ & $\mathbf{B}_{2} \mathbf{O}_{3}$ & $\mathbf{C a O}$ & $\mathbf{C r}_{2} \mathbf{O}_{3}$ & $(\mathbf{\Sigma F e})_{2} \mathbf{O}_{3}$ & $\mathbf{K}_{2} \mathbf{O}$ & $\mathbf{L i}_{2} \mathbf{O}$ & $\mathbf{M g O}$ \\
\hline Maximum & 13.68 & 12.00 & 5.5 & 1.20 & 16.19 & 4.00 & 7.50 & 6.02 \\
\hline Minimum & 1.68 & 4.90 & 0 & 0 & 3.80 & 0 & 0 & 0 \\
\hline & & & & & & & & \\
\hline & $\mathbf{M n O}$ & $\mathbf{N a}_{2} \mathbf{O}$ & $\mathbf{N i O}$ & $\mathbf{S i O}_{2}$ & $\mathbf{T i O}_{2}$ & $\mathbf{U}_{3} \mathbf{O}_{8}$ & $\mathbf{Z r O}_{2}$ & \multirow{2}{*}{} \\
\cline { 1 - 7 } Maximum & 3.14 & 22.74 & 3.00 & 60.00 & 5.00 & 5.53 & 6.24 & \\
\hline Minimum & 0 & 6.26 & 0 & 29.98 & 0 & 0 & 0 & \\
\hline \hline
\end{tabular}


One of the objectives of the current study is to determine whether the current spinel liquidus temperature model will remain applicable to DWPF glass when the SCIX streams are added. The addition of these streams will increase the concentrations of $\mathrm{TiO}_{2}$ and $\mathrm{ZrO}_{2}$ beyond the current model applicability ranges for these components (Table 1-1). This study will, through the generation of additional data, determine whether the current model is indeed applicable over these broader composition ranges, or whether the model coefficients (and particularly the distribution of $\mathrm{ZrO}_{2}$ among the melt precursors) need to be re-fit when the new data are considered. The types of crystallization that occur in the glasses when the SCIX streams are included will also be determined. A considerable change in the methodology used to model liquidus temperature would be necessary if the addition of the SCIX streams move the projected composition of the DWPF glass into a region where spinels are no longer the primary crystalline phase.

\subsection{Viscosity}

The viscosity of the glass, or its resistance to flow, is a critical parameter for DWPF processing. Viscosity impacts the melting rate of the feed, the rate of bubble release, the devitrification rate, convection and heat transfer, the rate of refractory corrosion and erosion, the ability to pour canisters, and the quality of the poured glass. ${ }^{15}$ To avoid detrimental impacts of the glass having a viscosity that is either too high or too low, a viscosity constraint of $20-110$ poise at the nominal melt temperature of $1150{ }^{\circ} \mathrm{C}$ was chosen for DWPF operation. ${ }^{16,17}$ The viscosity of the glass in the melter cannot be easily measured during operation, so a model that predicts the viscosity of a glass melt using its composition and melt temperature was developed for the DWPF in 1991. ${ }^{18,19}$ The model is based on glass structural considerations, and assumes that a pure $\mathrm{SiO}_{2}$ glass is fully polymerized (i.e., consists only of bridging oxygen bonds). ${ }^{15}$ Each mole of alkali oxide and each mole of $\mathrm{Fe}_{2} \mathrm{O}_{3}$ added to the glass are assumed to create two non-bridging oxygen bonds, and each mole of $\mathrm{B}_{2} \mathrm{O}_{3}$ is assumed to create one non-bridging oxygen bond. Each mole of $\mathrm{Al}_{2} \mathrm{O}_{3}$ is assumed to create two bridging oxygen bonds. These assumptions are expressed as a calculated non-bridging oxygen term, which is then related to the viscosity-temperature dependence of the Vogel-Fulcher-Tammann equation. ${ }^{15,20,21}$ The model was developed using the target compositions $^{\mathrm{a}}$ and measured viscosities of 41 glass compositions. A later study identified some bias in this first model. ${ }^{15}$ The coefficients of the model were therefore revised using the measured, rather than target compositions of 33 of the original 41 glass compositions. Eight of the original glass compositions were eliminated due to disparate compositions and inhomogeneity. ${ }^{15}$ The ranges of temperatures, viscosities, and compositions over which the DWPF viscosity model was developed are given in Table 1-3. The report noted that there was no need for $\mathrm{CaO}$ or $\mathrm{ZrO}_{2}$ terms in the model over the composition ranges used in development (Table 1-3). It was suggested that glass compositions with more than $3.5 \mathrm{wt} \% \mathrm{CaO}$ or more than $2 \mathrm{wt} \% \mathrm{ZrO}_{2}$ may necessitate the development of terms for these components, since they can significantly impact the viscosity of a glass at higher concentrations. ${ }^{21}$

\footnotetext{
${ }^{a}$ Measured composition data for these glasses were not available at the time the 1991 model was developed.
} 
Table 1-3. Temperature, Viscosity, and Composition Ranges (wt \%) for the DWPF Viscosity Model. (from Jantzen ${ }^{15}$ )

\begin{tabular}{|c|c|c|}
\hline Parameter & $\begin{array}{c}\text { Maximum } \\
\text { Value }\end{array}$ & $\begin{array}{c}\text { Minimum } \\
\text { Value }\end{array}$ \\
\hline Temperature $\left({ }^{\circ} \mathrm{C}\right)$ & 1491 & 873 \\
\hline Viscosity (poise) & 1122.02 & 10.23 \\
\hline $\mathrm{Fe}^{+2} / \Sigma \mathrm{Fe}$ & 0.47 & 0.00 \\
\hline $\mathrm{Al}_{2} \mathrm{O}_{3}$ & 13.90 & 0.00 \\
\hline $\mathrm{B}_{2} \mathrm{O}_{3}$ & 12.20 & 6.41 \\
\hline $\mathrm{BaO}$ & 0.20 & 0.00 \\
\hline $\mathrm{CaO}$ & 1.47 & 0.00 \\
\hline $\mathrm{Cr}_{2} \mathrm{O}_{3}$ & 0.09 & 0.00 \\
\hline $\mathrm{Cs}_{2} \mathrm{O}$ & 0.15 & 0.00 \\
\hline $\mathrm{CuO}$ & 0.33 & 0.00 \\
\hline $\mathrm{Cu}_{2} \mathrm{O}$ & 0.30 & 0.00 \\
\hline $\mathrm{FeO}$ & 7.14 & 0.00 \\
\hline $\mathrm{Fe}_{2} \mathrm{O}_{3}$ & 14.20 & 0.00 \\
\hline $\mathrm{K}_{2} \mathrm{O}$ & 5.73 & 0.00 \\
\hline $\mathrm{La}_{2} \mathrm{O}_{3}$ & 0.36 & 0.00 \\
\hline $\mathrm{Li}_{2} \mathrm{O}$ & 6.96 & 2.59 \\
\hline $\mathrm{MgO}$ & 2.92 & 0.49 \\
\hline $\mathrm{MnO}$ & 3.26 & 0.00 \\
\hline $\mathrm{Na}_{2} \mathrm{O}$ & 15.80 & 5.80 \\
\hline $\mathrm{NiO}$ & 2.97 & 0.00 \\
\hline $\mathrm{SiO}_{2}$ & 77.04 & 45.60 \\
\hline $\mathrm{SrO}$ & 0.07 & 0.00 \\
\hline $\mathrm{TiO}_{2}$ & 1.78 & 0.00 \\
\hline $\mathrm{ZnO}$ & 0.00 & 0.00 \\
\hline $\mathrm{ZrO}_{2}$ & 0.99 & 0.00 \\
\hline Sum of Oxides & 100.28 & 98.23 \\
\hline
\end{tabular}

The revised DWPF viscosity model was validated using data from approximately 170 glasses whose compositions fell within the ranges given in Table 1-4. In general, the model showed good applicability over this broader range of glass compositions. ${ }^{15}$ The study also evaluated data for uranium-containing glasses and determined that a uranium term is not needed in the revised DWPF viscosity model as long as $\mathrm{U}_{3} \mathrm{O}_{8}$ concentrations in glass remain $\leq 5.76 \mathrm{wt} \%{ }^{15}$ Note that glasses containing up to $3.1 \mathrm{wt} \% \mathrm{TiO}_{2}$ were included in this portion of the study, which appeared to have little or no impact on viscosity, although these data were not used for model validation (Table 1-4). ${ }^{15}$ 
Table 1-4. Temperature, Viscosity, and Composition Ranges (wt \%) for Validation of the DWPF Viscosity Model. (from Jantzen ${ }^{15}$ )

\begin{tabular}{|c|c|c|}
\hline Parameter & $\begin{array}{c}\text { Maximum } \\
\text { Value }\end{array}$ & $\begin{array}{c}\text { Minimum } \\
\text { Value }\end{array}$ \\
\hline Temperature $\left({ }^{\circ} \mathrm{C}\right)$ & 1350 & 803.35 \\
\hline Viscosity (poise) & 11,000 & 8.9 \\
\hline $\mathrm{Fe}^{+2} / \Sigma \mathrm{Fe}$ & 0.71 & 0.00 \\
\hline $\mathrm{Al}_{2} \mathrm{O}_{3}$ & 17.74 & 0.81 \\
\hline $\mathrm{B}_{2} \mathrm{O}_{3}$ & 13.25 & 4.90 \\
\hline $\mathrm{BaO}$ & 0.5 & 0.00 \\
\hline $\mathrm{CaO}$ & 3.2 & 0.02 \\
\hline $\mathrm{CdO}$ & 1.18 & 0.00 \\
\hline $\mathrm{Ce}_{2} \mathrm{O}_{3}$ & 0.67 & 0.00 \\
\hline $\mathrm{CoO}$ & 0.10 & 0.00 \\
\hline $\mathrm{Cr}_{2} \mathrm{O}_{3}$ & 0.82 & 0.00 \\
\hline $\mathrm{Cs}_{2} \mathrm{O}$ & 0.24 & 0.00 \\
\hline $\mathrm{CuO}$ & 0.23 & 0.00 \\
\hline $\mathrm{Cu}_{2} \mathrm{O}$ & 0.20 & 0.00 \\
\hline $\mathrm{FeO}$ & 8.62 & 0.00 \\
\hline $\mathrm{Fe}_{2} \mathrm{O}_{3}$ & 17.74 & 1.90 \\
\hline $\mathrm{K}_{2} \mathrm{O}$ & 3.86 & 0.00 \\
\hline $\mathrm{La}_{2} \mathrm{O}_{3}$ & 1.12 & 0.00 \\
\hline $\mathrm{Li}_{2} \mathrm{O}$ & 6.60 & 0.87 \\
\hline $\mathrm{MgO}$ & 4.80 & 0.00 \\
\hline $\mathrm{MnO}$ & 3.31 & 0.03 \\
\hline $\mathrm{MoO}_{3}$ & 0.51 & 0.00 \\
\hline $\mathrm{Na}_{2} \mathrm{O}$ & 16.8 & 6.41 \\
\hline $\mathrm{Nd}_{2} \mathrm{O}_{3}$ & 2.01 & 0.00 \\
\hline $\mathrm{NiO}$ & 3.01 & 0.00 \\
\hline $\mathrm{P}_{2} \mathrm{O}_{5}$ & 1.01 & 0.00 \\
\hline $\mathrm{PbO}$ & 0.19 & 0.00 \\
\hline $\mathrm{Pr}_{6} \mathrm{O}_{11}$ & 0.02 & 0.00 \\
\hline $\mathrm{Rh}_{2} \mathrm{O}_{3}$ & 0.02 & 0.00 \\
\hline $\mathrm{RuO}_{2}$ & 0.06 & 0.00 \\
\hline $\mathrm{SO}_{4}$ & 0.59 & 0.00 \\
\hline $\mathrm{Sb}_{2} \mathrm{O}_{3}$ & 0.01 & 0.00 \\
\hline $\mathrm{SiO}_{2}$ & 60.39 & 40.50 \\
\hline $\mathrm{Sm}_{2} \mathrm{O}_{3}$ & 0.02 & 0.00 \\
\hline $\mathrm{SrO}$ & 0.18 & 0.00 \\
\hline $\mathrm{TiO}_{2}$ & 1.43 & 0.00 \\
\hline $\mathrm{V}_{2} \mathrm{O}_{5}$ & 0.01 & 0.00 \\
\hline $\mathrm{Y}_{2} \mathrm{O}_{3}$ & 0.23 & 0.00 \\
\hline $\mathrm{ZnO}$ & 0.21 & 0.00 \\
\hline $\mathrm{ZrO}_{2}$ & 2.00 & 0.00 \\
\hline Sum of Oxides & 104.75 & 95.07 \\
\hline
\end{tabular}

One of the objectives of the current study is to determine whether the current DWPF viscosity model will remain applicable to DWPF glass when the SCIX streams are added. The addition of these streams will increase the concentrations of $\mathrm{TiO}_{2}$ and $\mathrm{ZrO}_{2}$ beyond the current model applicability ranges for these components (Table 1-3). This study will, through the generation of 
additional data, determine whether the current model is indeed applicable over these broader composition ranges, or whether the model coefficients need to be re-fit when the new data are considered. As noted during development of the current viscosity model, the higher $\mathrm{ZrO}_{2}$ concentrations expected from the SCIX streams may necessitate the incorporation of a term for $\mathrm{ZrO}_{2}$ into the non-bridging oxygen expression that is incorporated in the model.

\subsection{Chemical Durability}

DWPF uses the Thermodynamic Hydration Energy Reaction MOdel (THERMO) to predict the chemical durability of a glass from its composition and redox. ${ }^{22}$ The model was developed using data from the ASTM Product Consistency Test (PCT) Method-A. ${ }^{23}$ The durability of each Slurry Mix Evaporator (SME) batch at the DWPF must have a predicted PCT response that is at least two standard deviations more durable than the Environmental Assessment (EA) glass benchmark for repository acceptance. ${ }^{22}$

THERMO expresses the thermodynamic tendency of the components of a glass to hydrate, and each species has an individual thermodynamic hydration equation written for it. ${ }^{22}$ The hydrated species for each reaction were chosen to be consistent with those that form in neutral to basic solutions as predicted on known Eh-pH (Pourbaix) diagrams and as shown by experimental data. $^{22}$ THERMO was developed for completely amorphous glasses to avoid issues with mixed mechanism modeling, since crystalline phases can leach via different routes than the glass matrix. A free energy of hydration term, or $\Delta \mathrm{G}_{\mathrm{i}}$, has been developed for each of the components of a typical DWPF-type glass. ${ }^{\text {a }}$ The summation of these terms, weighted by their molar concentrations in a glass, gives the overall tendency of a glass to hydrate expressed as a preliminary glass dissolution estimator, $\Delta \mathrm{G}_{\mathrm{p}}{ }^{22}$

Although specific data sets were used for development and validation of THERMO, ${ }^{22,25}$ concentration ranges of the components of these glasses are not explicitly provided. In practice, the predictability of the durability of glasses for each sludge macrobatch is experimentally verified prior to vitrification at the DWPF. Therefore, it is one of the objectives of the current study to determine whether THERMO continues to satisfactorily predict the PCT response of DWPF-type glasses when the SCIX streams are included. The PCT will be performed on the glasses fabricated in the laboratory, and the measured results will be compared with the predictions from THERMO.

\section{$1.6 \underline{\mathrm{TiO}}_{2}$ Concentration Limits}

The concentration of $\mathrm{TiO}_{2}$ in DWPF glass is controlled by an individual limit in PCCS of 2.0 $\mathrm{wt} \%$. The original $\mathrm{TiO}_{2}$ concentration limit was set at $1.0 \mathrm{wt} \%$ for DWPF glass ${ }^{26}$ based on studies of the impact of $\mathrm{TiO}_{2}$ on crystallization. ${ }^{27,28}$ Work by Plodinec showed that $\mathrm{TiO}_{2}$ increased liquidus temperature, increased viscosity (and led to non-Newtonian behavior), increased crystalline content after slow cooling (which could lead to decreased chemical durability), and sharply decreased the melt rate of the DWPF-type glass compositions studied. ${ }^{27-29}$ Other studies have cautioned that the relatively high density of Ti may cause Ti-rich spinels to settle to the bottom of the melt pool. ${ }^{30}$

In 2003, a request was made to reevaluate the $\mathrm{DWPF} \mathrm{TiO}_{2}$ limit in order to allow higher $\mathrm{TiO}_{2}$ concentrations in glass. ${ }^{31}$ Data developed during an evaluation of the incorporation of CST and

\footnotetext{
${ }^{\text {a }}$ Note that a $\Delta \mathrm{G}_{\mathrm{i}}$ term for niobium has not yet been incorporated into PCCS since concentrations of $\mathrm{Nb}_{2} \mathrm{O}_{5}$ in $\mathrm{DWPF}$ glass to date have not been of significance. The addition of the SCIX streams will increase the concentration of $\mathrm{Nb}_{2} \mathrm{O}_{5}$ above this value. To address this, a recent study has provided an updated $\Delta \mathrm{G}_{\mathrm{i}}$ term for $\mathrm{Nb}_{2} \mathrm{O}_{5}$ that can be incorporated into PCCS as part of the preparations to receive the SCIX streams. ${ }^{24}$
} 
MST into 25 DWPF-type glasses served as the basis of the reevaluation. ${ }^{8,2}$ The data indicated that DWPF-type glasses with $\mathrm{TiO}_{2}$ concentrations up to $5.5 \mathrm{wt} \%$ can be produced that do not compromise durability even after slow cooling, which addressed some of the concerns in the work by Plodinec. ${ }^{29}$ The data also showed that the liquidus temperature of these glasses was below the DWPF limit. However, the review recommended that, since the model development range for the current liquidus temperature model only spans $\mathrm{TiO}_{2}$ concentrations from 0 to 1.8549 wt \% (Table 1-1), glasses with $\mathrm{TiO}_{2}$ concentrations outside this range should not be processed until additional data are used to either refine the model or extend the model's validation range. ${ }^{29}$

One of the objectives of the current study is to again revisit the $\mathrm{TiO}_{2}$ concentration limit for DWPF glass production. The concentration of $\mathrm{TiO}_{2}$ in glass will have to be increased significantly beyond $2 \mathrm{wt} \%$ in order to incorporate the SCIX streams based on recent composition projections. ${ }^{10}$ As described earlier, the impacts of increased $\mathrm{TiO}_{2}$ concentrations on the applicability of the current DWPF viscosity and liquidus temperature models will be evaluated. The impacts of $\mathrm{TiO}_{2}$ on crystallization during slow cooling will also be studied, since $\mathrm{TiO}_{2}$ is known to act as a seed for crystallization in many glass systems. ${ }^{32}$

The studies by Plodinec identified reductions in melt rate when $\mathrm{TiO}_{2}$ concentrations were increased. ${ }^{27,28}$ Lorier and Jantzen recommended that further melt rate studies be performed if $\mathrm{TiO}_{2}$ concentrations greater than $1 \mathrm{wt} \%$ are necessary in DWPF glass. ${ }^{29}$ While not within the scope of this report, melt rate impacts of these higher concentrations will be determined as part of the evaluation of potential impacts of incorporating SCIX streams in DWPF glass.

\subsection{Experimental Procedure}

\subsection{Selection of Glass Compositions}

\subsubsection{KT01- and KT02-series}

Two series of glass compositions, identified as the KT01-series and the KT02-series, were developed for the first portion of the study described in this report. Composition projections developed during the paper study evaluation of the addition of the SCIX streams were used as the basis for the two series. ${ }^{10}$ Compositions for sludge batches 8 through 17 were projected in the paper study report, both with and without the addition of material from the SWPF. ${ }^{a}$ The projections with CST going to Tank 40 were used, including the beginning, middle, and end compositions for each sludge batch, since the composition of the material in Tank 40 would change over time as CST is added and as material is transferred to the DWPF. This resulted in six cases for each of the ten sludge batches (i.e., the beginning, middle, and end of processing, both with and without the SWPF streams), giving a total of sixty sludge compositions. For the purposes of this study, each of the sludge compositions was then combined with Frit 418 at 40 wt $\%$ waste loading. ${ }^{b}$ The averages of the sixty values for each sludge and frit component (including the CST constituents) were used as a baseline glass composition. The minimum and maximum concentrations of each component were also considered. These values are given in Table 2-1.

\footnotetext{
${ }^{a}$ Note that the projected values used in this study have been revised slightly from those that appear in the paper study report, although they remain very similar.

${ }^{\mathrm{b}}$ Frit 418 is not necessarily an optimal frit for any of the projected sludge compositions, nor were attempts made to ensure that the resulting glasses were acceptable for DWPF processing based on PCCS model predictions. Frit 418 was chosen simply to provide a uniform frit composition for all of the sludge projections included in the study.
} 
Table 2-1. Projected Average, Minimum, and Maximum Concentrations (wt \%) of HLW Glass Components in Out-Year Processing at DWPF with SCIX and SWPF Streams.

\begin{tabular}{|c|c|c|c|}
\hline Oxide & Average & Minimum & Maximum \\
\hline $\mathrm{Al}_{2} \mathrm{O}_{3}$ & 5.69 & 4.19 & 8.42 \\
\hline $\mathrm{B}_{2} \mathrm{O}_{3}$ & 4.80 & 4.80 & 4.80 \\
\hline $\mathrm{BaO}$ & 0.08 & 0.06 & 0.11 \\
\hline $\mathrm{CaO}$ & 1.01 & 0.73 & 1.28 \\
\hline $\mathrm{Ce}_{2} \mathrm{O}_{3}$ & 0.21 & 0.07 & 0.39 \\
\hline $\mathrm{Cr}_{2} \mathrm{O}_{3}$ & 0.11 & 0.07 & 0.16 \\
\hline $\mathrm{CuO}$ & 0.03 & 0.02 & 0.05 \\
\hline $\mathrm{Fe}_{2} \mathrm{O}_{3}$ & 12.05 & 8.24 & 16.08 \\
\hline $\mathrm{K}_{2} \mathrm{O}$ & 0.07 & 0.05 & 0.13 \\
\hline $\mathrm{La}_{2} \mathrm{O}_{3}$ & 0.08 & 0.04 & 0.12 \\
\hline $\mathrm{Li}_{2} \mathrm{O}$ & 4.80 & 4.80 & 4.80 \\
\hline $\mathrm{MgO}$ & 0.15 & 0.10 & 0.22 \\
\hline $\mathrm{MnO}$ & 1.30 & 0.33 & 2.54 \\
\hline $\mathrm{Na}_{2} \mathrm{O}$ & 15.03 & 13.07 & 16.41 \\
\hline $\mathrm{Nb}_{2} \mathrm{O}_{5}$ & 0.43 & 0.01 & 1.67 \\
\hline $\mathrm{NiO}$ & 0.36 & 0.10 & 0.76 \\
\hline $\mathrm{PbO}$ & 0.12 & 0.05 & 0.20 \\
\hline $\mathrm{SO}_{4}{ }^{2-}$ & 0.08 & 0.01 & 0.19 \\
\hline $\mathrm{SiO}_{2}$ & 47.52 & 45.96 & 49.24 \\
\hline $\mathrm{ThO}_{2}$ & 0.23 & 0.00 & 1.15 \\
\hline $\mathrm{TiO}_{2}$ & 2.74 & 0.74 & 5.00 \\
\hline $\mathrm{U}_{3} \mathrm{O}_{8}$ & 2.53 & 0.23 & 7.32 \\
\hline $\mathrm{ZnO}$ & 0.05 & 0.02 & 0.09 \\
\hline $\mathrm{ZrO}_{2}$ & 0.52 & 0.18 & 1.40 \\
\hline
\end{tabular}

The KT01-series compositions were then derived from this baseline glass composition (the average values in Table 2-1) with the intent of investigating the impact of individual glass components on the retention of $\mathrm{TiO}_{2}$. The concentrations of $\mathrm{Al}_{2} \mathrm{O}_{3}, \mathrm{~B}_{2} \mathrm{O}_{3}, \mathrm{Fe}_{2} \mathrm{O}_{3}, \mathrm{~K}_{2} \mathrm{O}$, ${ }^{\mathrm{a}} \mathrm{Li}_{2} \mathrm{O}$, $\mathrm{Na}_{2} \mathrm{O}$, and $\mathrm{SiO}_{2}$ were adjusted from the baseline composition to determine whether these changes would affect the ability of the glass to accommodate increased $\mathrm{TiO}_{2}$ concentrations. The minimum and maximum values for these components, as shown in Table 2-1, were used for the adjustments from the baseline composition. In the case of $\mathrm{B}_{2} \mathrm{O}_{3}$ and $\mathrm{Li}_{2} \mathrm{O}$, which come only from the frit, the concentrations were adjusted to represent an increase in concentration of $50 \%$ (relative) for these components within the frit. The $\mathrm{U}_{3} \mathrm{O}_{8}$ and $\mathrm{ThO}_{2}$ components were removed so that the glasses could be handled in a non-radioactive laboratory. The concentration of $\mathrm{TiO}_{2}$ was forced to an elevated value of $8 \mathrm{wt} \%$ for each composition. The remaining components were then normalized to give a total of $100 \mathrm{wt} \%$. These steps resulted in 13 glass compositions for the KT01-series, as shown in Table 2-2.

A similar process was used to develop compositions for the KT02-series glasses. The intent was to again identify any impact of individual glass components on the retention of $\mathrm{TiO}_{2}$. The only difference from the KT01-series was the increase in $\mathrm{TiO}_{2}$ concentrations from $8 \mathrm{wt} \%$ to $12 \mathrm{wt} \%$

${ }^{a}$ The concentration of $\mathrm{K}_{2} \mathrm{O}$ was increased significantly beyond it's maximum value in the glass composition range (Table 2-1) since $\mathrm{K}_{2} \mathrm{O}$ has been reported to improve $\mathrm{TiO}_{2}$ retention in glass. ${ }^{32}$ A substitution of $\mathrm{K}_{2} \mathrm{O}$ for approximately $60 \%$ of the total $\mathrm{Na}_{2} \mathrm{O}$ and $\mathrm{Li}_{2} \mathrm{O}$ on a molar basis was used to develop the $\mathrm{K}_{2} \mathrm{O}$ concentration. It is noted that while these $\mathrm{K}_{2} \mathrm{O}$ concentrations were chosen to more clearly show any impact on $\mathrm{TiO}_{2}$ retention, they are impractically high for actual processing. 
for each of the glass compositions, with a subsequent normalization of the other components to $100 \mathrm{wt} \%$. The compositions of the KT02-series glasses are given in Table 2-3. The KT02-series glasses were not characterized as thoroughly as the KT01-series glasses since their $\mathrm{TiO}_{2}$ concentrations were quite high. As will be described below, X-ray Diffraction (XRD) was used to identify any obvious changes in the crystallization behavior of these glasses after the CCC when $\mathrm{TiO}_{2}$ concentrations were high, but chemical properties were not determined. Note that the KT01 and KT02 compositions do not necessarily pass the current PCCS constraints since they were developed for research and development efforts, rather than for specific DWPF compositions. 
Table 2-2. Target Compositions (wt \%) of the KT01-Series Glasses.

\begin{tabular}{||c|c|c|c|c|c|c|c|c||}
\hline Oxide & KT01-LA & KT01-HA & KT01-LF & KT01-HF & KT01-LN & KT01-HN & KT01-LS & KT01-HS \\
\hline $\mathrm{Al}_{2} \mathrm{O}_{3}$ & 4.19 & 8.42 & 5.78 & 5.24 & 5.65 & 5.41 & 5.57 & 5.18 \\
\hline $\mathrm{B}_{2} \mathrm{O}_{3}$ & 4.75 & 4.52 & 4.88 & 4.42 & 4.77 & 4.57 & 4.70 & 4.37 \\
\hline $\mathrm{BaO}$ & 0.08 & 0.08 & 0.08 & 0.08 & 0.08 & 0.08 & 0.08 & 0.08 \\
\hline $\mathrm{CaO}$ & 1.00 & 0.95 & 1.03 & 0.93 & 1.00 & 0.96 & 0.99 & 0.92 \\
\hline $\mathrm{Ce}_{2} \mathrm{O}_{3}$ & 0.21 & 0.20 & 0.22 & 0.19 & 0.21 & 0.20 & 0.21 & 0.19 \\
\hline $\mathrm{Cr}_{2} \mathrm{O}_{3}$ & 0.11 & 0.10 & 0.11 & 0.10 & 0.11 & 0.10 & 0.11 & 0.10 \\
\hline $\mathrm{CuO}$ & 0.03 & 0.03 & 0.03 & 0.03 & 0.03 & 0.03 & 0.03 & 0.03 \\
\hline $\mathrm{Fe}_{2} \mathrm{O}_{3}$ & 11.92 & 11.34 & 8.24 & 16.08 & 11.97 & 11.46 & 11.81 & 10.97 \\
\hline $\mathrm{K}_{2} \mathrm{O}$ & 0.07 & 0.07 & 0.08 & 0.07 & 0.07 & 0.07 & 0.07 & 0.07 \\
\hline $\mathrm{La}_{2} \mathrm{O}_{3}$ & 0.08 & 0.07 & 0.08 & 0.07 & 0.08 & 0.07 & 0.08 & 0.07 \\
\hline $\mathrm{Li}_{2} \mathrm{O}$ & 4.75 & 4.52 & 4.88 & 4.42 & 4.77 & 4.57 & 4.70 & 4.37 \\
\hline $\mathrm{MgO}$ & 0.15 & 0.14 & 0.15 & 0.14 & 0.15 & 0.15 & 0.15 & 0.14 \\
\hline $\mathrm{MnO}^{2}$ & 1.28 & 1.22 & 1.32 & 1.20 & 1.29 & 1.24 & 1.27 & 1.18 \\
\hline $\mathrm{Na}_{2} \mathrm{O}$ & 14.86 & 14.14 & 15.26 & 13.84 & 13.07 & 16.41 & 14.73 & 13.67 \\
\hline $\mathrm{Nb}_{2} \mathrm{O}_{5}$ & 0.43 & 0.41 & 0.44 & 0.40 & 0.43 & 0.41 & 0.42 & 0.39 \\
\hline $\mathrm{NiO}^{2}$ & 0.36 & 0.34 & 0.37 & 0.34 & 0.36 & 0.35 & 0.36 & 0.33 \\
\hline $\mathrm{PbO}^{2} \mathrm{O}$ & 0.12 & 0.11 & 0.12 & 0.11 & 0.12 & 0.11 & 0.11 & 0.11 \\
\hline $\mathrm{SO}_{4}{ }^{2-}$ & 0.07 & 0.07 & 0.08 & 0.07 & 0.08 & 0.07 & 0.07 & 0.07 \\
\hline $\mathrm{SiO}_{2}$ & 46.98 & 44.72 & 48.27 & 43.75 & 47.19 & 45.19 & 45.96 & 49.24 \\
\hline $\mathrm{TiO}_{2}$ & 8.00 & 8.00 & 8.00 & 8.00 & 8.00 & 8.00 & 8.00 & 8.00 \\
\hline $\mathrm{ZnO}^{2}$ & 0.05 & 0.05 & 0.05 & 0.05 & 0.05 & 0.05 & 0.05 & 0.05 \\
\hline $\mathrm{ZrO}_{2}$ & 0.52 & 0.49 & 0.53 & 0.48 & 0.52 & 0.50 & 0.51 & 0.48 \\
\hline
\end{tabular}


Table 2-2. Target Compositions (wt \%) of the KT01-Series Glasses. (continued)

\begin{tabular}{|c|c|c|c|c|c|}
\hline Oxide & " KT01-LL & "KT01-HL & "KT01-LB & "KT01-HB & " KT01-HK \\
\hline $\mathrm{Al}_{2} \mathrm{O}_{3}$ & 5.53 & 5.38 & 5.53 & 5.38 & 4.97 \\
\hline $\mathrm{B}_{2} \mathrm{O}_{3}$ & 4.67 & 4.54 & 4.80 & 7.20 & 4.20 \\
\hline $\mathrm{BaO}$ & 0.08 & 0.08 & 0.08 & 0.08 & 0.07 \\
\hline $\mathrm{CaO}$ & 0.98 & 0.96 & 0.98 & 0.96 & 0.89 \\
\hline $\mathrm{Ce}_{2} \mathrm{O}_{3}$ & 0.21 & 0.20 & 0.21 & 0.20 & 0.19 \\
\hline $\mathrm{Cr}_{2} \mathrm{O}_{3}$ & 0.11 & 0.10 & 0.11 & 0.10 & 0.10 \\
\hline $\mathrm{CuO}$ & 0.03 & 0.03 & 0.03 & 0.03 & 0.03 \\
\hline $\mathrm{Fe}_{2} \mathrm{O}_{3}$ & 11.72 & 11.39 & 11.72 & 11.39 & 10.54 \\
\hline $\mathrm{K}_{2} \mathrm{O}$ & 0.07 & 0.07 & 0.07 & 0.07 & 18.38 \\
\hline $\mathrm{La}_{2} \mathrm{O}_{3}$ & 0.08 & 0.07 & 0.08 & 0.07 & 0.07 \\
\hline $\mathrm{Li}_{2} \mathrm{O}$ & 4.80 & 7.20 & 4.67 & 4.54 & 1.28 \\
\hline $\mathrm{MgO}$ & 0.15 & 0.14 & 0.15 & 0.14 & 0.13 \\
\hline $\mathrm{MnO}$ & 1.26 & 1.23 & 1.26 & 1.23 & 1.14 \\
\hline $\mathrm{Na}_{2} \mathrm{O}$ & 14.61 & 14.20 & 14.61 & 14.20 & 7.09 \\
\hline $\mathrm{Nb}_{2} \mathrm{O}_{5}$ & 0.42 & 0.41 & 0.42 & 0.41 & 0.38 \\
\hline $\mathrm{NiO}$ & 0.35 & 0.34 & 0.35 & 0.34 & 0.32 \\
\hline $\mathrm{PbO}$ & 0.11 & 0.11 & 0.11 & 0.11 & 0.10 \\
\hline $\mathrm{SO}_{4}{ }^{2-}$ & 0.07 & 0.07 & 0.07 & 0.07 & 0.07 \\
\hline $\mathrm{SiO}_{2}$ & 46.19 & 44.92 & 46.19 & 44.92 & 41.56 \\
\hline $\mathrm{TiO}_{2}$ & 8.00 & 8.00 & 8.00 & 8.00 & 8.00 \\
\hline $\mathrm{ZnO}$ & 0.05 & 0.05 & 0.05 & 0.05 & 0.05 \\
\hline $\mathrm{ZrO}_{2}$ & 0.51 & 0.49 & 0.51 & 0.49 & 0.46 \\
\hline
\end{tabular}


Table 2-3. Target Compositions (wt \%) of the KT02-Series Glasses.

\begin{tabular}{|c|c|c|c|c|c|c|c|c||}
\hline \hline Oxide & KT02-LA & KT02-HA & KT02-LF & KT02-HF & KT02-LN & KT02-HN & KT02-LS & KT02-HS \\
\hline $\mathrm{Al}_{2} \mathrm{O}_{3}$ & 4.19 & 8.42 & 5.50 & 4.96 & 5.36 & 5.12 & 5.09 & 4.69 \\
\hline $\mathrm{B}_{2} \mathrm{O}_{3}$ & 4.53 & 4.30 & 4.64 & 4.19 & 4.53 & 4.32 & 4.30 & 3.96 \\
\hline $\mathrm{BaO}$ & 0.08 & 0.07 & 0.08 & 0.07 & 0.08 & 0.07 & 0.07 & 0.07 \\
\hline $\mathrm{CaO}$ & 0.95 & 0.91 & 0.98 & 0.88 & 0.95 & 0.91 & 0.91 & 0.83 \\
\hline $\mathrm{Ce}_{2} \mathrm{O}_{3}$ & 0.20 & 0.19 & 0.20 & 0.18 & 0.20 & 0.19 & 0.19 & 0.17 \\
\hline $\mathrm{Cr}_{2} \mathrm{O}_{3}$ & 0.10 & 0.10 & 0.11 & 0.10 & 0.10 & 0.10 & 0.10 & 0.09 \\
\hline $\mathrm{CuO}^{2}$ & 0.03 & 0.03 & 0.03 & 0.03 & 0.03 & 0.03 & 0.03 & 0.03 \\
\hline $\mathrm{Fe}_{2} \mathrm{O}_{3}$ & 11.37 & 10.80 & 8.24 & 16.08 & 11.36 & 10.86 & 10.78 & 9.94 \\
\hline $\mathrm{K}_{2} \mathrm{O}$ & 0.07 & 0.07 & 0.07 & 0.07 & 0.07 & 0.07 & 0.07 & 0.06 \\
\hline $\mathrm{La}_{2} \mathrm{O}_{3}$ & 0.07 & 0.07 & 0.08 & 0.07 & 0.07 & 0.07 & 0.07 & 0.06 \\
\hline $\mathrm{Li}_{2} \mathrm{O}$ & 4.53 & 4.30 & 4.64 & 4.19 & 4.53 & 4.32 & 4.30 & 3.96 \\
\hline $\mathrm{MgO}$ & 0.14 & 0.14 & 0.15 & 0.13 & 0.14 & 0.14 & 0.14 & 0.13 \\
\hline $\mathrm{MnO}^{\mathrm{Na} O} \mathrm{O}$ & 1.23 & 1.16 & 1.26 & 1.13 & 1.23 & 1.17 & 1.16 & 1.07 \\
\hline $\mathrm{Nb}_{2} \mathrm{O}_{5}$ & 0.41 & 0.39 & 0.42 & 0.38 & 0.41 & 0.39 & 0.39 & 0.36 \\
\hline $\mathrm{NiO}_{\mathrm{PbO}}$ & 0.34 & 0.33 & 0.35 & 0.32 & 0.34 & 0.33 & 0.33 & 0.30 \\
\hline $\mathrm{SO}_{4}$ & 0.11 & 0.10 & 0.11 & 0.10 & 0.11 & 0.11 & 0.10 & 0.10 \\
\hline $\mathrm{SiO} 2$ & 44.84 & 42.58 & 45.97 & 41.45 & 44.80 & 42.80 & 45.96 & 49.24 \\
\hline $\mathrm{TiO}_{2}$ & 12.00 & 12.00 & 12.00 & 12.00 & 12.00 & 12.00 & 12.00 & 12.00 \\
\hline $\mathrm{ZnO}$ & 0.05 & 0.05 & 0.05 & 0.05 & 0.05 & 0.05 & 0.05 & 0.04 \\
\hline $\mathrm{ZrO}_{2}$ & 0.49 & 0.47 & 0.51 & 0.46 & 0.49 & 0.47 & 0.47 & 0.43 \\
\hline
\end{tabular}


Table 2-3. Target Compositions (wt \%) of the KT02-Series Glasses. (continued)

\begin{tabular}{||c|c|c|c|c|c||}
\hline Oxide & KT02-LL & KT02-HL & KT02-LB & KT02-HB & KT02-HK \\
\hline $\mathrm{Al}_{2} \mathrm{O}_{3}$ & 5.28 & 5.12 & 5.28 & 5.12 & 4.97 \\
\hline $\mathrm{B}_{2} \mathrm{O}_{3}$ & 4.45 & 4.32 & 4.80 & 7.20 & 4.20 \\
\hline $\mathrm{BaO}$ & 0.08 & 0.07 & 0.08 & 0.07 & 0.07 \\
\hline $\mathrm{CaO}$ & 0.94 & 0.91 & 0.94 & 0.91 & 0.89 \\
\hline $\mathrm{Ce}_{2} \mathrm{O}_{3}$ & 0.20 & 0.19 & 0.20 & 0.19 & 0.19 \\
\hline $\mathrm{Cr}_{2} \mathrm{O}_{3}$ & 0.10 & 0.10 & 0.10 & 0.10 & 0.10 \\
\hline $\mathrm{CuO}$ & 0.03 & 0.03 & 0.03 & 0.03 & 0.03 \\
\hline $\mathrm{Fe}_{2} \mathrm{O}_{3}$ & 11.18 & 10.86 & 11.18 & 10.86 & 10.54 \\
\hline $\mathrm{K}_{2} \mathrm{O}$ & 0.07 & 0.07 & 0.07 & 0.07 & 18.38 \\
\hline $\mathrm{La}_{2} \mathrm{O}_{3}$ & 0.07 & 0.07 & 0.07 & 0.07 & 0.07 \\
\hline $\mathrm{Li}_{2} \mathrm{O}$ & 4.80 & 7.20 & 4.45 & 4.32 & 1.28 \\
\hline $\mathrm{MgO} \mathrm{O}$ & 0.14 & 0.14 & 0.14 & 0.14 & 0.13 \\
\hline $\mathrm{MnO}$ & 1.21 & 1.17 & 1.21 & 1.17 & 1.14 \\
\hline $\mathrm{Na}_{2} \mathrm{O}$ & 13.94 & 13.53 & 13.94 & 13.53 & 7.09 \\
\hline $\mathrm{Nb}_{2} \mathrm{O}_{5}$ & 0.40 & 0.39 & 0.40 & 0.39 & 0.38 \\
\hline $\mathrm{NiO}^{2}$ & 0.34 & 0.33 & 0.34 & 0.33 & 0.32 \\
\hline $\mathrm{PbO}_{\mathrm{SbO}}$ & 0.11 & 0.11 & 0.11 & 0.11 & 0.10 \\
\hline $\mathrm{SO}$ & 0.07 & 0.07 & 0.07 & 0.07 & 0.07 \\
\hline $\mathrm{SiO}$ & 44.07 & 42.80 & 44.07 & 42.80 & 41.56 \\
\hline $\mathrm{TiO}_{2}$ & 12.00 & 12.00 & 12.00 & 12.00 & 12.00 \\
\hline $\mathrm{ZnO}$ & 0.05 & 0.05 & 0.05 & 0.05 & 0.05 \\
\hline $\mathrm{ZrO} \mathrm{O}_{2}$ & 0.49 & 0.47 & 0.49 & 0.47 & 0.46 \\
\hline \hline
\end{tabular}




\subsubsection{KT03-series}

The KT03-series of compositions was developed to further investigate the potential impacts of the addition of the SCIX streams on glass properties. The average glass composition given in Table 2-1 was again used, with adjusted values (minimums and maximums) for the concentrations of $\mathrm{Al}_{2} \mathrm{O}_{3}, \mathrm{~B}_{2} \mathrm{O}_{3}, \mathrm{Fe}_{2} \mathrm{O}_{3}, \mathrm{~K}_{2} \mathrm{O}, \mathrm{Li}_{2} \mathrm{O}, \mathrm{Na}_{2} \mathrm{O}$, and $\mathrm{SiO}_{2}$ to identify any impacts of these individual components on the behavior of $\mathrm{TiO}_{2}$ in the glass. The $\mathrm{TiO}_{2}$ concentrations were fixed at an elevated value of $8 \mathrm{wt} \%$. The other major constituents of CST, $\mathrm{Nb}_{2} \mathrm{O}_{5}$ and $\mathrm{ZrO}_{2}$, were fixed at elevated concentrations of $3 \mathrm{wt} \%$ and $2.5 \mathrm{wt} \%$, respectively, to identify any interactive effects with $\mathrm{TiO}_{2}$. The resulting target compositions for the KT03-series glasses are given in Table 2-4. Note that the last two glasses in the series, KT03-HK and KT03-MK, include elevated concentrations of $\mathrm{K}_{2} \mathrm{O}$. The $\mathrm{K}_{2} \mathrm{O}$ concentrations in these glasses were developed using a molar substitution for $\mathrm{Li}_{2} \mathrm{O}$ and $\mathrm{Na}_{2} \mathrm{O}$ in amounts of approximately $60 \%$ and $30 \%$, respectively. The concentrations of $\mathrm{Nb}_{2} \mathrm{O}_{5}, \mathrm{TiO}_{2}$, and $\mathrm{ZrO}_{2}$ in these glasses are slightly reduced as a result of the normalization of the remaining oxides after the substitution with $\mathrm{K}_{2} \mathrm{O}$. Note that the KT03 compositions do not necessarily pass the current PCCS constraints since they were developed for research and development efforts, rather than for specific DWPF compositions.

Table 2-4. Target Compositions (wt \%) of the KT03-Series Glasses.

\begin{tabular}{|c|c|c|c|c|c|c|c||}
\hline \hline Oxide & KT03-LA & KT03-HA & KT03-LF & KT03-HF & KT03-LN & KT03-HN & KT03-LS \\
\hline $\mathrm{Al}_{2} \mathrm{O}_{3}$ & 4.19 & 8.42 & 5.46 & 4.91 & 5.32 & 5.08 & 5.01 \\
\hline $\mathrm{B}_{2} \mathrm{O}_{3}$ & 4.50 & 4.27 & 4.61 & 4.15 & 4.49 & 4.28 & 4.23 \\
\hline $\mathrm{BaO}$ & 0.08 & 0.07 & 0.08 & 0.07 & 0.08 & 0.07 & 0.07 \\
\hline $\mathrm{CaO}$ & 0.95 & 0.90 & 0.97 & 0.87 & 0.95 & 0.90 & 0.89 \\
\hline $\mathrm{Ce}_{2} \mathrm{O}_{3}$ & 0.20 & 0.19 & 0.20 & 0.18 & 0.20 & 0.19 & 0.19 \\
\hline $\mathrm{Cr}_{2} \mathrm{O}_{3}$ & 0.10 & 0.10 & 0.11 & 0.09 & 0.10 & 0.10 & 0.10 \\
\hline $\mathrm{CuO}$ & 0.03 & 0.03 & 0.03 & 0.03 & 0.03 & 0.03 & 0.03 \\
\hline $\mathrm{Fe}_{2} \mathrm{O}_{3}$ & 11.29 & 10.71 & 8.24 & 16.08 & 11.27 & 10.76 & 10.62 \\
\hline $\mathrm{K}_{2} \mathrm{O}$ & 0.07 & 0.07 & 0.07 & 0.06 & 0.07 & 0.07 & 0.07 \\
\hline $\mathrm{La}_{2} \mathrm{O}_{3}$ & 0.07 & 0.07 & 0.07 & 0.07 & 0.07 & 0.07 & 0.07 \\
\hline $\mathrm{Li}_{2} \mathrm{O}$ & 4.50 & 4.27 & 4.61 & 4.15 & 4.49 & 4.28 & 4.23 \\
\hline $\mathrm{MgO}^{\mathrm{MnO}}$ & 0.14 & 0.14 & 0.15 & 0.13 & 0.14 & 0.14 & 0.13 \\
\hline $\mathrm{Na}_{2} \mathrm{O}$ & 14.22 & 1.15 & 1.25 & 1.12 & 1.22 & 1.16 & 1.14 \\
\hline $\mathrm{Nb}_{2} \mathrm{O}_{5}$ & 3.00 & 13.35 & 14.43 & 12.98 & 13.07 & 16.41 & 13.24 \\
\hline $\mathrm{NiO}$ & 0.34 & 0.32 & 3.00 & 3.00 & 3.00 & 3.00 & 3.00 \\
\hline $\mathrm{PbO}^{2}$ & 0.11 & 0.10 & 0.11 & 0.10 & 0.11 & 0.10 & 0.10 \\
\hline $\mathrm{SO}_{4}$ & 0.07 & 0.07 & 0.07 & 0.07 & 0.07 & 0.07 & 0.07 \\
\hline $\mathrm{SiO}_{2}$ & 44.52 & 42.23 & 45.63 & 41.06 & 44.44 & 42.41 & 45.96 \\
\hline $\mathrm{TiO}_{2}$ & 8.00 & 8.00 & 8.00 & 8.00 & 8.00 & 8.00 & 8.00 \\
\hline $\mathrm{ZnO}^{\mathrm{ZrO} O}$ & 0.05 & 0.05 & 0.05 & 0.05 & 0.05 & 0.05 & 0.05 \\
\hline $\mathrm{ZrO}_{2}$ & 2.50 & 2.50 & 2.50 & 2.50 & 2.50 & 2.50 & 2.50 \\
\hline
\end{tabular}


Table 2-4. Target Compositions (wt \%) of the KT03-Series Glasses. (continued)

\begin{tabular}{||c|c|c|c|c|c|c|c|}
\hline Oxide & KT03-HS & KT03-LL & KT03-HL & KT03-LB & KT03-HB & KT03-HK & KT03-MK \\
\hline $\mathrm{Al}_{2} \mathrm{O}_{3}$ & 4.60 & 5.24 & 5.08 & 5.24 & 5.08 & 4.76 & 5.00 \\
\hline $\mathrm{B}_{2} \mathrm{O}_{3}$ & 3.89 & 4.42 & 4.29 & 4.80 & 7.20 & 4.02 & 4.22 \\
\hline $\mathrm{BaO}$ & 0.07 & 0.08 & 0.07 & 0.08 & 0.07 & 0.07 & 0.07 \\
\hline $\mathrm{CaO}$ & 0.82 & 0.93 & 0.90 & 0.93 & 0.90 & 0.85 & 0.89 \\
\hline $\mathrm{Ce}_{2} \mathrm{O}_{3}$ & 0.17 & 0.19 & 0.19 & 0.19 & 0.19 & 0.18 & 0.19 \\
\hline $\mathrm{Cr}_{2} \mathrm{O}_{3}$ & 0.09 & 0.10 & 0.10 & 0.10 & 0.10 & 0.09 & 0.10 \\
\hline $\mathrm{CuO}$ & 0.03 & 0.03 & 0.03 & 0.03 & 0.03 & 0.03 & 0.03 \\
\hline $\mathrm{Fe}_{2} \mathrm{O}_{3}$ & 9.76 & 11.09 & 10.77 & 11.09 & 10.77 & 10.08 & 10.59 \\
\hline $\mathrm{K}_{2} \mathrm{O}$ & 0.06 & 0.07 & 0.07 & 0.07 & 0.07 & 18.74 & 9.85 \\
\hline $\mathrm{La}_{2} \mathrm{O}_{3}$ & 0.06 & 0.07 & 0.07 & 0.07 & 0.07 & 0.06 & 0.07 \\
\hline $\mathrm{Li}_{2} \mathrm{O}$ & 3.89 & 4.80 & 7.20 & 4.42 & 4.29 & 1.04 & 2.65 \\
\hline $\mathrm{MgO}_{\mathrm{MnO}}$ & 0.12 & 0.14 & 0.14 & 0.14 & 0.14 & 0.13 & 0.13 \\
\hline $\mathrm{MnO}_{2} \mathrm{O}$ & 1.05 & 1.20 & 1.16 & 1.20 & 1.16 & 1.09 & 1.14 \\
\hline $\mathrm{Na}_{2} \mathrm{O}$ & 12.16 & 13.83 & 13.43 & 13.83 & 13.43 & 6.39 & 9.96 \\
\hline $\mathrm{Nb}_{2} \mathrm{O}_{5}$ & 3.00 & 3.00 & 3.00 & 3.00 & 3.00 & 2.71 & 2.85 \\
\hline $\mathrm{NiO}^{2}$ & 0.30 & 0.34 & 0.33 & 0.34 & 0.33 & 0.30 & 0.32 \\
\hline $\mathrm{PbO}^{2} \mathrm{O}$ & 0.09 & 0.11 & 0.10 & 0.11 & 0.10 & 0.10 & 0.10 \\
\hline $\mathrm{SO}_{4}$ & 0.06 & 0.07 & 0.07 & 0.07 & 0.07 & 0.06 & 0.07 \\
\hline $\mathrm{SiO}{ }_{2}$ & 49.24 & 43.74 & 42.46 & 43.74 & 42.46 & 39.75 & 41.76 \\
\hline $\mathrm{TiO}_{2}$ & 8.00 & 8.00 & 8.00 & 8.00 & 8.00 & 7.24 & 7.60 \\
\hline $\mathrm{ZnO}^{\mathrm{ZrO} O}$ & 0.04 & 0.05 & 0.05 & 0.05 & 0.05 & 0.04 & 0.05 \\
\hline \hline
\end{tabular}

\subsubsection{KT04-series}

The basis for the KT04-series compositions was changed from the average glass composition used previously (Table 2-1) to projections of individual sludge batches incorporating the SCIX streams. These projections were again very similar to those provided in the paper study report, ${ }^{10}$ with minor refinements. ${ }^{\text {a }}$ The most recent projections for sludge batches 8 through 17 were used, ${ }^{33}$ and CST additions to Tank 40 were projected at the accelerated DWPF processing rate of 75 Sludge Receipt and Adjustment Tank (SRAT) batches per year with the SWPF streams included. Note that the sludge projections did not include sulfate concentrations. The final SRAT batch composition for each sludge batch was used, since these cases represent the maximum concentrations of CST in the sludge. The resulting ten sludge composition projections are given in Table 2-5. Each projection is identified by the relevant sludge batch and SRAT batch number.

\footnotetext{
${ }^{a}$ The method used to calculate the number of SRAT batches required to process each sludge batch was modified, which resulted in minor changes to the projected compositions.
} 
Table 2-5. Projected Compositions (wt \%) of the Final SRAT Batches of Sludge Batches 8 through 17 Used to Develop the KT04-Series Glass Compositions.

\begin{tabular}{|c|c|c|c|c|c|c|c|c|c|c|}
\hline Oxide & "SB08-69 & "SB09-79 & "SB10-80 & "SB11-70 & "SB12-71 & "SB13-66 & "SB14-74 & SB15-91 & "SB16-38 & SB17-35 \\
\hline $\mathrm{Al}_{2} \mathrm{O}_{3}$ & 14.25 & 12.68 & 10.85 & 12.29 & 17.00 & 17.86 & 12.51 & 10.96 & 12.14 & 12.51 \\
\hline $\mathrm{BaO}$ & 0.17 & 0.17 & 0.17 & 0.17 & 0.17 & 0.17 & 0.17 & 0.17 & 0.17 & 0.17 \\
\hline $\mathrm{CaO}$ & 2.30 & 2.31 & 2.32 & 2.12 & 2.32 & 2.43 & 1.98 & 1.76 & 2.11 & 2.16 \\
\hline $\mathrm{Ce}_{2} \mathrm{O}_{3}$ & 0.70 & 0.70 & 0.62 & 0.53 & 0.35 & 0.27 & 0.17 & 0.17 & 0.44 & 0.54 \\
\hline $\mathrm{Cr}_{2} \mathrm{O}_{3}$ & 0.22 & 0.22 & 0.22 & 0.22 & 0.33 & 0.33 & 0.33 & 0.22 & 0.22 & 0.23 \\
\hline $\mathrm{CuO}$ & 0.09 & 0.09 & 0.09 & 0.09 & 0.09 & 0.09 & 0.09 & 0.09 & 0.09 & 0.10 \\
\hline $\mathrm{Fe}_{2} \mathrm{O}_{3}$ & 29.72 & 28.22 & 27.86 & 30.08 & 23.87 & 22.28 & 20.88 & 19.97 & 27.31 & 30.27 \\
\hline $\mathrm{K}_{2} \mathrm{O}$ & 0.09 & 0.09 & 0.09 & 0.09 & 0.18 & 0.27 & 0.18 & 0.18 & 0.18 & 0.09 \\
\hline $\mathrm{La}_{2} \mathrm{O}_{3}$ & 0.26 & 0.18 & 0.18 & 0.18 & 0.18 & 0.09 & 0.09 & 0.09 & 0.18 & 0.18 \\
\hline $\mathrm{MgO}$ & 0.37 & 0.37 & 0.37 & 0.38 & 0.38 & 0.25 & 0.25 & 0.25 & 0.25 & 0.26 \\
\hline $\mathrm{MnO}$ & 4.73 & 4.17 & 4.37 & 2.64 & 2.54 & 2.93 & 1.64 & 2.10 & 1.27 & 0.90 \\
\hline $\mathrm{Na}_{2} \mathrm{O}$ & 25.08 & 27.05 & 27.47 & 26.69 & 26.56 & 26.30 & 25.62 & 27.15 & 24.50 & 23.42 \\
\hline $\mathrm{Nb}_{2} \mathrm{O}_{5}$ & 2.54 & 2.68 & 2.66 & 2.61 & 2.67 & 2.53 & 2.66 & 2.83 & 1.88 & 1.75 \\
\hline $\mathrm{NiO}$ & 0.86 & 0.48 & 0.77 & 0.39 & 0.29 & 0.39 & 1.42 & 1.32 & 1.15 & 1.08 \\
\hline $\mathrm{PbO}$ & 0.40 & 0.32 & 0.32 & 0.24 & 0.16 & 0.16 & 0.16 & 0.16 & 0.33 & 0.33 \\
\hline $\mathrm{SiO}_{2}$ & 3.43 & 4.68 & 5.15 & 6.74 & 8.08 & 7.96 & 6.55 & 5.72 & 3.04 & 2.31 \\
\hline $\mathrm{ThO}_{2}$ & 0.43 & 1.54 & 2.14 & 0.60 & 0.00 & 0.00 & 0.00 & 0.00 & 0.00 & 0.00 \\
\hline $\mathrm{TiO}_{2}$ & 10.67 & 10.69 & 10.64 & 10.91 & 11.03 & 10.80 & 10.79 & 10.79 & 10.72 & 10.04 \\
\hline $\mathrm{U}_{3} \mathrm{O}_{8}$ & 1.41 & 0.80 & 1.16 & 0.54 & 1.25 & 2.32 & 11.97 & 13.54 & 12.13 & 11.87 \\
\hline $\mathrm{ZnO}$ & 0.00 & 0.09 & 0.09 & 0.19 & 0.09 & 0.19 & 0.19 & 0.09 & 0.09 & 0.10 \\
\hline $\mathrm{ZrO}_{2}$ & 2.27 & 2.47 & 2.46 & 2.32 & 2.46 & 2.36 & 2.35 & 2.47 & 1.78 & 1.70 \\
\hline
\end{tabular}


A single frit composition was identified that produced a PCCS Measurement Acceptability Region (MAR) acceptable glass at a target waste loading of $40 \mathrm{wt} \%$ with each of the sludge composition projections given in Table 2-5. The composition of this frit, which was labeled Frit 0607, is given in Table 2-6. The $\mathrm{U}_{3} \mathrm{O}_{8}$ and $\mathrm{ThO}_{2}$ components were removed from the sludge projections to support non-radioactive experiments. Each of the re-normalized sludge compositions was then combined with Frit 0607 at a waste loading of $40 \mathrm{wt} \%$ to give the target glass compositions for the KT04-series shown in Table 2-7.

Table 2-6. Composition of Frit 0607 (wt \%).

\begin{tabular}{|c|c|c|c|}
\hline $\mathbf{B}_{\mathbf{2}} \mathbf{O}_{3}$ & $\mathbf{L i}_{\mathbf{2}} \mathbf{O}$ & $\mathbf{N a}_{\mathbf{2}} \mathbf{O}$ & $\mathbf{S i O}_{\mathbf{2}}$ \\
\hline 10 & 6 & 5 & 79 \\
\hline
\end{tabular}


Table 2-7. Target Compositions (wt \%) for the KT04-Series Glasses.

\begin{tabular}{|c|c|c|c|c|c|c|c|c|c|c|}
\hline Oxide & " KT04-01 & " КT04-02 & " KT04-03 & " КT04-04 & " KT04-05 & " KT04-06 & " KT04-07 & " KT04-08 & " КT04-09 & " KT04-10 \\
\hline $\mathrm{Al}_{2} \mathrm{O}_{3}$ & 5.80 & 5.19 & 4.49 & 4.97 & 6.89 & 7.31 & 5.69 & 5.07 & 5.53 & 5.68 \\
\hline $\mathrm{B}_{2} \mathrm{O}_{3}$ & 6.00 & 6.00 & 6.00 & 6.00 & 6.00 & 6.00 & 6.00 & 6.00 & 6.00 & 6.00 \\
\hline $\mathrm{BaO}$ & 0.07 & 0.07 & 0.07 & 0.07 & 0.07 & 0.07 & 0.08 & 0.08 & 0.08 & 0.08 \\
\hline $\mathrm{CaO}$ & 0.94 & 0.95 & 0.96 & 0.86 & 0.94 & 1.00 & 0.90 & 0.81 & 0.96 & 0.98 \\
\hline $\mathrm{Ce}_{2} \mathrm{O}_{3}$ & 0.29 & 0.29 & 0.26 & 0.22 & 0.14 & 0.11 & 0.08 & 0.08 & 0.20 & 0.25 \\
\hline $\mathrm{Cr}_{2} \mathrm{O}_{3}$ & 0.09 & 0.09 & 0.09 & 0.09 & 0.13 & 0.14 & 0.15 & 0.10 & 0.10 & 0.10 \\
\hline $\mathrm{CuO}$ & 0.04 & 0.04 & 0.04 & 0.04 & 0.04 & 0.04 & 0.04 & 0.04 & 0.04 & 0.04 \\
\hline $\mathrm{Fe}_{2} \mathrm{O}_{3}$ & 12.11 & 11.56 & 11.53 & 12.17 & 9.67 & 9.12 & 9.49 & 9.24 & 12.43 & 13.74 \\
\hline $\mathrm{K}_{2} \mathrm{O}$ & 0.04 & 0.04 & 0.04 & 0.04 & 0.07 & 0.11 & 0.08 & 0.08 & 0.08 & 0.04 \\
\hline $\mathrm{La}_{2} \mathrm{O}_{3}$ & 0.11 & 0.07 & 0.07 & 0.07 & 0.07 & 0.04 & 0.04 & 0.04 & 0.08 & 0.08 \\
\hline $\mathrm{Li}_{2} \mathrm{O}$ & 3.60 & 3.60 & 3.60 & 3.60 & 3.60 & 3.60 & 3.60 & 3.60 & 3.60 & 3.60 \\
\hline $\mathrm{MgO}$ & 0.15 & 0.15 & 0.15 & 0.15 & 0.15 & 0.10 & 0.11 & 0.11 & 0.11 & 0.12 \\
\hline $\mathrm{MnO}$ & 1.93 & 1.71 & 1.81 & 1.07 & 1.03 & 1.20 & 0.74 & 0.97 & 0.58 & 0.41 \\
\hline $\mathrm{Na}_{2} \mathrm{O}$ & 13.22 & 14.08 & 14.36 & 13.80 & 13.76 & 13.77 & 14.64 & 15.56 & 14.15 & 13.63 \\
\hline $\mathrm{Nb}_{2} \mathrm{O}_{5}$ & 1.04 & 1.10 & 1.10 & 1.06 & 1.08 & 1.03 & 1.21 & 1.31 & 0.85 & 0.79 \\
\hline $\mathrm{NiO}$ & 0.35 & 0.20 & 0.32 & 0.16 & 0.12 & 0.16 & 0.65 & 0.61 & 0.52 & 0.49 \\
\hline $\mathrm{PbO}$ & 0.16 & 0.13 & 0.13 & 0.10 & 0.07 & 0.07 & 0.07 & 0.07 & 0.15 & 0.15 \\
\hline $\mathrm{SiO}_{2}$ & 48.80 & 49.32 & 49.53 & 50.13 & 50.67 & 50.66 & 50.38 & 50.04 & 48.79 & 48.45 \\
\hline $\mathrm{TiO}_{2}$ & 4.35 & 4.38 & 4.40 & 4.41 & 4.47 & 4.42 & 4.90 & 4.99 & 4.88 & 4.56 \\
\hline $\mathrm{ZnO}$ & 0.00 & 0.04 & 0.04 & 0.08 & 0.04 & 0.08 & 0.08 & 0.04 & 0.04 & 0.04 \\
\hline $\mathrm{ZrO}_{2}$ & 0.92 & 1.01 & 1.02 & 0.94 & 1.00 & 0.97 & 1.07 & 1.14 & 0.81 & 0.77 \\
\hline
\end{tabular}




\subsection{Glass Fabrication}

Each of the study glasses was prepared from the proper proportions of reagent-grade metal oxides, carbonates, and boric acid in $200 \mathrm{~g}$ batches. The raw materials were thoroughly mixed and placed into platinum/gold, $250 \mathrm{ml}$ crucibles. The batch was placed into a high-temperature furnace at the melt temperature of $1150{ }^{\circ} \mathrm{C}$. The crucible was removed from the furnace after an isothermal hold for 1 hour. The glass was poured onto a clean, stainless steel plate and allowed to air cool (quench). The glass pour patty was used as a sampling stock for the various property measurements described below.

Approximately $25 \mathrm{~g}$ of each glass was heat-treated to simulate cooling along the centerline of a DWPF-type canister ${ }^{34}$ to gauge the effects of thermal history on the product performance. This cooling schedule is referred to as the CCC heat treatment. Visual observations of both quenched and CCC glasses were documented. ${ }^{\mathrm{a}}$

\subsection{Compositional Analysis}

To confirm that the as-fabricated glasses met the target compositions, a representative sample from each quenched glass in the KT01, KT03, and KT04-series was submitted to the Process Science Analytical Laboratory (PSAL) for chemical analysis under the auspices of an analytical plan. ${ }^{35-37}$ The KT02-series glasses were not measured. Two dissolution techniques, sodium peroxide fusion (PF) and lithium-metaborate fusion (LM), were used to prepare the glass samples, in duplicate, for analysis. Each of the samples was analyzed, twice for each element of interest, by Inductively Coupled Plasma - Atomic Emission Spectroscopy (ICP-AES). Glass standards (Batch 1) were also intermittently measured to assess the performance of the ICP-AES instrument over the course of these analyses.

\subsection{X-Ray Diffraction Analysis}

Representative samples of each quenched and CCC glass were submitted to Analytical Development (AD) for XRD analysis. Samples were run under conditions providing a detection limit of approximately $0.5 \mathrm{vol} \%$. That is, if crystals (or unincorporated batch material) were present at $0.5 \mathrm{vol} \%$ or greater, the diffractometer would not only be capable of detecting the crystals but would also allow a qualitative determination of the type of crystal(s) present. Otherwise, a characteristically high background signal (amorphous hump) devoid of crystalline peaks indicates that the glass product is free of crystallization, suggesting either a completely amorphous product or that the degree of crystallization is below the detection limit.

\subsection{Product Consistency Test}

The PCT Method-A ${ }^{23}$ was performed in triplicate on each KT01, KT03, and KT04-series quenched and CCC glass to assess chemical durability. Also included in the experimental test matrix was the EA benchmark glass, ${ }^{38}$ the Approved Reference Material (ARM) glass, ${ }^{22}$ and blanks from the sample cleaning batch. Samples were ground, washed, and prepared according to the standard procedure. ${ }^{23}$ Fifteen milliliters of Type-I ASTM water were added to $1.5 \mathrm{~g}$ of glass in stainless steel vessels. The vessels were closed, sealed, and placed in an oven at $90 \pm 2{ }^{\circ} \mathrm{C}$ where the samples were maintained at temperature for 7 days. Once cooled, the resulting solutions were sampled (filtered and acidified), then labeled and analyzed by PSAL under the auspices of an analytical plan. ${ }^{39-41}$ Samples of a multi-element, standard solution were also included in the analytical plan as a check on the accuracy of the ICP-AES instrument used for

\footnotetext{
${ }^{a}$ See notebook SRNL-NB-2010-00027.
} 
these measurements. Normalized release rates were calculated based on the target and measured compositions using the average of the common logarithms of the leachate concentrations.

\section{$2.6 \underline{\text { Viscosity }}$}

The viscosity of select glasses was measured following Procedure A of the ASTM C 965 standard. ${ }^{42}$ Harrop and Orton high temperature rotating spindle viscometers were used with platinum crucibles and spindles. The viscometers were specially designed to operate with small quantities of glass to support measurements of radioactive glasses when necessary. ${ }^{43,44}$ A well characterized standard glass was used to determine the appropriate spindle constants. ${ }^{44,45}$ Measurements were taken over a range of temperatures from 1050 to $1250{ }^{\circ} \mathrm{C}$ in $50{ }^{\circ} \mathrm{C}$ intervals. Measurements at $1150^{\circ} \mathrm{C}$ were taken at three different times during the procedure to provide an opportunity to identify the effects of any crystallization or volatilization that may have occurred during the test. The data were fit to a Fulcher equation ${ }^{20,21}$ to provide a measured viscosity value at the nominal DWPF melt temperature of $1150^{\circ} \mathrm{C}$.

\subsection{Liquidus Temperature}

The liquidus temperatures $\left(\mathrm{T}_{\mathrm{L}}\right)$ of select study glasses were determined using the isothermal heat treatment method. ${ }^{46}$ The temperature profile of the furnace was carefully determined and periodically verified. ${ }^{47}$ All thermocouples and temperature measurement devices were calibrated and periodically verified by the SRNL Measurement and Test Equipment (M\&TE) program. A standard glass composition was incorporated into the test glass matrix as a method of verifying the measured data. ${ }^{\mathrm{a}}$ Polished samples of each quenched glass were observed via optical microscopy prior to $T_{L}$ measurement to determine whether any preexisting crystals were present. Quenched glasses that were found to contain crystals were excluded from testing. The study glasses were ground, washed, dried, and heat treated in platinum boats with tight fitting lids following the procedure. ${ }^{46}$ The glasses were air quenched after being removed from the furnace, then sectioned and polished for microscopy. ${ }^{48}$ Any bulk crystallization that occurred during the isothermal heat treatments was identified by optical microscopy. The procedure was repeated over various temperatures to determine the $T_{L}$ to within a narrow range of tolerance.

Liquidus temperatures were determined for the KT01-series glasses. Liquidus temperatures for the KT02-series glasses were not measured due to their unrealistically high $\mathrm{TiO}_{2}$ concentrations. In the interest of reducing the time necessary for the measurements, the $\mathrm{T}_{\mathrm{L}}$ for the KT03 and KT04-series glasses were estimated. The isothermal heat treatment method described above was continued, although a smaller number of heat treatments were performed in order to estimate the $\mathrm{T}_{\mathrm{L}}$ for each glass over a broader range of temperature while supporting comparisons with the model predicted $\mathrm{T}_{\mathrm{L}}$ values. Liquidus temperature estimates for the KT03 and KT04-series glasses are not yet complete, and the results will be reported in a separate technical report.

\subsection{Results and Discussion}

\subsection{Homogeneity}

The homogeneity of each glass sample was assessed via XRD and visual observations. The results will be discussed below for each series of glasses. The potential impacts of any crystallization that was identified will be discussed during the later description of the measured properties of the glasses (i.e., viscosity, liquidus temperature, etc.).

\footnotetext{
a The glass standard is identified as 'Unknown Glass A' from the Pacific Northwest National Laboratory liquidus temperature round robin study.
} 


\subsubsection{KT01-Series}

Visual observations of the quenched versions of the KT01-series glasses identified no visible crystallization. $^{\text {a }}$ All of the quenched glasses were found to be amorphous by XRD. Visual observations of the CCC versions of the KT01-series glasses identified minor surface crystallization, indicated by a dull or hazy appearance. Crystallization within the bulk of the glass was only observed for compositions KT01-HL and KT01-HF. XRD results were in agreement with the visual observations, with crystallization being identified in compositions KT01-HL and KT01-HF. Composition KT01-HL contained magnetite $\left(\mathrm{Fe}^{2+} \mathrm{Fe}_{2}{ }^{2+} \mathrm{O}_{8}\right)$ and lithium silicate $\left(\mathrm{Li}_{2} \mathrm{SiO}_{3}\right)$, likely due to the elevated concentration of $\mathrm{Li}_{2} \mathrm{O}$ in this glass (see Table 2-2). Composition KT01-HF contained magnetite, likely because of the elevated concentration of $\mathrm{Fe}_{2} \mathrm{O}_{3}$ in this glass.

\subsubsection{KT02-Series}

Visual observations of the quenched versions of the KT02-series glasses identified some minor surface crystallization on some of the glasses, visible as a small number of light brown streaks. All of the quenched glasses were amorphous by XRD, indicating that the volume fraction of the observed surface crystallization was very small. Visual observations of the CCC versions of the KT02-series identified varying degrees of surface crystallization on all of the glasses. Most of the glasses were also crystallized in the bulk, with the exception of compositions KT02-LA, KT02-LF, KT02-HN, and KT02-HK. The XRD results for the CCC glasses were in agreement with these observations: crystalline phases were identified in all of the glasses except for compositions KT02-LA, KT02-LF, KT02-HN, and KT02-HK.

The KT02 compositions were selected to explore the impact of varying the concentrations of the major components of the glass on the retention of $\mathrm{TiO}_{2}$ (see Section 2.1.1). The XRD results for the KT02 glasses are summarized in Table 3-1, which offers some insight into the impact of compositional changes on the propensity for titanium to crystallize out of the glass. As mentioned earlier, all of the quenched glasses were XRD amorphous. Titanium containing crystalline phases formed in all of the compositions except for the low $\mathrm{Al}_{2} \mathrm{O}_{3}$ concentration glass, the low $\mathrm{Fe}_{2} \mathrm{O}_{3}$ concentration glass, the high $\mathrm{Na}_{2} \mathrm{O}$ concentration glass, and the high $\mathrm{K}_{2} \mathrm{O}$ concentration glass. ${ }^{\mathrm{b}}$ In general, the impacts of these components will need additional investigation before drawing further conclusions (i.e., a larger number of compositions should be fabricated and characterized since the effects of an individual component are likely to be strongly influenced by overall composition).

\footnotetext{
${ }^{\text {a }}$ See laboratory notebook SRNL-NB-2010-00027 for a complete record of the visual observations and XRD data.

${ }^{b}$ The CCC version of glass KT02-LS contained an unidentified phase that may or may not contain $\mathrm{TiO}_{2}$. Further characterization of this glass is in progress to identify this phase.
} 
Table 3-1. Summary of XRD Results for the KT02-Series Glasses.

\begin{tabular}{|c|c|c|c|}
\hline Glass ID & $\begin{array}{c}\text { Composition } \\
\text { Note }\end{array}$ & $\begin{array}{c}\text { Heat } \\
\text { Treatment }\end{array}$ & XRD Results \\
\hline \multirow{2}{*}{ KT02-HA } & \multirow{2}{*}{ High $\mathrm{Al}_{2} \mathrm{O}_{3}$} & quenched & amorphous \\
\hline & & $\mathrm{CCC}$ & $\mathrm{LiFeTiO}_{4}$, unidentified phase \\
\hline \multirow{2}{*}{ KT02-LA } & \multirow{2}{*}{ Low $\mathrm{Al}_{2} \mathrm{O}_{3}$} & quenched & amorphous \\
\hline & & $\mathrm{CCC}$ & amorphous \\
\hline \multirow{2}{*}{ KT02-HB } & \multirow{2}{*}{ High $\mathrm{B}_{2} \mathrm{O}_{3}$} & quenched & amorphous \\
\hline & & $\mathrm{CCC}$ & $\mathrm{LiFeTiO}_{4}$, unidentified phase \\
\hline \multirow{2}{*}{ KT02-LB } & \multirow{2}{*}{ Low $\mathrm{B}_{2} \mathrm{O}_{3}$} & quenched & amorphous \\
\hline & & $\mathrm{CCC}$ & $\mathrm{LiFeTiO}_{4}$, unidentified phase \\
\hline \multirow{2}{*}{ KT02-HF } & \multirow{2}{*}{ High $\mathrm{Fe}_{2} \mathrm{O}_{3}$} & quenched & amorphous \\
\hline & & $\mathrm{CCC}$ & $\mathrm{LiFeTiO}_{4}, \mathrm{Fe}_{9} \mathrm{TiO}_{15}$ \\
\hline \multirow{2}{*}{ KT02-LF } & \multirow{2}{*}{ Low $\mathrm{Fe}_{2} \mathrm{O}_{3}$} & quenched & amorphous \\
\hline & & $\mathrm{CCC}$ & amorphous \\
\hline \multirow{2}{*}{ KT02-HL } & \multirow{2}{*}{ High $\mathrm{Li}_{2} \mathrm{O}$} & quenched & amorphous \\
\hline & & $\mathrm{CCC}$ & $\mathrm{LiFeTiO}_{4}, \mathrm{Li}_{2} \mathrm{SiO}_{3}$ \\
\hline \multirow{2}{*}{ KT02-LL } & \multirow{2}{*}{ Low $\mathrm{Li}_{2} \mathrm{O}$} & quenched & amorphous \\
\hline & & $\mathrm{CCC}$ & $\mathrm{LiFeTiO}_{4}$, unidentified phase \\
\hline \multirow{2}{*}{ KT02-HN } & \multirow{2}{*}{ High $\mathrm{Na}_{2} \mathrm{O}$} & quenched & amorphous \\
\hline & & $\mathrm{CCC}$ & amorphous \\
\hline \multirow{2}{*}{ KT02-LN } & \multirow{2}{*}{ Low $\mathrm{Na}_{2} \mathrm{O}$} & quenched & amorphous \\
\hline & & $\mathrm{CCC}$ & $\mathrm{LiFeTiO}_{4}$, unidentified phase \\
\hline \multirow{2}{*}{ KT02-HS } & \multirow{2}{*}{ High $\mathrm{SiO}_{2}$} & quenched & amorphous \\
\hline & & $\mathrm{CCC}$ & Rutile $\left(\mathrm{TiO}_{2}\right)$, Hematite $\left(\mathrm{Fe}_{2} \mathrm{O}_{3}\right)$ \\
\hline \multirow{2}{*}{ KT02-LS } & \multirow{2}{*}{ Low $\mathrm{SiO}_{2}$} & quenched & amorphous \\
\hline & & $\mathrm{CCC}$ & unidentified phase \\
\hline \multirow{2}{*}{ KT02-HK } & \multirow{2}{*}{ High $\mathrm{K}_{2} \mathrm{O}$} & quenched & amorphous \\
\hline & & $\mathrm{CCC}$ & amorphous \\
\hline
\end{tabular}

\subsubsection{KT03-Series}

Visual observations of the quenched versions of the KT03-series glasses identified no visible crystallization, and the XRD results indicated that all of the quenched glasses were amorphous. Visual observations of the CCC versions of the glasses indicated surface crystallization on all of the compositions. The XRD results for the CCC glasses showed that four of the compositions, KT03-LN, KT03-LB, KT03-LL, and KT03-HF, contained magnetite and trevorite $\left(\mathrm{NiFe}_{2} \mathrm{O}_{4}\right)$. Two of the glasses were highly devitrified: KT03-HL contained trevorite $\left(\mathrm{NiFe}_{2} \mathrm{O}_{4}\right)$ and KT03-HA contained magnetite with another unidentified phase.

\subsubsection{KT04-Series}

Visual observations of the quenched versions of the KT04-series glasses identified no visible crystallization, and the XRD results indicated that all of the quenched glasses were amorphous. Visual observations of the $\mathrm{CCC}$ versions of the glasses indicated a very minor amount of surface crystallization on some of the compositions. All of the CCC glasses were amorphous by XRD.

\subsection{Chemical Composition}

In this section, the measured versus targeted compositions of the study glasses are presented and compared. Measurements for samples of the Batch 1 standard glass that were included in the 
analytical plans along with the study glasses are also discussed. The elemental concentrations were converted to oxide concentrations by multiplying the values for each element by the gravimetric factor for the corresponding oxide. During this process, an elemental concentration that was determined to be below the detection limit of the analytical procedures used was reduced to half of that detection limit as the oxide concentration was determined. In the sections that follow, the analytical sequences of the measurements are explored, the measurements of the standard are investigated, the measurements for each glass are reviewed, the average chemical compositions for each glass are determined, and comparisons are made between the measurements and the targeted compositions of the glasses.

\subsubsection{KT01-Series}

Table A-1 and Table A-2 in Appendix A provide the elemental concentration measurements from the KT01 glasses that were prepared using LM, and Table A-3 in Appendix A provides the measurements from the samples of these glasses prepared using PF. Measurements for samples of the standard Batch 1 glass that were included in the analytical plan along with the study glasses are also provided in these tables. Exhibit A-1 in Appendix A provides plots in analytical sequence of the sample measurements generated by PSAL for each oxide over both preparation methods. The plots are in analytical sequence with different symbols and colors being used to represent each of the study glasses and the standard glass. In general, there does not appear to be any gross patterns or trends due to the analytical sequence. However, there is an obvious issue with the measured $\mathrm{TiO}_{2}$ concentration for the samples from glass KT01-HA. It appears that $\mathrm{TiO}_{2}$ was omitted from the batching process for this glass. A second version of this glass was batched and identified as KT01-HA2. The corrected glass was included in the analytical work with the KT03-series, as described in the analytical plans for that series. ${ }^{36,40}$ The original KT01-HA glass was discarded for all measurements. Further opportunity for a review of the measurements for each glass is provided in the discussions that follow.

Exhibit A-2 in Appendix A provides plots of the oxide concentration measurements by Glass ID (including the Batch 1 standard) by analytical solution or Lab ID for both preparation methods for the KT01-series. The different symbols and colors being used to represent the glasses are discernable in this exhibit. These plots show the individual measurements across the duplicates of each preparation method and the two ICP-AES calibrations for each glass for each oxide. The results are grouped by analytical block and arranged by targeted concentration to facilitate the interpretation of the measurements. A review of the plots presented in these exhibits reveals the repeatability of the four individual values for each oxide for each glass. There appears to be good repeatability of these measurements for each of the oxides for each of the glasses, with the exception of KT01-HA. The lack of $\mathrm{TiO}_{2}$ in this glass pushes the measured concentrations of most of the other oxides above their target values. Again, this glass was discarded and replaced with KT01-HA2, which will be discussed as part of the KT03-series data. The data suggest no other significant issues in the batching of the KT01 glasses or in the analytical process used to provide representative measurements of their compositions.

Exhibit A-3 in Appendix A provides statistical analyses of the results for the Batch 1 standard that was included with the KT01 glasses by analytical block/sub-block for each oxide of interest over both preparation methods. The results include analysis of variance (ANOVA) investigations looking for statistically significant differences among the means of these groups for each of the oxides. The reference values for the oxide concentrations of the standard are given in the header for each set of measurements in the exhibit. The results from the statistical tests for the Batch 1 standard included with the KT01 glasses may be summarized as follows: $\mathrm{Al}_{2} \mathrm{O}_{3}, \mathrm{BaO}, \mathrm{CaO}$, $\mathrm{Cr}_{2} \mathrm{O}_{3}, \mathrm{Fe}_{2} \mathrm{O}_{3}, \mathrm{~K}_{2} \mathrm{O}, \mathrm{Li}_{2} \mathrm{O}, \mathrm{MgO}, \mathrm{MnO}, \mathrm{NiO}, \mathrm{SiO}_{2}$, and $\mathrm{TiO}_{2}$ have measurements that indicate an 
ICP-AES calibration effect on the block averages at the 5\% significance level. While statistically significant, the practical impact of these calibration effects is minimal.

All of the measurements for each oxide for each KT01 glass (i.e., all of the measurements in Appendix A Table A-1, Table A-2, and Table A-3) were averaged to determine a representative chemical composition for each glass. A sum of oxides was also computed for each glass based upon the measured values. Exhibit A-4 in Appendix A provides plots showing results for each glass for each oxide to help highlight the comparisons among the measured and targeted values. Some observations from the plots of Exhibit A-4 are offered: The $\mathrm{K}_{2} \mathrm{O}$ concentration for glass KT01-HK is slightly below the target. The concentrations of $\mathrm{BaO}, \mathrm{Ce}_{2} \mathrm{O}_{3}, \mathrm{La}_{2} \mathrm{O}_{3}, \mathrm{Na}_{2} \mathrm{O}, \mathrm{NiO}$, $\mathrm{PbO}, \mathrm{SiO}_{2}$, and $\mathrm{ZrO}_{2}$ were slightly low for most of the $\mathrm{KT} 01$ glasses, as was the sum of oxides. The concentration of $\mathrm{Nb}_{2} \mathrm{O}_{5}$ was slightly high for all of the KT01 glasses. The $\mathrm{SO}_{4}$ measurements were hindered by the detection limit of the instrument. The composition of glass KT01-HA is erroneous due to the lack of $\mathrm{TiO}_{2}$, as discussed earlier. In general, there appear to have been only minor difficulties in meeting the targeted concentrations for the KT01 glasses.

Table A-4 in Appendix A provides a summary of the average measured compositions as well as the targeted compositions and the associated differences and relative differences. Note that the targeted sum of oxides for the Batch 1 standard does not sum to $100 \%$ due to an incomplete coverage of the oxides in this glass. All of the sums of oxides for the KT01 glasses fall within the PCCS acceptable interval of 95 to $105 \mathrm{wt} \%$. Entries in Table A-4 show the relative differences between the measured values and the targeted values. These differences are shaded when they are greater than or equal to 5\%. Overall, these comparisons between the measured and targeted compositions again suggest only minor difficulties in hitting the targeted compositions for the KT01 glasses.

\subsubsection{KT03-Series}

Table B-1 and Table B-2 in Appendix B provide the elemental concentration measurements from the KT03 glasses that were prepared using LM, and Table B-3 in Appendix B provides the measurements from the samples of these glasses prepared using PF. Measurements for samples of the standard Batch 1 glass that were included in the analytical plan along with the study glasses are also provided in these tables. Exhibit B-1 in Appendix B provides plots in analytical sequence of the sample measurements generated by PSAL for each oxide over both preparation methods. The plots are in analytical sequence with different symbols and colors being used to represent each of the study glasses and the standard glass. In general, there does not appear to be any gross patterns or trends due to the analytical sequence. Further opportunity for a review of the measurements for each glass is provided in the discussions that follow.

Exhibit B-2 in Appendix B provides plots of the oxide concentration measurements by Glass ID (including the Batch 1 standard) by analytical solution or Lab ID for both preparation methods for the KT03-series. The different symbols and colors being used to represent the glasses are discernable in this exhibit. These plots show the individual measurements across the duplicates of each preparation method and the two ICP-AES calibrations for each glass for each oxide. The results are grouped by analytical block and arranged by targeted concentration to facilitate the interpretation of the measurements. A review of the plots presented in these exhibits reveals the repeatability of the four individual values for each oxide for each glass. There appears to be good repeatability of these measurements for each of the oxides for each of the glasses. Some minor issues are apparent, including a high concentration of $\mathrm{BaO}$ in glass KT03-LA. There is a minor amount of scatter in the $\mathrm{Cr}_{2} \mathrm{O}_{3}$ values, with a possible preparation issue for glass KT03-HK. There is a possible calibration issue with the $\mathrm{Na}_{2} \mathrm{O}$ measurements for glass KT03-LN. Concentrations of $\mathrm{Nb}_{2} \mathrm{O}_{5}$ are slightly low for several of the glasses. There are possible 
preparation issues for the $\mathrm{NiO}$ measurements for glasses KT03-HA and KT03-HN. The $\mathrm{ZrO}_{2}$ concentrations are slightly low for most of the glasses. Despite these minor issues, the data suggest no significant problems in the batching of the KT03 glasses or in the analytical process used to provide representative measurements of their compositions. Note that there were no issues with the corrected version of the KT01 series glass KT01-HA2. This version of the glass will therefore be used in support of further characterization of that composition.

Exhibit B-3 in Appendix B provides statistical analyses of the results for the Batch 1 standard that was included with the KT03 glasses by analytical block/sub-block for each oxide of interest over both preparation methods. The results include ANOVA investigations looking for statistically significant differences among the means of these groups for each of the oxides. The reference values for the oxide concentrations of the standard are given in the header for each set of measurements in the exhibit. The results from the statistical tests for the Batch 1 standard included with the KT03 glasses may be summarized as follows: $\mathrm{Al}_{2} \mathrm{O}_{3}, \mathrm{BaO}, \mathrm{CaO}, \mathrm{Ce}_{2} \mathrm{O}_{3}, \mathrm{Cr}_{2} \mathrm{O}_{3}$, $\mathrm{Li}_{2} \mathrm{O}, \mathrm{MgO}$, and $\mathrm{NiO}$ have measurements that indicate an ICP-AES calibration effect on the block averages at the $5 \%$ significance level. While statistically significant, the practical impact of these calibration effects is minimal.

All of the measurements for each oxide for each KT03 glass (i.e., all of the measurements in Appendix B, Table B-1, Table B-2, and Table B-3) were averaged to determine a representative chemical composition for each glass. A sum of oxides was also computed for each glass based upon the measured values. Exhibit B-4 in Appendix B provides plots showing results for each glass for each oxide to help highlight the comparisons among the measured and targeted values. Some observations from the plots of Exhibit B-4 are offered: The $\mathrm{Al}_{2} \mathrm{O}_{3}$ concentration is slightly high for glass KT03-HF. The $\mathrm{BaO}$ concentration is high for glass KT03-LA. The CaO concentration is slightly high for most of the glasses. There is minor scatter in the $\mathrm{Cr}_{2} \mathrm{O}_{3}, \mathrm{NiO}$, and $\mathrm{SiO}_{2}$ values. The $\mathrm{Fe}_{2} \mathrm{O}_{3}, \mathrm{MnO}, \mathrm{Nb}_{2} \mathrm{O}_{5}, \mathrm{TiO}_{2}$, and $\mathrm{ZrO}_{2}$ concentrations are slightly low for some of the glasses. The $\mathrm{K}_{2} \mathrm{O}$ concentration is slightly low for glass $\mathrm{KT} 03-\mathrm{HK}$. The $\mathrm{MgO}$ concentration is slightly low for glass KT03-MK and the Batch 1 standard. The $\mathrm{SO}_{4}$ measurements were hindered by the detection limit of the instrument. In general, there appear to have been only minor difficulties in meeting the targeted concentrations for the KT03 glasses and glass KT01-HA2.

Table B-4 in Appendix B provides a summary of the average measured compositions as well as the targeted compositions and the associated differences and relative differences. Note that the targeted sum of oxides for the Batch 1 standard does not sum to $100 \%$ due to an incomplete coverage of the oxides in this glass. All of the sums of oxides for the KT03 glasses fall within the PCCS acceptable interval of 95 to $105 \mathrm{wt} \%$. Entries in Table B-4 show the relative differences between the measured values and the targeted values. These differences are shaded when they are greater than or equal to 5\%. Overall, these comparisons between the measured and targeted compositions again suggest only minor difficulties in hitting the targeted compositions for the KT03 glasses and glass KT01-HA2.

\subsubsection{KT04-Series}

Table C-1 and Table C-2 in Appendix C provide the elemental concentration measurements from the KT04 glasses that were prepared using LM, and Table C-3 in Appendix C provides the measurements from the samples of these glasses prepared using PF. Measurements for samples of the standard Batch 1 glass that were included in the analytical plan along with the study glasses are also provided in these tables. Exhibit $\mathrm{C}-1$ in Appendix $\mathrm{C}$ provides plots in analytical sequence of the sample measurements generated by PSAL for each oxide over both preparation methods. The plots are in analytical sequence with different symbols and colors being used to 
represent each of the study glasses and the standard glass. In general, there does not appear to be any gross patterns or trends due to the analytical sequence. Further opportunity for a review of the measurements for each glass is provided in the discussions that follow.

Exhibit C-2 in Appendix C provides plots of the oxide concentration measurements by Glass ID (including the Batch 1 standard) by analytical solution or Lab ID for both preparation methods for the KT04-series. The different symbols and colors being used to represent the glasses are discernable in this exhibit. These plots show the individual measurements across the duplicates of each preparation method and the two ICP-AES calibrations for each glass for each oxide. The results are grouped by analytical block and arranged by targeted concentration to facilitate the interpretation of the measurements. A review of the plots presented in these exhibits reveals the repeatability of the four individual values for each oxide for each glass. There appears to be good repeatability of these measurements for each of the oxides for each of the glasses. Some minor issues are apparent, including minor calibration differences for $\mathrm{B}_{2} \mathrm{O}_{3}, \mathrm{Li}_{2} \mathrm{O}, \mathrm{Na}_{2} \mathrm{O}$, and $\mathrm{SiO}_{2}$ for some of the glasses. Despite these minor issues, the data suggest no significant problems in the batching of the KT04 glasses or in the analytical process used to provide representative measurements of their compositions.

Exhibit C-3 in Appendix C provides statistical analyses of the results for the Batch 1 standard that was included with the KT04 glasses by analytical block/sub-block for each oxide of interest over both preparation methods. The results include ANOVA investigations looking for statistically significant differences among the means of these groups for each of the oxides. The reference values for the oxide concentrations of the standard are given in the header for each set of measurements in the exhibit. The results from the statistical tests for the Batch 1 standard included with the KT04 glasses may be summarized as follows: $\mathrm{BaO}, \mathrm{Cr}_{2} \mathrm{O}_{3}, \mathrm{~K}_{2} \mathrm{O}, \mathrm{Li}_{2} \mathrm{O}$, and $\mathrm{ZrO}_{2}$ have measurements that indicate an ICP-AES calibration effect on the block averages at the $5 \%$ significance level. While statistically significant, the practical impact of these calibration effects is minimal.

All of the measurements for each oxide for each KT04 glass (i.e., all of the measurements in Appendix C Table C-1, Table C-2, and Table C-3) were averaged to determine a representative chemical composition for each glass. A sum of oxides was also computed for each glass based upon the measured values. Exhibit C-4 in Appendix C provides plots showing results for each glass for each oxide to help highlight the comparisons among the measured and targeted values. Some observations from the plots of Exhibit C-4 are offered: The $\mathrm{Al}_{2} \mathrm{O}_{3}$ concentrations are slightly above target. The $\mathrm{B}_{2} \mathrm{O}_{3}, \mathrm{Fe}_{2} \mathrm{O}_{3}, \mathrm{SiO}_{2}$, and $\mathrm{ZrO}_{2}$ concentrations are slightly low for all of the glasses. The $\mathrm{CaO}, \mathrm{Li}_{2} \mathrm{O}$, and $\mathrm{Na}_{2} \mathrm{O}$ concentrations are slightly high for some of the glasses. There is minor scatter in the $\mathrm{Cr}_{2} \mathrm{O}_{3}$ values. The concentrations of $\mathrm{Nb}_{2} \mathrm{O}_{5}, \mathrm{NiO}$, and $\mathrm{PbO}$ are slightly low for some of the glasses. In general, there appear to have been only minor difficulties in meeting the targeted concentrations for the KT04 glasses.

Table C-4 in Appendix C provides a summary of the average measured compositions as well as the targeted compositions and the associated differences and relative differences. Note that the targeted sum of oxides for the Batch 1 standard does not sum to $100 \%$ due to an incomplete coverage of the oxides in this glass. All of the sums of oxides for the KT04 glasses fall within the PCCS acceptable interval of 95 to $105 \mathrm{wt} \%$. Entries in Table C-4 show the relative differences between the measured values and the targeted values. These differences are shaded when they are greater than or equal to 5\%. Overall, these comparisons between the measured and targeted compositions again suggest only minor difficulties in hitting the targeted compositions for the KT04 glasses. 


\subsection{Durability}

The measurements generated by the PCTs are presented and reviewed in the following sections. For each series of glasses below, the analytical sequence of the measurements is explored, the measurements of the standards are investigated and used to assess the overall accuracy of the ICP-AES measurement process, the measurements for each glass are reviewed, plots are provided that explore the effects of heat treatment on the PCTs for these glasses, the PCTs are normalized using the compositions (targeted and measured) discussed in Section 3.2, and the normalized PCTs are compared to durability predictions for these compositions generated from the current DWPF models. ${ }^{3}$

One of the quality control checkpoints for the PCT procedure is solution mass loss over the course of the seven day test. None of the vessels in the KT01, KT03, or KT04 PCTs had any issues with mass loss. The ratio of leachant volume to the mass of ground glass was also confirmed to be correct for each vessel. All of the measurements of the ARM glass fell within the control ranges. ${ }^{22}$

\subsubsection{KT01-Series}

Table D-1 in Appendix D provides the elemental leachate concentration measurements determined by the PSAL for the solution samples generated by the PCTs for the KT01 glasses. The values were adjusted for the dilution factors: the values for the study glasses, the blanks, and the ARM glass in Table D-1 were multiplied by 1.6667 to determine the values in parts per million and the values for EA were multiplied by 16.6667. Table D-1 also provides the resulting ppm measurements.

Exhibit D-1 in Appendix D provides plots of the leachate (ppm) concentrations in analytical sequence as generated by the PSAL for all of the data from the KT01 PCTs. Different colors and symbols are used for each of the study glasses and standards. No issues are seen in these plots.

Exhibit D-2 in Appendix D provides analyses of the PSAL measurements of the samples of the multi-element standard solution by analytical set and ICP-AES calibration block for the KT01series. An ANOVA investigating for statistically significant differences among the block averages for these samples for each element of interest is included in the exhibit. A statistically significant (at a 5\% level) difference among the averages of these measurements was indicated for $\mathrm{Li}$ and $\mathrm{Si}$. However, no attempt was made to bias correct for these effects since averaging the measured concentrations for each set of triplicates in the PCT helps to minimize the impact of any potential ICP-AES bias effects.

Exhibit D-3 in Appendix D provides plots of the leachate concentrations for each type of submitted sample: the study glasses by heat treatment and the standards (EA, ARM, the multielement solution standard, and blanks). The common logarithm plots allow for the assessment of the repeatability of the measurements, which suggest only minor scatter in the triplicate values for some analytes for some of the glasses.

The PCT leachate concentrations were normalized using the target and measured cation compositions of the glasses to obtain $\mathrm{g} / \mathrm{L}$ leachate concentrations following the procedure. ${ }^{23}$ Exhibit D-4 in Appendix D provides scatter plots for these results and offers an opportunity to investigate the consistency in the leaching across the elements for the KT01 glasses. All combinations of the normalizations of the PCTs (i.e., those generated using the targeted and measured compositional views) and both heat treatments are represented in the series of scatter plots. Consistency in the leaching across the elements is typically demonstrated by a high degree 
of linear correlation among the values for pairs of these elements. The smallest correlation in this plot is that for $\mathrm{Na}$ and $\mathrm{Si}$ with a value of $\sim 82 \%$, indicating relatively linear correlations for all of the element pairs.

Table D-2 in Appendix D summarizes the normalized PCTs for the KT01 glasses. The PCTs are listed by heat treatment and compositional view for each glass. The KT01 glasses all had NL [B] values that were well below the $16.695 \mathrm{~g} / \mathrm{L}$ value of the benchmark EA glass. The highest $\mathrm{NL}$ [B] value was for glass $\mathrm{KT01}-\mathrm{HL}$, the high $\mathrm{Li}_{2} \mathrm{O}$ composition, with values of $1.2 \mathrm{~g} / \mathrm{L}$ and 1.5 $\mathrm{g} / \mathrm{L}$ for the quenched and CCC versions of this glass, respectively, normalized to the measured composition.

Exhibit D-5 in Appendix D provides plots showing comparisons of the normalized PCT responses for the two heat treatments for each glass. A review of these plots shows only minor differences in normalized release for the KT01 glasses as a function of heat treatment. Glass KT01-HL shows an increase in normalized release for Li and $\mathrm{Si}$ after the CCC heat treatment, although these values remain far below those of the benchmark EA glass. The difference in PCT response is likely due to the presence of lithium silicate $\left(\mathrm{Li}_{2} \mathrm{SiO}_{3}\right)$ identified in the $\mathrm{CCC}$ version of KT01-HL (see Section 3.1.1).

The predictability of the KT01 PCT responses was evaluated using the DWPF durability model. The predicted PCT values, determined using the targeted and measured compositions of the KT01 glasses, were compared with the normalized PCT responses. Exhibit D-6 in Appendix D provides plots of the DWPF models for $\mathrm{B}, \mathrm{Li}, \mathrm{Na}$, and $\mathrm{Si}$ that relate the logarithm of the normalized PCT value (for each element of interest) to a linear function of a free energy of hydration term $\left(\Delta \mathrm{G}_{\mathrm{p}}\right.$, in $\mathrm{kcal} / 100 \mathrm{~g}$ glass $)$ derived from all of the compositional views and heat treatments of the KT01 glasses. Prediction limits at a 95\% confidence for an individual PCT result are also plotted along with the linear fit. The EA and ARM results are indicated on these plots as well. All but one of the KT01 glasses fall within the prediction limits of the DWPF model. Glass KT01-HK (the high $\mathrm{K}_{2} \mathrm{O}$ concentration composition) has a PCT response that falls below the lower prediction limit; however, the PCT response of this glass remains considerably lower than that of the benchmark EA glass.

\subsubsection{KT03-Series}

Table E-1 in Appendix E provides the elemental leachate concentration measurements determined by the PSAL for the solution samples generated by the PCTs for the KT03 glasses. Note that the corrected version of glass KT01-HA, labeled KT01-HA2, is also included with the PCT analyses of the KT03-series glasses. The values were adjusted for the dilution factors: the values for the study glasses, the blanks, and the ARM glass in Table E-1 were multiplied by 1.6667 to determine the values in parts per million and the values for EA were multiplied by 16.6667. Table E-1 also provides the resulting ppm measurements.

Exhibit E-1 in Appendix E provides plots of the leachate (ppm) concentrations in analytical sequence as generated by the PSAL for all of the data from the KT03 PCTs. Different colors and symbols are used for each of the study glasses and standards. No issues are seen in these plots.

Exhibit E-2 in Appendix E provides analyses of the PSAL measurements of the samples of the multi-element standard solution by analytical set and ICP-AES calibration block for the KT03series. An ANOVA investigating for statistically significant differences among the block averages for these samples for each element of interest is included in the exhibit. No statistically significant (at a 5\% level) difference among the averages of these measurements was indicated. 
Exhibit E-3 in Appendix E provides plots of the leachate concentrations for each type of submitted sample: the study glasses by heat treatment and the standards (EA, ARM, the multielement solution standard, and blanks). The common logarithm plots allow for the assessment of the repeatability of the measurements, which suggest very little scatter in the triplicate values for some analytes for some of the glasses.

The PCT leachate concentrations were normalized using the target and measured cation compositions of the glasses to obtain $\mathrm{g} / \mathrm{L}$ leachate concentrations following the procedure. ${ }^{23}$ Exhibit E-4 in Appendix E provides scatter plots for these results and offers an opportunity to investigate the consistency in the leaching across the elements for the KT03 glasses. The smallest correlation in this plot is that for $\mathrm{Na}$ and $\mathrm{Si}$ with a value of $\sim 68 \%$. This non-linearity will not have a significant impact on the outcome of the study since all of the glasses are highly durable.

Table E-2 in Appendix E summarizes the normalized PCTs for the KT03 glasses. The PCTs are listed by heat treatment and compositional view for each glass. The KT03 glasses all had NL [B] values that were well below the $16.695 \mathrm{~g} / \mathrm{L}$ value of the benchmark EA glass. The highest $\mathrm{NL}$ [B] value was for the quenched version of glass KT03-HK, the high $\mathrm{K}_{2} \mathrm{O}$ composition, with a value of $1.4 \mathrm{~g} / \mathrm{L}$ normalized to the measured composition.

Exhibit E-5 in Appendix E provides plots showing comparisons of the normalized PCT responses for the two heat treatments for each glass. A review of these plots shows only minor differences in normalized release for the KT03 glasses as a function of heat treatment. Glass KT03-HK shows a decrease in normalized release for $\mathrm{B}$ and $\mathrm{Na}$ and an increase in normalized release for $\mathrm{Si}$ after the CCC heat treatment. Glass KT03-HL shows an increase in normalized release for Li and Si after the CCC heat treatment. The differences in these PCT responses with heat treatment may be due to crystallization (see Section 3.1.3), although normalized releases for both of these glasses remain far below those of the benchmark EA glass.

The predictability of the KT03 PCT responses was evaluated using the DWPF durability model. The predicted PCT values, determined using the targeted and measured compositions of the KT03 glasses, were compared with the normalized PCT responses. Exhibit E-6 in Appendix E provides plots of the DWPF models for $\mathrm{B}, \mathrm{Li}, \mathrm{Na}$, and $\mathrm{Si}$ that relate the logarithm of the normalized PCT value (for each element of interest) to the $\Delta \mathrm{G}_{\mathrm{p}}$ term derived from all of the compositional views and heat treatments of the KT03 glasses. Note that glass KT01-HA2 is also included in these plots. Prediction limits at a 95\% confidence for an individual PCT result are also plotted along with the linear fit. The EA and ARM results are indicated on these plots as well. All but two of the KT03 glasses fall within the prediction limits of the DWPF model. Glasses KT03-HK and KT03-MK have PCT responses that fall below the lower prediction limit; however, the PCT responses of these glasses remain considerably lower than that of the benchmark EA glass. As seen in the KT01 plots (Section 3.3.1), there is an issue with the predictability of these high $\mathrm{K}_{2} \mathrm{O}$ concentration glasses. However, this lack of model applicability may be of little practical importance since $\mathrm{K}_{2} \mathrm{O}$ concentrations of this magnitude are unlikely for actual compositions to be processed at the DWPF.

\subsubsection{KT04-Series}

Table F-1 in Appendix F provides the elemental leachate concentration measurements determined by the PSAL for the solution samples generated by the PCTs for the KT04 glasses. The values were adjusted for the dilution factors: the values for the study glasses, the blanks, and the ARM glass in Table F-1 were multiplied by 1.6667 to determine the values in parts per million and the values for EA were multiplied by 16.6667. Table F-1 also provides the resulting ppm measurements. 
Exhibit F-1 in Appendix F provides plots of the leachate (ppm) concentrations in analytical sequence as generated by the PSAL for all of the data from the KT04 PCTs. Different colors and symbols are used for each of the study glasses and standards. No issues are seen in these plots.

Exhibit F-2 in Appendix F provides analyses of the PSAL measurements of the samples of the multi-element standard solution by analytical set and ICP-AES calibration block for the KT04series. An ANOVA investigating for statistically significant differences among the block averages for these samples for each element of interest is included in the exhibit. No statistically significant (at a 5\% level) difference among the averages of these measurements was indicated.

Exhibit F-3 in Appendix F provides plots of the leachate concentrations for each type of submitted sample: the study glasses by heat treatment and the standards (EA, ARM, the multielement solution standard, and blanks). The common logarithm plots allow for the assessment of the repeatability of the measurements, which suggest only minor scatter in the triplicate values for some analytes for some of the glasses.

The PCT leachate concentrations were normalized using the target and measured cation compositions of the glasses to obtain $\mathrm{g} / \mathrm{L}$ leachate concentrations following the procedure. ${ }^{23}$ Exhibit F-4 in Appendix F provides scatter plots for these results and offers an opportunity to investigate the consistency in the leaching across the elements for the KT04 glasses. The smallest correlation in this plot is that for $\mathrm{B}$ and $\mathrm{Si}$ with a value of $\sim 98 \%$, indicating very linear correlations for all of the element pairs.

Table F-2 in Appendix F summarizes the normalized PCTs for the KT04 glasses. The PCTs are listed by heat treatment and compositional view for each glass. The KT04 glasses all had NL [B] values that were well below the $16.695 \mathrm{~g} / \mathrm{L}$ value of the benchmark EA glass, and in fact all below $1.0 \mathrm{~g} / \mathrm{L}$. The highest NL [B] value was for glass KT04-03, with values of $0.61 \mathrm{~g} / \mathrm{L}$ and $0.62 \mathrm{~g} / \mathrm{L}$ for the quenched and CCC versions of this glass, respectively, normalized to the measured composition.

Exhibit F-5 in Appendix F provides plots showing comparisons of the normalized PCT responses for the two heat treatments for each glass. A review of these plots shows only minor differences in normalized release for the KT04 glasses as a function of heat treatment, with no significant differences observed. This can likely be attributed to the lack of measurable bulk crystallization in the KT04 glasses (see Section 3.1.4).

The predictability of the KT04 PCT responses was evaluated using the DWPF durability model. The predicted PCT values, determined using the targeted and measured compositions of the KT04 glasses, were compared with the normalized PCT responses. Exhibit F-6 in Appendix F provides plots of the DWPF models for $\mathrm{B}, \mathrm{Li}, \mathrm{Na}$, and $\mathrm{Si}$ that relate the logarithm of the normalized PCT value (for each element of interest) to the $\Delta \mathrm{G}_{\mathrm{p}}$ term derived from all of the compositional views and heat treatments of the KT04 glasses. Prediction limits at a 95\% confidence for an individual PCT result are also plotted along with the linear fit. The EA and ARM results are indicated on these plots as well. All of the KT04 glasses fall within the prediction limits of the DWPF model, regardless of compositional view or heat treatment, indicating good predictability for these compositions.

\section{$3.4 \underline{\text { Viscosity }}$}

Viscosity data were collected for all of the glasses in the KT01, KT03, and KT04-series. Note that data for the corrected version of composition KT01-HA, labeled KT01-HA2, are used in this 
discussion. The measured viscosity at $1150{ }^{\circ} \mathrm{C}$ was determined by fitting the data for each glass to the Fulcher equation. ${ }^{20,21}$ Complete data from the fitting of Fulcher equations are given in Appendix G for the KT01-series glasses, Appendix H for the KT03-series glasses, and Appendix I for the KT04-series glasses. The results of the Fulcher fits were used to calculate a measured viscosity value for each glass at $1150{ }^{\circ} \mathrm{C}$. These values are given in Table J-1 of Appendix J. The measured values are displayed graphically versus the model predicted values in Exhibit J-1 and Exhibit J-2 in Appendix J, and below in Figure 3-1.

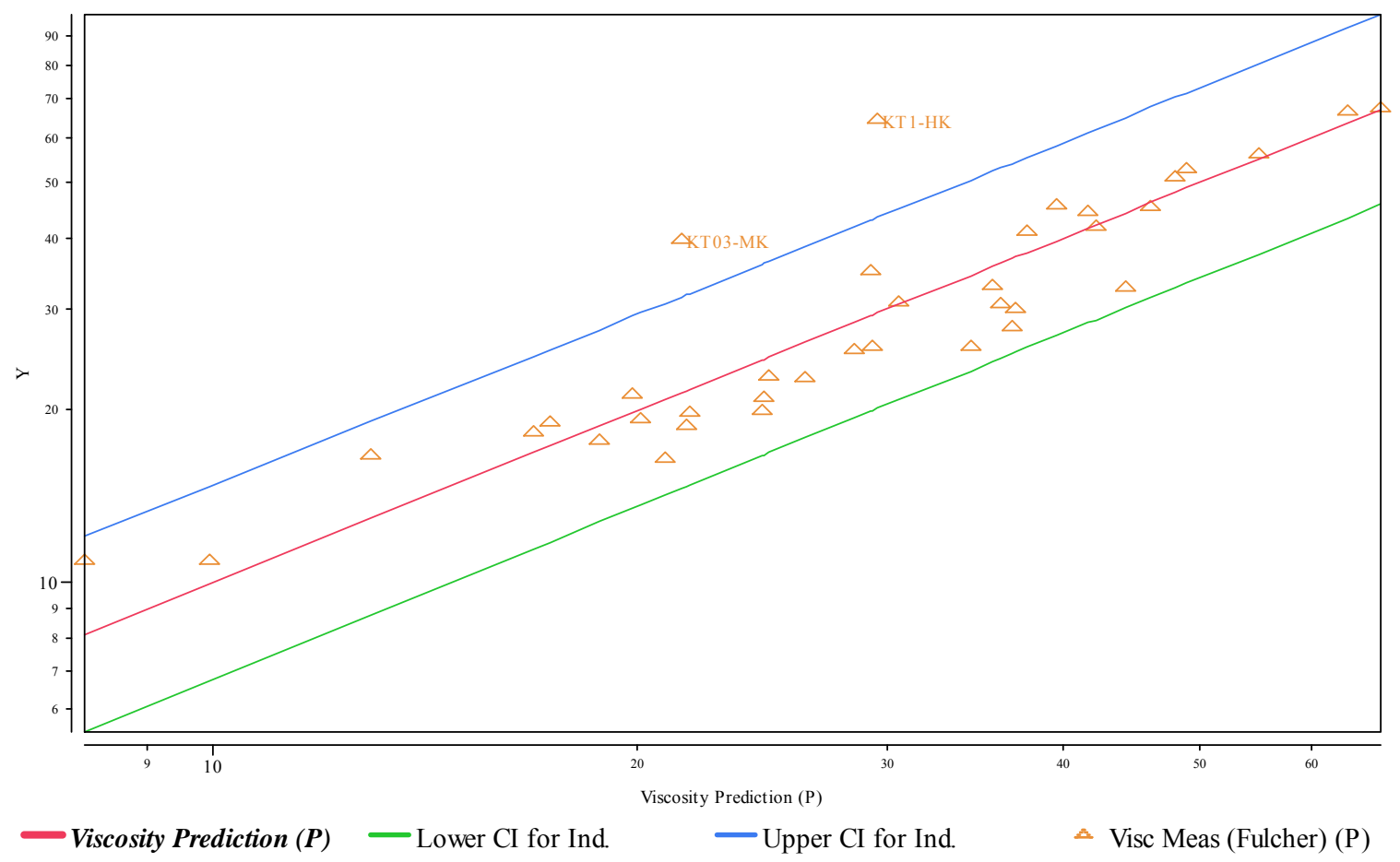

Figure 3-1. Measured Versus Predicted Viscosities at $1150{ }^{\circ} \mathrm{C}$ for the KT01, KT03, and KT04-Series Glasses, Based on the Measured Compositions.

Figure 3-1 shows that all but two of the KT01, KT03, and KT04-series glasses had measured viscosities that were predictable using the current DWPF viscosity model (based on the measured compositions). The two glasses with viscosities that fall above the upper confidence intervals for the model prediction are the high $\mathrm{K}_{2} \mathrm{O}$ concentration glasses KT01-HK and KT03-MK. These glasses have measured $\mathrm{K}_{2} \mathrm{O}$ concentrations of 16.4 and $9.9 \mathrm{wt} \%$, respectively. These concentrations are above the DWPF viscosity model development range 0 to $5.73 \mathrm{wt} \% \mathrm{~K}_{2} \mathrm{O}$ (see Table 1-3). Interestingly, the KT03-HK composition, with a measured $\mathrm{K}_{2} \mathrm{O}$ concentration of 17.6 wt $\%$, had a measured viscosity that was well predicted by the current DWPF model. While these results point to a lack of applicability for the DWPF viscosity model for increased $\mathrm{K}_{2} \mathrm{O}$ concentrations, it is important to note that these high $\mathrm{K}_{2} \mathrm{O}$ concentration glasses were developed to determine potential impacts of $\mathrm{K}_{2} \mathrm{O}$ on the retention of $\mathrm{TiO}_{2}$ and that their $\mathrm{K}_{2} \mathrm{O}$ concentrations are impractically high for actual DWPF processing. Overall, the measured viscosity values of the KT01, KT03, and KT04-series glasses are well predicted by the current DWPF viscosity model. 


\subsection{Liquidus Temperature}

Liquidus temperatures were measured for the KT01-series glasses. Glass KT01-HK was excluded from the measurements since a small amount of bulk crystallization was visible in the glass by optical microscopy after quenching from $1150{ }^{\circ} \mathrm{C}$. Liquidus temperature estimates for glass KT01-HA2 and the KT03 and KT04-series glasses are still underway. Measured liquidus temperatures for the KT01-series glasses are presented in Figure 3-2 and compared with the predicted values from the DWPF model (based on the measured compositions of the glasses).

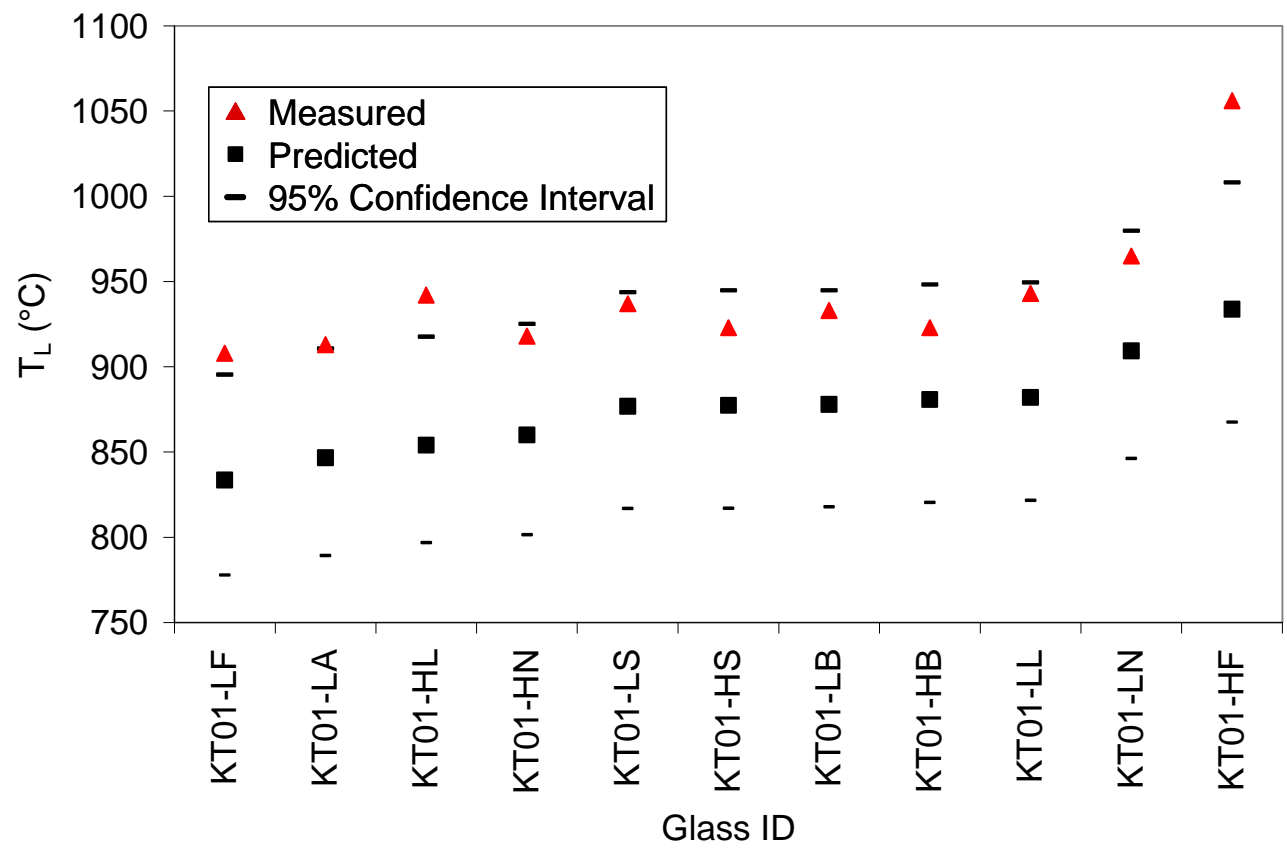

Figure 3-2. Predicted and Measured Liquidus Temperatures for the KT01-Series Glasses, Based on the Measured Compositions.

The results in Figure 3-2 are mixed with regard to the predictability of the $T_{L}$ for each glass. All of the measured $\mathrm{T}_{\mathrm{L}}$ values are higher than the model predicted values, although most fall within the $95 \%$ confidence intervals. Compositions KT01-LF, KT01-LA, KT01-HL, and KT01-HF have measured $T_{L}$ values that are above the upper $95 \%$ confidence bounds on the predicted values. The concentrations of some of the components in these glasses, particularly $\mathrm{TiO}_{2}$, fall outside the region of applicability of the current $\mathrm{T}_{\mathrm{L}}$ model (Table 1-1). These results indicate that the model may need to be adjusted in order to more correctly predict the $\mathrm{T}_{\mathrm{L}}$ of glasses when the SCIX streams are incorporated, although further data are necessary (and are currently being collected) for a more complete assessment.

\subsection{Summary}

Four series of glass compositions were selected, fabricated, and characterized as part of a study to determine the impacts of the addition of CST and MST from the SCIX process on the DWPF glass waste form and the applicability of the DWPF process control models. The KT01 and KT02-series of glasses were chosen to allow for the identification of the influence of the concentrations of major components of the glass on the retention of $\mathrm{TiO}_{2}$. The $\mathrm{KT} 03$ series of glasses was chosen to allow for the identification of these influences when higher $\mathrm{Nb}_{2} \mathrm{O}_{5}$ and $\mathrm{ZrO}_{2}$ concentrations are included along with $\mathrm{TiO}_{2}$. The $\mathrm{KT} 04$ series of glasses was chosen to 
investigate the properties and performance of glasses based on the best available projections of actual compositions to be processed at the DWPF (i.e., future sludge batches including the SCIX streams).

The glasses were fabricated in the laboratory without the radioactive components. They were characterized using XRD to identify crystallization, ICP-AES to verify chemical compositions, and the PCT to measure durability. The viscosity and liquidus temperature of several of the glasses were also measured.

The XRD results showed no titanium containing phases in the KT01-series glasses, regardless of whether they had been air quenched or slowly cooled. The target $\mathrm{TiO}_{2}$ concentration had to be increased to $12 \mathrm{wt} \%$ in glass in the KT02-series before any compositional impacts on $\mathrm{TiO}_{2}$ retention (e.g., the formation of titanium containing crystals) were apparent. Titanium containing crystalline phases formed in the slowly cooled versions of all of the KT02 compositions except for the low $\mathrm{Al}_{2} \mathrm{O}_{3}$ concentration glass, the low $\mathrm{Fe}_{2} \mathrm{O}_{3}$ concentration glass, the high $\mathrm{Na}_{2} \mathrm{O}$ concentration glass, and the high $\mathrm{K}_{2} \mathrm{O}$ concentration glass. The impacts of these components will need additional investigation before drawing further conclusions (i.e., a larger number of compositions should be fabricated and characterized). However, one should keep in mind that the $12 \mathrm{wt} \% \mathrm{TiO}_{2}$ concentrations in these glasses are unrealistic for DWPF processing with the SCIX streams and material from SWPF. Spinels were identified in some of the KT03 glasses after the CCC heat treatment. The spinels did not adversely impact the durability of the glasses but will be important for liquidus temperature determinations. The KT04-series glasses were amorphous regardless of heat treatment.

No issues were found upon review of the chemical composition measurements. Glass KT01-HA was identified as having been incorrectly batched. This composition was replaced for subsequent analyses with a corrected version, identified as KT01-HA2.

No issues were found upon review of the PCT measurements. All of the glasses studied were considerably more durable than the benchmark EA glass. The measured PCT responses were compared with the predicted values from the current DWPF durability model. One of the KT01series and two of the KT03-series glasses had measured PCT responses that were outside the lower bound of the durability model. All three of these glasses had intentionally high $\mathrm{K}_{2} \mathrm{O}$ concentrations (to evaluate the impact of $\mathrm{K}_{2} \mathrm{O}$ on $\mathrm{TiO}_{2}$ retention), which may indicate a lack of applicability for the durability model in this composition region. However, this is likely of little practical importance since $\mathrm{K}_{2} \mathrm{O}$ concentrations of this magnitude are unrealistic for actual compositions to be processed at the DWPF. All of the KT04 glasses had durabilities that were predictable regardless of heat treatment or compositional view.

All but two of the KT01, KT03, and KT04-series glasses had measured viscosities that were predictable using the current DWPF viscosity model (based on the measured compositions). The viscosities of two of the high $\mathrm{K}_{2} \mathrm{O}$ concentration glasses, KT01-HK and KT03-MK, fell above the upper confidence intervals for the model prediction. These glasses have $\mathrm{K}_{2} \mathrm{O}$ concentrations that are above the DWPF viscosity model development range for $\mathrm{K}_{2} \mathrm{O}$. Another high $\mathrm{K}_{2} \mathrm{O}$ concentration glass, the KT03-HK composition, had a measured viscosity that was well predicted by the current DWPF model. While these results point to a lack of applicability for the DWPF viscosity model for increased $\mathrm{K}_{2} \mathrm{O}$ concentrations, it is again important to note that these $\mathrm{K}_{2} \mathrm{O}$ concentrations are unrealistic for actual DWPF processing. Overall, the measured viscosity values of the KT01, KT03, and KT04-series glasses are well predicted by the current DWPF viscosity model. 
The results of $T_{L}$ measurements for the KT01-series glasses were mixed with regard to the predictability of the $T_{L}$ for each glass. All of the measured $T_{L}$ values were higher than the model predicted values, although most fell within the $95 \%$ confidence intervals. Compositions KT01-LF, KT01-LA, KT01-HL, and KT01-HF had measured $\mathrm{T}_{\mathrm{L}}$ values that were above the upper $95 \%$ confidence bounds on the predicted values. The concentrations of some of the components in these glasses, particularly $\mathrm{TiO}_{2}$, fall outside the region of applicability of the current $\mathrm{T}_{\mathrm{L}}$ model. These results indicate that the model may need to be adjusted in order to more correctly predict the $\mathrm{T}_{\mathrm{L}}$ of glasses when the SCIX streams are incorporated, although further data are necessary for a more complete assessment. Liquidus temperature measurements for the KT03 and KT04-series glasses are underway.

\subsection{Recommendations and Path Forward}

Overall, the results presented in this report show an ability to incorporate the anticipated SCIX streams into the DWPF-type glass compositions studied. Additional experiments are needed to determine whether to extend the validation range of the DWPF process control models or whether refitting of the models will be necessary. Liquidus temperature measurements are continuing for the KT03 and KT04 glasses, and should be performed for any additional compositions developed for this study.

Several additional sets of experimental glasses are being fabricated and characterized to provide further information on potential impacts of the SCIX streams on DWPF glass, including:

- A study of glass compositions previously shown to crystallize titanium containing phases at lower $\mathrm{TiO}_{2}$ concentrations

- A study of glass compositions covering a broader range of potential sludge compositions that remain acceptable for processing by the current DWPF process control models

- A study of glass compositions incorporating noble metals, which may serve as nucleation sites for titanium containing crystalline phases

- A study of glass compositions containing uranium and thorium, which may impact the retention of $\mathrm{TiO}_{2}$ or other glass properties

At the completion of these studies, all of the data generated will be reviewed with regard to applicability of the DWPF PCCS model and recommendations will be made as to whether the validation ranges of the current models can be extended, or whether some or all of the models need to be refit to allow for the incorporation of the SCIX streams. As changes are made to the projected sludge compositions and the volume of the SCIX material, additional evaluations should be performed.

\subsection{References}

1. Fellinger, T. L., "Technical Task Request: DWPF Glass Evaluation for the Introduction of MSP Products into Future Sludge Batches," U.S. Department of Energy Report HLW-DWPFTTR-2010-0009, Revision A, Savannah River Remediation, Aiken, SC (2010).

2. Fox, K. M., "Task Technical and Quality Assurance Plan for Evaluation of the Introduction of Modular Salt Processing Products to Defense Waste Processing Facility Glass for Future Sludge Batches," U.S. Department of Energy Report SRNL-RP-2010-00560, Revision 0, Savannah River National Laboratory, Aiken, SC (2010).

3. Edwards, T. B., K. G. Brown and R. L. Postles, "SME Acceptability Determination for DWPF Process Control," U.S. Department of Energy Report WSRC-TR-95-00364, Revision 5, Washington Savannah River Company, Aiken, SC (2006). 
4. Edwards, T. B. and J. R. Harbour, "Composition and Property Measurements for CST Phase 1 Glasses," U.S. Department of Energy Report WSRC-TR-99-00245, Revision 0, Westinghouse Savannah River Company, Aiken, SC (1999).

5. Edwards, T. B. and J. R. Harbour, "Composition and Property Measurements for CST Phase 2 Glasses," U.S. Department of Energy Report WSRC-TR-99-00289, Revision 0, Westinghouse Savannah River Company, Aiken, SC (1999).

6. Edwards, T. B. and J. R. Harbour, "Composition and Property Measurements for CST Phase 3 Glasses," U.S. Department of Energy Report WSRC-TR-99-00291, Revision 0, Westinghouse Savannah River Company, Aiken, SC (1999).

7. Edwards, T. B. and J. R. Harbour, "Composition and Property Measurements for CST Phase 4 Glasses," U.S. Department of Energy Report WSRC-TR-99-00293, Revision 0, Westinghouse Savannah River Company, Aiken, SC (1999).

8. Edwards, T. B., J. R. Harbour and R. J. Workman, "Summary of Results for CST Glass Study: Composition and Property Measurements," U.S. Department of Energy Report WSRC-TR-9900324, Revision 0, Westinghouse Savannah River Company, Aiken, SC (1999).

9. Peeler, D. K. and T. B. Edwards, "High-Level Review of the Impacts of CST and RF on DWPF Processing: A Glass Formulation Perspective," U.S. Department of Energy Memorandum SRNL-PSE-2007-00177, Washington Savannah River Company, Aiken, SC (2007).

10. Fox, K. M., T. B. Edwards, M. E. Stone and D. C. Koopman, "Paper Study Evaluations of the Introduction of Small Column Ion Exchange (SCIX) Waste Streams to the Defense Waste Processing Facility," U.S. Department of Energy Report SRNL-STI-2010-00297, Revision 0, Savannah River National Laboratory, Aiken, SC (2010).

11. Brown, K. G., C. M. Jantzen and G. Ritzhaupt, "Relating Liquidus Temperature to Composition for Defense Waste Processing Facility (DWPF) Process Control," U.S. Department of Energy Report WSRC-TR-2001-00520, Revision 0, Westinghouse Savannah River Company, Aiken, SC (2001).

12. Peeler, D. K. and T. B. Edwards, "Impact of Reducing the $100^{\circ} \mathrm{C}$ Liquidus Temperature Offset on Waste Loading Targets," U.S. Department of Energy Report SRNL-STI-2010-00528, Revision 0, Savannah River National Laboratory, Aiken, SC (2010).

13. Jantzen, C. M. and K. G. Brown, "Predicting the Spinel-Nepheline Liquidus for Application to Nuclear Waste Glass Processing. Part II: Quasicrystalline Freezing Point Depression Model," Journal of the American Ceramic Society, 90 [6] 1880-1891 (2007).

14. Jantzen, C. M. and K. G. Brown, "Predicting the Spinel-Nepheline Liquidus for Application to Nuclear Waste Glass Processing. Part I: Primary Phase Analysis, Liquidus Measurement, and Quasicrystalline Approach," Journal of the American Ceramic Society, 90 [6] 1866-1879 (2007).

15. Jantzen, C. M., "The Impacts of Uranium and Thorium on the Defense Waste Processing Facility (DWPF) Viscosity Model," U.S. Department of Energy Report WSRC-TR-2004-00311, Revision 0, Westinghouse Savannah River Company, Aiken, SC (2005). 
16. Plodinec, M. J. and J. R. Wiley, "Viscosity of Glass Melts Containing Simulated SRP Waste," U.S. Department of Energy Report DP-1507, Savannah River Laboratory, E.I. duPont deNemours \& Co., Aiken, SC (1978).

17. Smith, M. E., "Reevalutaion of DWPF High Viscosity Constraint: SRTC ITS Position," U.S. Department of Energy Memorandum SRT-GFM-99-0011, Savannah River Technology Center, Aiken, SC (April 30, 1999).

18. Jantzen, C. M., "Relationship of Glass Composition to Glass Viscosity, Resistivity, Liquidus Temperature, and Durability: First Principles Process-Product Models for Vitrification of Nuclear Waste"; pp. 37-51 in Ceramic Transactions, Vol. 23, Proceedings of the 5th International Symposium on Ceramics in Nuclear Waste Management. Edited by G. G. Wicks, D. F. Bickford and R. Bunnell. The American Ceramic Society, Westerville, 1991.

19. Jantzen, C. M., K. G. Brown and M. J. Plodinec, "The Relationship Between Glass Viscosity, Glass Resistivity, and Composition: A First Principles Model for Vitrification of Nuclear Waste," U.S. Department of Energy Report WSRC-MS-90-41, Revision 1, Westinghouse Savannah River Company, Aiken, SC (1990).

20. Fulcher, G. S., "Analysis of Recent Measurements of the Viscosity of Glasses," Journal of the American Ceramic Society, 8 [6] 339-355 (1925).

21. Fulcher, G. S., "Analysis of Recent Measurements of the Viscosity of Glasses, II," Journal of the American Ceramic Society, 8 [12] 789-794 (1925).

22. Jantzen, C. M., J. B. Picket, K. G. Brown, T. B. Edwards and D. C. Beam, "Process/Product Models for the Defense Waste Processing Facility (DWPF): Part I. Predicting Glass Durability from Composition Using a Thermodynamic Hydration Energy Reaction Model (THERMO)," U.S. Department of Energy Report WSRC-TR-93-672, Revision 1, Westinghouse Savannah River Company, Aiken, SC (1995).

23. ASTM, "Standard Test Methods for Determining Chemical Durability of Nuclear Waste Glasses: The Product Consistency Test (PCT)," ASTM C-1285, (2002).

24. Jantzen, C. M., "Free Energy of Hydration of Niobium Oxide," U.S. Department of Energy Report SRNL-TR-2010-00125, Revision 0, Savannah River National Laboratory, Aiken, SC (2010).

25. Jantzen, C. M., J. B. Picket, K. G. Brown, T. B. Edwards and D. C. Beam, "Process/Product Models for the Defense Waste Processing Facility (DWPF): Part II. Predicting Glass Durability from Composition Using a Thermodynamic Hydration Energy Reaction Model (THERMO)," U.S. Department of Energy Report WSRC-TR-93-672, Revision 1, Westinghouse Savannah River Company, Aiken, SC (1995).

26. Bickford, D. F., A. Applewhite-Ramsey, C. M. Jantzen and K. G. Brown, "Control of Radioactive Waste Glass Melters: I, Preliminary General Limits at Savannah River," Journal of the American Ceramic Society, 73 [10] 2896-2902 (1990).

27. Plodinec, M. J., "Development of Glass Compositions for Immobilization of SRP Waste," U.S. Department of Energy Report DP-1517, Savannah River Laboratory, Aiken, SC (1979). 
28. Plodinec, M. J., "Improved Glass Compositions for Immobilization of SRP Waste"; pp. 223229 in Vol. 2, Proceedings of the International Symposium on the Scientific Basis for Nuclear Waste Management. Edited by C. J. M. Northrup, Jr. Plenum Press, New York, 1980.

29. Lorier, T. H. and C. M. Jantzen, "Evaluation of the $\mathrm{TiO}_{2}$ Limit for DWPF Glass," U.S. Department of Energy Report WSRC-TR-2003-00396, Revision 0, Westinghouse Savannah River Company, Aiken, SC (2003).

30. Volf, M. B., Chemical Approach to Glass, Glass Science and Technology, Vol. 7, Elsevier Scientific Publishers, New York (1984).

31. Lilliston, G., "HLW Technical Task Request," U.S. Department of Energy Report SP-TTR2003-00004, Westinghouse Savannah River Company, Aiken, SC (2003).

32. Langowski, M. H., "The Incorporation of P, S, Cr, F, Cl, I, Mn, Ti, U, and Bi into Simulated Nuclear Waste Glasses: Literature Study," U.S. Department of Energy Report PNNL-10980 UC512, Pacific Northwest National Laboratory, Richland, WA (1996).

33. Chew, D. P. and B. A. Hamm, "Liquid Waste System Plan," U.S. Department of Energy Report SRR-LWP-2009-00001, Revision 15, Savannah River Remediation, Aiken, SC (2010).

34. Marra, S. L. and C. M. Jantzen, "Characterization of Projected DWPF Glass Heat Treated to Simulate Canister Centerline Cooling," U.S. Department of Energy Report WSRC-TR-92-142, Revision 1, Westinghouse Savannah River Company, Aiken, SC (1993).

35. Edwards, T. B., "Analytical Plans for Measuring the Chemical Compositions of Glasses from the Study of the Impact of MSP Streams on DWPF Wasteforms," U.S. Department of Energy Memorandum SRNL-L5200-2010-00013, Savannah River National Laboratory, Aiken, SC (2010).

36. Edwards, T. B., "An Analytical Plan for Measuring the Chemical Compositions of the KT03Series of Glasses from the Study of the Impact of SCIX Streams on DWPF Wasteforms," U.S. Department of Energy Memorandum SRNL-L5200-2010-00017, Revision 1, Savannah River National Laboratory, Aiken, SC (2010).

37. Edwards, T. B., "An Analytical Plan for Measuring the Chemical Compositions of the KT04Series of Glasses from the Study of the Impact of SCIX Streams on DWPF Wasteforms," U.S. Department of Energy Memorandum Savannah River National Laboratory, Aiken, SC (2010).

38. Jantzen, C. M., N. E. Bibler, D. C. Beam, C. L. Crawford and M. A. Pickett, "Characterization of the Defense Waste Processing Facility (DWPF) Environmental Assessment (EA) Glass Standard Reference Material," U.S. Department of Energy Report WSRC-TR-92-346, Revision 1, Westinghouse Savannah River Company, Aiken, SC (1993).

39. Edwards, T. B., "Analytical Plans for Measuring the PCT Solutions of Glasses from the Study of the Impact of MSP Streams on DWPF Wasteforms," U.S. Department of Energy Memorandum SRNL-L5200-2010-00014, Savannah River National Laboratory, Aiken, SC (2010).

40. Edwards, T. B., "An Analytical Plan for Measuring the PCT Solutions of the KT03 Series of Glasses from the Study of the Impact of SCIX Streams on DWPF Wasteforms," U.S. Department of Energy Memorandum SRNL-L5200-2010-00020, Revision 1, Savannah River National Laboratory, Aiken, SC (2010). 
41. Edwards, T. B., "An Analytical Plan for Measuring the PCT Solutions of the KT04 Series of Glasses from the Study of the Impact of SCIX Streams on DWPF Wasteforms," U.S. Department of Energy Memorandum SRNL-L5200-2010-00027, Savannah River National Laboratory, Aiken, $\mathrm{SC}(2010)$.

42. ASTM, "Standard Practice for Measuring Viscosity of Glass Above the Softening Point," ASTM C-965, (2007).

43. Schumacher, R. F. and D. K. Peeler, "Establishment of Harrop, High-Temperature Viscometer," U.S. Department of Energy Report WSRC-RP-98-00737, Revision 0, Westinghouse Savannah River Company, Aiken, SC (1998).

44. Schumacher, R. F., R. J. Workman and T. B. Edwards, "Calibration and Measurement of the Viscosity of DWPF Start-Up Glass," U.S. Department of Energy Report WSRC-RP-2000-00874, Revision 0, Westinghouse Savannah River Company, Aiken, SC (2001).

45. Crum, J. V., R. L. Russell, M. J. Schweiger, D. E. Smith, J. D. Vienna, T. B. Edwards, C. M. Jantzen, D. K. Peeler, R. F. Schumacher and R. J. Workman, "DWPF Startup Frit Viscosity Measurement Round Robin Results," Pacific Northwest National Laboratory, (Unpublished).

46. “Isothermal Liquidus Temperature Measurement Procedure,” Manual L29, ITS-0025, Savannah River National Laboratory, Aiken, SC (2009).

47. “Furnace Functional Check,” Manual L29, ITS-0096, Savannah River National Laboratory, Aiken, SC (2009).

48. “Microscopy Sample Preparation,” Manual L29, ITS-0017, Savannah River National Laboratory, Aiken, SC (2008). 
SRNL-STI-2010-00566

Revision 0

Appendix A. Data Supporting the Chemical Composition Measurements of the KT01-Series Glasses 
Table A-1 PSAL Chemical Composition Measurements of the KT01-Series of Glasses Using LM Preparation Method (Part 1).

\begin{tabular}{|c|c|c|c|c|c|c|c|c|c|c|c|c|}
\hline Glass ID & Block & Sub-Blk & Sequence & Lab ID & Ba (wt\%) & Ca (wt\%) & Ce (wt\%) & Cr (wt\%) & Cu (wt \%) & K (wt\%) & La (wt\%) & Mg (wt\%) \\
\hline Batch 1 & 1 & 1 & 1 & BCHLM111 & 0.124 & 0.836 & $<0.010$ & 0.076 & 0.305 & 2.94 & $<0.010$ & 0.812 \\
\hline KT1-HS & 1 & 1 & 2 & P05LM21 & 0.062 & 0.671 & 0.148 & 0.065 & 0.029 & 0.064 & 0.050 & 0.080 \\
\hline KT1-LL & 1 & 1 & 3 & P10LM21 & 0.064 & 0.702 & 0.159 & 0.073 & 0.032 & 0.055 & 0.054 & 0.087 \\
\hline KT1-LF & 1 & 1 & 4 & P04LM21 & 0.069 & 0.726 & 0.166 & 0.078 & 0.029 & 0.064 & 0.056 & 0.092 \\
\hline KT1-LL & 1 & 1 & 5 & P10LM11 & 0.066 & 0.702 & 0.159 & 0.076 & 0.031 & 0.052 & 0.055 & 0.091 \\
\hline KT1-HS & 1 & 1 & 6 & P05LM11 & 0.062 & 0.650 & 0.146 & 0.067 & 0.028 & 0.056 & 0.051 & 0.083 \\
\hline KT1-HN & 1 & 1 & 7 & P02LM21 & 0.065 & 0.668 & 0.158 & 0.082 & 0.033 & 0.073 & 0.053 & 0.087 \\
\hline KT1-HF & 1 & 1 & 8 & P09LM11 & 0.061 & 0.667 & 0.148 & 0.071 & 0.027 & 0.056 & 0.050 & 0.082 \\
\hline Batch 1 & 1 & 1 & 9 & BCHLM112 & 0.126 & 0.827 & $<0.010$ & 0.079 & 0.302 & 3.01 & $<0.010$ & 0.825 \\
\hline KT1-LF & 1 & 1 & 10 & P04LM11 & 0.070 & 0.716 & 0.166 & 0.078 & 0.029 & 0.061 & 0.056 & 0.093 \\
\hline KT1-LN & 1 & 1 & 11 & P06LM11 & 0.070 & 0.712 & 0.168 & 0.080 & 0.031 & 0.054 & 0.057 & 0.094 \\
\hline KT1-HN & 1 & 1 & 12 & P02LM11 & 0.066 & 0.655 & 0.157 & 0.083 & 0.032 & 0.072 & 0.052 & 0.088 \\
\hline KT1-HF & 1 & 1 & 13 & P09LM21 & 0.063 & 0.668 & 0.151 & 0.072 & 0.027 & 0.054 & 0.051 & 0.084 \\
\hline KT1-LN & 1 & 1 & 14 & P06LM21 & 0.069 & 0.694 & 0.166 & 0.080 & 0.030 & 0.054 & 0.057 & 0.094 \\
\hline KT1-HK & 1 & 1 & 15 & P01LM11 & 0.061 & 0.609 & 0.144 & 0.078 & 0.034 & 13.8 & 0.048 & 0.076 \\
\hline KT1-HK & 1 & 1 & 16 & P01LM21 & 0.062 & 0.613 & 0.146 & 0.083 & 0.035 & 14.0 & 0.049 & 0.079 \\
\hline Batch 1 & 1 & 1 & 17 & BCHLM113 & 0.127 & 0.808 & $<0.010$ & 0.079 & 0.298 & 3.03 & $<0.010$ & 0.847 \\
\hline Batch 1 & 1 & 2 & 1 & BCHLM121 & 0.123 & 0.839 & $<0.010$ & 0.075 & 0.306 & 2.93 & $<0.010$ & 0.807 \\
\hline KT1-LF & 1 & 2 & 2 & P04LM12 & 0.067 & 0.741 & 0.171 & 0.074 & 0.031 & 0.067 & 0.055 & 0.088 \\
\hline KT1-HN & 1 & 2 & 3 & P02LM12 & 0.063 & 0.674 & 0.160 & 0.078 & 0.034 & 0.078 & 0.052 & 0.083 \\
\hline KT1-HF & 1 & 2 & 4 & P09LM22 & 0.062 & 0.687 & 0.155 & 0.069 & 0.028 & 0.059 & 0.051 & 0.080 \\
\hline KT1-HS & 1 & 2 & 5 & P05LM12 & 0.060 & 0.675 & 0.151 & 0.063 & 0.029 & 0.062 & 0.049 & 0.078 \\
\hline KT1-HN & 1 & 2 & 6 & P02LM22 & 0.062 & 0.689 & 0.161 & 0.078 & 0.035 & 0.080 & 0.052 & 0.082 \\
\hline KT1-LN & 1 & 2 & 7 & P06LM12 & 0.067 & 0.741 & 0.171 & 0.076 & 0.032 & 0.060 & 0.056 & 0.088 \\
\hline KT1-LL & 1 & 2 & 8 & P10LM12 & 0.063 & 0.729 & 0.162 & 0.073 & 0.033 & 0.058 & 0.055 & 0.086 \\
\hline Batch 1 & 1 & 2 & 9 & BCHLM122 & 0.121 & 0.855 & $<0.010$ & 0.075 & 0.309 & 3.03 & $<0.010$ & 0.787 \\
\hline KT1-LL & 1 & 2 & 10 & P10LM22 & 0.064 & 0.710 & 0.160 & 0.071 & 0.033 & 0.058 & 0.054 & 0.085 \\
\hline KT1-HK & 1 & 2 & 11 & P01LM12 & 0.059 & 0.621 & 0.146 & 0.075 & 0.036 & 13.2 & 0.048 & 0.073 \\
\hline KT1-LF & 1 & 2 & 12 & P04LM22 & 0.067 & 0.740 & 0.166 & 0.074 & 0.030 & 0.068 & 0.055 & 0.088 \\
\hline KT1-HF & 1 & 2 & 13 & P09LM12 & 0.059 & 0.679 & 0.149 & 0.067 & 0.028 & 0.060 & 0.050 & 0.078 \\
\hline KT1-HS & 1 & 2 & 14 & P05LM22 & 0.061 & 0.673 & 0.147 & 0.064 & 0.029 & 0.067 & 0.050 & 0.079 \\
\hline KT1-LN & 1 & 2 & 15 & P06LM22 & 0.067 & 0.717 & 0.168 & 0.076 & 0.031 & 0.059 & 0.056 & 0.089 \\
\hline KT1-HK & 1 & 2 & 16 & P01LM22 & 0.062 & 0.638 & 0.149 & 0.079 & 0.036 & 13.4 & 0.048 & 0.075 \\
\hline Batch 1 & 1 & 2 & 17 & BCHLM123 & 0.122 & 0.838 & $<0.010$ & 0.075 & 0.303 & 2.99 & $<0.010$ & 0.803 \\
\hline Batch 1 & 2 & 1 & 1 & BCHLM211 & 0.123 & 0.844 & $<0.010$ & 0.075 & 0.308 & 2.86 & $<0.010$ & 0.807 \\
\hline KT1-LB & 2 & 1 & 2 & P12LM11 & 0.066 & 0.708 & 0.169 & 0.073 & 0.034 & 0.061 & 0.055 & 0.088 \\
\hline KT1-HL & 2 & 1 & 3 & P13LM11 & 0.062 & 0.698 & 0.159 & 0.069 & 0.028 & 0.058 & 0.053 & 0.085 \\
\hline KT1-LA & 2 & 1 & 4 & P07LM21 & 0.063 & 0.743 & 0.163 & 0.069 & 0.034 & 0.081 & 0.052 & 0.085 \\
\hline KT1-LB & 2 & 1 & 5 & P12LM21 & 0.063 & 0.721 & 0.168 & 0.071 & 0.035 & 0.062 & 0.055 & 0.085 \\
\hline KT1-LS & 2 & 1 & 6 & P11LM21 & 0.063 & 0.725 & 0.168 & 0.067 & 0.029 & 0.063 & 0.052 & 0.086 \\
\hline KT1-LA & 2 & 1 & 7 & P07LM11 & 0.067 & 0.721 & 0.171 & 0.073 & 0.034 & 0.070 & 0.054 & 0.090 \\
\hline KT1-HB & 2 & 1 & 8 & P08LM21 & 0.063 & 0.685 & 0.162 & 0.070 & 0.034 & 0.056 & 0.053 & 0.085 \\
\hline Batch 1 & 2 & 1 & 9 & BCHLM212 & 0.121 & 0.848 & $<0.010$ & 0.074 & 0.308 & 2.83 & $<0.010$ & 0.802 \\
\hline KT1-HL & 2 & 1 & 10 & P13LM21 & 0.063 & 0.698 & 0.160 & 0.069 & 0.029 & 0.057 & 0.053 & 0.085 \\
\hline KT1-HB & 2 & 1 & 11 & P08LM11 & 0.062 & 0.698 & 0.161 & 0.069 & 0.035 & 0.057 & 0.053 & 0.085 \\
\hline KT1-HA & 2 & 1 & 12 & P03LM11 & 0.068 & 0.732 & 0.176 & 0.075 & 0.036 & 0.058 & 0.058 & 0.094 \\
\hline KT1-LS & 2 & 1 & 13 & P11LM11 & 0.065 & 0.719 & 0.170 & 0.069 & 0.029 & 0.062 & 0.052 & 0.087 \\
\hline KT1-HA & 2 & 1 & 14 & P03LM21 & 0.068 & 0.730 & 0.176 & 0.078 & 0.035 & 0.058 & 0.058 & 0.095 \\
\hline Batch 1 & 2 & 1 & 15 & BCHLM213 & 0.125 & 0.830 & $<0.010$ & 0.077 & 0.306 & 2.75 & $<0.010$ & 0.818 \\
\hline Batch 1 & 2 & 2 & 1 & BCHLM221 & 0.119 & 0.857 & $<0.010$ & 0.072 & 0.310 & 2.89 & $<0.010$ & 0.788 \\
\hline KT1-HB & 2 & 2 & 2 & P08LM22 & 0.061 & 0.692 & 0.162 & 0.068 & 0.036 & 0.059 & 0.053 & 0.082 \\
\hline KT1-LA & 2 & 2 & 3 & P07LM12 & 0.067 & 0.729 & 0.171 & 0.071 & 0.036 & 0.073 & 0.055 & 0.087 \\
\hline KT1-HA & 2 & 2 & 4 & P03LM22 & 0.066 & 0.748 & 0.174 & 0.075 & 0.038 & 0.063 & 0.058 & 0.090 \\
\hline KT1-LS & 2 & 2 & 5 & P11LM22 & 0.064 & 0.726 & 0.167 & 0.066 & 0.031 & 0.065 & 0.052 & 0.084 \\
\hline KT1-HA & 2 & 2 & 6 & P03LM12 & 0.066 & 0.741 & 0.174 & 0.072 & 0.038 & 0.062 & 0.057 & 0.090 \\
\hline KT1-HL & 2 & 2 & 7 & P13LM12 & 0.061 & 0.705 & 0.159 & 0.067 & 0.030 & 0.062 & 0.053 & 0.082 \\
\hline KT1-HB & 2 & 2 & 8 & P08LM12 & 0.062 & 0.700 & 0.160 & 0.068 & 0.037 & 0.061 & 0.053 & 0.083 \\
\hline Batch 1 & 2 & 2 & 9 & BCHLM222 & 0.119 & 0.864 & $<0.010$ & 0.072 & 0.311 & 2.92 & $<0.010$ & 0.789 \\
\hline KT1-LS & 2 & 2 & 10 & P11LM12 & 0.063 & 0.723 & 0.168 & 0.066 & 0.031 & 0.067 & 0.052 & 0.083 \\
\hline KT1-LB & 2 & 2 & 11 & P12LM22 & 0.063 & 0.714 & 0.167 & 0.069 & 0.036 & 0.064 & 0.055 & 0.083 \\
\hline KT1-LA & 2 & 2 & 12 & P07LM22 & 0.063 & 0.738 & 0.161 & 0.067 & 0.036 & 0.083 & 0.052 & 0.083 \\
\hline KT1-LB & 2 & 2 & 13 & P12LM12 & 0.064 & 0.716 & 0.168 & 0.070 & 0.036 & 0.065 & 0.055 & 0.084 \\
\hline KT1-HL & 2 & 2 & 14 & P13LM22 & 0.062 & 0.702 & 0.158 & 0.067 & 0.031 & 0.060 & 0.053 & 0.082 \\
\hline Batch 1 & 2 & 2 & 15 & BCHLM223 & 0.120 & 0.846 & $<0.010$ & 0.073 & 0.306 & 2.89 & $<0.010$ & 0.801 \\
\hline
\end{tabular}


Table A-2. PSAL Chemical Composition Measurements of the KT01-Series of Glasses Using LM Preparation Method (Part 2).

\begin{tabular}{|c|c|c|c|c|c|c|c|c|c|c|c|c|c|c|}
\hline Glass ID & Block & $\begin{array}{l}\text { Sub- } \\
\text { Blk }\end{array}$ & Sequence & Lab ID & $\begin{array}{c}\mathrm{Mn} \\
(\mathrm{wt} \%)\end{array}$ & $\begin{array}{c}\mathrm{Na} \\
(\mathrm{wt} \%)\end{array}$ & $\begin{array}{c}\mathrm{Nb} \\
(\mathrm{wt} \%)\end{array}$ & $\begin{array}{c}\mathrm{Ni} \\
(\mathrm{wt} \%)\end{array}$ & $\begin{array}{c}\mathrm{Pb} \\
(\mathrm{wt} \%)\end{array}$ & $\begin{array}{c}\mathrm{S} \\
(\mathrm{wt} \%)\end{array}$ & $\begin{array}{c}\mathrm{Si} \\
(\mathrm{wt} \%)\end{array}$ & $\begin{array}{c}\mathrm{Ti} \\
(\mathrm{wt} \%)\end{array}$ & $\begin{array}{c}\mathrm{Zn} \\
(\mathrm{wt} \%)\end{array}$ & $\begin{array}{c}\mathrm{Zr} \\
(\mathrm{wt} \%)\end{array}$ \\
\hline Batch 1 & 1 & 1 & 1 & BCHLM111 & 1.32 & 6.54 & $<0.010$ & 0.532 & $<0.010$ & $<0.100$ & 23.4 & 0.418 & $<0.010$ & 0.066 \\
\hline KT1-HS & 1 & 1 & 2 & P05LM21 & 0.906 & 10 & 0.295 & 0.225 & 0.085 & $<0.100$ & 22.8 & 4.67 & 0.039 & 0.328 \\
\hline KT1-LL & 1 & 1 & 3 & P10LM21 & 0.967 & 10.5 & 0.313 & 0.249 & 0.094 & $<0.100$ & 21.1 & 4.69 & 0.048 & 0.354 \\
\hline KT1-LF & 1 & 1 & 4 & P04LM21 & 1.02 & 11 & 0.33 & 0.264 & 0.095 & $<0.100$ & 22.3 & 4.54 & 0.041 & 0.372 \\
\hline KT1-LL & 1 & 1 & 5 & P10LM11 & 0.963 & 10.4 & 0.32 & 0.257 & 0.098 & $<0.100$ & 21.1 & 4.64 & 0.04 & 0.361 \\
\hline KT1-HS & 1 & 1 & 6 & P05LM11 & 0.9 & 9.69 & 0.301 & 0.234 & 0.088 & $<0.100$ & 22.6 & 4.66 & 0.036 & 0.333 \\
\hline KT1-HN & 1 & 1 & 7 & P02LM21 & 0.949 & 11.7 & 0.308 & 0.26 & 0.096 & $<0.100$ & 20.9 & 4.69 & 0.039 & 0.347 \\
\hline KT1-HF & 1 & 1 & 8 & P09LM11 & 0.921 & 9.88 & 0.294 & 0.229 & 0.088 & $<0.100$ & 20.2 & 4.66 & 0.035 & 0.334 \\
\hline Batch 1 & 1 & 1 & 9 & BCHLM112 & 1.33 & 6.44 & $<0.010$ & 0.542 & $<0.010$ & $<0.100$ & 23.6 & 0.418 & $<0.010$ & 0.066 \\
\hline KT1-LF & 1 & 1 & 10 & P04LM11 & 1.01 & 10.9 & 0.332 & 0.268 & 0.098 & $<0.100$ & 22.6 & 4.59 & 0.043 & 0.376 \\
\hline KT1-LN & 1 & 1 & 11 & P06LM11 & 0.983 & 9.31 & 0.335 & 0.269 & 0.1 & $<0.100$ & 21.9 & 4.67 & 0.044 & 0.376 \\
\hline KT1-HN & 1 & 1 & 12 & P02LM11 & 0.928 & 11.6 & 0.306 & 0.26 & 0.097 & $<0.100$ & 20.7 & 4.6 & 0.038 & 0.348 \\
\hline KT1-HF & 1 & 1 & 13 & P09LM21 & 0.914 & 9.86 & 0.301 & 0.234 & 0.091 & $<0.100$ & 20.5 & 4.68 & 0.036 & 0.342 \\
\hline KT1-LN & 1 & 1 & 14 & P06LM21 & 0.992 & 9.29 & 0.333 & 0.266 & 0.1 & $<0.100$ & 22.1 & 4.65 & 0.052 & 0.373 \\
\hline KT1-HK & 1 & 1 & 15 & P01LM11 & 0.866 & 5.14 & 0.286 & 0.235 & 0.086 & $<0.100$ & 19.3 & 4.68 & 0.033 & 0.315 \\
\hline KT1-HK & 1 & 1 & 16 & P01LM21 & 0.877 & 5.29 & 0.288 & 0.243 & 0.089 & $<0.100$ & 19.7 & 4.71 & 0.034 & 0.316 \\
\hline Batch 1 & 1 & 1 & 17 & BCHLM113 & 1.33 & 6.25 & $<0.010$ & 0.55 & $<0.010$ & $<0.100$ & 23.4 & 0.414 & $<0.010$ & 0.067 \\
\hline Batch 1 & 1 & 2 & 1 & BCHLM121 & 1.3 & 6.46 & $<0.010$ & 0.527 & $<0.010$ & $<0.100$ & 23.5 & 0.398 & $<0.010$ & 0.065 \\
\hline KT1-LF & 1 & 2 & 2 & P04LM12 & 1.01 & 10.9 & 0.322 & 0.258 & 0.095 & $<0.100$ & 22.8 & 4.64 & 0.041 & 0.374 \\
\hline KT1-HN & 1 & 2 & 3 & P02LM12 & 0.922 & 11.8 & 0.295 & 0.251 & 0.094 & $<0.100$ & 21 & 4.66 & 0.036 & 0.345 \\
\hline KT1-HF & 1 & 2 & 4 & P09LM22 & 0.916 & 9.86 & 0.288 & 0.226 & 0.089 & $<0.100$ & 20.8 & 4.76 & 0.034 & 0.34 \\
\hline KT1-HS & 1 & 2 & 5 & P05LM12 & 0.901 & 9.87 & 0.29 & 0.225 & 0.085 & $<0.100$ & 22.9 & 4.74 & 0.034 & 0.331 \\
\hline KT1-HN & 1 & 2 & 6 & P02LM22 & 0.942 & 12 & 0.297 & 0.25 & 0.093 & $<0.100$ & 20.9 & 4.74 & 0.036 & 0.344 \\
\hline KT1-LN & 1 & 2 & 7 & P06LM12 & 0.987 & 9.41 & 0.323 & 0.259 & 0.096 & $<0.100$ & 21.9 & 4.74 & 0.042 & 0.372 \\
\hline KT1-LL & 1 & 2 & 8 & P10LM12 & 0.966 & 10.5 & 0.309 & 0.249 & 0.093 & $<0.100$ & 21.4 & 4.78 & 0.038 & 0.359 \\
\hline Batch 1 & 1 & 2 & 9 & BCHLM122 & 1.31 & 6.5 & $<0.010$ & 0.526 & $<0.010$ & $<0.100$ & 23.7 & 0.409 & $<0.010$ & 0.066 \\
\hline KT1-LL & 1 & 2 & 10 & P10LM22 & 0.942 & 10.6 & 0.3 & 0.245 & 0.093 & $<0.100$ & 21.2 & 4.65 & 0.047 & 0.353 \\
\hline KT1-HK & 1 & 2 & 11 & P01LM12 & 0.848 & 5.3 & 0.275 & 0.228 & 0.085 & $<0.100$ & 19.1 & 4.58 & 0.031 & 0.314 \\
\hline KT1-LF & 1 & 2 & 12 & P04LM22 & 1.01 & 11.2 & 0.315 & 0.256 & 0.092 & $<0.100$ & 22.3 & 4.54 & 0.038 & 0.369 \\
\hline KT1-HF & 1 & 2 & 13 & P09LM12 & 0.901 & 9.96 & 0.281 & 0.221 & 0.086 & $<0.100$ & 19.9 & 4.67 & 0.033 & 0.329 \\
\hline KT1-HS & 1 & 2 & 14 & P05LM22 & 0.898 & 9.93 & 0.282 & 0.222 & 0.084 & $<0.100$ & 22.7 & 4.69 & 0.038 & 0.326 \\
\hline KT1-LN & 1 & 2 & 15 & P06LM22 & 0.985 & 9.44 & 0.32 & 0.259 & 0.098 & $<0.100$ & 21.7 & 4.65 & 0.05 & 0.37 \\
\hline KT1-HK & 1 & 2 & 16 & P01LM22 & 0.872 & 5.47 & 0.278 & 0.234 & 0.086 & $<0.100$ & 19.2 & 4.71 & 0.032 & 0.316 \\
\hline Batch 1 & 1 & 2 & 17 & BCHLM123 & 1.32 & 6.49 & $<0.010$ & 0.522 & $<0.010$ & $<0.100$ & 23.6 & 0.405 & $<0.010$ & 0.066 \\
\hline Batch 1 & 2 & 1 & 1 & BCHLM211 & 1.28 & 6.54 & $<0.010$ & 0.531 & $<0.010$ & $<0.100$ & 23.1 & 0.397 & $<0.010$ & 0.065 \\
\hline KT1-LB & 2 & 1 & 2 & P12LM11 & 0.973 & 10.6 & 0.324 & 0.253 & 0.1 & $<0.100$ & 21.5 & 4.52 & 0.039 & 0.37 \\
\hline KT1-HL & 2 & 1 & 3 & P13LM11 & 0.932 & 10.2 & 0.311 & 0.244 & 0.09 & $<0.100$ & 20.5 & 4.6 & 0.035 & 0.349 \\
\hline KT1-LA & 2 & 1 & 4 & P07LM21 & 0.966 & 10.7 & 0.317 & 0.243 & 0.092 & $<0.100$ & 21.5 & 4.58 & 0.04 & 0.36 \\
\hline KT1-LB & 2 & 1 & 5 & P12LM21 & 0.966 & 10.6 & 0.323 & 0.246 & 0.097 & $<0.100$ & 21.6 & 4.54 & 0.038 & 0.368 \\
\hline KT1-LS & 2 & 1 & 6 & P11LM21 & 0.969 & 10.8 & 0.325 & 0.248 & 0.095 & $<0.100$ & 21.5 & 4.63 & 0.036 & 0.367 \\
\hline KT1-LA & 2 & 1 & 7 & P07LM11 & 0.978 & 11.4 & 0.334 & 0.263 & 0.1 & $<0.100$ & 21.7 & 4.61 & 0.039 & 0.375 \\
\hline KT1-HB & 2 & 1 & 8 & P08LM21 & 0.927 & 10.3 & 0.312 & 0.246 & 0.092 & $<0.100$ & 20.8 & 4.56 & 0.038 & 0.355 \\
\hline Batch 1 & 2 & 1 & 9 & BCHLM212 & 1.3 & 6.41 & $<0.010$ & 0.525 & $<0.010$ & $<0.100$ & 23.1 & 0.381 & $<0.010$ & 0.066 \\
\hline KT1-HL & 2 & 1 & 10 & P13LM21 & 0.963 & 10.3 & 0.309 & 0.244 & 0.091 & $<0.100$ & 21.1 & 4.7 & 0.035 & 0.348 \\
\hline KT1-HB & 2 & 1 & 11 & P08LM11 & 0.941 & 10.3 & 0.301 & 0.24 & 0.09 & $<0.100$ & 20.6 & 4.47 & 0.039 & 0.352 \\
\hline KT1-HA & 2 & 1 & 12 & P03LM11 & 1.04 & 11.3 & 0.332 & 0.267 & 0.106 & $<0.100$ & 22.4 & $<0.010$ & 0.04 & 0.388 \\
\hline KT1-LS & 2 & 1 & 13 & P11LM11 & 0.991 & 10.7 & 0.321 & 0.25 & 0.098 & $<0.100$ & 20.9 & 4.54 & 0.037 & 0.37 \\
\hline KT1-HA & 2 & 1 & 14 & P03LM21 & 1.03 & 11.4 & 0.334 & 0.24 & 0.106 & $<0.100$ & 22.3 & $<0.010$ & 0.039 & 0.388 \\
\hline Batch 1 & 2 & 1 & 15 & BCHLM213 & 1.3 & 6.45 & $<0.010$ & 0.537 & $<0.010$ & $<0.100$ & 22.6 & 0.378 & $<0.010$ & 0.065 \\
\hline Batch 1 & 2 & 2 & 1 & BCHLM221 & 1.31 & 6.42 & $<0.010$ & 0.519 & $<0.010$ & $<0.100$ & 22.9 & 0.387 & $<0.010$ & 0.064 \\
\hline KT1-HB & 2 & 2 & 2 & P08LM22 & 0.942 & 10.2 & 0.315 & 0.244 & 0.09 & $<0.100$ & 20.6 & 4.71 & 0.036 & 0.352 \\
\hline KT1-LA & 2 & 2 & 3 & P07LM12 & 0.992 & 11.1 & 0.336 & 0.261 & 0.097 & $<0.100$ & 21.6 & 4.72 & 0.038 & 0.374 \\
\hline KT1-HA & 2 & 2 & 4 & P03LM22 & 1.03 & 11.1 & 0.34 & 0.236 & 0.102 & $<0.100$ & 22.7 & $<0.010$ & 0.037 & 0.382 \\
\hline KT1-LS & 2 & 2 & 5 & P11LM22 & 0.981 & 10.5 & 0.325 & 0.248 & 0.094 & $<0.100$ & 21.3 & 4.74 & 0.035 & 0.364 \\
\hline KT1-HA & 2 & 2 & 6 & P03LM12 & 1.03 & 11 & 0.337 & 0.263 & 0.101 & $<0.100$ & 22.5 & $<0.010$ & 0.038 & 0.382 \\
\hline KT1-HL & 2 & 2 & 7 & P13LM12 & 0.937 & 10.2 & 0.309 & 0.24 & 0.087 & $<0.100$ & 20.4 & 4.7 & 0.033 & 0.347 \\
\hline KT1-HB & 2 & 2 & 8 & P08LM12 & 0.94 & 10.1 & 0.307 & 0.239 & 0.088 & $<0.100$ & 20.6 & 4.57 & 0.038 & 0.35 \\
\hline Batch 1 & 2 & 2 & 9 & BCHLM222 & 1.32 & 6.42 & $<0.010$ & 0.516 & $<0.010$ & $<0.100$ & 23.1 & 0.387 & $<0.010$ & 0.066 \\
\hline KT1-LS & 2 & 2 & 10 & P11LM12 & 0.98 & 11 & 0.323 & 0.245 & 0.095 & $<0.100$ & 21.3 & 4.71 & 0.034 & 0.362 \\
\hline KT1-LB & 2 & 2 & 11 & P12LM22 & 0.973 & 10.7 & 0.322 & 0.243 & 0.096 & $<0.100$ & 21.3 & 4.59 & 0.036 & 0.362 \\
\hline KT1-LA & 2 & 2 & 12 & P07LM22 & 0.971 & 10.8 & 0.315 & 0.24 & 0.09 & $<0.100$ & 21.5 & 4.63 & 0.038 & 0.355 \\
\hline KT1-LB & 2 & 2 & 13 & P12LM12 & 0.972 & 10.6 & 0.325 & 0.247 & 0.096 & $<0.100$ & 21.4 & 4.61 & 0.037 & 0.366 \\
\hline KT1-HL & 2 & 2 & 14 & P13LM22 & 0.952 & 10.3 & 0.311 & 0.241 & 0.088 & $<0.100$ & 20.6 & 4.73 & 0.033 & 0.345 \\
\hline Batch 1 & 2 & 2 & 15 & BCHLM223 & 1.31 & 6.54 & $<0.010$ & 0.524 & $<0.010$ & $<0.100$ & 23 & 0.376 & $<0.010$ & 0.065 \\
\hline
\end{tabular}


Table A-3. PSAL Chemical Composition Measurements of the KT01-Series of Glasses Using PF Preparation Method.

\begin{tabular}{|c|c|c|c|c|c|c|c|c|}
\hline Glass ID & Block & Sub-Blk & Sequence & Lab ID & Al (wt\%) & B (wt \%) & Fe (wt \%) & Li (wt\%) \\
\hline Batch 1 & 1 & 1 & 1 & BCHPF111 & 2.52 & 2.58 & 8.97 & 2.06 \\
\hline KT1-HF & 1 & 1 & 2 & P09PF11 & 2.76 & 1.37 & 11.0 & 2.00 \\
\hline KT1-LF & 1 & 1 & 3 & P04PF11 & 3.11 & 1.53 & 5.60 & 2.25 \\
\hline KT1-LF & 1 & 1 & 4 & P04PF21 & 3.11 & 1.56 & 5.61 & 2.26 \\
\hline KT1-HS & 1 & 1 & 5 & P05PF21 & 2.79 & 1.39 & 7.70 & 2.02 \\
\hline KT1-HK & 1 & 1 & 6 & P01PF11 & 2.77 & 1.37 & 7.53 & 0.65 \\
\hline KT1-HA & 1 & 1 & 7 & P03PF11 & 4.87 & 1.55 & 8.18 & 2.24 \\
\hline KT1-HS & 1 & 1 & 8 & P05PF11 & 2.78 & 1.37 & 7.51 & 2.01 \\
\hline Batch 1 & 1 & 1 & 9 & BCHPF112 & 2.51 & 2.43 & 8.93 & 2.07 \\
\hline KT1-HF & 1 & 1 & 10 & P09PF21 & 2.77 & 1.45 & 11.0 & 2.02 \\
\hline KT1-LL & 1 & 1 & 11 & P10PF21 & 2.93 & 1.51 & 7.98 & 2.19 \\
\hline KT1-HN & 1 & 1 & 12 & P02PF21 & 2.88 & 1.47 & 7.95 & 2.10 \\
\hline KT1-HN & 1 & 1 & 13 & P02PF11 & 2.89 & 1.48 & 7.88 & 2.09 \\
\hline KT1-HK & 1 & 1 & 14 & P01PF21 & 2.71 & 1.38 & 7.41 & 0.65 \\
\hline KT1-LL & 1 & 1 & 15 & P10PF11 & 2.98 & 1.52 & 8.11 & 2.22 \\
\hline KT1-HA & 1 & 1 & 16 & P03PF21 & 4.94 & 1.58 & 8.27 & 2.25 \\
\hline Batch 1 & 1 & 1 & 17 & BCHPF113 & 2.51 & 2.44 & 8.90 & 2.06 \\
\hline Batch 1 & 1 & 2 & 1 & BCHPF121 & 2.53 & 2.51 & 8.90 & 2.04 \\
\hline KT1-HA & 1 & 2 & 2 & P03PF12 & 4.96 & 1.61 & 8.25 & 2.25 \\
\hline KT1-LL & 1 & 2 & 3 & P10PF12 & 2.94 & 1.49 & 7.93 & 2.17 \\
\hline KT1-HS & 1 & 2 & 4 & P05PF22 & 2.79 & 1.39 & 7.62 & 2.00 \\
\hline KT1-LL & 1 & 2 & 5 & P10PF22 & 2.96 & 1.47 & 7.97 & 2.18 \\
\hline KT1-HN & 1 & 2 & 6 & P02PF22 & 2.84 & 1.41 & 7.80 & 2.03 \\
\hline KT1-HA & 1 & 2 & 7 & P03PF22 & 4.99 & 1.55 & 8.34 & 2.24 \\
\hline KT1-LF & 1 & 2 & 8 & P04PF22 & 3.14 & 1.55 & 5.72 & 2.25 \\
\hline Batch 1 & 1 & 2 & 9 & BCHPF122 & 2.53 & 2.39 & 8.96 & 2.04 \\
\hline KT1-HS & 1 & 2 & 10 & P05PF12 & 2.86 & 1.41 & 7.66 & 2.03 \\
\hline KT1-HK & 1 & 2 & 11 & P01PF22 & 2.70 & 1.33 & 7.37 & 0.63 \\
\hline KT1-HF & 1 & 2 & 12 & P09PF12 & 2.76 & 1.37 & 11.0 & 1.99 \\
\hline KT1-HK & 1 & 2 & 13 & P01PF12 & 2.78 & 1.36 & 7.60 & 0.64 \\
\hline KT1-LF & 1 & 2 & 14 & P04PF12 & 3.15 & 1.55 & 5.63 & 2.24 \\
\hline KT1-HF & 1 & 2 & 15 & P09PF22 & 2.80 & 1.38 & 11.0 & 2.00 \\
\hline KT1-HN & 1 & 2 & 16 & P02PF12 & 2.93 & 1.44 & 7.91 & 2.08 \\
\hline Batch 1 & 1 & 2 & 17 & BCHPF 123 & 2.52 & 2.40 & 8.93 & 2.04 \\
\hline Batch 1 & 2 & 1 & 1 & BCHPF211 & 2.48 & 2.48 & 8.89 & 2.03 \\
\hline KT1-LB & 2 & 1 & 2 & $\begin{array}{l}\text { P12PF11 } \\
\end{array}$ & 3.00 & 1.56 & 7.99 & 2.16 \\
\hline KT1-HB & 2 & 1 & 3 & P08PF21 & 2.82 & 2.20 & 7.55 & 2.02 \\
\hline KT1-LN & 2 & 1 & 4 & P06PF11 & 3.01 & 1.51 & 8.17 & 2.17 \\
\hline KT1-HB & 2 & 1 & 5 & P08PF11 & 2.83 & 2.22 & 7.80 & 2.04 \\
\hline KT1-LS & 2 & 1 & 6 & P11PF21 & 2.92 & 1.45 & 8.01 & 2.12 \\
\hline KT1-LN & 2 & 1 & 7 & P06PF21 & 2.98 & 1.46 & 8.02 & 2.15 \\
\hline KT1-LA & 2 & 1 & 8 & P07PF21 & 2.21 & 1.45 & 8.16 & 2.13 \\
\hline Batch 1 & 2 & 1 & 9 & BCHPF212 & 2.45 & 2.37 & 8.83 & 2.01 \\
\hline KT1-HL & 2 & 1 & 10 & P13PF21 & 2.87 & 1.46 & 7.95 & 3.29 \\
\hline KT1-HL & 2 & 1 & 11 & P13PF11 & 2.85 & 1.43 & 7.79 & 3.25 \\
\hline KT1-LB & 2 & 1 & 12 & P12PF21 & 2.90 & 1.47 & 7.81 & 2.09 \\
\hline KT1-LS & 2 & 1 & 13 & P11PF11 & 2.96 & 1.45 & 7.93 & 2.14 \\
\hline KT1-LA & 2 & 1 & 14 & P07PF11 & 2.17 & 1.41 & 7.91 & 2.09 \\
\hline Batch 1 & 2 & 1 & 15 & BCHPF213 & 2.45 & 2.36 & 8.84 & 2.01 \\
\hline Batch 1 & 2 & 2 & 1 & BCHPF221 & 2.53 & 2.53 & 8.96 & 2.07 \\
\hline KT1-LB & 2 & 2 & 2 & P12PF22 & 2.93 & 1.54 & 7.86 & 2.12 \\
\hline KT1-LA & 2 & 2 & 3 & P07PF22 & 2.25 & 1.51 & 8.20 & 2.16 \\
\hline KT1-HB & 2 & 2 & 4 & P08PF12 & 2.89 & 2.28 & 7.85 & 2.09 \\
\hline KT1-HL & 2 & 2 & 5 & P13PF22 & 2.86 & 1.45 & 8.00 & 3.31 \\
\hline KT1-LA & 2 & 2 & 6 & P07PF12 & 2.20 & 1.44 & 7.91 & 2.12 \\
\hline KT1-HL & 2 & 2 & 7 & P13PF12 & 2.90 & 1.44 & 7.91 & 3.33 \\
\hline KT1-LB & 2 & 2 & 8 & P12PF12 & 2.97 & 1.50 & 7.99 & 2.16 \\
\hline Batch 1 & 2 & 2 & 9 & BCHPF222 & 2.48 & 2.39 & 8.90 & 2.04 \\
\hline KT1-LN & 2 & 2 & 10 & P06PF12 & 3.00 & 1.53 & 8.19 & 2.18 \\
\hline KT1-LS & 2 & 2 & 11 & P11PF22 & 2.96 & 1.49 & 8.06 & 2.14 \\
\hline KT1-HB & 2 & 2 & 12 & P08PF22 & 2.83 & 2.22 & 7.69 & 2.05 \\
\hline KT1-LS & 2 & 2 & 13 & P11PF12 & 2.97 & 1.48 & 8.06 & 2.15 \\
\hline KT1-LN & 2 & 2 & 14 & P06PF22 & 2.99 & 1.49 & 8.08 & 2.17 \\
\hline Batch 1 & 2 & 2 & 15 & BCHPF223 & 2.49 & 2.42 & 8.93 & 2.05 \\
\hline
\end{tabular}


Table A-4. Comparison of Measured versus Targeted Composition for KT01 Glasses.

\begin{tabular}{|c|c|c|c|c|c|}
\hline Glass ID & Oxide & $\begin{array}{c}\text { Measured } \\
\text { (wt\%) }\end{array}$ & $\begin{array}{c}\text { Targeted } \\
\text { (wt\%) }\end{array}$ & $\begin{array}{c}\text { Diff of } \\
\text { Measured }\end{array}$ & $\begin{array}{c}\text { \% Diff of } \\
\text { Measured }\end{array}$ \\
\hline Batch 1 & $\mathrm{Al}_{2} \mathrm{O}_{3}$ & 4.7238 & 4.8770 & -0.1533 & $-3.1 \%$ \\
\hline Batch 1 & $\mathrm{~B}_{2} \mathrm{O}_{3}$ & 7.8619 & 7.7770 & 0.0849 & $1.1 \%$ \\
\hline Batch 1 & $\mathrm{BaO}$ & 0.1368 & 0.1510 & -0.0142 & $-9.4 \%$ \\
\hline Batch 1 & $\mathrm{CaO}$ & 1.1767 & 1.2200 & -0.0433 & $-3.5 \%$ \\
\hline Batch 1 & $\mathrm{Ce}_{2} \mathrm{O}_{3}$ & 0.0059 & 0.0000 & 0.0059 & \\
\hline Batch 1 & $\mathrm{Cr}_{2} \mathrm{O}_{3}$ & 0.1099 & 0.1070 & 0.0029 & $2.7 \%$ \\
\hline Batch 1 & $\mathrm{CuO}$ & 0.3831 & 0.3990 & -0.0159 & $-4.0 \%$ \\
\hline Batch 1 & $\mathrm{Fe}_{2} \mathrm{O}_{3}$ & 12.7410 & 12.8390 & -0.0980 & $-0.8 \%$ \\
\hline Batch 1 & $\mathrm{~K}_{2} \mathrm{O}$ & 3.5204 & 3.3270 & 0.1934 & $5.8 \%$ \\
\hline Batch 1 & $\mathrm{La}_{2} \mathrm{O}_{3}$ & 0.0059 & 0.0000 & 0.0059 & \\
\hline Batch 1 & $\mathrm{Li}_{2} \mathrm{O}$ & 4.3991 & 4.4290 & -0.0299 & $-0.7 \%$ \\
\hline Batch 1 & $\mathrm{MgO}$ & 1.3385 & 1.4190 & -0.0805 & $-5.7 \%$ \\
\hline Batch 1 & $\mathrm{MnO}$ & 1.6925 & 1.7260 & -0.0335 & $-1.9 \%$ \\
\hline Batch 1 & $\mathrm{Na}_{2} \mathrm{O}$ & 8.7013 & 9.0030 & -0.3017 & $-3.4 \%$ \\
\hline Batch 1 & $\mathrm{Nb}_{2} \mathrm{O}_{5}$ & 0.0072 & 0.0000 & 0.0072 & \\
\hline Batch 1 & $\mathrm{NiO}$ & 0.6735 & 0.7510 & -0.0775 & $-10.3 \%$ \\
\hline Batch 1 & $\mathrm{PbO}$ & 0.0054 & 0.0000 & 0.0054 & \\
\hline Batch 1 & $\mathrm{SiO}_{2}$ & 49.7387 & 50.2200 & -0.4813 & $-1.0 \%$ \\
\hline Batch 1 & $\mathrm{SO}_{4}$ & 0.1498 & 0.0000 & 0.1498 & \\
\hline Batch 1 & $\mathrm{TiO}_{2}$ & 0.6628 & 0.6770 & -0.0142 & $-2.1 \%$ \\
\hline Batch 1 & $\mathrm{ZnO}$ & 0.0062 & 0.0000 & 0.0062 & \\
\hline Batch 1 & $\mathrm{ZrO}_{2}$ & 0.0886 & 0.0980 & -0.0094 & $-9.6 \%$ \\
\hline Batch 1 & Sum & 98.1289 & 99.0200 & -0.8911 & $-0.9 \%$ \\
\hline KT1-HA & $\mathrm{Al}_{2} \mathrm{O}_{3}$ & 9.3341 & 8.4200 & 0.9141 & $10.9 \%$ \\
\hline KT1-HA & $\mathrm{B}_{2} \mathrm{O}_{3}$ & 5.0633 & 4.5200 & 0.5433 & $12.0 \%$ \\
\hline KT1-HA & $\mathrm{BaO}$ & 0.0748 & 0.0800 & -0.0052 & $-6.5 \%$ \\
\hline KT1-HA & $\mathrm{CaO}$ & 1.0323 & 0.9500 & 0.0823 & $8.7 \%$ \\
\hline KT1-HA & $\mathrm{Ce}_{2} \mathrm{O}_{3}$ & 0.2050 & 0.2000 & 0.0050 & $2.5 \%$ \\
\hline KT1-HA & $\mathrm{Cr}_{2} \mathrm{O}_{3}$ & 0.1096 & 0.1000 & 0.0096 & $9.6 \%$ \\
\hline KT1-HA & $\mathrm{CuO}$ & 0.0460 & 0.0300 & 0.0160 & $53.3 \%$ \\
\hline KT1-HA & $\mathrm{Fe}_{2} \mathrm{O}_{3}$ & 11.8093 & 11.3400 & 0.4693 & $4.1 \%$ \\
\hline KT1-HA & $\mathrm{K}_{2} \mathrm{O}$ & 0.0726 & 0.0700 & 0.0026 & $3.7 \%$ \\
\hline KT1-HA & $\mathrm{La}_{2} \mathrm{O}_{3}$ & 0.0677 & 0.0700 & -0.0023 & $-3.2 \%$ \\
\hline KT1-HA & $\mathrm{Li}_{2} \mathrm{O}$ & 4.8333 & 4.5200 & 0.3133 & $6.9 \%$ \\
\hline KT1-HA & $\mathrm{MgO}$ & 0.1530 & 0.1400 & 0.0130 & $9.3 \%$ \\
\hline KT1-HA & $\mathrm{MnO}$ & 1.3332 & 1.2200 & 0.1132 & $9.3 \%$ \\
\hline KT1-HA & $\mathrm{Na}_{2} \mathrm{O}$ & 15.0976 & 14.1400 & 0.9576 & $6.8 \%$ \\
\hline KT1-HA & $\mathrm{Nb}_{2} \mathrm{O}_{5}$ & 0.4803 & 0.4100 & 0.0703 & $17.1 \%$ \\
\hline KT1-HA & $\mathrm{NiO}$ & 0.3200 & 0.3400 & -0.0200 & $-5.9 \%$ \\
\hline KT1-HA & $\mathrm{PbO}$ & 0.1118 & 0.1100 & 0.0018 & $1.6 \%$ \\
\hline KT1-HA & $\mathrm{SiO}_{2}$ & 48.0808 & 44.7200 & 3.3608 & $7.5 \%$ \\
\hline KT1-HA & $\mathrm{SO}_{4}$ & 0.1498 & 0.0700 & 0.0798 & $114.0 \%$ \\
\hline KT1-HA & $\mathrm{TiO}_{2}$ & 0.0083 & 8.0000 & -7.9917 & $-99.9 \%$ \\
\hline KT1-HA & $\mathrm{ZnO}$ & 0.0479 & 0.0500 & -0.0021 & $-4.2 \%$ \\
\hline KT1-HA & $\mathrm{ZrO}_{2}$ & 0.5201 & 0.4900 & 0.0301 & $6.1 \%$ \\
\hline KT1-HA & Sum & 98.9507 & 99.9900 & -1.0393 & $-1.0 \%$ \\
\hline KT1-HB & $\mathrm{Al}_{2} \mathrm{O}_{3}$ & 5.3709 & 5.3800 & -0.0091 & $-0.2 \%$ \\
\hline KT1-HB & $\mathrm{B}_{2} \mathrm{O}_{3}$ & 7.1804 & 7.2000 & -0.0196 & $-0.3 \%$ \\
\hline KT1-HB & $\mathrm{BaO}$ & 0.0692 & 0.0800 & -0.0108 & $-13.5 \%$ \\
\hline KT1-HB & $\mathrm{CaO}$ & 0.9707 & 0.9600 & 0.0107 & $1.1 \%$ \\
\hline KT1-HB & $\mathrm{Ce}_{2} \mathrm{O}_{3}$ & 0.1889 & 0.2000 & -0.0111 & $-5.6 \%$ \\
\hline KT1-HB & $\mathrm{Cr}_{2} \mathrm{O}_{3}$ & 0.1005 & 0.1000 & 0.0005 & $0.5 \%$ \\
\hline KT1-HB & $\mathrm{CuO}$ & 0.0444 & 0.0300 & 0.0144 & $48.1 \%$ \\
\hline KT1-HB & $\mathrm{Fe}_{2} \mathrm{O}_{3}$ & 11.0409 & 11.3900 & -0.3491 & $-3.1 \%$ \\
\hline KT1-HB & $\mathrm{K}_{2} \mathrm{O}$ & 0.0702 & 0.0700 & 0.0002 & $0.2 \%$ \\
\hline KT1-HB & $\mathrm{La}_{2} \mathrm{O}_{3}$ & 0.0622 & 0.0700 & -0.0078 & $-11.2 \%$ \\
\hline KT1-HB & $\mathrm{Li}_{2} \mathrm{O}$ & 4.4134 & 4.5400 & -0.1266 & $-2.8 \%$ \\
\hline KT1-HB & $\mathrm{MgO}$ & 0.1389 & 0.1400 & -0.0011 & $-0.8 \%$ \\
\hline KT1-HB & $\mathrm{MnO}$ & 1.2105 & 1.2300 & -0.0195 & $-1.6 \%$ \\
\hline KT1-HB & $\mathrm{Na}_{2} \mathrm{O}$ & 13.7833 & 14.2000 & -0.4167 & $-2.9 \%$ \\
\hline KT1-HB & $\mathrm{Nb}_{2} \mathrm{O}_{5}$ & 0.4417 & 0.4100 & 0.0317 & $7.7 \%$ \\
\hline
\end{tabular}


Table A-4. Comparison of Measured versus Targeted Composition for KT01 Glasses. (continued)

\begin{tabular}{|c|c|c|c|c|c|}
\hline Glass ID & Oxide & $\begin{array}{c}\text { Measured } \\
(w t \%)\end{array}$ & $\begin{array}{c}\text { Targeted } \\
\text { (wt\%) }\end{array}$ & $\begin{array}{c}\text { Diff of } \\
\text { Measured }\end{array}$ & $\begin{array}{l}\text { \% Diff of } \\
\text { Measured }\end{array}$ \\
\hline KT1-HB & $\mathrm{NiO}$ & 0.3083 & 0.3400 & -0.0317 & $-9.3 \%$ \\
\hline KT1-HB & $\mathrm{PbO}$ & 0.0969 & 0.1100 & -0.0131 & $-11.9 \%$ \\
\hline KT1-HB & $\mathrm{SiO}_{2}$ & 44.1765 & 44.9200 & -0.7435 & $-1.7 \%$ \\
\hline KT1-HB & $\mathrm{SO}_{4}$ & 0.1498 & 0.0700 & 0.0798 & $114.0 \%$ \\
\hline KT1-HB & $\mathrm{TiO}_{2}$ & 7.6353 & 8.0000 & -0.3647 & $-4.6 \%$ \\
\hline KT1-HB & $\mathrm{ZnO}$ & 0.0470 & 0.0500 & -0.0030 & $-6.0 \%$ \\
\hline KT1-HB & $\mathrm{ZrO}_{2}$ & 0.4758 & 0.4900 & -0.0142 & $-2.9 \%$ \\
\hline KT1-HB & Sum & 97.9756 & 99.9800 & -2.0044 & $-2.0 \%$ \\
\hline KT1-HF & $\mathrm{Al}_{2} \mathrm{O}_{3}$ & 5.2386 & 5.2400 & -0.0014 & $0.0 \%$ \\
\hline KT1-HF & $\mathrm{B}_{2} \mathrm{O}_{3}$ & 4.4837 & 4.4200 & 0.0637 & $1.4 \%$ \\
\hline KT1-HF & $\mathrm{BaO}$ & 0.0684 & 0.0800 & -0.0116 & $-14.5 \%$ \\
\hline KT1-HF & $\mathrm{CaO}$ & 0.9448 & 0.9300 & 0.0148 & $1.6 \%$ \\
\hline KT1-HF & $\mathrm{Ce}_{2} \mathrm{O}_{3}$ & 0.1766 & 0.1900 & -0.0134 & $-7.1 \%$ \\
\hline KT1-HF & $\mathrm{Cr}_{2} \mathrm{O}_{3}$ & 0.1019 & 0.1000 & 0.0019 & $1.9 \%$ \\
\hline KT1-HF & $\mathrm{CuO}$ & 0.0344 & 0.0300 & 0.0044 & $14.7 \%$ \\
\hline KT1-HF & $\mathrm{Fe}_{2} \mathrm{O}_{3}$ & 15.7267 & 16.0800 & -0.3533 & $-2.2 \%$ \\
\hline KT1-HF & $\mathrm{K}_{2} \mathrm{O}$ & 0.0690 & 0.0700 & -0.0010 & $-1.5 \%$ \\
\hline KT1-HF & $\mathrm{La}_{2} \mathrm{O}_{3}$ & 0.0592 & 0.0700 & -0.0108 & $-15.4 \%$ \\
\hline KT1-HF & $\mathrm{Li}_{2} \mathrm{O}$ & 4.3112 & 4.4200 & -0.1088 & $-2.5 \%$ \\
\hline KT1-HF & $\mathrm{MgO}$ & 0.1343 & 0.1400 & -0.0057 & $-4.1 \%$ \\
\hline KT1-HF & $\mathrm{MnO}$ & 1.1789 & 1.2000 & -0.0211 & $-1.8 \%$ \\
\hline KT1-HF & $\mathrm{Na}_{2} \mathrm{O}$ & 13.3317 & 13.8400 & -0.5083 & $-3.7 \%$ \\
\hline KT1-HF & $\mathrm{Nb}_{2} \mathrm{O}_{5}$ & 0.4163 & 0.4000 & 0.0163 & $4.1 \%$ \\
\hline KT1-HF & $\mathrm{NiO}$ & 0.2895 & 0.3400 & -0.0505 & $-14.9 \%$ \\
\hline KT1-HF & $\mathrm{PbO}$ & 0.0953 & 0.1100 & -0.0147 & $-13.3 \%$ \\
\hline KT1-HF & $\mathrm{SiO}_{2}$ & 43.5348 & 43.7500 & -0.2152 & $-0.5 \%$ \\
\hline KT1-HF & $\mathrm{SO}_{4}$ & 0.1498 & 0.0700 & 0.0798 & $114.0 \%$ \\
\hline KT1-HF & $\mathrm{TiO}_{2}$ & 7.8271 & 8.0000 & -0.1729 & $-2.2 \%$ \\
\hline KT1-HF & $\mathrm{ZnO}$ & 0.0429 & 0.0500 & -0.0071 & $-14.1 \%$ \\
\hline KT1-HF & $\mathrm{ZrO}_{2}$ & 0.4542 & 0.4800 & -0.0258 & $-5.4 \%$ \\
\hline KT1-HF & Sum & 98.6694 & 100.0100 & -1.3406 & $-1.3 \%$ \\
\hline KT1-HK & $\mathrm{Al}_{2} \mathrm{O}_{3}$ & 5.1772 & 4.9700 & 0.2072 & $4.2 \%$ \\
\hline KT1-HK & $\mathrm{B}_{2} \mathrm{O}_{3}$ & 4.3791 & 4.2000 & 0.1791 & $4.3 \%$ \\
\hline KT1-HK & $\mathrm{BaO}$ & 0.0681 & 0.0700 & -0.0019 & $-2.7 \%$ \\
\hline KT1-HK & $\mathrm{CaO}$ & 0.8679 & 0.8900 & -0.0221 & $-2.5 \%$ \\
\hline KT1-HK & $\mathrm{Ce}_{2} \mathrm{O}_{3}$ & 0.1713 & 0.1900 & -0.0187 & $-9.8 \%$ \\
\hline KT1-HK & $\mathrm{Cr}_{2} \mathrm{O}_{3}$ & 0.1151 & 0.1000 & 0.0151 & $15.1 \%$ \\
\hline KT1-HK & $\mathrm{CuO}$ & 0.0441 & 0.0300 & 0.0141 & $47.1 \%$ \\
\hline KT1-HK & $\mathrm{Fe}_{2} \mathrm{O}_{3}$ & 10.6906 & 10.5400 & 0.1506 & $1.4 \%$ \\
\hline KT1-HK & $\mathrm{K}_{2} \mathrm{O}$ & 16.3826 & 18.3800 & -1.9974 & $-10.9 \%$ \\
\hline KT1-HK & $\mathrm{La}_{2} \mathrm{O}_{3}$ & 0.0566 & 0.0700 & -0.0134 & $-19.2 \%$ \\
\hline KT1-HK & $\mathrm{Li}_{2} \mathrm{O}$ & 1.3832 & 1.2800 & 0.1032 & $8.1 \%$ \\
\hline KT1-HK & $\mathrm{MgO}$ & 0.1256 & 0.1300 & -0.0044 & $-3.4 \%$ \\
\hline KT1-HK & $\mathrm{MnO}$ & 1.1179 & 1.1400 & -0.0221 & $-1.9 \%$ \\
\hline KT1-HK & $\mathrm{Na}_{2} \mathrm{O}$ & 7.1444 & 7.0900 & 0.0544 & $0.8 \%$ \\
\hline KT1-HK & $\mathrm{Nb}_{2} \mathrm{O}_{5}$ & 0.4030 & 0.3800 & 0.0230 & $6.1 \%$ \\
\hline KT1-HK & $\mathrm{NiO}$ & 0.2990 & 0.3200 & -0.0210 & $-6.6 \%$ \\
\hline KT1-HK & $\mathrm{PbO}$ & 0.0932 & 0.1000 & -0.0068 & $-6.8 \%$ \\
\hline KT1-HK & $\mathrm{SiO}_{2}$ & 41.3420 & 41.5600 & -0.2180 & $-0.5 \%$ \\
\hline KT1-HK & $\mathrm{SO}_{4}$ & 0.1498 & 0.0700 & 0.0798 & $114.0 \%$ \\
\hline KT1-HK & $\mathrm{TiO}_{2}$ & 7.7896 & 8.0000 & -0.2104 & $-2.6 \%$ \\
\hline KT1-HK & $\mathrm{ZnO}$ & 0.0405 & 0.0500 & -0.0095 & $-19.1 \%$ \\
\hline KT1-HK & $\mathrm{ZrO}_{2}$ & 0.4258 & 0.4600 & -0.0342 & $-7.4 \%$ \\
\hline KT1-HK & Sum & 98.2665 & 100.0200 & -1.7535 & $-1.8 \%$ \\
\hline KT1-HL & $\mathrm{Al}_{2} \mathrm{O}_{3}$ & 5.4229 & 5.3800 & 0.0429 & $0.8 \%$ \\
\hline KT1-HL & $\mathrm{B}_{2} \mathrm{O}_{3}$ & 4.6528 & 4.5400 & 0.1128 & $2.5 \%$ \\
\hline KT1-HL & $\mathrm{BaO}$ & 0.0692 & 0.0800 & -0.0108 & $-13.5 \%$ \\
\hline KT1-HL & $\mathrm{CaO}$ & 0.9805 & 0.9600 & 0.0205 & $2.1 \%$ \\
\hline KT1-HL & $\mathrm{Ce}_{2} \mathrm{O}_{3}$ & 0.1862 & 0.2000 & -0.0138 & $-6.9 \%$ \\
\hline KT1-HL & $\mathrm{Cr}_{2} \mathrm{O}_{3}$ & 0.0994 & 0.1000 & -0.0006 & $-0.6 \%$ \\
\hline
\end{tabular}


Table A-4. Comparison of Measured versus Targeted Composition for KT01 Glasses. (continued)

\begin{tabular}{|c|c|c|c|c|c|}
\hline Glass ID & Oxide & $\begin{array}{c}\text { Measured } \\
(w \mathbf{t} \%)\end{array}$ & $\begin{array}{c}\text { Targeted } \\
\text { (wt \%) }\end{array}$ & $\begin{array}{c}\text { Diff of } \\
\text { Measured }\end{array}$ & $\begin{array}{l}\text { \% Diff of } \\
\text { Measured }\end{array}$ \\
\hline KT1-HL & $\mathrm{CuO}$ & 0.0369 & 0.0300 & 0.0069 & $23.1 \%$ \\
\hline KT1-HL & $\mathrm{Fe}_{2} \mathrm{O}_{3}$ & 11.3125 & 11.3900 & -0.0775 & $-0.7 \%$ \\
\hline KT1-HL & $\mathrm{K}_{2} \mathrm{O}$ & 0.0714 & 0.0700 & 0.0014 & $2.0 \%$ \\
\hline KT1-HL & $\mathrm{La}_{2} \mathrm{O}_{3}$ & 0.0622 & 0.0700 & -0.0078 & $-11.2 \%$ \\
\hline KT1-HL & $\mathrm{Li}_{2} \mathrm{O}$ & 7.0938 & 7.2000 & -0.1062 & $-1.5 \%$ \\
\hline KT1-HL & $\mathrm{MgO}$ & 0.1385 & 0.1400 & -0.0015 & $-1.1 \%$ \\
\hline KT1-HL & $\mathrm{MnO}$ & 1.2215 & 1.2300 & -0.0085 & $-0.7 \%$ \\
\hline KT1-HL & $\mathrm{Na}_{2} \mathrm{O}$ & 13.8170 & 14.2000 & -0.3830 & $-2.7 \%$ \\
\hline KT1-HL & $\mathrm{Nb}_{2} \mathrm{O}_{5}$ & 0.4435 & 0.4100 & 0.0335 & $8.2 \%$ \\
\hline KT1-HL & $\mathrm{NiO}$ & 0.3083 & 0.3400 & -0.0317 & $-9.3 \%$ \\
\hline KT1-HL & $\mathrm{PbO}$ & 0.0959 & 0.1100 & -0.0141 & $-12.8 \%$ \\
\hline KT1-HL & $\mathrm{SiO}_{2}$ & 44.1765 & 44.9200 & -0.7435 & $-1.7 \%$ \\
\hline KT1-HL & $\mathrm{SO}_{4}$ & 0.1498 & 0.0700 & 0.0798 & $114.0 \%$ \\
\hline KT1-HL & $\mathrm{TiO}_{2}$ & 7.8104 & 8.0000 & -0.1896 & $-2.4 \%$ \\
\hline KT1-HL & $\mathrm{ZnO}$ & 0.0423 & 0.0500 & -0.0077 & $-15.4 \%$ \\
\hline KT1-HL & $\mathrm{ZrO}_{2}$ & 0.4691 & 0.4900 & -0.0209 & $-4.3 \%$ \\
\hline KT1-HL & Sum & 98.6604 & 99.9800 & -1.3196 & $-1.3 \%$ \\
\hline KT1-HN & $\mathrm{Al}_{2} \mathrm{O}_{3}$ & 5.4512 & 5.4100 & 0.0412 & $0.8 \%$ \\
\hline KT1-HN & $\mathrm{B}_{2} \mathrm{O}_{3}$ & 4.6689 & 4.5700 & 0.0989 & $2.2 \%$ \\
\hline KT1-HN & $\mathrm{BaO}$ & 0.0715 & 0.0800 & -0.0085 & $-10.7 \%$ \\
\hline KT1-HN & $\mathrm{CaO}$ & 0.9396 & 0.9600 & -0.0204 & $-2.1 \%$ \\
\hline KT1-HN & $\mathrm{Ce}_{2} \mathrm{O}_{3}$ & 0.1862 & 0.2000 & -0.0138 & $-6.9 \%$ \\
\hline KT1-HN & $\mathrm{Cr}_{2} \mathrm{O}_{3}$ & 0.1173 & 0.1000 & 0.0173 & $17.3 \%$ \\
\hline KT1-HN & $\mathrm{CuO}$ & 0.0419 & 0.0300 & 0.0119 & $39.8 \%$ \\
\hline KT1-HN & $\mathrm{Fe}_{2} \mathrm{O}_{3}$ & 11.2732 & 11.4600 & -0.1868 & $-1.6 \%$ \\
\hline KT1-HN & $\mathrm{K}_{2} \mathrm{O}$ & 0.0912 & 0.0700 & 0.0212 & $30.4 \%$ \\
\hline KT1-HN & $\mathrm{La}_{2} \mathrm{O}_{3}$ & 0.0613 & 0.0700 & -0.0087 & $-12.5 \%$ \\
\hline KT1-HN & $\mathrm{Li}_{2} \mathrm{O}$ & 4.4673 & 4.5700 & -0.1027 & $-2.2 \%$ \\
\hline KT1-HN & $\mathrm{MgO}$ & 0.1410 & 0.1500 & -0.0090 & $-6.0 \%$ \\
\hline KT1-HN & $\mathrm{MnO}$ & 1.2076 & 1.2400 & -0.0324 & $-2.6 \%$ \\
\hline KT1-HN & $\mathrm{Na}_{2} \mathrm{O}$ & 15.8727 & 16.4100 & -0.5373 & $-3.3 \%$ \\
\hline KT1-HN & $\mathrm{Nb}_{2} \mathrm{O}_{5}$ & 0.4313 & 0.4100 & 0.0213 & $5.2 \%$ \\
\hline KT1-HN & $\mathrm{NiO}$ & 0.3248 & 0.3500 & -0.0252 & $-7.2 \%$ \\
\hline KT1-HN & $\mathrm{PbO}$ & 0.1023 & 0.1100 & -0.0077 & $-7.0 \%$ \\
\hline KT1-HN & $\mathrm{SiO}_{2}$ & 44.6579 & 45.1900 & -0.5321 & $-1.2 \%$ \\
\hline KT1-HN & $\mathrm{SO}_{4}$ & 0.1498 & 0.0700 & 0.0798 & $114.0 \%$ \\
\hline KT1-HN & $\mathrm{TiO}_{2}$ & 7.7937 & 8.0000 & -0.2063 & $-2.6 \%$ \\
\hline KT1-HN & $\mathrm{ZnO}$ & 0.0464 & 0.0500 & -0.0036 & $-7.3 \%$ \\
\hline KT1-HN & $\mathrm{ZrO}_{2}$ & 0.4674 & 0.5000 & -0.0326 & $-6.5 \%$ \\
\hline KT1-HN & Sum & 98.5644 & 100.0000 & -1.4356 & $-1.4 \%$ \\
\hline KT1-HS & $\mathrm{Al}_{2} \mathrm{O}_{3}$ & 5.3000 & 5.1800 & 0.1200 & $2.3 \%$ \\
\hline KT1-HS & $\mathrm{B}_{2} \mathrm{O}_{3}$ & 4.4757 & 4.3700 & 0.1057 & $2.4 \%$ \\
\hline KT1-HS & $\mathrm{BaO}$ & 0.0684 & 0.0800 & -0.0116 & $-14.5 \%$ \\
\hline KT1-HS & $\mathrm{CaO}$ & 0.9336 & 0.9200 & 0.0136 & $1.5 \%$ \\
\hline KT1-HS & $\mathrm{Ce}_{2} \mathrm{O}_{3}$ & 0.1734 & 0.1900 & -0.0166 & $-8.8 \%$ \\
\hline KT1-HS & $\mathrm{Cr}_{2} \mathrm{O}_{3}$ & 0.0946 & 0.1000 & -0.0054 & $-5.4 \%$ \\
\hline KT1-HS & $\mathrm{CuO}$ & 0.0360 & 0.0300 & 0.0060 & $20.0 \%$ \\
\hline KT1-HS & $\mathrm{Fe}_{2} \mathrm{O}_{3}$ & 10.8979 & 10.9700 & -0.0721 & $-0.7 \%$ \\
\hline KT1-HS & $\mathrm{K}_{2} \mathrm{O}$ & 0.0750 & 0.0700 & 0.0050 & $7.1 \%$ \\
\hline KT1-HS & $\mathrm{La}_{2} \mathrm{O}_{3}$ & 0.0586 & 0.0700 & -0.0114 & $-16.2 \%$ \\
\hline KT1-HS & $\mathrm{Li}_{2} \mathrm{O}$ & 4.3381 & 4.3700 & -0.0319 & $-0.7 \%$ \\
\hline KT1-HS & $\mathrm{MgO}$ & 0.1327 & 0.1400 & -0.0073 & $-5.2 \%$ \\
\hline KT1-HS & $\mathrm{MnO}$ & 1.1637 & 1.1800 & -0.0163 & $-1.4 \%$ \\
\hline KT1-HS & $\mathrm{Na}_{2} \mathrm{O}$ & 13.3081 & 13.6700 & -0.3619 & $-2.6 \%$ \\
\hline KT1-HS & $\mathrm{Nb}_{2} \mathrm{O}_{5}$ & 0.4177 & 0.3900 & 0.0277 & $7.1 \%$ \\
\hline KT1-HS & $\mathrm{NiO}$ & 0.2882 & 0.3300 & -0.0418 & $-12.7 \%$ \\
\hline KT1-HS & $\mathrm{PbO}$ & 0.0921 & 0.1100 & -0.0179 & $-16.3 \%$ \\
\hline KT1-HS & $\mathrm{SiO}_{2}$ & 48.6691 & 49.2400 & -0.5709 & $-1.2 \%$ \\
\hline KT1-HS & $\mathrm{SO}_{4}$ & 0.1498 & 0.0700 & 0.0798 & $114.0 \%$ \\
\hline
\end{tabular}


Table A-4. Comparison of Measured versus Targeted Composition for KT01 Glasses. (continued)

\begin{tabular}{|c|c|c|c|c|c|}
\hline Glass ID & Oxide & $\begin{array}{c}\text { Measured } \\
(w t \%)\end{array}$ & $\begin{array}{c}\text { Targeted } \\
\text { (wt\%) }\end{array}$ & $\begin{array}{c}\text { Diff of } \\
\text { Measured }\end{array}$ & $\begin{array}{l}\text { \% Diff of } \\
\text { Measured }\end{array}$ \\
\hline KT1-HS & $\mathrm{TiO}_{2}$ & 7.8229 & 8.0000 & -0.1771 & $-2.2 \%$ \\
\hline KT1-HS & $\mathrm{ZnO}$ & 0.0457 & 0.0500 & -0.0043 & $-8.5 \%$ \\
\hline KT1-HS & $\mathrm{ZrO}_{2}$ & 0.4451 & 0.4800 & -0.0349 & $-7.3 \%$ \\
\hline KT1-HS & Sum & 98.9864 & 100.0100 & -1.0236 & $-1.0 \%$ \\
\hline KT1-LA & $\mathrm{Al}_{2} \mathrm{O}_{3}$ & 4.1711 & 4.1900 & -0.0189 & $-0.5 \%$ \\
\hline KT1-LA & $\mathrm{B}_{2} \mathrm{O}_{3}$ & 4.6769 & 4.7500 & -0.0731 & $-1.5 \%$ \\
\hline KT1-LA & $\mathrm{BaO}$ & 0.0726 & 0.0800 & -0.0074 & $-9.3 \%$ \\
\hline KT1-LA & $\mathrm{CaO}$ & 1.0253 & 1.0000 & 0.0253 & $2.5 \%$ \\
\hline KT1-LA & $\mathrm{Ce}_{2} \mathrm{O}_{3}$ & 0.1950 & 0.2100 & -0.0150 & $-7.1 \%$ \\
\hline KT1-LA & $\mathrm{Cr}_{2} \mathrm{O}_{3}$ & 0.1023 & 0.1100 & -0.0077 & $-7.0 \%$ \\
\hline KT1-LA & $\mathrm{CuO}$ & 0.0438 & 0.0300 & 0.0138 & $46.0 \%$ \\
\hline KT1-LA & $\mathrm{Fe}_{2} \mathrm{O}_{3}$ & 11.5019 & 11.9200 & -0.4181 & $-3.5 \%$ \\
\hline KT1-LA & $\mathrm{K}_{2} \mathrm{O}$ & 0.0925 & 0.0700 & 0.0225 & $32.1 \%$ \\
\hline KT1-LA & $\mathrm{La}_{2} \mathrm{O}_{3}$ & 0.0625 & 0.0800 & -0.0175 & $-21.9 \%$ \\
\hline KT1-LA & $\mathrm{Li}_{2} \mathrm{O}$ & 4.5749 & 4.7500 & -0.1751 & $-3.7 \%$ \\
\hline KT1-LA & $\mathrm{MgO}$ & 0.1430 & 0.1500 & -0.0070 & $-4.6 \%$ \\
\hline KT1-LA & $\mathrm{MnO}$ & 1.2612 & 1.2800 & -0.0188 & $-1.5 \%$ \\
\hline KT1-LA & $\mathrm{Na}_{2} \mathrm{O}$ & 14.8280 & 14.8600 & -0.0320 & $-0.2 \%$ \\
\hline KT1-LA & $\mathrm{Nb}_{2} \mathrm{O}_{5}$ & 0.4656 & 0.4300 & 0.0356 & $8.3 \%$ \\
\hline KT1-LA & $\mathrm{NiO}$ & 0.3204 & 0.3600 & -0.0396 & $-11.0 \%$ \\
\hline KT1-LA & $\mathrm{PbO}$ & 0.1021 & 0.1200 & -0.0179 & $-14.9 \%$ \\
\hline KT1-LA & $\mathrm{SiO}_{2}$ & 46.1554 & 46.9800 & -0.8246 & $-1.8 \%$ \\
\hline KT1-LA & $\mathrm{SO}_{4}$ & 0.1498 & 0.0700 & 0.0798 & $114.0 \%$ \\
\hline KT1-LA & $\mathrm{TiO}_{2}$ & 7.7312 & 8.0000 & -0.2688 & $-3.4 \%$ \\
\hline KT1-LA & $\mathrm{ZnO}$ & 0.0482 & 0.0500 & -0.0018 & $-3.5 \%$ \\
\hline KT1-LA & $\mathrm{ZrO}_{2}$ & 0.4944 & 0.5200 & -0.0256 & $-4.9 \%$ \\
\hline KT1-LA & Sum & 98.2180 & 100.0100 & -1.7920 & $-1.8 \%$ \\
\hline KT1-LB & $\mathrm{Al}_{2} \mathrm{O}_{3}$ & 5.5740 & 5.5300 & 0.0440 & $0.8 \%$ \\
\hline KT1-LB & $\mathrm{B}_{2} \mathrm{O}_{3}$ & 4.8862 & 4.8000 & 0.0862 & $1.8 \%$ \\
\hline KT1-LB & $\mathrm{BaO}$ & 0.0715 & 0.0800 & -0.0085 & $-10.7 \%$ \\
\hline KT1-LB & $\mathrm{CaO}$ & 1.0001 & 0.9800 & 0.0201 & $2.0 \%$ \\
\hline KT1-LB & $\mathrm{Ce}_{2} \mathrm{O}_{3}$ & 0.1968 & 0.2100 & -0.0132 & $-6.3 \%$ \\
\hline KT1-LB & $\mathrm{Cr}_{2} \mathrm{O}_{3}$ & 0.1034 & 0.1100 & -0.0066 & $-6.0 \%$ \\
\hline KT1-LB & $\mathrm{CuO}$ & 0.0441 & 0.0300 & 0.0141 & $47.1 \%$ \\
\hline KT1-LB & $\mathrm{Fe}_{2} \mathrm{O}_{3}$ & 11.3125 & 11.7200 & -0.4075 & $-3.5 \%$ \\
\hline KT1-LB & $\mathrm{K}_{2} \mathrm{O}$ & 0.0759 & 0.0700 & 0.0059 & $8.4 \%$ \\
\hline KT1-LB & $\mathrm{La}_{2} \mathrm{O}_{3}$ & 0.0645 & 0.0800 & -0.0155 & $-19.4 \%$ \\
\hline KT1-LB & $\mathrm{Li}_{2} \mathrm{O}$ & 4.5911 & 4.6700 & -0.0789 & $-1.7 \%$ \\
\hline KT1-LB & $\mathrm{MgO}$ & 0.1410 & 0.1500 & -0.0090 & $-6.0 \%$ \\
\hline KT1-LB & $\mathrm{MnO}$ & 1.2538 & 1.2600 & -0.0062 & $-0.5 \%$ \\
\hline KT1-LB & $\mathrm{Na}_{2} \mathrm{O}$ & 14.3225 & 14.6100 & -0.2875 & $-2.0 \%$ \\
\hline KT1-LB & $\mathrm{Nb}_{2} \mathrm{O}_{5}$ & 0.4628 & 0.4200 & 0.0428 & $10.2 \%$ \\
\hline KT1-LB & $\mathrm{NiO}$ & 0.3146 & 0.3500 & -0.0354 & $-10.1 \%$ \\
\hline KT1-LB & $\mathrm{PbO}$ & 0.1048 & 0.1100 & -0.0052 & $-4.8 \%$ \\
\hline KT1-LB & $\mathrm{SiO}_{2}$ & 45.8880 & 46.1900 & -0.3020 & $-0.7 \%$ \\
\hline KT1-LB & $\mathrm{SO}_{4}$ & 0.1498 & 0.0700 & 0.0798 & $114.0 \%$ \\
\hline KT1-LB & $\mathrm{TiO}_{2}$ & 7.6144 & 8.0000 & -0.3856 & $-4.8 \%$ \\
\hline KT1-LB & $\mathrm{ZnO}$ & 0.0467 & 0.0500 & -0.0033 & $-6.6 \%$ \\
\hline KT1-LB & $\mathrm{ZrO}_{2}$ & 0.4951 & 0.5100 & -0.0149 & $-2.9 \%$ \\
\hline KT1-LB & Sum & 98.7133 & 100.0000 & -1.2867 & $-1.3 \%$ \\
\hline KT1-LF & $\mathrm{Al}_{2} \mathrm{O}_{3}$ & 5.9094 & 5.7800 & 0.1294 & $2.2 \%$ \\
\hline KT1-LF & $\mathrm{B}_{2} \mathrm{O}_{3}$ & 4.9828 & 4.8800 & 0.1028 & $2.1 \%$ \\
\hline KT1-LF & $\mathrm{BaO}$ & 0.0762 & 0.0800 & -0.0038 & $-4.7 \%$ \\
\hline KT1-LF & $\mathrm{CaO}$ & 1.0225 & 1.0300 & -0.0075 & $-0.7 \%$ \\
\hline KT1-LF & $\mathrm{Ce}_{2} \mathrm{O}_{3}$ & 0.1959 & 0.2200 & -0.0241 & $-11.0 \%$ \\
\hline KT1-LF & $\mathrm{Cr}_{2} \mathrm{O}_{3}$ & 0.1111 & 0.1100 & 0.0011 & $1.0 \%$ \\
\hline KT1-LF & $\mathrm{CuO}$ & 0.0372 & 0.0300 & 0.0072 & $24.1 \%$ \\
\hline KT1-LF & $\mathrm{Fe}_{2} \mathrm{O}_{3}$ & 8.0635 & 8.2400 & -0.1765 & $-2.1 \%$ \\
\hline KT1-LF & $\mathrm{K}_{2} \mathrm{O}$ & 0.0783 & 0.0800 & -0.0017 & $-2.1 \%$ \\
\hline
\end{tabular}


Table A-4. Comparison of Measured versus Targeted Composition for KT01 Glasses. (continued)

\begin{tabular}{|c|c|c|c|c|c|}
\hline Glass ID & Oxide & $\begin{array}{c}\text { Measured } \\
\text { (wt\%) }\end{array}$ & $\begin{array}{c}\text { Targeted } \\
\text { (wt\%) }\end{array}$ & $\begin{array}{c}\text { Diff of } \\
\text { Measured }\end{array}$ & $\begin{array}{l}\text { \% Diff of } \\
\text { Measured }\end{array}$ \\
\hline KT1-LF & $\mathrm{La}_{2} \mathrm{O}_{3}$ & 0.0651 & 0.0800 & -0.0149 & $-18.6 \%$ \\
\hline KT1-LF & $\mathrm{Li}_{2} \mathrm{O}$ & 4.8440 & 4.8800 & -0.0360 & $-0.7 \%$ \\
\hline KT1-LF & $\mathrm{MgO}$ & 0.1497 & 0.1500 & -0.0003 & $-0.2 \%$ \\
\hline KT1-LF & $\mathrm{MnO}$ & 1.3073 & 1.3200 & -0.0127 & $-1.0 \%$ \\
\hline KT1-LF & $\mathrm{Na}_{2} \mathrm{O}$ & 14.8280 & 15.2600 & -0.4320 & $-2.8 \%$ \\
\hline KT1-LF & $\mathrm{Nb}_{2} \mathrm{O}_{5}$ & 0.4646 & 0.4400 & 0.0246 & $5.6 \%$ \\
\hline KT1-LF & $\mathrm{NiO}$ & 0.3328 & 0.3700 & -0.0372 & $-10.1 \%$ \\
\hline KT1-LF & $\mathrm{PbO}$ & 0.1023 & 0.1200 & -0.0177 & $-14.7 \%$ \\
\hline KT1-LF & $\mathrm{SiO}_{2}$ & 48.1343 & 48.2700 & -0.1358 & $-0.3 \%$ \\
\hline KT1-LF & $\mathrm{SO}_{4}$ & 0.1498 & 0.0800 & 0.0698 & $87.2 \%$ \\
\hline KT1-LF & $\mathrm{TiO}_{2}$ & 7.6353 & 8.0000 & -0.3647 & $-4.6 \%$ \\
\hline KT1-LF & $\mathrm{ZnO}$ & 0.0507 & 0.0500 & 0.0007 & $1.5 \%$ \\
\hline KT1-LF & $\mathrm{ZrO}_{2}$ & 0.5035 & 0.5300 & -0.0265 & $-5.0 \%$ \\
\hline KT1-LF & Sum & 99.0442 & 100.0000 & -0.9558 & $-1.0 \%$ \\
\hline KT1-LL & $\mathrm{Al}_{2} \mathrm{O}_{3}$ & 5.5787 & 5.5300 & 0.0487 & $0.9 \%$ \\
\hline KT1-LL & $\mathrm{B}_{2} \mathrm{O}_{3}$ & 4.8218 & 4.6700 & 0.1518 & $3.3 \%$ \\
\hline KT1-LL & $\mathrm{BaO}$ & 0.0717 & 0.0800 & -0.0083 & $-10.3 \%$ \\
\hline KT1-LL & $\mathrm{CaO}$ & 0.9945 & 0.9800 & 0.0145 & $1.5 \%$ \\
\hline KT1-LL & $\mathrm{Ce}_{2} \mathrm{O}_{3}$ & 0.1874 & 0.2100 & -0.0226 & $-10.8 \%$ \\
\hline KT1-LL & $\mathrm{Cr}_{2} \mathrm{O}_{3}$ & 0.1071 & 0.1100 & -0.0029 & $-2.7 \%$ \\
\hline KT1-LL & $\mathrm{CuO}$ & 0.0404 & 0.0300 & 0.0104 & $34.6 \%$ \\
\hline KT1-LL & $\mathrm{Fe}_{2} \mathrm{O}_{3}$ & 11.4340 & 11.7200 & -0.2860 & $-2.4 \%$ \\
\hline KT1-LL & $\mathrm{K}_{2} \mathrm{O}$ & 0.0672 & 0.0700 & -0.0028 & $-4.1 \%$ \\
\hline KT1-LL & $\mathrm{La}_{2} \mathrm{O}_{3}$ & 0.0639 & 0.0800 & -0.0161 & $-20.1 \%$ \\
\hline KT1-LL & $\mathrm{Li}_{2} \mathrm{O}$ & 4.7149 & 4.8000 & -0.0851 & $-1.8 \%$ \\
\hline KT1-LL & $\mathrm{MgO}$ & 0.1447 & 0.1500 & -0.0053 & $-3.5 \%$ \\
\hline KT1-LL & $\mathrm{MnO}$ & 1.2389 & 1.2600 & -0.0211 & $-1.7 \%$ \\
\hline KT1-LL & $\mathrm{Na}_{2} \mathrm{O}$ & 14.1540 & 14.6100 & -0.4560 & $-3.1 \%$ \\
\hline KT1-LL & $\mathrm{Nb}_{2} \mathrm{O}_{5}$ & 0.4442 & 0.4200 & 0.0242 & $5.8 \%$ \\
\hline KT1-LL & $\mathrm{NiO}$ & 0.3181 & 0.3500 & -0.0319 & $-9.1 \%$ \\
\hline KT1-LL & $\mathrm{PbO}$ & 0.1018 & 0.1100 & -0.0082 & $-7.5 \%$ \\
\hline KT1-LL & $\mathrm{SiO}_{2}$ & 45.3532 & 46.1900 & -0.8368 & $-1.8 \%$ \\
\hline KT1-LL & $\mathrm{SO}_{4}$ & 0.1498 & 0.0700 & 0.0798 & $114.0 \%$ \\
\hline KT1-LL & $\mathrm{TiO}_{2}$ & 7.8229 & 8.0000 & -0.1771 & $-2.2 \%$ \\
\hline KT1-LL & $\mathrm{ZnO}$ & 0.0538 & 0.0500 & 0.0038 & $7.7 \%$ \\
\hline KT1-LL & $\mathrm{ZrO}_{2}$ & 0.4819 & 0.5100 & -0.0281 & $-5.5 \%$ \\
\hline KT1-LL & Sum & 98.3449 & 100.0000 & -1.6551 & $-1.7 \%$ \\
\hline KT1-LN & $\mathrm{Al}_{2} \mathrm{O}_{3}$ & 5.6591 & 5.6500 & 0.0091 & $0.2 \%$ \\
\hline KT1-LN & $\mathrm{B}_{2} \mathrm{O}_{3}$ & 4.8218 & 4.7700 & 0.0518 & $1.1 \%$ \\
\hline KT1-LN & $\mathrm{BaO}$ & 0.0762 & 0.0800 & -0.0038 & $-4.7 \%$ \\
\hline KT1-LN & $\mathrm{CaO}$ & 1.0018 & 1.0000 & 0.0018 & $0.2 \%$ \\
\hline KT1-LN & $\mathrm{Ce}_{2} \mathrm{O}_{3}$ & 0.1971 & 0.2100 & -0.0129 & $-6.2 \%$ \\
\hline KT1-LN & $\mathrm{Cr}_{2} \mathrm{O}_{3}$ & 0.1140 & 0.1100 & 0.0040 & $3.6 \%$ \\
\hline KT1-LN & $\mathrm{CuO}$ & 0.0388 & 0.0300 & 0.0088 & $29.4 \%$ \\
\hline KT1-LN & $\mathrm{Fe}_{2} \mathrm{O}_{3}$ & 11.6020 & 11.9700 & -0.3680 & $-3.1 \%$ \\
\hline KT1-LN & $\mathrm{K}_{2} \mathrm{O}$ & 0.0684 & 0.0700 & -0.0016 & $-2.3 \%$ \\
\hline KT1-LN & $\mathrm{La}_{2} \mathrm{O}_{3}$ & 0.0663 & 0.0800 & -0.0137 & $-17.2 \%$ \\
\hline KT1-LN & $\mathrm{Li}_{2} \mathrm{O}$ & 4.6664 & 4.7700 & -0.1036 & $-2.2 \%$ \\
\hline KT1-LN & $\mathrm{MgO}$ & 0.1513 & 0.1500 & 0.0013 & $0.9 \%$ \\
\hline KT1-LN & $\mathrm{MnO}$ & 1.2741 & 1.2900 & -0.0159 & $-1.2 \%$ \\
\hline KT1-LN & $\mathrm{Na}_{2} \mathrm{O}$ & 12.6207 & 13.0700 & -0.4494 & $-3.4 \%$ \\
\hline KT1-LN & $\mathrm{Nb}_{2} \mathrm{O}_{5}$ & 0.4688 & 0.4300 & 0.0388 & $9.0 \%$ \\
\hline KT1-LN & $\mathrm{NiO}$ & 0.3350 & 0.3600 & -0.0250 & $-6.9 \%$ \\
\hline KT1-LN & $\mathrm{PbO}$ & 0.1061 & 0.1200 & -0.0139 & $-11.6 \%$ \\
\hline KT1-LN & $\mathrm{SiO}_{2}$ & 46.8507 & 47.1900 & -0.3393 & $-0.7 \%$ \\
\hline KT1-LN & $\mathrm{SO}_{4}$ & 0.1498 & 0.0800 & 0.0698 & $87.2 \%$ \\
\hline KT1-LN & $\mathrm{TiO}_{2}$ & 7.8021 & 8.0000 & -0.1979 & $-2.5 \%$ \\
\hline KT1-LN & $\mathrm{ZnO}$ & 0.0585 & 0.0500 & 0.0085 & $17.0 \%$ \\
\hline KT1-LN & $\mathrm{ZrO}_{2}$ & 0.5035 & 0.5200 & -0.0165 & $-3.2 \%$ \\
\hline
\end{tabular}


Table A-4. Comparison of Measured versus Targeted Composition for KT01 Glasses. (continued)

\begin{tabular}{|c|c|c|c|c|c|}
\hline Glass ID & Oxide & $\begin{array}{c}\text { Measured } \\
\text { (wt\%) }\end{array}$ & $\begin{array}{c}\text { Targeted } \\
(\mathrm{wt} \%)\end{array}$ & $\begin{array}{c}\text { Diff of } \\
\text { Measured }\end{array}$ & $\begin{array}{l}\text { \% Diff of } \\
\text { Measured }\end{array}$ \\
\hline KT1-LN & Sum & 98.6324 & 100.0000 & -1.3676 & $-1.4 \%$ \\
\hline KT1-LS & $\mathrm{Al}_{2} \mathrm{O}_{3}$ & 5.5787 & 5.5700 & 0.0087 & $0.2 \%$ \\
\hline KT1-LS & $\mathrm{B}_{2} \mathrm{O}_{3}$ & 4.7252 & 4.7000 & 0.0252 & $0.5 \%$ \\
\hline KT1-LS & $\mathrm{BaO}$ & 0.0712 & 0.0800 & -0.0088 & $-11.0 \%$ \\
\hline KT1-LS & $\mathrm{CaO}$ & 1.0120 & 0.9900 & 0.0220 & $2.2 \%$ \\
\hline KT1-LS & $\mathrm{Ce}_{2} \mathrm{O}_{3}$ & 0.1971 & 0.2100 & -0.0129 & $-6.2 \%$ \\
\hline KT1-LS & $\mathrm{Cr}_{2} \mathrm{O}_{3}$ & 0.0979 & 0.1100 & -0.0121 & $-11.0 \%$ \\
\hline KT1-LS & $\mathrm{CuO}$ & 0.0376 & 0.0300 & 0.0076 & $25.2 \%$ \\
\hline KT1-LS & $\mathrm{Fe}_{2} \mathrm{O}_{3}$ & 11.4590 & 11.8100 & -0.3510 & $-3.0 \%$ \\
\hline KT1-LS & $\mathrm{K}_{2} \mathrm{O}$ & 0.0774 & 0.0700 & 0.0074 & $10.6 \%$ \\
\hline KT1-LS & $\mathrm{La}_{2} \mathrm{O}_{3}$ & 0.0610 & 0.0800 & -0.0190 & $-23.8 \%$ \\
\hline KT1-LS & $\mathrm{Li}_{2} \mathrm{O}$ & 4.6018 & 4.7000 & -0.0982 & $-2.1 \%$ \\
\hline KT1-LS & $\mathrm{MgO}$ & 0.1410 & 0.1500 & -0.0090 & $-6.0 \%$ \\
\hline KT1-LS & $\mathrm{MnO}$ & 1.2657 & 1.2700 & -0.0043 & $-0.3 \%$ \\
\hline KT1-LS & $\mathrm{Na}_{2} \mathrm{O}$ & 14.4910 & 14.7300 & -0.2390 & $-1.6 \%$ \\
\hline KT1-LS & $\mathrm{Nb}_{2} \mathrm{O}_{5}$ & 0.4628 & 0.4200 & 0.0428 & $10.2 \%$ \\
\hline KT1-LS & $\mathrm{NiO}$ & 0.3153 & 0.3600 & -0.0447 & $-12.4 \%$ \\
\hline KT1-LS & $\mathrm{PbO}$ & 0.1029 & 0.1100 & -0.0071 & $-6.5 \%$ \\
\hline KT1-LS & $\mathrm{SiO}_{2}$ & 45.4601 & 45.9600 & -0.4999 & $-1.1 \%$ \\
\hline KT1-LS & $\mathrm{SO}_{4}$ & 0.1498 & 0.0700 & 0.0798 & $114.0 \%$ \\
\hline KT1-LS & $\mathrm{TiO}_{2}$ & 7.7645 & 8.0000 & -0.2355 & $-2.9 \%$ \\
\hline KT1-LS & $\mathrm{ZnO}$ & 0.0442 & 0.0500 & -0.0058 & $-11.6 \%$ \\
\hline KT1-LS & $\mathrm{ZrO}_{2}$ & 0.4941 & 0.5100 & -0.0159 & $-3.1 \%$ \\
\hline KT1-LS & Sum & 98.6102 & 99.9800 & -1.3698 & $-1.4 \%$ \\
\hline
\end{tabular}


Exhibit A-1. Measurements in Analytical Sequence for KT01-Series by Preparation Method by Oxide.

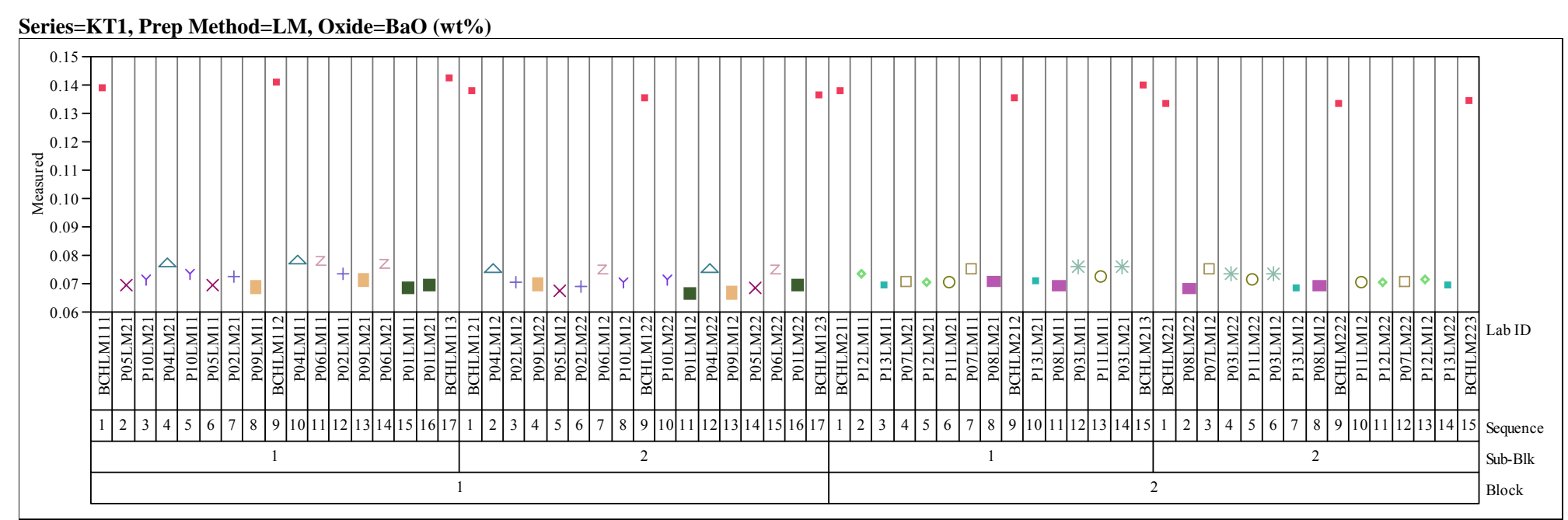

Series=KT1, Prep Method=LM, Oxide=CaO (wt\%)

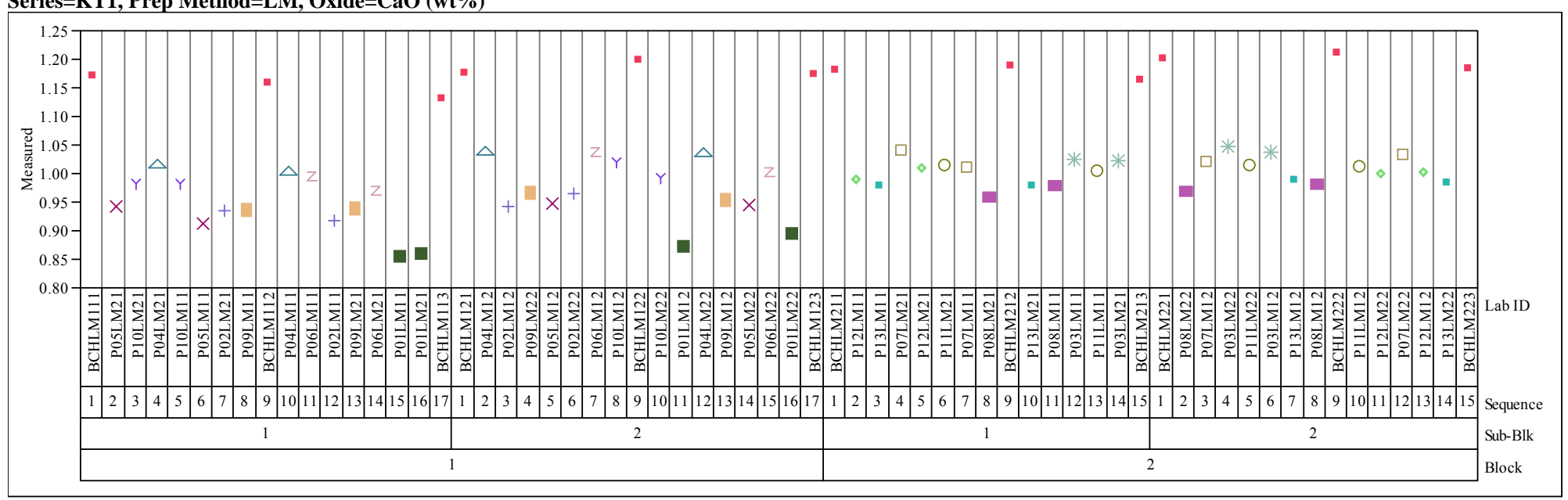


Exhibit A-1. Measurements in Analytical Sequence for KT01-Series by Preparation Method by Oxide. (continued) Series=KT1, Prep Method=LM, Oxide=Ce2O3 (wt\%)

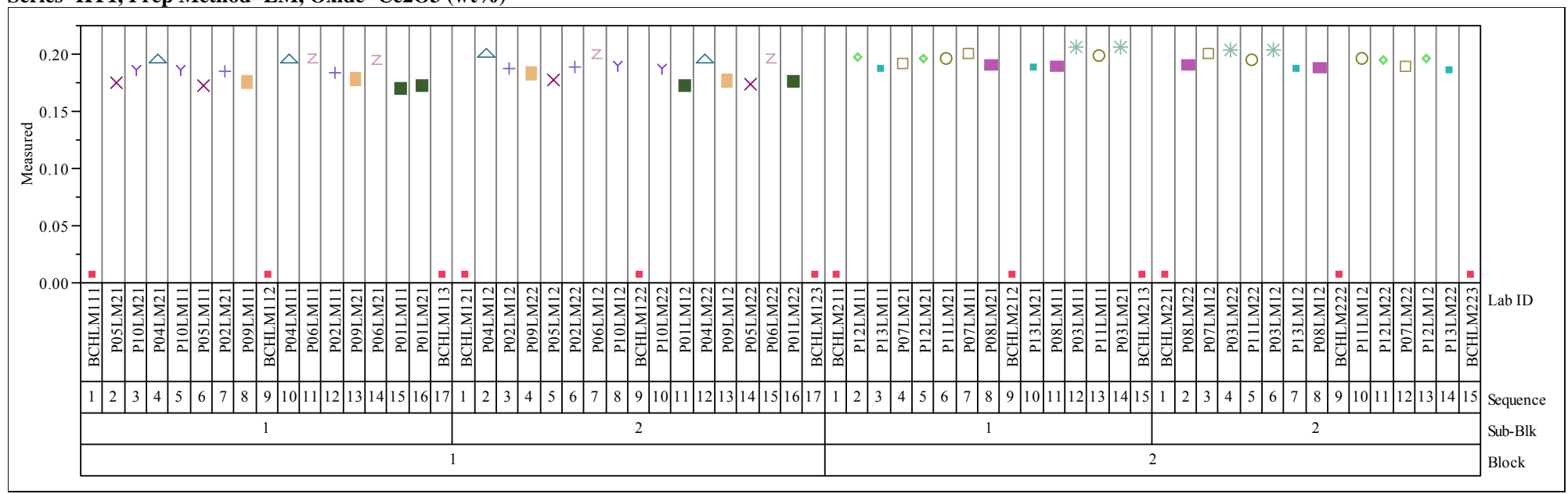

Series=KT1, Prep Method=LM, Oxide=Cr2O3 (wt \%)

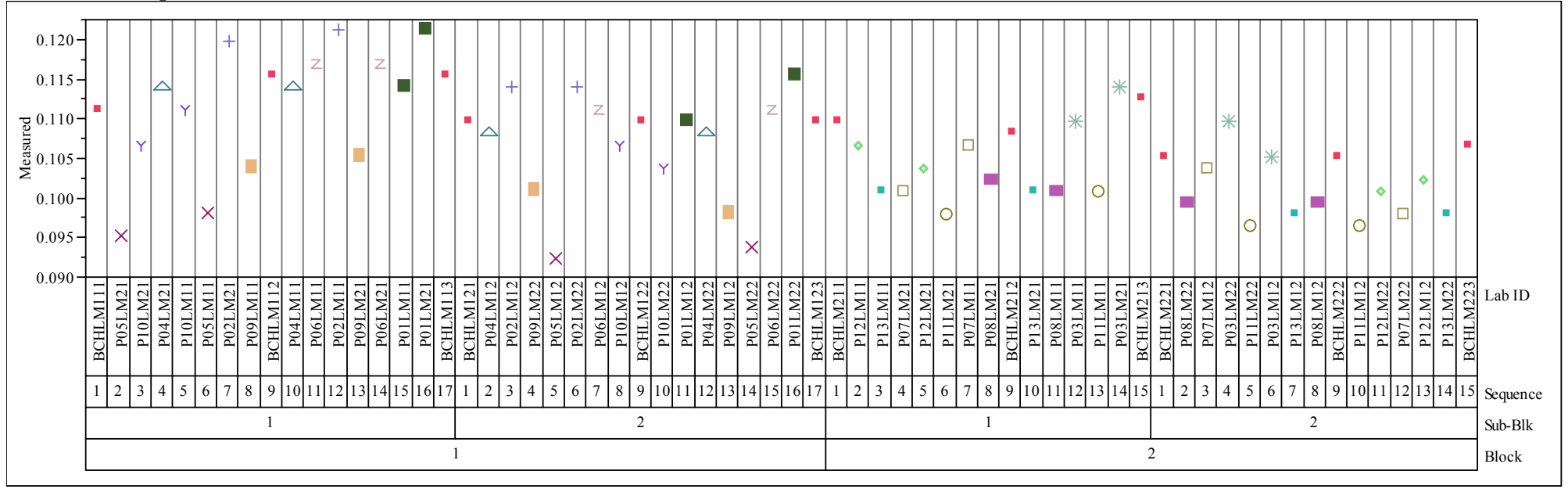


Exhibit A-1. Measurements in Analytical Sequence for KT01-Series by Preparation Method by Oxide. (continued) Series=KT1, Prep Method=LM, Oxide=CuO (wt\%)

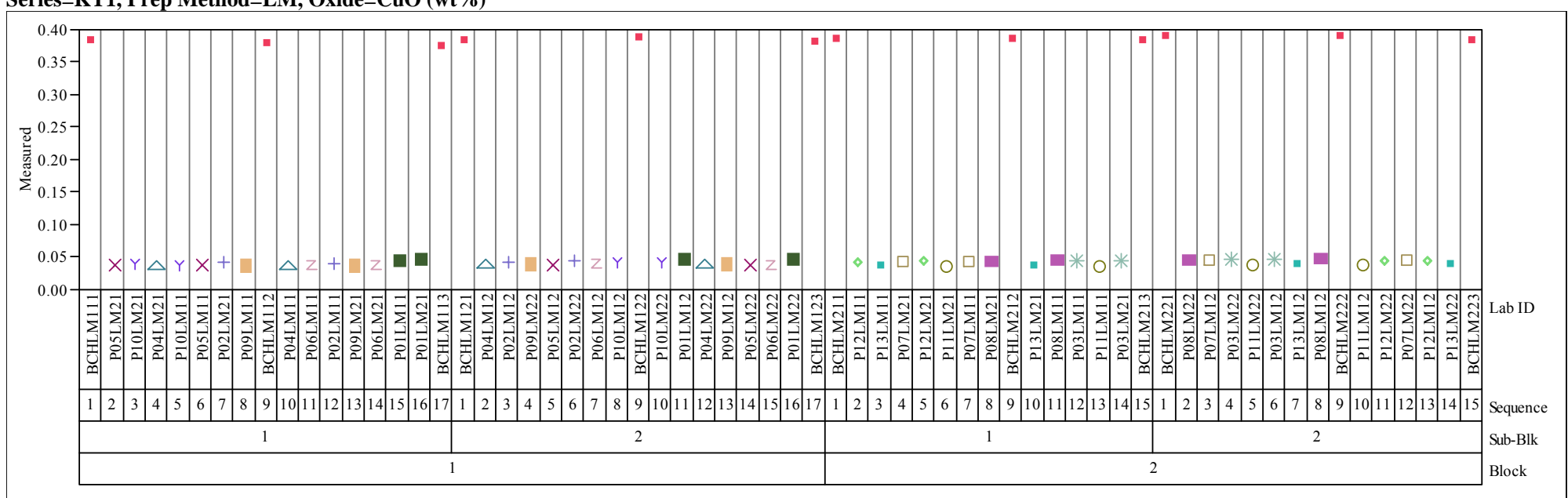

Series=KT1, Prep Method=LM, Oxide=K2O (wt\%)

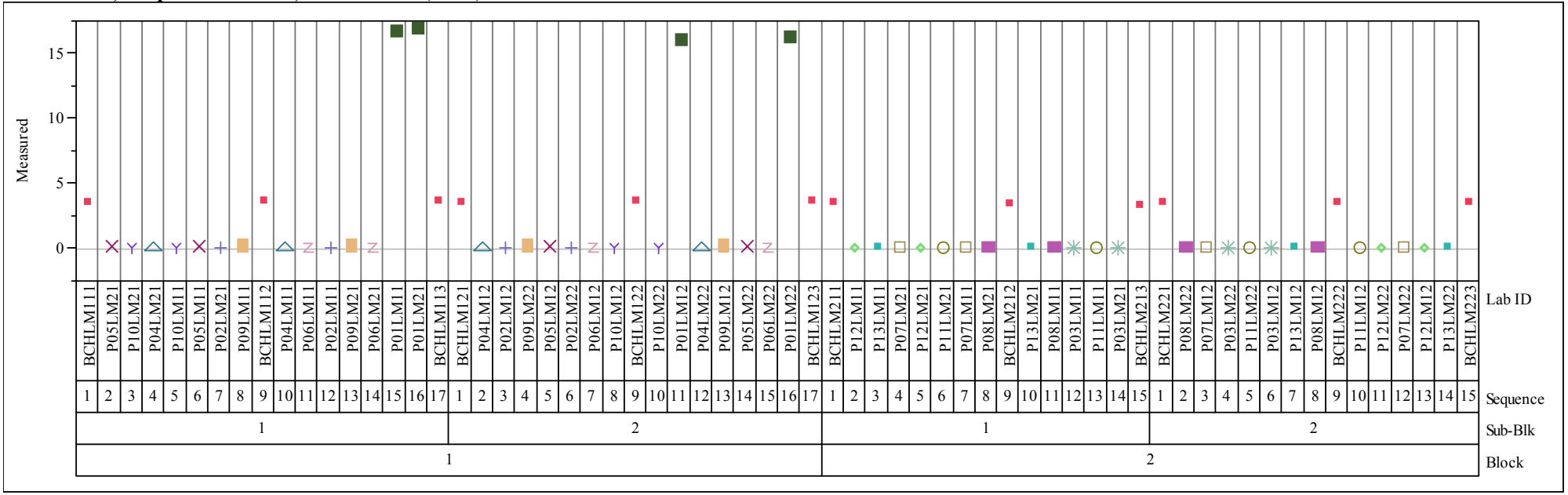


Exhibit A-1. Measurements in Analytical Sequence for KT01-Series by Preparation Method by Oxide. (continued) Series=KT1, Prep Method=LM, Oxide=La2O3 (wt $\%$ )

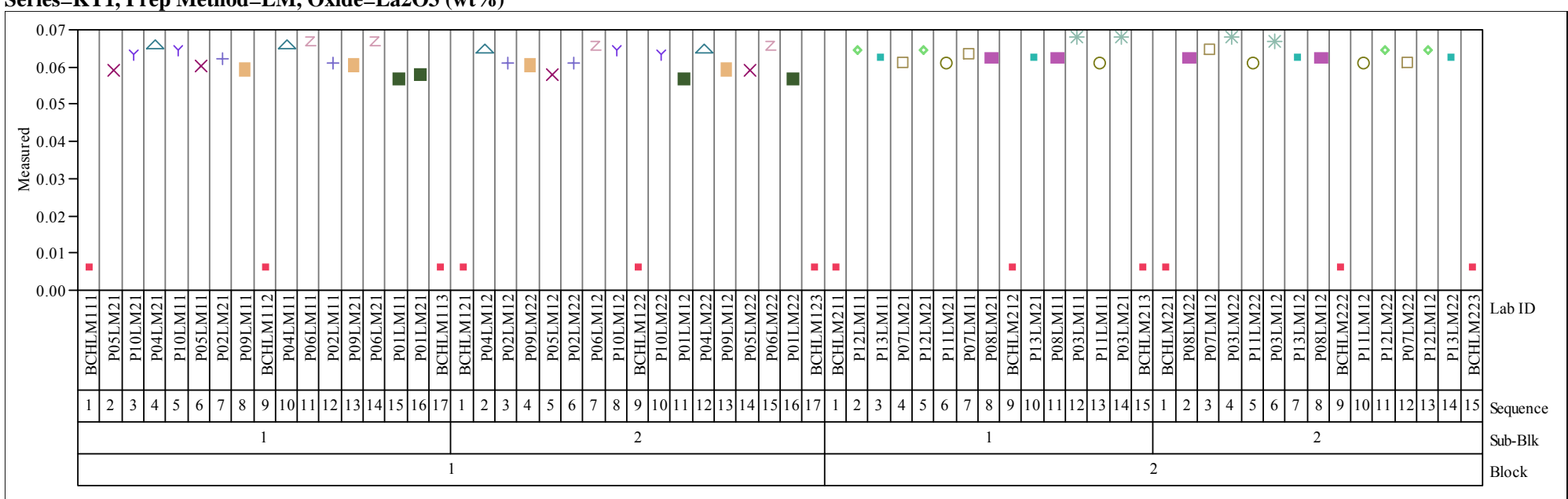

Series=KT1, Prep Method $=$ LM, Oxide $=$ MgO (wt $\%)$

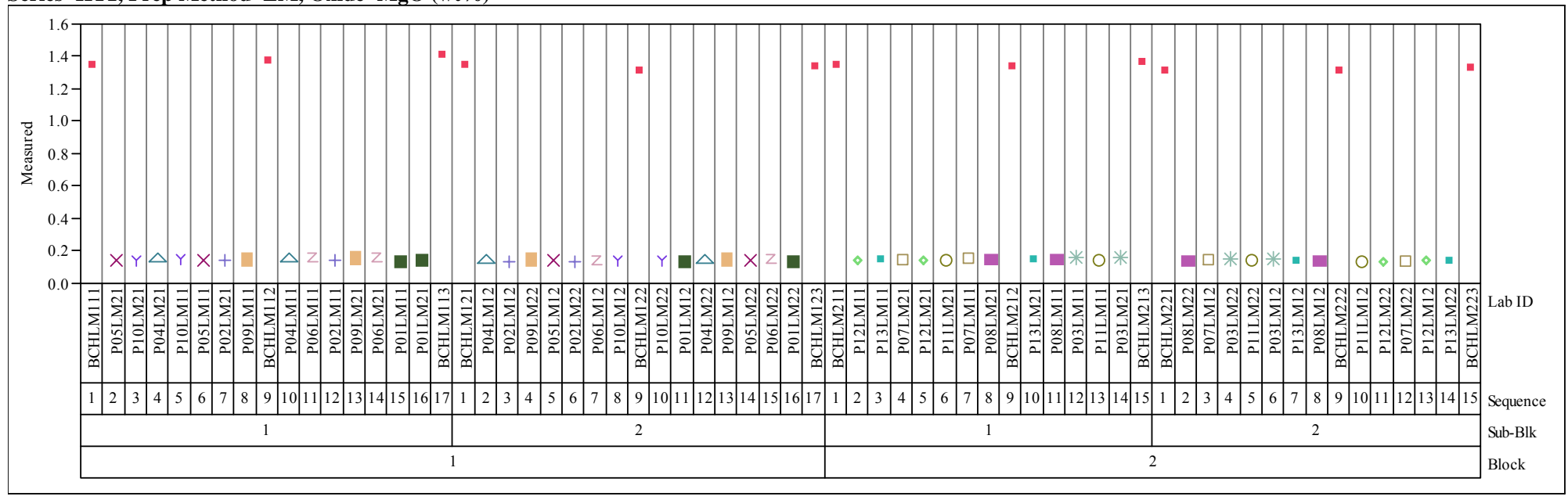


Exhibit A-1. Measurements in Analytical Sequence for KT01-Series by Preparation Method by Oxide. (continued) Series=KT1, Prep Method=LM, Oxide=MnO (wt\%)

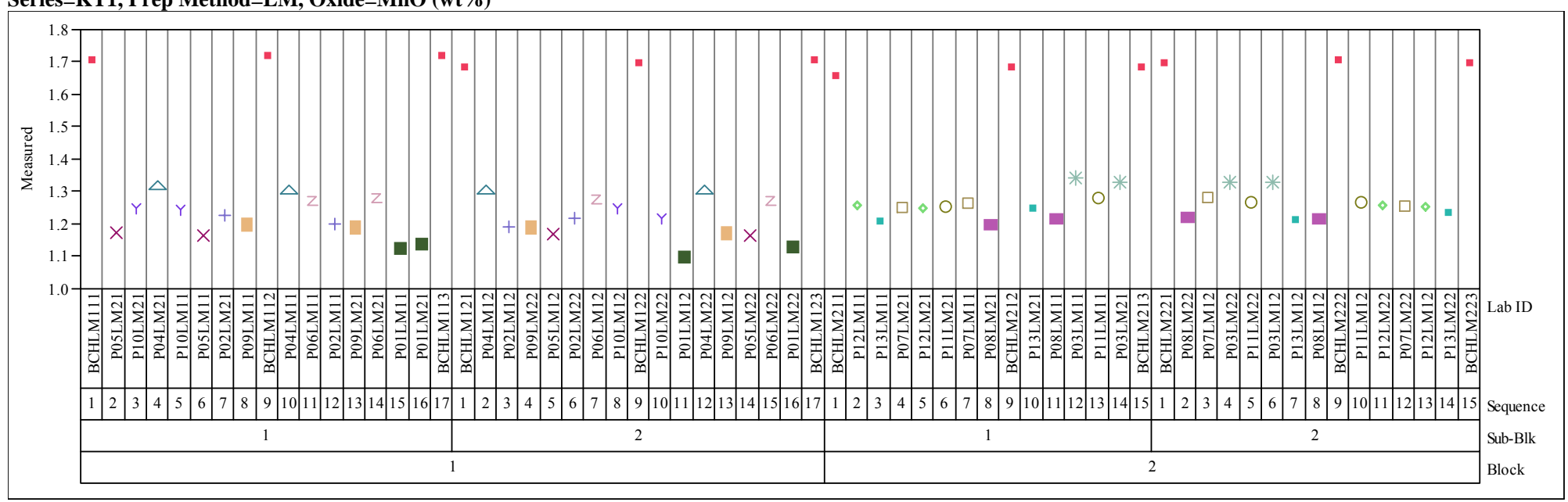

Series=KT1, Prep Method=LM, Oxide=Na2O (wt\%)

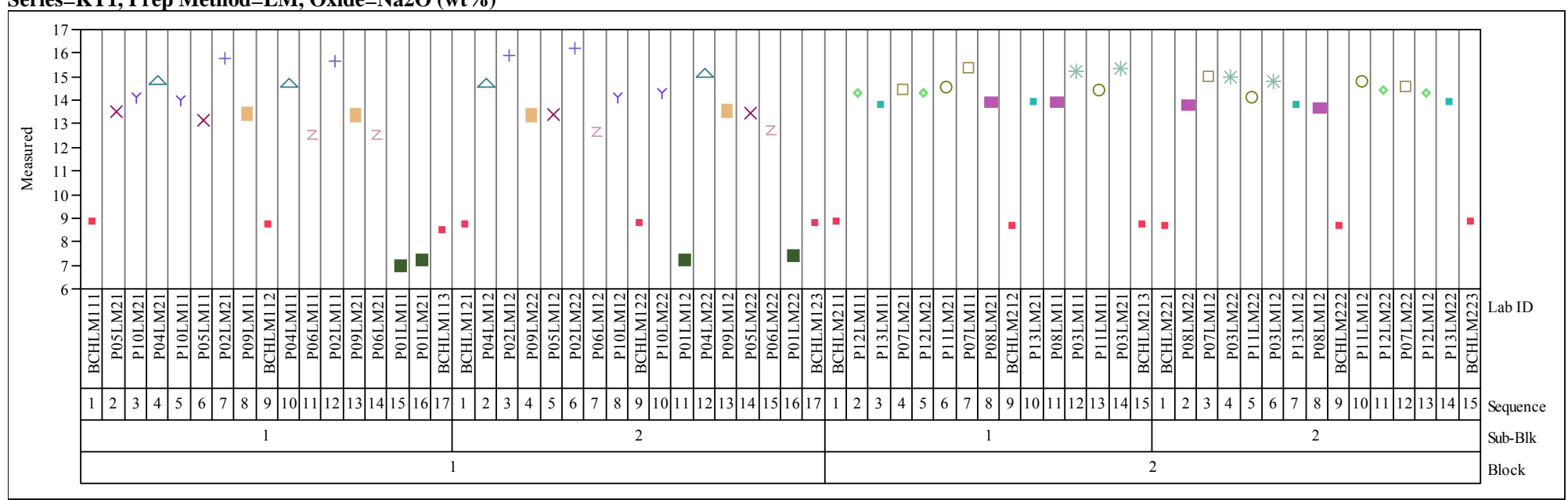


Exhibit A-1. Measurements in Analytical Sequence for KT01-Series by Preparation Method by Oxide. (continued) Series=KT1, Prep Method=LM, Oxide=Nb2O5 (wt\%)

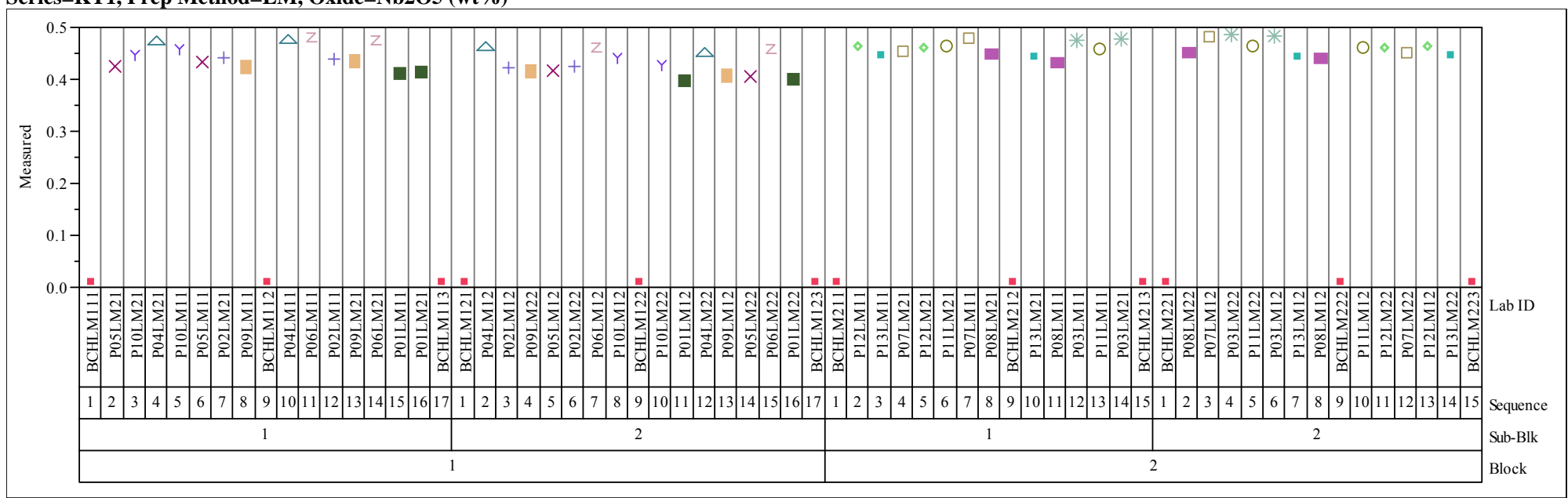

Series=KT1, Prep Method=LM, Oxide=NiO (wt\%)

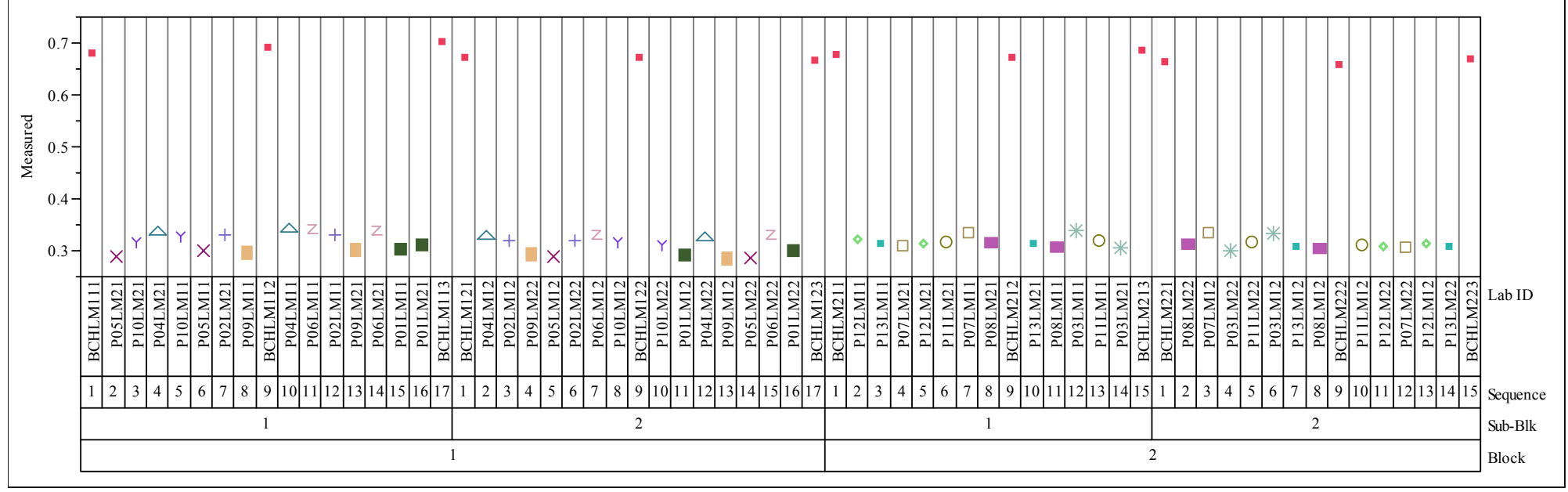


Exhibit A-1. Measurements in Analytical Sequence for KT01-Series by Preparation Method by Oxide. (continued) Series=KT1, Prep Method=LM, Oxide=PbO (wt $\%$ )

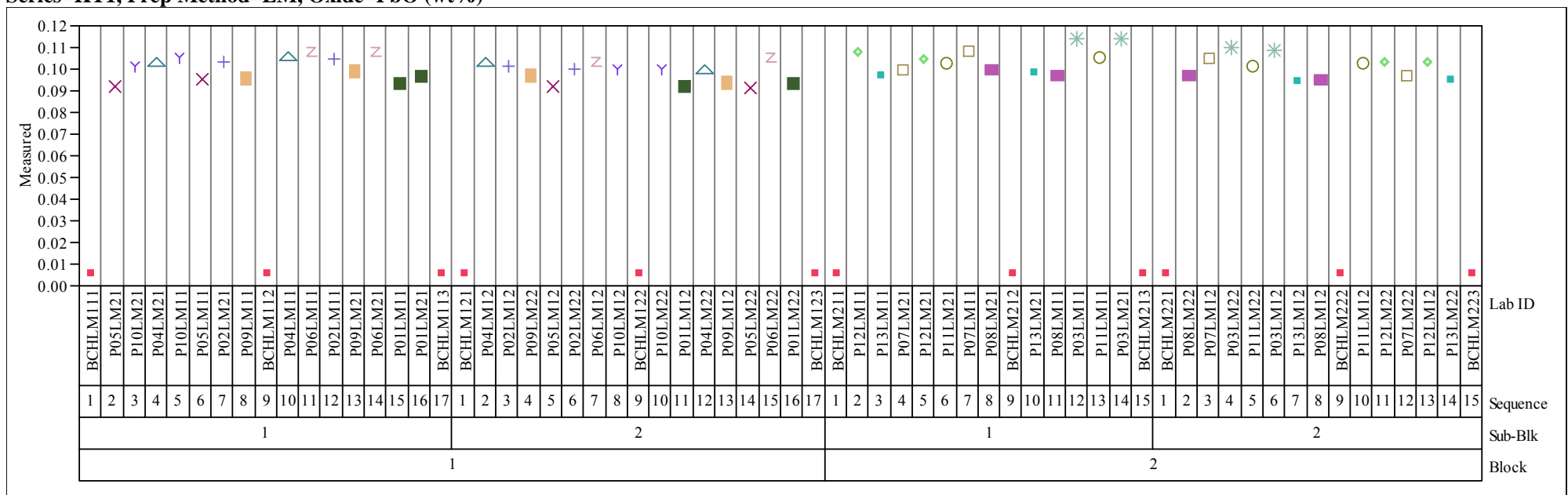

Series=KT1, Prep Method=LM, Oxide=SiO2 (wt\%)

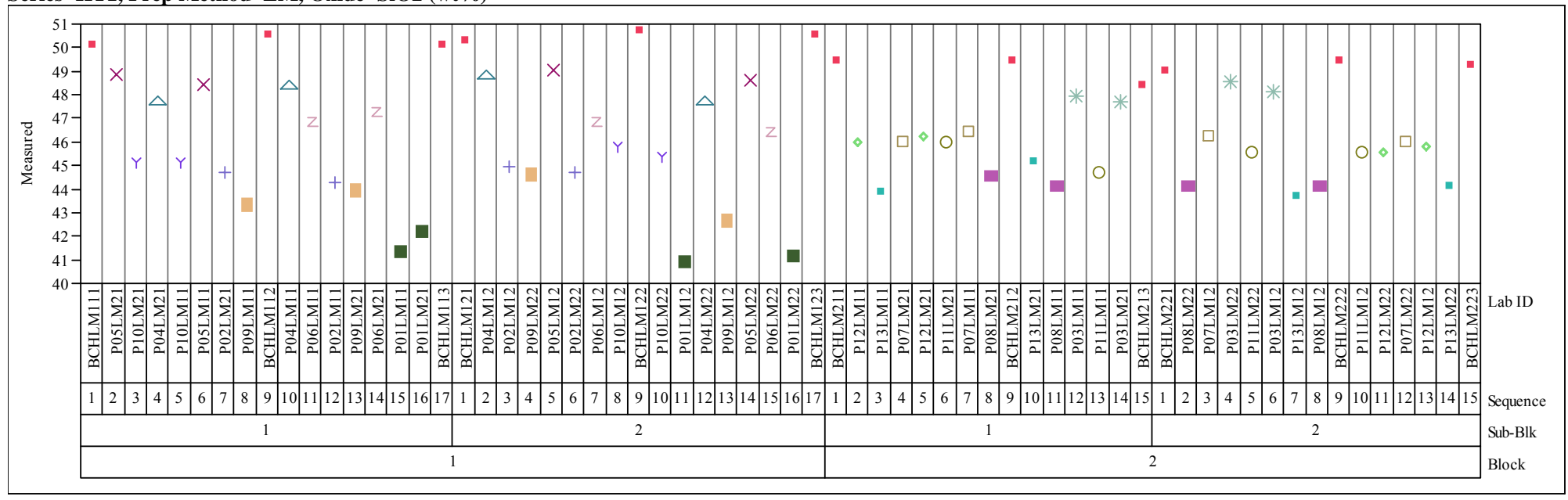


Exhibit A-1. Measurements in Analytical Sequence for KT01-Series by Preparation Method by Oxide. (continued) Series=KT1, Prep Method=LM, Oxide=SO4 (wt\%)

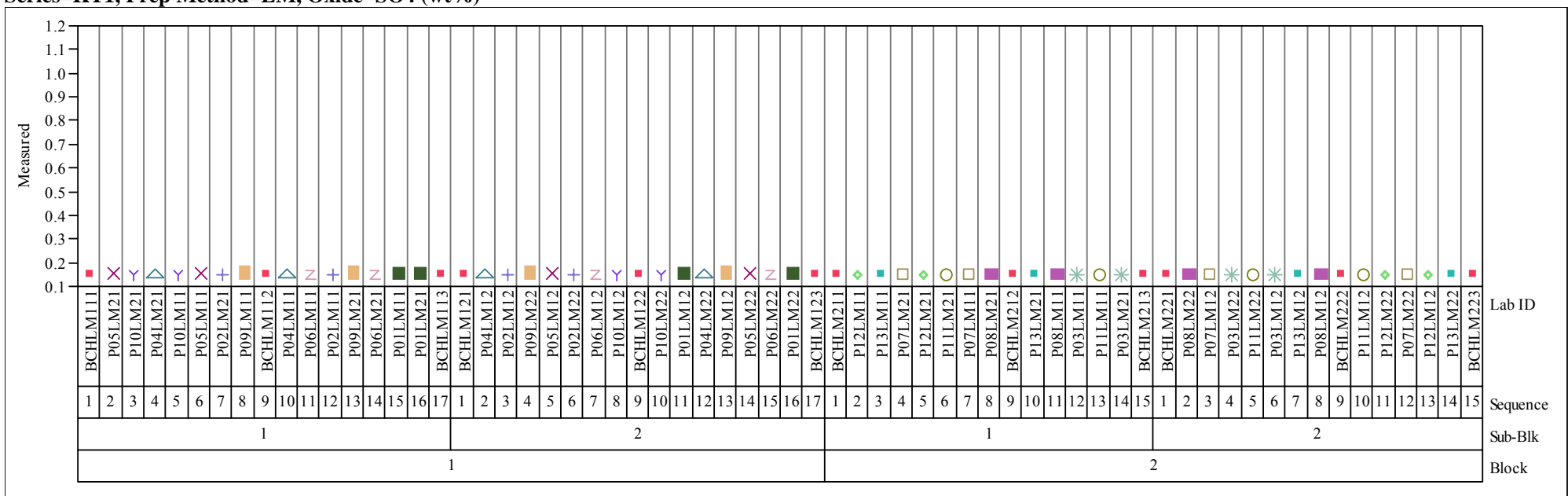

Series=KT1, Prep Method=LM, Oxide=TiO2 (wt\%)

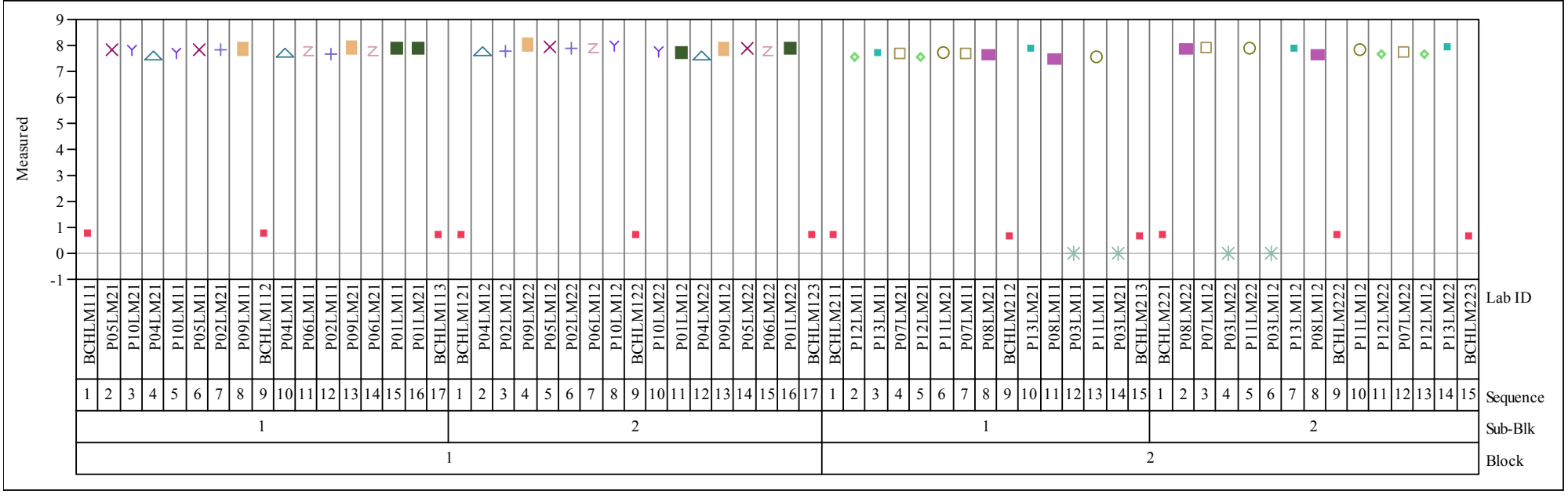


Exhibit A-1. Measurements in Analytical Sequence for KT01-Series by Preparation Method by Oxide. (continued) Series=KT1, Prep Method=LM, Oxide=ZnO (wt\%)

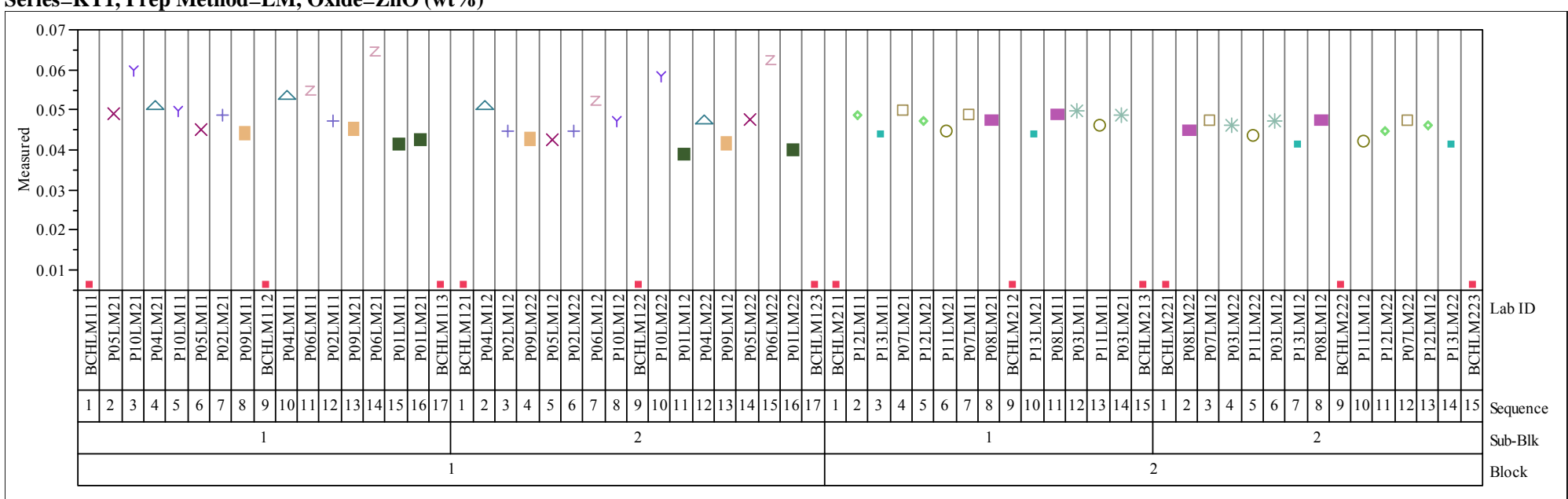

Series=KT1, Prep Method=LM, Oxide=ZrO2 (wt\%)

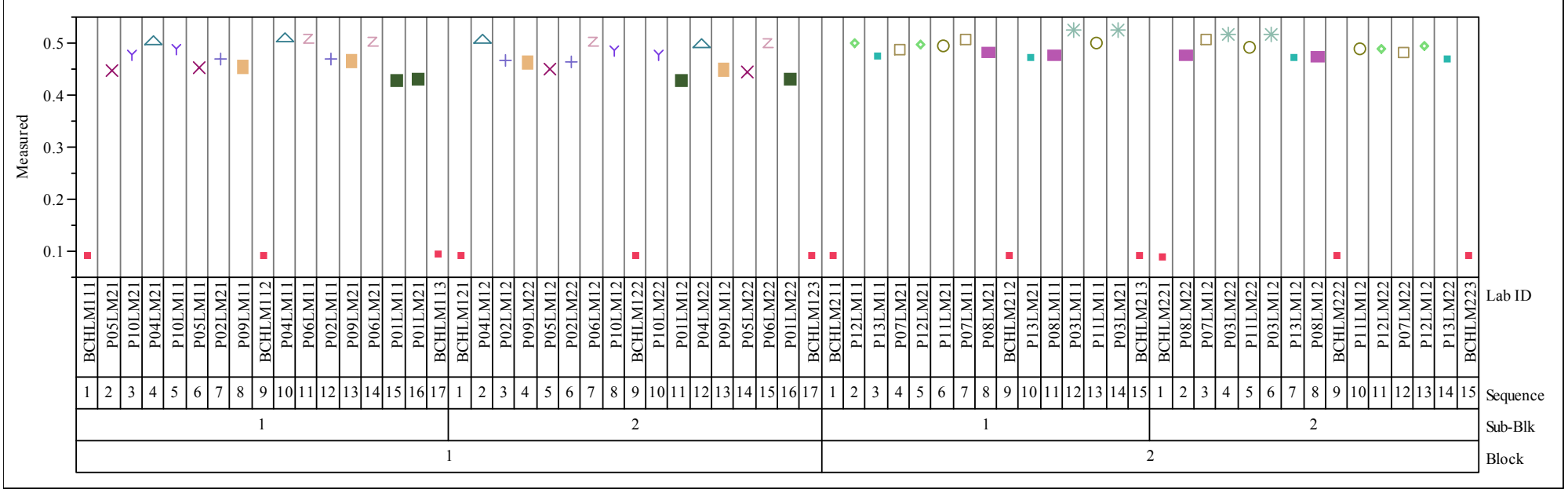


Exhibit A-1. Measurements in Analytical Sequence for KT01-Series by Preparation Method by Oxide. (continued) Series=KT1, Prep Method=PF, Oxide=Al2O3 (wt\%)

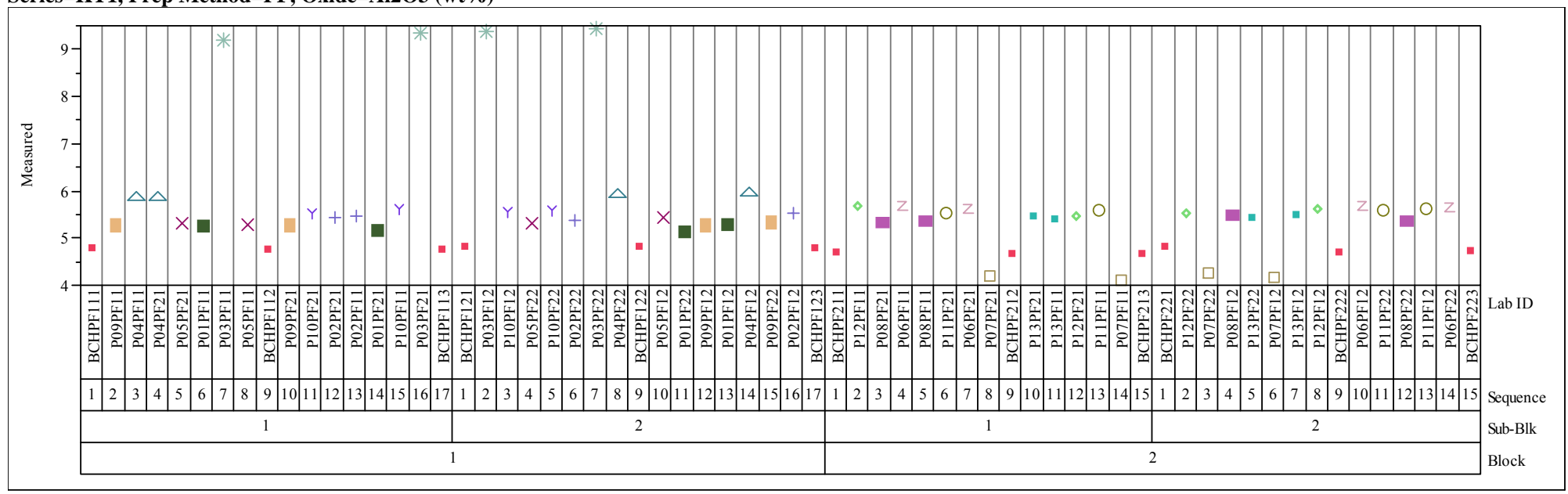

Series=KT1, Prep Method=PF, Oxide=B2O3 (wt \%)

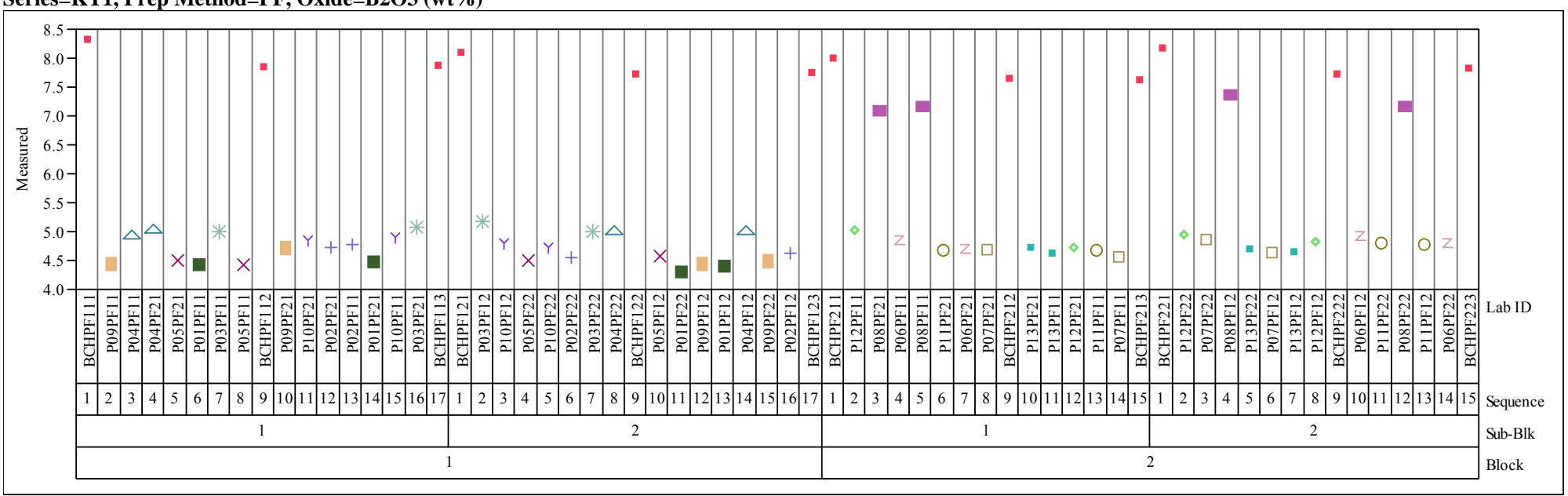


Exhibit A-1. Measurements in Analytical Sequence for KT01-Series by Preparation Method by Oxide. (continued) Series=KT1, Prep Method=PF, Oxide=Fe2O3 (wt\%)

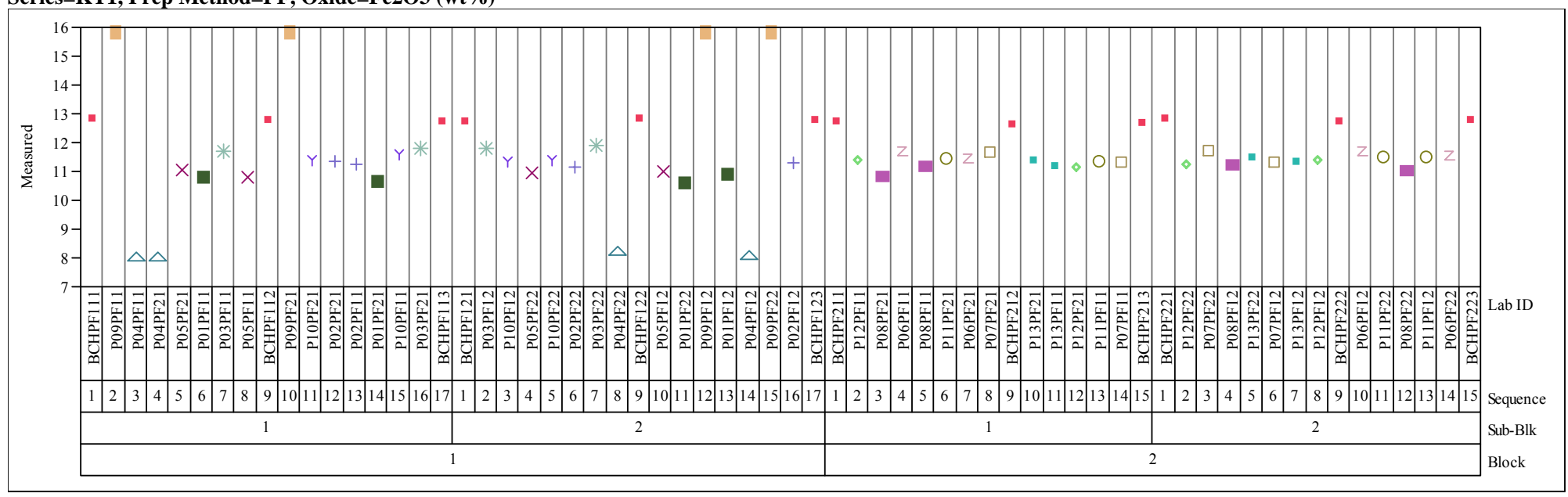

Series=KT1, Prep Method=PF, Oxide=Li2O (wt\%)

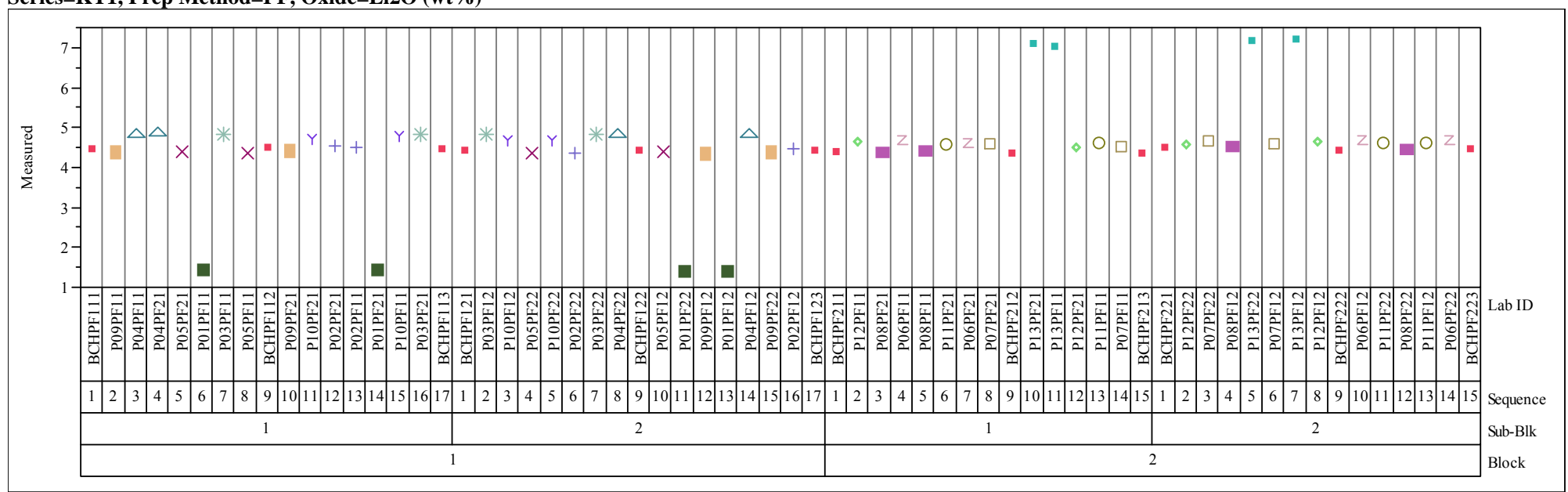


Exhibit A-2. Measurements for Each KT01-Series Glass ID by Preparation Method by Oxide.

Series=KT1, Prep Method=LM, Oxide=BaO (wt \%)

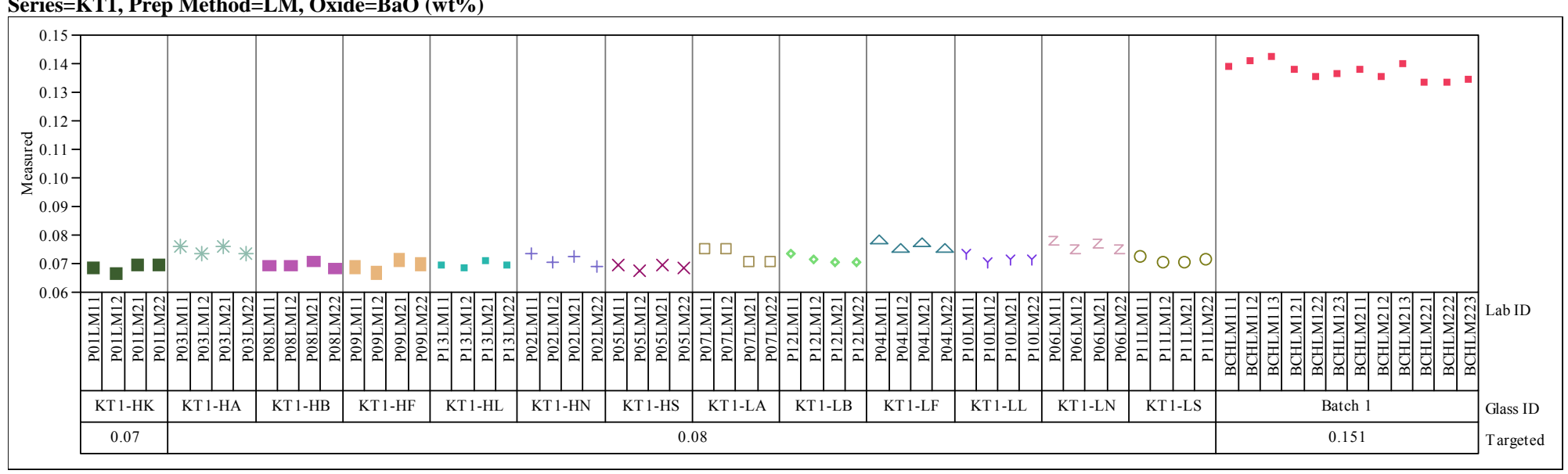

Series=KT1, Prep Method=LM, Oxide=CaO (wt \%)

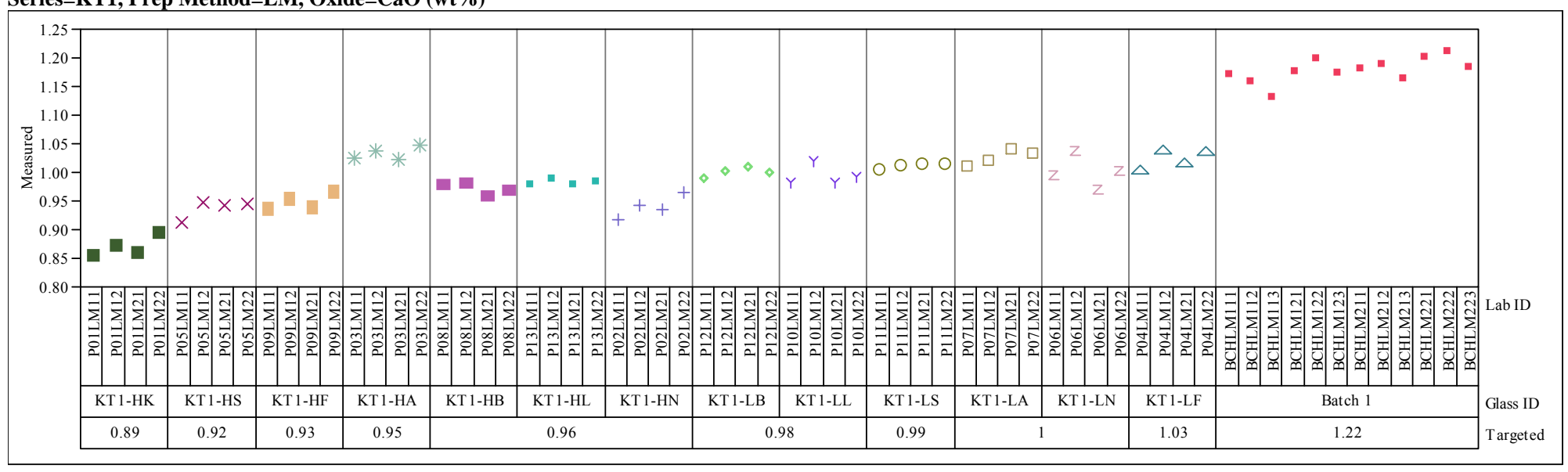




\section{Exhibit A-2. Measurements for Each KT01-Series Glass ID by Preparation Method by Oxide. (continued)}

Series=KT1, Prep Method=LM, Oxide=Ce2O3 (wt \%)

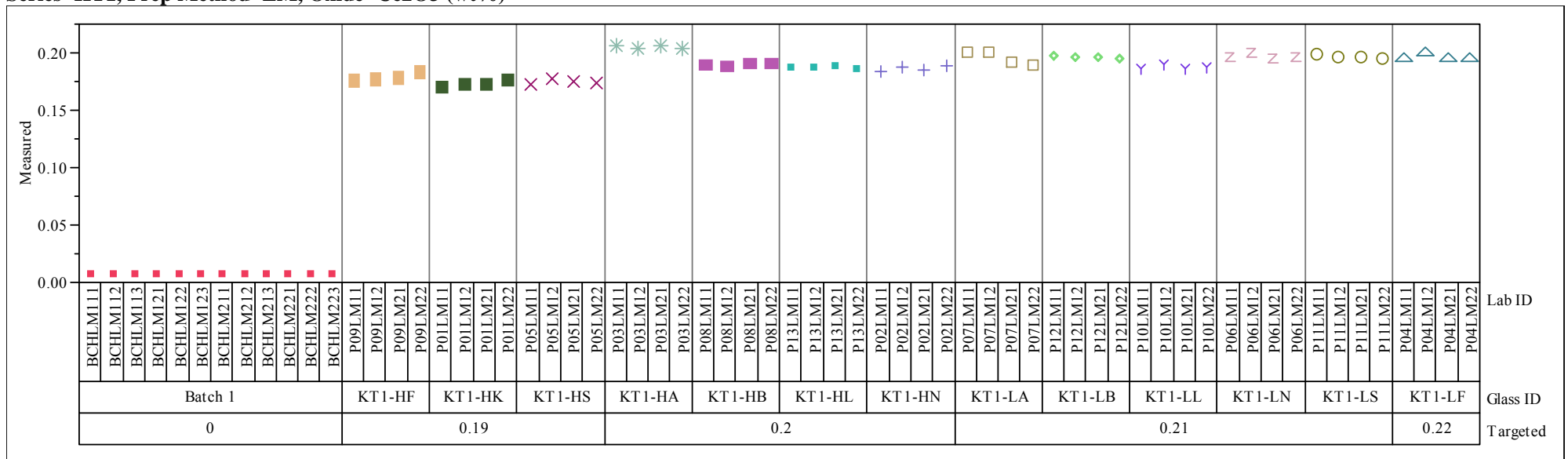

Series=KT1, Prep Method=LM, Oxide=Cr2O3 (wt \%)

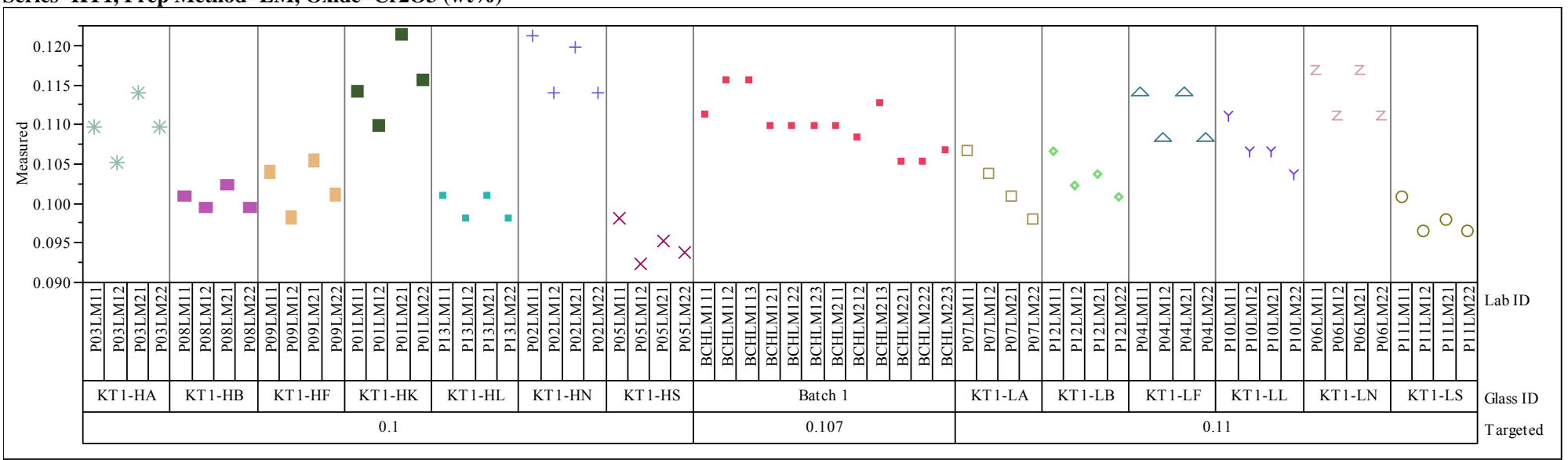


Exhibit A-2. Measurements for Each KT01-Series Glass ID by Preparation Method by Oxide. (continued) Series=KT1, Prep Method=LM, Oxide=CuO (wt\%)

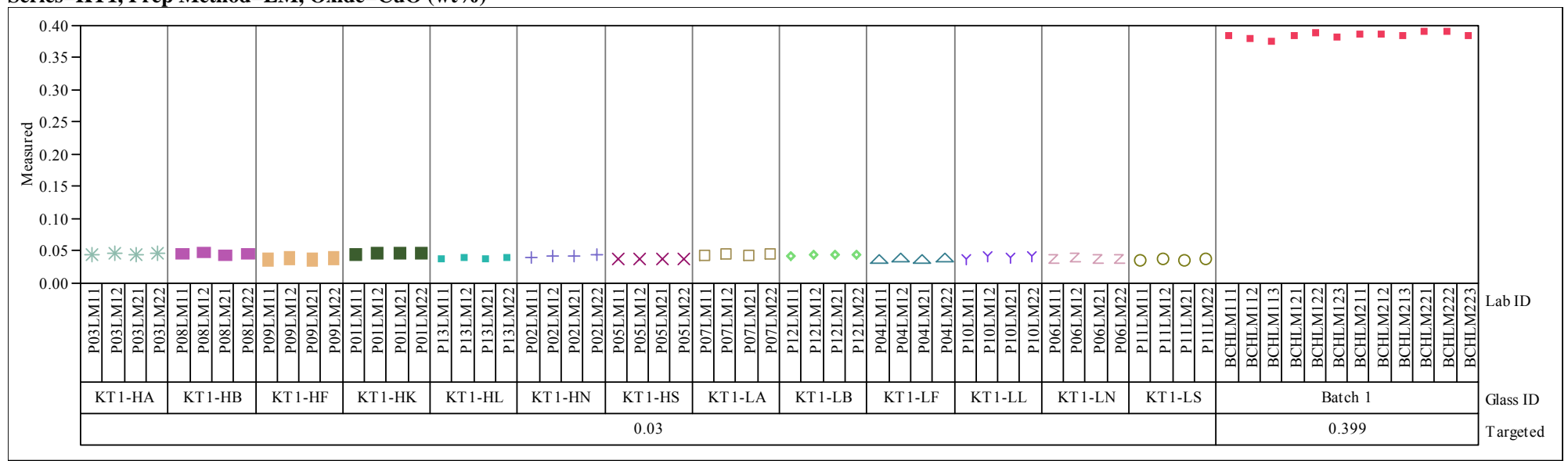

Series=KT1, Prep Method=LM, Oxide=K2O (wt \%)

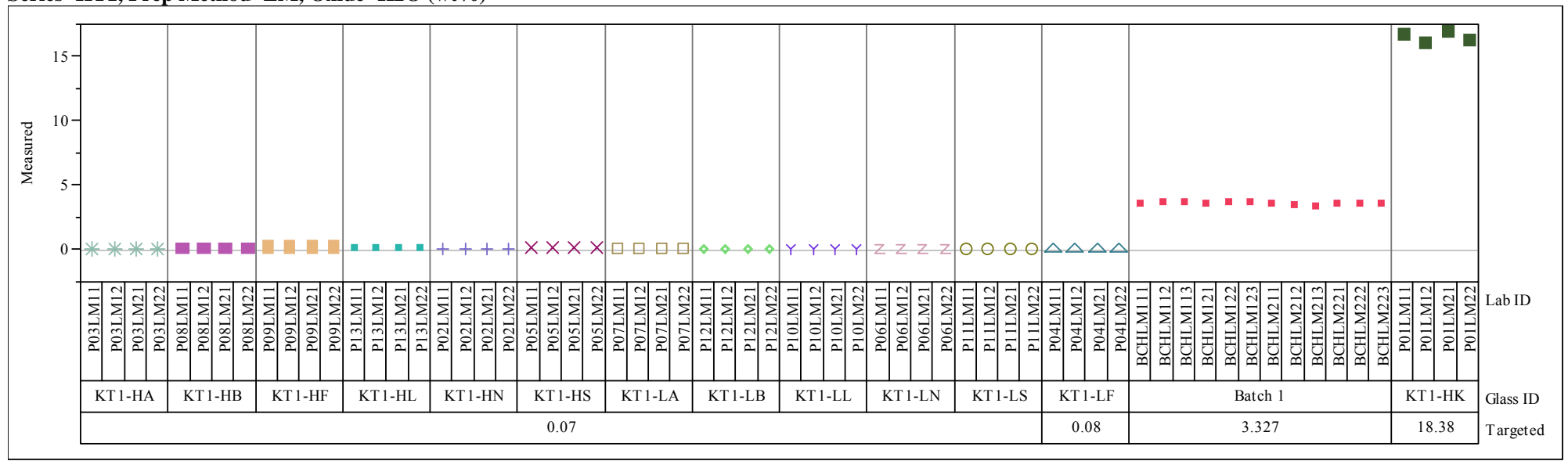


Exhibit A-2. Measurements for Each KT01-Series Glass ID by Preparation Method by Oxide. (continued) Series=KT1, Prep Method=LM, Oxide=La2O3 (wt $\%)$

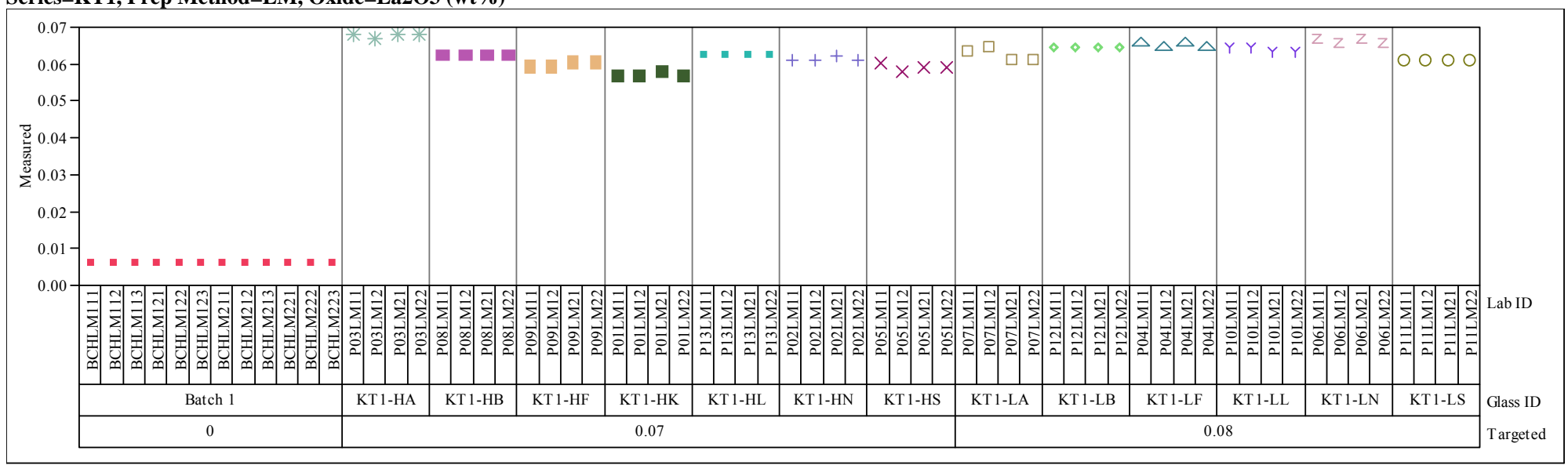

Series=KT1, Prep Method=LM, Oxide=MgO (wt \%)

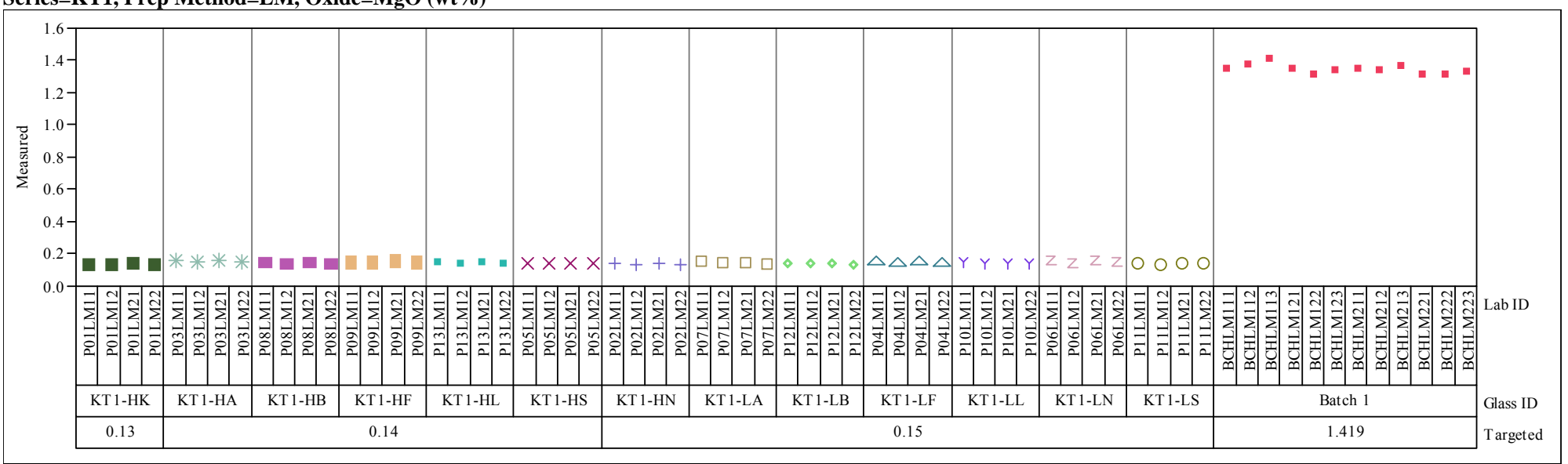


Exhibit A-2. Measurements for Each KT01-Series Glass ID by Preparation Method by Oxide. (continued) Series=KT1, Prep Method=LM, Oxide=MnO (wt\%)

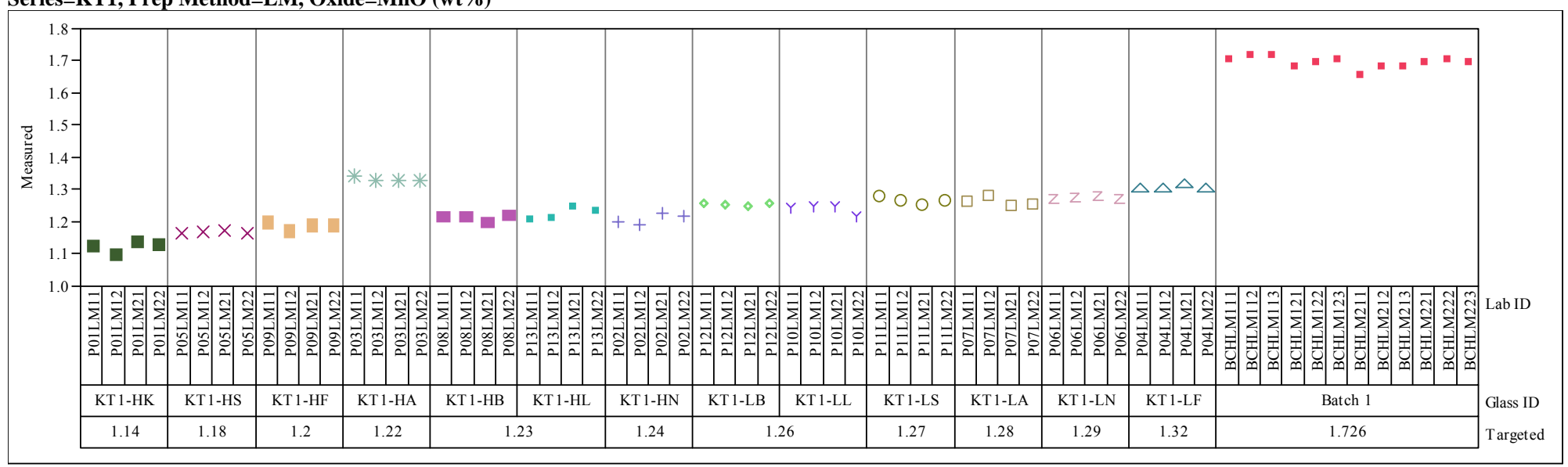

Series=KT1, Prep Method=LM, Oxide=Na2O (wt\%)

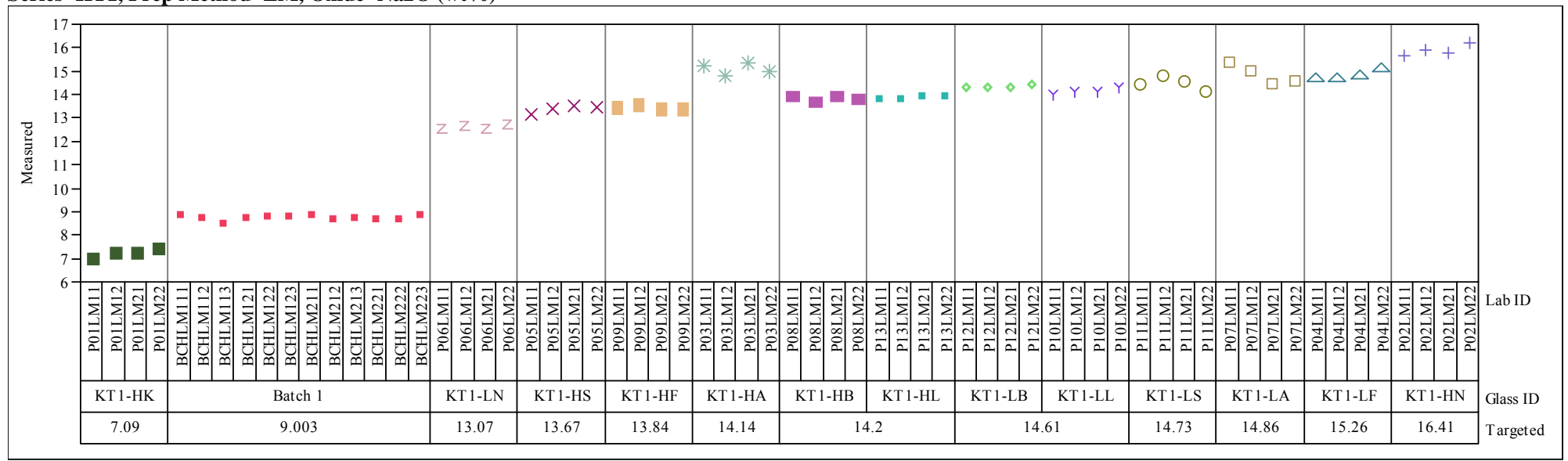


Exhibit A-2. Measurements for Each KT01-Series Glass ID by Preparation Method by Oxide. (continued) Series=KT1, Prep Method=LM, Oxide=Nb2O5 (wt\%)

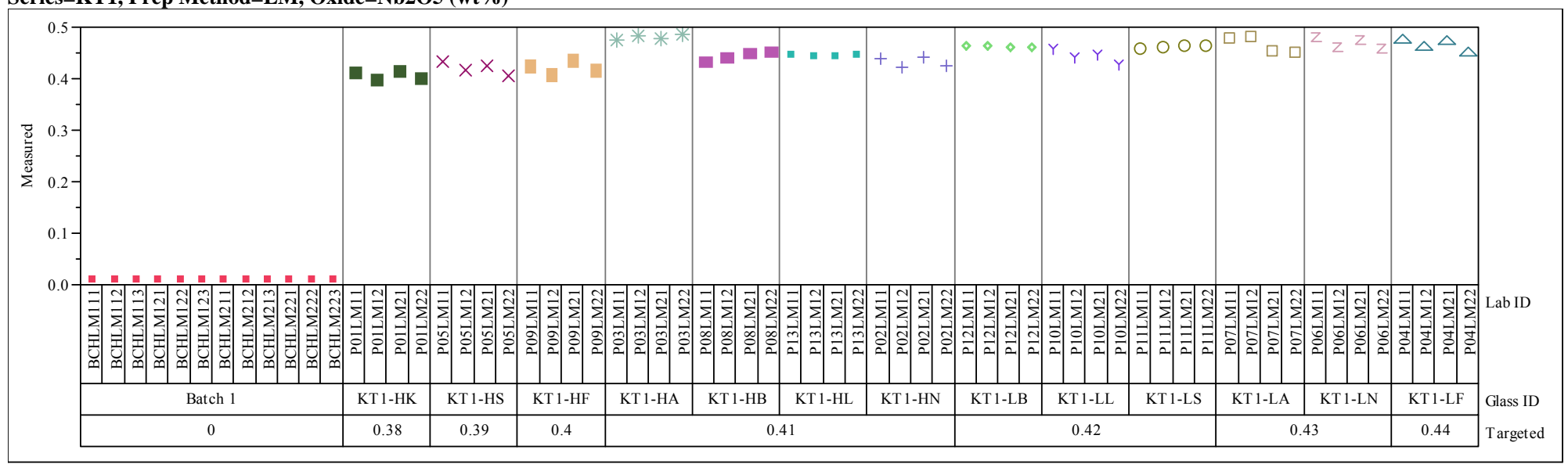

Series=KT1, Prep Method=LM, Oxide=NiO (wt\%)

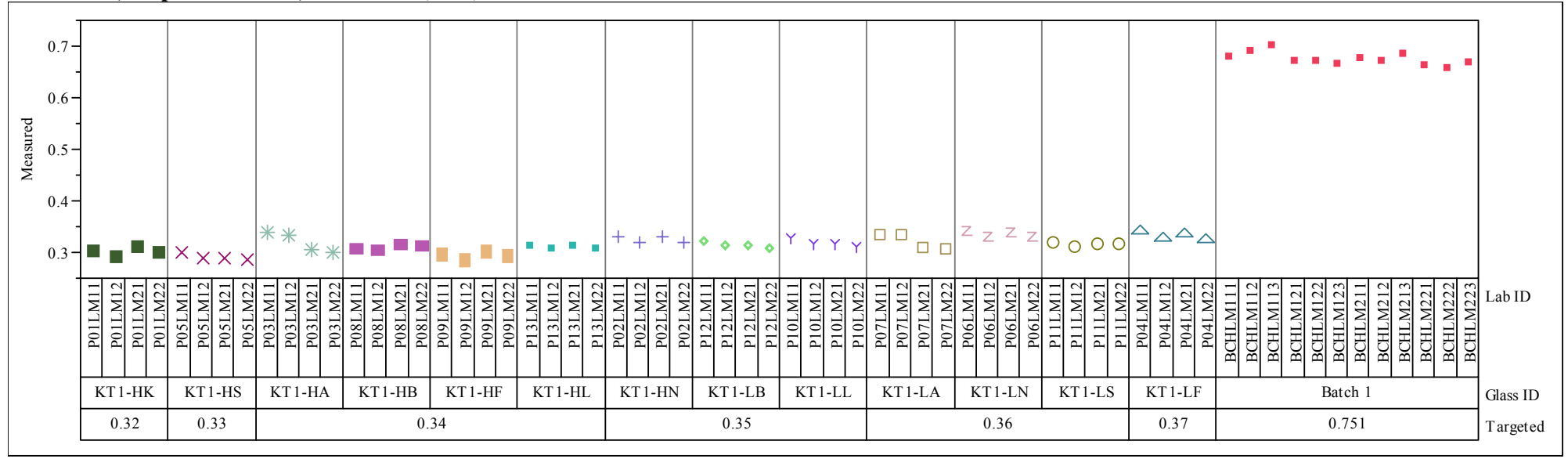


Exhibit A-2. Measurements for Each KT01-Series Glass ID by Preparation Method by Oxide. (continued) Series=KT1, Prep Method=LM, Oxide=PbO (wt\%)

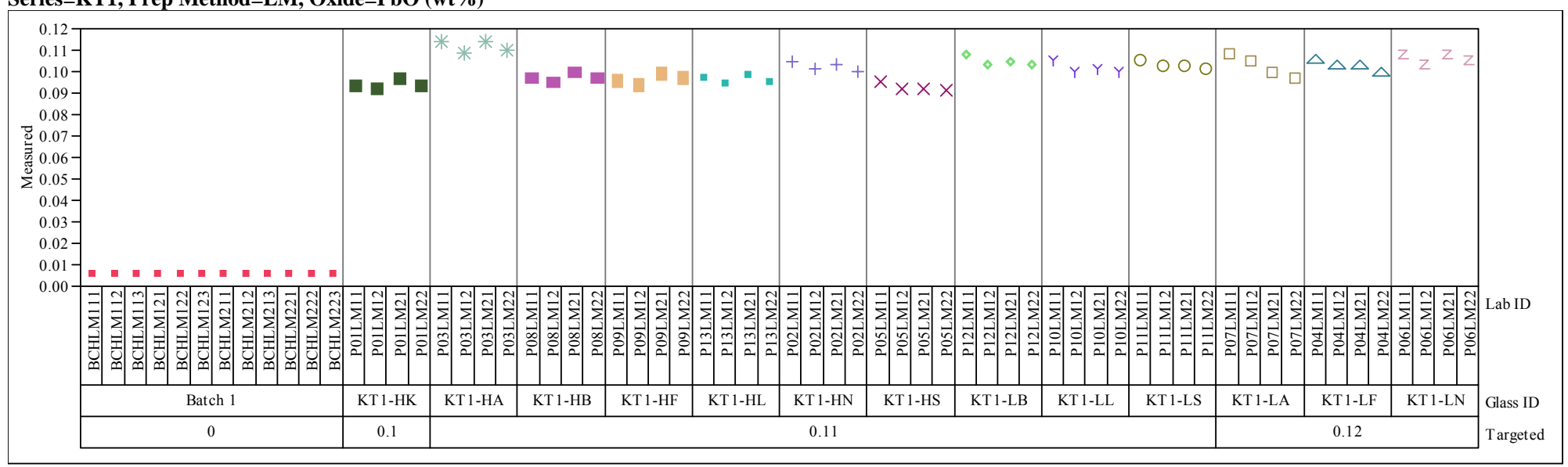

Series=KT1, Prep Method=LM, Oxide=SiO2 (wt \%)

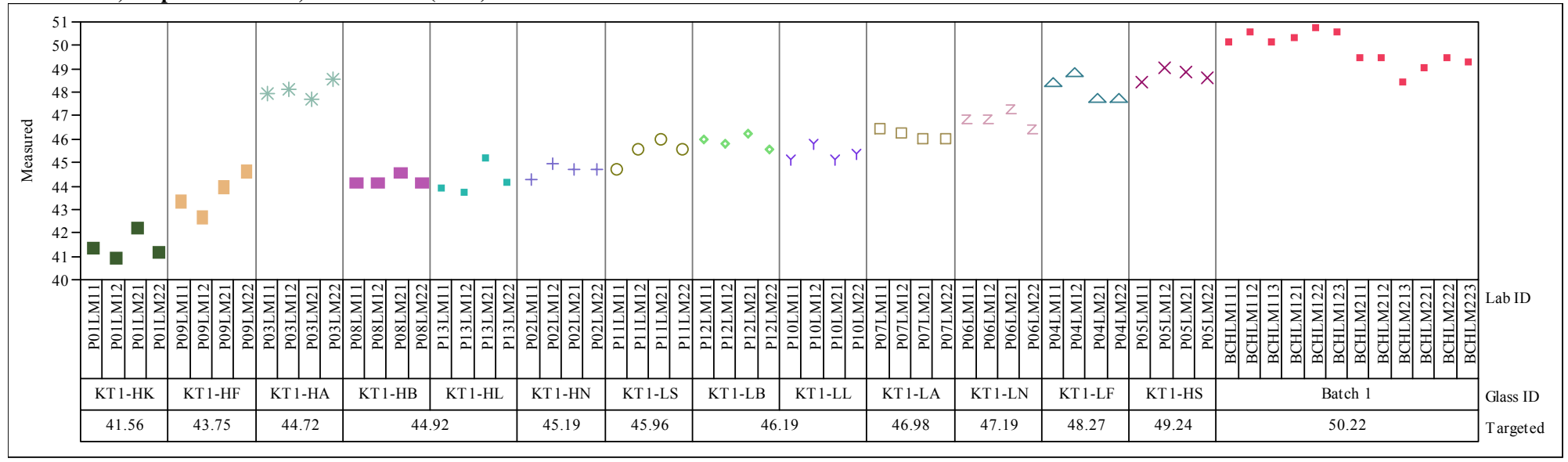


Exhibit A-2. Measurements for Each KT01-Series Glass ID by Preparation Method by Oxide. (continued) Series=KT1, Prep Method=LM, Oxide=SO4 (wt\%)

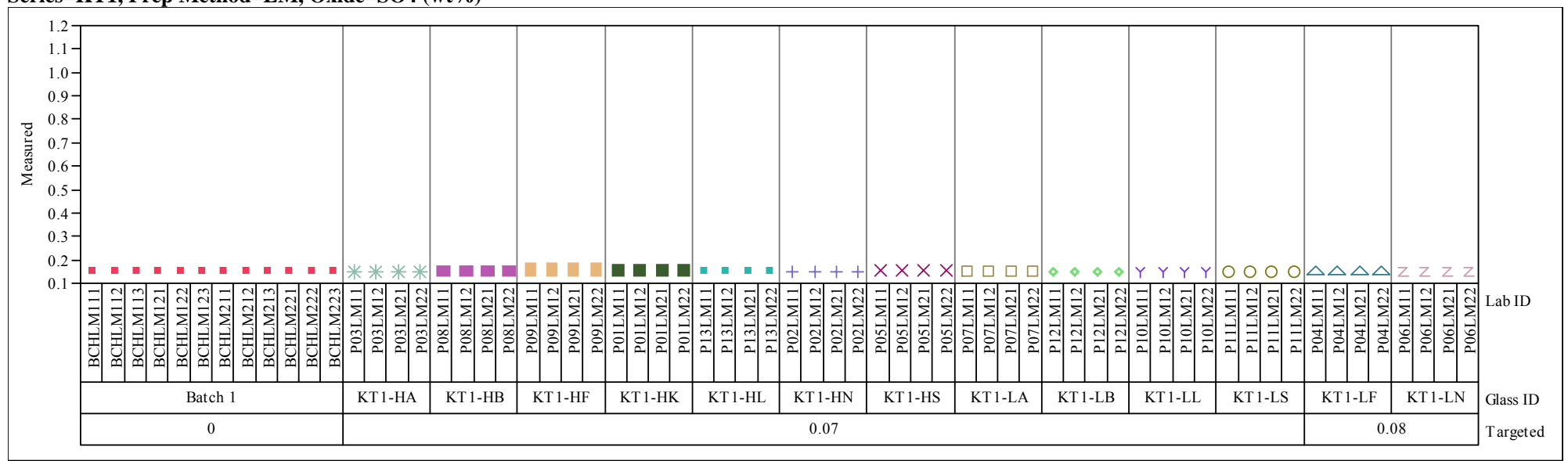

Series=KT1, Prep Method=LM, Oxide=TiO2 (wt \%)

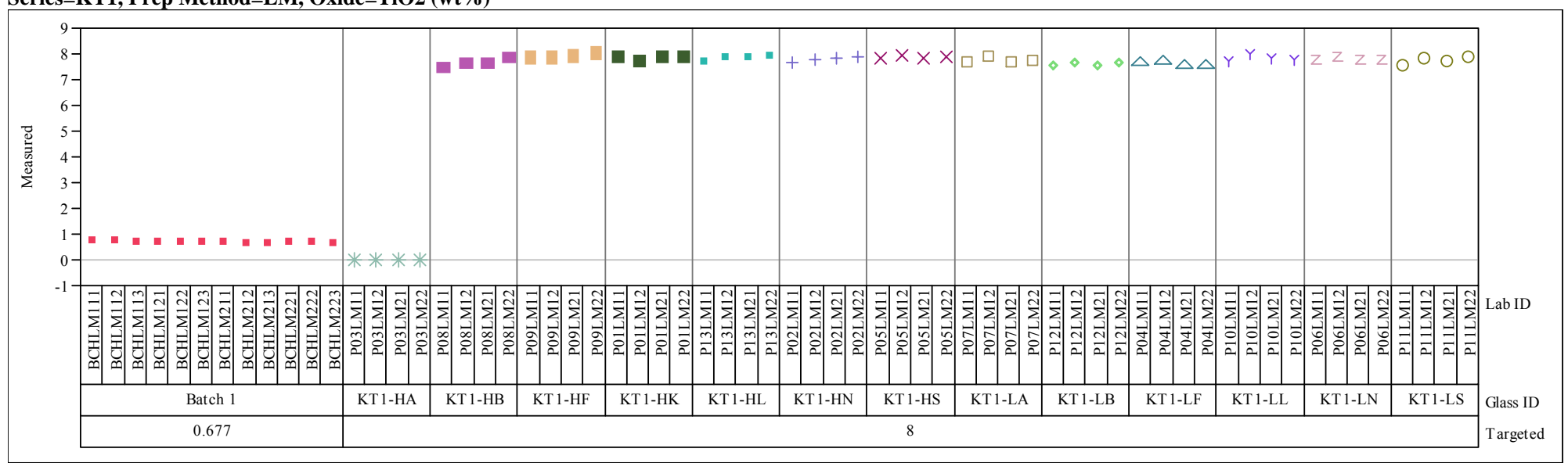


Exhibit A-2. Measurements for Each KT01-Series Glass ID by Preparation Method by Oxide. (continued) Series=KT1, Prep Method=LM, Oxide=ZnO (wt\%)

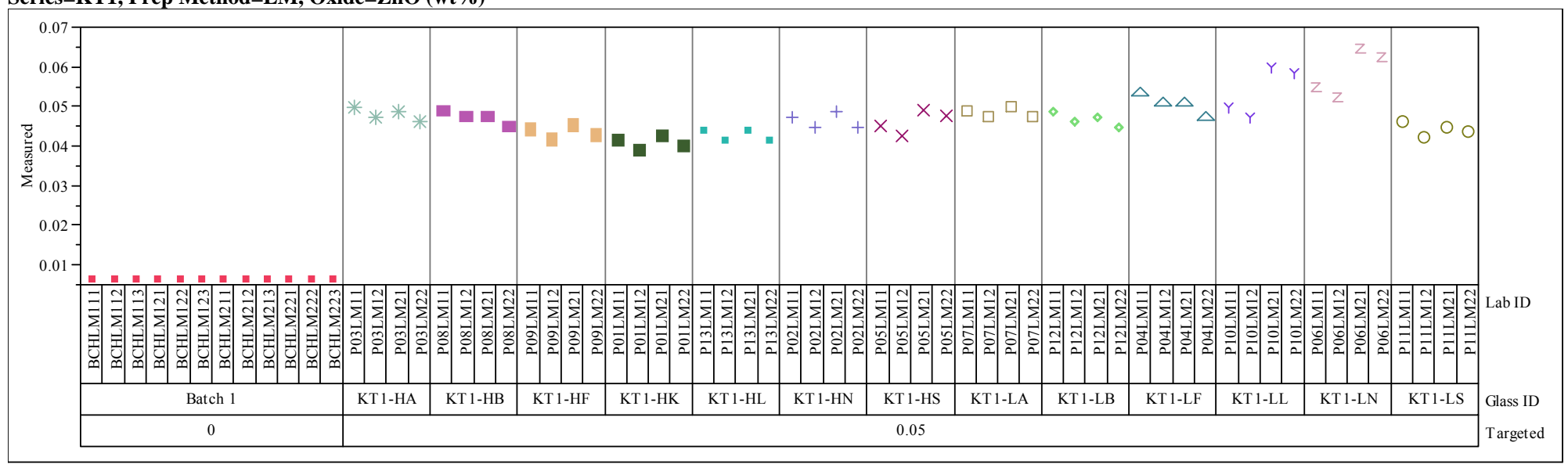

Series=KT1, Prep Method=LM, Oxide=ZrO2 (wt\%)

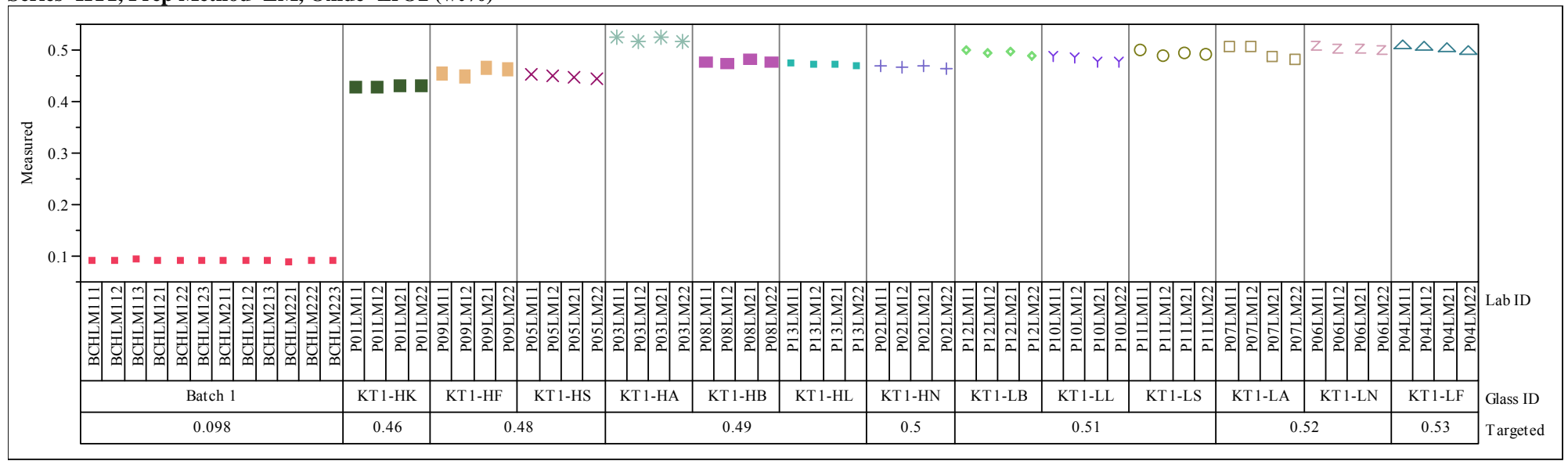


Exhibit A-2. Measurements for Each KT01-Series Glass ID by Preparation Method by Oxide. (continued) Series=KT1, Prep Method=PF, Oxide=Al2O3 (wt\%)

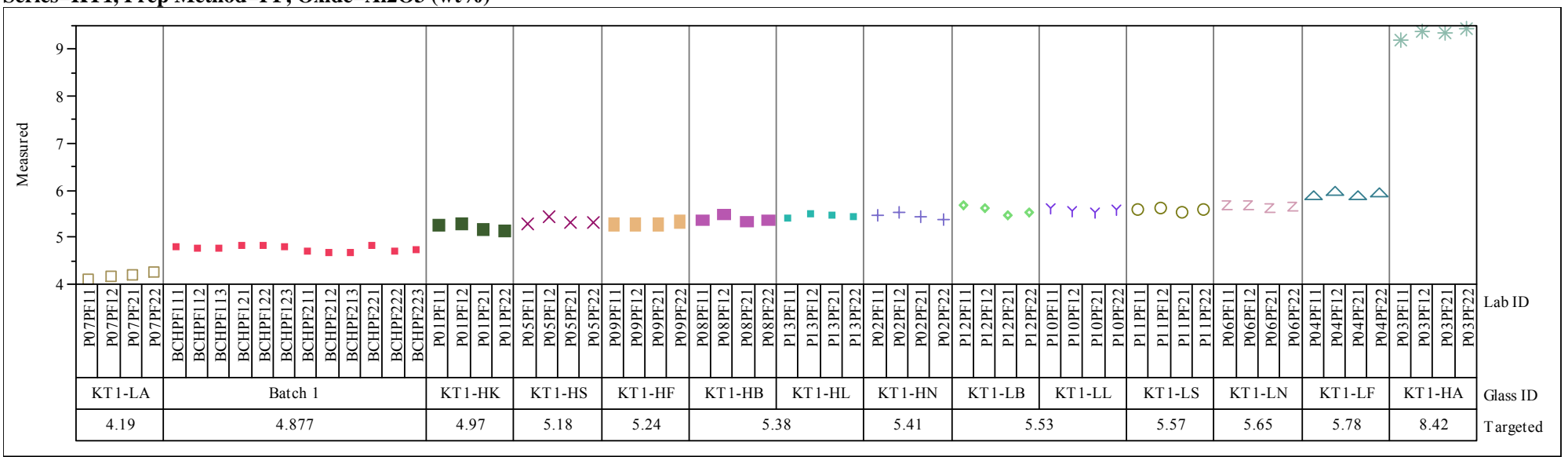

Series=KT1, Prep Method=PF, Oxide=B2O3 (wt \%)

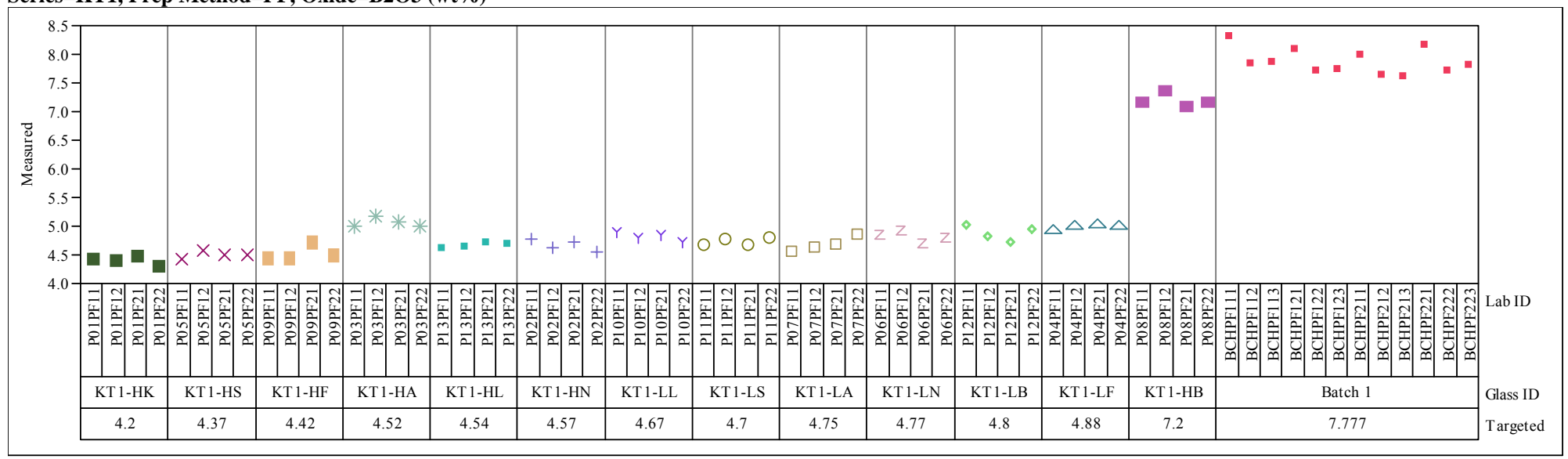


Exhibit A-2. Measurements for Each KT01-Series Glass ID by Preparation Method by Oxide. (continued) Series=KT1, Prep Method=PF, Oxide=Fe2O3 (wt\%)

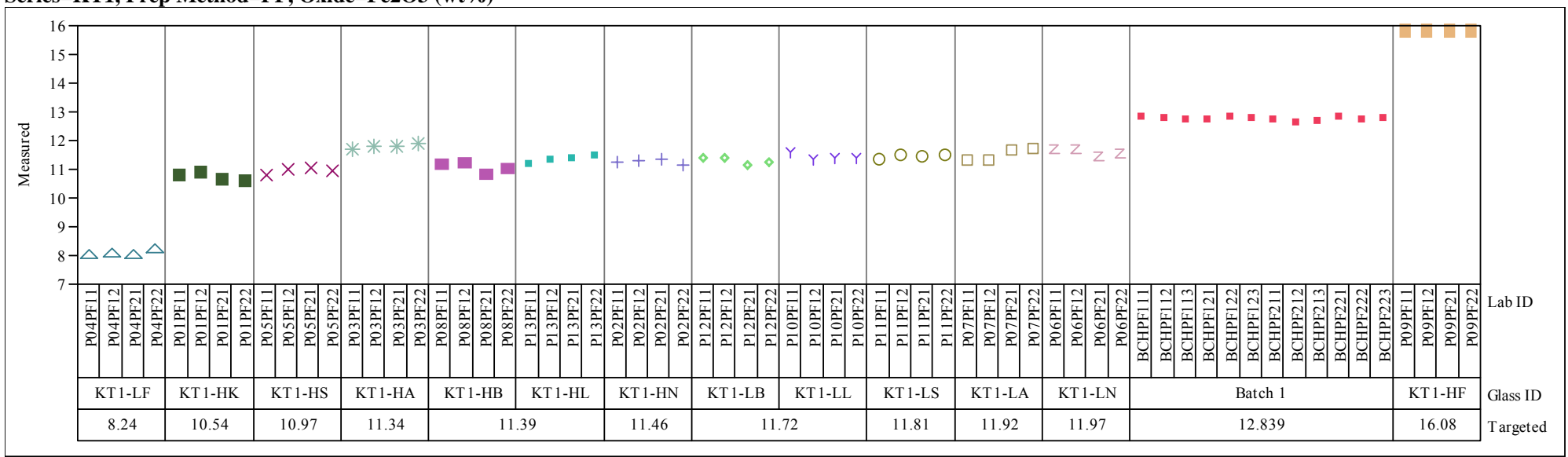

Series=KT1, Prep Method=PF, Oxide=Li2O (wt \%)

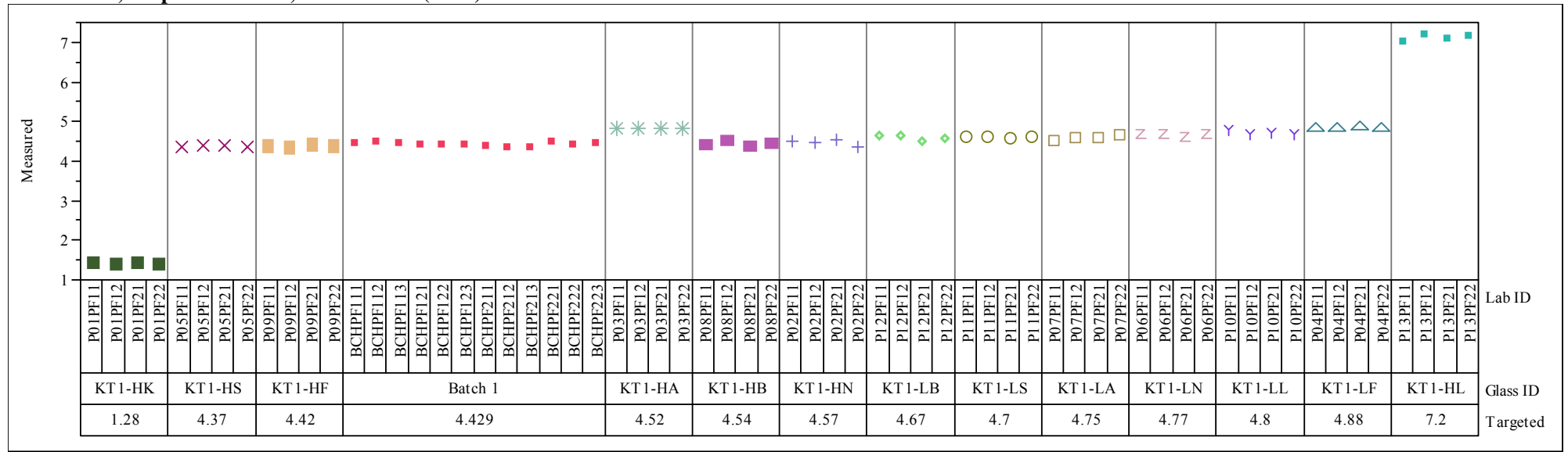


Exhibit A-3. Statistical Evaluation of the ICP-AES Calibration Effects from the KT01 Batch 1 Results by Oxide.

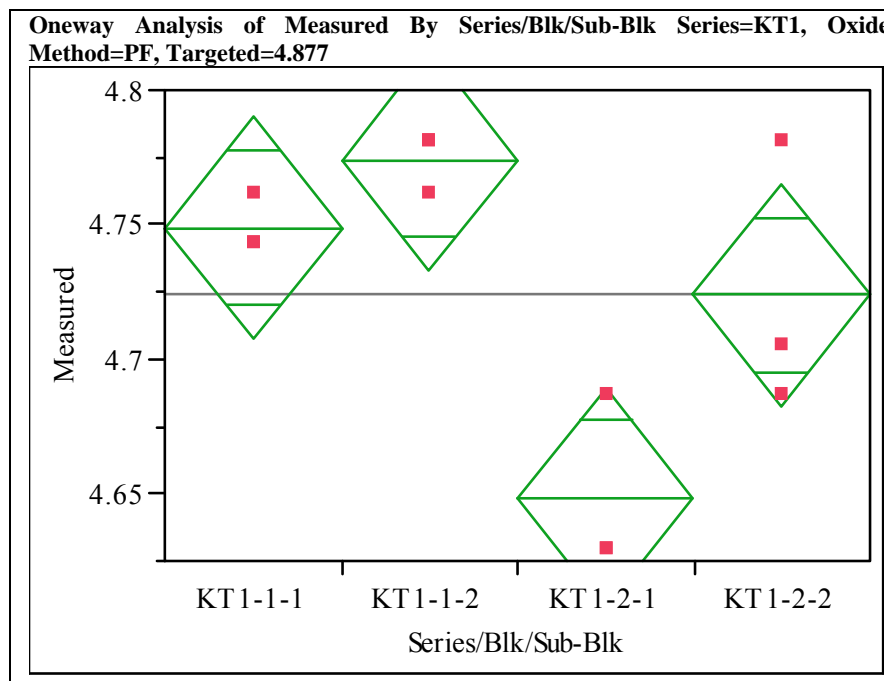

\section{Oneway Anova}

Summary of Fit

$\begin{array}{ll}\text { Rsquare } & 0.777778 \\ \text { Adj Rsquare } & 0.694444 \\ \text { Rot } & 0.030855\end{array}$

$\begin{array}{ll}\text { Adj Rsquare } & 0.694444 \\ \text { Root Mean Square Error } & 0.030855\end{array}$

Mean of Response $\quad 4.72375$

Observations (or Sum Wgts)

Analysis of Variance

Source DF Sum of Squares Mean Square F Ratio Prob > F

$\begin{array}{lrrrrr}\text { Series/Blk/Sub-Blk } & 3 & 0.02665757 & 0.008886 & 9.3333 & 0.0054\end{array}$

Error

C. Total

$\begin{array}{rr}8 & 0.00761645 \\ 11 & 0.03427402\end{array}$

Means for Oneway Anova

Level Number Mean Std Error Lower 95\% Upper 95\%

$\begin{array}{llllll}\text { KT1-1-1 } & 3 & 4.74894 & 0.01781 & 4.7079 & 4.7900 \\ \text { KT1-1-2 } & 3 & 4.7748 & 0.01781 & 4.7331 & 4.8152\end{array}$

\begin{tabular}{llllll} 
KT1-1-2 & 3 & 4.77414 & 0.01781 & 4.7331 & 4.8152 \\
\hline
\end{tabular}

$\begin{array}{llllll}\text { KT1-2-1 } & 3 & 4.64817 & 0.01781 & 4.6071 & 4.6893 \\ \text { KT1-2-2 } & 3 & 4.72375 & 0.01781 & 4.6827 & 4.7648\end{array}$

Std Error uses a pooled estimate of error variance

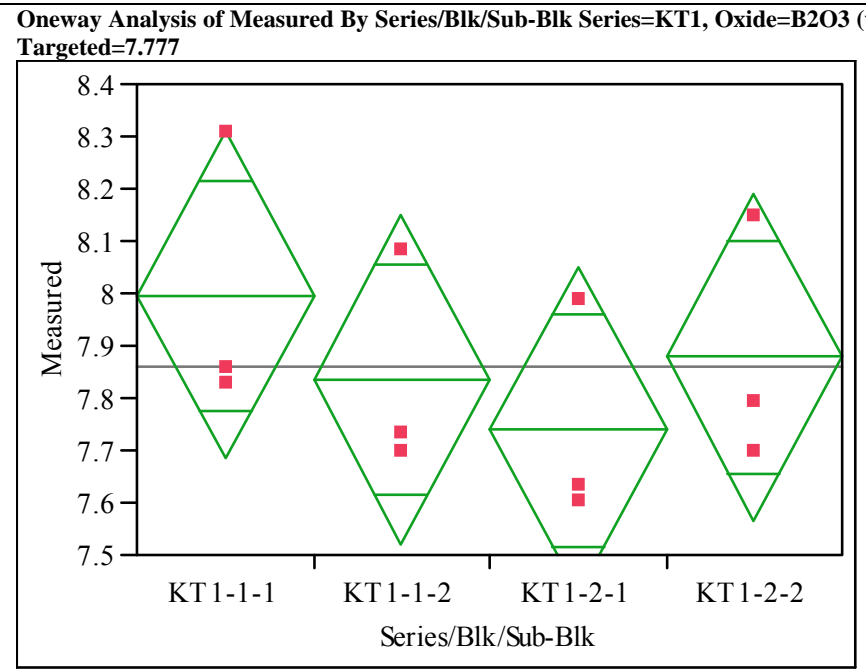

\section{Oneway Anova}

Summary of Fit

$\begin{array}{ll}\text { Rsquare } & 0.188332 \\ \text { Adj Rsquare } & -0.11604 \\ \text { Rot } & 0.235148\end{array}$

$\begin{array}{ll} & -0.11604 \\ \text { Adj Rsquare } & 0.235148\end{array}$

Root Mean Square Error $\quad 0.235148$

Observations (or Sum Wgts) 7.861923

Analysis of Variance

Source DF Sum of Squares Mean Square F Ratio Prob $>$ F

$\begin{array}{lrrrrr} & & & & & \\ \text { Source } & & & & & \\ \text { Series/Blk/Sub-Blk } & 3 & 0.10264078 & 0.034214 & 0.6188 & 0.6222\end{array}$

$\begin{array}{lrrr}\text { Error } & 8 & 0.44235759 & 0.055295 \\ \text { C. Total } & 11 & 0.54499837 & \end{array}$

Means for Oneway Anova

Level Number Mean Std Error Lower 95\% Upper 95\%

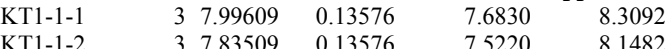

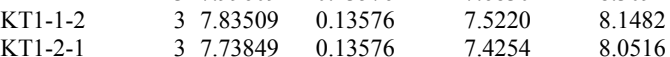

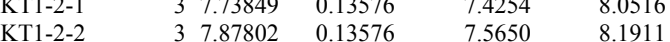

Std Error uses a pooled estimate of error variance 
Exhibit A-3. Statistical Evaluation of the ICP-AES Calibration Effects from the KT01 Batch 1 Results by Oxide. (continued)

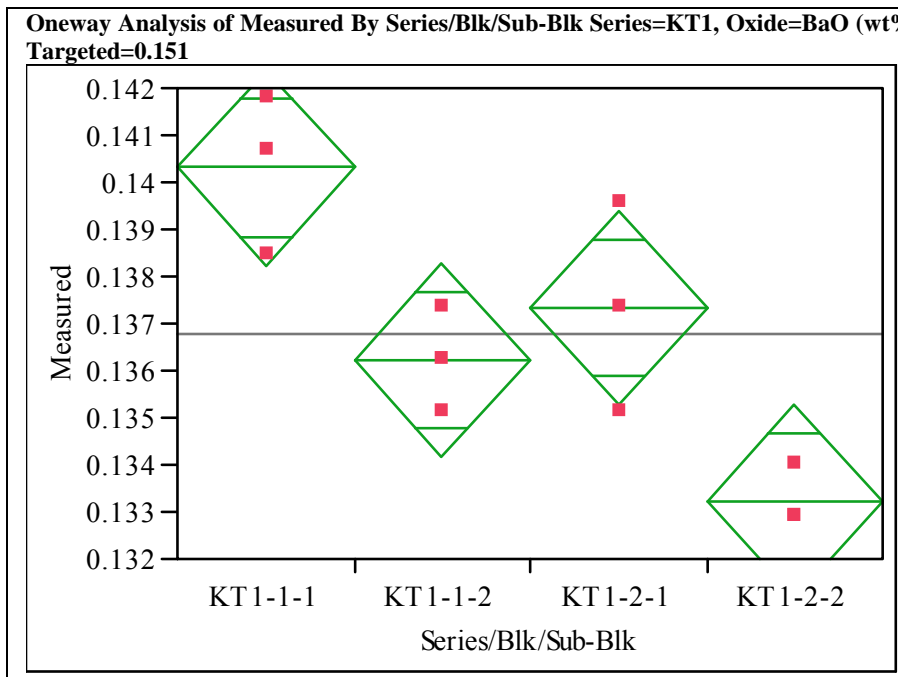

\section{Oneway Anova}

Summary of Fit

$\begin{array}{lr}\text { Rsquare } & 0.800866\end{array}$

0.72619
Adj Rsquare
Root Mean Square Error $\quad 0.001546$

$\begin{array}{ll}\text { Root Mean Square Error } & 0.001546 \\ \text { Mean of Response } & 0.136771\end{array}$

$\begin{array}{lr}\text { Mean of Response } & 0.136771 \\ \text { Observations (or Sum Wgts) } & 12\end{array}$

Analysis of Variance

$\begin{array}{lrrrrr}\text { Source } & \text { DF } & \text { Sum of Squares } & \text { Mean Square } & \text { F Ratio } & \text { Prob }>\text { F } \\ \text { Series/Blk/Sub-Blk } & 3 & 0.00007687 & 0.000026 & 10.7246 & 0.0035\end{array}$

Error

$\begin{array}{rrr}3 & 0.00007687 & 0.000026 \\ 8 & 0.00001911 & 2.389 \mathrm{e}-6\end{array}$

C. Tota

Means for Oneway Anova

Level Number Mean Std Error Lower 95\% Upper 95\%

$\begin{array}{lllrrr}\text { KT1-1-1 } & 3 & 0.140307 & 0.00089 & 0.13825 & 0.14236\end{array}$

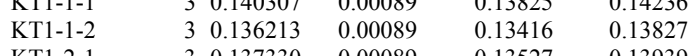

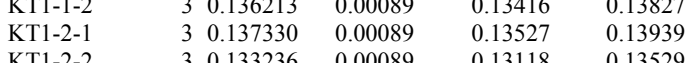

Std Error uses a pooled estimate of error variance

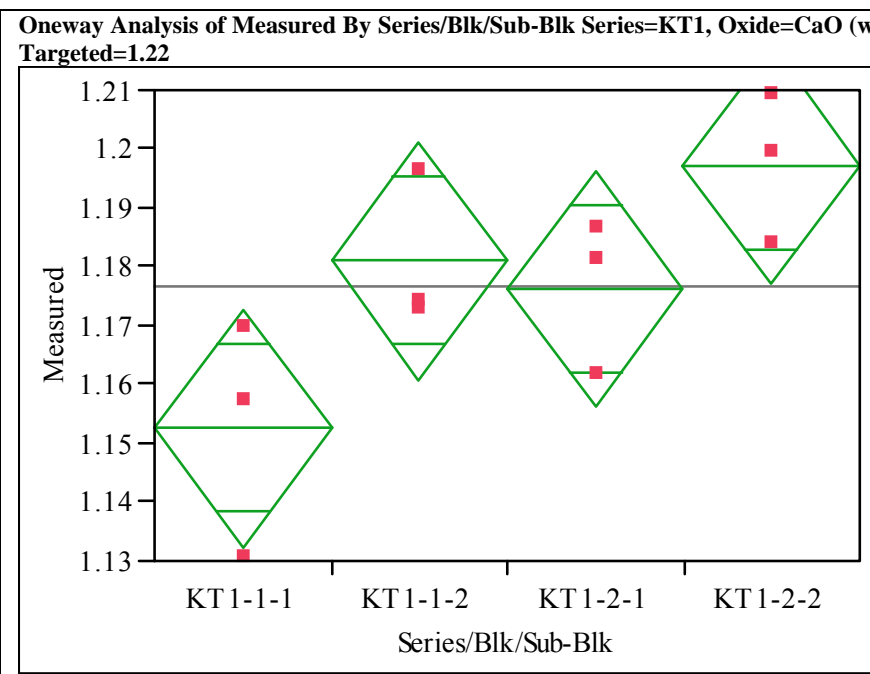

\section{Oneway Anova}

Summary of Fit

$\begin{array}{ll}\text { Rsquare } & 0.627592 \\ \text { Adj Rsquare } & 0.487939 \\ \text { Root Mean Square Error } & 0.015118 \\ \text { Mean of Response } & 1.176727\end{array}$

Observations (or Sum Wgts)

Analysis of Variance

$\begin{array}{lrrrrr}\text { Source } & \text { DF } & \text { Sum of Squares } & \text { Mean Square } & \text { F Ratio } & \text { Prob }>\text { F } \\ \text { Series/Blk/Sub-Blk } & 3 & 0.00308152 & 0.001027 & 4.4939 & 0.0396\end{array}$

$\begin{array}{lrrr}\text { Error } & 8 & 0.00182855 & 0.000229 \\ \text { C. Total } & 11 & 0.00491006 & \end{array}$

Means for Oneway Anova

Level Number Mean Std Error Lower 95\% Upper 95\%

$\begin{array}{lrrrrr}\text { Level } & \text { Number } & \text { Mean } & \text { Std Error } & \text { Lower 95\% } & \text { Upper 95\% } \\ \text { KT1-1-1 } & 3 & 1.15247 & 0.00873 & 1.1323 & 1.1726\end{array}$

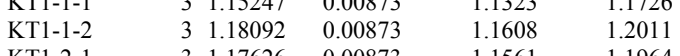

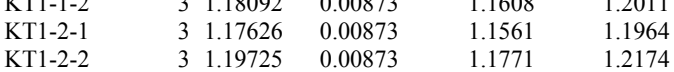

Std Error uses a pooled estimate of error variance 
Exhibit A-3. Statistical Evaluation of the ICP-AES Calibration Effects from the KT01 Batch 1 Results by Oxide. (continued)

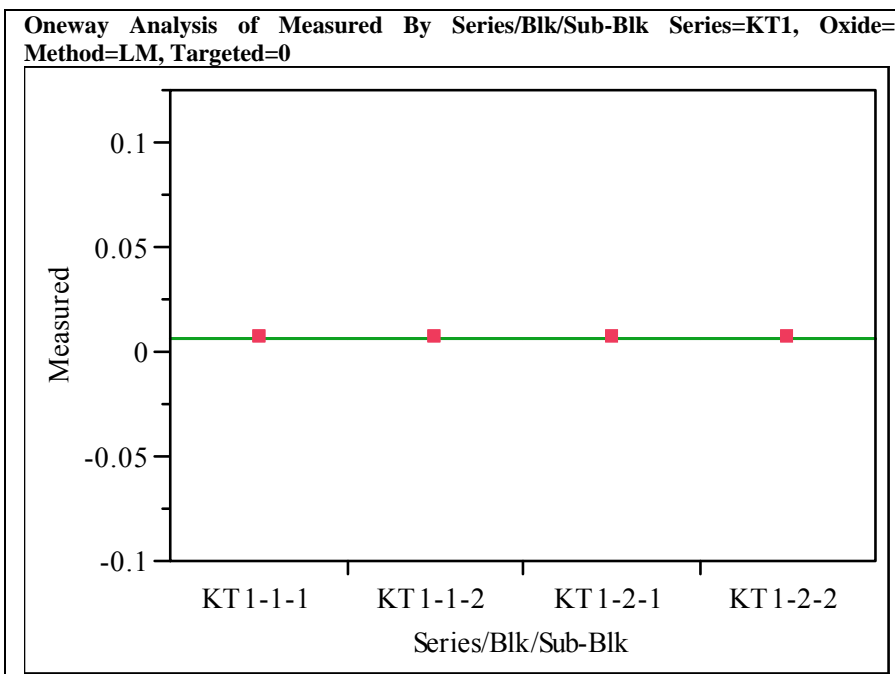

\section{Oneway Anova}

Summary of Fit

Rsquare

Adj Rsquare

$\begin{array}{lr}\text { Root Mean Square Error } & 0 \\ \text { Mean of Response } & 0.005857\end{array}$

Analysis of Variance

Source DF Sum of Squares Mean Square F Ratio Prob $>$ F

$\begin{array}{lrrr}\text { Source } & \text { DF } & \text { Sum of Squares } & \text { Mean Square } \\ \text { Series/Blk/Sub-Blk } & 3 & 0 & 0 \\ \text { Error } & 8 & 0 & 0\end{array}$

Error

Means for Oneway Anova

Level Number Mean Std Error Lower 95\% Upper 95\%

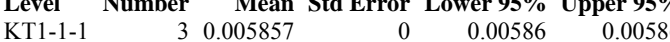

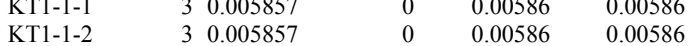

$\begin{array}{llllll}\text { KT1-1-1-2 } & 3 & 0.005857 & 0 & 0.00586 & 0.00586 \\ \text { KT1-2-1 } & 3 & 0.005857 & 0 & 0.00586 & 0.00586\end{array}$

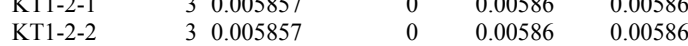

Std Error uses a pooled estimate of error variance

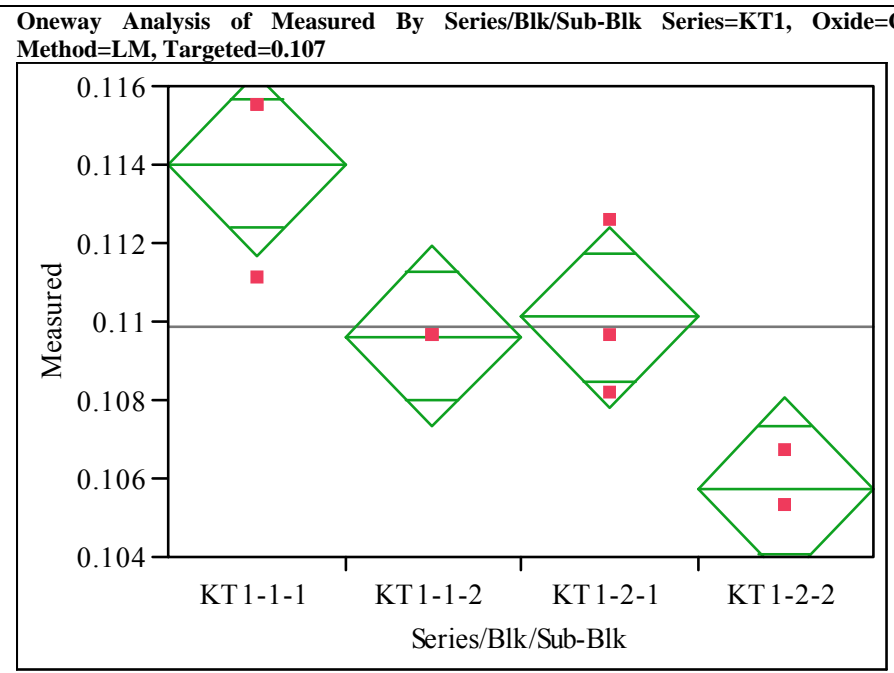

\section{Oneway Anova}

Summary of Fit

$\begin{array}{ll}\text { Rsquare } & 0.810056\end{array}$

0.738827
Adj Rsquare
Root Mean Square Error $\quad 0.00174$

Root Mean Square Error $\quad 0.00174$

Observations (or Sum Wgts)

Analysis of Variance

Source DF Sum of Squares Mean Square F Ratio Prob $>$ F

$\begin{array}{llllll}\text { Series/Blk/Sub-Blk } & 3 & 0.00010325 & 0.000034 & 11.3725 & 0.0029\end{array}$

$\begin{array}{lrrr}\text { Error } & 8 & 0.00002421 & 3.026 \mathrm{e}-6\end{array}$

Means for Oneway Anova

Level Number Mean Std Error Lower 95\% Upper 95\%

\begin{tabular}{lrrrrr} 
KT1-1-1 & 3 & 0.114005 & 0.00100 & 0.11169 & 0.11632 \\
\hline
\end{tabular}

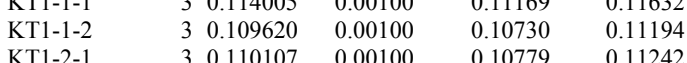

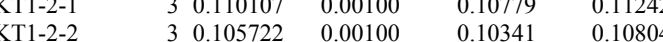

Std Error uses a pooled estimate of error variance 
Exhibit A-3. Statistical Evaluation of the ICP-AES Calibration Effects from the KT01 Batch 1 Results by Oxide. (continued)

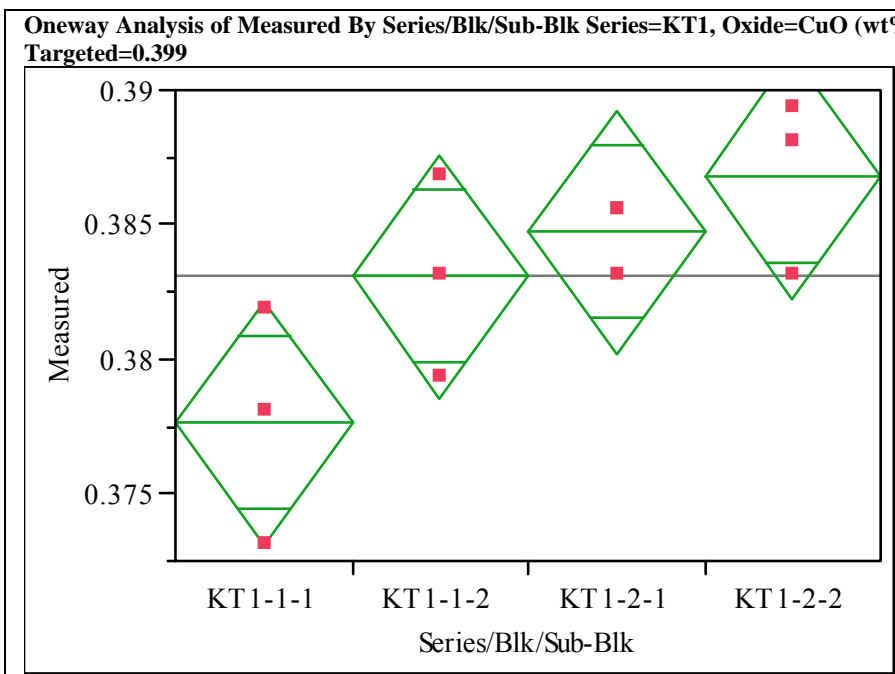

\section{Oneway Anova}

Summary of Fit

$\begin{array}{ll}\text { Rsquare } & 0.599099\end{array}$

$\begin{array}{ll}\text { Adj Rsquare } & 0.448761 \\ \text { Root Mean Square Error } & 0.003409\end{array}$

$\begin{array}{ll}\text { Root Mean Square Error } & 0.003409 \\ \text { Mean of Response } & 0.383051\end{array}$

$\begin{array}{lr}\text { Mean of Response } & 0.383051 \\ \text { Observations (or Sum Wgts) } & 12\end{array}$

Analysis of Variance

$\begin{array}{lrrrrr}\text { Source } & \text { DF } & \text { Sum of Squares } & \text { Mean Square } & \text { F Ratio } & \text { Prob }>\text { F } \\ \text { Series/Blk/Sub-Blk } & 3 & 0.00013894 & 0.000046 & 3.9850 & 0.0523\end{array}$

Error

$\begin{array}{ll}3 & 0.00013894 \\ 8 & 0.00009298\end{array}$

0.000012

C. Total

0.00023192

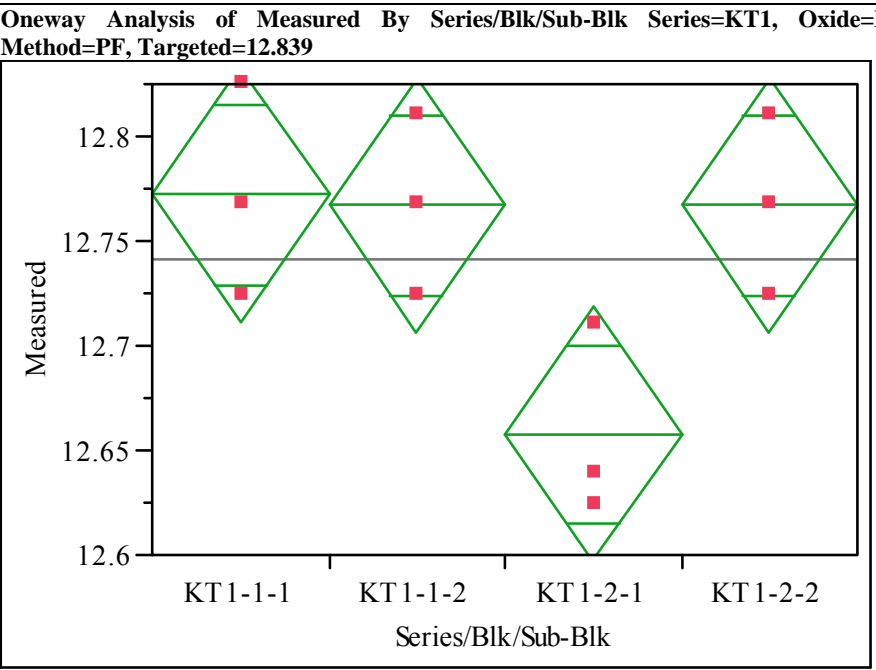

\section{Oneway Anova}

Summary of Fit

$\begin{array}{lr}\text { Rsquare } & 0.62634 \\ \text { Adj Rsquare } & 0.486217 \\ \text { Root Mean Square Error } & 0.045586 \\ \text { Mean of Response } & 12.74101 \\ \text { Obse } & \end{array}$

Observations (or Sum Wgts)

Analysis of Variance

Source DF Sum of Squares Mean Square F Ratio Prob > F

$\begin{array}{lrrrrr} & & & \\ \text { Series/Blk/Sub-Blk } & 3 & 0.02786711 & 0.009289 & 4.4699 & 0.0401\end{array}$

$\begin{array}{lrr}\text { Error } & 8 & 0.01662488 \\ \text { C. } & 11 & 0.04449198\end{array}$

0.002078

Means for Oneway Anova

Level Number Mean Std Error Lower 95\% Upper 95\%

$\begin{array}{llllll}\text { KT1-1-1 } & 3 & 0.377626 & 0.00197 & 0.37309 & 0.38217\end{array}$

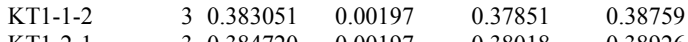

$\begin{array}{llllll}\text { KT1-2-1 } & 3 & 0.384720 & 0.00197 & 0.38018 & 0.38926 \\ \text { KT1-2-2 } & 3 & 0.386806 & 0.00197 & 0.38227 & 0.3913\end{array}$

Std Error uses a pooled estimate of error variance

Means for Oneway Anova

Level Number Mean Std Error Lower 95\% Upper 95\%

$\begin{array}{lrrrrr}\text { KT1-1-1 } & 3 & 12.7720 & 0.02632 & 12.711 & 12.833\end{array}$

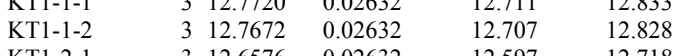

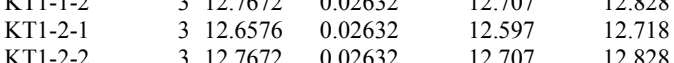

Std Error uses a pooled estimate of error variance 
Exhibit A-3. Statistical Evaluation of the ICP-AES Calibration Effects from the KT01 Batch 1 Results by Oxide. (continued)

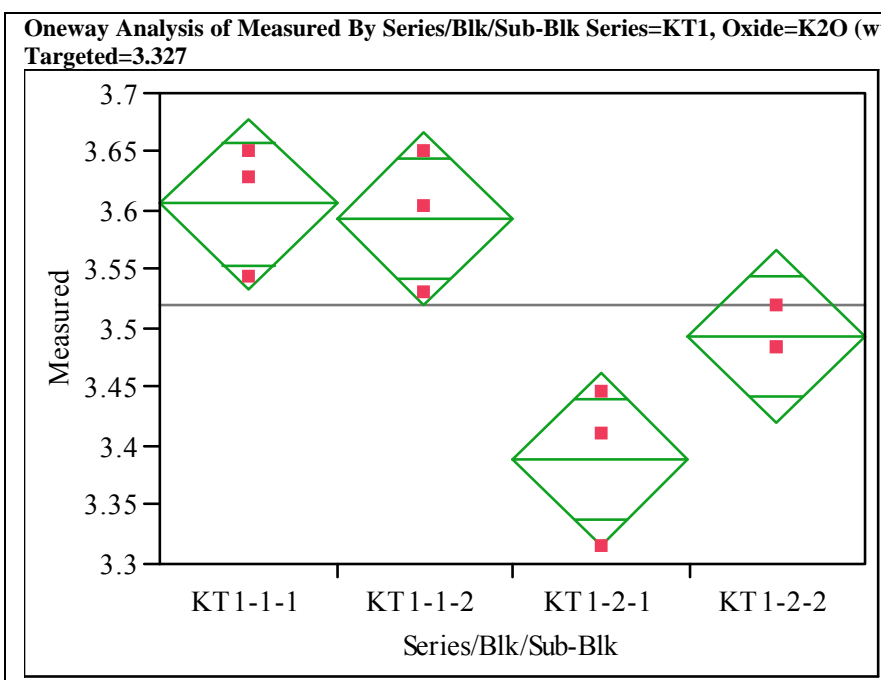

\section{Oneway Anova}

Summary of Fit

$\begin{array}{ll}\text { Rsquare } & 0.792565 \\ & 0.714777\end{array}$

$\begin{array}{ll}\text { Adj Rsquare } & 0.714777 \\ \text { Root Mean Square Error } & 0.054872\end{array}$

Root Mean Square Error $\quad 0.054872$

$\begin{array}{lr}\text { Mean of Response } & 3.520444 \\ \text { Observations (or Sum Wgts) } & 12\end{array}$

Analysis of Variance

Source DF Sum of Squares Mean Square F Ratio Prob > F

$\begin{array}{lllllll}0.030678 & 10.1888 & 0.0042\end{array}$

Error

3 . 0.0920335

0.00301

C. Total

$11 \quad 0.11612117$

Oneway Analysis of Measured By Series/Blk/Sub-Blk Series=KT1, Oxide=La2O3 (wt\%), Prep Method $=$ LM, Targeted $=0$

Means for Oneway Anova

Level Number Mean Std Error Lower 95\% Upper 95\%

$\begin{array}{lllrrr}\text { KT1-1-1 } & 3 & 3.60577 & 0.03168 & 3.5327 & 3.6788\end{array}$

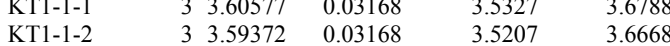

$\begin{array}{llllll}\text { KT1-1-2 } & 3 & 3.59372 & 0.03168 & 3.5207 & 3.6668 \\ \text { KT1-2-1 } & 3 & 3.38894 & 0.03168 & 3.3159 & 3.4620\end{array}$

$\begin{array}{llllll}\text { KT1-2-2 } & 3 & 3.388934 & 0.03168 & 3.3159 & 3.4620 \\ \text { KT1-2 } & & & 0.03168 & 3.4203 & 3.5664\end{array}$

Std Error uses a pooled estimate of error variance

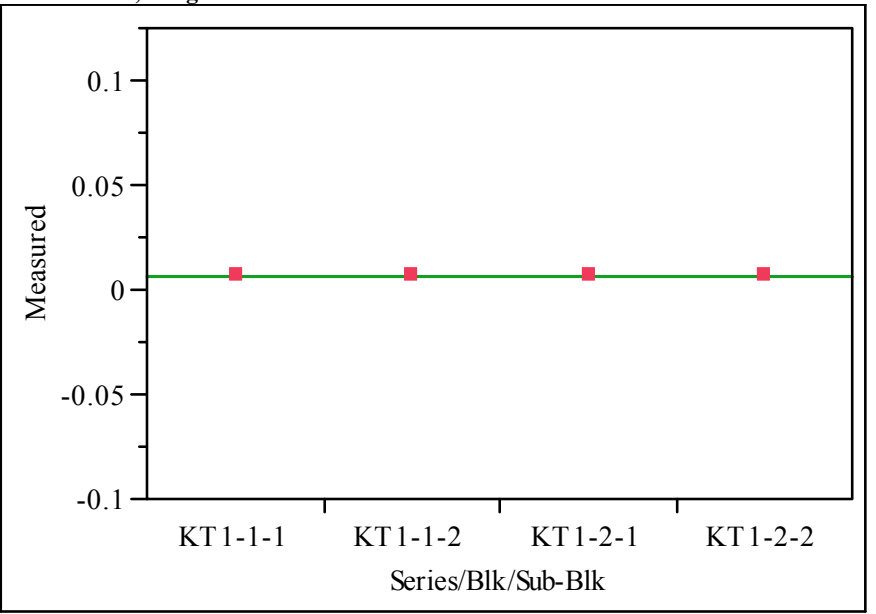

\section{Oneway Anova}

Summary of Fit

Rsquare

Adj Rsquare

Root Mean Square Error $\quad 0$

Observations (or Sum Wgts)

Analysis of Variance

Source DF Sum of Squares Mean Square F Ratio Prob $>$ F

$\begin{array}{llcr}\text { Series/Blk/Sub-Blk } & 3 & 0 & 0 \\ \text { Error } & 8 & 0 & 0\end{array}$

$\begin{array}{lrr}\text { Error } & 8 & 0 \\ \text { C. Total } & 11 & 0\end{array}$

Means for Oneway Anova

Level Number Mean Std Error Lower 95\% Upper 95\%

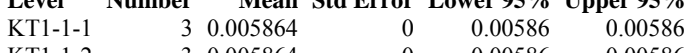

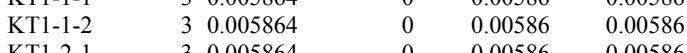

$\begin{array}{llllll}\text { KT1-1-1-2 } & 3 & 0.005864 & 0 & 0.00586 & 0.00586 \\ \text { KT1-2-1 } & 3 & 0.005864 & 0 & 0.00586 & 0.00586 \\ \text { KT1-2-2 } & 3 & 0.005864 & 0 & 0.00586 & 0.00586\end{array}$

Std Error uses a pooled estimate of error variance 
Exhibit A-3. Statistical Evaluation of the ICP-AES Calibration Effects from the KT01 Batch 1 Results by Oxide. (continued)

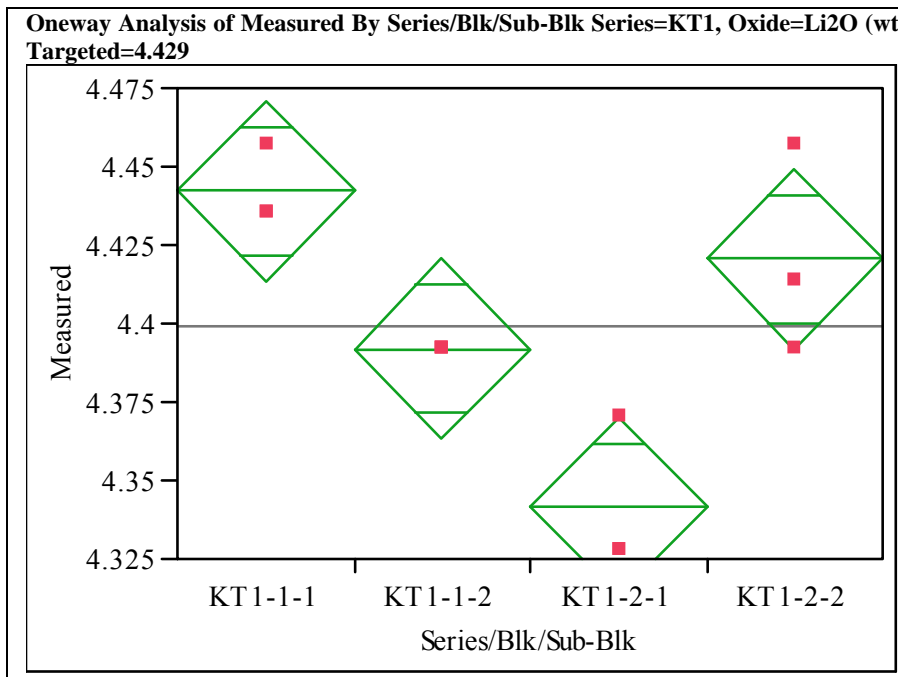

\section{Oneway Anova}

Summary of Fit

Rsquare $\quad 0.820896$

$\begin{array}{ll}\text { Adj Rsquare } & 0.753731 \\ \text { Root Mean Square Error } & 0.021529\end{array}$

$\begin{array}{ll}\text { Root Mean Square Error } & 0.021529 \\ \text { Mean of Response } & 4.399092\end{array}$

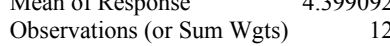

Analysis of Variance

Source DF Sum of Squares Mean Square F Ratio Prob $>$ F

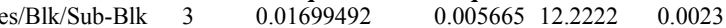

Error

3 . 0.01699492

0.000463

C. Total

Oneway Analysis of Measured By Series/Blk/Sub-Blk Series=KT1, Oxide=MgO (wt\%), Prep Method=LM, Targeted $\mathbf{1} \mathbf{1 . 4 1 9}$

Level Number Mean Std Error Lower 95\% Upper 95\%

\begin{tabular}{lrrrrr} 
KT1-1-1 & 3 & 4.44215 & 0.01243 & 4.4135 & 4.4708 \\
\hline
\end{tabular}

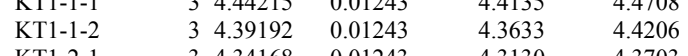

$\begin{array}{llllll}\text { KT1-2-1 } & 3 & 4.34168 & 0.01243 & 4.3130 & 4.3703\end{array}$

Std Error uses a pooled estimate of error variance

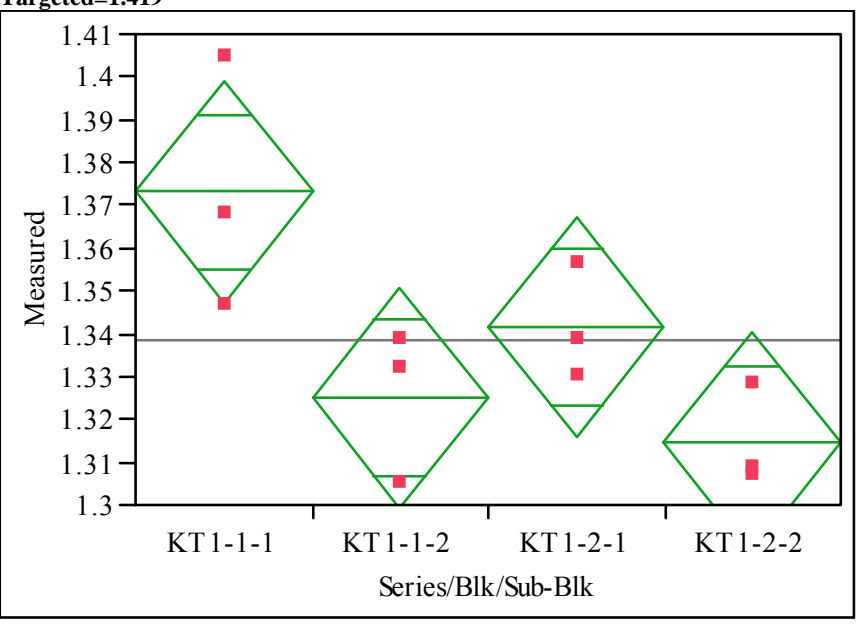

\section{Oneway Anova}

Summary of Fit

$\begin{array}{ll}\text { Rsquare } & 0.663125 \\ \text { Adj Rsquare } & 0.536797\end{array}$

0.6336797
Root Mean Square Error $\quad 0.019345$

Root Mean Square Error $\quad 0.019345$

Observations (or Sum Wgts)

Analysis of Variance

Source DF Sum of Squares Mean Square F Ratio Prob $>$ F

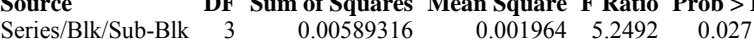

$\begin{array}{lrrr}\text { Error } & 8 & 0.00299379 & 0.000374 \\ \text { C. Total } & 11 & 0.00888695 & \end{array}$

Means for Oneway Anova

Level Number Mean Std Error Lower 95\% Upper 95\%

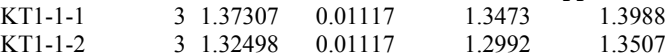

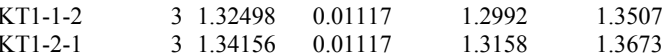

$\begin{array}{llllll}\text { KT1-2-1 } & 3 & 1.34156 & 0.0117 & 1.3158 & 1.3673 \\ \text { KT1-2-2 } & 3 & 1.31448 & 0.01117 & 1.2887 & 1.3402\end{array}$

Std Error uses a pooled estimate of error variance 
Exhibit A-3. Statistical Evaluation of the ICP-AES Calibration Effects from the KT01 Batch 1 Results by Oxide. (continued)

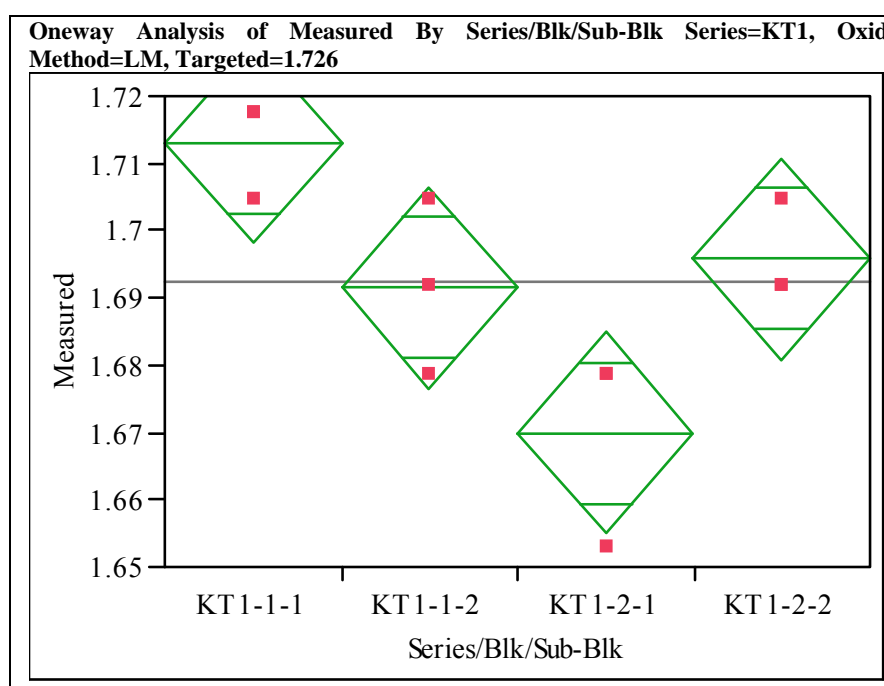

\section{Oneway Anova}

Summary of Fit

Rsquare $\quad 0.738182$

$\begin{array}{lr}\text { Adj Rsquare } & 0.64 \\ \text { Rot Mean Square Error } \quad 0.011182\end{array}$

Root Mean Square Error $\quad 0.011182$

Mean of Response 1.692548

Analysis of Variance

Source DF Sum of Squares Mean Square F Ratio Prob $>$ F

$\begin{array}{lllllll} & 0.00282034 & 0.000940 & 7.5185 & 0.0103\end{array}$

Error

$3 \quad 0.00282034$

0.000125

C. Total

$11 \quad 0.00382066$

Means for Oneway Anova

Level Number Mean Std Error Lower 95\% Upper 95\%

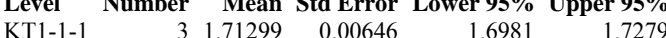

$\begin{array}{llllll}\text { KT1-1-1 } & 3 & 1.712147 & 0.00646 & 1.6981 & 1.7279 \\ \text { KT1-1-2 } & 3 & 1.69147 & 0.00646 & 1.6766 & 1.7064\end{array}$

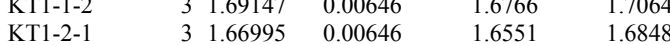

$\begin{array}{llllll}\text { KT1-2-1 } & 3 & 1.66995 & 0.00646 & 1.6551 & 1.6848 \\ \text { KT1-2-2 } & 3 & 1.69578 & 0.00646 & 1.6809 & 1.7107\end{array}$

Std Error uses a pooled estimate of error variance

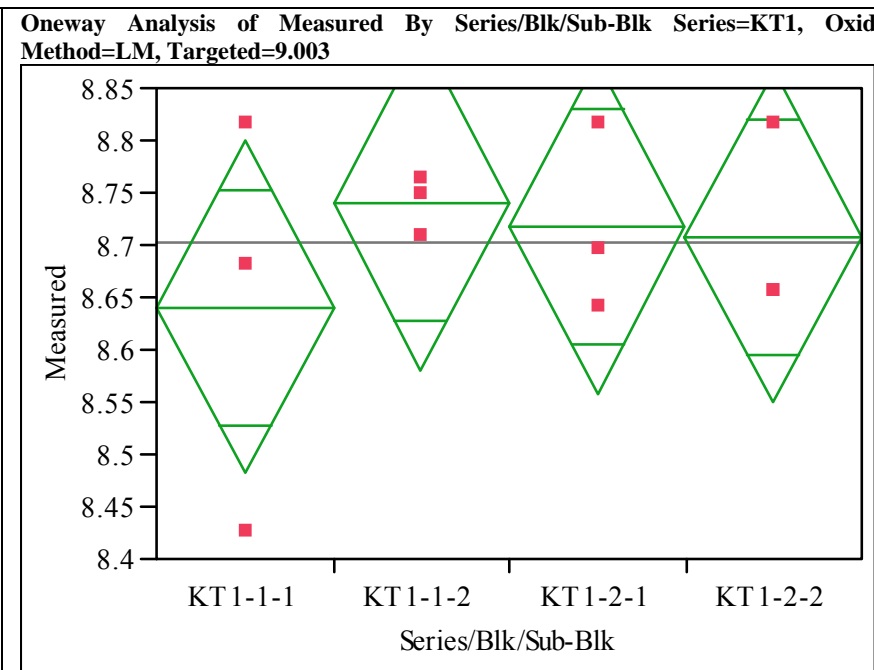

\section{Oneway Anova}

Summary of Fit

$\begin{array}{ll}\text { Rsquare } & 0.125058 \\ \text { Adj Rsquare } & -0.20305\end{array}$

$\begin{array}{lr} & -1250385 \\ \text { Root Mean Square Error } \quad 0.11937\end{array}$

Root Mean Square Error $\quad 0.11937$

Observations (or Sum Wgts)

Analysis of Variance

Source DF Sum of Squares Mean Square F Ratio Prob $>$ F

$\begin{array}{lrrrrr}\text { Source } & & & & & \\ \text { Series/Blk/Sub-Blk } & 3 & 0.01629337 & 0.005431 & 0.3812 & 0.7695\end{array}$

$\begin{array}{lrrr}\text { Error } & 8 & 0.11399299 & 0.014249 \\ \text { C. Total } & 11 & 0.13028636 & \end{array}$

Means for Oneway Anova

Level Number Mean Std Error Lower 95\% Upper 95\%

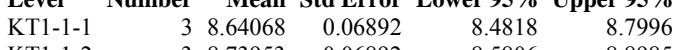

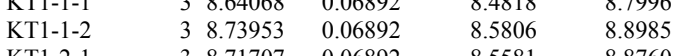

$\begin{array}{llllll}\text { KT1-1-2 } & 3 & 8.73953 & 0.06892 & 8.5806 & 8.8985 \\ \text { KT1-2-1 } & 3 & 8.71707 & 0.06892 & 8.5581 & 8.8760 \\ \text { KT1-2 } & 3 & 8.70808 & 0.06892 & 8.5492 & 8.8670\end{array}$

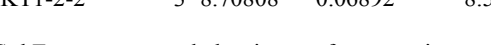

Std Error uses a pooled estimate of error variance 
Exhibit A-3. Statistical Evaluation of the ICP-AES Calibration Effects from the KT01 Batch 1 Results by Oxide. (continued)

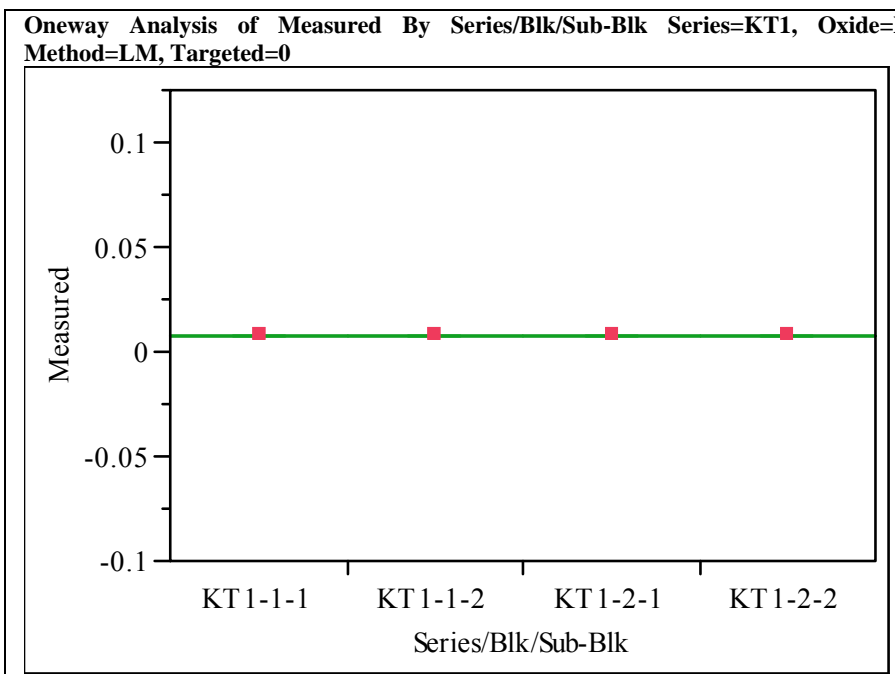

\section{Oneway Anova}

Summary of Fit

Rsquare

Adj Rsquare $\quad-00$

Root Mean Square Error $\quad \begin{array}{rr}-0.375 \\ 1.06-18\end{array}$

$\begin{array}{rr}1.06 \mathrm{e}-18 \\ \text { Mean of Response } & 0.007153\end{array}$

Observations (or Sum Wgts) 0.007153

Analysis of Variance

Source DF Sum of Squares Mean Square F Ratio Prob $>$ F

$\begin{array}{lrrrrr}\text { Series/Blk/Sub-Blk } & 3 & 0 & 0 & 0.0000 & 1.0000 \\ \text { Error } & 8 & 9.0278 \mathrm{e}-36 & 1.128 \mathrm{e}-36 & & \end{array}$

Error

$8 \quad 9.0278 \mathrm{e}-36 \quad 1.128 \mathrm{e}-36$

C. Total

$9.0278 \mathrm{e}-36$

Means for Oneway Anova

Level Number Mean Std Error Lower 95\% Upper 95\%

$\begin{array}{lrrrrr}\text { KT1-1-1 } & 3 & 0.007153 & 6.133 \mathrm{e}-19 & 0.00715 & 0.00715 \\ \text { KT1-1-2 } & 3 & 0.007153 & 6.133 \mathrm{e}-19 & 0.00715 & 0.00715\end{array}$

$\begin{array}{llllll}\text { KT1-1-2 } & 3 & 0.007153 & 6.133 \mathrm{e}-19 & 0.00715 & 0.00715 \\ \text { KT1-1 } & 3 & 0.007153 & 6.133 \mathrm{e}-19 & 0.00715 & 0.00715\end{array}$

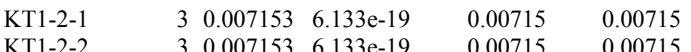

Std Error uses a pooled estimate of error variance

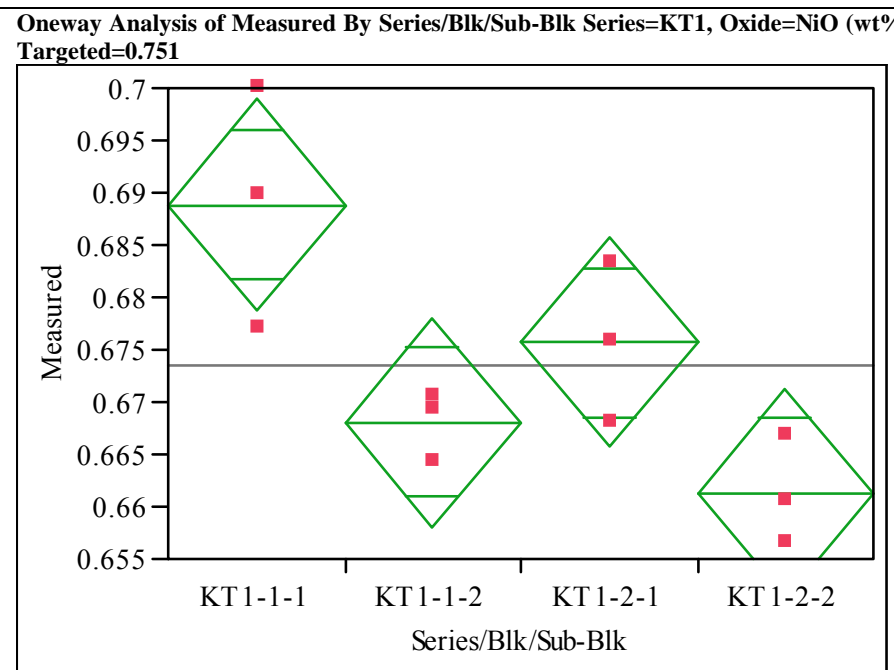

\section{Oneway Anova}

Summary of Fit

$\begin{array}{ll}\text { Rsquare } & 0.734152 \\ \text { Adj Rsquare } & 0.634459 \\ \text { Root Mean Square Error } & 0.007546 \\ \text { Mean of Response } & 0.673471\end{array}$

Observations (or Sum Wgts)

Analysis of Variance

Source DF Sum of Squares Mean Square F Ratio Prob $>$ F

$\begin{array}{lllllll}\text { Series/Blk/Sub-Blk } & 3 & 0.00125803 & 0.000419 & 7.3641 & 0.0109\end{array}$

$\begin{array}{lrrr}\text { Error } & 8 & 0.00045555 & 0.000057 \\ \text { C. Total } & 11 & 0.00171358 & \end{array}$

Means for Oneway Anov

Level Number Mean Std Error Lower 95\% Upper 95\%

$\begin{array}{lllllll}\text { KT1-1-1 } & & 3 & 0.688847 & 0.00436 & 0.67880 & 0.69889 \\ \text { KT1-1-2 } & & 3 & 0.668063 & 0.0043 & 0.65802 & 0.67811\end{array}$

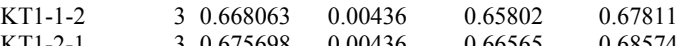

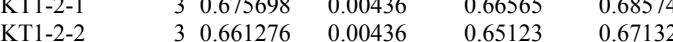

Std Error uses a pooled estimate of error variance 
Exhibit A-3. Statistical Evaluation of the ICP-AES Calibration Effects from the KT01 Batch 1 Results by Oxide. (continued)

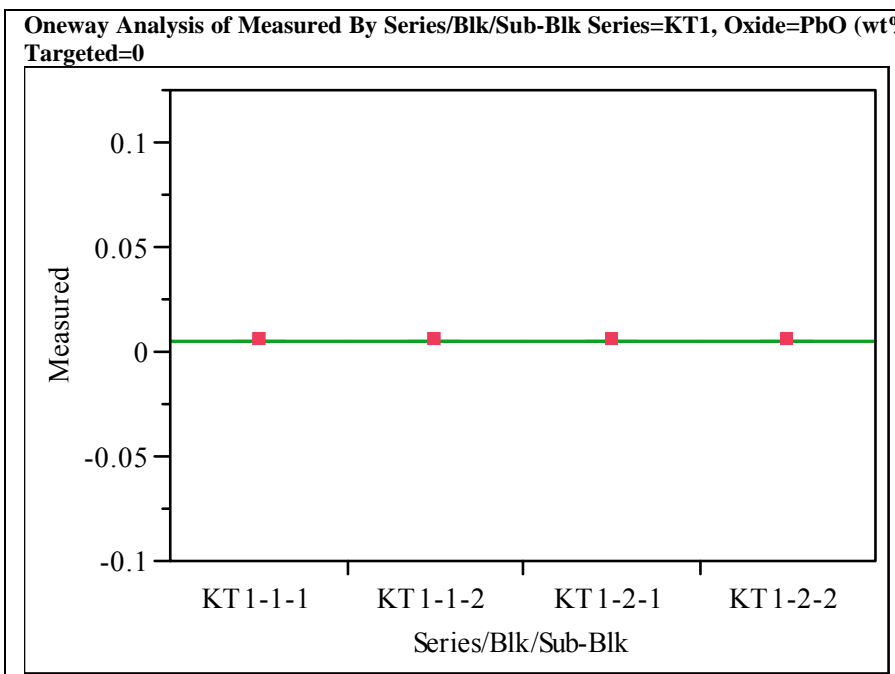

\section{Oneway Anova}

Summary of Fit

Rsquare

$\begin{array}{lr} & 0 \\ \text { Adj Rsquare } & -0.375\end{array}$

Root Mean Square Error $\quad \begin{array}{r}-0.375 \\ 1.06 \mathrm{e}-18\end{array}$

$\begin{array}{lr}\text { Mean of Response } & 1.06 \mathrm{e}-18 \\ 0.005386 & -12\end{array}$

Observations (or Sum Wgts) 0.005386

Anservions (or Sim whe

Source DF Sum of Squares Mean Square F Ratio Prob $>$ F

$\begin{array}{lrrrrr}\text { Series/Blk/Sub-Blk } & 3 & 0 & 0 & 0.0000 & 1.0000 \\ \text { Error } & 8 & 9.0278 \mathrm{e}-36 & 1.128 \mathrm{e}-36 & & \end{array}$

Error

$\begin{array}{lrr}8 & 9.0278 \mathrm{e}-36 & 1.128 \mathrm{e}-36\end{array}$

C. Total

$9.0278 \mathrm{e}-36$

Means for Oneway Anova

Level Number Mean Std Error Lower 95\% Upper 95\%

$\begin{array}{lrrrrr}\text { KT1-1-1 } & 3 & 0.005386 & 6.133 \mathrm{e}-19 & 0.00539 & 0.00539\end{array}$

$\begin{array}{llllll}\text { KT1-1-1 } & 3 & 0.005386 & 6.133 \mathrm{e}-19 & 0.00539 & 0.00539 \\ \text { KT1-1-2 } & 3 & 0.005386 & 6.133 \mathrm{e}-19 & 0.00539 & 0.00539 \\ \text { KT1-2-1 } & 3 & 0.005386 & 6.133 \mathrm{e}-19 & 0.00539 & 0.00539\end{array}$

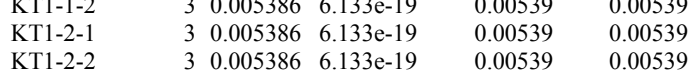

0.0053

(1)

Std Error uses a pooled estimate of error variance

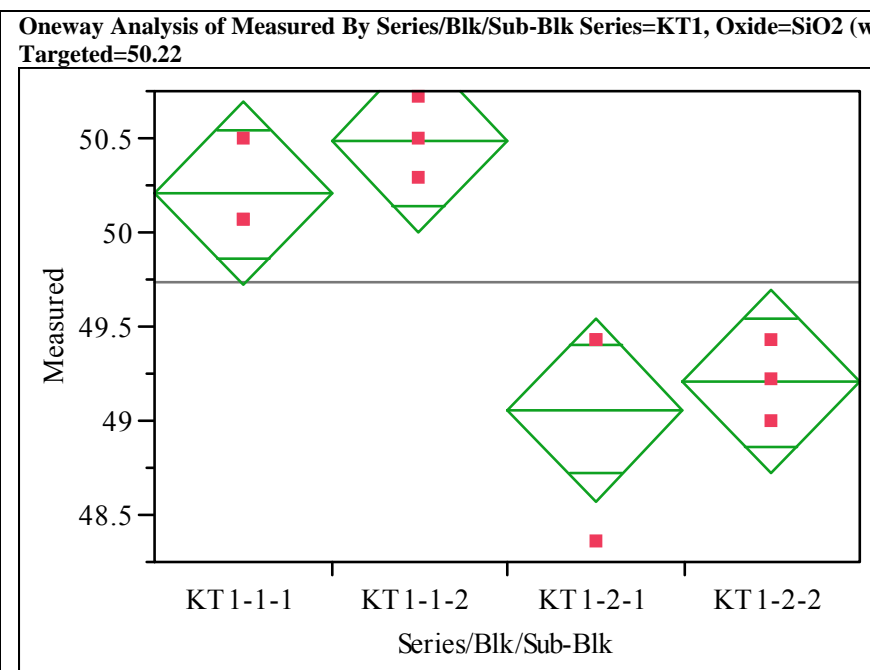

\section{Oneway Anova}

Summary of Fit

$\begin{array}{lr}\text { Rsquare } & 0.810298 \\ \text { Adj Rsquare } & 0.73916\end{array}$

$\begin{array}{lr}\text { Adj Rsquare } & 0.73916 \\ \text { Root Mean Square Error } \quad 0.365355\end{array}$

$\begin{array}{ll}\text { Root Mean Square Error } & 0.365355 \\ \text { Mean of Response } & 49.73873\end{array}$

\begin{tabular}{lr} 
Observations (or Sum Wgts) & 49.73873 \\
\hline
\end{tabular}

Analysis of Variance

Source DF Sum of Squares Mean Square F Ratio Prob > F

$\begin{array}{lrrrrr}\text { Source } & & \text { DF Sum of Squares } & \text { Mean Square } & \text { F Ratio } & \text { Prob }>\text { F } \\ \text { Series/Blk/Sub-Blk } & 3 & 4.5613491 & 1.52045 & 11.3905 & 0.0029\end{array}$

$\begin{array}{lrrr}\text { Error } & 8 & 1.0678744 & 0.13348 \\ \text { C. Total } & 11 & 5.6292235 & \end{array}$

Means for Oneway Anova

Level Number Mean Std Error Lower 95\% Upper 95\%

$\begin{array}{llllll}\text { KT1-1-1 } & 3 & 50.2022 & 0.21094 & 49.716 & 50.689 \\ \text { KT1-1-2 } & 3 & 50.875 & 0.2094 & 50.001 & 50.974\end{array}$

$\begin{array}{llllll}\text { KT1-1-2 } & 3 & 50.4875 & 0.21094 & 50.001 & 50.974 \\ \text { KT1-2-1 } & 3 & 49.0613 & 0.21094 & 48.575 & 49.548\end{array}$

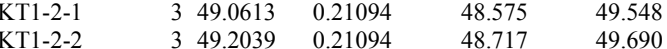

Std Error uses a pooled estimate of error variance 
Exhibit A-3. Statistical Evaluation of the ICP-AES Calibration Effects from the KT01 Batch 1 Results by Oxide. (continued)

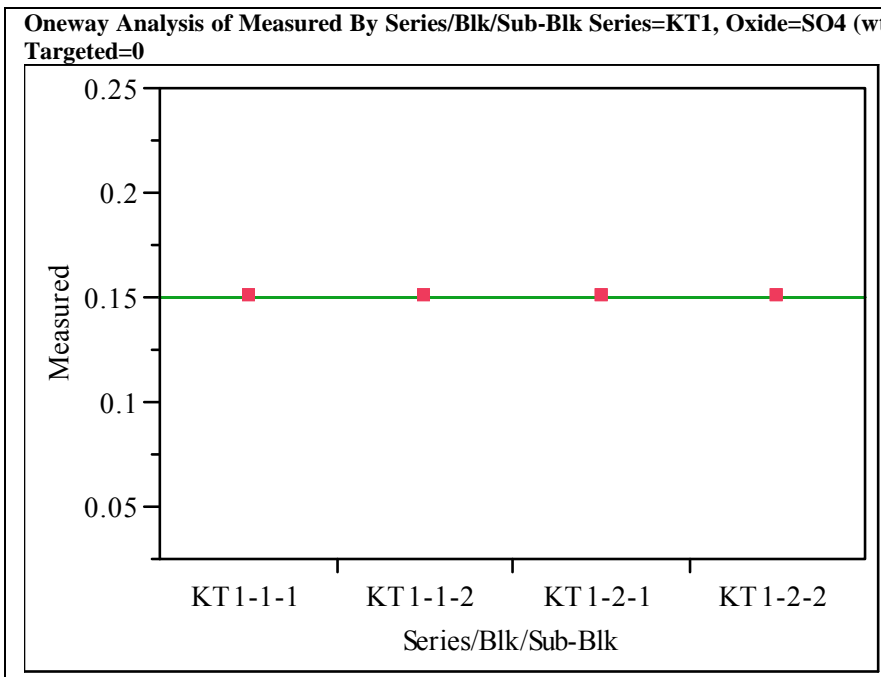

\section{Oneway Anova}

Summary of Fit

Rsquare

Adj Rsquare

$\begin{array}{lr}\text { Root Mean Square Error } & 0 \\ \text { Mean of Response } & 0.149795\end{array}$

Observations (or Sum W

Analysis of Variance

Source DF Sum of Squares Mean Square F Ratio Prob $>$ F

$\begin{array}{llll}\text { Series/Blk/Sub-Blk } & 3 & 0 & 0 \\ \text { Error } & 8 & 0 & 0\end{array}$

Error

Means for Oneway Anova

Level Number Mean Std Error Lower 95\% Upper 95\%

$\begin{array}{lrrrrr}\text { KT1-1-1 } & 3 & 0.149795 & 0 & 0.14980 & 0.14980\end{array}$

$\begin{array}{llllll}\text { KT1-1-1 } & 3 & 0.149795 & 0 & 0.14980 & 0.14980 \\ \text { KT1-1-2 } & 3 & 0.149795 & 0 & 0.14980 & 0.14980\end{array}$

$\begin{array}{llllll}\text { KT1-1-2 } & 3 & 0.149795 & 0 & 0.14980 & 0.14980 \\ \text { KT1-2-1 } & 3 & 0.149795 & 0 & 0.14980 & 0.14980 \\ \text { KT1-2-2 } & 3 & 0.149795 & 0 & 0.14980 & 0.14980\end{array}$

Std Error uses a pooled estimate of error variance

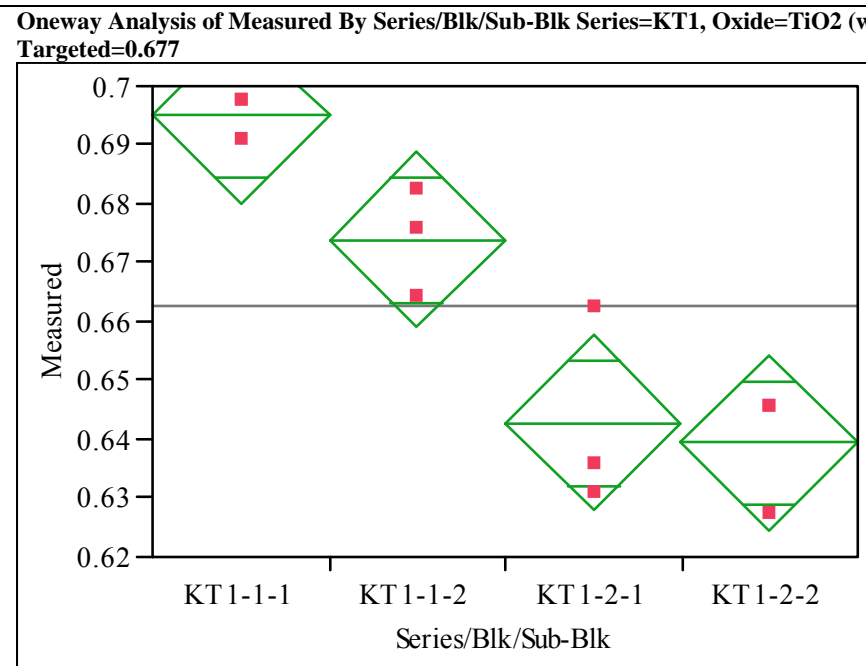

\section{Oneway Anova}

Summary of Fit

$\begin{array}{lr}\text { Rsquare } & 0.862705 \\ \text { Adj Rsquare } & 0.81122\end{array}$

$\begin{array}{ll}\text { Adj Rsquare } & 0.81122 \\ \text { Root Mean Square Error } & 0.01122\end{array}$

Root Mean Square Error $\quad 0.01122$
Mean of Response

Observations (or Sum Wgts) 0.662752

Analysis of Variance

Source DF Sum of Squares Mean Square F Ratio Prob $>$ F

$\begin{array}{llllll}\text { Series/Blk/Sub-Blk } & 3 & 0.00632863 & 0.002110 & 16.7563 & 0.0008\end{array}$

$\begin{array}{lrrr}\text { Error } & 8 & 0.00100717 & 0.000126\end{array}$

Means for Oneway Anova

Level Number Mean Std Error Lower 95\% Upper 95\%

\begin{tabular}{lrrrrr} 
LT $1-1-1$ & 3 & 0.695000 & 0.00648 & 0.68006 & 0.70994 \\
\hline
\end{tabular}

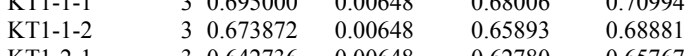

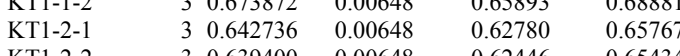

$\begin{array}{llllll}\text { KT1-2-2 } & 3 & 0.639400 & 0.00648 & 0.62446 & 0.65434\end{array}$

Std Error uses a pooled estimate of error variance 
Exhibit A-3. Statistical Evaluation of the ICP-AES Calibration Effects from the KT01 Batch 1 Results by Oxide. (continued)

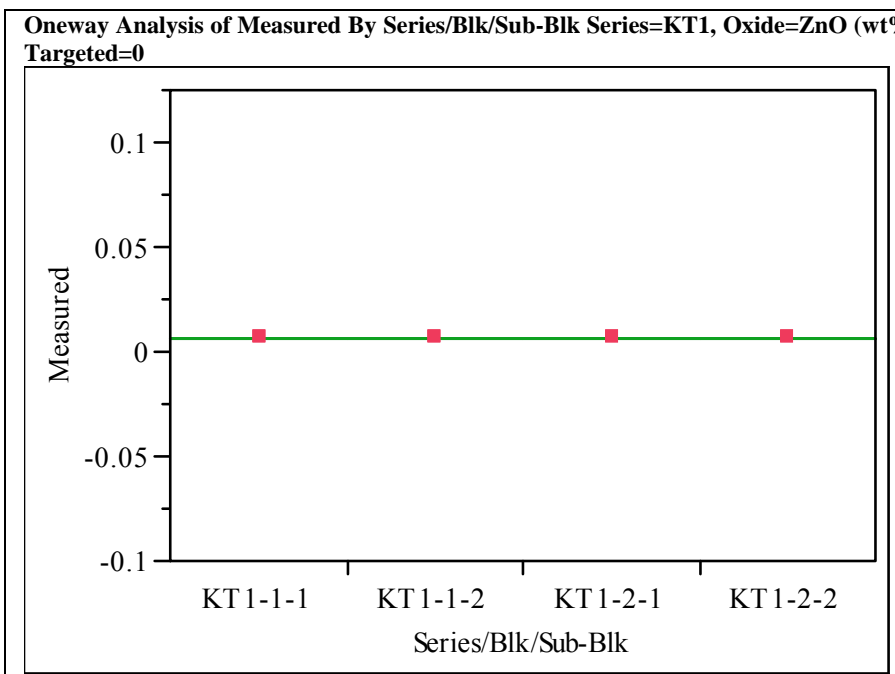

\section{Oneway Anova}

Summary of Fit

Rsquare

Adj Rsquare

$\begin{array}{lr}\text { Root Mean Square Error } & 0 \\ \text { Mean of Response } & 0.006224\end{array}$

Analysis of Variance

Source DF Sum of Squares Mean Square F Ratio Prob > F

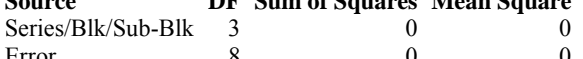

Error $\quad 8$

Means for Oneway Anova

Level Number Mean Std Error Lower 95\% Upper 95\%

$\begin{array}{lrrrrr}\text { LTevel } & \text { Number } & \text { Mean } & \text { Std Error Lower } & \text { 95\% } & \text { Upper } 95 \% \\ \text { KT1-1 } & 3 & 0.006224 & 0 & 0.00622 & 0.00622\end{array}$

$\begin{array}{llllll}\text { KT1-1-1 } & 3 & 0.006224 & 0 & 0.00622 & 0.00622 \\ \text { KT1-1-2 } & 3 & 0.006224 & 0 & 0.00622 & 0.00622\end{array}$

$\begin{array}{llllll}\text { KT1-1-1 } & 3 & 0.006224 & 0 & 0.00622 & 0.00622 \\ \text { KT1-2-1 } & 3 & 0.006224 & 0 & 0.00622 & 0.00622\end{array}$

Std Error uses a pooled estimate of error variance

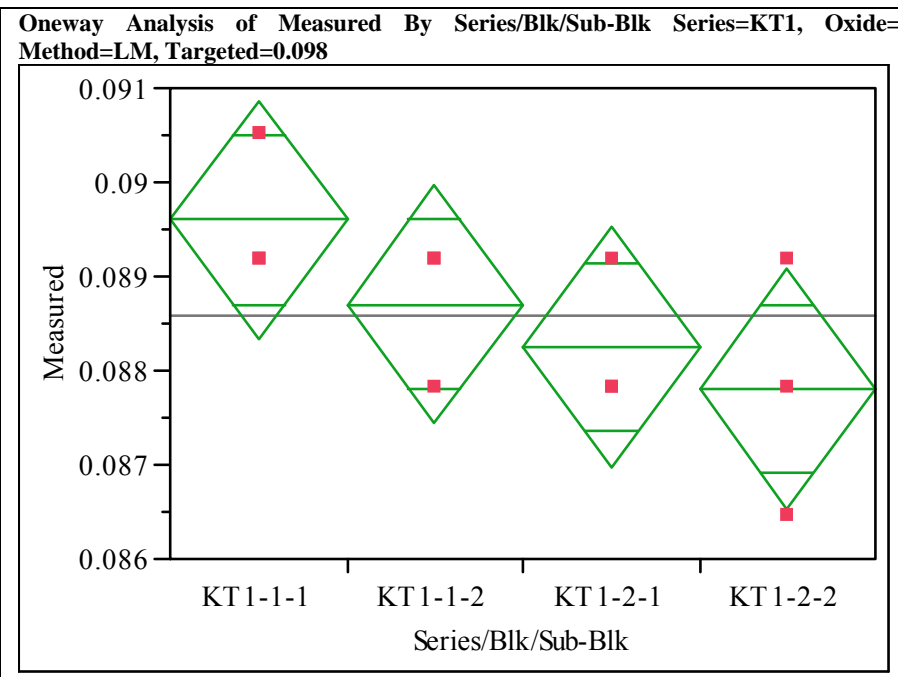

\section{Oneway Anova}

Summary of Fit

$\begin{array}{ll}\text { Rsquare } & 0.421687 \\ \text { Adj Rsquare } & 0.204819\end{array}$

0.204819
Root Mean Square Error $\quad 0.000955$

Root Mean Square Error $\quad 0.000955$
Mean of Response

Observations (or Sum Wgts)

Analysis of Variance

Source DF Sum of Squares Mean Square F Ratio Prob $>$ F

$\begin{array}{llllll}\text { Series/Blk/Sub-Blk } & 3 & 0.00000532 & 1.774 \mathrm{e}-6 & 1.9444 & 0.2011\end{array}$

$\begin{array}{lrr}\text { Error } & 8 & 0.00000730 \\ \text { C. Total } & 11 & 0.00001262\end{array}$

Means for Oneway Anova

Level Number Mean Std Error Lower 95\% Upper 95\%

$\begin{array}{lrrrrr}\text { LT1-1-1 } & 3 & 0.089603 & 0.00055 & 0.08833 & 0.09087 \\ \text { KT } & 3 & 0.088703 & 0.0055 & 0.0873 & 0.08997\end{array}$

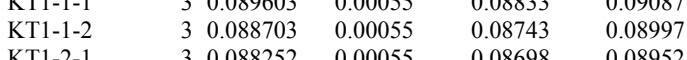

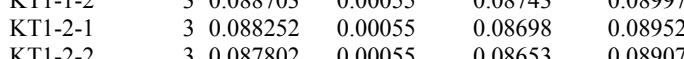

Std Error uses a pooled estimate of error variance 
Exhibit A-4. Plots of Measured versus Targeted Concentrations by Glass ID by Oxide.

Overlay Plot Series=KT1, Oxide=Al2O3 (wt\%)

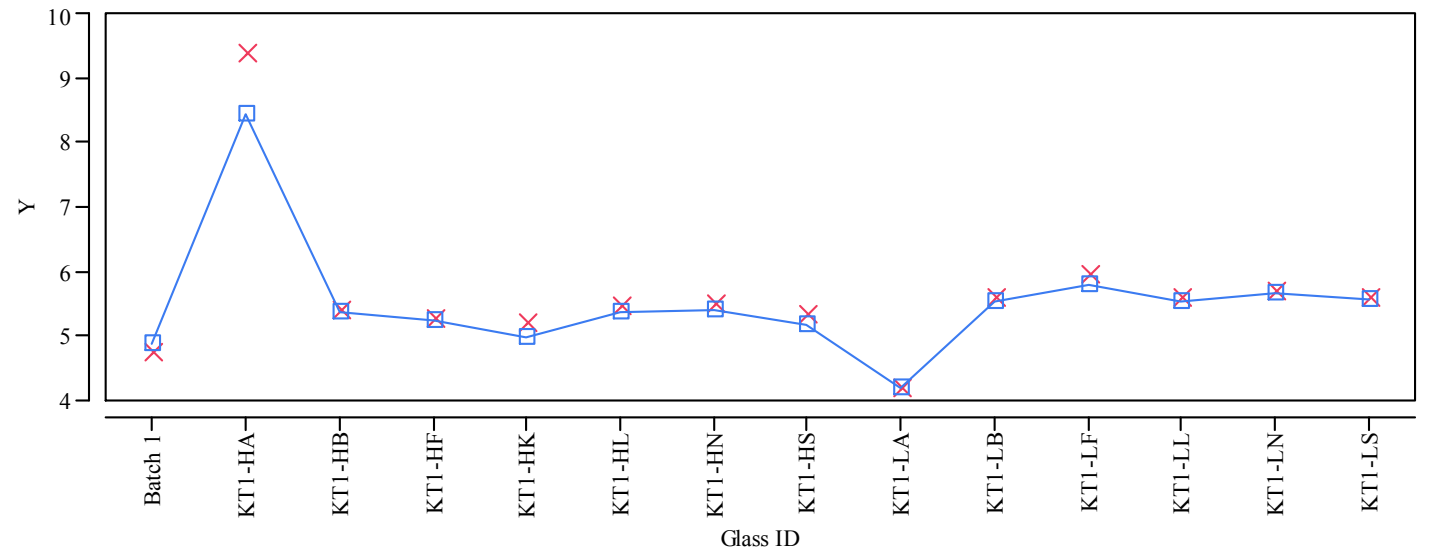

Overlay Plot Series=KT1, Oxide=B2O3 (wt \%)

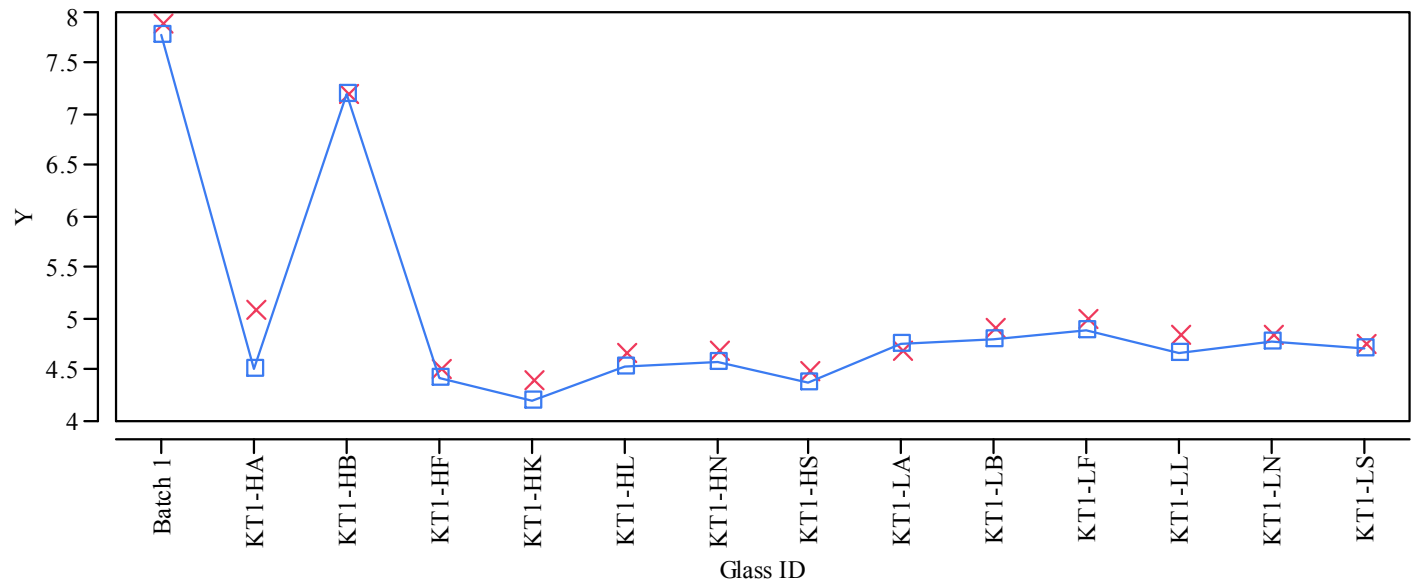

Overlay Plot Series=KT1, Oxide=BaO (wt \%)

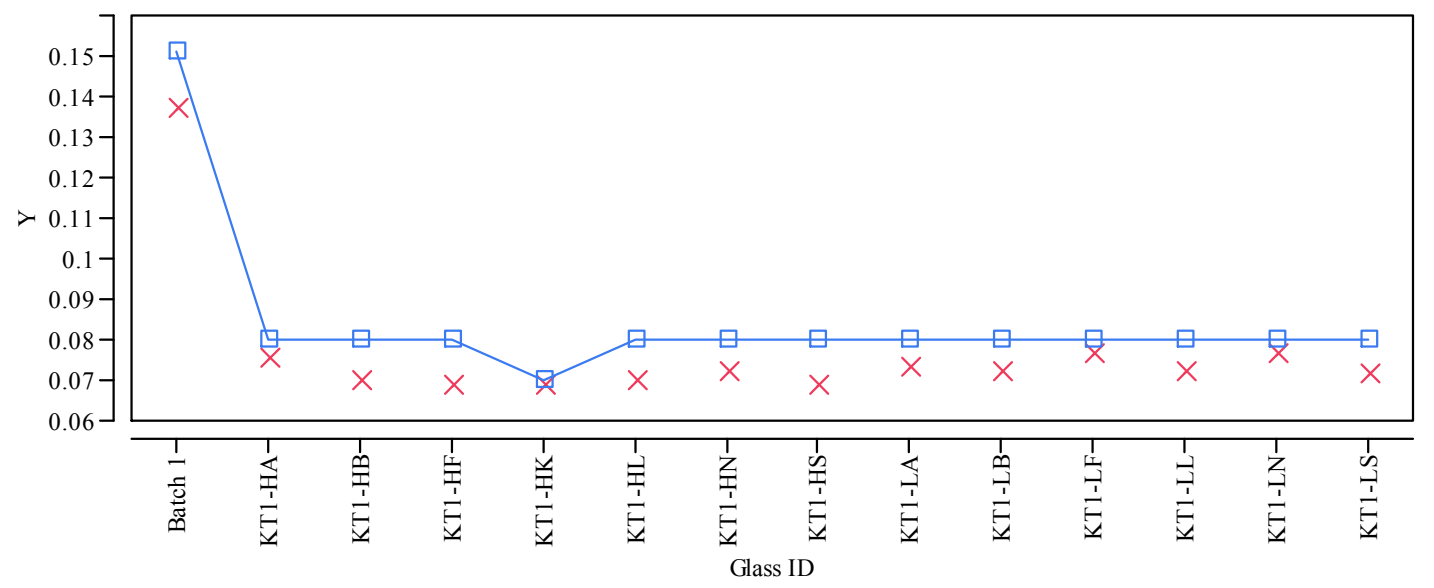

Y $\times$ Measured $\square$ - Targeted 
Exhibit A-4. Plots of Measured versus Targeted Concentrations by Glass ID by Oxide. (continued)

\section{Overlay Plot Series=KT1, Oxide=CaO $($ wt $\%)$}

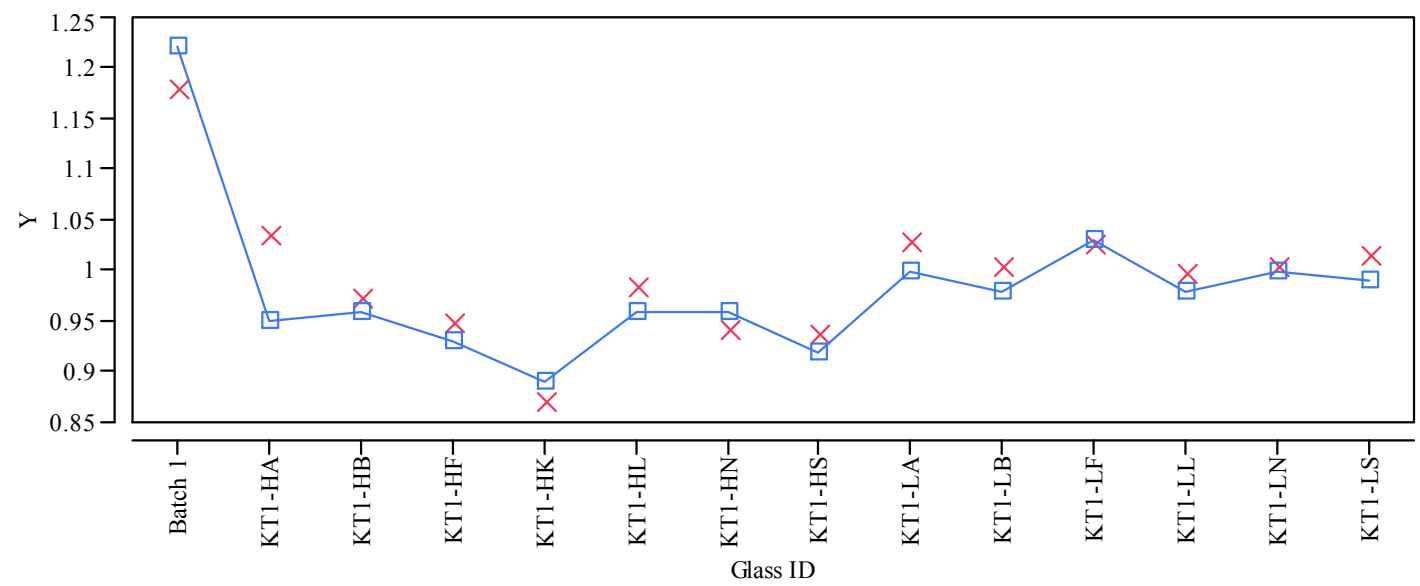

\section{Overlay Plot Series=KT1, Oxide=Ce2O3 (wt \%)}

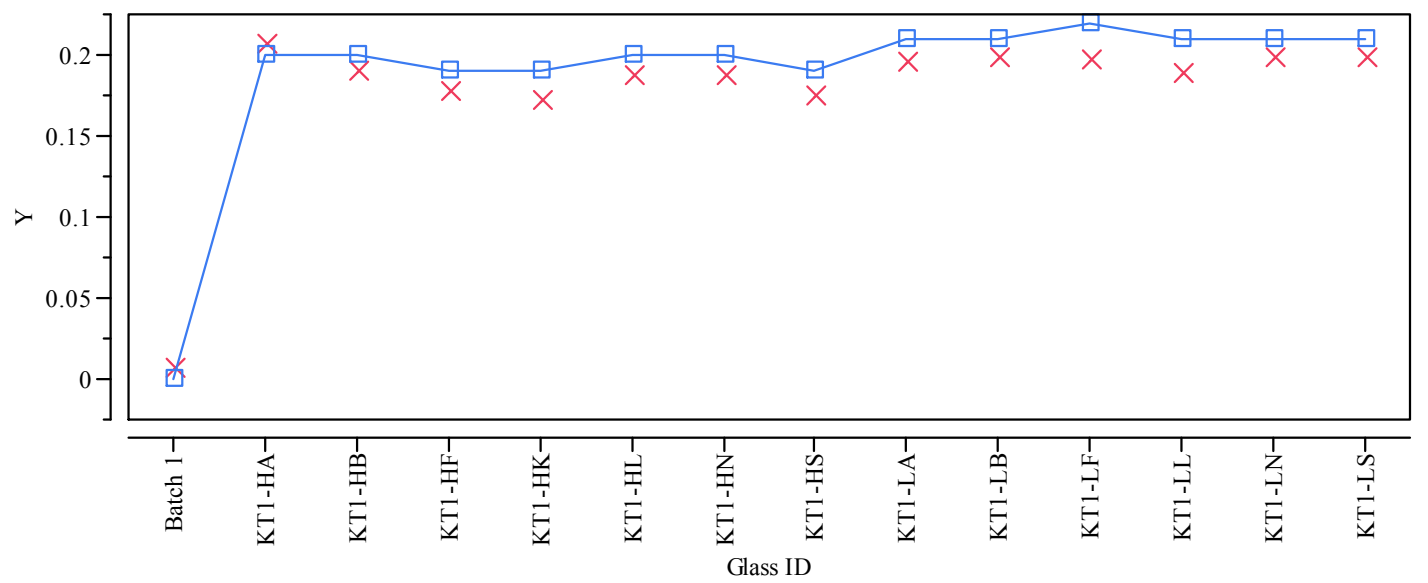

Overlay Plot Series=KT1, Oxide=Cr2O3 (wt \%)

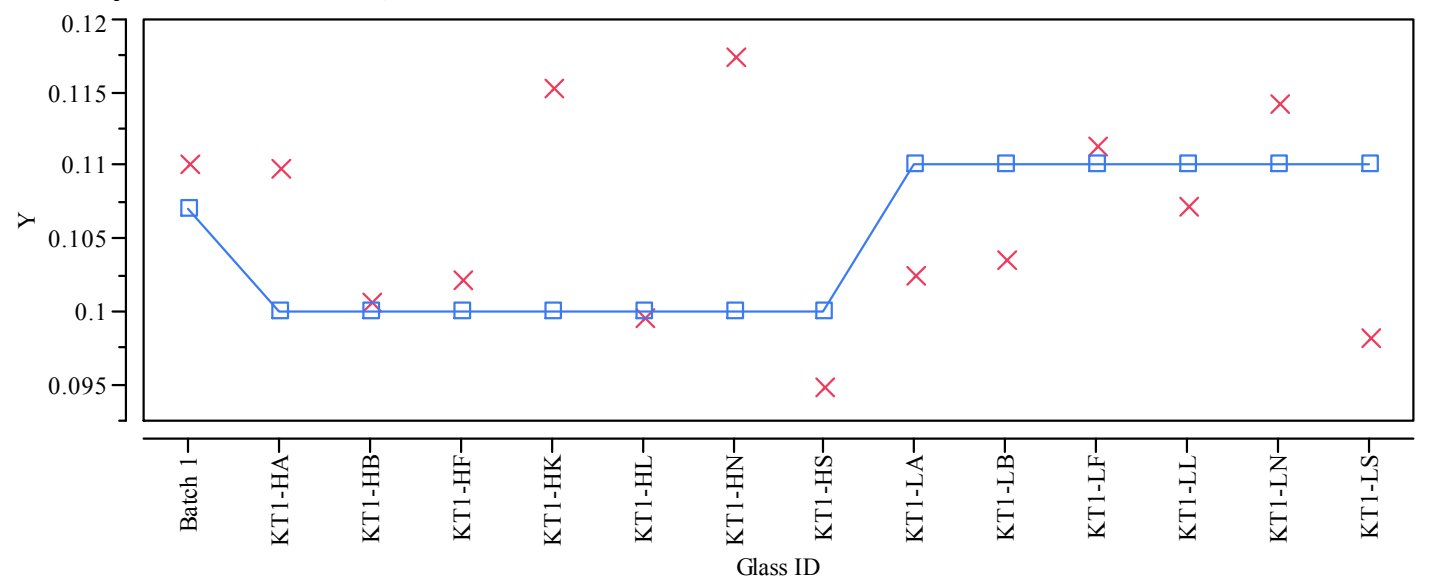

$\mathrm{Y} \times$ Measured $\square$ - Targeted 
Exhibit A-4. Plots of Measured versus Targeted Concentrations by Glass ID by Oxide. (continued)

\section{Overlay Plot Series=KT1, Oxide $=\mathrm{CuO}(\mathrm{wt} \%)$}

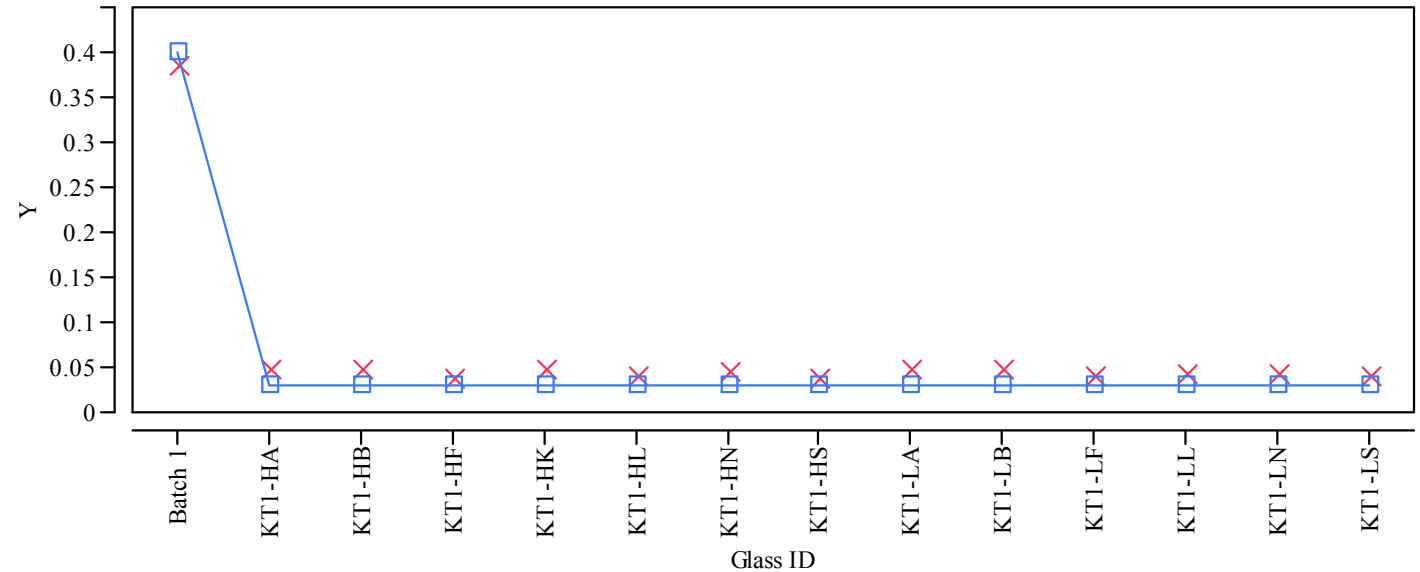

\section{Overlay Plot Series=KT1, Oxide=Fe2O3 (wt \%)}

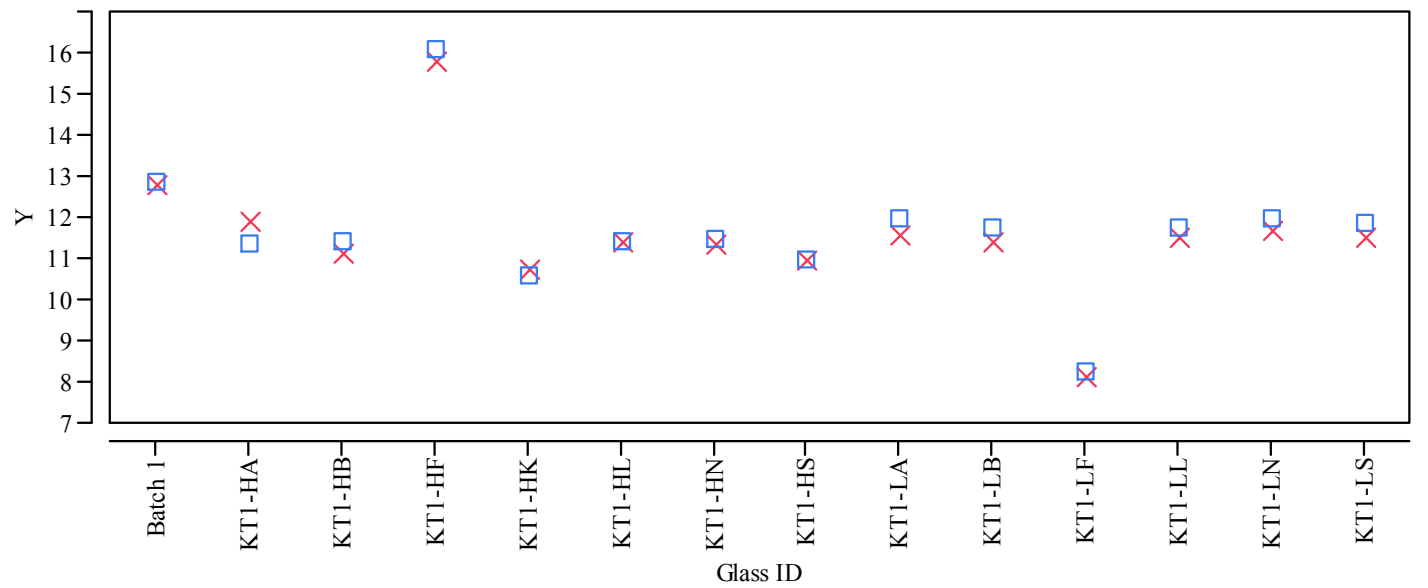

\section{Overlay Plot Series=KT1, Oxide=K2O (wt\%)}

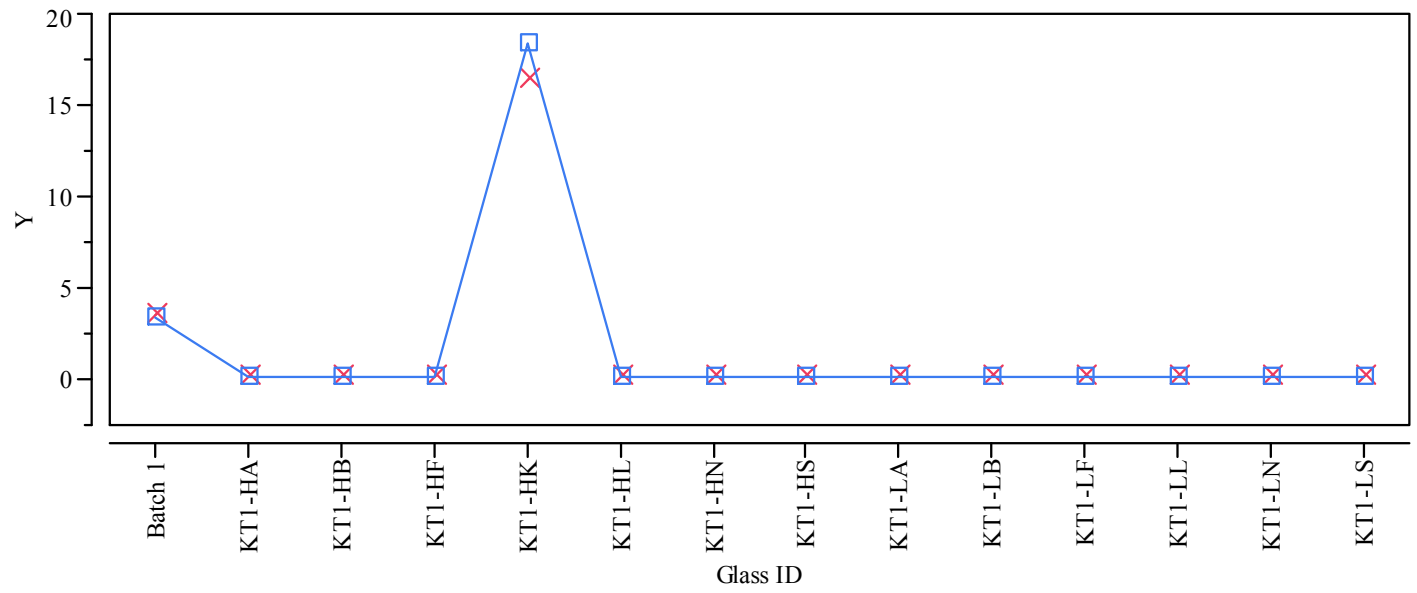

$\mathrm{Y} \times$ Measured $\square$ - Targeted 
Exhibit A-4. Plots of Measured versus Targeted Concentrations by Glass ID by Oxide. (continued)

Overlay Plot Series=KT1, Oxide=La2O3 (wt\%)

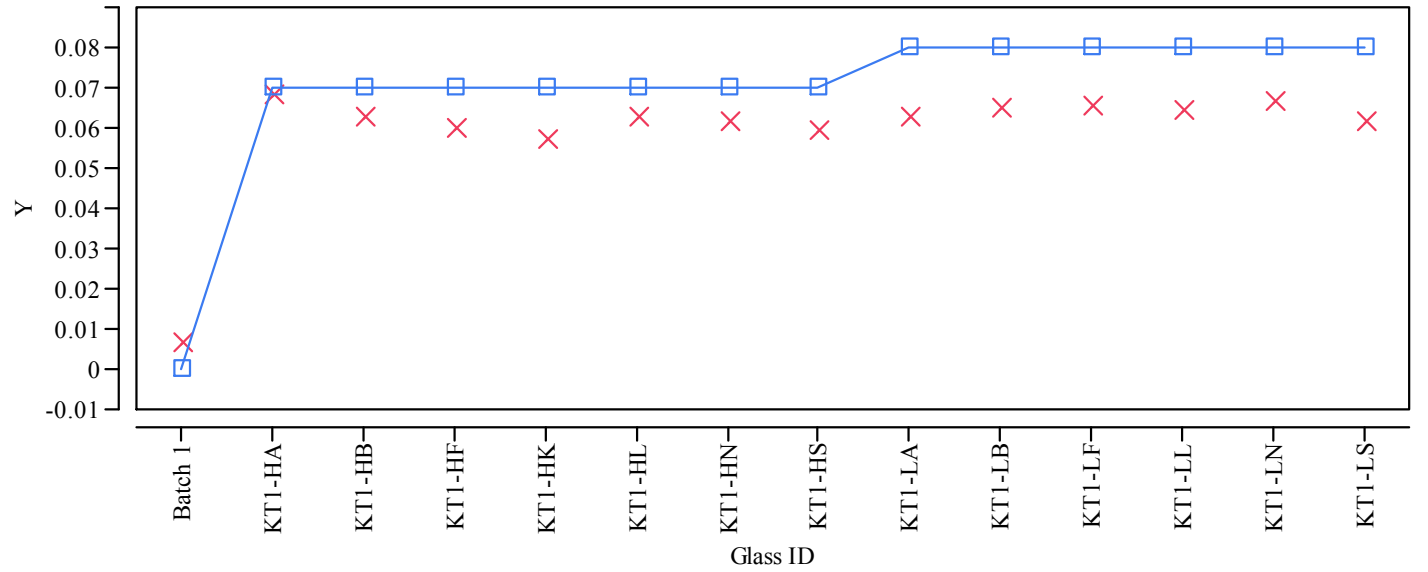

Overlay Plot Series=KT1, Oxide=Li2O (wt\%)

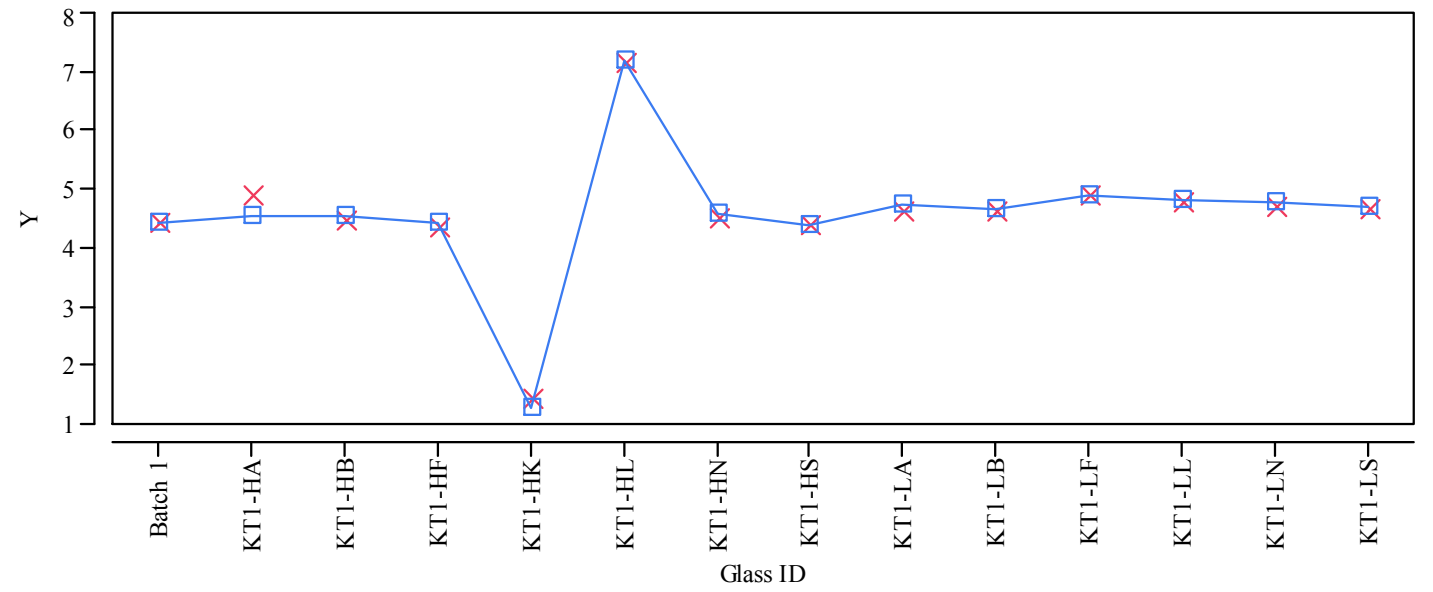

Overlay Plot Series=KT1, Oxide=MgO (wt\%)

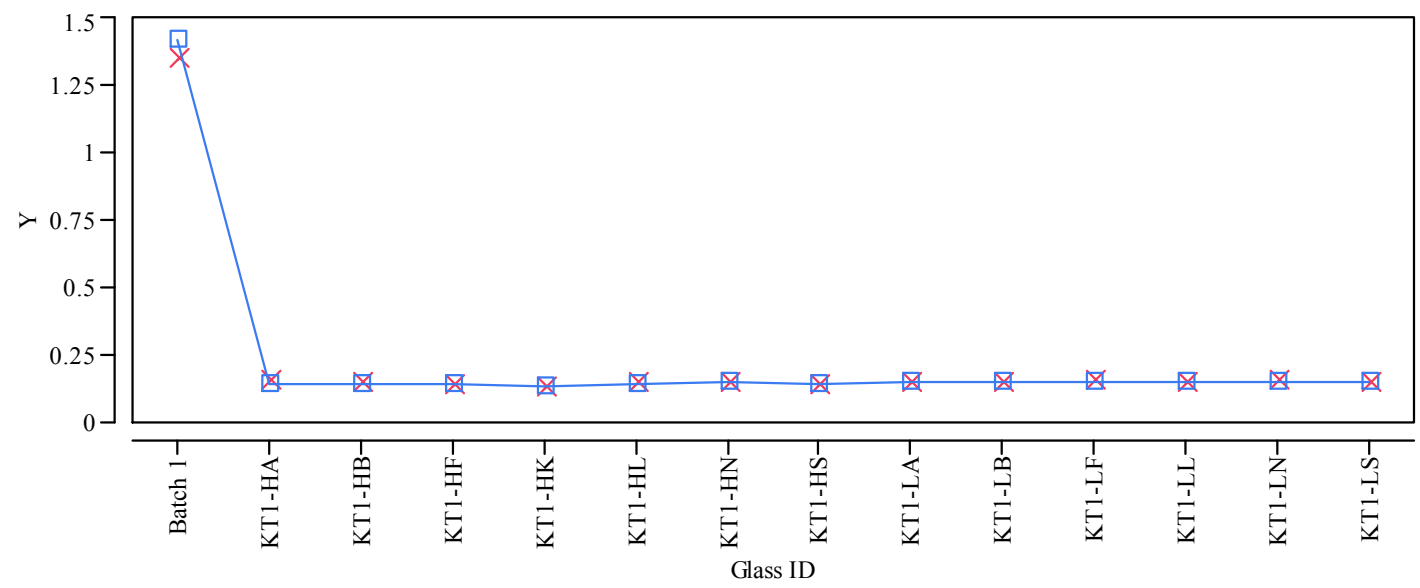

Y $\times$ Measured $\square-$ Targeted 
Exhibit A-4. Plots of Measured versus Targeted Concentrations by Glass ID by Oxide. (continued)

Overlay Plot Series=KT1, Oxide=MnO (wt\%)

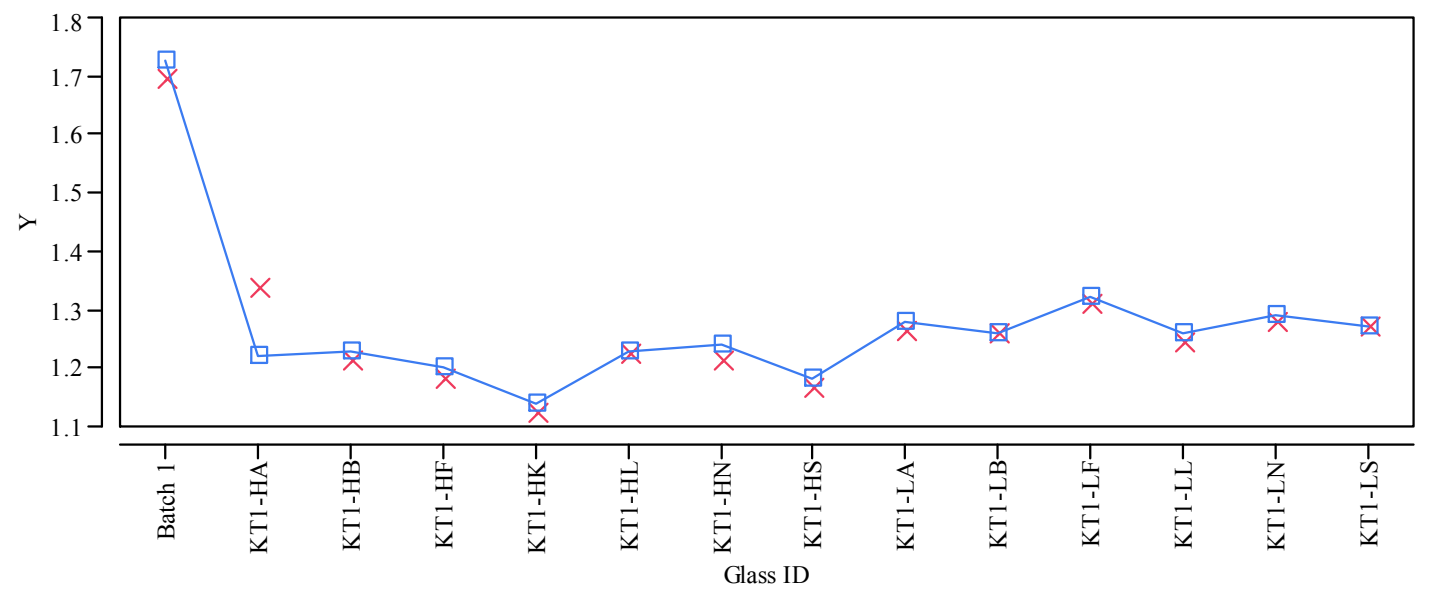

Overlay Plot Series=KT1, Oxide=Na2O (wt\%)

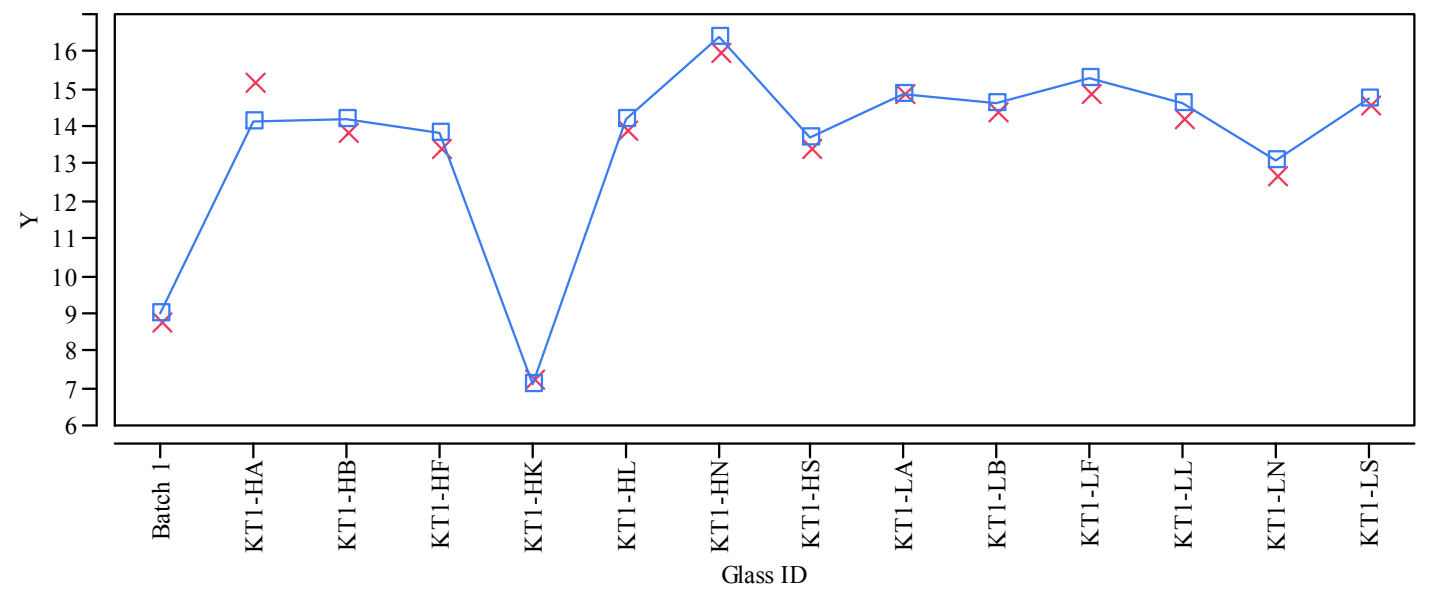

Overlay Plot Series=KT1, Oxide=Nb2O5 (wt \%)

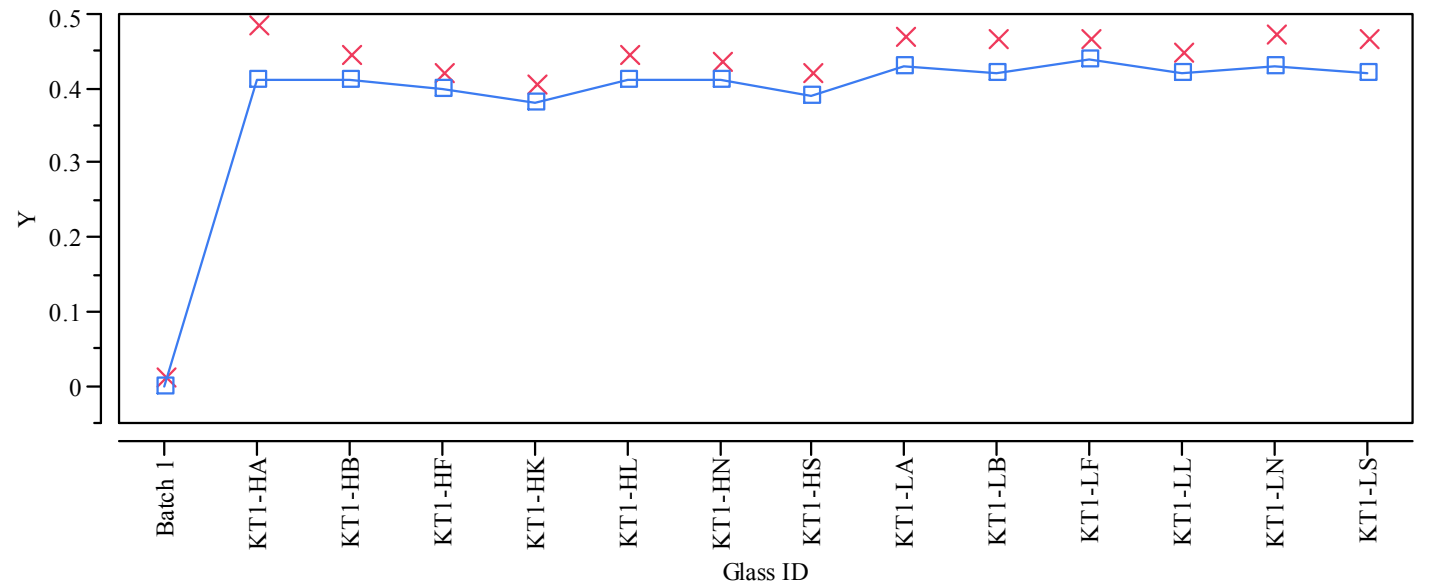

$\mathrm{Y} \times$ Measured $\square$ - Targeted 
Exhibit A-4. Plots of Measured versus Targeted Concentrations by Glass ID by Oxide. (continued)

Overlay Plot Series=KT1, Oxide=NiO (wt \%)

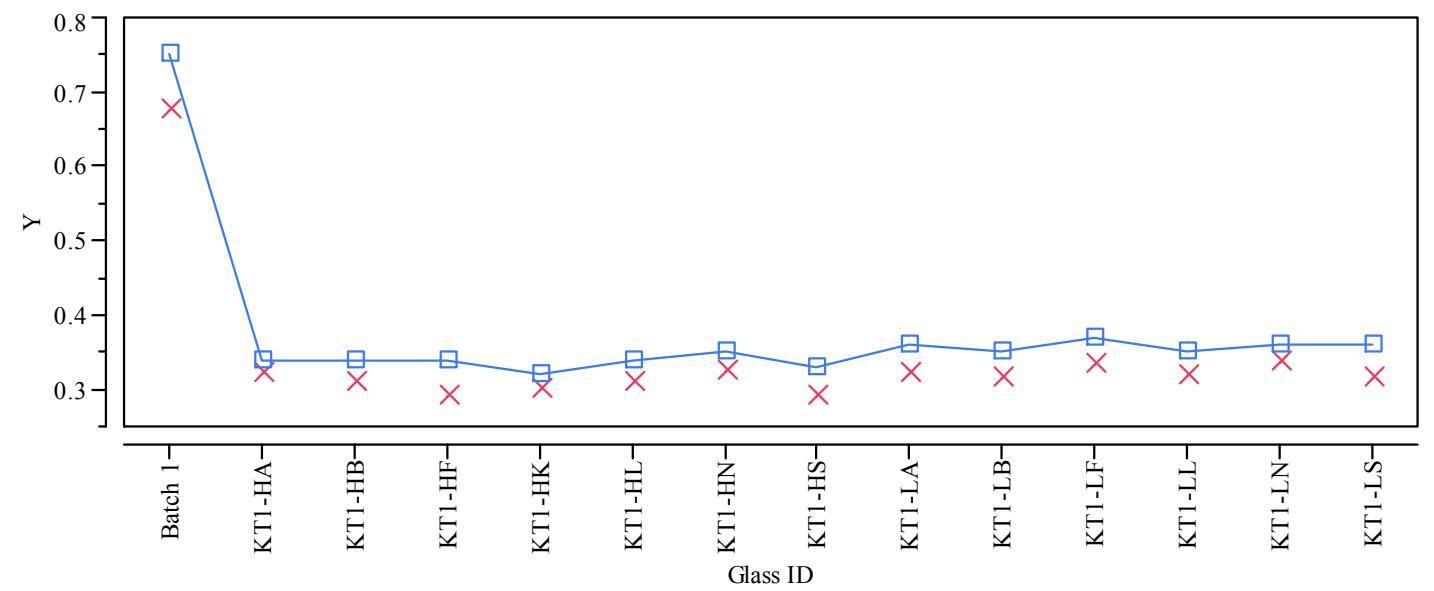

Overlay Plot Series=KT1, Oxide=PbO (wt \%)

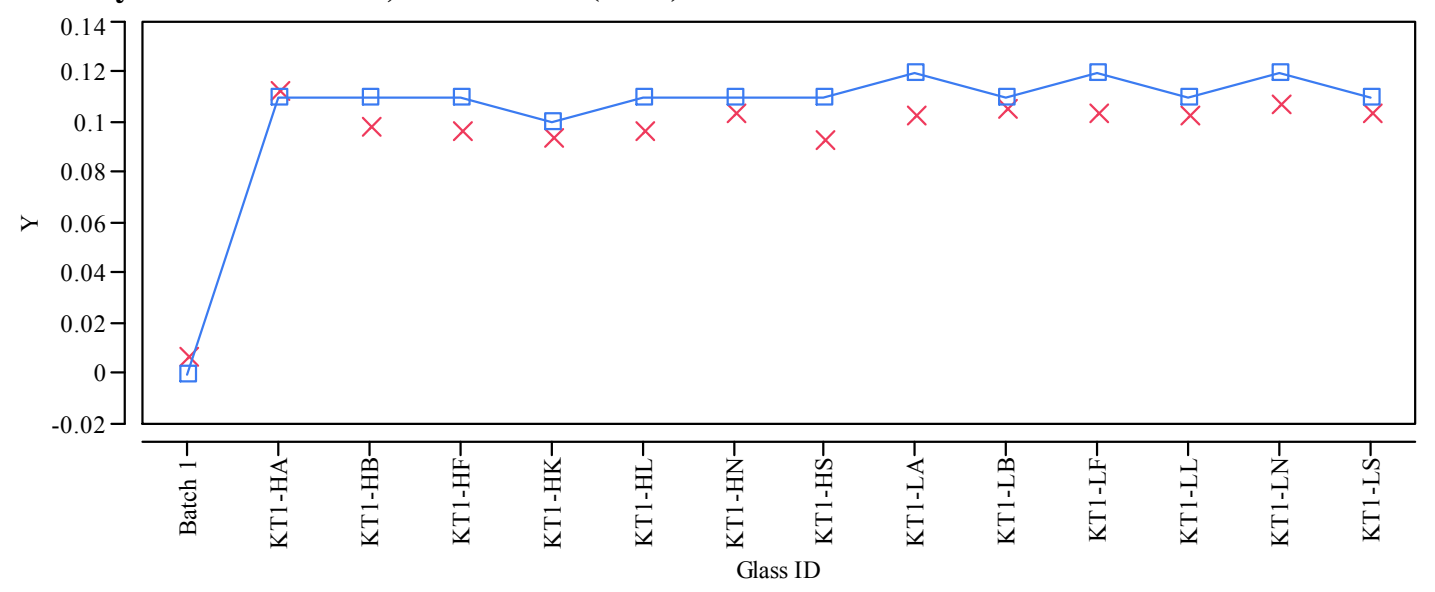

Overlay Plot Series=KT1, Oxide=SiO2 (wt\%)

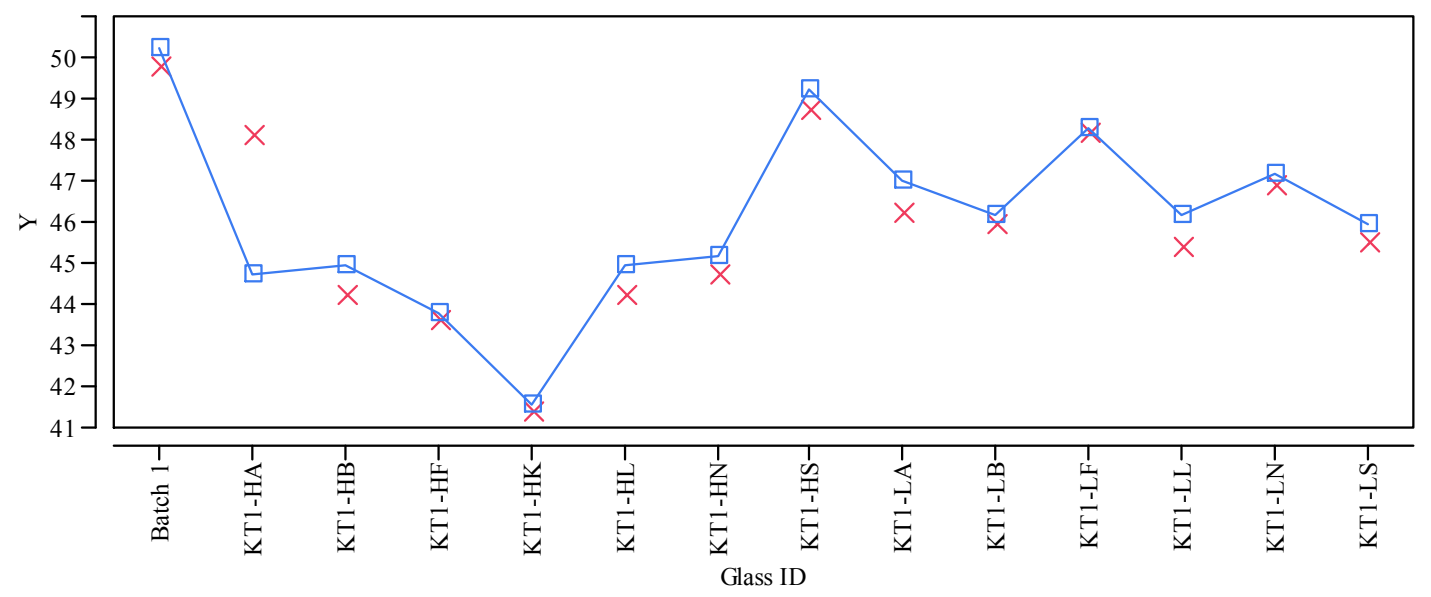

$\mathrm{Y} \times$ Measured $\square$ - Targeted 
Exhibit A-4. Plots of Measured versus Targeted Concentrations by Glass ID by Oxide. (continued)

\section{Overlay Plot Series=KT1, Oxide=SO4 (wt \%)}

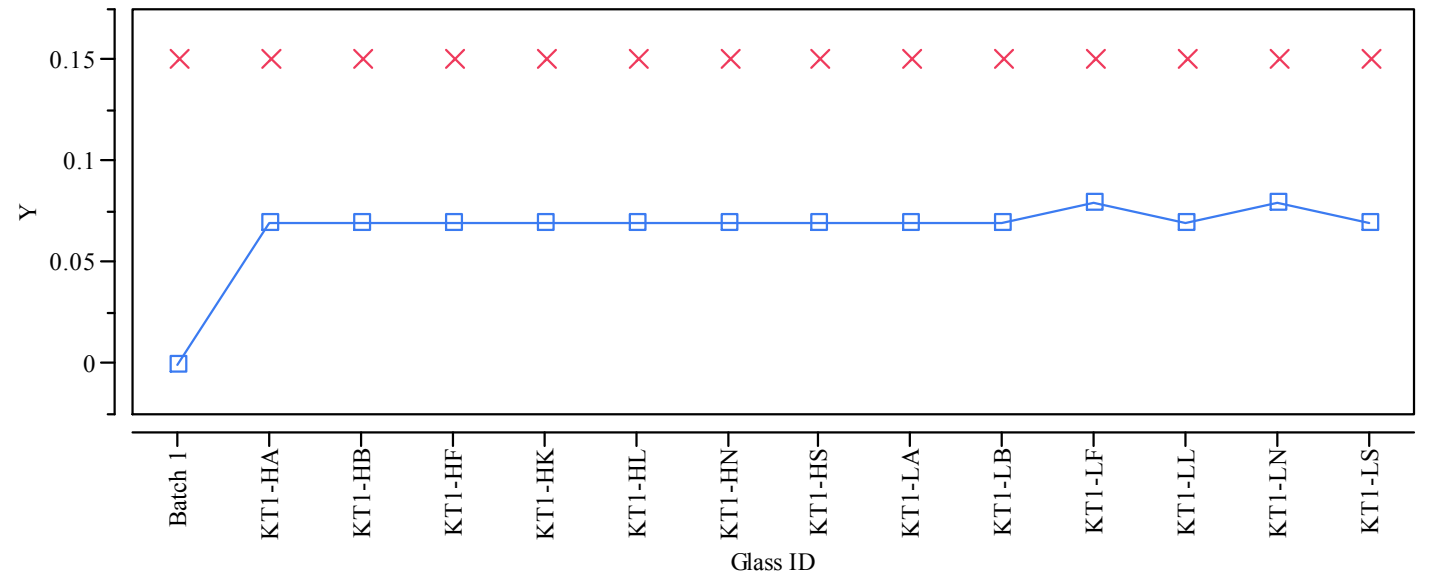

\section{Overlay Plot Series=KT1, Oxide=TiO2 (wt \%)}

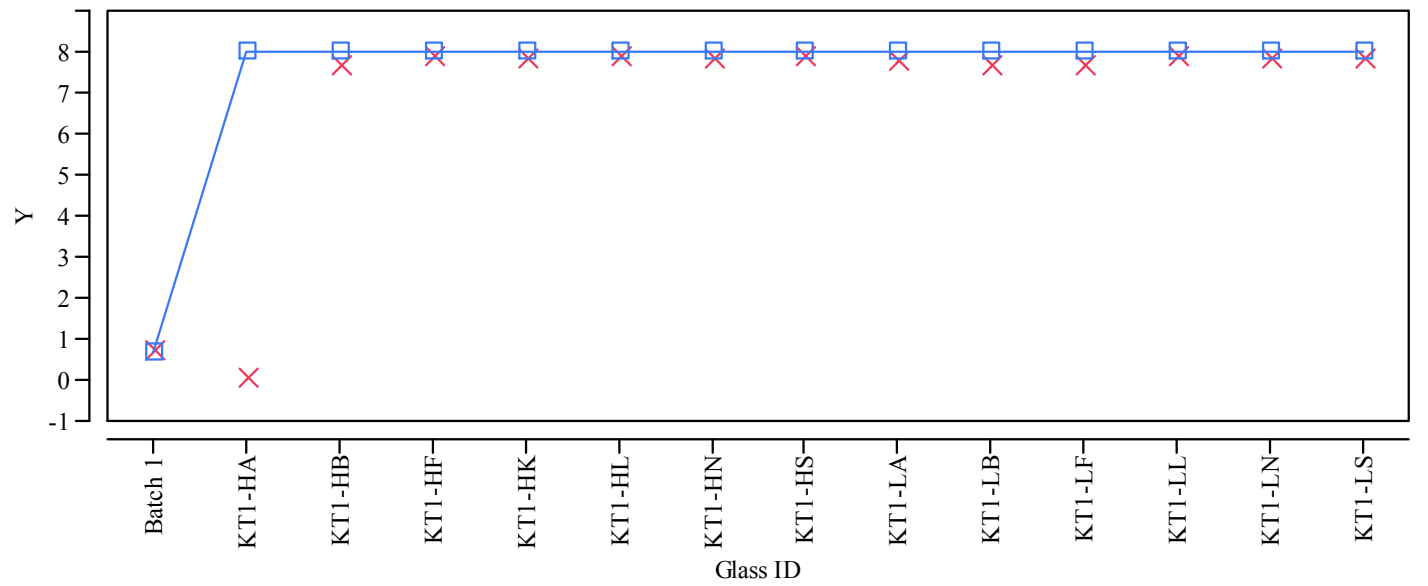

\section{Overlay Plot Series=KT1, Oxide=ZnO (wt\%)}

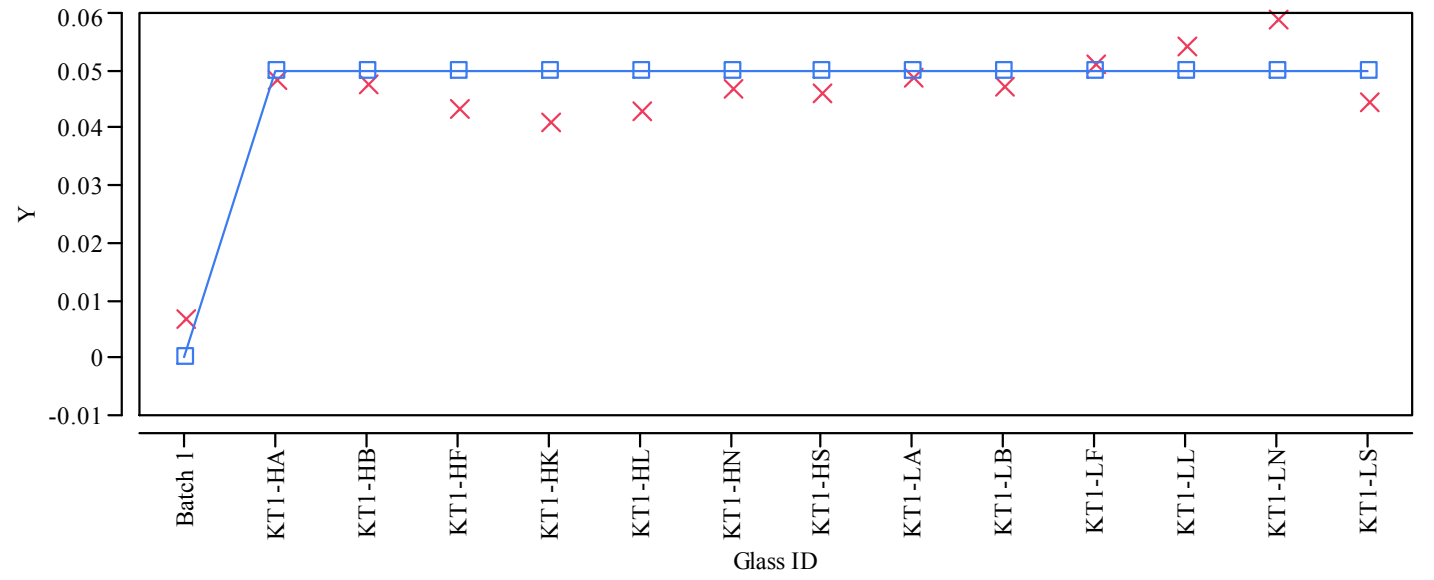

Y $\times$ Measured $\square$ - Targeted 
Exhibit A-4. Plots of Measured versus Targeted Concentrations by Glass ID by Oxide. (continued)

Overlay Plot Series=KT1, Oxide=ZrO2 (wt\%)

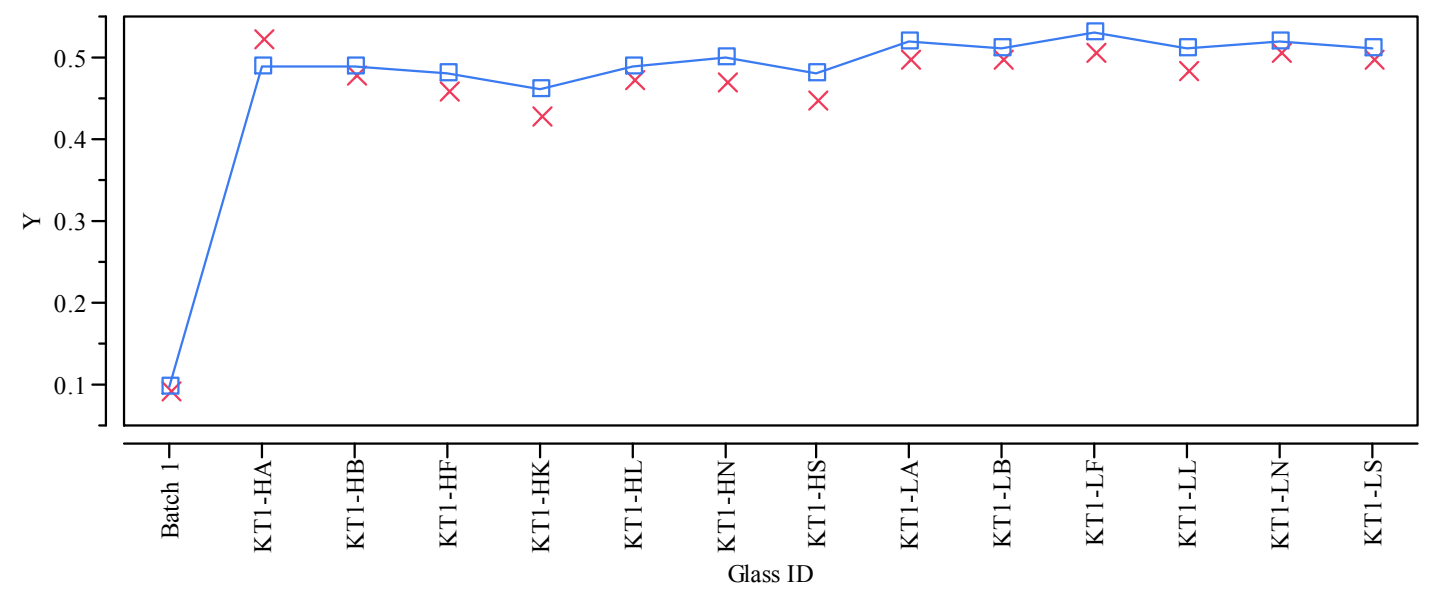

Overlay Plot Series=KT1, Oxide=Sum of Oxides

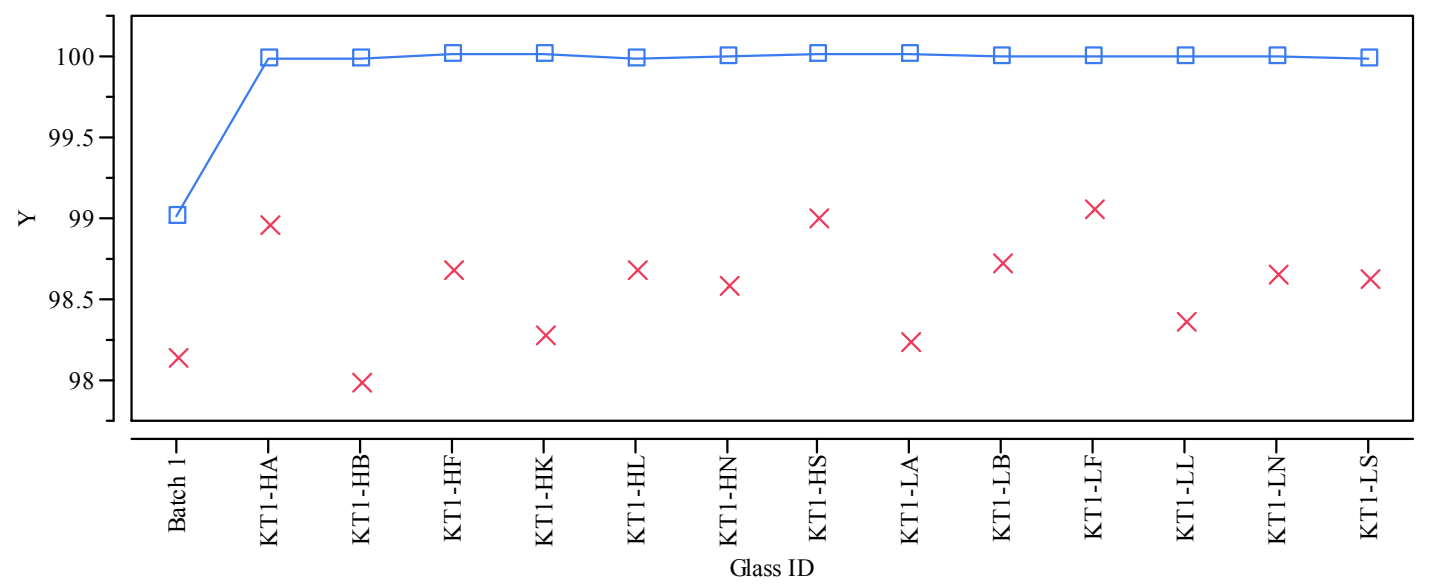

$\mathrm{Y} \times$ Measured $\square$ - Targeted 
SRNL-STI-2010-00566

Revision 0

Appendix B. Data Supporting the Chemical Composition Measurements of the KT03-Series Glasses 
Table B-1. PSAL Chemical Composition Measurements of the KT03-Series of Glasses Using LM Preparation Method (Part 1).

\begin{tabular}{|c|c|c|c|c|c|c|c|c|c|c|c|c|c|}
\hline Glass ID & Block & Sub-Blk & Seq & Lab ID & $\begin{array}{c}\text { Ba } \\
\text { (wt\%) }\end{array}$ & $\begin{array}{c}\mathrm{Ca} \\
(\mathrm{wt} \%)\end{array}$ & $\begin{array}{c}\mathrm{Ce} \\
(\mathrm{wt} \%)\end{array}$ & $\begin{array}{c}\mathrm{Cr} \\
(\mathrm{wt} \%)\end{array}$ & $\begin{array}{c}\mathrm{Cu} \\
(w t \%)\end{array}$ & $\begin{array}{c}\mathrm{K} \\
\text { (wt\%) }\end{array}$ & $\begin{array}{c}\text { La } \\
\text { (wt\%) }\end{array}$ & $\begin{array}{c}\text { Mg } \\
\text { (wt\%) }\end{array}$ & $\begin{array}{c}\text { Mn } \\
\text { (wt\%) }\end{array}$ \\
\hline Batch 1 & 1 & 1 & 1 & BCHLM111 & 0.124 & 0.863 & 0.004 & 0.073 & 0.317 & 3.00 & $<0.010$ & 0.807 & 1.34 \\
\hline KT03-HS & 1 & 1 & 2 & U05LM21 & 0.060 & 0.585 & 0.154 & 0.051 & 0.041 & 0.051 & 0.043 & 0.078 & 0.801 \\
\hline KT03-LB & 1 & 1 & 3 & U10LM21 & 0.062 & 0.678 & 0.164 & 0.080 & 0.046 & 0.075 & 0.050 & 0.085 & 0.868 \\
\hline KT03-HN & 1 & 1 & 4 & U04LM21 & 0.060 & 0.653 & 0.160 & 0.068 & 0.034 & 0.061 & 0.048 & 0.083 & 0.841 \\
\hline KT03-LB & 1 & 1 & 5 & U10LM11 & 0.062 & 0.688 & 0.165 & 0.065 & 0.044 & 0.078 & 0.049 & 0.085 & 0.844 \\
\hline KT03-HS & 1 & 1 & 6 & U05LM11 & 0.060 & 0.587 & 0.151 & 0.056 & 0.038 & 0.052 & 0.043 & 0.079 & 0.799 \\
\hline KT03-MK & 1 & 1 & 7 & U02LM21 & 0.059 & 0.642 & 0.162 & 0.065 & 0.038 & 8.12 & 0.047 & 0.041 & 0.827 \\
\hline KT03-LA & 1 & 1 & 8 & U09LM11 & 0.647 & 0.706 & 0.165 & 0.070 & 0.041 & 0.069 & 0.050 & 0.086 & 0.854 \\
\hline KT03-LL & 1 & 1 & 9 & U15LM21 & 0.058 & 0.680 & 0.164 & 0.064 & 0.039 & 0.064 & 0.050 & 0.085 & 0.853 \\
\hline Batch 1 & 1 & 1 & 10 & BCHLM112 & 0.122 & 0.853 & 0.004 & 0.070 & 0.314 & 2.99 & $<0.010$ & 0.787 & 1.30 \\
\hline KT03-HN & 1 & 1 & 11 & U04LM11 & 0.059 & 0.655 & 0.159 & 0.076 & 0.059 & 0.059 & 0.048 & 0.082 & 0.837 \\
\hline KT03-LS & 1 & 1 & 12 & U06LM11 & 0.058 & 0.644 & 0.160 & 0.068 & 0.040 & 0.088 & 0.046 & 0.078 & 0.824 \\
\hline KT03-MK & 1 & 1 & 13 & U02LM11 & 0.058 & 0.656 & 0.158 & 0.065 & 0.034 & 8.12 & 0.046 & 0.040 & 0.812 \\
\hline KT03-LA & 1 & 1 & 14 & U09LM21 & 0.615 & 0.715 & 0.163 & 0.061 & 0.046 & 0.070 & 0.049 & 0.082 & 0.828 \\
\hline KT03-LS & 1 & 1 & 15 & U06LM21 & 0.056 & 0.658 & 0.157 & 0.081 & 0.038 & 0.098 & 0.045 & 0.075 & 0.801 \\
\hline KT03-LL & 1 & 1 & 16 & U15LM11 & 0.057 & 0.687 & 0.162 & 0.058 & 0.040 & 0.062 & 0.049 & 0.083 & 0.844 \\
\hline KT1-HA2 & 1 & 1 & 17 & U01LM11 & 0.070 & 0.667 & 0.167 & 0.057 & 0.034 & 0.053 & 0.052 & 0.087 & 0.905 \\
\hline KT1-HA2 & 1 & 1 & 18 & U01LM21 & 0.069 & 0.698 & 0.163 & 0.061 & 0.030 & 0.060 & 0.052 & 0.086 & 0.885 \\
\hline Batch 1 & 1 & 1 & 19 & BCHLM113 & 0.119 & 0.854 & 0.004 & 0.070 & 0.315 & 3.05 & $<0.010$ & 0.779 & 1.28 \\
\hline Batch 1 & 1 & 2 & 1 & BCHLM111 & 0.126 & 0.852 & 0.005 & 0.072 & 0.314 & 3.06 & $<0.010$ & 0.809 & 1.37 \\
\hline KT03-HN & 1 & 2 & 2 & U04LM12 & 0.062 & 0.650 & 0.163 & 0.078 & 0.057 & 0.058 & 0.051 & 0.085 & 0.857 \\
\hline KT03-MK & 1 & 2 & 3 & U02LM12 & 0.061 & 0.651 & 0.159 & 0.067 & 0.033 & 8.34 & 0.048 & 0.042 & 0.832 \\
\hline KT03-LA & 1 & 2 & 4 & U09LM22 & 0.637 & 0.709 & 0.166 & 0.062 & 0.045 & 0.069 & 0.052 & 0.085 & 0.849 \\
\hline KT03-LL & 1 & 2 & 5 & U15LM12 & 0.060 & 0.684 & 0.165 & 0.060 & 0.039 & 0.061 & 0.052 & 0.087 & 0.866 \\
\hline KT03-MK & 1 & 2 & 6 & U02LM22 & 0.061 & 0.638 & 0.160 & 0.065 & 0.037 & 8.24 & 0.048 & 0.042 & 0.829 \\
\hline KT03-LS & 1 & 2 & 7 & U06LM12 & 0.060 & 0.642 & 0.162 & 0.069 & 0.039 & 0.088 & 0.048 & 0.080 & 0.828 \\
\hline KT03-LB & 1 & 2 & 8 & U10LM12 & 0.063 & 0.687 & 0.165 & 0.064 & 0.043 & 0.077 & 0.051 & 0.085 & 0.847 \\
\hline KT03-HS & 1 & 2 & 9 & U05LM12 & 0.060 & 0.589 & 0.152 & 0.056 & 0.037 & 0.051 & 0.044 & 0.079 & 0.792 \\
\hline Batch 1 & 1 & 2 & 10 & BCHLM122 & 0.124 & 0.856 & 0.005 & 0.072 & 0.315 & 3.08 & $<0.010$ & 0.805 & 1.32 \\
\hline KT03-LB & 1 & 2 & 11 & U10LM22 & 0.063 & 0.679 & 0.165 & 0.081 & 0.045 & 0.074 & 0.052 & 0.085 & 0.862 \\
\hline KT1-HA2 & 1 & 2 & 12 & U01LM12 & 0.073 & 0.673 & 0.170 & 0.058 & 0.033 & 0.052 & 0.055 & 0.090 & 0.927 \\
\hline KT03-HN & 1 & 2 & 13 & U04LM22 & 0.059 & 0.657 & 0.159 & 0.068 & 0.033 & 0.060 & 0.049 & 0.081 & 0.831 \\
\hline KT03-LA & 1 & 2 & 14 & U09LM12 & 0.631 & 0.706 & 0.165 & 0.069 & 0.040 & 0.068 & 0.051 & 0.084 & 0.834 \\
\hline KT03-LL & 1 & 2 & 15 & U15LM22 & 0.059 & 0.676 & 0.164 & 0.063 & 0.038 & 0.063 & 0.051 & 0.085 & 0.838 \\
\hline KT03-HS & 1 & 2 & 16 & U05LM22 & 0.061 & 0.584 & 0.154 & 0.050 & 0.040 & 0.050 & 0.045 & 0.078 & 0.791 \\
\hline KT03-LS & 1 & 2 & 17 & U06LM22 & 0.058 & 0.654 & 0.158 & 0.082 & 0.037 & 0.095 & 0.047 & 0.077 & 0.807 \\
\hline KT1-HA2 & 1 & 2 & 18 & U01LM22 & 0.071 & 0.699 & 0.165 & 0.061 & 0.029 & 0.060 & 0.054 & 0.086 & 0.895 \\
\hline Batch 1 & 1 & 2 & 19 & BCHLM123 & 0.122 & 0.853 & 0.005 & 0.071 & 0.313 & 3.06 & $<0.010$ & 0.785 & 1.32 \\
\hline Batch 1 & 2 & 1 & 1 & BCHLM211 & 0.130 & 0.840 & 0.007 & 0.075 & 0.310 & 3.18 & $<0.010$ & 0.823 & 1.31 \\
\hline KT03-LF & 2 & 1 & 2 & U12LM11 & 0.072 & 0.725 & 0.172 & 0.071 & 0.050 & 0.076 & 0.056 & 0.093 & 0.915 \\
\hline KT03-HL & 2 & 1 & 3 & U13LM11 & 0.063 & 0.684 & 0.159 & 0.086 & 0.036 & 0.068 & 0.050 & 0.085 & 0.823 \\
\hline KT03-LN & 2 & 1 & 4 & U07LM21 & 0.066 & 0.713 & 0.166 & 0.069 & 0.039 & 0.070 & 0.053 & 0.086 & 0.866 \\
\hline KT03-LF & 2 & 1 & 5 & U12LM21 & 0.071 & 0.686 & 0.181 & 0.067 & 0.042 & 0.069 & 0.057 & 0.097 & 0.966 \\
\hline KT03-HK & 2 & 1 & 6 & U11LM21 & 0.060 & 0.615 & 0.155 & 0.063 & 0.044 & 13.72 & 0.049 & 0.079 & 0.807 \\
\hline KT03-LN & 2 & 1 & 7 & U07LM11 & 0.064 & 0.711 & 0.165 & 0.064 & 0.041 & 0.076 & 0.052 & 0.084 & 0.852 \\
\hline KT03-HB & 2 & 1 & 8 & U08LM21 & 0.063 & 0.657 & 0.165 & 0.064 & 0.045 & 0.057 & 0.052 & 0.085 & 0.845 \\
\hline KT03-HL & 2 & 1 & 9 & U13LM21 & 0.063 & 0.659 & 0.162 & 0.069 & 0.040 & 0.058 & 0.051 & 0.086 & 0.837 \\
\hline Batch 1 & 2 & 1 & 10 & BCHLM212 & 0.126 & 0.850 & 0.007 & 0.074 & 0.315 & 2.87 & $<0.010$ & 0.804 & 1.36 \\
\hline KT03-HA & 2 & 1 & 11 & U14LM11 & 0.062 & 0.660 & 0.161 & 0.070 & 0.033 & 0.059 & 0.051 & 0.083 & 0.813 \\
\hline KT03-HB & 2 & 1 & 12 & U08LM11 & 0.062 & 0.647 & 0.161 & 0.065 & 0.047 & 0.056 & 0.051 & 0.082 & 0.832 \\
\hline KT03-HF & 2 & 1 & 13 & U03LM11 & 0.062 & 0.630 & 0.160 & 0.062 & 0.054 & 0.058 & 0.051 & 0.086 & 0.816 \\
\hline KT03-HK & 2 & 1 & 14 & U11LM11 & 0.056 & 0.623 & 0.147 & 0.117 & 0.041 & 14.35 & 0.045 & 0.073 & 0.759 \\
\hline KT03-HF & 2 & 1 & 15 & U03LM21 & 0.061 & 0.631 & 0.159 & 0.062 & 0.045 & 0.060 & 0.051 & 0.082 & 0.807 \\
\hline KT03-HA & 2 & 1 & 16 & U14LM21 & 0.061 & 0.662 & 0.158 & 0.059 & 0.047 & 0.074 & 0.050 & 0.081 & 0.812 \\
\hline Batch 1 & 2 & 1 & 17 & BCHLM213 & 0.126 & 0.848 & 0.007 & 0.073 & 0.311 & 3.14 & $<0.010$ & 0.799 & 1.31 \\
\hline Batch 1 & 2 & 2 & 1 & BCHLM221 & 0.121 & 0.854 & 0.005 & 0.070 & 0.312 & 2.95 & $<0.010$ & 0.774 & 1.35 \\
\hline KT03-HB & 2 & 2 & 2 & U08LM22 & 0.061 & 0.651 & 0.162 & 0.062 & 0.043 & 0.058 & 0.049 & 0.084 & 0.831 \\
\hline KT03-LN & 2 & 2 & 3 & U07LM12 & 0.062 & 0.710 & 0.161 & 0.061 & 0.039 & 0.077 & 0.050 & 0.082 & 0.833 \\
\hline KT03-HF & 2 & 2 & 4 & U03LM22 & 0.061 & 0.633 & 0.160 & 0.062 & 0.043 & 0.061 & 0.051 & 0.084 & 0.832 \\
\hline KT03-HK & 2 & 2 & 5 & U11LM22 & 0.056 & 0.622 & 0.152 & 0.058 & 0.042 & 15.02 & 0.046 & 0.075 & 0.781 \\
\hline KT03-HF & 2 & 2 & 6 & U03LM12 & 0.059 & 0.634 & 0.158 & 0.060 & 0.053 & 0.060 & 0.049 & 0.084 & 0.804 \\
\hline KT03-HL & 2 & 2 & 7 & U13LM12 & 0.058 & 0.690 & 0.153 & 0.080 & 0.034 & 0.071 & 0.047 & 0.079 & 0.798 \\
\hline KT03-HB & 2 & 2 & 8 & U08LM12 & 0.062 & 0.645 & 0.163 & 0.064 & 0.045 & 0.056 & 0.050 & 0.085 & 0.840 \\
\hline KT03-HA & 2 & 2 & 9 & U14LM22 & 0.060 & 0.665 & 0.158 & 0.057 & 0.045 & 0.075 & 0.049 & 0.082 & 0.825 \\
\hline Batch 1 & 2 & 2 & 10 & BCHLM222 & 0.122 & 0.861 & 0.005 & 0.069 & 0.314 & 3.08 & $<0.010$ & 0.776 & 1.39 \\
\hline KT03-LF & 2 & 2 & 11 & U12LM22 & 0.064 & 0.696 & 0.175 & 0.060 & 0.040 & 0.073 & 0.053 & 0.088 & 0.904 \\
\hline
\end{tabular}


Table B-1. PSAL Chemical Composition Measurements of the KT03-Series of Glasses Using LM Preparation Method (Part 1). (continued)

\begin{tabular}{|c|c|c|c|c|c|c|c|c|c|c|c|c|c|}
\hline Glass ID & Block & Sub-Blk & Seq & Lab ID & $\begin{array}{c}\text { Ba } \\
(w t \%)\end{array}$ & $\begin{array}{c}\mathrm{Ca} \\
(\mathrm{wt} \%)\end{array}$ & $\begin{array}{c}\mathrm{Ce} \\
(\mathrm{wt} \%)\end{array}$ & $\begin{array}{c}\mathrm{Cr} \\
(\mathrm{wt} \%)\end{array}$ & $\begin{array}{c}\mathrm{Cu} \\
(\mathrm{wt} \%)\end{array}$ & $\begin{array}{c}\mathbf{K} \\
(\mathbf{w t} \%)\end{array}$ & $\begin{array}{c}\text { La } \\
(w t \%)\end{array}$ & $\begin{array}{c}\mathrm{Mg} \\
(\mathrm{wt} \%)\end{array}$ & $\begin{array}{c}\text { Mn } \\
\text { (wt\%) }\end{array}$ \\
\hline KT03-LN & 2 & 2 & 12 & U07LM22 & 0.059 & 0.717 & 0.159 & 0.061 & 0.037 & 0.073 & 0.049 & 0.078 & 0.797 \\
\hline KT03-LF & 2 & 2 & 13 & U12LM12 & 0.063 & 0.737 & 0.165 & 0.062 & 0.048 & 0.081 & 0.052 & 0.082 & 0.829 \\
\hline KT03-HA & 2 & 2 & 14 & U14LM12 & 0.059 & 0.672 & 0.158 & 0.066 & 0.031 & 0.061 & 0.049 & 0.079 & 0.802 \\
\hline KT03-HL & 2 & 2 & 15 & U13LM22 & 0.057 & 0.671 & 0.156 & 0.062 & 0.038 & 0.062 & 0.047 & 0.078 & 0.798 \\
\hline KT03-HK & 2 & 2 & 16 & U11LM12 & 0.052 & 0.629 & 0.144 & 0.113 & 0.039 & 15.29 & 0.043 & 0.068 & 0.738 \\
\hline Batch 1 & 2 & 2 & 17 & BCHLM223 & 0.119 & 0.861 & 0.005 & 0.068 & 0.310 & 3.18 & $<0.010$ & 0.754 & 1.35 \\
\hline
\end{tabular}




\section{Table B-2. PSAL Chemical Composition Measurements of the KT03-Series of Glasses} Using LM Preparation Method (Part 2).

\begin{tabular}{|c|c|c|c|c|c|c|c|c|c|c|c|c|c|}
\hline Glass ID & Block & \begin{tabular}{|l|} 
Sub-Blk \\
\end{tabular} & Seq & Lab ID & Mn (wt\%) & Na (wt\%) & Nb (wt\%) & Ni (wt\%) & Pb (wt\%) & S (wt\%) & Ti (wt\%) & Zn (wt\%) & Zr (wt\%) \\
\hline Batch 1 & 1 & 1 & 1 & BCHLM111 & 1.34 & 6.71 & $<0.100$ & 0.520 & $<0.010$ & $<0.100$ & 0.391 & $<0.010$ & 0.071 \\
\hline KT03-HS & 1 & 1 & 2 & U05LM21 & 0.801 & 9.06 & 1.96 & 0.219 & 0.095 & $<0.100$ & 4.50 & 0.038 & 1.73 \\
\hline KT03-LB & 1 & 1 & 3 & U10LM21 & 0.868 & 9.85 & 1.70 & 0.239 & 0.100 & $<0.100$ & 4.49 & 0.042 & 1.51 \\
\hline KT03-HN & 1 & 1 & 4 & U04LM21 & 0.841 & 12.0 & 1.85 & 0.228 & 0.099 & $<0.100$ & 4.56 & 0.039 & 1.66 \\
\hline KT03-LB & 1 & 1 & 5 & U10LM11 & 0.844 & 9.84 & 1.94 & 0.235 & 0.097 & $<0.100$ & 4.55 & 0.041 & 1.71 \\
\hline KT03-HS & 1 & 1 & 6 & U05LM11 & 0.799 & 9.08 & 1.68 & 0.221 & 0.093 & $<0.100$ & 4.35 & 0.038 & 1.42 \\
\hline KT03-MK & 1 & 1 & 7 & U02LM21 & 0.827 & 7.54 & 1.85 & 0.221 & 0.095 & $<0.100$ & 4.25 & 0.034 & 1.62 \\
\hline KT03-LA & 1 & 1 & 8 & U09LM11 & 0.854 & 10.3 & 1.85 & 0.220 & 0.094 & $<0.100$ & 4.46 & 0.043 & 1.65 \\
\hline KT03-LL & 1 & 1 & 9 & U15LM21 & 0.853 & 10.1 & 1.83 & 0.228 & 0.097 & $<0.100$ & 4.50 & 0.041 & 1.58 \\
\hline Batch 1 & 1 & 1 & 10 & BCHLM112 & 1.30 & 6.69 & $<0.100$ & 0.509 & $<0.010$ & $<0.100$ & 0.381 & $<0.010$ & 0.077 \\
\hline KT03-HN & 1 & 1 & 11 & U04LM11 & 0.837 & 11.60 & 1.79 & 0.333 & 0.095 & $<0.100$ & 4.52 & 0.046 & 1.55 \\
\hline KT03-LS & 1 & 1 & 12 & U06LM11 & 0.824 & 9.46 & 1.91 & 0.220 & 0.094 & $<0.100$ & 4.48 & 0.038 & 1.67 \\
\hline KT03-MK & 1 & 1 & 13 & U02LM11 & 0.812 & 7.39 & 1.83 & 0.215 & 0.094 & $<0.100$ & 4.30 & 0.034 & 1.60 \\
\hline KT03-LA & 1 & 1 & \begin{tabular}{|l|}
14 \\
\end{tabular} & U09LM21 & 0.828 & 10.2 & 1.93 & 0.214 & 0.090 & $<0.100$ & 4.47 & 0.043 & 1.69 \\
\hline KT03-LS & 1 & 1 & 15 & U06LM21 & 0.801 & 9.64 & 1.91 & 0.208 & 0.090 & $<0.100$ & 4.53 & 0.037 & 1.69 \\
\hline KT03-LL & 1 & 1 & 16 & U15LM11 & 0.844 & 10.1 & 1.91 & 0.225 & 0.097 & $<0.100$ & 4.51 & 0.038 & 1.68 \\
\hline KT1-HA2 & 1 & 1 & 17 & U01LM11 & 0.905 & 10.3 & 0.271 & 0.244 & 0.081 & $<0.100$ & 4.43 & 0.038 & 0.353 \\
\hline KT1-HA2 & 1 & 1 & 18 & U01LM21 & 0.885 & 10.5 & 0.274 & 0.234 & 0.078 & $<0.100$ & 4.45 & 0.039 & 0.352 \\
\hline Batch 1 & 1 & 1 & 19 & BCHLM113 & 1.28 & 6.77 & $<0.100$ & 0.502 & $<0.010$ & $<0.100$ & 0.380 & $<0.010$ & 0.075 \\
\hline Batch 1 & 1 & 2 & 1 & BCHLM111 & 1.37 & 6.96 & $<0.100$ & 0.520 & $<0.010$ & $<0.100$ & 0.390 & $<0.010$ & 0.069 \\
\hline KT03-HN & 1 & 2 & 2 & U04LM12 & 0.857 & 11.9 & 1.65 & 0.340 & 0.097 & $<0.100$ & 4.72 & 0.046 & 1.64 \\
\hline KT03-MK & 1 & 2 & 3 & U02LM12 & 0.832 & 7.66 & 1.61 & 0.220 & 0.096 & $<0.100$ & 4.50 & 0.035 & 1.66 \\
\hline KT03-LA & 1 & 2 & 4 & U09LM22 & 0.849 & 10.6 & 1.76 & 0.221 & 0.092 & $<0.100$ & 4.67 & 0.043 & 1.79 \\
\hline KT03-LL & 1 & 2 & 5 & U15LM12 & 0.866 & 10.3 & 1.78 & 0.232 & 0.100 & $<0.100$ & 4.74 & 0.039 & 1.79 \\
\hline KT03-MK & 1 & 2 & 6 & U02LM22 & 0.829 & 7.55 & 1.72 & 0.222 & 0.098 & $<0.100$ & 4.50 & 0.034 & 1.74 \\
\hline KT03-LS & 1 & 2 & 7 & U06LM12 & 0.828 & 9.90 & 1.76 & 0.222 & 0.095 & $<0.100$ & 4.72 & 0.038 & 1.81 \\
\hline KT03-LB & 1 & 2 & 8 & U10LM12 & 0.847 & 10.4 & 1.79 & 0.234 & 0.098 & $<0.100$ & 4.75 & 0.041 & 1.82 \\
\hline KT03-HS & 1 & 2 & 9 & U05LM12 & 0.792 & 9.28 & 1.53 & 0.219 & 0.092 & $<0.100$ & 4.57 & 0.037 & 1.50 \\
\hline Batch 1 & 1 & 2 & 10 & BCHLM122 & 1.32 & 6.96 & $<0.100$ & 0.511 & $<0.010$ & $<0.100$ & 0.387 & $<0.010$ & 0.071 \\
\hline KT03-LB & 1 & 2 & \begin{tabular}{|l|}
11 \\
\end{tabular} & U10LM22 & 0.862 & 10.6 & 1.56 & 0.237 & 0.099 & $<0.100$ & 4.63 & 0.042 & 1.57 \\
\hline KT1-HA2 & 1 & 2 & 12 & U01LM12 & 0.927 & 10.8 & 0.278 & 0.248 & 0.082 & $<0.100$ & 4.66 & 0.038 & 0.356 \\
\hline KT03-HN & 1 & 2 & 13 & U04LM22 & 0.831 & 12.3 & 1.73 & 0.224 & 0.094 & $<0.100$ & 4.73 & 0.039 & 1.73 \\
\hline KT03-LA & 1 & 2 & 14 & U09LM12 & 0.834 & 11.2 & 1.78 & 0.217 & 0.091 & $<0.100$ & 4.68 & 0.041 & 1.78 \\
\hline KT03-LL & 1 & 2 & 15 & U15LM22 & 0.838 & 10.3 & 1.63 & 0.227 & 0.097 & $<0.100$ & 4.64 & 0.040 & 1.64 \\
\hline KT03-HS & 1 & 2 & 16 & U05LM22 & 0.791 & 9.34 & 1.77 & 0.218 & 0.094 & $<0.100$ & 4.63 & 0.037 & 1.80 \\
\hline KT03-LS & 1 & 2 & 17 & U06LM22 & 0.807 & 9.93 & 1.77 & 0.212 & 0.091 & $<0.100$ & 4.69 & 0.037 & 1.78 \\
\hline KT1-HA2 & 1 & 2 & 18 & U01LM22 & 0.895 & 10.8 & 0.28 & 0.235 & 0.078 & $<0.100$ & 4.70 & 0.038 & 0.351 \\
\hline Batch 1 & 1 & 2 & 19 & BCHLM123 & 1.32 & 6.72 & $<0.100$ & 0.505 & $<0.010$ & $<0.100$ & 0.382 & $<0.010$ & 0.069 \\
\hline Batch 1 & 2 & 1 & 1 & BCHLM211 & 1.31 & 6.99 & $<0.100$ & 0.528 & $<0.010$ & $<0.100$ & 0.393 & $<0.010$ & 0.071 \\
\hline KT03-LF & 2 & 1 & 2 & U12LM11 & 0.915 & 10.4 & 1.46 & 0.281 & 0.101 & $<0.100$ & 4.44 & 0.044 & 1.37 \\
\hline KT03-HL & 2 & 1 & 3 & U13LM11 & 0.823 & 10.2 & 1.50 & 0.222 & 0.090 & $<0.100$ & 4.53 & 0.040 & 1.43 \\
\hline KT03-LN & 2 & 1 & 4 & U07LM21 & 0.866 & 9.24 & 1.96 & 0.224 & 0.095 & $<0.100$ & 4.59 & 0.043 & 1.74 \\
\hline KT03-LF & 2 & 1 & 5 & U12LM21 & 0.966 & 10.4 & 1.95 & 0.264 & 0.111 & $<0.100$ & 4.61 & 0.046 & 1.76 \\
\hline KT03-HK & 2 & 1 & 6 & U11LM21 & 0.807 & 4.52 & 1.86 & 0.214 & 0.096 & $<0.100$ & 4.12 & 0.042 & 1.63 \\
\hline KT03-LN & 2 & 1 & 7 & U07LM11 & 0.852 & 9.16 & 1.98 & 0.221 & 0.095 & $<0.100$ & 4.52 & 0.043 & 1.76 \\
\hline KT03-HB & 2 & 1 & 8 & U08LM21 & 0.845 & 9.44 & 1.92 & 0.222 & 0.094 & $<0.100$ & 4.64 & 0.046 & 1.75 \\
\hline KT03-HL & 2 & 1 & 9 & U13LM21 & 0.837 & 9.71 & 1.60 & 0.231 & 0.095 & $<0.100$ & 4.61 & 0.040 & 1.38 \\
\hline Batch 1 & 2 & 1 & 10 & BCHLM212 & 1.36 & 6.38 & $<0.100$ & 0.519 & $<0.010$ & $<0.100$ & 0.388 & $<0.010$ & 0.074 \\
\hline KT03-HA & 2 & 1 & 11 & U14LM11 & 0.813 & 9.46 & 1.80 & 0.214 & 0.092 & $<0.100$ & 4.65 & 0.037 & 1.58 \\
\hline KT03-HB & 2 & 1 & 12 & U08LM11 & 0.832 & 9.62 & 2.01 & 0.218 & 0.096 & $<0.100$ & 4.66 & 0.045 & 1.79 \\
\hline KT03-HF & 2 & 1 & 13 & U03LM11 & 0.816 & 9.31 & 2.04 & 0.232 & 0.094 & $<0.100$ & 4.61 & 0.037 & 1.79 \\
\hline KT03-HK & 2 & 1 & 14 & U11LM11 & 0.759 & 4.65 & 1.71 & 0.194 & 0.088 & $<0.100$ & 4.14 & 0.039 & 1.55 \\
\hline KT03-HF & 2 & 1 & 15 & U03LM21 & 0.807 & 9.40 & 2.00 & 0.226 & 0.095 & $<0.100$ & 4.60 & 0.037 & 1.77 \\
\hline KT03-HA & 2 & 1 & 16 & U14LM21 & 0.812 & 9.91 & 1.82 & 0.402 & 0.089 & $<0.100$ & 4.64 & 0.037 & 1.69 \\
\hline Batch 1 & 2 & 1 & 17 & BCHLM213 & 1.31 & 6.69 & $<0.100$ & 0.516 & $<0.010$ & $<0.100$ & 0.385 & $<0.010$ & 0.072 \\
\hline Batch 1 & 2 & 2 & 1 & BCHLM221 & 1.35 & 6.56 & $<0.100$ & 0.499 & $<0.010$ & $<0.100$ & 0.379 & $<0.010$ & 0.067 \\
\hline KT03-HB & 2 & 2 & 2 & U08LM22 & 0.831 & 10.6 & 2.16 & 0.219 & 0.093 & $<0.100$ & 5.06 & 0.043 & 1.90 \\
\hline KT03-LN & 2 & 2 & 3 & U07LM12 & 0.833 & 10.5 & 2.18 & 0.216 & 0.092 & $<0.100$ & 5.10 & 0.040 & 1.93 \\
\hline KT03-HF & 2 & 2 & 4 & U03LM22 & 0.832 & 9.54 & 1.97 & 0.230 & 0.094 & $<0.100$ & 4.66 & 0.036 & 1.74 \\
\hline KT03-HK & 2 & 2 & 5 & U11LM22 & 0.781 & 4.77 & 1.84 & 0.205 & 0.094 & $<0.100$ & 4.33 & 0.038 & 1.60 \\
\hline KT03-HF & 2 & 2 & 6 & U03LM12 & 0.804 & 9.67 & 2.01 & 0.228 & 0.091 & $<0.100$ & 4.71 & 0.035 & 1.78 \\
\hline KT03-HL & 2 & 2 & 7 & U13LM12 & 0.798 & 10.1 & 1.47 & 0.209 & 0.083 & $<0.100$ & 4.57 & 0.035 & 1.39 \\
\hline KT03-HB & 2 & 2 & 8 & U08LM12 & 0.840 & 9.81 & 1.96 & 0.224 & 0.094 & $<0.100$ & 4.61 & 0.044 & 1.74 \\
\hline KT03-HA & 2 & 2 & 9 & U14LM22 & 0.825 & 9.92 & 1.78 & 0.404 & 0.088 & $<0.100$ & 4.63 & 0.036 & 1.65 \\
\hline Batch 1 & 2 & 2 & 10 & BCHLM222 & 1.39 & 6.85 & $<0.100$ & 0.502 & $<0.010$ & $<0.100$ & 0.378 & $<0.010$ & 0.071 \\
\hline KT03-LF & 2 & 2 & 11 & U12LM22 & 0.904 & 11.3 & 1.95 & 0.245 & 0.105 & $<0.100$ & 4.76 & 0.040 & 1.75 \\
\hline KT03-LN & 2 & 2 & 12 & U07LM22 & 0.797 & 10.3 & 2.00 & 0.205 & 0.088 & $<0.100$ & 4.75 & 0.038 & 1.77 \\
\hline KT03-LF & 2 & 2 & 13 & U12LM12 & 0.829 & 11.1 & 1.50 & 0.252 & 0.092 & $<0.100$ & 4.67 & 0.037 & 1.40 \\
\hline
\end{tabular}


SRNL-STI-2010-00566

Revision 0

Table B-2. PSAL Chemical Composition Measurements of the KT03-Series of Glasses Using LM Preparation Method (Part 2). (continued)

\begin{tabular}{|c|c|c|c|c|c|c|c|c|c|c|c|c|c|}
\hline Glass ID & Block & \begin{tabular}{|l|} 
Sub-Blk \\
\end{tabular} & Seq & Lab ID & Mn (wt\%) & $\mathrm{Na}(\mathrm{wt} \%)$ & Nb (wt \%) & Ni (wt\%) & Pb (wt\%) & S (wt \%) & Ti (wt\%) & Zn (wt\%) & Zr (wt\%) \\
\hline KT03-HA & 2 & \begin{tabular}{|l|}
2 \\
\end{tabular} & \begin{tabular}{|l|}
14 \\
\end{tabular} & U14LM12 & 0.802 & 10.6 & 1.83 & 0.206 & 0.088 & $<0.100$ & 4.73 & 0.033 & 1.60 \\
\hline KT03-HL & 2 & 2 & 15 & U13LM22 & 0.798 & 10.3 & 1.57 & 0.214 & 0.089 & $<0.100$ & 4.81 & 0.035 & 1.34 \\
\hline KT03-HK & 2 & 2 & 16 & U11LM12 & 0.738 & 4.81 & 1.69 & 0.185 & 0.084 & $<0.100$ & 4.31 & 0.035 & 1.52 \\
\hline Batch 1 & 2 & 2 & 17 & BCHLM223 & 1.35 & 6.95 & $<0.100$ & 0.490 & $<0.010$ & $<0.100$ & 0.377 & $<0.010$ & 0.070 \\
\hline
\end{tabular}


Table B-3. PSAL Chemical Composition Measurements of the KT03-Series of Glasses Using PF Preparation Method.

\begin{tabular}{|c|c|c|c|c|c|c|c|c|c|}
\hline Glass ID & Block & Sub-Blk & Sequence & Lab ID & $\mathrm{Al}(\mathrm{wt} \%)$ & B (wt\%) & Fe (wt \%) & Li (wt\%) & Si (wt\%) \\
\hline Batch 1 & 1 & 1 & 1 & BCHPF111 & 2.54 & 2.45 & 9.19 & 2.10 & 23.4 \\
\hline KT03-LA & 1 & 1 & 2 & U09PF11 & 2.27 & 1.37 & 7.74 & 2.08 & 20.0 \\
\hline KT03-HN & 1 & 1 & 3 & U04PF11 & 2.75 & 1.32 & 7.49 & 2.00 & 19.1 \\
\hline KT03-HS & 1 & 1 & 4 & U05PF21 & 2.54 & 1.18 & 6.61 & 1.81 & 21.2 \\
\hline KT1-HA2 & 1 & 1 & 5 & U01PF11 & 4.64 & 1.38 & 7.15 & 2.14 & 20.2 \\
\hline KT03-HF & 1 & 1 & 6 & U03PF11 & 2.81 & 1.25 & 10.65 & 1.93 & 19.0 \\
\hline KT03-HS & 1 & 1 & 7 & U05PF11 & 2.55 & 1.17 & 6.55 & 1.81 & 22.1 \\
\hline KT03-LA & 1 & 1 & 8 & U09PF21 & 2.33 & 1.36 & 7.92 & 2.08 & 19.2 \\
\hline KT03-HN & 1 & 1 & 9 & U04PF21 & 2.75 & 1.25 & 7.01 & 1.99 & 19.1 \\
\hline Batch 1 & 1 & 1 & 10 & BCHPF112 & 2.55 & 2.38 & 8.78 & 2.10 & 23.0 \\
\hline KT03-LL & 1 & 1 & 11 & U15PF11 & 2.82 & 1.37 & 7.51 & 2.20 & 20.0 \\
\hline KT03-LB & 1 & 1 & 12 & U10PF21 & 2.83 & 1.45 & 7.35 & 2.05 & 20.5 \\
\hline KT03-MK & 1 & 1 & 13 & U02PF21 & 2.72 & 1.26 & 6.97 & 1.24 & 19.2 \\
\hline KT03-MK & 1 & 1 & 14 & U02PF11 & 2.72 & 1.27 & 6.85 & 1.24 & 18.7 \\
\hline KT1-HA2 & 1 & 1 & 15 & U01PF21 & 4.55 & 1.38 & 7.00 & 2.10 & 20.1 \\
\hline KT03-LL & 1 & 1 & 16 & U15PF21 & 2.92 & 1.32 & 7.43 & 2.19 & 19.6 \\
\hline KT03-LB & 1 & 1 & 17 & U10PF11 & 2.84 & 1.44 & 7.28 & 2.06 & 20.3 \\
\hline KT03-HF & 1 & 1 & 18 & U03PF21 & 2.64 & 1.24 & 10.35 & 1.90 & 18.9 \\
\hline Batch 1 & 1 & 1 & 19 & BCHPF113 & 2.54 & 2.35 & 8.53 & 2.09 & 23.8 \\
\hline Batch 1 & 1 & 2 & 1 & BCHPF121 & 2.50 & 2.45 & 9.12 & 2.07 & 23.2 \\
\hline KT03-HF & 1 & 2 & 2 & U03PF12 & 2.78 & 1.27 & 11.18 & 1.92 & 18.9 \\
\hline KT03-LB & 1 & 2 & 3 & U10PF12 & 2.74 & 1.46 & 7.89 & 2.01 & 20.2 \\
\hline KT03-LL & 1 & 2 & 4 & U15PF22 & 2.88 & 1.36 & 7.99 & 2.18 & 19.9 \\
\hline KT03-LB & 1 & 2 & 5 & U10PF22 & 2.75 & 1.44 & 7.76 & 2.02 & 20.1 \\
\hline KT03-MK & 1 & 2 & 6 & U02PF22 & 2.66 & 1.27 & 7.35 & 1.23 & 19.0 \\
\hline KT03-HF & 1 & 2 & 7 & U03PF22 & 2.62 & 1.23 & 11.10 & 1.91 & 18.7 \\
\hline KT03-HN & 1 & 2 & 8 & U04PF22 & 2.71 & 1.26 & 7.42 & 1.98 & 19.0 \\
\hline KT03-LL & 1 & 2 & 9 & U15PF12 & 2.77 & 1.32 & 7.83 & 2.19 & 19.5 \\
\hline Batch 1 & 1 & 2 & 10 & BCHPF122 & 2.50 & 2.33 & 9.87 & 2.07 & 22.9 \\
\hline KT03-HS & 1 & 2 & 11 & U05PF12 & 2.52 & 1.21 & 6.83 & 1.81 & 22.2 \\
\hline KT1-HA2 & 1 & 2 & 12 & U01PF22 & 4.54 & 1.39 & 7.58 & 2.11 & 20.3 \\
\hline KT03-LA & 1 & 2 & 13 & U09PF12 & 2.23 & 1.36 & 7.97 & 2.05 & 19.9 \\
\hline KT1-HA2 & 1 & 2 & 14 & U01PF12 & 4.47 & 1.39 & 7.63 & 2.09 & 20.1 \\
\hline KT03-HN & 1 & 2 & 15 & U04PF12 & 2.70 & 1.29 & 7.58 & 1.98 & 18.9 \\
\hline KT03-HS & 1 & 2 & 16 & U05PF22 & 2.49 & 1.15 & 6.82 & 1.80 & 21.9 \\
\hline KT03-LA & 1 & 2 & 17 & U09PF22 & 2.30 & 1.32 & 8.46 & 2.06 & 19.6 \\
\hline KT03-MK & 1 & 2 & 18 & U02PF12 & 2.68 & 1.25 & 7.24 & 1.24 & 18.4 \\
\hline Batch 1 & 1 & 2 & 19 & BCHPF123 & 2.50 & 2.31 & 9.09 & 2.06 & 22.6 \\
\hline Batch 1 & 2 & 1 & 1 & BCHPF211 & 2.52 & 2.47 & 8.36 & 2.08 & 22.2 \\
\hline KT03-HA & 2 & 1 & 2 & U14PF11 & 4.50 & 1.40 & 6.98 & 2.01 & 19.3 \\
\hline KT03-HB & 2 & 1 & 3 & U08PF21 & 2.73 & 2.23 & 7.11 & 2.01 & 19.5 \\
\hline KT03-LS & 2 & 1 & 4 & U06PF11 & 2.74 & 1.32 & 6.90 & 2.01 & 20.8 \\
\hline KT03-HB & 2 & 1 & 5 & U08PF11 & 2.75 & 2.17 & 6.92 & 2.01 & 19.4 \\
\hline KT03-HK & 2 & 1 & 6 & U11PF21 & 2.60 & 1.25 & 6.67 & 0.57 & 18.2 \\
\hline KT03-LS & 2 & 1 & 7 & U06PF21 & 2.70 & 1.29 & 6.79 & 1.98 & 20.6 \\
\hline KT03-LN & 2 & 1 & 8 & U07PF21 & 2.82 & 1.34 & 7.75 & 2.06 & 20.4 \\
\hline KT03-LF & 2 & 1 & 9 & U12PF11 & 2.88 & 1.38 & 5.60 & 2.12 & 20.8 \\
\hline Batch 1 & 2 & 1 & 10 & BCHPF212 & 2.47 & 2.26 & 8.75 & 2.05 & 22.8 \\
\hline KT03-HL & 2 & 1 & 11 & U13PF21 & 2.68 & 1.39 & 7.48 & 3.29 & 19.5 \\
\hline KT03-HA & 2 & 1 & 12 & U14PF21 & 4.45 & 1.34 & 6.95 & 1.99 & 19.4 \\
\hline KT03-HL & 2 & 1 & 13 & U13PF11 & 2.71 & 1.33 & 7.32 & 3.34 & 19.7 \\
\hline KT03-LF & 2 & 1 & 14 & U12PF21 & 2.88 & 1.39 & 5.74 & 2.14 & 21.1 \\
\hline KT03-HK & 2 & 1 & 15 & U11PF11 & 2.52 & 1.19 & 6.76 & 0.56 & 18.3 \\
\hline KT03-LN & 2 & 1 & 16 & U07PF11 & 2.84 & 1.35 & 7.71 & 2.09 & 20.6 \\
\hline Batch 1 & 2 & 1 & 17 & BCHPF213 & 2.49 & 2.29 & 8.75 & 2.09 & 23.0 \\
\hline Batch 1 & 2 & 2 & 1 & BCHPF221 & 2.30 & 2.32 & 8.23 & 1.90 & 21.2 \\
\hline KT03-LF & 2 & 2 & 2 & U12PF22 & 2.93 & 1.47 & 5.79 & 2.12 & 21.1 \\
\hline KT03-LN & 2 & 2 & 3 & U07PF22 & 2.88 & 1.40 & 7.80 & 2.06 & 20.6 \\
\hline KT03-HB & 2 & 2 & 4 & U08PF12 & 2.69 & 2.18 & 7.39 & 1.94 & 19.8 \\
\hline KT03-HL & 2 & 2 & 5 & U13PF22 & 2.71 & 1.29 & 7.60 & 3.28 & 19.5 \\
\hline KT03-LN & 2 & 2 & 6 & U07PF12 & 2.85 & 1.35 & 7.97 & 2.05 & 20.7 \\
\hline KT03-HL & 2 & 2 & 7 & U13PF12 & 2.73 & 1.30 & 7.57 & 3.31 & 19.9 \\
\hline KT03-HA & 2 & 2 & 8 & U14PF12 & 4.48 & 1.25 & 7.35 & 1.95 & 19.9 \\
\hline KT03-HK & 2 & 2 & 9 & U11PF22 & 2.58 & 1.17 & 6.91 & 0.52 & 18.9 \\
\hline Batch 1 & 2 & 2 & 10 & BCHPF222 & 2.46 & 2.28 & 9.05 & 2.01 & 23.1 \\
\hline KT03-LS & 2 & 2 & 11 & U06PF12 & 2.67 & 1.33 & 7.42 & 1.94 & 21.8 \\
\hline KT03-HA & 2 & 2 & 12 & U14PF22 & 4.41 & 1.28 & 7.20 & 1.93 & 19.7 \\
\hline
\end{tabular}


SRNL-STI-2010-00566

Revision 0

Table B-3. PSAL Chemical Composition Measurements of the KT03-Series of Glasses Using PF Preparation Method. (continued)

\begin{tabular}{|c|c|c|c|c|c|c|c|c|c|}
\hline Glass ID & Block & Sub-Blk & Sequence & Lab ID & Al (wt \%) & B (wt \%) & Fe (wt\%) & Li (wt\%) & Si (wt \%) \\
\hline KT03-HB & 2 & 2 & 13 & U08PF22 & 2.68 & 2.16 & 7.37 & 1.94 & 20.2 \\
\hline KT03-HK & 2 & 2 & 14 & U11PF12 & 2.55 & 1.18 & 6.84 & 0.52 & 18.8 \\
\hline KT03-LS & 2 & 2 & 15 & U06PF22 & 2.69 & 1.24 & 7.04 & 1.94 & 21.4 \\
\hline KT03-LF & 2 & 2 & 16 & U12PF12 & 2.93 & 1.34 & 5.50 & 2.12 & 21.2 \\
\hline Batch 1 & 2 & 2 & 17 & BCHPF223 & 2.39 & 2.21 & 8.44 & 1.98 & 22.3 \\
\hline
\end{tabular}


Table B-4. Comparison of Measured versus Targeted Composition for KT03 Glasses.

\begin{tabular}{|c|c|c|c|c|c|}
\hline Glass ID & Oxide & $\begin{array}{c}\text { Measured } \\
(\mathbf{w t} \%)\end{array}$ & $\begin{array}{c}\text { Targeted } \\
\text { (wt\%) }\end{array}$ & $\begin{array}{c}\text { Difference of Measured } \\
\text { versus Targeted }\end{array}$ & $\begin{array}{c}\text { \% Difference of } \\
\text { Measured versus } \\
\text { Targeted }\end{array}$ \\
\hline Batch 1 & $\mathrm{Al}_{2} \mathrm{O}_{3}$ & 4.6860 & 4.8770 & -0.1910 & $-3.9 \%$ \\
\hline Batch 1 & $\mathrm{~B}_{2} \mathrm{O}_{3}$ & 7.5399 & 7.7770 & -0.2371 & $-3.0 \%$ \\
\hline Batch 1 & $\mathrm{BaO}$ & 0.1378 & 0.1510 & -0.0132 & $-8.7 \%$ \\
\hline Batch 1 & $\mathrm{CaO}$ & 1.1946 & 1.2200 & -0.0254 & $-2.1 \%$ \\
\hline Batch 1 & $\mathrm{Ce}_{2} \mathrm{O}_{3}$ & 0.0061 & 0.0000 & 0.0061 & \\
\hline Batch 1 & $\mathrm{Cr}_{2} \mathrm{O}_{3}$ & 0.1044 & 0.1070 & -0.0026 & $-2.4 \%$ \\
\hline Batch 1 & $\mathrm{CuO}$ & 0.3922 & 0.3990 & -0.0068 & $-1.7 \%$ \\
\hline Batch 1 & $\mathrm{Fe}_{2} \mathrm{O}_{3}$ & 12.6481 & 12.8390 & -0.1909 & $-1.5 \%$ \\
\hline Batch 1 & $\mathrm{~K}_{2} \mathrm{O}$ & 3.6780 & 3.3270 & 0.3510 & $10.6 \%$ \\
\hline Batch 1 & $\mathrm{La}_{2} \mathrm{O}_{3}$ & 0.0059 & 0.0000 & 0.0059 & \\
\hline Batch 1 & $\mathrm{Li}_{2} \mathrm{O}$ & 4.4134 & 4.4290 & -0.0156 & $-0.4 \%$ \\
\hline Batch 1 & $\mathrm{MgO}$ & 1.3131 & 1.4190 & -0.1059 & $-7.5 \%$ \\
\hline Batch 1 & $\mathrm{MnO}$ & 1.7216 & 1.7260 & -0.0044 & $-0.3 \%$ \\
\hline Batch 1 & $\mathrm{Na}_{2} \mathrm{O}$ & 9.1248 & 9.0030 & 0.1218 & $1.4 \%$ \\
\hline Batch 1 & $\mathrm{Nb}_{2} \mathrm{O}_{5}$ & 0.0715 & 0.0000 & 0.0715 & \\
\hline Batch 1 & $\mathrm{NiO}$ & 0.6491 & 0.7510 & -0.1019 & $-13.6 \%$ \\
\hline Batch 1 & $\mathrm{PbO}$ & 0.0054 & 0.0000 & 0.0054 & \\
\hline Batch 1 & $\mathrm{SiO}_{2}$ & 48.7582 & 50.2200 & -1.4618 & $-2.9 \%$ \\
\hline Batch 1 & $\mathrm{SO}_{4}$ & 0.1498 & 0.0000 & 0.1498 & \\
\hline Batch 1 & $\mathrm{TiO}_{2}$ & 0.6409 & 0.6770 & -0.0361 & $-5.3 \%$ \\
\hline Batch 1 & $\mathrm{ZnO}$ & 0.0062 & 0.0000 & 0.0062 & \\
\hline Batch 1 & $\mathrm{ZrO}_{2}$ & 0.0965 & 0.0980 & -0.0015 & $-1.6 \%$ \\
\hline Batch 1 & Sum & 97.3436 & 99.0200 & -1.6764 & $-1.7 \%$ \\
\hline KT03-HA & $\mathrm{Al}_{2} \mathrm{O}_{3}$ & 8.4272 & 8.4200 & 0.0072 & $0.1 \%$ \\
\hline KT03-HA & $\mathrm{B}_{2} \mathrm{O}_{3}$ & 4.2422 & 4.2700 & -0.0278 & $-0.7 \%$ \\
\hline KT03-HA & $\mathrm{BaO}$ & 0.0675 & 0.0700 & -0.0025 & $-3.5 \%$ \\
\hline KT03-HA & $\mathrm{CaO}$ & 0.9301 & 0.9000 & 0.0301 & $3.3 \%$ \\
\hline KT03-HA & $\mathrm{Ce}_{2} \mathrm{O}_{3}$ & 0.1859 & 0.1900 & -0.0041 & $-2.1 \%$ \\
\hline KT03-HA & $\mathrm{Cr}_{2} \mathrm{O}_{3}$ & 0.0921 & 0.1000 & -0.0079 & $-7.9 \%$ \\
\hline KT03-HA & $\mathrm{CuO}$ & 0.0488 & 0.0300 & 0.0188 & $62.7 \%$ \\
\hline KT03-HA & $\mathrm{Fe}_{2} \mathrm{O}_{3}$ & 10.1795 & 10.7100 & -0.5305 & $-5.0 \%$ \\
\hline KT03-HA & $\mathrm{K}_{2} \mathrm{O}$ & 0.0810 & 0.0700 & 0.0110 & $15.7 \%$ \\
\hline KT03-HA & $\mathrm{La}_{2} \mathrm{O}_{3}$ & 0.0583 & 0.0700 & -0.0117 & $-16.6 \%$ \\
\hline KT03-HA & $\mathrm{Li}_{2} \mathrm{O}$ & 4.2412 & 4.2700 & -0.0288 & $-0.7 \%$ \\
\hline KT03-HA & $\mathrm{MgO}$ & 0.1347 & 0.1400 & -0.0053 & $-3.8 \%$ \\
\hline KT03-HA & $\mathrm{MnO}$ & 1.0497 & 1.1500 & -0.1003 & $-8.7 \%$ \\
\hline KT03-HA & $\mathrm{Na}_{2} \mathrm{O}$ & 13.4429 & 13.3500 & 0.0929 & $0.7 \%$ \\
\hline KT03-HA & $\mathrm{Nb}_{2} \mathrm{O}_{5}$ & 2.5856 & 3.0000 & -0.4144 & $-13.8 \%$ \\
\hline KT03-HA & $\mathrm{NiO}$ & 0.3900 & 0.3200 & 0.0700 & $21.9 \%$ \\
\hline KT03-HA & $\mathrm{PbO}$ & 0.0961 & 0.1000 & -0.0039 & $-3.9 \%$ \\
\hline KT03-HA & $\mathrm{SiO}_{2}$ & 41.8768 & 42.2300 & -0.3532 & $-0.8 \%$ \\
\hline KT03-HA & $\mathrm{SO}_{4}$ & 0.1498 & 0.0700 & 0.0798 & $114.0 \%$ \\
\hline KT03-HA & $\mathrm{TiO}_{2}$ & 7.7771 & 8.0000 & -0.2230 & $-2.8 \%$ \\
\hline KT03-HA & $\mathrm{ZnO}$ & 0.0445 & 0.0500 & -0.0055 & $-11.0 \%$ \\
\hline KT03-HA & $\mathrm{ZrO}_{2}$ & 2.2018 & 2.5000 & -0.2982 & $-11.9 \%$ \\
\hline KT03-HA & Sum & 98.3031 & 100.0100 & -1.7069 & $-1.7 \%$ \\
\hline KT03-HB & $\mathrm{Al}_{2} \mathrm{O}_{3}$ & 5.1253 & 5.0800 & 0.0453 & $0.9 \%$ \\
\hline KT03-HB & $\mathrm{B}_{2} \mathrm{O}_{3}$ & 7.0355 & 7.2000 & -0.1645 & $-2.3 \%$ \\
\hline KT03-HB & $\mathrm{BaO}$ & 0.0692 & 0.0700 & -0.0008 & $-1.1 \%$ \\
\hline KT03-HB & $\mathrm{CaO}$ & 0.9095 & 0.9000 & 0.0095 & $1.1 \%$ \\
\hline KT03-HB & $\mathrm{Ce}_{2} \mathrm{O}_{3}$ & 0.1906 & 0.1900 & 0.0006 & $0.3 \%$ \\
\hline KT03-HB & $\mathrm{Cr}_{2} \mathrm{O}_{3}$ & 0.0932 & 0.1000 & -0.0068 & $-6.8 \%$ \\
\hline KT03-HB & $\mathrm{CuO}$ & 0.0563 & 0.0300 & 0.0263 & $87.8 \%$ \\
\hline KT03-HB & $\mathrm{Fe}_{2} \mathrm{O}_{3}$ & 10.2903 & 10.7700 & -0.4797 & $-4.5 \%$ \\
\hline KT03-HB & $\mathrm{K}_{2} \mathrm{O}$ & 0.0684 & 0.0700 & -0.0016 & $-2.3 \%$ \\
\hline KT03-HB & $\mathrm{La}_{2} \mathrm{O}_{3}$ & 0.0592 & 0.0700 & -0.0108 & $-15.4 \%$ \\
\hline KT03-HB & $\mathrm{Li}_{2} \mathrm{O}$ & 4.2520 & 4.2900 & -0.0380 & $-0.9 \%$ \\
\hline KT03-HB & $\mathrm{MgO}$ & 0.1393 & 0.1400 & -0.0007 & $-0.5 \%$ \\
\hline KT03-HB & $\mathrm{MnO}$ & 1.0807 & 1.1600 & -0.0793 & $-6.8 \%$ \\
\hline
\end{tabular}


Table B-4. Comparison of Measured versus Targeted Composition for KT03 Glasses. (continued)

\begin{tabular}{|c|c|c|c|c|c|}
\hline Glass ID & Oxide & $\begin{array}{l}\text { Measured } \\
(w t \%)\end{array}$ & $\begin{array}{c}\text { Targeted } \\
(\mathbf{w t} \%)\end{array}$ & $\begin{array}{c}\text { Difference of Measured } \\
\text { versus Targeted }\end{array}$ & $\begin{array}{c}\text { \% Difference of } \\
\text { Measured versus } \\
\text { Targeted }\end{array}$ \\
\hline KT03-HB & $\mathrm{Na}_{2} \mathrm{O}$ & 13.3014 & 13.4300 & -0.1286 & $-1.0 \%$ \\
\hline KT03-HB & $\mathrm{Nb}_{2} \mathrm{O}_{5}$ & 2.8789 & 3.0000 & -0.1211 & $-4.0 \%$ \\
\hline KT03-HB & $\mathrm{NiO}$ & 0.2809 & 0.3300 & -0.0491 & $-14.9 \%$ \\
\hline KT03-HB & $\mathrm{PbO}$ & 0.1015 & 0.1000 & 0.0015 & $1.5 \%$ \\
\hline KT03-HB & $\mathrm{SiO}_{2}$ & 42.1977 & 42.4600 & -0.2623 & $-0.6 \%$ \\
\hline KT03-HB & $\mathrm{SO}_{4}$ & 0.1498 & 0.0700 & 0.0798 & $114.0 \%$ \\
\hline KT03-HB & $\mathrm{TiO}_{2}$ & 7.9105 & 8.0000 & -0.0895 & $-1.1 \%$ \\
\hline KT03-HB & $\mathrm{ZnO}$ & 0.0554 & 0.0500 & 0.0054 & $10.8 \%$ \\
\hline KT03-HB & $\mathrm{ZrO}_{2}$ & 2.4247 & 2.5000 & -0.0753 & $-3.0 \%$ \\
\hline KT03-HB & Sum & 98.6702 & 100.0100 & -1.3398 & $-1.3 \%$ \\
\hline KT03-HF & $\mathrm{Al}_{2} \mathrm{O}_{3}$ & 5.1253 & 4.9100 & 0.2153 & $4.4 \%$ \\
\hline KT03-HF & $\mathrm{B}_{2} \mathrm{O}_{3}$ & 4.0168 & 4.1500 & -0.1332 & $-3.2 \%$ \\
\hline KT03-HF & $\mathrm{BaO}$ & 0.0678 & 0.0700 & -0.0022 & $-3.1 \%$ \\
\hline KT03-HF & $\mathrm{CaO}$ & 0.8843 & 0.8700 & 0.0143 & $1.6 \%$ \\
\hline KT03-HF & $\mathrm{Ce}_{2} \mathrm{O}_{3}$ & 0.1865 & 0.1800 & 0.0065 & $3.6 \%$ \\
\hline KT03-HF & $\mathrm{Cr}_{2} \mathrm{O}_{3}$ & 0.0899 & 0.0900 & -0.0001 & $-0.1 \%$ \\
\hline KT03-HF & $\mathrm{CuO}$ & 0.0610 & 0.0300 & 0.0310 & $103.4 \%$ \\
\hline KT03-HF & $\mathrm{Fe}_{2} \mathrm{O}_{3}$ & 15.4694 & 16.0800 & -0.6106 & $-3.8 \%$ \\
\hline KT03-HF & $\mathrm{K}_{2} \mathrm{O}$ & 0.0720 & 0.0600 & 0.0120 & $20.0 \%$ \\
\hline KT03-HF & $\mathrm{La}_{2} \mathrm{O}_{3}$ & 0.0592 & 0.0700 & -0.0108 & $-15.4 \%$ \\
\hline KT03-HF & $\mathrm{Li}_{2} \mathrm{O}$ & 4.1228 & 4.1500 & -0.0272 & $-0.7 \%$ \\
\hline KT03-HF & $\mathrm{MgO}$ & 0.1393 & 0.1300 & 0.0093 & $7.2 \%$ \\
\hline KT03-HF & $\mathrm{MnO}$ & 1.0520 & 1.1200 & -0.0680 & $-6.1 \%$ \\
\hline KT03-HF & $\mathrm{Na}_{2} \mathrm{O}$ & 12.7790 & 12.9800 & -0.2010 & $-1.5 \%$ \\
\hline KT03-HF & $\mathrm{Nb}_{2} \mathrm{O}_{5}$ & 2.8682 & 3.0000 & -0.1318 & $-4.4 \%$ \\
\hline KT03-HF & $\mathrm{NiO}$ & 0.2914 & 0.3100 & -0.0186 & $-6.0 \%$ \\
\hline KT03-HF & $\mathrm{PbO}$ & 0.1007 & 0.1000 & 0.0007 & $0.7 \%$ \\
\hline KT03-HF & $\mathrm{SiO}_{2}$ & 40.3793 & 41.0600 & -0.6807 & $-1.7 \%$ \\
\hline KT03-HF & $\mathrm{SO}_{4}$ & 0.1498 & 0.0700 & 0.0798 & $114.0 \%$ \\
\hline KT03-HF & $\mathrm{TiO}_{2}$ & 7.7479 & 8.0000 & -0.2521 & $-3.2 \%$ \\
\hline KT03-HF & $\mathrm{ZnO}$ & 0.0451 & 0.0500 & -0.0049 & $-9.8 \%$ \\
\hline KT03-HF & $\mathrm{ZrO}_{2}$ & 2.3909 & 2.5000 & -0.1091 & $-4.4 \%$ \\
\hline KT03-HF & Sum & 98.0986 & 99.9800 & -1.8814 & $-1.9 \%$ \\
\hline KT03-HK & $\mathrm{Al}_{2} \mathrm{O}_{3}$ & 4.8418 & 4.7600 & 0.0818 & $1.7 \%$ \\
\hline KT03-HK & $\mathrm{B}_{2} \mathrm{O}_{3}$ & 3.8558 & 4.0200 & -0.1642 & $-4.1 \%$ \\
\hline KT03-HK & $\mathrm{BaO}$ & 0.0625 & 0.0700 & -0.0075 & $-10.7 \%$ \\
\hline KT03-HK & $\mathrm{CaO}$ & 0.8707 & 0.8500 & 0.0207 & $2.4 \%$ \\
\hline KT03-HK & $\mathrm{Ce}_{2} \mathrm{O}_{3}$ & 0.1751 & 0.1800 & -0.0049 & $-2.7 \%$ \\
\hline KT03-HK & $\mathrm{Cr}_{2} \mathrm{O}_{3}$ & 0.1283 & 0.0900 & 0.0383 & $42.5 \%$ \\
\hline KT03-HK & $\mathrm{CuO}$ & 0.0519 & 0.0300 & 0.0219 & $73.2 \%$ \\
\hline KT03-HK & $\mathrm{Fe}_{2} \mathrm{O}_{3}$ & 9.7148 & 10.0800 & -0.3652 & $-3.6 \%$ \\
\hline KT03-HK & $\mathrm{K}_{2} \mathrm{O}$ & 17.5811 & 18.7400 & -1.1589 & $-6.2 \%$ \\
\hline KT03-HK & $\mathrm{La}_{2} \mathrm{O}_{3}$ & 0.0537 & 0.0600 & -0.0063 & $-10.6 \%$ \\
\hline KT03-HK & $\mathrm{Li}_{2} \mathrm{O}$ & 1.1679 & 1.0400 & 0.1279 & $12.3 \%$ \\
\hline KT03-HK & $\mathrm{MgO}$ & 0.1223 & 0.1300 & -0.0077 & $-5.9 \%$ \\
\hline KT03-HK & $\mathrm{MnO}$ & 0.9958 & 1.0900 & -0.0942 & $-8.6 \%$ \\
\hline KT03-HK & $\mathrm{Na}_{2} \mathrm{O}$ & 6.3188 & 6.3900 & -0.0713 & $-1.1 \%$ \\
\hline KT03-HK & $\mathrm{Nb}_{2} \mathrm{O}_{5}$ & 2.5391 & 2.7100 & -0.1709 & $-6.3 \%$ \\
\hline KT03-HK & $\mathrm{NiO}$ & 0.2539 & 0.3000 & -0.0461 & $-15.4 \%$ \\
\hline KT03-HK & $\mathrm{PbO}$ & 0.0975 & 0.1000 & -0.0025 & $-2.5 \%$ \\
\hline KT03-HK & $\mathrm{SiO}_{2}$ & 39.6840 & 39.7500 & -0.0660 & $-0.2 \%$ \\
\hline KT03-HK & $\mathrm{SO}_{4}$ & 0.1498 & 0.0600 & 0.0898 & $149.7 \%$ \\
\hline KT03-HK & $\mathrm{TiO}_{2}$ & 7.0473 & 7.2400 & -0.1927 & $-2.7 \%$ \\
\hline KT03-HK & $\mathrm{ZnO}$ & 0.0479 & 0.0400 & 0.0079 & $19.8 \%$ \\
\hline KT03-HK & $\mathrm{ZrO}_{2}$ & 2.1275 & 2.2600 & -0.1325 & $-5.9 \%$ \\
\hline KT03-HK & Sum & 97.8876 & 99.9900 & -2.1024 & $-2.1 \%$ \\
\hline KT03-HL & $\mathrm{Al}_{2} \mathrm{O}_{3}$ & 5.1158 & 5.0800 & 0.0358 & $0.7 \%$ \\
\hline KT03-HL & $\mathrm{B}_{2} \mathrm{O}_{3}$ & 4.2744 & 4.2900 & -0.0156 & $-0.4 \%$ \\
\hline
\end{tabular}


Table B-4. Comparison of Measured versus Targeted Composition for KT03 Glasses. (continued)

\begin{tabular}{|c|c|c|c|c|c|}
\hline Glass ID & Oxide & $\begin{array}{l}\text { Measured } \\
(w t \%)\end{array}$ & $\begin{array}{c}\text { Targeted } \\
(\mathbf{w t} \%)\end{array}$ & $\begin{array}{c}\text { Difference of Measured } \\
\text { versus Targeted }\end{array}$ & $\begin{array}{c}\text { \% Difference of } \\
\text { Measured versus } \\
\text { Targeted }\end{array}$ \\
\hline KT03-HL & $\mathrm{BaO}$ & 0.0673 & 0.0700 & -0.0027 & $-3.9 \%$ \\
\hline KT03-HL & $\mathrm{CaO}$ & 0.9459 & 0.9000 & 0.0459 & $5.1 \%$ \\
\hline KT03-HL & $\mathrm{Ce}_{2} \mathrm{O}_{3}$ & 0.1845 & 0.1900 & -0.0055 & $-2.9 \%$ \\
\hline KT03-HL & $\mathrm{Cr}_{2} \mathrm{O}_{3}$ & 0.1085 & 0.1000 & 0.0085 & $8.5 \%$ \\
\hline KT03-HL & $\mathrm{CuO}$ & 0.0463 & 0.0300 & 0.0163 & $54.4 \%$ \\
\hline KT03-HL & $\mathrm{Fe}_{2} \mathrm{O}_{3}$ & 10.7120 & 10.7700 & -0.0580 & $-0.5 \%$ \\
\hline KT03-HL & $\mathrm{K}_{2} \mathrm{O}$ & 0.0780 & 0.0700 & 0.0080 & $11.4 \%$ \\
\hline KT03-HL & $\mathrm{La}_{2} \mathrm{O}_{3}$ & 0.0572 & 0.0700 & -0.0128 & $-18.3 \%$ \\
\hline KT03-HL & $\mathrm{Li}_{2} \mathrm{O}$ & 7.1153 & 7.2000 & -0.0847 & $-1.2 \%$ \\
\hline KT03-HL & $\mathrm{MgO}$ & 0.1360 & 0.1400 & -0.0040 & $-2.9 \%$ \\
\hline KT03-HL & $\mathrm{MnO}$ & 1.0510 & 1.1600 & -0.1090 & $-9.4 \%$ \\
\hline KT03-HL & $\mathrm{Na}_{2} \mathrm{O}$ & 13.5845 & 13.4300 & 0.1545 & $1.2 \%$ \\
\hline KT03-HL & $\mathrm{Nb}_{2} \mathrm{O}_{5}$ & 2.1958 & 3.0000 & -0.8042 & $-26.8 \%$ \\
\hline KT03-HL & $\mathrm{NiO}$ & 0.2787 & 0.3300 & -0.0513 & $-15.6 \%$ \\
\hline KT03-HL & $\mathrm{PbO}$ & 0.0961 & 0.1000 & -0.0039 & $-3.9 \%$ \\
\hline KT03-HL & $\mathrm{SiO}_{2}$ & 42.0372 & 42.4600 & -0.4228 & $-1.0 \%$ \\
\hline KT03-HL & $\mathrm{SO}_{4}$ & 0.1498 & 0.0700 & 0.0798 & $114.0 \%$ \\
\hline KT03-HL & $\mathrm{TiO}_{2}$ & 7.7228 & 8.0000 & -0.2772 & $-3.5 \%$ \\
\hline KT03-HL & $\mathrm{ZnO}$ & 0.0467 & 0.0500 & -0.0033 & $-6.6 \%$ \\
\hline KT03-HL & $\mathrm{ZrO}_{2}$ & 1.8709 & 2.5000 & -0.6291 & $-25.2 \%$ \\
\hline KT03-HL & Sum & 97.8748 & 100.0100 & -2.1352 & $-2.1 \%$ \\
\hline KT03-HN & $\mathrm{Al}_{2} \mathrm{O}_{3}$ & 5.1536 & 5.0800 & 0.0736 & $1.4 \%$ \\
\hline KT03-HN & $\mathrm{B}_{2} \mathrm{O}_{3}$ & 4.1215 & 4.2800 & -0.1585 & $-3.7 \%$ \\
\hline KT03-HN & $\mathrm{BaO}$ & 0.0670 & 0.0700 & -0.0030 & $-4.3 \%$ \\
\hline KT03-HN & $\mathrm{CaO}$ & 0.9147 & 0.9000 & 0.0147 & $1.6 \%$ \\
\hline KT03-HN & $\mathrm{Ce}_{2} \mathrm{O}_{3}$ & 0.1877 & 0.1900 & -0.0023 & $-1.2 \%$ \\
\hline KT03-HN & $\mathrm{Cr}_{2} \mathrm{O}_{3}$ & 0.1060 & 0.1000 & 0.0060 & $6.0 \%$ \\
\hline KT03-HN & $\mathrm{CuO}$ & 0.0573 & 0.0300 & 0.0273 & $90.9 \%$ \\
\hline KT03-HN & $\mathrm{Fe}_{2} \mathrm{O}_{3}$ & 10.5440 & 10.7600 & -0.2160 & $-2.0 \%$ \\
\hline KT03-HN & $\mathrm{K}_{2} \mathrm{O}$ & 0.0717 & 0.0700 & 0.0017 & $2.4 \%$ \\
\hline KT03-HN & $\mathrm{La}_{2} \mathrm{O}_{3}$ & 0.0575 & 0.0700 & -0.0125 & $-17.9 \%$ \\
\hline KT03-HN & $\mathrm{Li}_{2} \mathrm{O}$ & 4.2789 & 4.2800 & -0.0011 & $0.0 \%$ \\
\hline KT03-HN & $\mathrm{MgO}$ & 0.1372 & 0.1400 & -0.0028 & $-2.0 \%$ \\
\hline KT03-HN & $\mathrm{MnO}$ & 1.0865 & 1.1600 & -0.0735 & $-6.3 \%$ \\
\hline KT03-HN & $\mathrm{Na}_{2} \mathrm{O}$ & 16.1086 & 16.4100 & -0.3014 & $-1.8 \%$ \\
\hline KT03-HN & $\mathrm{Nb}_{2} \mathrm{O}_{5}$ & 2.5105 & 3.0000 & -0.4895 & $-16.3 \%$ \\
\hline KT03-HN & $\mathrm{NiO}$ & 0.3579 & 0.3300 & 0.0279 & $8.5 \%$ \\
\hline KT03-HN & $\mathrm{PbO}$ & 0.1037 & 0.1000 & 0.0037 & $3.7 \%$ \\
\hline KT03-HN & $\mathrm{SiO}_{2}$ & 40.7002 & 42.4100 & -1.7098 & $-4.0 \%$ \\
\hline KT03-HN & $\mathrm{SO}_{4}$ & 0.1498 & 0.0700 & 0.0798 & $114.0 \%$ \\
\hline KT03-HN & $\mathrm{TiO}_{2}$ & 7.7270 & 8.0000 & -0.2730 & $-3.4 \%$ \\
\hline KT03-HN & $\mathrm{ZnO}$ & 0.0529 & 0.0500 & 0.0029 & $5.8 \%$ \\
\hline KT03-HN & $\mathrm{ZrO}_{2}$ & 2.2221 & 2.5000 & -0.2779 & $-11.1 \%$ \\
\hline KT03-HN & Sum & 96.7162 & 100.0000 & -3.2838 & $-3.3 \%$ \\
\hline KT03-HS & $\mathrm{Al}_{2} \mathrm{O}_{3}$ & 4.7710 & 4.6000 & 0.1710 & $3.7 \%$ \\
\hline KT03-HS & $\mathrm{B}_{2} \mathrm{O}_{3}$ & 3.7914 & 3.8900 & -0.0986 & $-2.5 \%$ \\
\hline KT03-HS & $\mathrm{BaO}$ & 0.0673 & 0.0700 & -0.0027 & $-3.9 \%$ \\
\hline KT03-HS & $\mathrm{CaO}$ & 0.8203 & 0.8200 & 0.0003 & $0.0 \%$ \\
\hline KT03-HS & $\mathrm{Ce}_{2} \mathrm{O}_{3}$ & 0.1789 & 0.1700 & 0.0089 & $5.2 \%$ \\
\hline KT03-HS & $\mathrm{Cr}_{2} \mathrm{O}_{3}$ & 0.0778 & 0.0900 & -0.0122 & $-13.5 \%$ \\
\hline KT03-HS & $\mathrm{CuO}$ & 0.0488 & 0.0300 & 0.0188 & $62.7 \%$ \\
\hline KT03-HS & $\mathrm{Fe}_{2} \mathrm{O}_{3}$ & 9.5826 & 9.7600 & -0.1774 & $-1.8 \%$ \\
\hline KT03-HS & $\mathrm{K}_{2} \mathrm{O}$ & 0.0614 & 0.0600 & 0.0014 & $2.4 \%$ \\
\hline KT03-HS & $\mathrm{La}_{2} \mathrm{O}_{3}$ & 0.0513 & 0.0600 & -0.0087 & $-14.5 \%$ \\
\hline KT03-HS & $\mathrm{Li}_{2} \mathrm{O}$ & 3.8914 & 3.8900 & 0.0014 & $0.0 \%$ \\
\hline KT03-HS & $\mathrm{MgO}$ & 0.1302 & 0.1200 & 0.0102 & $8.5 \%$ \\
\hline KT03-HS & $\mathrm{MnO}$ & 1.0275 & 1.0500 & -0.0225 & $-2.1 \%$ \\
\hline KT03-HS & $\mathrm{Na}_{2} \mathrm{O}$ & 12.3881 & 12.1600 & 0.2281 & $1.9 \%$ \\
\hline
\end{tabular}


Table B-4. Comparison of Measured versus Targeted Composition for KT03 Glasses. (continued)

\begin{tabular}{|c|c|c|c|c|c|}
\hline Glass ID & Oxide & $\begin{array}{l}\text { Measured } \\
(\mathbf{w t} \%)\end{array}$ & $\begin{array}{l}\text { Targeted } \\
\text { (wt\%) }\end{array}$ & $\begin{array}{c}\text { Difference of Measured } \\
\text { versus Targeted }\end{array}$ & $\begin{array}{c}\text { \% Difference of } \\
\text { Measured versus } \\
\text { Targeted } \\
\end{array}$ \\
\hline KT03-HS & $\mathrm{Nb}_{2} \mathrm{O}_{5}$ & 2.4819 & 3.0000 & -0.5181 & $-17.3 \%$ \\
\hline KT03-HS & $\mathrm{NiO}$ & 0.2790 & 0.3000 & -0.0210 & $-7.0 \%$ \\
\hline KT03-HS & $\mathrm{PbO}$ & 0.1007 & 0.0900 & 0.0107 & $11.9 \%$ \\
\hline KT03-HS & $\mathrm{SiO}_{2}$ & 46.7437 & 49.2400 & -2.4963 & $-5.1 \%$ \\
\hline KT03-HS & $\mathrm{SO}_{4}$ & 0.1498 & 0.0600 & 0.0898 & $149.7 \%$ \\
\hline KT03-HS & $\mathrm{TiO}_{2}$ & 7.5269 & 8.0000 & -0.4732 & $-5.9 \%$ \\
\hline KT03-HS & $\mathrm{ZnO}$ & 0.0467 & 0.0400 & 0.0067 & $16.7 \%$ \\
\hline KT03-HS & $\mathrm{ZrO}_{2}$ & 2.1782 & 2.5000 & -0.3218 & $-12.9 \%$ \\
\hline KT03-HS & Sum & 96.3948 & 100.0000 & -3.6052 & $-3.6 \%$ \\
\hline KT03-LA & $\mathrm{Al}_{2} \mathrm{O}_{3}$ & 4.3128 & 4.1900 & 0.1228 & $2.9 \%$ \\
\hline KT03-LA & $\mathrm{B}_{2} \mathrm{O}_{3}$ & 4.3549 & 4.5000 & -0.1451 & $-3.2 \%$ \\
\hline KT03-LA & $\mathrm{BaO}$ & 0.7062 & 0.0800 & 0.6262 & $782.7 \%$ \\
\hline KT03-LA & $\mathrm{CaO}$ & 0.9920 & 0.9500 & 0.0420 & $4.4 \%$ \\
\hline KT03-LA & $\mathrm{Ce}_{2} \mathrm{O}_{3}$ & 0.1930 & 0.2000 & -0.0070 & $-3.5 \%$ \\
\hline KT03-LA & $\mathrm{Cr}_{2} \mathrm{O}_{3}$ & 0.0957 & 0.1000 & -0.0043 & $-4.3 \%$ \\
\hline KT03-LA & $\mathrm{CuO}$ & 0.0538 & 0.0300 & 0.0238 & $79.4 \%$ \\
\hline KT03-LA & $\mathrm{Fe}_{2} \mathrm{O}_{3}$ & 11.4698 & 11.2900 & 0.1798 & $1.6 \%$ \\
\hline KT03-LA & $\mathrm{K}_{2} \mathrm{O}$ & 0.0831 & 0.0700 & 0.0131 & $18.7 \%$ \\
\hline KT03-LA & $\mathrm{La}_{2} \mathrm{O}_{3}$ & 0.0592 & 0.0700 & -0.0108 & $-15.4 \%$ \\
\hline KT03-LA & $\mathrm{Li}_{2} \mathrm{O}$ & 4.4511 & 4.5000 & -0.0489 & $-1.1 \%$ \\
\hline KT03-LA & $\mathrm{MgO}$ & 0.1397 & 0.1400 & -0.0003 & $-0.2 \%$ \\
\hline KT03-LA & $\mathrm{MnO}$ & 1.0862 & 1.2200 & -0.1338 & $-11.0 \%$ \\
\hline KT03-LA & $\mathrm{Na}_{2} \mathrm{O}$ & 14.2551 & 14.0800 & 0.1751 & $1.2 \%$ \\
\hline KT03-LA & $\mathrm{Nb}_{2} \mathrm{O}_{5}$ & 2.6178 & 3.0000 & -0.3822 & $-12.7 \%$ \\
\hline KT03-LA & $\mathrm{NiO}$ & 0.2774 & 0.3400 & -0.0626 & $-18.4 \%$ \\
\hline KT03-LA & $\mathrm{PbO}$ & 0.0988 & 0.1100 & -0.0112 & $-10.2 \%$ \\
\hline KT03-LA & $\mathrm{SiO}_{2}$ & 42.0907 & 44.5200 & -2.4293 & $-5.5 \%$ \\
\hline KT03-LA & $\mathrm{SO}_{4}$ & 0.1498 & 0.0700 & 0.0798 & $114.0 \%$ \\
\hline KT03-LA & $\mathrm{TiO}_{2}$ & 7.6228 & 8.0000 & -0.3772 & $-4.7 \%$ \\
\hline KT03-LA & $\mathrm{ZnO}$ & 0.0529 & 0.0500 & 0.0029 & $5.8 \%$ \\
\hline KT03-LA & $\mathrm{ZrO}_{2}$ & 2.3335 & 2.5000 & -0.1665 & $-6.7 \%$ \\
\hline KT03-LA & Sum & 97.4965 & 100.0100 & -2.5135 & $-2.5 \%$ \\
\hline KT03-LB & $\mathrm{Al}_{2} \mathrm{O}_{3}$ & 5.2717 & 5.2400 & 0.0317 & $0.6 \%$ \\
\hline KT03-LB & $\mathrm{B}_{2} \mathrm{O}_{3}$ & 4.6608 & 4.8000 & -0.1392 & $-2.9 \%$ \\
\hline KT03-LB & $\mathrm{BaO}$ & 0.0698 & 0.0800 & -0.0102 & $-12.8 \%$ \\
\hline KT03-LB & $\mathrm{CaO}$ & 0.9557 & 0.9300 & 0.0257 & $2.8 \%$ \\
\hline KT03-LB & $\mathrm{Ce}_{2} \mathrm{O}_{3}$ & 0.1930 & 0.1900 & 0.0030 & $1.6 \%$ \\
\hline KT03-LB & $\mathrm{Cr}_{2} \mathrm{O}_{3}$ & 0.1060 & 0.1000 & 0.0060 & $6.0 \%$ \\
\hline KT03-LB & $\mathrm{CuO}$ & 0.0557 & 0.0300 & 0.0257 & $85.7 \%$ \\
\hline KT03-LB & $\mathrm{Fe}_{2} \mathrm{O}_{3}$ & 10.8228 & 11.0900 & -0.2672 & $-2.4 \%$ \\
\hline KT03-LB & $\mathrm{K}_{2} \mathrm{O}$ & 0.0915 & 0.0700 & 0.0215 & $30.8 \%$ \\
\hline KT03-LB & $\mathrm{La}_{2} \mathrm{O}_{3}$ & 0.0592 & 0.0700 & -0.0108 & $-15.4 \%$ \\
\hline KT03-LB & $\mathrm{Li}_{2} \mathrm{O}$ & 4.3812 & 4.4200 & -0.0388 & $-0.9 \%$ \\
\hline KT03-LB & $\mathrm{MgO}$ & 0.1410 & 0.1400 & 0.0010 & $0.7 \%$ \\
\hline KT03-LB & $\mathrm{MnO}$ & 1.1043 & 1.2000 & -0.0957 & $-8.0 \%$ \\
\hline KT03-LB & $\mathrm{Na}_{2} \mathrm{O}$ & 13.7125 & 13.8300 & -0.1175 & $-0.8 \%$ \\
\hline KT03-LB & $\mathrm{Nb}_{2} \mathrm{O}_{5}$ & 2.4998 & 3.0000 & -0.5002 & $-16.7 \%$ \\
\hline KT03-LB & $\mathrm{NiO}$ & 0.3006 & 0.3400 & -0.0394 & $-11.6 \%$ \\
\hline KT03-LB & $\mathrm{PbO}$ & 0.1061 & 0.1100 & -0.0039 & $-3.5 \%$ \\
\hline KT03-LB & $\mathrm{SiO}_{2}$ & 43.3743 & 43.7400 & -0.3657 & $-0.8 \%$ \\
\hline KT03-LB & $\mathrm{SO}_{4}$ & 0.1498 & 0.0700 & 0.0798 & $114.0 \%$ \\
\hline KT03-LB & $\mathrm{TiO}_{2}$ & 7.6811 & 8.0000 & -0.3189 & $-4.0 \%$ \\
\hline KT03-LB & $\mathrm{ZnO}$ & 0.0517 & 0.0500 & 0.0017 & $3.3 \%$ \\
\hline KT03-LB & $\mathrm{ZrO}_{2}$ & 2.2322 & 2.5000 & -0.2678 & $-10.7 \%$ \\
\hline KT03-LB & Sum & 98.0208 & 100.0000 & -1.9792 & $-2.0 \%$ \\
\hline KT03-LF & $\mathrm{Al}_{2} \mathrm{O}_{3}$ & 5.4890 & 5.4600 & 0.0290 & $0.5 \%$ \\
\hline KT03-LF & $\mathrm{B}_{2} \mathrm{O}_{3}$ & 4.4918 & 4.6100 & -0.1182 & $-2.6 \%$ \\
\hline KT03-LF & $\mathrm{BaO}$ & 0.0754 & 0.0800 & -0.0046 & $-5.8 \%$ \\
\hline
\end{tabular}


Table B-4. Comparison of Measured versus Targeted Composition for KT03 Glasses. (continued)

\begin{tabular}{|c|c|c|c|c|c|}
\hline Glass ID & Oxide & $\begin{array}{l}\text { Measured } \\
(w t \%)\end{array}$ & $\begin{array}{c}\text { Targeted } \\
(\mathbf{w t} \%)\end{array}$ & $\begin{array}{c}\text { Difference of Measured } \\
\text { versus Targeted }\end{array}$ & $\begin{array}{c}\text { \% Difference of } \\
\text { Measured versus } \\
\text { Targeted }\end{array}$ \\
\hline KT03-LF & $\mathrm{CaO}$ & 0.9948 & 0.9700 & 0.0248 & $2.6 \%$ \\
\hline KT03-LF & $\mathrm{Ce}_{2} \mathrm{O}_{3}$ & 0.2029 & 0.2000 & 0.0029 & $1.5 \%$ \\
\hline KT03-LF & $\mathrm{Cr}_{2} \mathrm{O}_{3}$ & 0.0950 & 0.1100 & -0.0150 & $-13.6 \%$ \\
\hline KT03-LF & $\mathrm{CuO}$ & 0.0563 & 0.0300 & 0.0263 & $87.8 \%$ \\
\hline KT03-LF & $\mathrm{Fe}_{2} \mathrm{O}_{3}$ & 8.0885 & 8.2400 & -0.1515 & $-1.8 \%$ \\
\hline KT03-LF & $\mathrm{K}_{2} \mathrm{O}$ & 0.0900 & 0.0700 & 0.0200 & $28.6 \%$ \\
\hline KT03-LF & $\mathrm{La}_{2} \mathrm{O}_{3}$ & 0.0639 & 0.0700 & -0.0061 & $-8.7 \%$ \\
\hline KT03-LF & $\mathrm{Li}_{2} \mathrm{O}$ & 4.5749 & 4.6100 & -0.0351 & $-0.8 \%$ \\
\hline KT03-LF & $\mathrm{MgO}$ & 0.1492 & 0.1500 & -0.0008 & $-0.5 \%$ \\
\hline KT03-LF & $\mathrm{MnO}$ & 1.1666 & 1.2500 & -0.0834 & $-6.7 \%$ \\
\hline KT03-LF & $\mathrm{Na}_{2} \mathrm{O}$ & 14.5584 & 14.4300 & 0.1284 & $0.9 \%$ \\
\hline KT03-LF & $\mathrm{Nb}_{2} \mathrm{O}_{5}$ & 2.4533 & 3.0000 & -0.5467 & $-18.2 \%$ \\
\hline KT03-LF & $\mathrm{NiO}$ & 0.3315 & 0.3500 & -0.0185 & $-5.3 \%$ \\
\hline KT03-LF & $\mathrm{PbO}$ & 0.1101 & 0.1100 & 0.0001 & $0.1 \%$ \\
\hline KT03-LF & $\mathrm{SiO}_{2}$ & 45.0323 & 45.6300 & -0.5977 & $-1.3 \%$ \\
\hline KT03-LF & $\mathrm{SO}_{4}$ & 0.1498 & 0.0700 & 0.0798 & $114.0 \%$ \\
\hline KT03-LF & $\mathrm{TiO}_{2}$ & 7.7062 & 8.0000 & -0.2938 & $-3.7 \%$ \\
\hline KT03-LF & $\mathrm{ZnO}$ & 0.0520 & 0.0500 & 0.0020 & $3.9 \%$ \\
\hline KT03-LF & $\mathrm{ZrO}_{2}$ & 2.1208 & 2.5000 & -0.3792 & $-15.2 \%$ \\
\hline KT03-LF & Sum & 98.0527 & 99.9900 & -1.9373 & $-1.9 \%$ \\
\hline KT03-LL & $\mathrm{Al}_{2} \mathrm{O}_{3}$ & 5.3804 & 5.2400 & 0.1404 & $2.7 \%$ \\
\hline KT03-LL & $\mathrm{B}_{2} \mathrm{O}_{3}$ & 4.3227 & 4.4200 & -0.0973 & $-2.2 \%$ \\
\hline KT03-LL & $\mathrm{BaO}$ & 0.0653 & 0.0800 & -0.0147 & $-18.4 \%$ \\
\hline KT03-LL & $\mathrm{CaO}$ & 0.9539 & 0.9300 & 0.0239 & $2.6 \%$ \\
\hline KT03-LL & $\mathrm{Ce}_{2} \mathrm{O}_{3}$ & 0.1918 & 0.1900 & 0.0018 & $0.9 \%$ \\
\hline KT03-LL & $\mathrm{Cr}_{2} \mathrm{O}_{3}$ & 0.0895 & 0.1000 & -0.0105 & $-10.5 \%$ \\
\hline KT03-LL & $\mathrm{CuO}$ & 0.0488 & 0.0300 & 0.0188 & $62.7 \%$ \\
\hline KT03-LL & $\mathrm{Fe}_{2} \mathrm{O}_{3}$ & 10.9944 & 11.0900 & -0.0956 & $-0.9 \%$ \\
\hline KT03-LL & $\mathrm{K}_{2} \mathrm{O}$ & 0.0753 & 0.0700 & 0.0053 & $7.6 \%$ \\
\hline KT03-LL & $\mathrm{La}_{2} \mathrm{O}_{3}$ & 0.0592 & 0.0700 & -0.0108 & $-15.4 \%$ \\
\hline KT03-LL & $\mathrm{Li}_{2} \mathrm{O}$ & 4.7149 & 4.8000 & -0.0851 & $-1.8 \%$ \\
\hline KT03-LL & $\mathrm{MgO}$ & 0.1410 & 0.1400 & 0.0010 & $0.7 \%$ \\
\hline KT03-LL & $\mathrm{MnO}$ & 1.0978 & 1.2000 & -0.1022 & $-8.5 \%$ \\
\hline KT03-LL & $\mathrm{Na}_{2} \mathrm{O}$ & 13.7496 & 13.8300 & -0.0804 & $-0.6 \%$ \\
\hline KT03-LL & $\mathrm{Nb}_{2} \mathrm{O}_{5}$ & 2.5570 & 3.0000 & -0.4430 & $-14.8 \%$ \\
\hline KT03-LL & $\mathrm{NiO}$ & 0.2901 & 0.3400 & -0.0499 & $-14.7 \%$ \\
\hline KT03-LL & $\mathrm{PbO}$ & 0.1053 & 0.1100 & -0.0047 & $-4.3 \%$ \\
\hline KT03-LL & $\mathrm{SiO}_{2}$ & 42.2512 & 43.7400 & -1.4888 & $-3.4 \%$ \\
\hline KT03-LL & $\mathrm{SO}_{4}$ & 0.1498 & 0.0700 & 0.0798 & $114.0 \%$ \\
\hline KT03-LL & $\mathrm{TiO}_{2}$ & 7.6686 & 8.0000 & -0.3314 & $-4.1 \%$ \\
\hline KT03-LL & $\mathrm{ZnO}$ & 0.0492 & 0.0500 & -0.0008 & $-1.7 \%$ \\
\hline KT03-LL & $\mathrm{ZrO}_{2}$ & 2.2592 & 2.5000 & -0.2408 & $-9.6 \%$ \\
\hline KT03-LL & Sum & 97.2150 & 100.0000 & -2.7850 & $-2.8 \%$ \\
\hline KT03-LN & $\mathrm{Al}_{2} \mathrm{O}_{3}$ & 5.3804 & 5.3200 & 0.0604 & $1.1 \%$ \\
\hline KT03-LN & $\mathrm{B}_{2} \mathrm{O}_{3}$ & 4.3791 & 4.4900 & -0.1109 & $-2.5 \%$ \\
\hline KT03-LN & $\mathrm{BaO}$ & 0.0701 & 0.0800 & -0.0099 & $-12.4 \%$ \\
\hline KT03-LN & $\mathrm{CaO}$ & 0.9973 & 0.9500 & 0.0473 & $5.0 \%$ \\
\hline KT03-LN & $\mathrm{Ce}_{2} \mathrm{O}_{3}$ & 0.1906 & 0.2000 & -0.0094 & $-4.7 \%$ \\
\hline KT03-LN & $\mathrm{Cr}_{2} \mathrm{O}_{3}$ & 0.0932 & 0.1000 & -0.0068 & $-6.8 \%$ \\
\hline KT03-LN & $\mathrm{CuO}$ & 0.0488 & 0.0300 & 0.0188 & $62.7 \%$ \\
\hline KT03-LN & $\mathrm{Fe}_{2} \mathrm{O}_{3}$ & 11.1624 & 11.2700 & -0.1076 & $-1.0 \%$ \\
\hline KT03-LN & $\mathrm{K}_{2} \mathrm{O}$ & 0.0891 & 0.0700 & 0.0191 & $27.3 \%$ \\
\hline KT03-LN & $\mathrm{La}_{2} \mathrm{O}_{3}$ & 0.0598 & 0.0700 & -0.0102 & $-14.6 \%$ \\
\hline KT03-LN & $\mathrm{Li}_{2} \mathrm{O}$ & 4.4457 & 4.4900 & -0.0443 & $-1.0 \%$ \\
\hline KT03-LN & $\mathrm{MgO}$ & 0.1368 & 0.1400 & -0.0032 & $-2.3 \%$ \\
\hline KT03-LN & $\mathrm{MnO}$ & 1.0807 & 1.2200 & -0.1393 & $-11.4 \%$ \\
\hline KT03-LN & $\mathrm{Na}_{2} \mathrm{O}$ & 13.2104 & 13.0700 & 0.1404 & $1.1 \%$ \\
\hline KT03-LN & $\mathrm{Nb}_{2} \mathrm{O}_{5}$ & 2.9039 & 3.0000 & -0.0961 & $-3.2 \%$ \\
\hline
\end{tabular}


Table B-4. Comparison of Measured versus Targeted Composition for KT03 Glasses. (continued)

\begin{tabular}{|c|c|c|c|c|c|}
\hline Glass ID & Oxide & $\begin{array}{l}\text { Measured } \\
(\mathbf{w t} \%)\end{array}$ & $\begin{array}{l}\text { Targeted } \\
\text { (wt\%) }\end{array}$ & $\begin{array}{l}\text { Difference of Measured } \\
\text { versus Targeted }\end{array}$ & $\begin{array}{c}\text { \% Difference of } \\
\text { Measured versus } \\
\text { Targeted } \\
\end{array}$ \\
\hline KT03-LN & $\mathrm{NiO}$ & 0.2755 & 0.3400 & -0.0645 & $-19.0 \%$ \\
\hline KT03-LN & $\mathrm{PbO}$ & 0.0996 & 0.1100 & -0.0104 & $-9.4 \%$ \\
\hline KT03-LN & $\mathrm{SiO}_{2}$ & 44.0161 & 44.4400 & -0.4239 & $-1.0 \%$ \\
\hline KT03-LN & $\mathrm{SO}_{4}$ & 0.1498 & 0.0700 & 0.0798 & $114.0 \%$ \\
\hline KT03-LN & $\mathrm{TiO}_{2}$ & 7.9063 & 8.0000 & -0.0937 & $-1.2 \%$ \\
\hline KT03-LN & $\mathrm{ZnO}$ & 0.0510 & 0.0500 & 0.0010 & $2.1 \%$ \\
\hline KT03-LN & $\mathrm{ZrO}_{2}$ & 2.4314 & 2.5000 & -0.0686 & $-2.7 \%$ \\
\hline KT03-LN & Sum & 99.1781 & 100.0100 & -0.8319 & $-0.8 \%$ \\
\hline KT03-LS & $\mathrm{Al}_{2} \mathrm{O}_{3}$ & 5.1017 & 5.0100 & 0.0917 & $1.8 \%$ \\
\hline KT03-LS & $\mathrm{B}_{2} \mathrm{O}_{3}$ & 4.1698 & 4.2300 & -0.0602 & $-1.4 \%$ \\
\hline KT03-LS & $\mathrm{BaO}$ & 0.0648 & 0.0700 & -0.0052 & $-7.5 \%$ \\
\hline KT03-LS & $\mathrm{CaO}$ & 0.9088 & 0.8900 & 0.0188 & $2.1 \%$ \\
\hline KT03-LS & $\mathrm{Ce}_{2} \mathrm{O}_{3}$ & 0.1865 & 0.1900 & -0.0035 & $-1.8 \%$ \\
\hline KT03-LS & $\mathrm{Cr}_{2} \mathrm{O}_{3}$ & 0.1096 & 0.1000 & 0.0096 & $9.6 \%$ \\
\hline KT03-LS & $\mathrm{CuO}$ & 0.0482 & 0.0300 & 0.0182 & $60.6 \%$ \\
\hline KT03-LS & $\mathrm{Fe}_{2} \mathrm{O}_{3}$ & 10.0615 & 10.6200 & -0.5585 & $-5.3 \%$ \\
\hline KT03-LS & $\mathrm{K}_{2} \mathrm{O}$ & 0.1111 & 0.0700 & 0.0411 & $58.7 \%$ \\
\hline KT03-LS & $\mathrm{La}_{2} \mathrm{O}_{3}$ & 0.0545 & 0.0700 & -0.0155 & $-22.1 \%$ \\
\hline KT03-LS & $\mathrm{Li}_{2} \mathrm{O}$ & 4.2358 & 4.2300 & 0.0058 & $0.1 \%$ \\
\hline KT03-LS & $\mathrm{MgO}$ & 0.1285 & 0.1300 & -0.0015 & $-1.1 \%$ \\
\hline KT03-LS & $\mathrm{MnO}$ & 1.0523 & 1.1400 & -0.0877 & $-7.7 \%$ \\
\hline KT03-LS & $\mathrm{Na}_{2} \mathrm{O}$ & 13.1194 & 13.2400 & -0.1206 & $-0.9 \%$ \\
\hline KT03-LS & $\mathrm{Nb}_{2} \mathrm{O}_{5}$ & 2.6285 & 3.0000 & -0.3715 & $-12.4 \%$ \\
\hline KT03-LS & $\mathrm{NiO}$ & 0.2742 & 0.3200 & -0.0458 & $-14.3 \%$ \\
\hline KT03-LS & $\mathrm{PbO}$ & 0.0996 & 0.1000 & -0.0004 & $-0.4 \%$ \\
\hline KT03-LS & $\mathrm{SiO}_{2}$ & 45.2462 & 45.9600 & -0.7138 & $-1.6 \%$ \\
\hline KT03-LS & $\mathrm{SO}_{4}$ & 0.1498 & 0.0700 & 0.0798 & $114.0 \%$ \\
\hline KT03-LS & $\mathrm{TiO}_{2}$ & 7.6811 & 8.0000 & -0.3189 & $-4.0 \%$ \\
\hline KT03-LS & $\mathrm{ZnO}$ & 0.0467 & 0.0500 & -0.0033 & $-6.6 \%$ \\
\hline KT03-LS & $\mathrm{ZrO}_{2}$ & 2.3470 & 2.5000 & -0.1530 & $-6.1 \%$ \\
\hline KT03-LS & Sum & 97.8258 & 100.0200 & -2.1942 & $-2.2 \%$ \\
\hline KT03-MK & $\mathrm{Al}_{2} \mathrm{O}_{3}$ & 5.0922 & 5.0000 & 0.0922 & $1.8 \%$ \\
\hline KT03-MK & $\mathrm{B}_{2} \mathrm{O}_{3}$ & 4.0651 & 4.2200 & -0.1549 & $-3.7 \%$ \\
\hline KT03-MK & $\mathrm{BaO}$ & 0.0667 & 0.0700 & -0.0033 & $-4.7 \%$ \\
\hline KT03-MK & $\mathrm{CaO}$ & 0.9049 & 0.8900 & 0.0149 & $1.7 \%$ \\
\hline KT03-MK & $\mathrm{Ce}_{2} \mathrm{O}_{3}$ & 0.1871 & 0.1900 & -0.0029 & $-1.5 \%$ \\
\hline KT03-MK & $\mathrm{Cr}_{2} \mathrm{O}_{3}$ & 0.0957 & 0.1000 & -0.0043 & $-4.3 \%$ \\
\hline KT03-MK & $\mathrm{CuO}$ & 0.0444 & 0.0300 & 0.0144 & $48.1 \%$ \\
\hline KT03-MK & $\mathrm{Fe}_{2} \mathrm{O}_{3}$ & 10.1544 & 10.5900 & -0.4356 & $-4.1 \%$ \\
\hline KT03-MK & $\mathrm{K}_{2} \mathrm{O}$ & 9.8837 & 9.8500 & 0.0337 & $0.3 \%$ \\
\hline KT03-MK & $\mathrm{La}_{2} \mathrm{O}_{3}$ & 0.0554 & 0.0700 & -0.0146 & $-20.8 \%$ \\
\hline KT03-MK & $\mathrm{Li}_{2} \mathrm{O}$ & 2.6642 & 2.6500 & 0.0142 & $0.5 \%$ \\
\hline KT03-MK & $\mathrm{MgO}$ & 0.0684 & 0.1300 & -0.0616 & $-47.4 \%$ \\
\hline KT03-MK & $\mathrm{MnO}$ & 1.0652 & 1.1400 & -0.0748 & $-6.6 \%$ \\
\hline KT03-MK & $\mathrm{Na}_{2} \mathrm{O}$ & 10.1572 & 9.9600 & 0.1972 & $2.0 \%$ \\
\hline KT03-MK & $\mathrm{Nb}_{2} \mathrm{O}_{5}$ & 2.5070 & 2.8500 & -0.3430 & $-12.0 \%$ \\
\hline KT03-MK & $\mathrm{NiO}$ & 0.2793 & 0.3200 & -0.0407 & $-12.7 \%$ \\
\hline KT03-MK & $\mathrm{PbO}$ & 0.1031 & 0.1000 & 0.0031 & $3.1 \%$ \\
\hline KT03-MK & $\mathrm{SiO}_{2}$ & 40.2723 & 41.7600 & -1.4877 & $-3.6 \%$ \\
\hline KT03-MK & $\mathrm{SO}_{4}$ & 0.1498 & 0.0700 & 0.0798 & $114.0 \%$ \\
\hline KT03-MK & $\mathrm{TiO}_{2}$ & 7.3184 & 7.6000 & -0.2817 & $-3.7 \%$ \\
\hline KT03-MK & $\mathrm{ZnO}$ & 0.0426 & 0.0500 & -0.0074 & $-14.7 \%$ \\
\hline KT03-MK & $\mathrm{ZrO}_{2}$ & 2.2356 & 2.3800 & -0.1444 & $-6.1 \%$ \\
\hline KT03-MK & Sum & 97.4130 & 100.0200 & -2.6070 & $-2.6 \%$ \\
\hline KT1-HA2 & $\mathrm{Al}_{2} \mathrm{O}_{3}$ & 8.5972 & 8.4200 & 0.1772 & $2.1 \%$ \\
\hline KT1-HA2 & $\mathrm{B}_{2} \mathrm{O}_{3}$ & 4.4596 & 4.5200 & -0.0604 & $-1.3 \%$ \\
\hline KT1-HA2 & $\mathrm{BaO}$ & 0.0790 & 0.0800 & -0.0010 & $-1.3 \%$ \\
\hline KT1-HA2 & $\mathrm{CaO}$ & 0.9574 & 0.9500 & 0.0074 & $0.8 \%$ \\
\hline
\end{tabular}


Table B-4. Comparison of Measured versus Targeted Composition for KT03 Glasses. (continued)

\begin{tabular}{|c|c|c|c|c|c|}
\hline Glass ID & Oxide & $\begin{array}{l}\text { Measured } \\
(w \mathbf{t} \%)\end{array}$ & $\begin{array}{l}\text { Targeted } \\
(\mathbf{w t} \%)\end{array}$ & $\begin{array}{c}\text { Difference of Measured } \\
\text { versus Targeted }\end{array}$ & $\begin{array}{c}\text { \% Difference of } \\
\text { Measured versus } \\
\text { Targeted }\end{array}$ \\
\hline KT1-HA2 & $\mathrm{Ce}_{2} \mathrm{O}_{3}$ & 0.1947 & 0.2000 & -0.0053 & $-2.6 \%$ \\
\hline KT1-HA2 & $\mathrm{Cr}_{2} \mathrm{O}_{3}$ & 0.0866 & 0.1000 & -0.0134 & $-13.4 \%$ \\
\hline KT1-HA2 & $\mathrm{CuO}$ & 0.0394 & 0.0300 & 0.0094 & $31.4 \%$ \\
\hline KT1-HA2 & $\mathrm{Fe}_{2} \mathrm{O}_{3}$ & 10.4940 & 11.3400 & -0.8460 & $-7.5 \%$ \\
\hline KT1-HA2 & $\mathrm{K}_{2} \mathrm{O}$ & 0.0678 & 0.0700 & -0.0022 & $-3.2 \%$ \\
\hline KT1-HA2 & $\mathrm{La}_{2} \mathrm{O}_{3}$ & 0.0625 & 0.0700 & -0.0075 & $-10.8 \%$ \\
\hline KT1-HA2 & $\mathrm{Li}_{2} \mathrm{O}$ & 4.5426 & 4.5200 & 0.0226 & $0.5 \%$ \\
\hline KT1-HA2 & $\mathrm{MgO}$ & 0.1447 & 0.1400 & 0.0047 & $3.3 \%$ \\
\hline KT1-HA2 & $\mathrm{MnO}$ & 1.1660 & 1.2200 & -0.0540 & $-4.4 \%$ \\
\hline KT1-HA2 & $\mathrm{Na}_{2} \mathrm{O}$ & 14.2888 & 14.1400 & 0.1488 & $1.1 \%$ \\
\hline KT1-HA2 & $\mathrm{Nb}_{2} \mathrm{O}_{5}$ & 0.3945 & 0.4100 & -0.0155 & $-3.8 \%$ \\
\hline KT1-HA2 & $\mathrm{NiO}$ & 0.3057 & 0.3400 & -0.0343 & $-10.1 \%$ \\
\hline KT1-HA2 & $\mathrm{PbO}$ & 0.0859 & 0.1100 & -0.0241 & $-21.9 \%$ \\
\hline KT1-HA2 & $\mathrm{SiO}_{2}$ & 43.1604 & 44.7200 & -1.5596 & $-3.5 \%$ \\
\hline KT1-HA2 & $\mathrm{SO}_{4}$ & 0.1498 & 0.0700 & 0.0798 & $114.0 \%$ \\
\hline KT1-HA2 & $\mathrm{TiO}_{2}$ & 7.6061 & 8.0000 & -0.3939 & $-4.9 \%$ \\
\hline KT1-HA2 & $\mathrm{ZnO}$ & 0.0476 & 0.0500 & -0.0024 & $-4.8 \%$ \\
\hline KT1-HA2 & $\mathrm{ZrO}_{2}$ & 0.4768 & 0.4900 & -0.0132 & $-2.7 \%$ \\
\hline KT1-HA2 & Sum & 97.4070 & 99.9900 & -2.5830 & $-2.6 \%$ \\
\hline
\end{tabular}


Exhibit B-1. Measurements in Analytical Sequence for KT03-Series by Preparation Method by Oxide.

Series=KT3, Prep Method=LM, Oxide=BaO (wt $\%)$

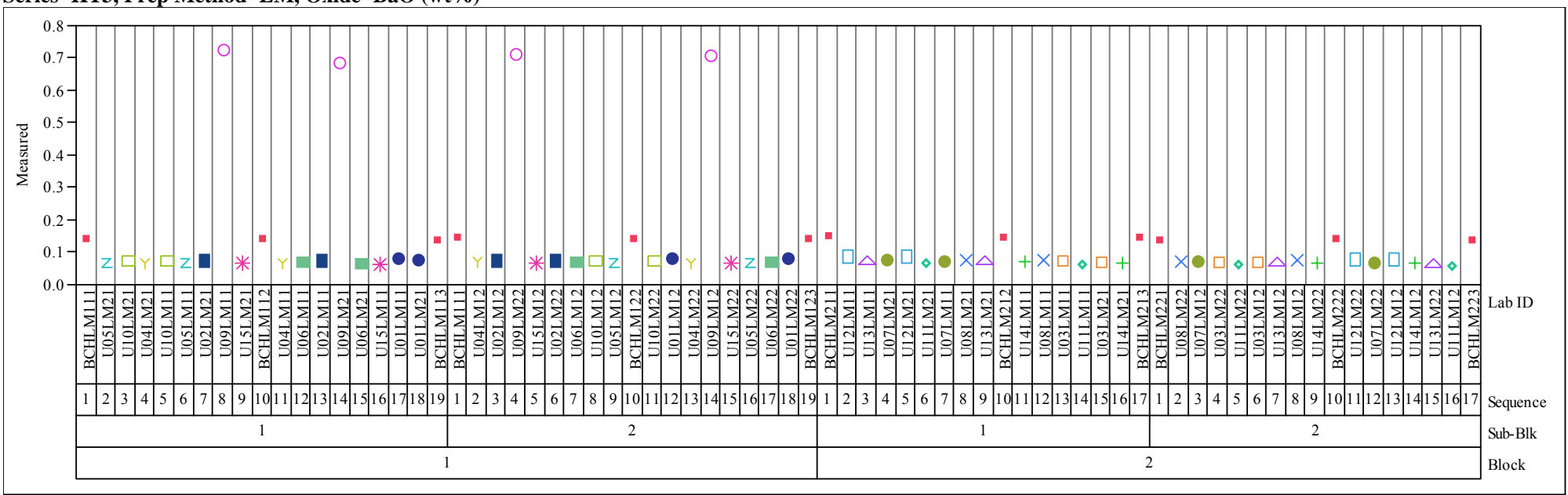

Series $=$ KT3, Prep Method $=$ LM, Oxide $=\mathrm{CaO}(\mathrm{wt} \%)$

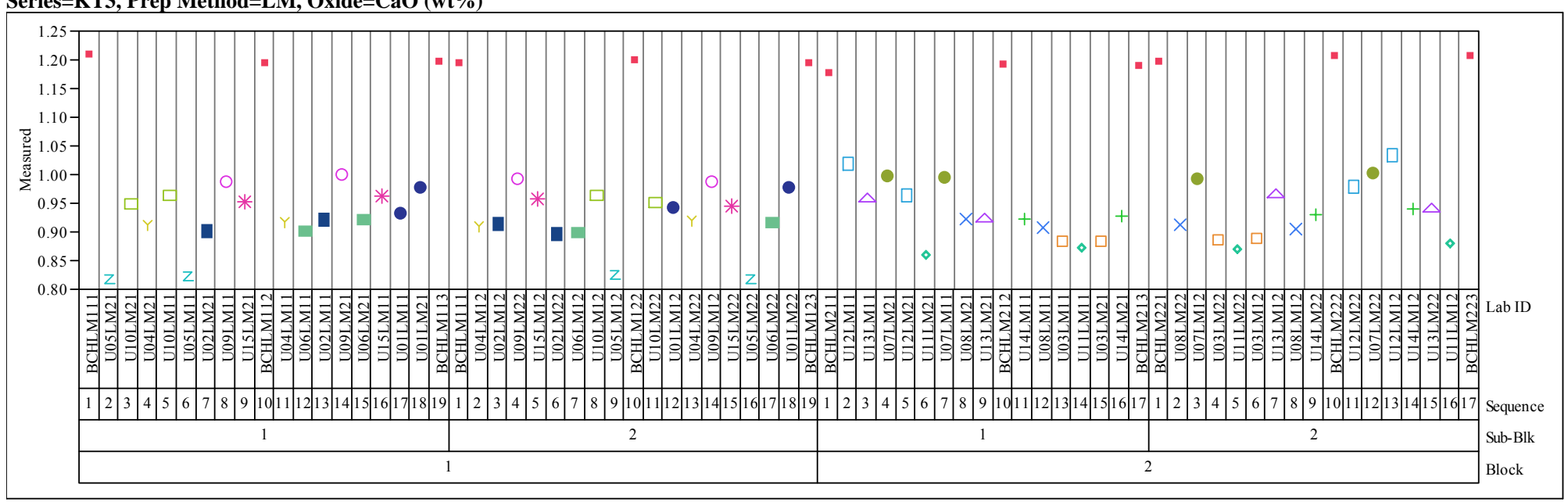


Exhibit B-1. Measurements in Analytical Sequence for KT03-Series by Preparation Method by Oxide. (continued) Series $=$ KT3, Prep Method $=L M$, Oxide $=\mathrm{Ce} 2 \mathrm{O} 3(w \mathrm{t} \%)$
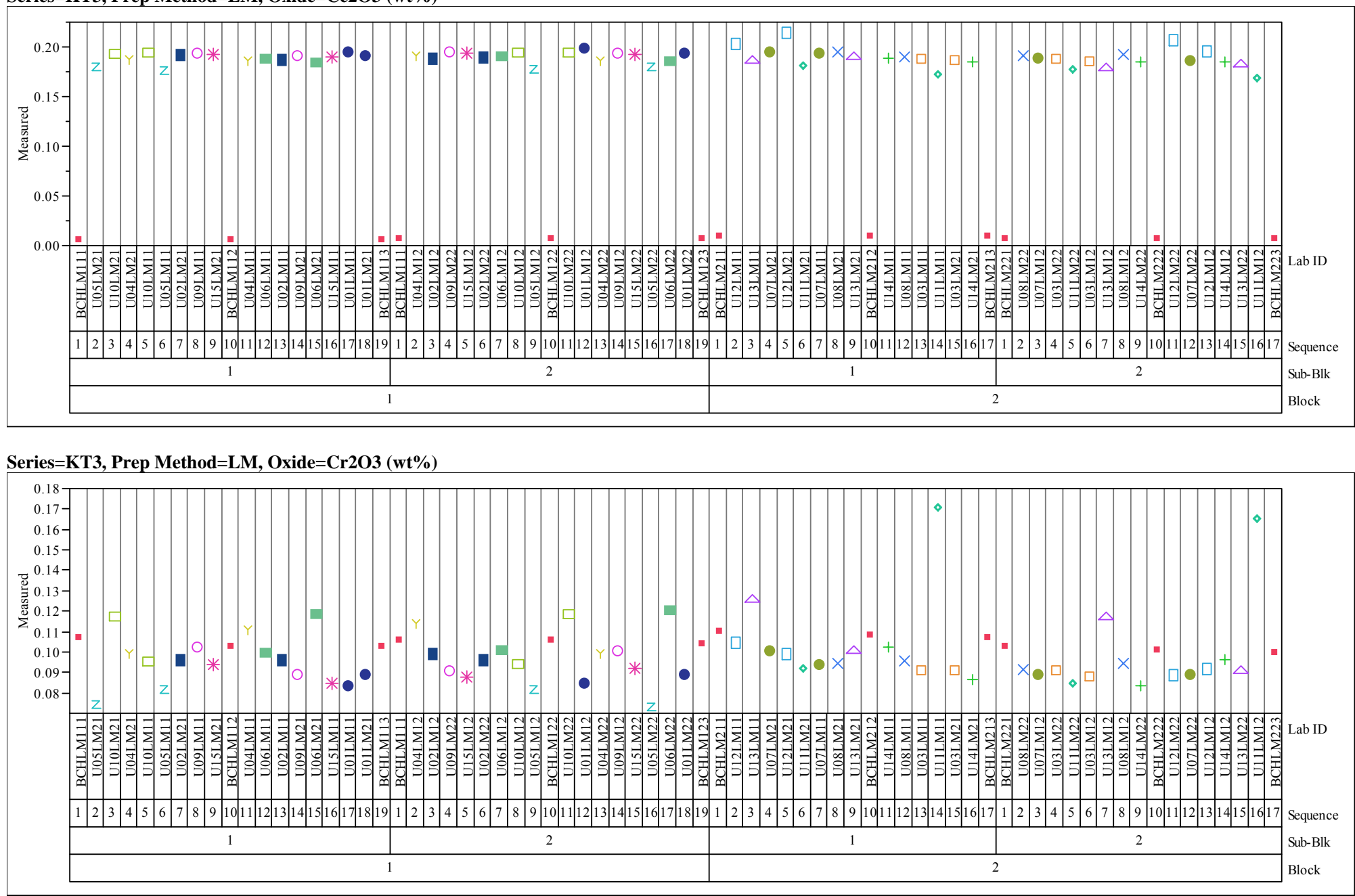
Exhibit B-1. Measurements in Analytical Sequence for KT03-Series by Preparation Method by Oxide. (continued) Series=KT3, Prep Method=LM, Oxide=CuO (wt\%)

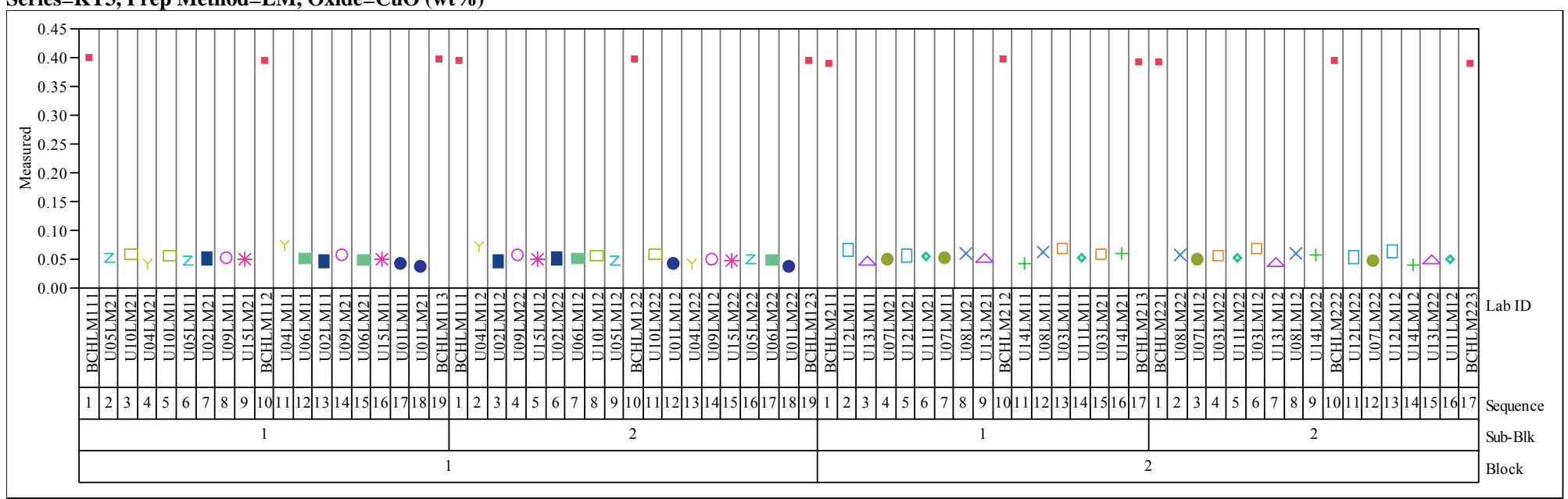

Series=KT3, Prep Method=LM, Oxide=K2O (wt\%)

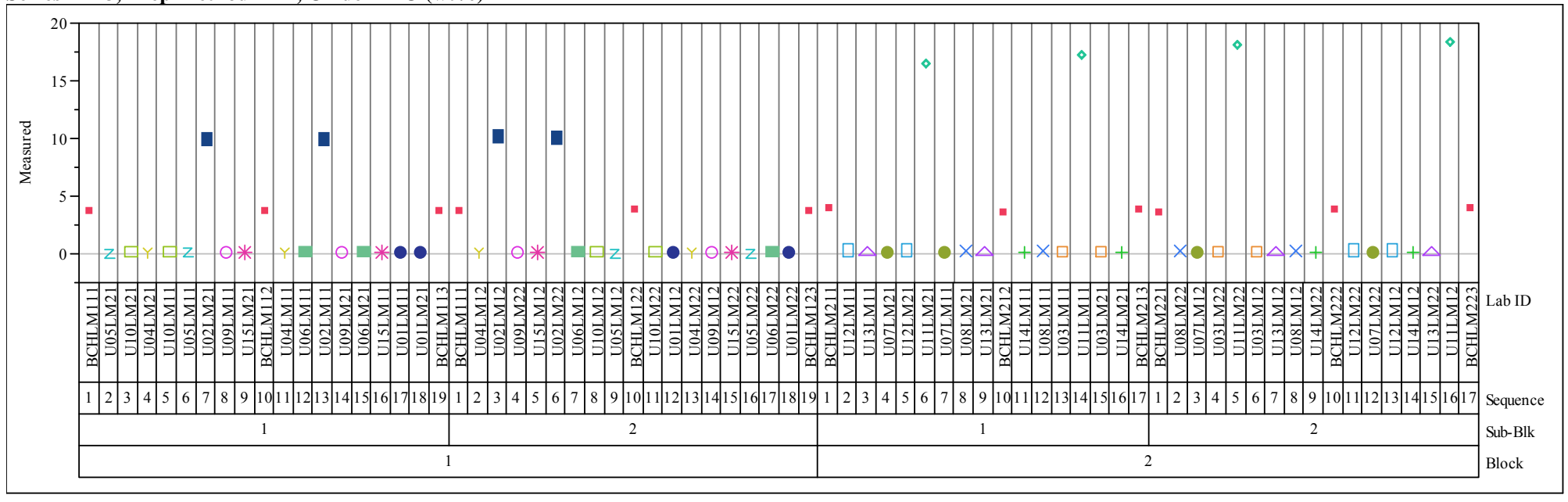


Exhibit B-1. Measurements in Analytical Sequence for KT03-Series by Preparation Method by Oxide. (continued) Series=KT3, Prep Method=LM, Oxide=La2O3 (wt $\%)$

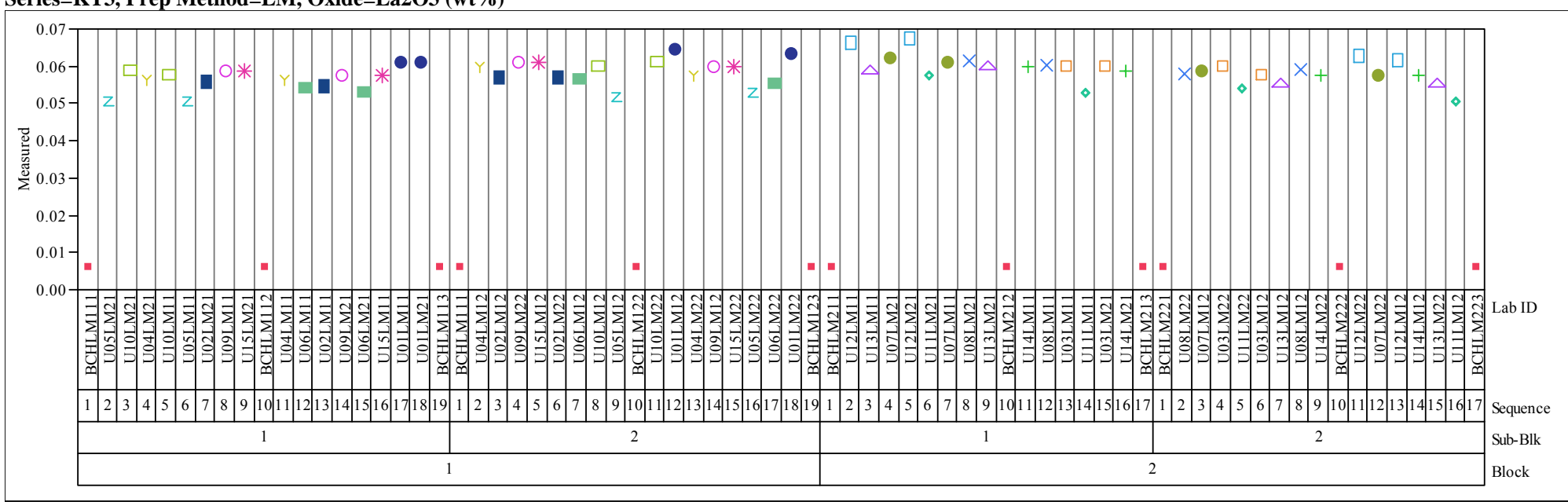

Series=KT3, Prep Method=LM, Oxide=MgO (wt\%)

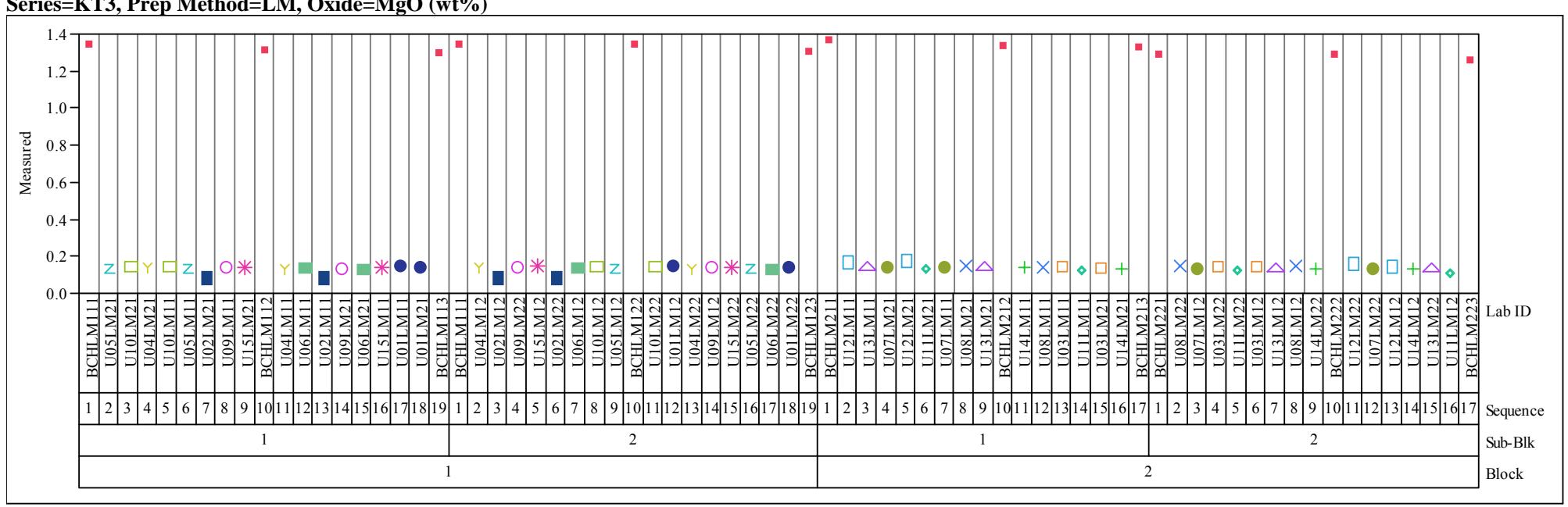


Exhibit B-1. Measurements in Analytical Sequence for KT03-Series by Preparation Method by Oxide. (continued) Series=KT3, Prep Method=LM, Oxide=MnO (wt\%)

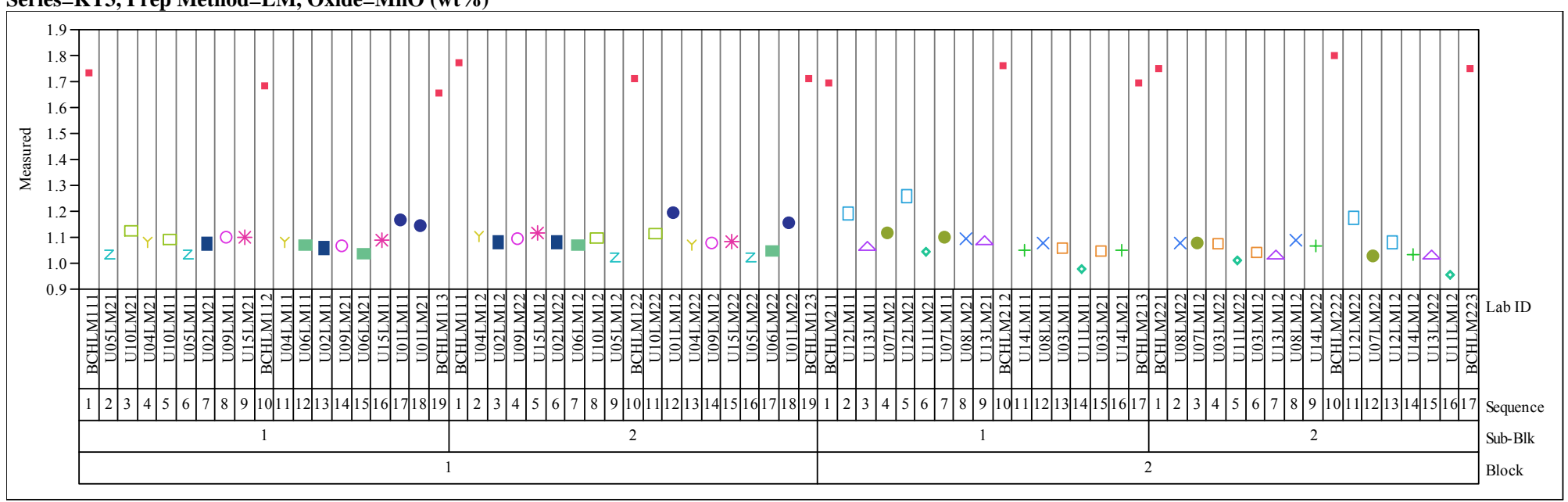

Series=KT3, Prep Method=LM, Oxide=Na2O (wt\%)

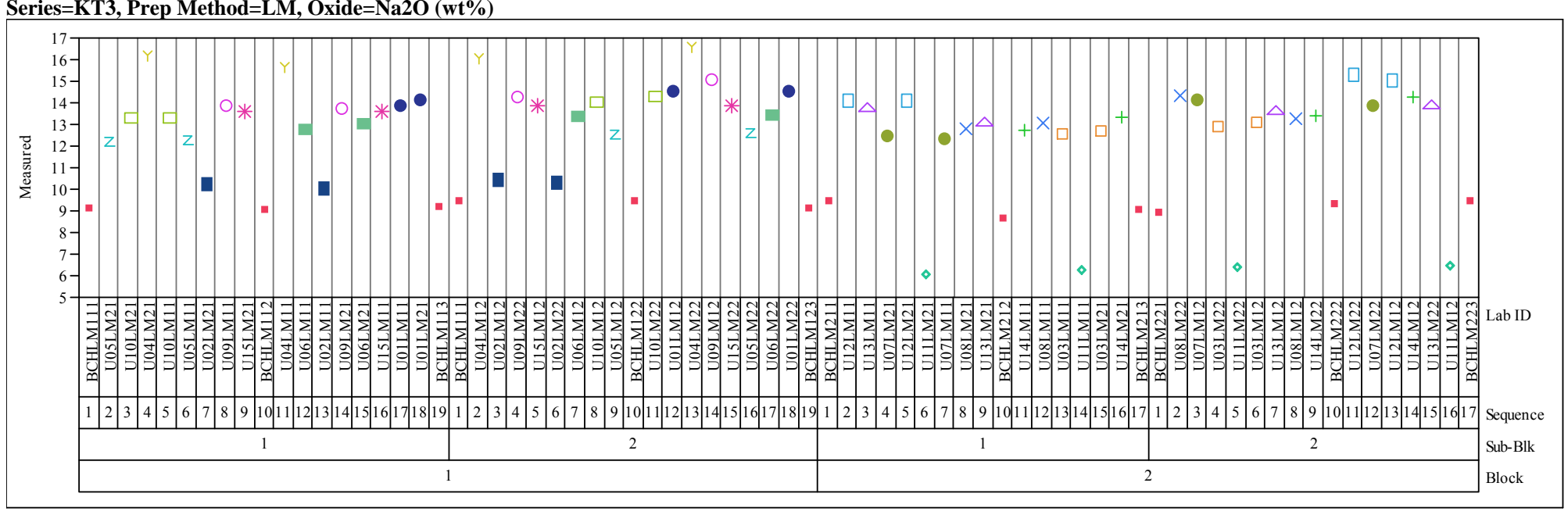


Exhibit B-1. Measurements in Analytical Sequence for KT03-Series by Preparation Method by Oxide. (continued) Series=KT3, Prep Method=LM, Oxide=Nb2O5 (wt\%)

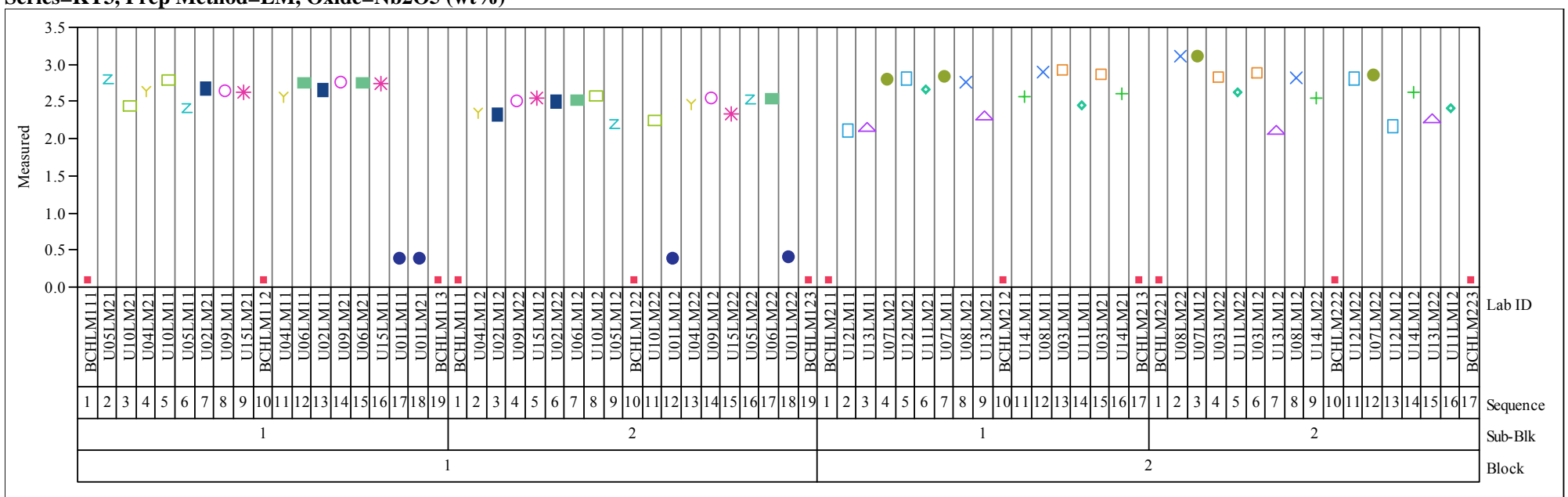

Series=KT3, Prep Method=LM, Oxide=NiO (wt $\%)$

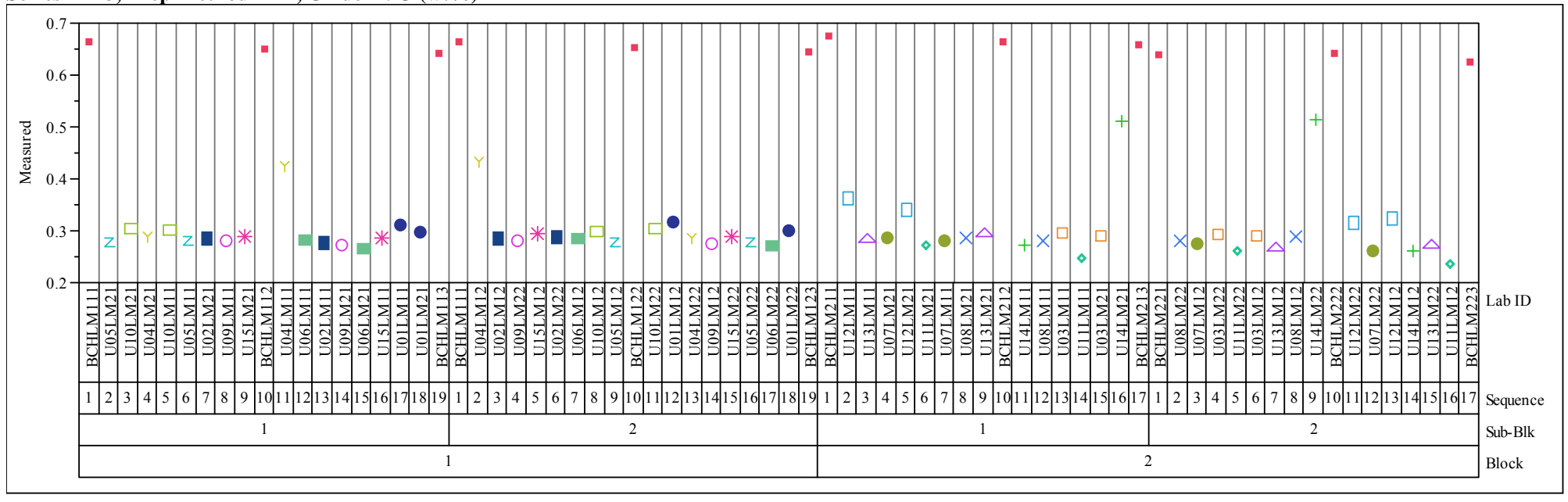


Exhibit B-1. Measurements in Analytical Sequence for KT03-Series by Preparation Method by Oxide. (continued) Series=KT3, Prep Method=LM, Oxide=PbO (wt $\%)$

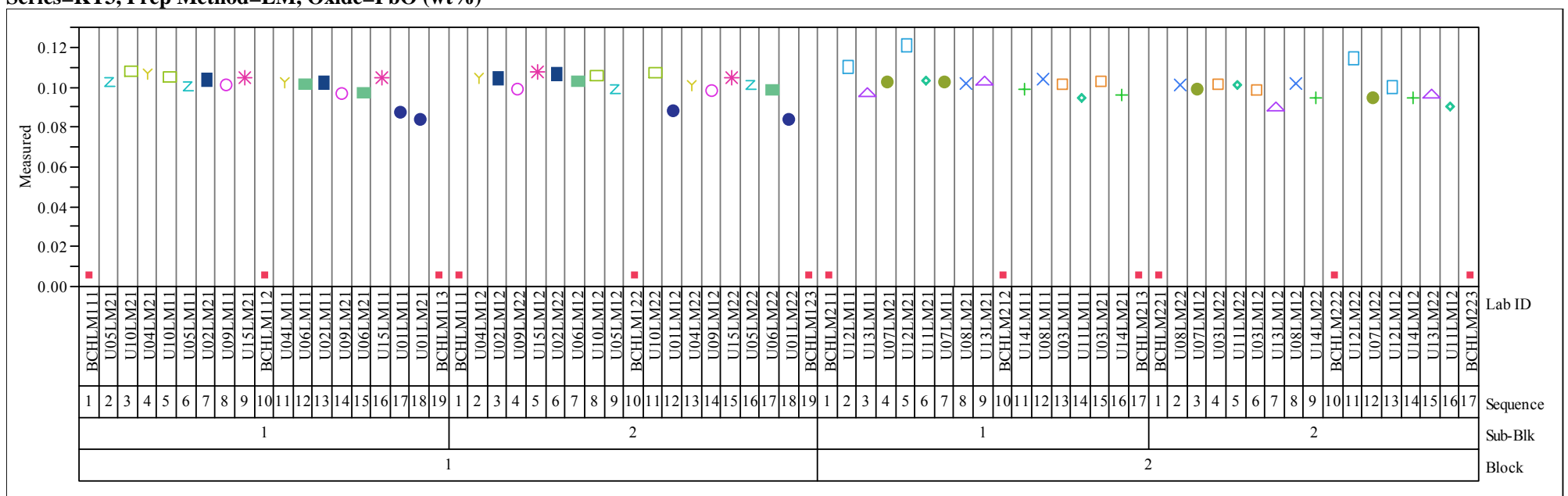

Series=KT3, Prep Method=LM, Oxide=SO4 (wt\%)

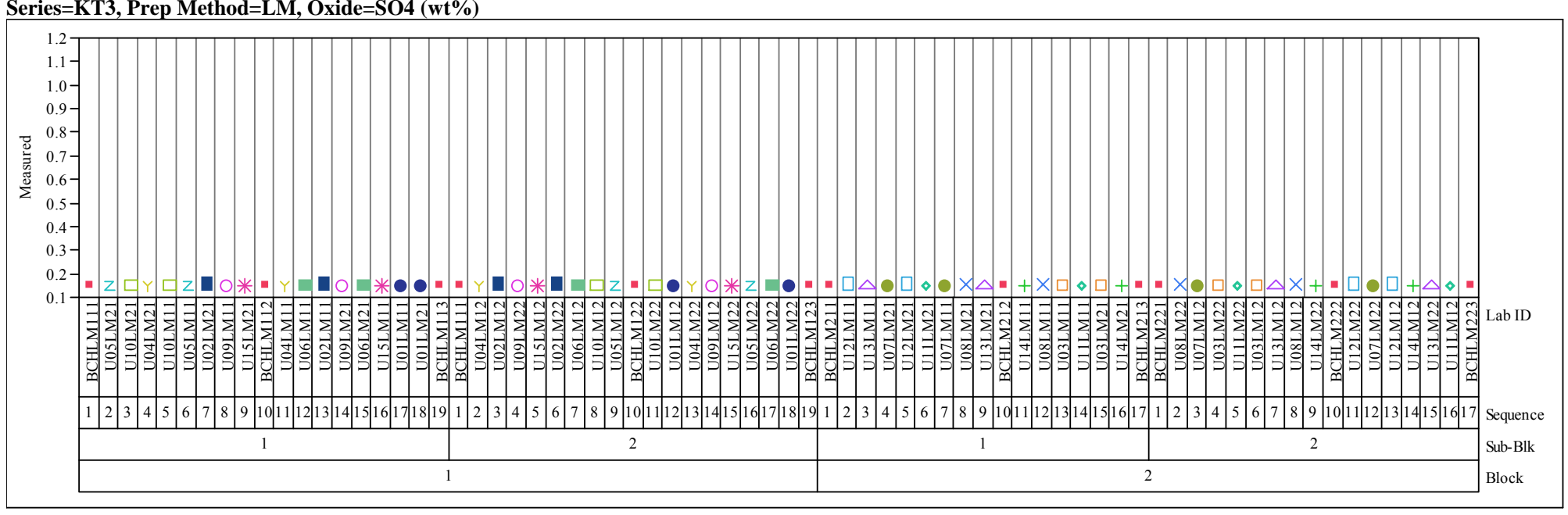


Exhibit B-1. Measurements in Analytical Sequence for KT03-Series by Preparation Method by Oxide. (continued) Series=KT3, Prep Method=LM, Oxide=TiO2 (wt $\%)$

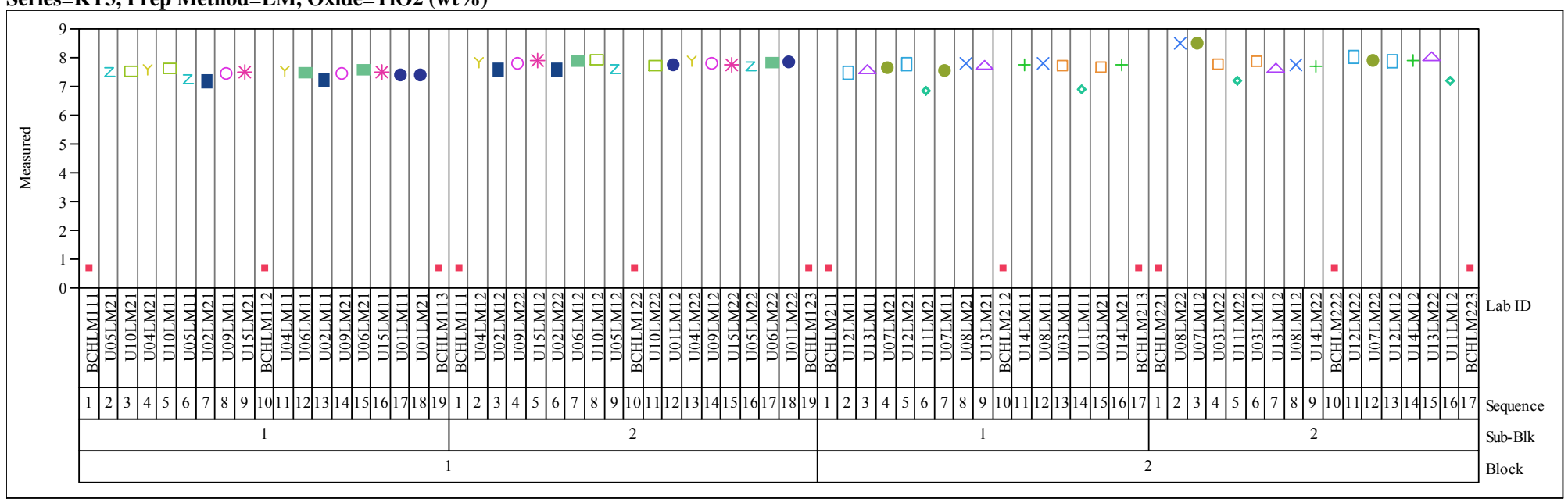

Series=KT3, Prep Method=LM, Oxide=ZnO (wt\%)

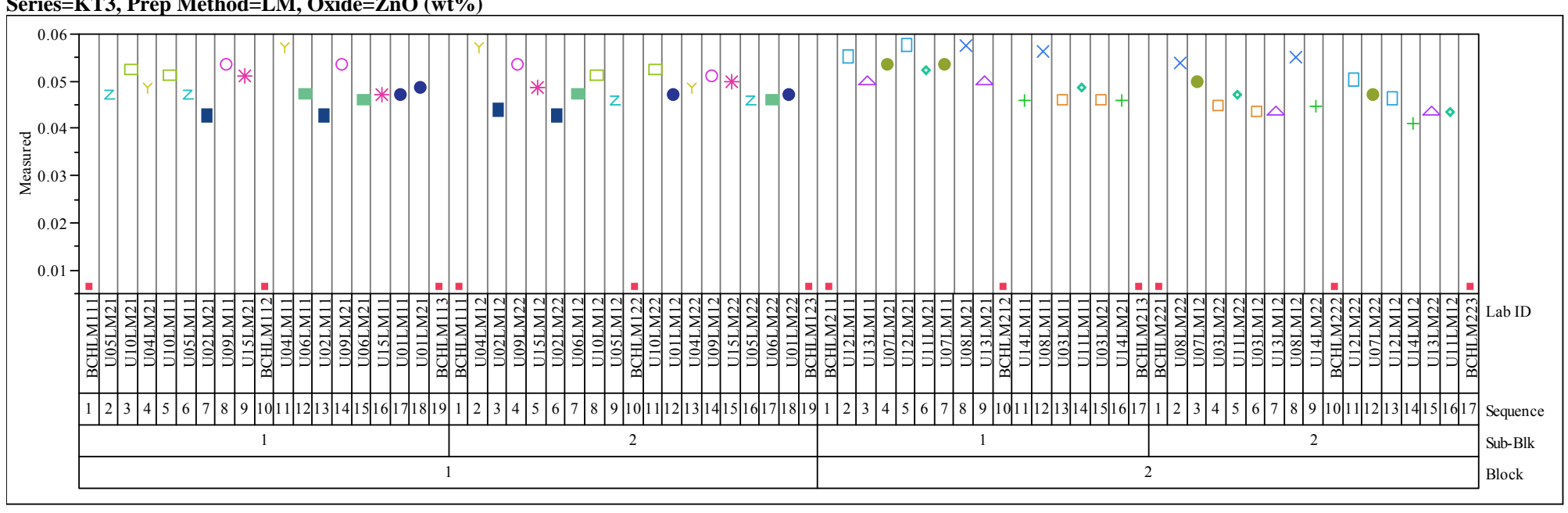


Exhibit B-1. Measurements in Analytical Sequence for KT03-Series by Preparation Method by Oxide. (continued) Series=KT3, Prep Method=LM, Oxide=ZrO2 (wt\%)

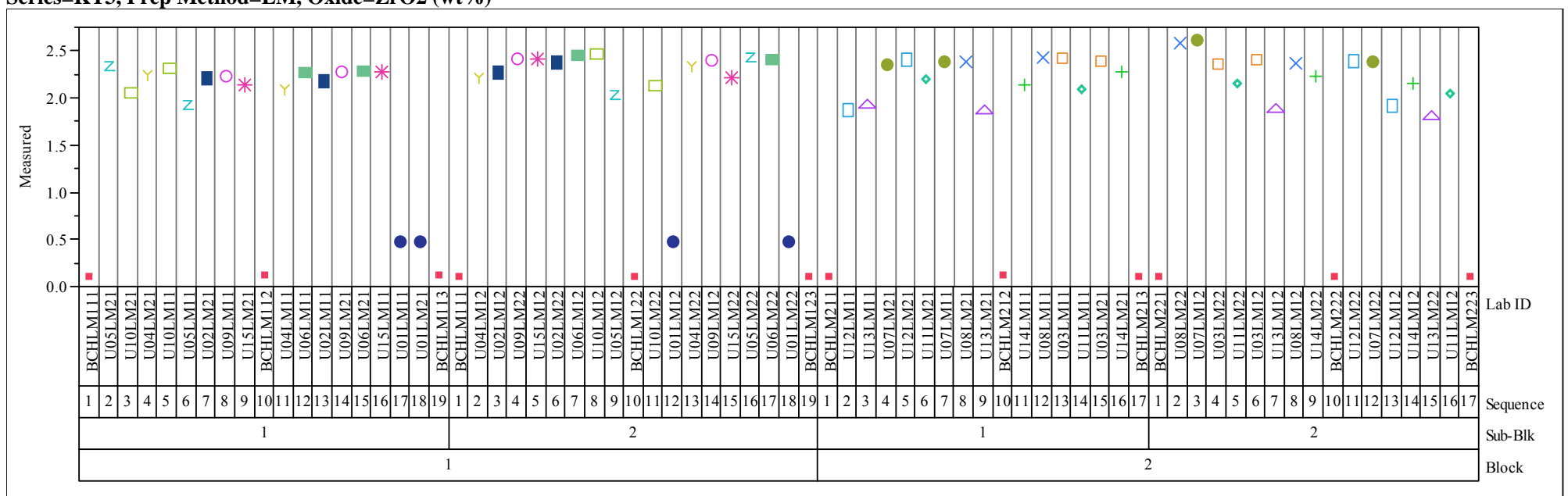

Series=KT3, Prep Method=PF, Oxide=Al2O3 (wt\%)

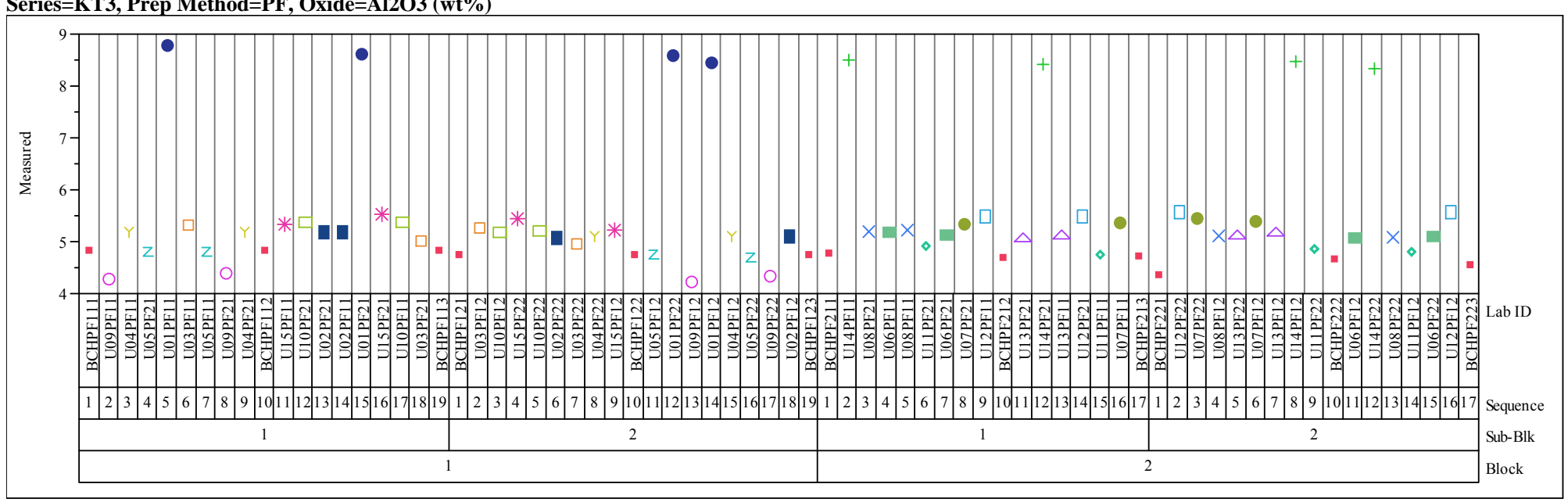


Exhibit B-1. Measurements in Analytical Sequence for KT03-Series by Preparation Method by Oxide. (continued) Series=KT3, Prep Method=PF, Oxide=B2O3 (wt\%)

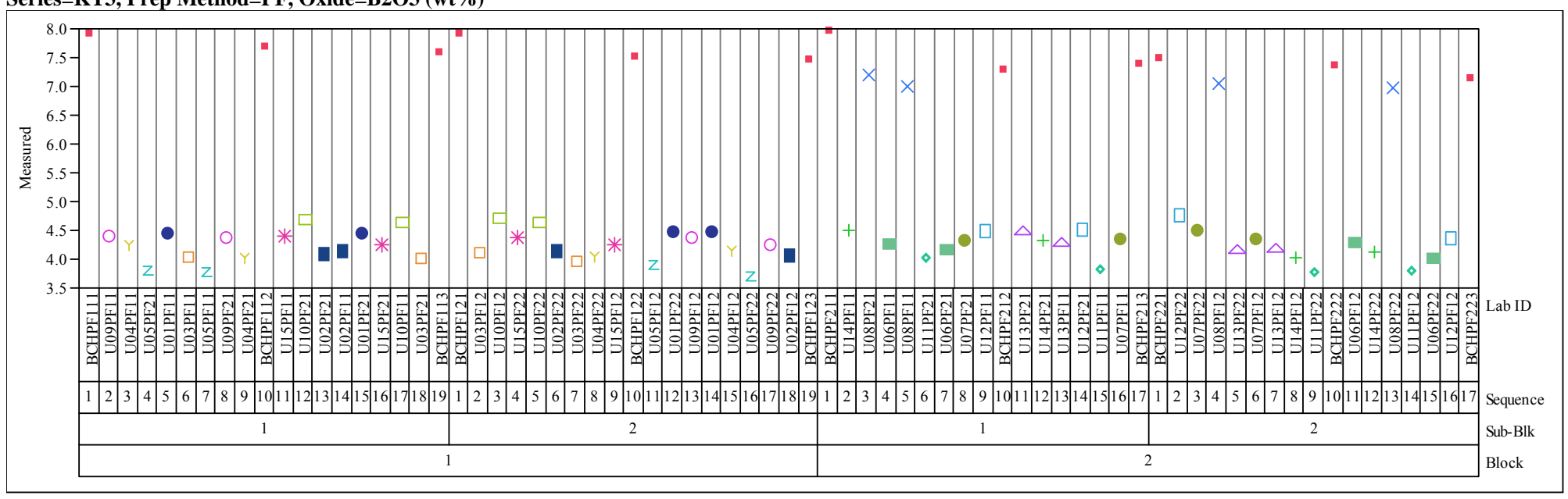

Series=KT3, Prep Method=PF, Oxide=Fe2O3 (wt \%)

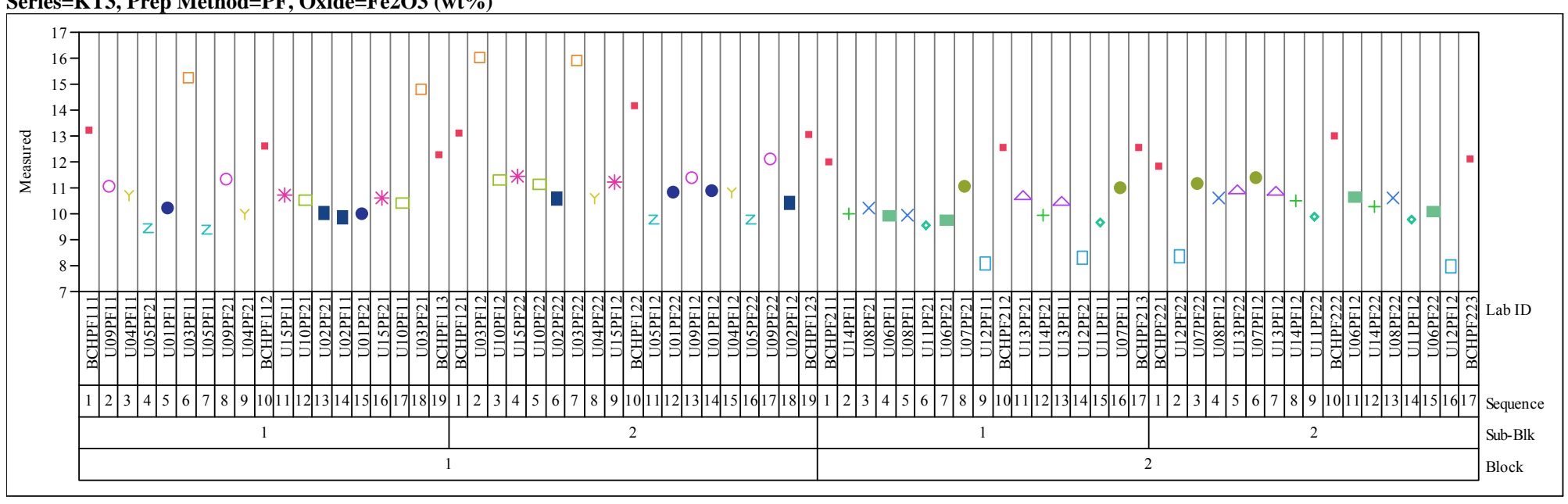


Exhibit B-1. Measurements in Analytical Sequence for KT03-Series by Preparation Method by Oxide. (continued) Series=KT3, Prep Method=PF, Oxide=Li2O (wt\%)

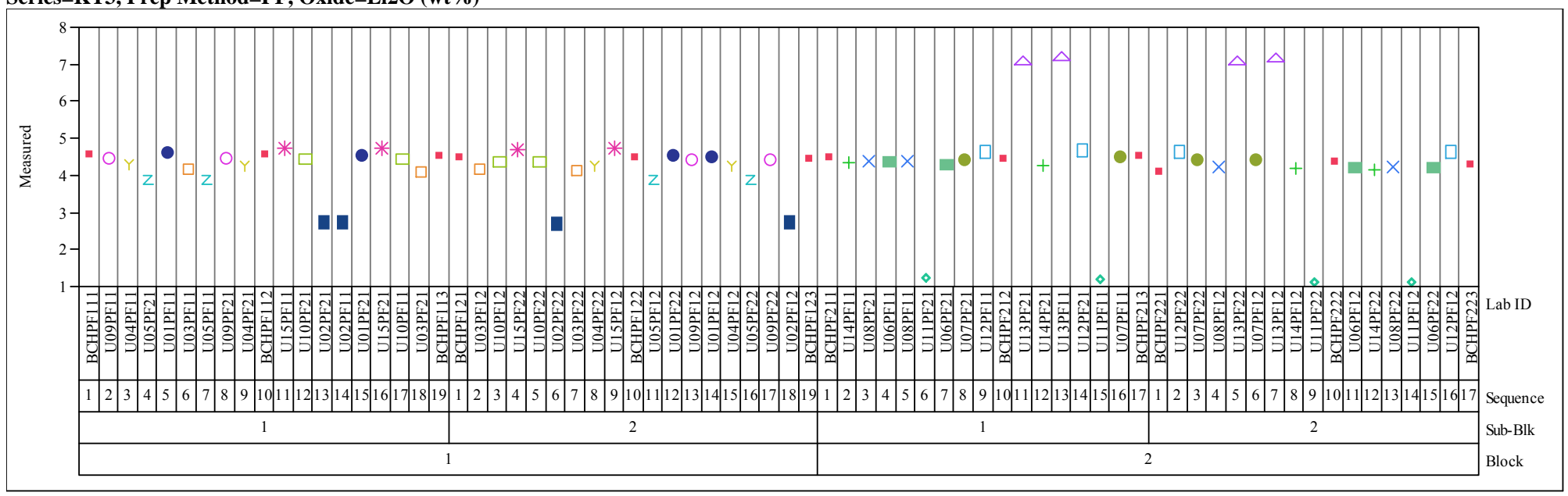

Series=KT3, Prep Method=PF, Oxide=SiO2 (wt \%)

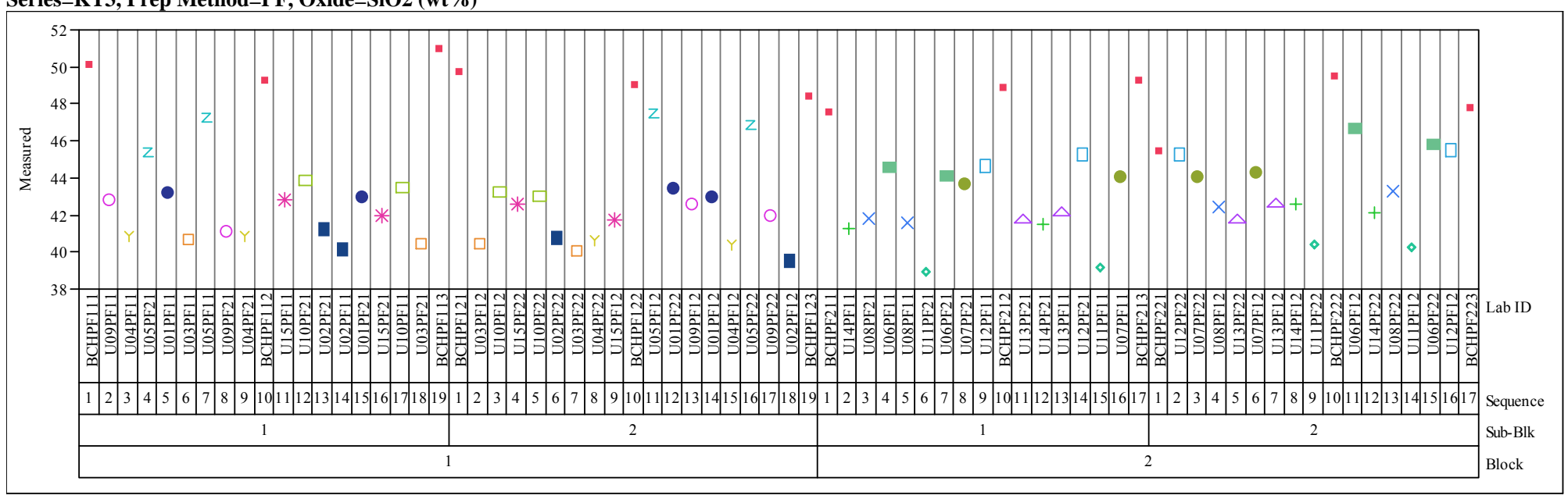


Exhibit B-2 Measurements for Each KT03-Series Glass ID by Preparation Method by Oxide.

Series=KT3, Oxide=Al2O3 (wt\%), Prep Method=PF

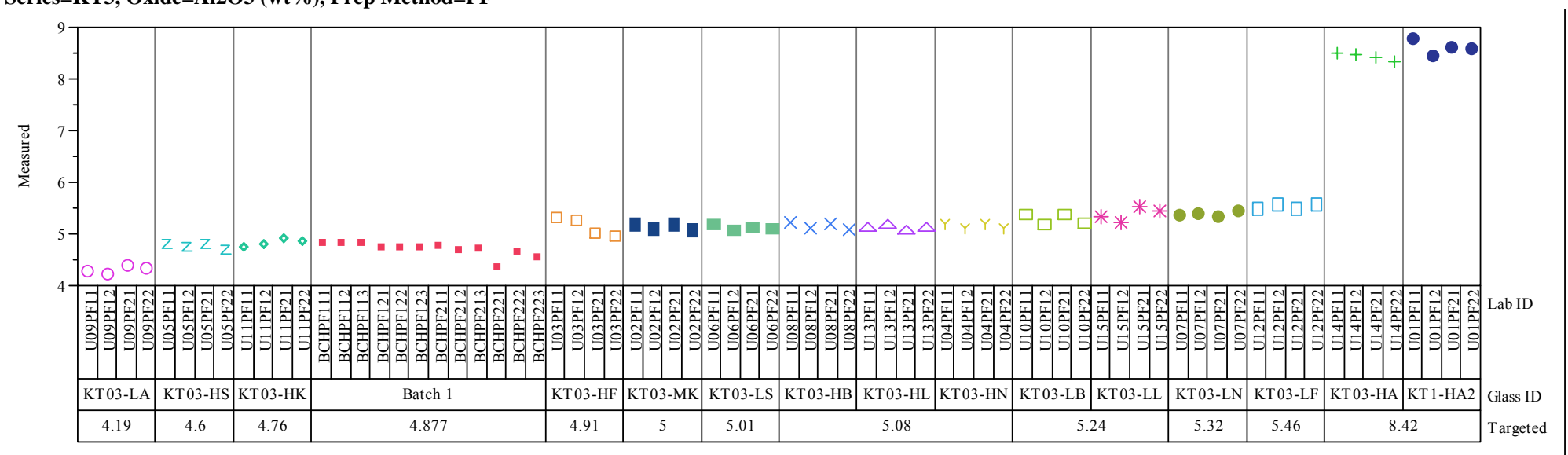

Series=KT3, Oxide=B2O3 (wt\%), Prep Method=PF

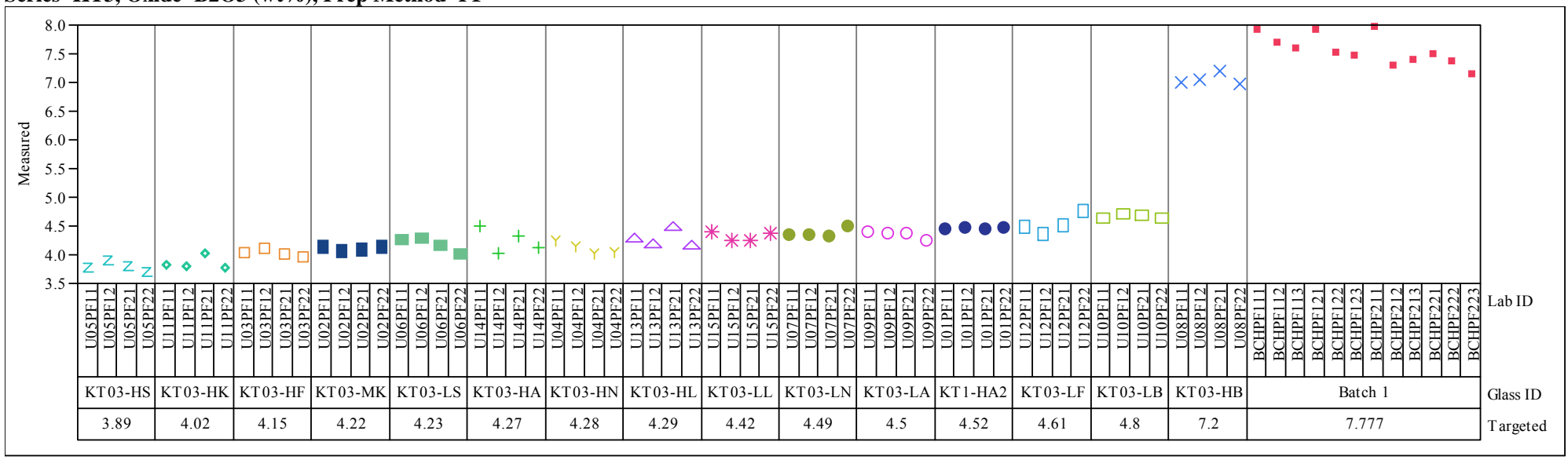


Exhibit B-2 Measurements for Each KT03-Series Glass ID by Preparation Method by Oxide. (continued) Series $=$ KT3, Oxide $=$ BaO (wt\%), Prep Method $=$ LM

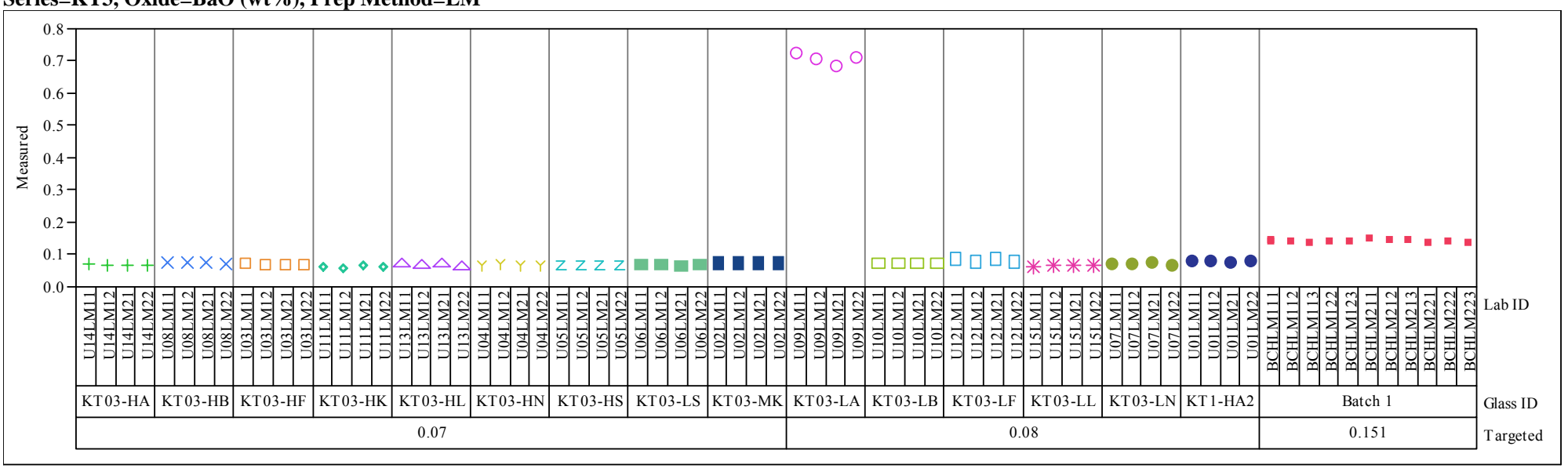

Series=KT3, Oxide=CaO (wt \%), Prep Method=LM

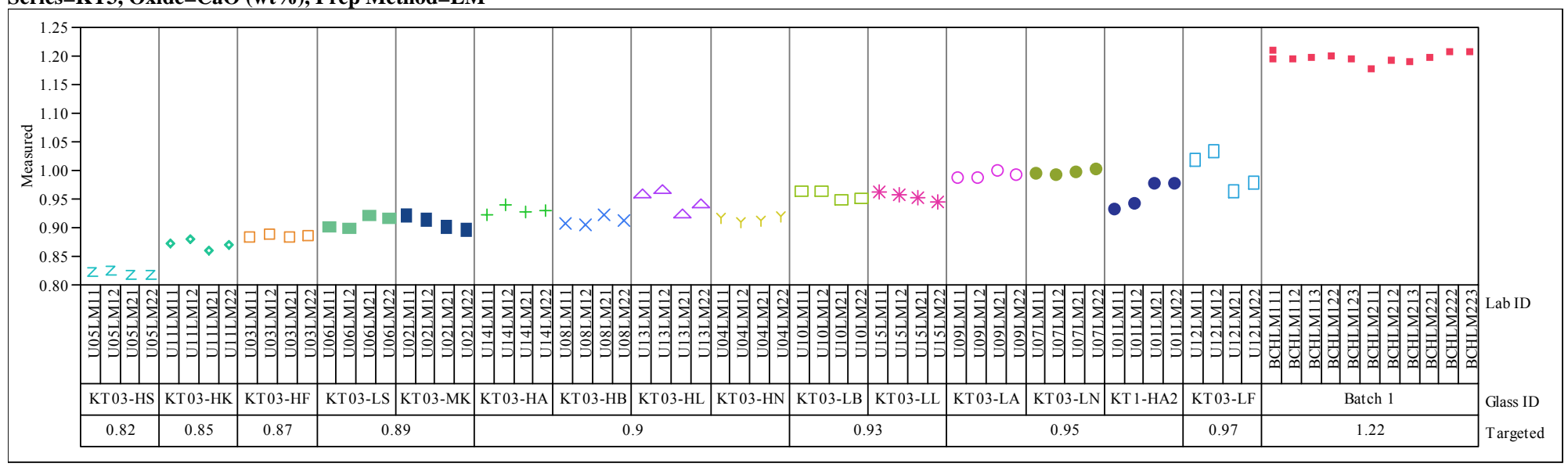


Exhibit B-2 Measurements for Each KT03-Series Glass ID by Preparation Method by Oxide. (continued) Series $=\mathrm{KT} 3$, Oxide=Ce2O3 (wt\%), Prep Method $=$ LM

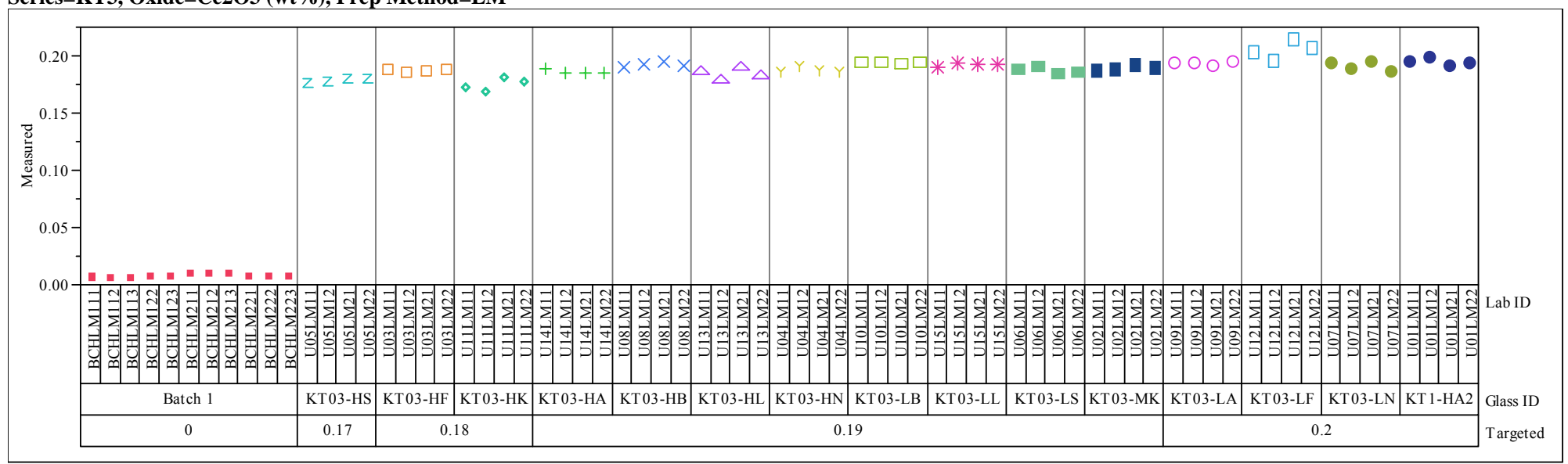

Series $=$ KT3, Oxide $=$ Cr2O3 (wt \%), Prep Method $=$ LM

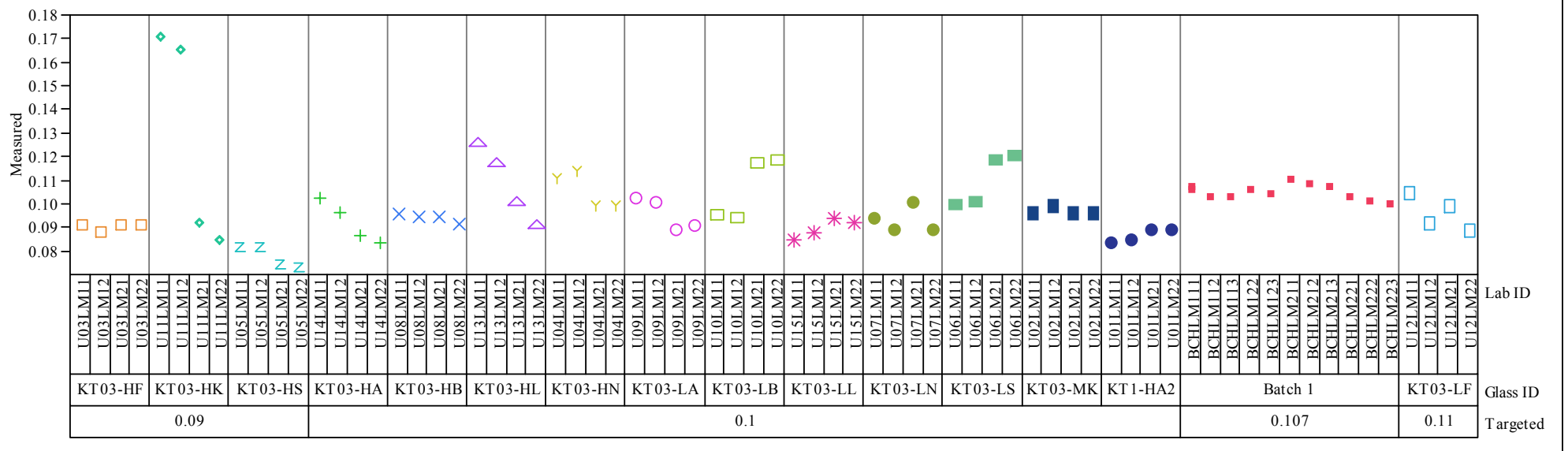


Exhibit B-2 Measurements for Each KT03-Series Glass ID by Preparation Method by Oxide. (continued) Series $=$ KT3, Oxide $=\mathbf{C u O}$ ( $w t \%$ ), Prep Method $=$ LM

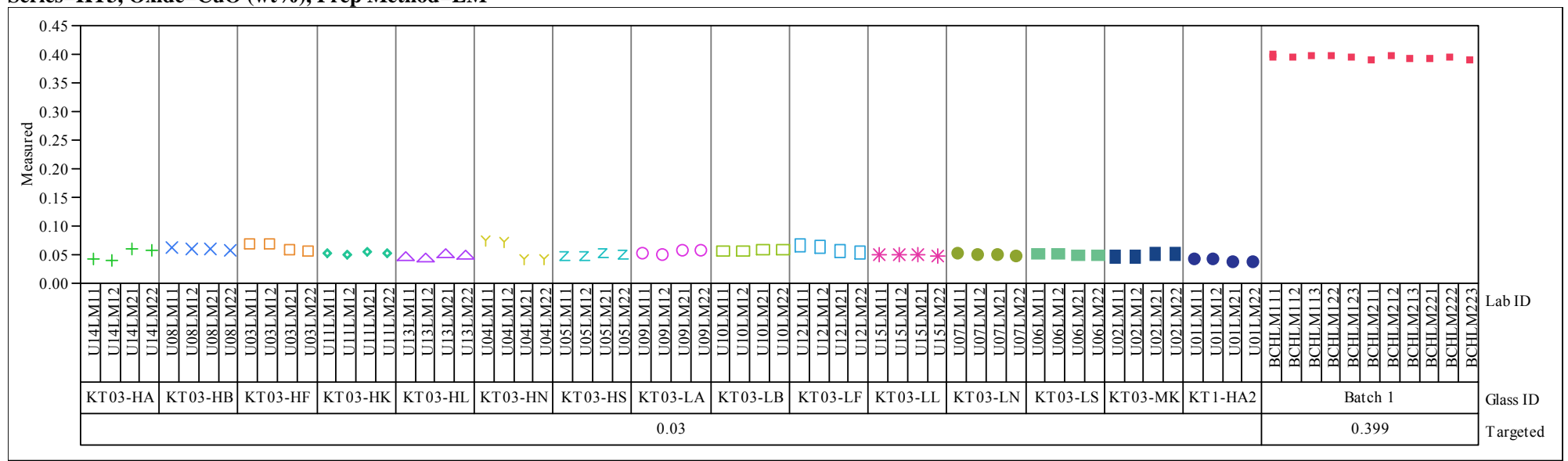

Series=KT3, Oxide=Fe2O3 (wt \%), Prep Method=PF

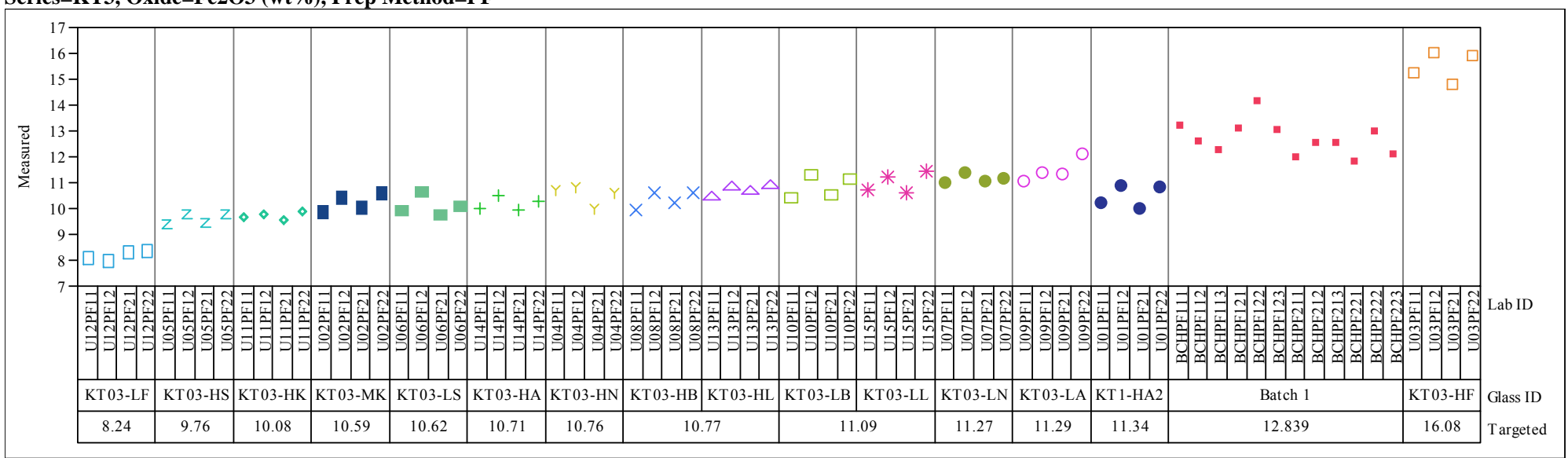


Exhibit B-2 Measurements for Each KT03-Series Glass ID by Preparation Method by Oxide. (continued) Series=KT3, Oxide=K2O ( $w$ t\%), Prep Method=LM

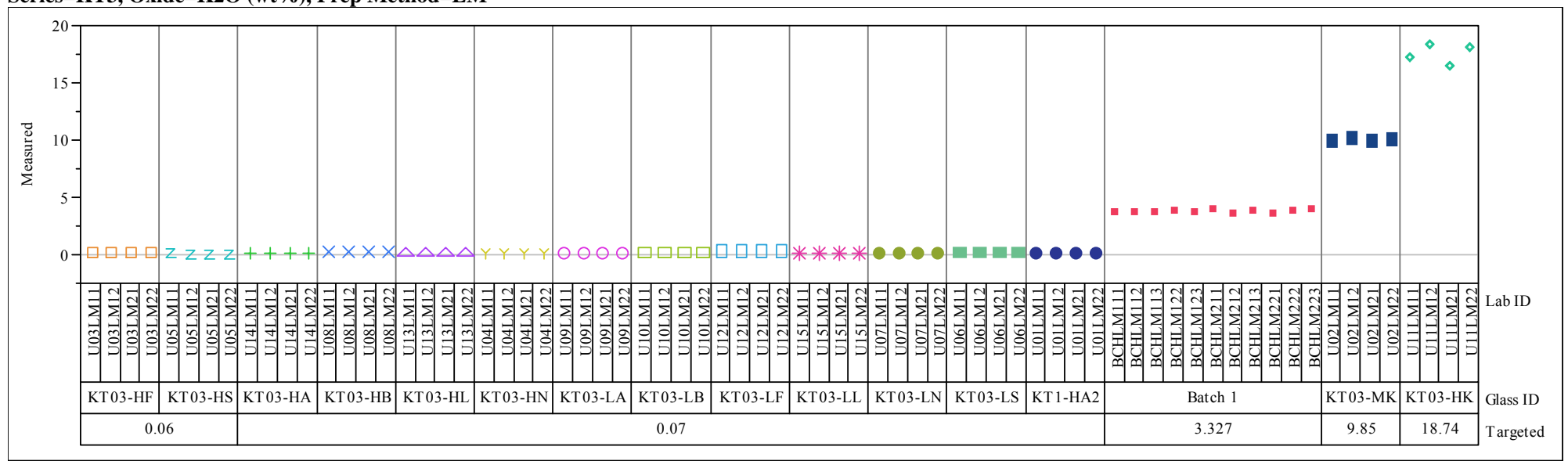

Series=KT3, Oxide=La2O3 (wt\%), Prep Method $=$ LM

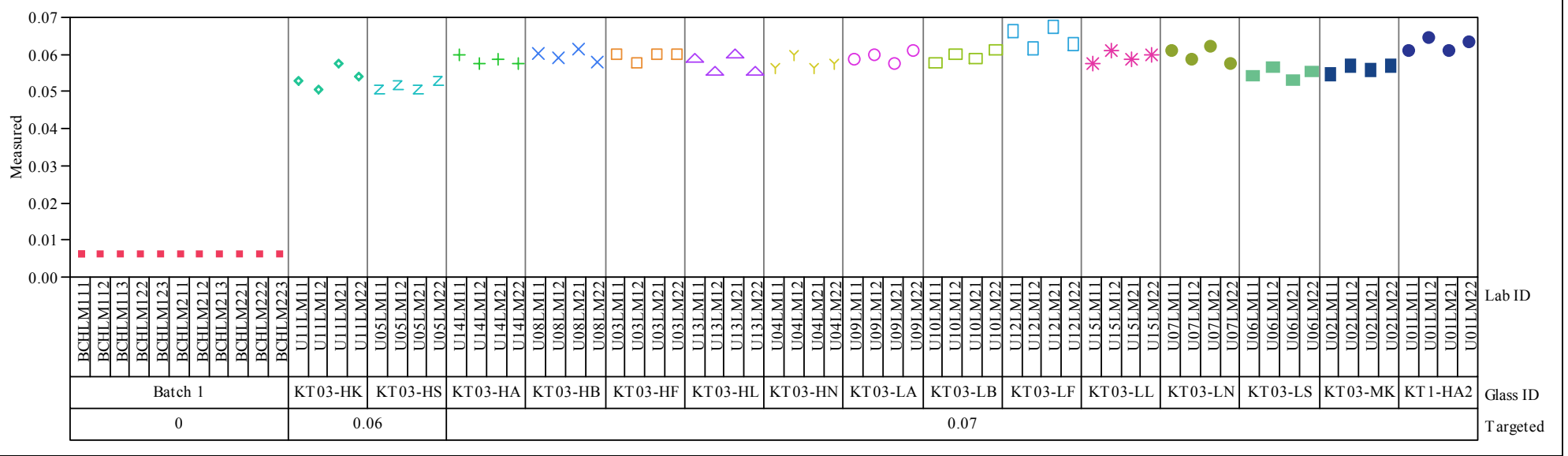


Exhibit B-2 Measurements for Each KT03-Series Glass ID by Preparation Method by Oxide. (continued) Series $=K T 3$, Oxide $=$ Li2O ( $w$ t \%), Prep Method $=$ PF

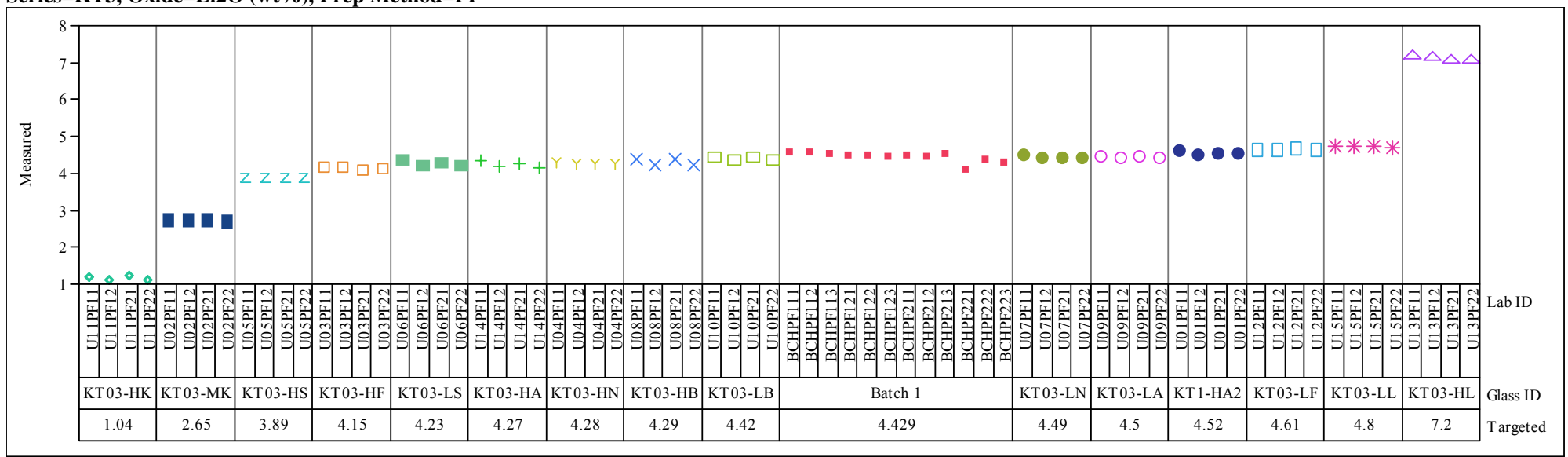

Series=KT3, Oxide=MgO (wt\%), Prep Method=LM

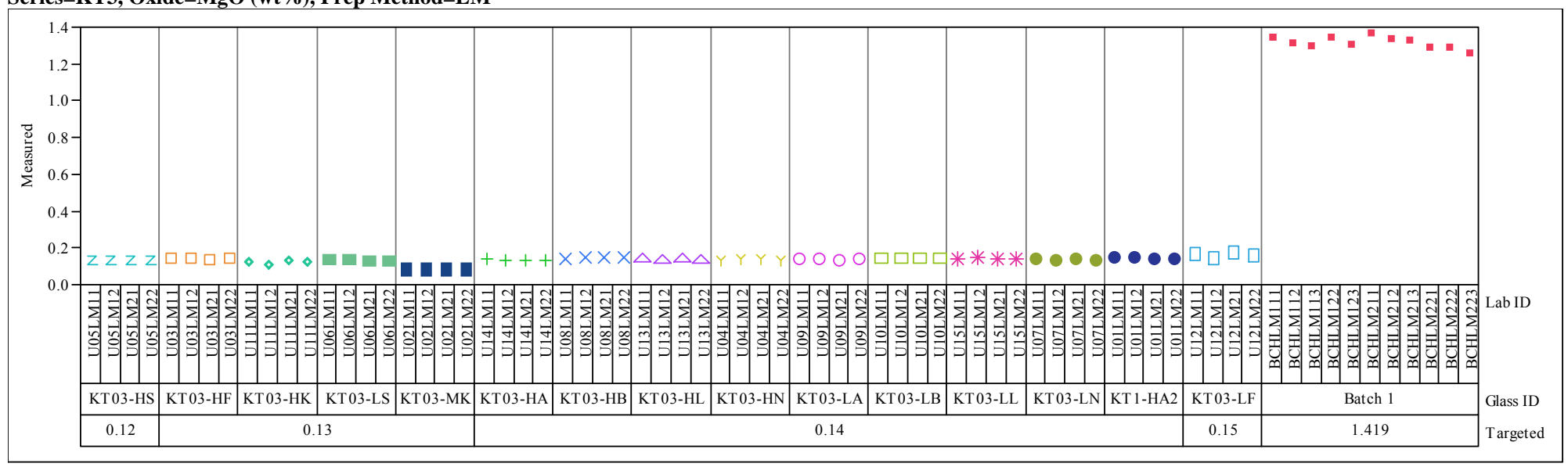


Exhibit B-2 Measurements for Each KT03-Series Glass ID by Preparation Method by Oxide. (continued) Series=KT3, Oxide=MnO (wt\%), Prep Method=LM

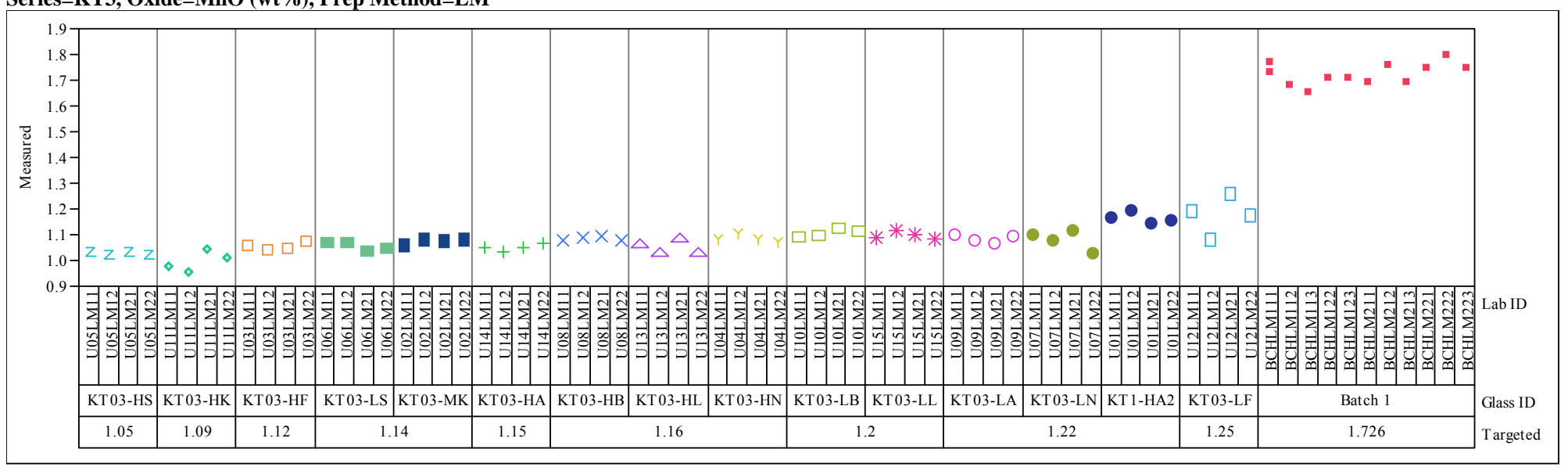

Series=KT3, Oxide=Na2O (wt \%), Prep Method=LM

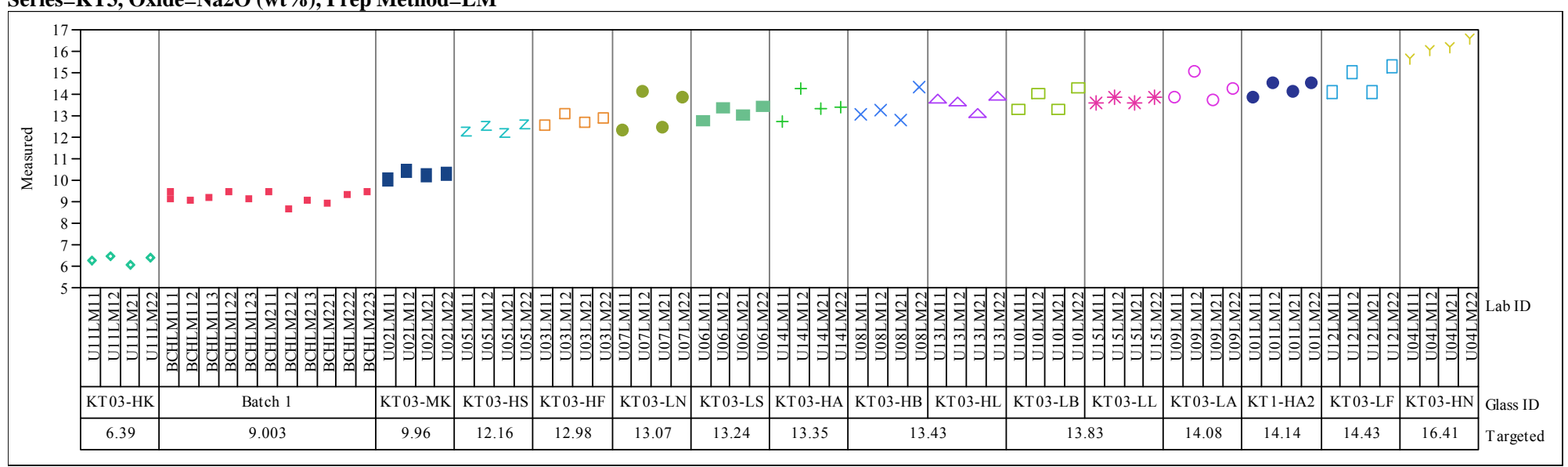


Exhibit B-2 Measurements for Each KT03-Series Glass ID by Preparation Method by Oxide. (continued) Series $=$ KT3, Oxide $=\mathrm{Nb} 2 \mathrm{O} 5$ (wt\%), Prep Method $=$ LM

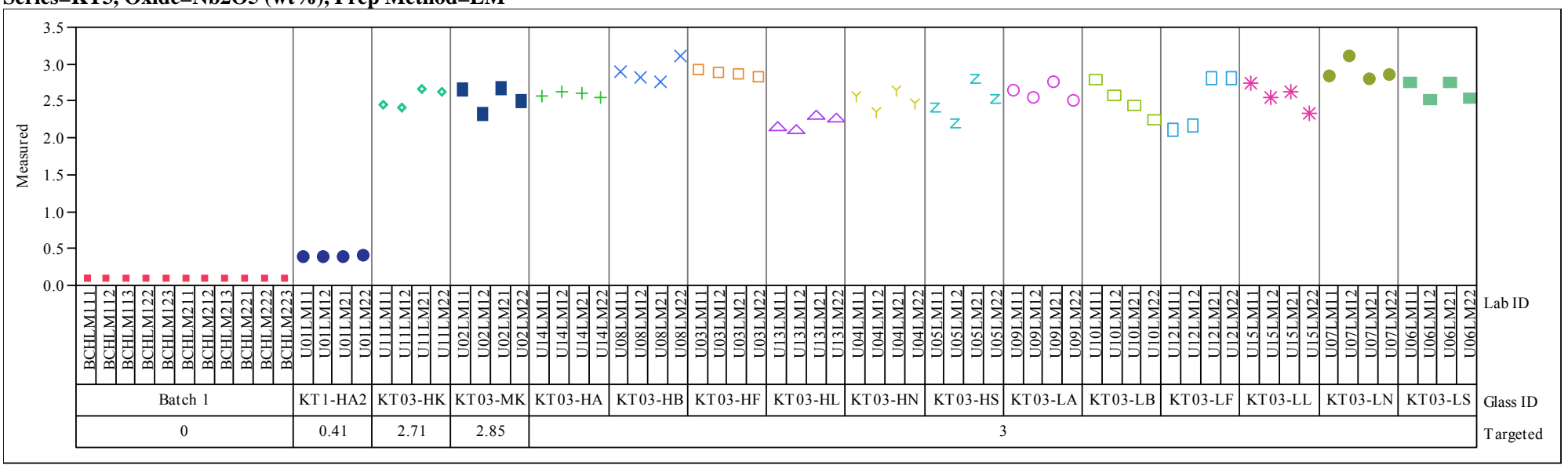

Series=KT3, Oxide=NiO (wt\%), Prep Method=LM

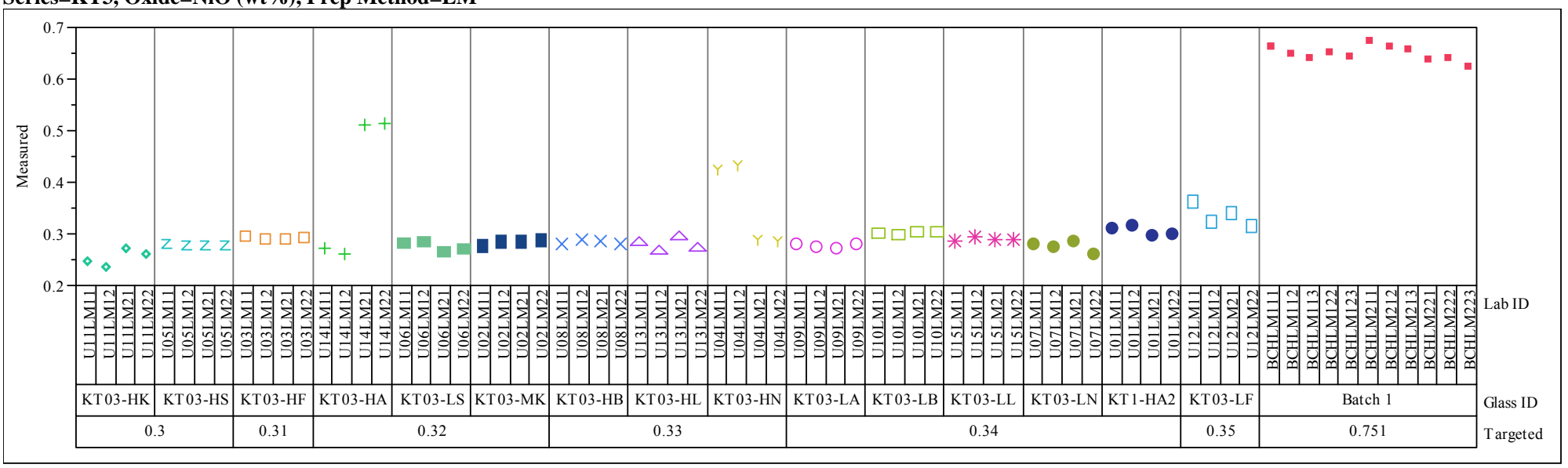


Exhibit B-2 Measurements for Each KT03-Series Glass ID by Preparation Method by Oxide. (continued) Series $=$ KT3, Oxide=PbO (wt\%), Prep Method=LM

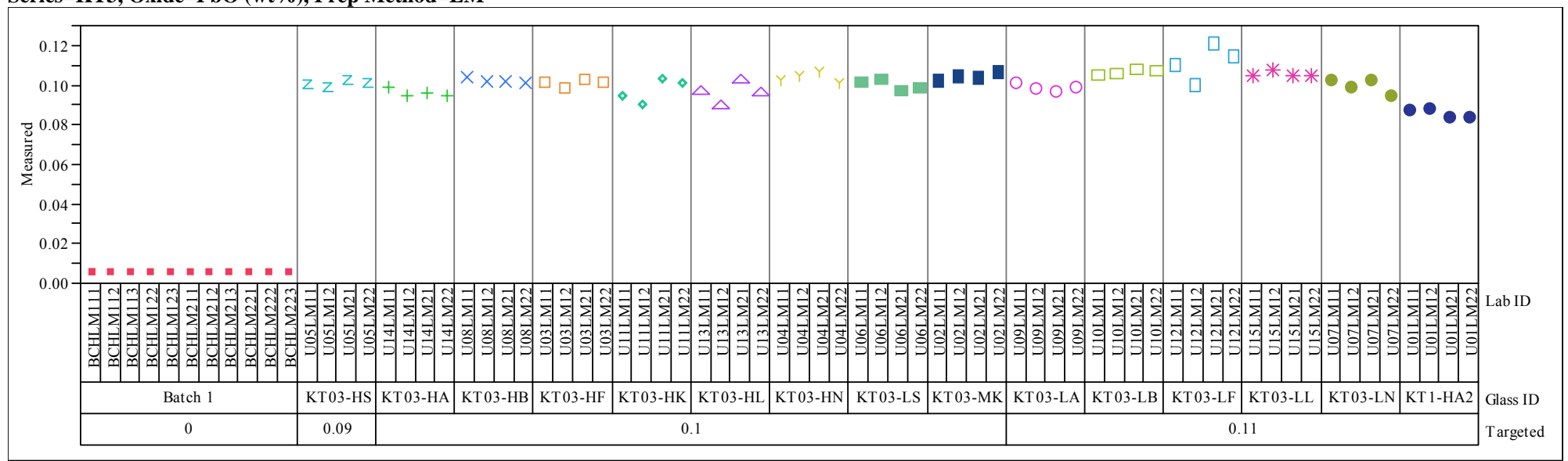

Series=KT3, Oxide=SiO2 (wt\%), Prep Method=PF

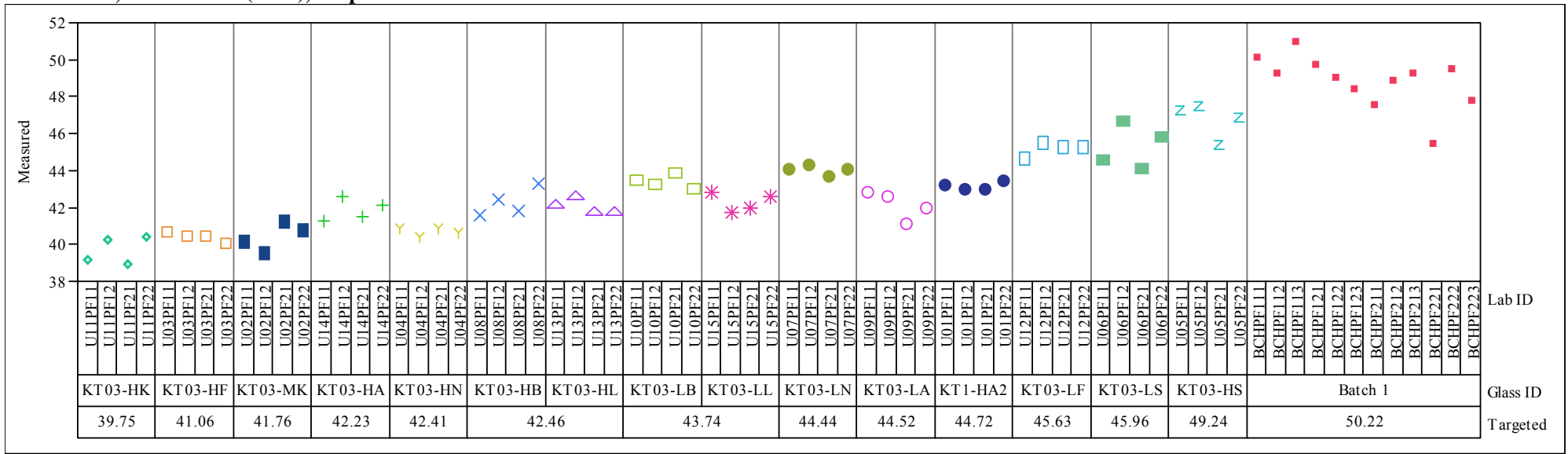


Exhibit B-2 Measurements for Each KT03-Series Glass ID by Preparation Method by Oxide. (continued) Series $=K T 3$, Oxide $=S O 4$ ( $w t \%)$, Prep Method $=$ LM

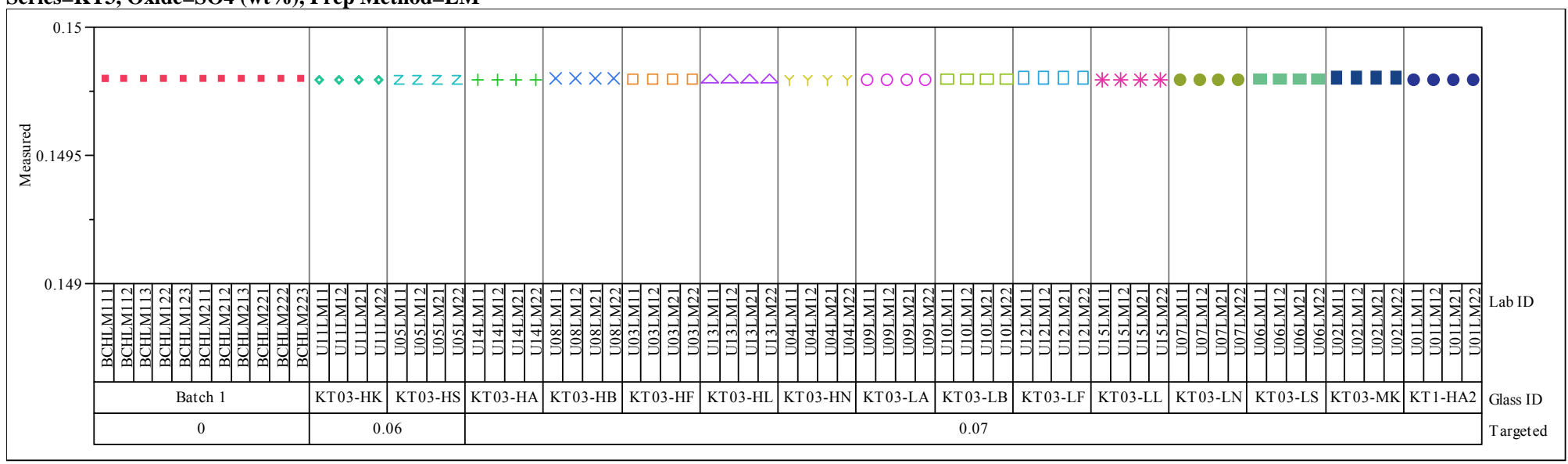

Series=KT3, Oxide=TiO2 (wt\%), Prep Method=LM

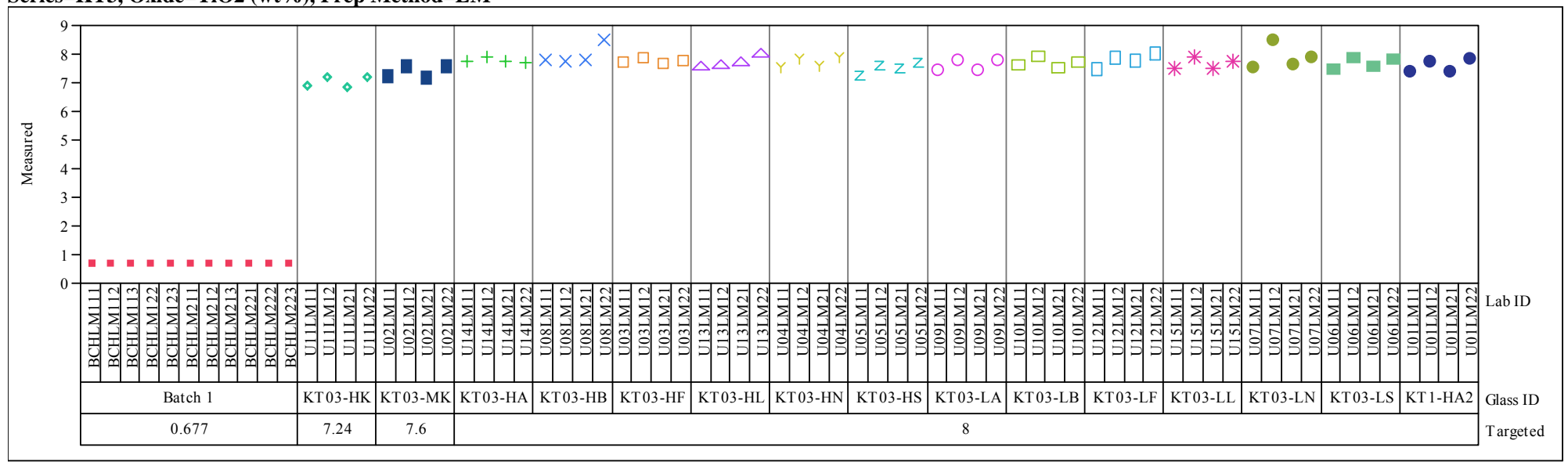


Exhibit B-2 Measurements for Each KT03-Series Glass ID by Preparation Method by Oxide. (continued) Series $=$ KT3, Oxide $=\mathrm{ZnO}$ (wt\%), Prep Method $=$ LM

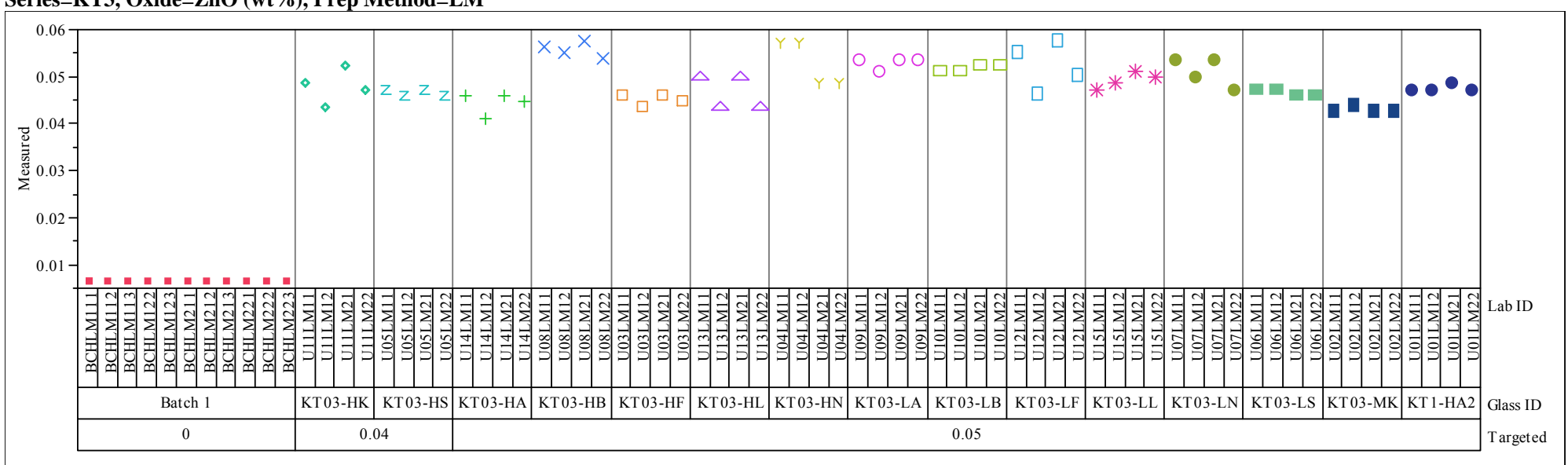

Series=KT3, Oxide=ZrO2 (wt\%), Prep Method=LM

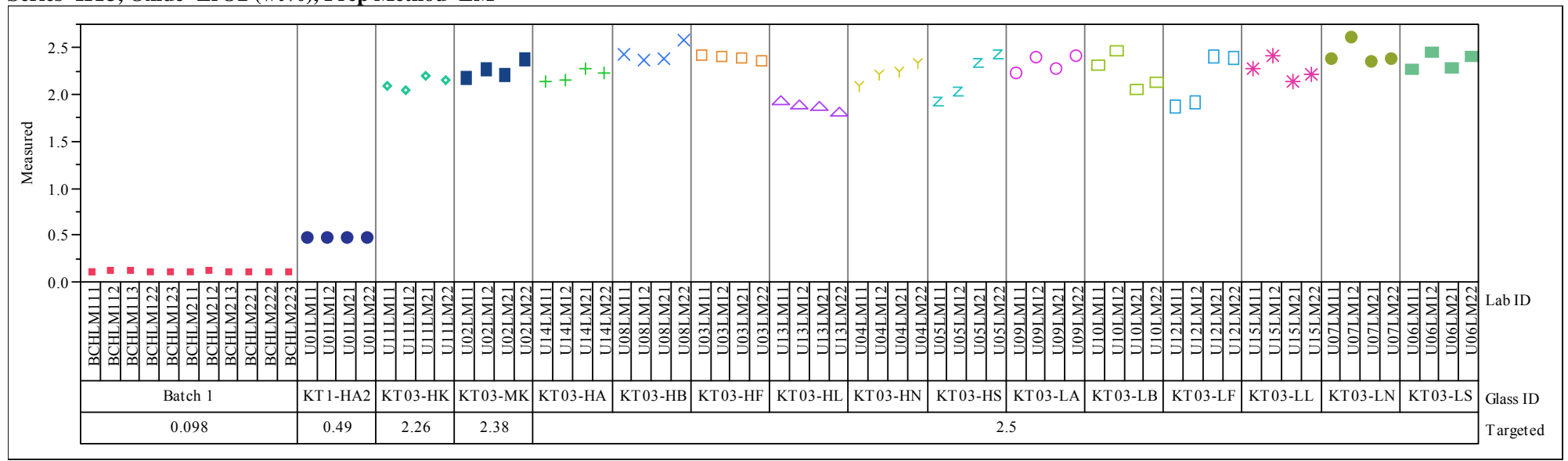


Exhibit B-3. Statistical Evaluation of the ICP-AES Calibration Effects from the KT03 Batch 1 Results by Oxide.

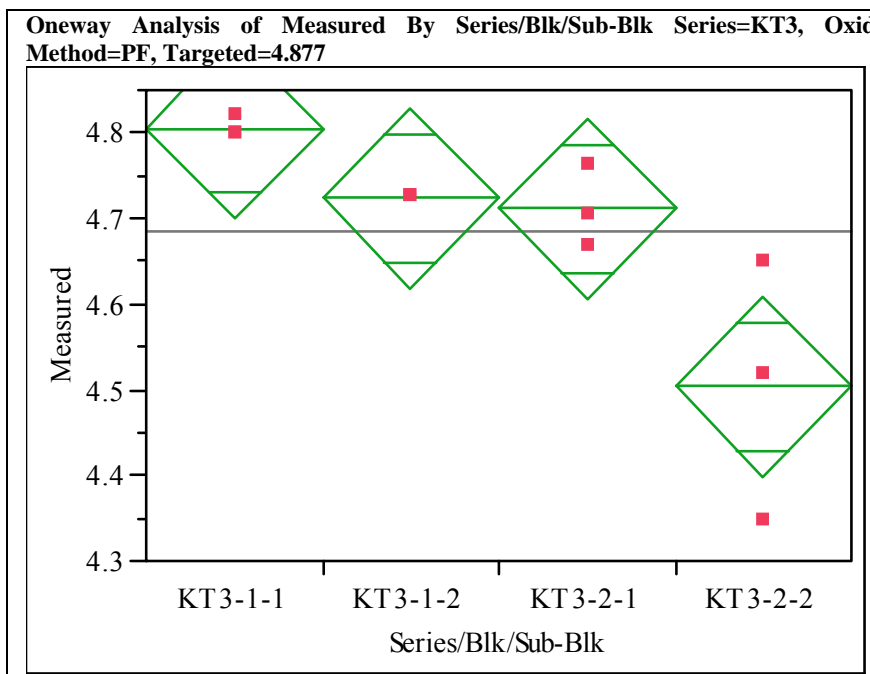

\section{Oneway Anova}

Summary of Fit

$\begin{array}{ll}\text { Rsquare } & 0.746429\end{array}$

$\begin{array}{ll}\text { Adj Rsquare } & 0.651339 \\ \text { Root Mean Square Error } & 0.079606\end{array}$

Root Mean Square Error $\quad \begin{array}{r}0.079606 \\ \text { Mean of Response }\end{array}$

Mean of Response
Observations (or Sum Wgts)

Analysis of Variance

Source DF Sum of Squares Mean Square F Ratio Prob > F

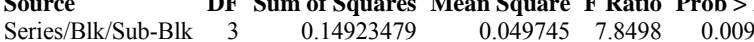

$\begin{array}{lrrr}\text { Series/Blk/Sub-Blk } & 3 & 0.14923479 & 0.049745 \\ \text { Error } & 8 & 0.05069699 & 0.006337\end{array}$

$\begin{array}{rr}8 & 0.05069699 \\ 11 & 0.19993177\end{array}$

Means for Oneway Anova

Level Number Mean Std Error Lower 95\% Upper 95\%

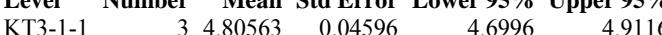

$\begin{array}{llllll}\text { KT3-1-1 } & 3 & 4.80563 & 0.04596 & 4.6996 & 4.9116 \\ \text { KT3-1-2 } & 3 & 4.72375 & 0.04596 & 4.6178 & 4.8297\end{array}$

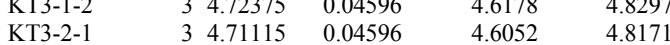

$\begin{array}{llllll}\text { KT3-2-2 } & 3 & 4.50331 & 0.04596 & 4.3973 & 4.6093\end{array}$

Std Error uses a pooled estimate of error variance

Oneway Analysis of Measured By Series/Blk/Sub-Blk Series=KT3, Oxide=B2O3 (wt\%), Prep Method=PF, Targeted $=7.777$

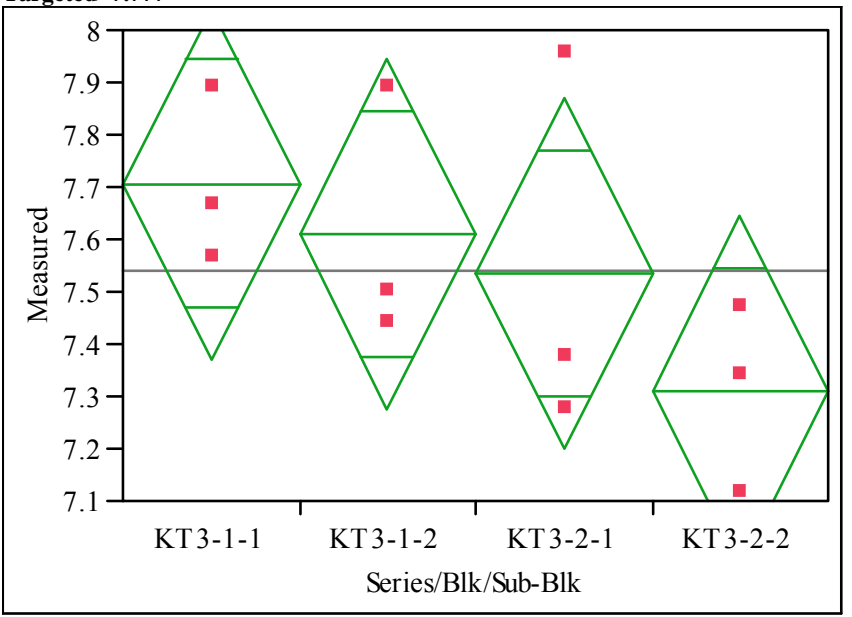

\section{Oneway Anova}

Summary of Fit

$\begin{array}{ll}\text { Rsquare } & 0.337562 \\ \text { Adj Rsquare } & 0.089148\end{array}$

Adj Rsquare $\quad \begin{array}{rr}0.089148 \\ \text { Root Mean Square Error } \quad 0.25131\end{array}$

Root Mean Square Error $\quad 0.25131$
Mean of Response

Observations (or Sum Wgts)

Analysis of Variance

Source DF Sum of Squares Mean Square F Ratio Prob $>$ F

$\begin{array}{lrrrrr}\text { Source } & \text { DF } & \text { Sum of Squares } & \text { Mean Square } & \text { F Ratio } & \text { Prob }>\text { F } \\ \text { Series/Blk/Sub-Blk } & 3 & 0.25746594 & 0.085822 & 1.3589 & 0.3229\end{array}$

$\begin{array}{lrrr}\text { Error } & 8 & 0.50525531 & 0.063157 \\ \text { C. Total } & 11 & 0.76272125 & \end{array}$

Means for Oneway Anova

Level Number Mean Std Error Lower 95\% Upper 95\%

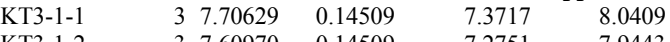

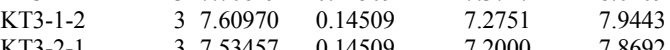

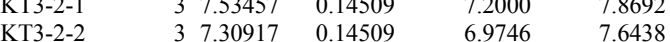

Std Error uses a pooled estimate of error variance 
Exhibit B-3. Statistical Evaluation of the ICP-AES Calibration Effects from the KT03 Batch 1 Results by Oxide. (continued)

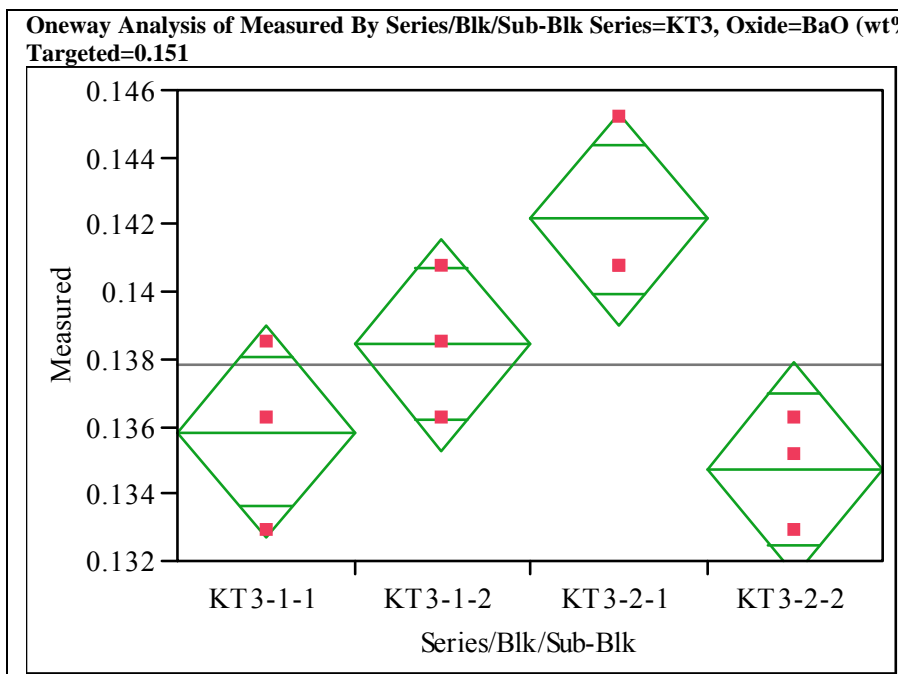

\section{Oneway Anova}

Summary of Fit

Rsquare $\quad 0.68673$

Adj Rsquare $\quad 0.569253$

$\begin{array}{ll}\text { Root Mean Square Error } & 0.002368 \\ \text { Mean of Response } & 0.137795\end{array}$

Mean of Response 0.137795

Analysis of Variance

Source DF Sum of Squares Mean Square F Ratio Prob $>$ F

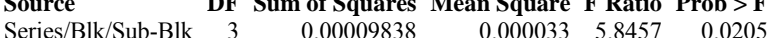

$\begin{array}{lrrr}\text { Error } & 8 & 0.00009838 & 0.000033 \\ \text { C. Total } & 11 & 0.0004488 & 5.61 \mathrm{e}-6\end{array}$

Means for Oneway Anova

Level Number Mean Std Error Lower 95\% Upper 95\%

$\begin{array}{llllrr}\text { KT3-1-1 } & 3 & 0.135841 & 0.00137 & 0.13269 & 0.13899\end{array}$

$\begin{array}{llllll}\text { KT3-1-1 } & 3 & 0.135841 & 0.00137 & 0.13269 & 0.13899 \\ \text { KT-1-2 } & 3 & 0.138446 & 0.00137 & 0.13529 & 0.14160\end{array}$

$\begin{array}{llllll}\text { KT3-2-1 } & 3 & 0.142168 & 0.00137 & 0.13901 & 0.14532 \\ \text { KT3-2-2 } & 3 & 0.134724 & 0.00137 & 0.13157 & 0.13788\end{array}$

Std Error uses a pooled estimate of error variance

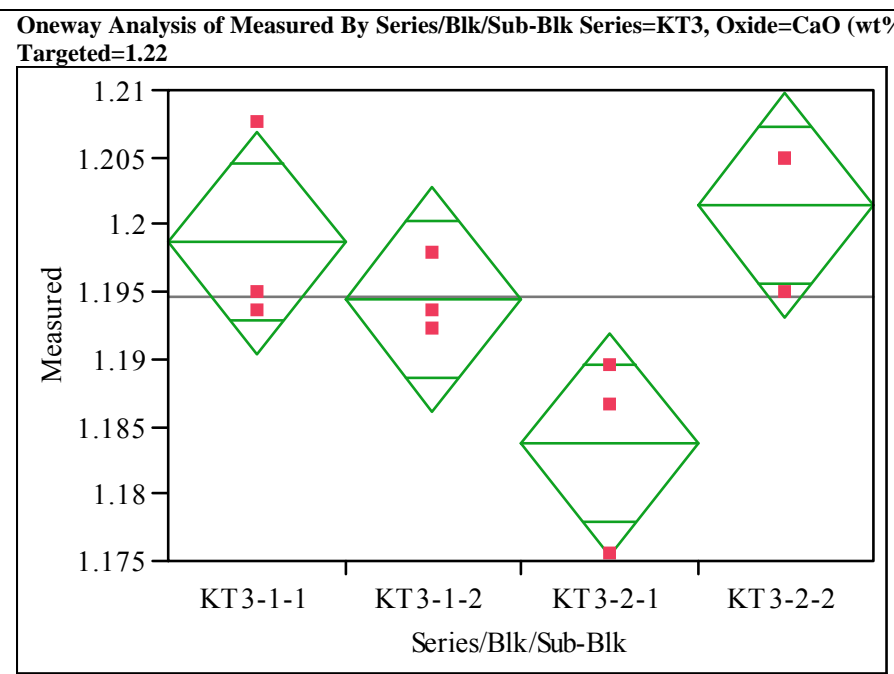

\section{Oneway Anova}

Summary of Fit

$\begin{array}{ll}\text { Rsquare } & 0.637822 \\ \text { Adj Rsquare } & 0.502006\end{array}$

$\begin{array}{ll}\text { Adj Rsquare } & 0.502006 \\ \text { Root Mean Square Error } & 0.006218\end{array}$

Root Mean Square Error $\quad 0.006218$

Observations (or Sum Wgts)

Analysis of Variance

Source DF Sum of Squares Mean Square F Ratio Prob $>$ F

$\begin{array}{lrrrrr} & & & & \\ \text { Series/Blk/Sub-Blk } & 3 & 0.00054475 & 0.000182 & 4.6962 & 0.0357\end{array}$

$\begin{array}{lrrr}\text { Error } & 8 & 0.00030933 & 0.000039\end{array}$

$\begin{array}{lrr}\text { C. Total } & 11 & 0.00085407\end{array}$

Means for Oneway Anova

Level Number Mean Std Error Lower 95\% Upper 95\%

$\begin{array}{lrrrrr}\text { KT3-1-1 } & 3 & \text { Mean } & \text { Std Error } & \text { Lower 95\% } & \text { Upper 95\% } \\ \text { KT } & 0.00359 & 1.1904 & 1.2069\end{array}$

$\begin{array}{llllll}\text { KT3-1-1 } & 3 & 1.19865 & 0.00359 & 1.1904 & 1.2069 \\ \text { KT3-1-2 } & 3 & 1.19445 & 0.00359 & 1.1862 & 1.2027\end{array}$

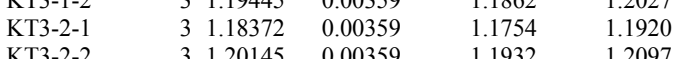

KT3-2-2 3 1.20145 0.003590

Std Error uses a pooled estimate of error variance 
Exhibit B-3. Statistical Evaluation of the ICP-AES Calibration Effects from the KT03 Batch 1 Results by Oxide. (continued)

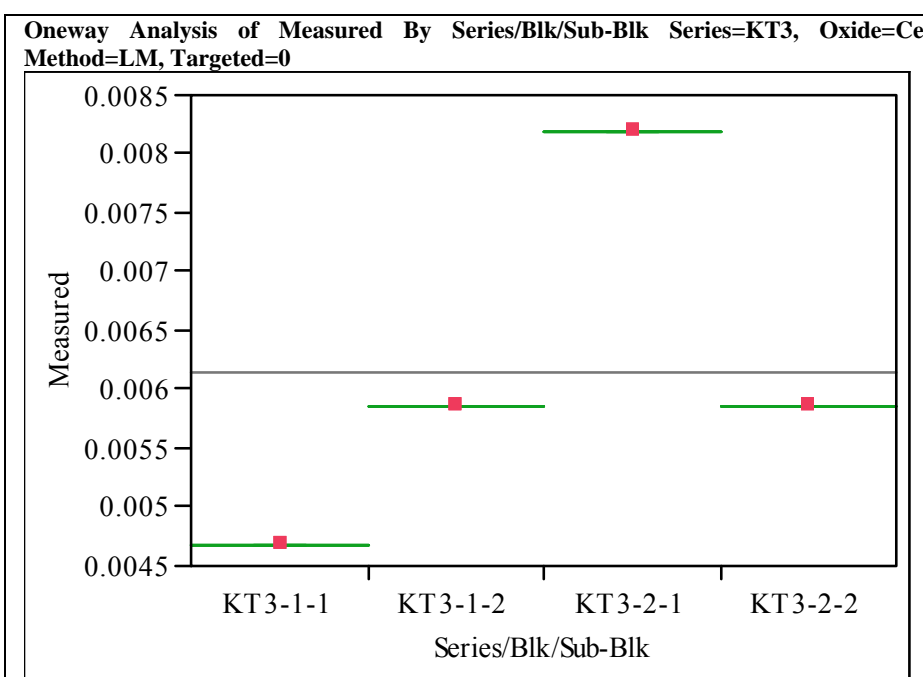

\section{Oneway Anova}

Summary of Fit

Rsquare

Adj Rsquare $\quad 1$

Root Mean Square Error $\quad 2.06 \mathrm{e}-11$

Mean of Response 0.006149

Observations (or Sum whe

Source DF Sum of Squares Mean Square $\quad$ F Ratio Prob $>$ F

$\begin{array}{lrrrrr}\text { Source } & \text { DF } & \text { Sum of Squares } & \text { Mean Square } & \text { F Ratio } & \text { Prob }>\text { F } \\ \text { Series/Blk/Sub-Blk } & 3 & 0.00001955 & 6.5167 \mathrm{e}-6 & 1.54 \mathrm{e}+16 & <.0001\end{array}$

$\begin{array}{lrrr}\text { Error } & 3 & 0.00001955 & 6.5167 \mathrm{e}-6 \\ \text { C. Total } & 8 & 3.3881 \mathrm{e}-21 & 4.235 \mathrm{e}-22\end{array}$

Means for Oneway Anova

Level Number Mean Std Error Lower 95\% Upper 95\%

$\begin{array}{lrrrrr}\text { KT3-1-1 } & 3 & 0.004685 & 1.188 \mathrm{e}-11 & 0.00469 & 0.00469\end{array}$

$\begin{array}{llllll}\text { KT3-1-1 } & 3 & 0.004685 & 1.188 \mathrm{e}-11 & 0.00469 & 0.00469 \\ \text { KT3-1-2 } & 3 & 0.005857 & 1.188 \mathrm{e}-11 & 0.00586 & 0.00586\end{array}$

$\begin{array}{llllll}\text { KT3-1-2 } & 3 & 0.005857 & 1.188 \mathrm{e}-11 & 0.00586 & 0.00586 \\ \text { KT3-2-1 } & 3 & 0.008199 & 1.188 \mathrm{e}-11 & 0.00820 & 0.00820 \\ \text { KT3-2-2 } & 3 & 0.005857 & 1.188 \mathrm{e}-11 & 0.00586 & 0.00586\end{array}$

Std Error uses a pooled estimate of error variance

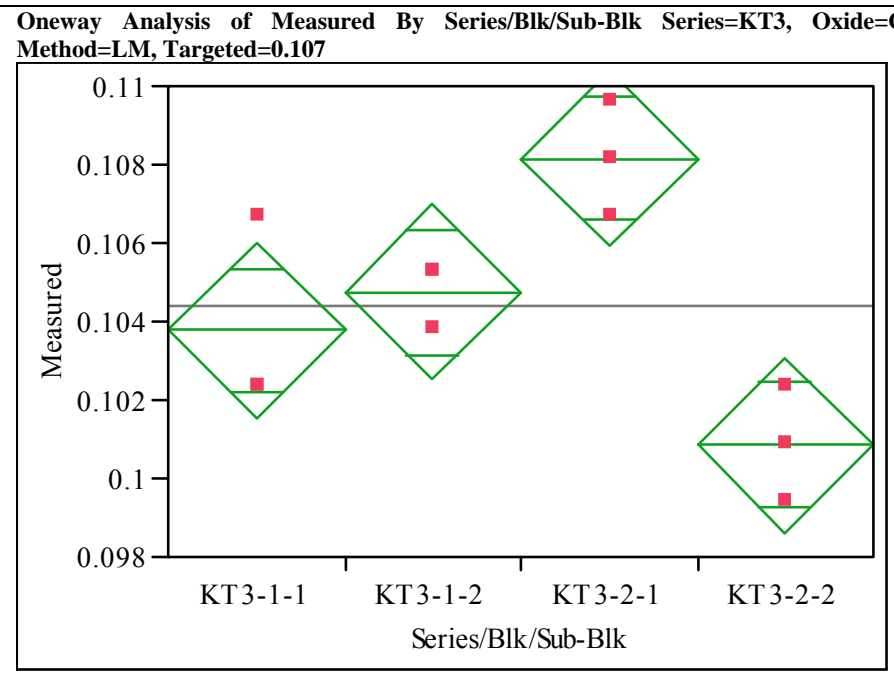

\section{Oneway Anova}

Summary of Fit

$\begin{array}{lr}\text { Rsquare } & 0.781942 \\ \text { Adj Rsquare } & 0.70017 \\ \text { Root Mean Square Error } & 0.001688 \\ \text { Mean of Response } & 0.104383 \\ \text { Obser }\end{array}$

Observations (or Sum Wgts)

Analysis of Variance

Source DF Sum of Squares Mean Square F Ratio Prob $>$ F

$\begin{array}{lrrrrr} & & & & & \\ \text { Source } & & & & & \\ \text { Series/Blk/Sub-Blk } & 3 & 0.00008171 & 0.000027 & 9.5625 & 0.0051\end{array}$

$\begin{array}{lrrr}\text { Error } & 8 & 0.00002279 & 2.848 \mathrm{e}-6 \\ \text { C. Total } & 11 & 0.00010450 & \end{array}$

Means for Oneway Anova

$\begin{array}{lrrrrr}\text { Level } & \text { Number } & \text { Mean } & \text { Std Error } & \text { Lower 95\% } & \text { Upper 95\% } \\ \text { KT3-1-1 } & 3 & 0.103774 & 0.00097 & 0.10153 & 0.10602\end{array}$

$\begin{array}{llllll}\text { KT3-1-1 } & & 0.103774 & 0.00097 & 0.10153 & 0.10602 \\ \text { KT3-1-2 } & 3 & 0.104748 & 0.00097 & 0.10250 & 0.10699\end{array}$

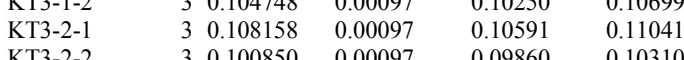

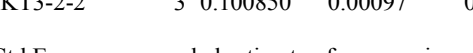

Std Error uses a pooled estimate of error variance 
Exhibit B-3. Statistical Evaluation of the ICP-AES Calibration Effects from the KT03 Batch 1 Results by Oxide. (continued)

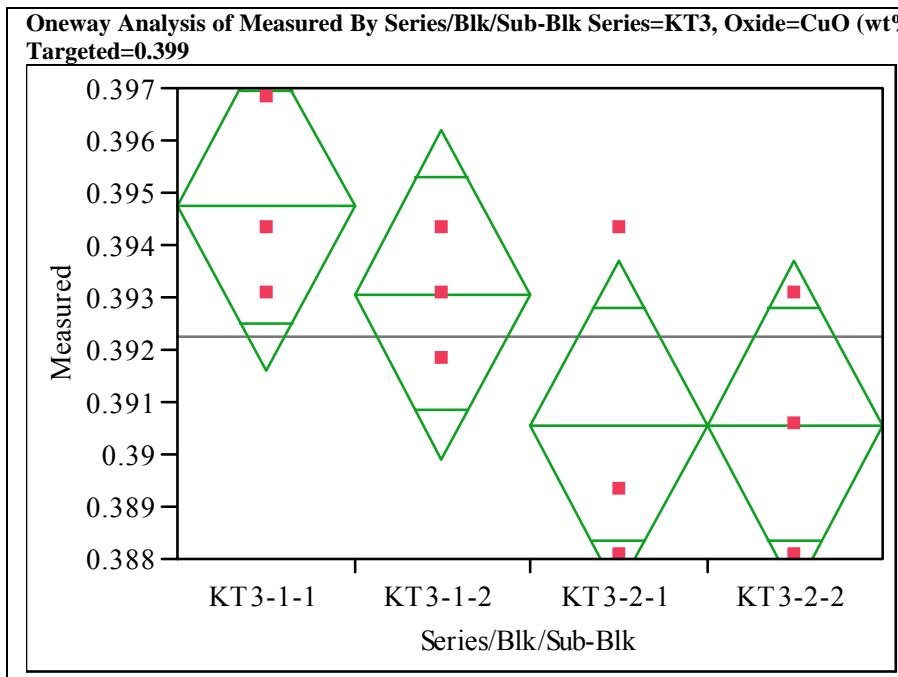

\section{Oneway Anova}

Summary of Fit

Rsquare $\quad 0.455696$

$\begin{array}{lr}\text { Adj Rsquare } & 0.251582 \\ \text { Rot } & 0.00237\end{array}$

Root Mean Square Error $\quad 0.0023$

Mean of Response 0.39223

Analysis of Variance

$\begin{array}{lrrrrr}\text { Source } & \text { DF } & \text { Sum of Squares } & \text { Mean Square } & \text { F Ratio } & \text { Prob }>\text { F } \\ \text { Series/Blk/Sub-Blk } & 3 & 0.00003761 & 0.000013 & 2.2326 & 0.1618\end{array}$

$\begin{array}{lrrr}\text { Error } & 3 & 0.00003761 & 0.000013 \\ \text { Cros } & 8 & 0.0004492 & 5.615 \mathrm{e}-6\end{array}$

$\begin{array}{rl}8 & 0.00004492 \\ 11 & 0.00008253\end{array}$

Means for Oneway Anova

Level Number Mean Std Error Lower 95\% Upper 95\%

$\begin{array}{lllrrr}\text { KT3-1-1 } & 3 & 0.394734 & 0.00137 & 0.39158 & 0.39789\end{array}$

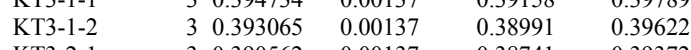

$\begin{array}{llllll}\text { KT3-1-2 } & 3 & 0.393065 & 0.00137 & 0.38991 & 0.39622 \\ \text { KT3-2-1 } & 3 & 0.390562 & 0.00137 & 0.38741 & 0.39372\end{array}$

$\begin{array}{llllll}\text { KT3-2-2 } & 3 & 0.390562 & 0.00137 & 0.38741 & 0.39372\end{array}$

Std Error uses a pooled estimate of error variance

Oneway Analysis of Measured By Series/Blk/Sub-Blk Series=KT3, Oxide=Fe2O3 (wt\%), Prep Method=PF, Targeted=12.839

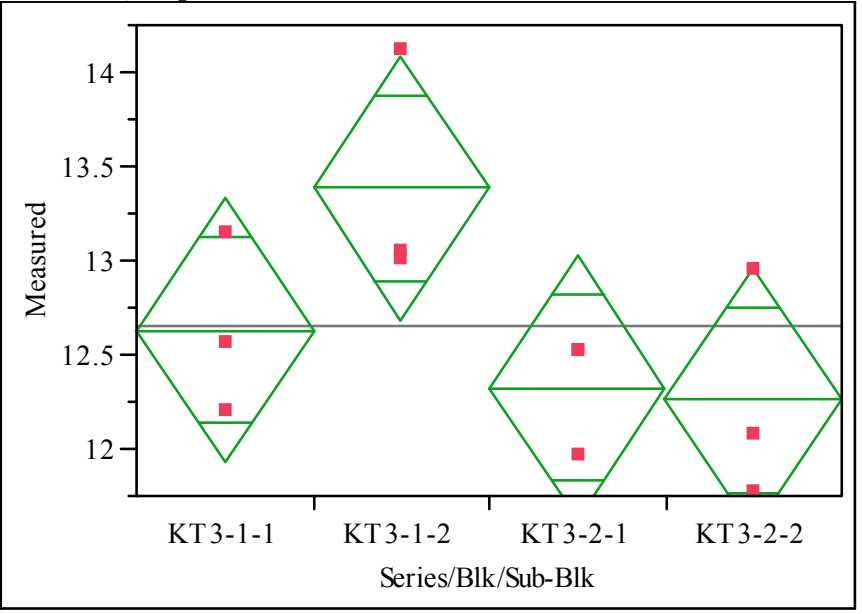

\section{Oneway Anova}

Summary of Fit

$\begin{array}{ll}\text { Rsquare } & 0.520567\end{array}$

0.34078
Adj Rsquare

$\begin{array}{ll}\text { Root Mean Square Error } & 0.524559 \\ \text { Mean of Response } & 12.64808\end{array}$

Observations (or Sum Wgts) 12.64808

Analysis of Variance

Source DF Sum of Squares Mean Square F Ratio Prob $>$ F

$\begin{array}{lrrrrr}\text { Source } & \text { DF } & \text { Sum of Squares } & \text { Mean Square } & \text { F Ratio } & \text { Prob }>\text { F } \\ \text { Series/Blk/Sub-Blk } & 3 & 2.3901666 & 0.796722 & 2.8955 & 0.1019\end{array}$

$\begin{array}{lrrr}\text { Error } & 8 & 2.2012971 & 0.275162 \\ \text { C. Total } & 11 & 4.5914636 & \end{array}$$$
0.275162
$$

Means for Oneway Anova

Level Number Mean Std Error Lower 95\% Upper 95\%

$\begin{array}{llllll}\text { KT3-1-1 } & 3 & 12.6290 & 0.30285 & 11.931 & 13.327 \\ \text { KT31-2 } & 3 & 13.3820 & 0.3285 & 12.684 & 14.080\end{array}$

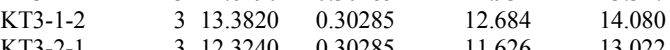

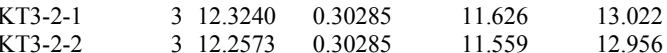

Std Error uses a pooled estimate of error variance 
Exhibit B-3. Statistical Evaluation of the ICP-AES Calibration Effects from the KT03 Batch 1 Results by Oxide. (continued)

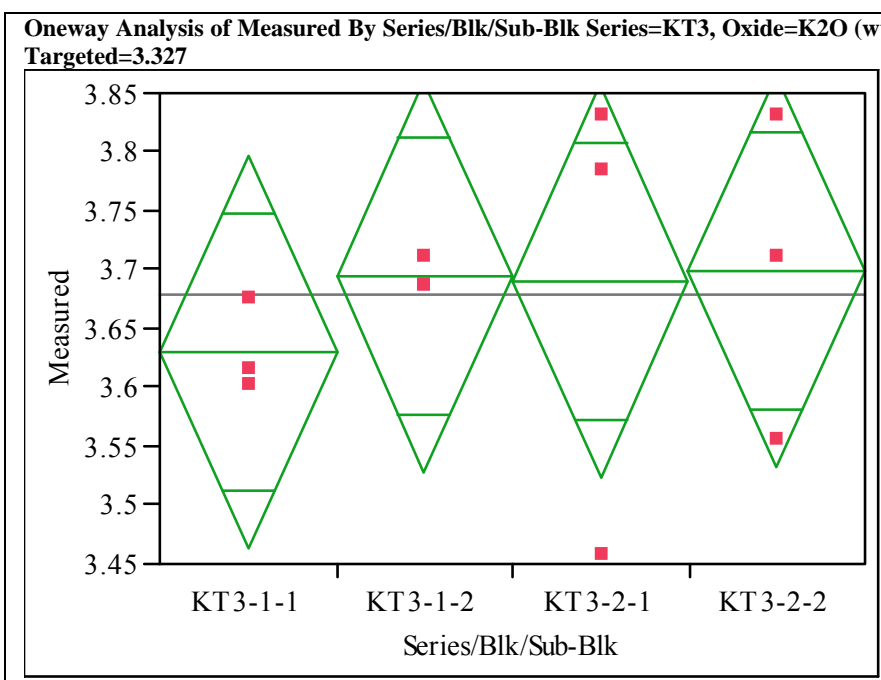

\section{Oneway Anova}

Summary of Fit

Rsquare $\quad 0.070087$

$\begin{array}{lr}\text { Adj Rsquare } & -0.27863 \\ \text { Root Mean Square Error } \quad 0.12475\end{array}$

Root Mean Square Error $\quad 0.12475$
Mean of Response

Mean of Response 3.678045

Analysis of Variance

$\begin{array}{lrrrrr}\text { Source } & \text { DF } & \text { Sum of Squares } & \text { Mean Square } & \text { F Ratio } & \text { Prob }>\text { F } \\ \text { Series/Blk/Sub-Blk } & 3 & 0.00938353 & 0.003128 & 0.2010 & 0.8928\end{array}$

$\begin{array}{lrrr}\text { Series/Blk/Sub-Blk } & 3 & 0.00938353 & 0.003128 \\ \text { Error } & 8 & 0.12450105 & 0.015563\end{array}$

110.13388458

Means for Oneway Anova

Level Number Mean Std Error Lower 95\% Upper 95\%

\begin{tabular}{lrrrrr} 
KT3-1-1 & 3 & 3.62986 & 0.07202 & 3.4638 & 3.7960 \\
\hline & 3 & 3.69411 & 0.07202 & 3.5280 & 3.8602
\end{tabular}

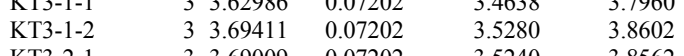

$\begin{array}{llllll}\text { KT3-1-2 } & 3 & 3.69411 & 0.07202 & 3.5280 & 3.8602 \\ \text { KT3-2-1 } & 3 & 3.69009 & 0.07202 & 3.5240 & 3.8562 \\ \text { KT3-2 } & 3 & 3.69812 & 0.07202 & 3.5320 & 3.8642\end{array}$

Std Error uses a pooled estimate of error variance

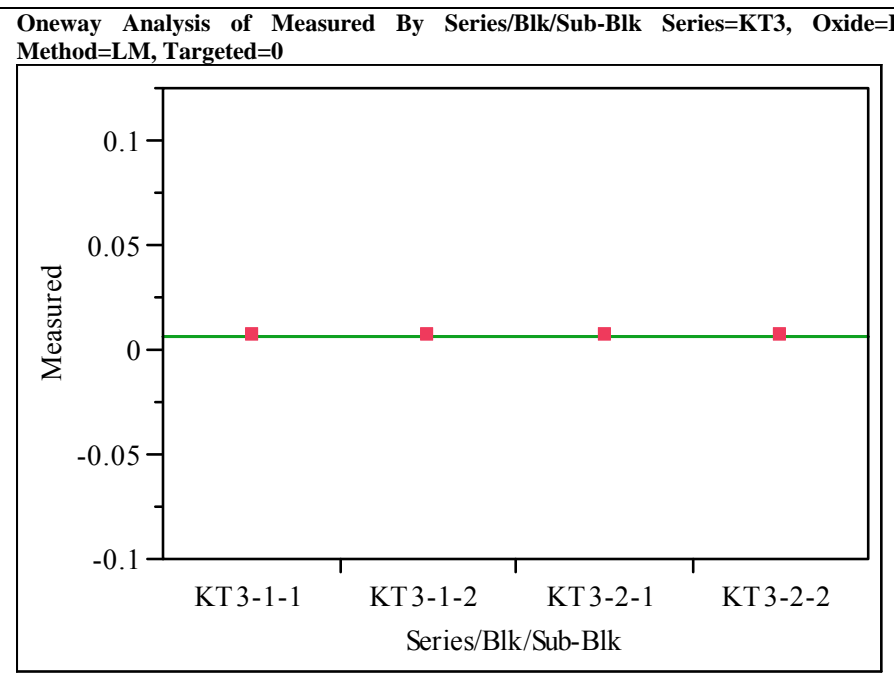

\section{Oneway Anova}

Summary of Fit

\section{Rsquare}

\section{Adj Rsquare}

Root Mean Square Error $\quad 0$

Observations (or Sum Wots) 0.005864

Analysis of Variance

Source DF Sum of Squares Mean Square F Ratio Prob $>$ F

$\begin{array}{lrrr}\text { Source } & \text { DF } & \text { Sum of Squares } & \text { Mean Square } \\ \text { Series/Blk/Sub-Blk } & 3 & 0 & 0 \\ \text { Error } & 8 & 0 & 0 \\ \text { C. Total } & 11 & 0 & \end{array}$

Means for Oneway Anova

Level Number Mean Std Error Lower 95\% Upper 95\%

$\begin{array}{lrrrrr}\text { LT3-1-1 } & 3 & 0.005864 & 0 & 0.00586 & 0.00586 \\ \text { KT3-1- } & 3 & 0.005864 & 0 & 0.00586 & 0.00586\end{array}$

$\begin{array}{llllll}\text { KT3-1-1 } & 3 & 0.005864 & 0 & 0.00586 & 0.00586 \\ \text { KT3-1-2 } & 3 & 0.005864 & 0 & 0.00586 & 0.00586 \\ \text { KT3 }-2-1 & 3 & 0.005864 & 0 & 0.00586 & 0.00586\end{array}$

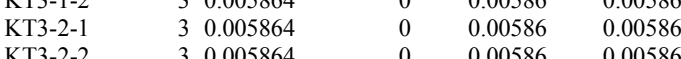

Std Error uses a pooled estimate of error variance 
Exhibit B-3. Statistical Evaluation of the ICP-AES Calibration Effects from the KT03 Batch 1 Results by Oxide. (continued)

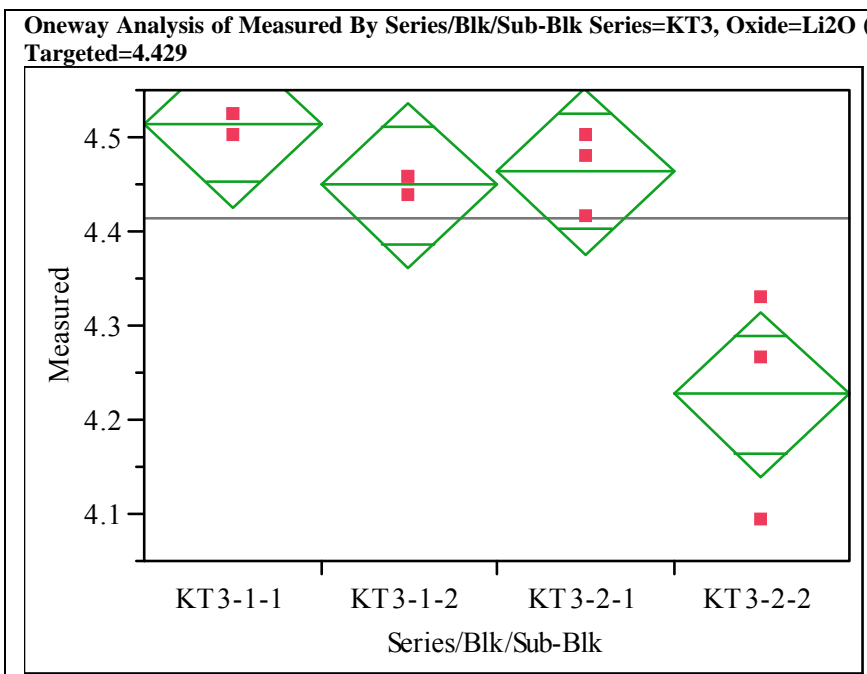

\section{Oneway Anova}

Summary of Fit

\begin{tabular}{ll} 
Rsquare & 0.808547 \\
\hline Adj Rsqure & 0.736752
\end{tabular}

$\begin{array}{ll}\text { Adj Rsquare } & 0.736752 \\ \text { Root Mean Square Error } & 0.065772\end{array}$

$\begin{array}{lr}\text { Root Mean Square Error } & 0.065772 \\ \text { Mean of Response } & 4.413445 \\ \text { Observations (or Sum Wgts) } & 12\end{array}$

Analysis of Variance

Source DF Sum of Squares Mean Square F Ratio Prob $>$ F

$\begin{array}{llllll}\text { Source } & & \text { DF } & & & \\ \text { Series/Blk/Sub-Blk } & 3 & 0.14615632 & 0.048719 & 11.2619 & 0.0030\end{array}$

$\begin{array}{lrrr}\text { Error } & 8 & 0.03460784 & 0.048719 \\ \text { C. } & 11 & 0.18076416 & \end{array}$

$11 \quad 0.18076416$

Means for Oneway Anova

Level Number Mean Std Error Lower 95\% Upper 95\%

\begin{tabular}{lrrrrr} 
KT3-1-1 & 3 & 4.51391 & 0.03797 & 4.4263 & 4.6015 \\
\hline
\end{tabular}

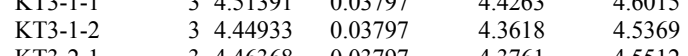

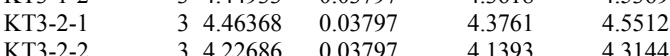

Std Error uses a pooled estimate of error variance

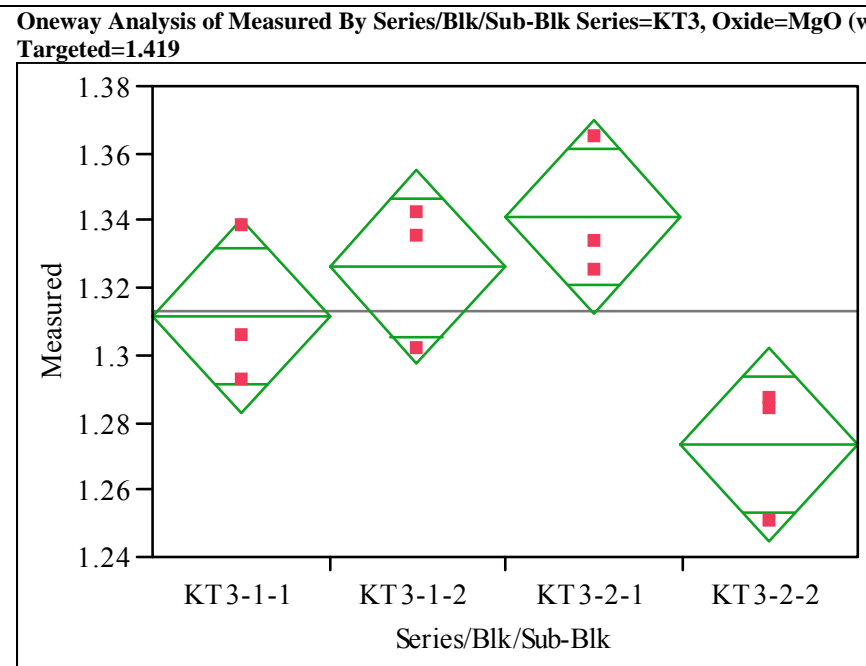

\section{Oneway Anova}

Summary of Fit

$\begin{array}{ll}\text { Rsquare } & 0.667777 \\ \text { Adj Rsquare } & 0.543193\end{array}$

0.6677193
Adj Rsquare

$\begin{array}{lr}\text { Root Mean Square Error } \quad 0.021648 \\ \text { Mean of Response } & 1313097\end{array}$

Observations (or Sum Wgts)

Analysis of Variance

Source DF Sum of Squares Mean Square F Ratio Prob $>$ F

$\begin{array}{lrrrrr} & \text { DF } & & & & \\ \text { Source } & & & & & \\ \text { Series/Blk/Sub-Blk } & 3 & 0.00753580 & 0.002512 & 5.3601 & 0.0257\end{array}$

$\begin{array}{lrrr}\text { Error } & 8 & 0.00374911 & 0.000469\end{array}$

$\begin{array}{lrr}\text { C. Total } & 11 & 0.01128491\end{array}$

Means for Oneway Anova

Level Number Mean Std Error Lower 95\% Upper 95\%

\begin{tabular}{lrrrrr} 
KT3-1-1 & 3 & 1.31172 & 0.01250 & 1.2829 & 1.3405 \\
\hline
\end{tabular}

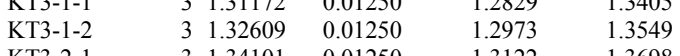

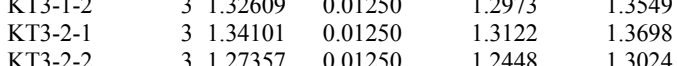

KT

Std Error uses a pooled estimate of error variance 
Exhibit B-3. Statistical Evaluation of the ICP-AES Calibration Effects from the KT03 Batch 1 Results by Oxide. (continued)

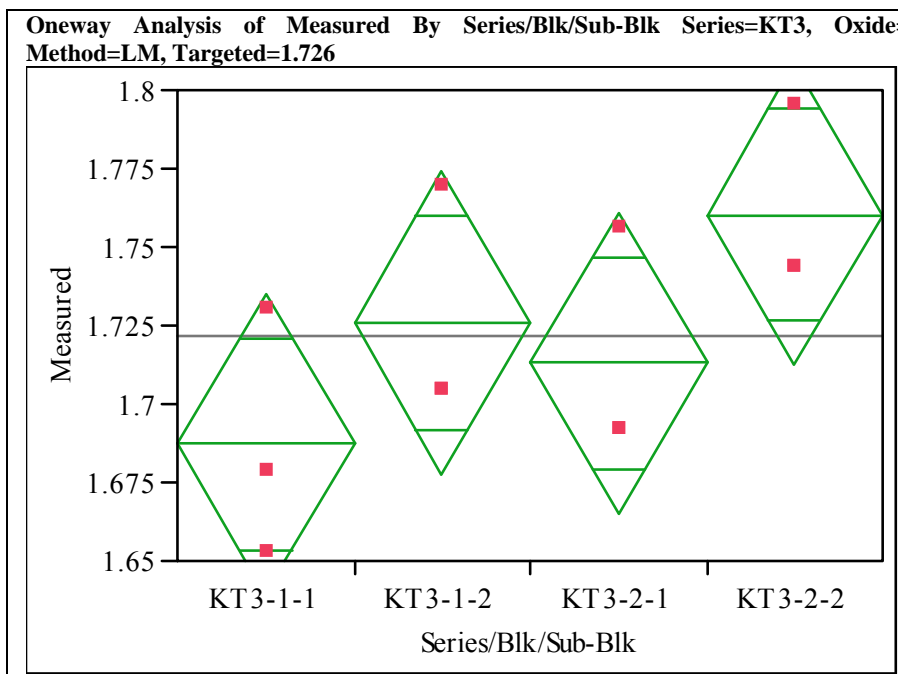

\section{Oneway Anova}

Summary of Fit

$\begin{array}{ll}\text { Rsquare } & 0.443787 \\ \text { Adj Rsquare } & 0.235207\end{array}$

0.235207
Root Mean Square Error $\quad 0.036138$

Root Mean Square Error $\quad 0.036138$

$\begin{array}{lr}\text { Mean of Response } & 1.7216 \\ \text { Observations (or Sum Wgts) } & 12\end{array}$

Analysis of Variance

Source DF Sum of Squares Mean Square F Ratio Prob $>$ F

$\begin{array}{llllll}\text { Series/Blk/Sub-Blk } & 3 & 0.00833599 & 0.002779 & 2.1277 & 0.1749\end{array}$

$\begin{array}{lrrr}\text { Error } & 8 & 0.01044777 & 0.001306 \\ \text { C. Total } & 11 & 0.01878376 & \end{array}$

110.01878376

Means for Oneway Anova

Level Number Mean Std Error Lower 95\% Upper 95\%

$\begin{array}{lrrrrr}\text { KT3-1-1 } & 3 & 1.68717 & 0.02086 & 1.6391 & 1.7353\end{array}$

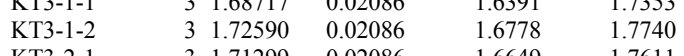

$\begin{array}{llllll}\text { KT3-2-1 } & 3 & 1.71299 & 0.02086 & 1.6649 & 1.7611\end{array}$

Std Error uses a pooled estimate of error variance

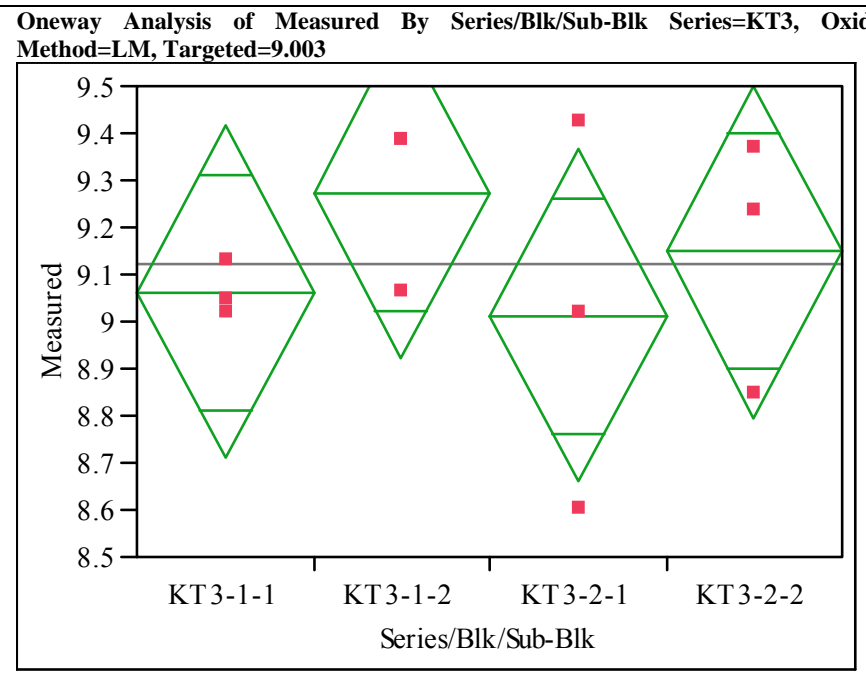

\section{Oneway Anova}

Summary of Fit

$\begin{array}{ll}\text { Rsquare } & 0.172211 \\ \text { Adj Rsquare } & -0.13821\end{array}$

Adj Rsquare $\quad-0.13821$

Root Mean Square Error $\quad 0.265354$

Observations (or Sum Wgts)

Analysis of Variance

Source DF Sum of Squares Mean Square F Ratio Prob $>$ F

$\begin{array}{lrrrrr} & & & & & \\ \text { Series/Blk/Sub-Blk } & 3 & 0.11718807 & 0.039063 & 0.5548 & 0.6593\end{array}$

$\begin{array}{lrrr}\text { Error } & 8 & 0.56330224 & 0.070413 \\ \text { C. Total } & 11 & 0.68049031 & \end{array}$

Means for Oneway Anova

Level Number Mean Std Error Lower 95\% Upper 95\%

$\begin{array}{lrrrrr}\text { KT3-1-1 } & 3 & 9.06305 & 0.15320 & 8.7098 & 9.4163\end{array}$

$\begin{array}{llllll}\text { KT3-1-1 } & 3 & 9.06305 & 0.15320 & 8.7098 & 9.4163 \\ \text { KT3-1-2 } & 3 & 9.27424 & 0.15320 & 8.9210 & 9.6275 \\ \text { KT3-2-1 } & 3 & 9.01363 & 0.15320 & 8.6603 & 9.3669\end{array}$

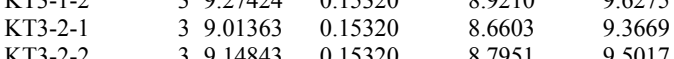

KT3-2-2

Std Error uses a pooled estimate of error variance 
Exhibit B-3. Statistical Evaluation of the ICP-AES Calibration Effects from the KT03 Batch 1 Results by Oxide. (continued)

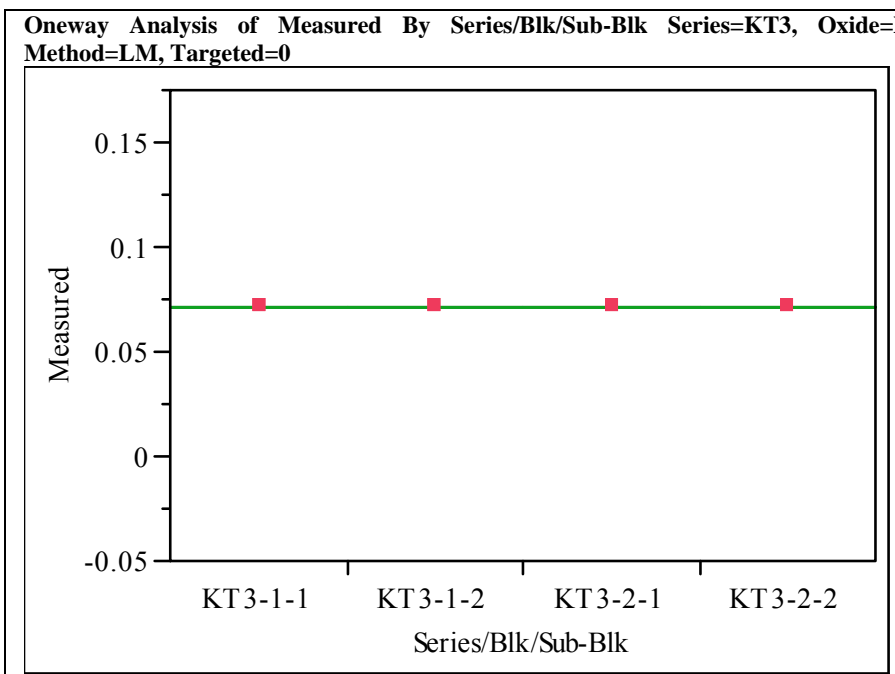

\section{Oneway Anova}

Summary of Fit

Rsquare

Adj Rsquare

$\begin{array}{lr}\text { Root Mean Square Error } & 0 \\ \text { Mean of Response } & 0.071525\end{array}$

Mean of Res

0.071525

Observations (or Sum Wgs)

Analysis of Variance

Source DF Sum of Squares Mean Square F Ratio Prob $>$ F

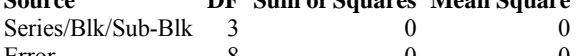

$\begin{array}{lll}\text { Error } & 8 \\ \text { C. } & & \end{array}$

$\begin{array}{rl}8 & 0 \\ 11 & 0\end{array}$

0

Means for Oneway Anova

Level Number Mean Std Error Lower 95\% Upper 95\%

$\begin{array}{lrrrrr}\text { KT3-1-1 } & 3 & 0.071525 & 0 & 0.07153 & 0.07153 \\ \text { KT3-1-2 } & 3 & 0.071525 & 0 & 0.07153 & 0.07153\end{array}$

$\begin{array}{llllll}\text { KT3-1-2 } & 3 & 0.071525 & 0 & 0.07153 & 0.07153 \\ \text { KT3-2 } & 3 & 0.071525 & 0 & 0.07153 & 0.07153\end{array}$

$\begin{array}{llllll}\text { KT3-2-1 } & 3 & 0.071525 & 0 & 0.07153 & 0.07153 \\ \text { KT3-2-2 } & 3 & 0.071525 & 0 & 0.07153 & 0.07153\end{array}$

Std Error uses a pooled estimate of error variance

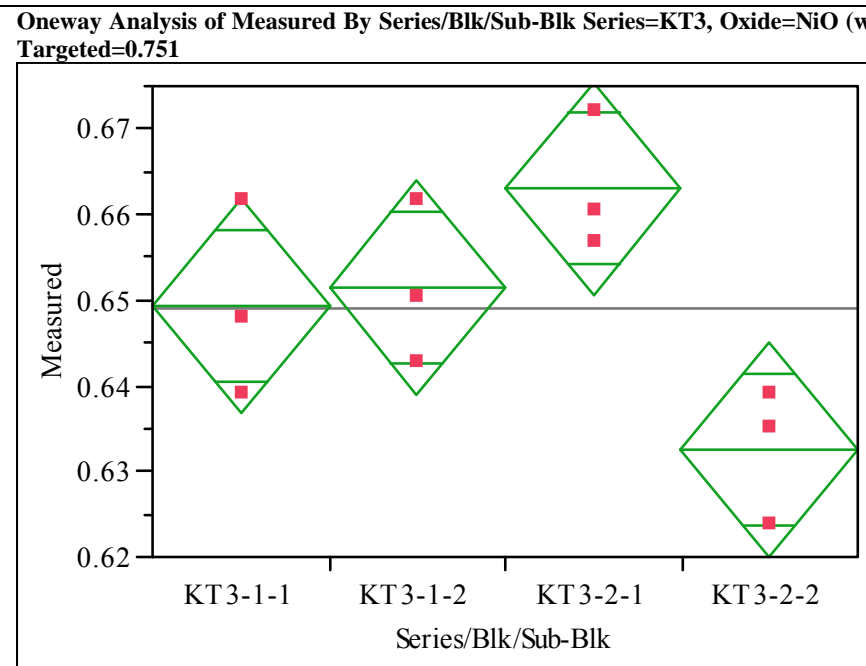

\section{Oneway Anova}

Summary of Fit
Rsquare
$\begin{array}{ll}\text { Rsquare } & 0.669936 \\ \text { Adj Rsquare } & 0.546162\end{array}$
Root Mean Square Error $\quad 0.00938$
0.649081

Observations (or Sum Wgts)

Analysis of Variance

Source DF Sum of Squares Mean Square F Ratio Prob $>$ F

$\begin{array}{llllll}\text { Series/Blk/Sub-Blk } & 3 & 0.00142859 & 0.000476 & 5.4126 & 0.0250\end{array}$

$\begin{array}{lrr}\text { Error } & 8 & 0.00070384 \\ \text { C. } & 11 & 0.00213243\end{array}$

0.00008

Means for Oneway Anova

Level Number Mean Std Error Lower 95\% Upper 95\%

\begin{tabular}{llllll} 
KT3-1-1 & 3 & 0.649399 & 0.00542 & 0.63691 & 0.66189 \\
\hline KT3-1-2 & 3 & 0.651529 & 0.00542 & 0.63903 & 0.66401
\end{tabular}

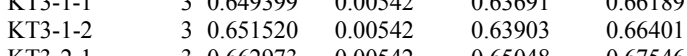

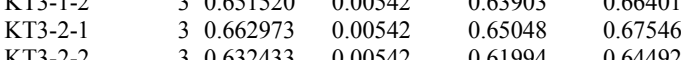

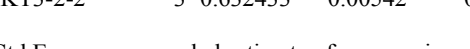

Std Error uses a pooled estimate of error variance 
Exhibit B-3. Statistical Evaluation of the ICP-AES Calibration Effects from the KT03 Batch 1 Results by Oxide. (continued)

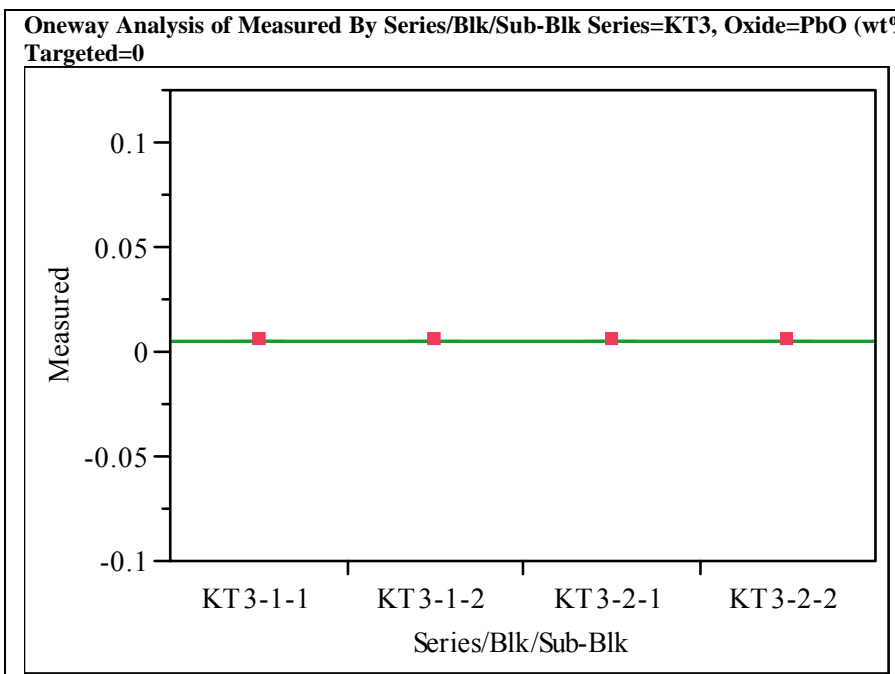

\section{Oneway Anova}

Summary of Fit

Rsquare

$\begin{array}{lr} & 0 \\ \text { Adj Rsquare } & -0.375\end{array}$

Root Mean Square Error $\quad \begin{array}{r}-0.375 \\ 1.06 \mathrm{e}-18\end{array}$

Mean of Response $\quad 0.005386$

Observations (or Sum Wgts) 0.005386

Observations (or Sum wh

Source DF Sum of Squares Mean Square F Ratio Prob $>$ F

$\begin{array}{lrrr}\text { Series/Blk/Sub-Blk } & 3 & 0 & 0 \\ \text { Error } & 8 & 9.0278 \mathrm{e}-36 & 1.128 \mathrm{e}-36\end{array}$

Error

Means for Oneway Anova

Level Number Mean Std Error Lower 95\% Upper 95\%

\begin{tabular}{lrrrrr}
\hline KT3-1-1 & 3 & 0.005386 & $6.133 \mathrm{e}-19$ & 0.00539 & 0.00539
\end{tabular}

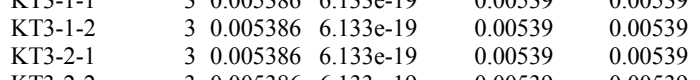

KT3-2-2

$\begin{array}{llll}3 & 0.005386 & 6.133 \mathrm{e}-19\end{array}$

0.0053

0053

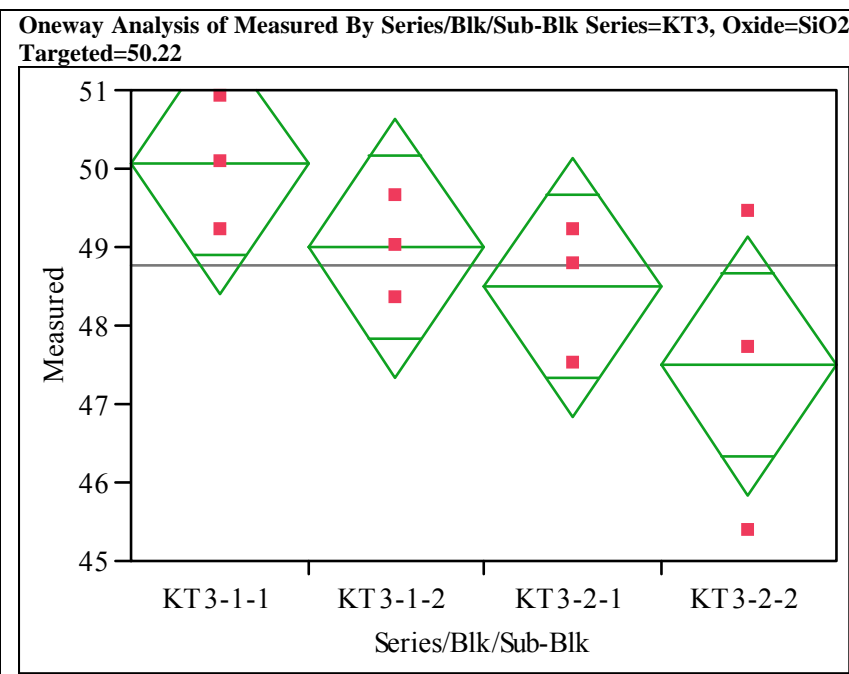

\section{Oneway Anova}

Summary of Fit

$\begin{array}{ll}\text { Rsquare } & 0.456799 \\ \text { Adj Rsquare } & 0.253098\end{array}$

0.253098
Adj Rsquare

Root Mean Square Error $\quad 1.235125$

$\begin{array}{lr}\text { Mean of Response } & 48.75821 \\ \text { Observations (or Sum Wgts) } & 12\end{array}$

Analysis of Variance

Source DF Sum of Squares Mean Square F Ratio Prob $>$ F

$\begin{array}{lrrrrr} & & & \\ \text { Series/Blk/Sub-Blk } & 3 & 10.263036 & 3.42101 & 2.2425 & 0.1606\end{array}$

$\begin{array}{lrrr}\text { Error } & 8 & 12.204279 & 1.52553\end{array}$

$\begin{array}{lrr}\text { C. Total } & 11 & 22.467314\end{array}$

Means for Oneway Anova

Level Number Mean Std Error Lower 95\% Upper 95\%

\begin{tabular}{lrrrrr} 
Level & Number & Mean & Std Error & Lower 95\% & Upper 95\% \\
KT3-1-1 & 3 & 50.0596 & 0.71310 & 48.415 & 51.704 \\
\hline
\end{tabular}

$\begin{array}{llllll}\text { KT3-1-1 } & 3 & 50.0596 & 0.71310 & 48.415 & 51.704 \\ \text { KT3-1-2 } & 3 & 48.9900 & 0.71310 & 47.346 & 50.634\end{array}$

\begin{tabular}{llllll} 
KT3-1-2 & 3 & 48.9900 & 0.71310 & 47.346 & 50.634 \\
KT3-2-1 & 3 & 48.4908 & 0.71310 & 46.846 & 50.135 \\
\hline
\end{tabular}

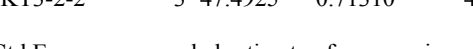

Std Error uses a pooled estimate of error variance 
Exhibit B-3. Statistical Evaluation of the ICP-AES Calibration Effects from the KT03 Batch 1 Results by Oxide. (continued)

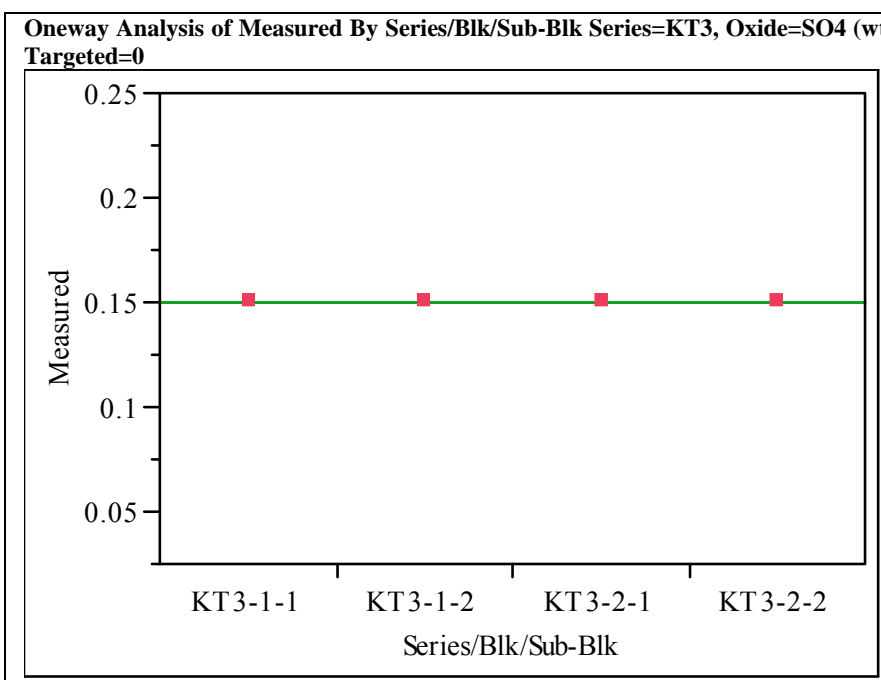

Oneway Anova

Oneway Anova
Summary of Fit

Rsquare

Adj Rsquare

Root Mean Square Error $\quad 0$

Mean of Response

0.149795

Observations (or Sum Wgts)

Analysis of Variance

Source DF Sum of Squares Mean Square F Ratio Prob $>$ F

$\begin{array}{lrrr}\text { Source } & \text { DF } & \text { Sum of Squares } & \text { Mean Square } \\ \text { Series/Blk/Sub-Blk } & 3 & 0 & 0 \\ \text { Error } & 8 & 0 & 0 \\ \text { C } & 8 & 0 & \end{array}$

Error $\quad 8$

$\begin{array}{rr}8 & 0 \\ 11 & 0\end{array}$

C. Total

Means for Oneway Anova

Level Number Mean Std Error Lower 95\% Upper 95\%

$\begin{array}{llllll}\text { KT3-1-1 } & 3 & 0.149795 & 0 & 0.14980 & 0.14980 \\ \text { KT3-1-2 } & 3 & 0.149795 & 0 & 0.14980 & 0.14980\end{array}$

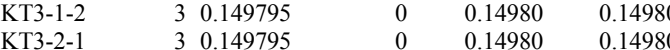

$\begin{array}{llllll}\text { KT3-2-1 } & 3 & 0.149955 & 0 & 0.14980 & 0.14980 \\ \text { KT3-2-2 } & 3 & 0.149795 & 0 & 0.14980 & 0.14980\end{array}$

Std Error uses a pooled estimate of error variance

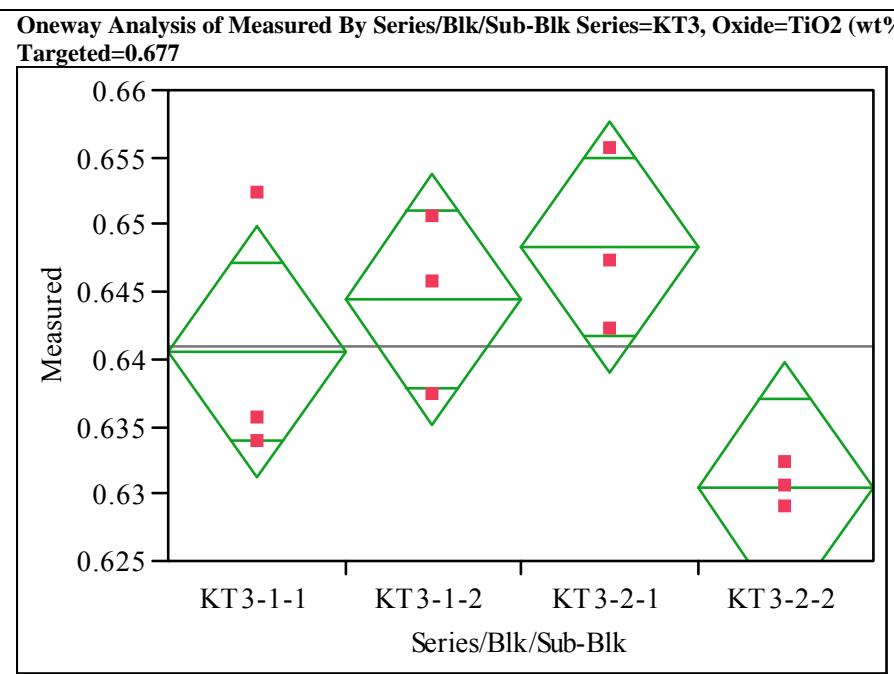

\section{Oneway Anova}

Summary of Fit

$\begin{array}{ll}\text { Rsquare } & 0.572041 \\ \text { Adj Rsquare } & 0.411557\end{array}$

$\begin{array}{ll}\text { Adj Rsquare } & 0.411557 \\ \text { Root Mean Square Error } & 0.007011\end{array}$

Root Mean Square Error $\quad 0.007011$

Observations (or Sum Wgts)

Analysis of Variance

Source DF Sum of Squares Mean Square F Ratio Prob $>$ F

$\begin{array}{lrrrrr} & & & \\ \text { Sories/Blk/Sub-Blk } & 3 & 0.00052561 & 0.000175 & 3.5645 & 0.0669\end{array}$

$\begin{array}{lrrr}\text { Error } & 8 & 0.00039322 & 0.000049\end{array}$

$\begin{array}{lrr}\text { C. Total } & 11 & 0.00091883\end{array}$

Means for Oneway Anova

Level Number Mean Std Error Lower 95\% Upper 95\%

\begin{tabular}{lrrrrr} 
KT3-1-1 $^{2}$ & 3 & 0.640512 & 0.00405 & 0.63118 & 0.64985 \\
\hline
\end{tabular}

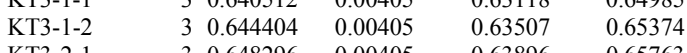

$\begin{array}{llllll}\text { KT3-1-2 } & 3 & 0.644404 & 0.00405 & 0.63507 & 0.65374 \\ \text { KT3-2-1 } & 3 & 0.648296 & 0.00405 & 0.63896 & 0.65763\end{array}$

$\begin{array}{llllll}\text { KT3-2-2 } & 3 & 0.630504 & 0.00405 & 0.62117 & 0.63984\end{array}$

Std Error uses a pooled estimate of error variance 
Exhibit B-3. Statistical Evaluation of the ICP-AES Calibration Effects from the KT03 Batch 1 Results by Oxide. (continued)

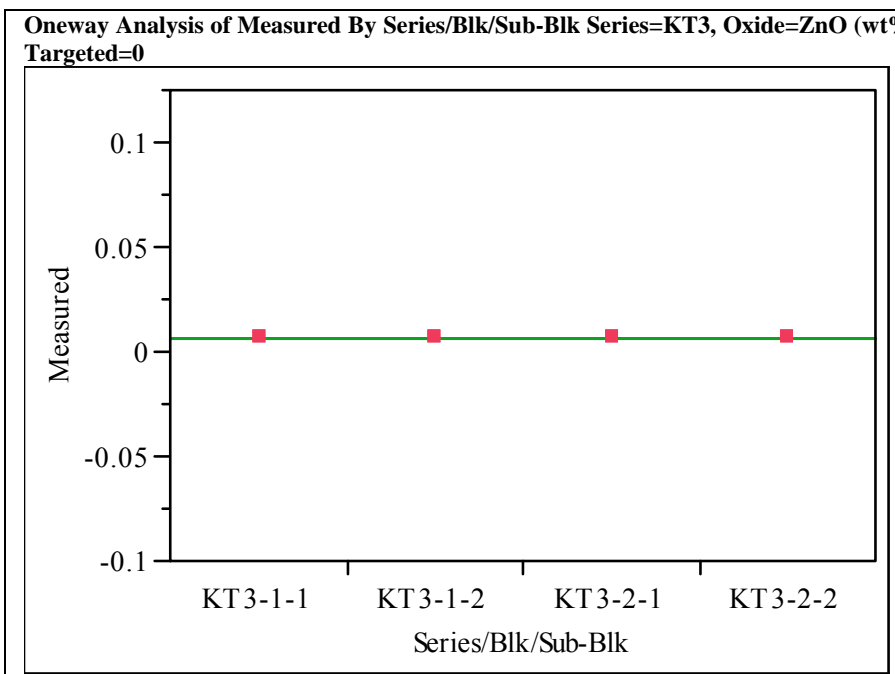

\section{Oneway Anova}

Summary of Fit

Rsquare

Adj Rsquare

$\begin{array}{lr}\text { Root Mean Square Error } & 0 \\ \text { Mean of Response } & 0.006224\end{array}$

Analysis of Variance

Source DF Sum of Squares Mean Square F Ratio Prob $>$ F

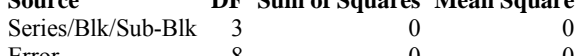

$\begin{array}{ll}\text { Series/Blk/Sub-Blk } & 3 \\ \text { Error } & 8\end{array}$

$\begin{array}{rl}8 & 0 \\ 11 & 0\end{array}$

C. Total

Means for Oneway Anova

Level Number Mean Std Error Lower 95\% Upper 95\%

$\begin{array}{llllll}\text { KT3-1-1 } & 3 & 0.006224 & 0 & 0.00622 & 0.00622\end{array}$

$\begin{array}{llllll}\text { KT3-1-2 } & 3 & 0.006224 & 0 & 0.00622 & 0.00622\end{array}$

$\begin{array}{llllll}\text { KT3-2-1 } & 3 & 0.006224 & 0 & 0.00622 & 0.00622 \\ \text { KT3-2-2 } & 3 & 0.006224 & 0 & 0.00622 & 0.00622\end{array}$

Std Error uses a pooled estimate of error variance

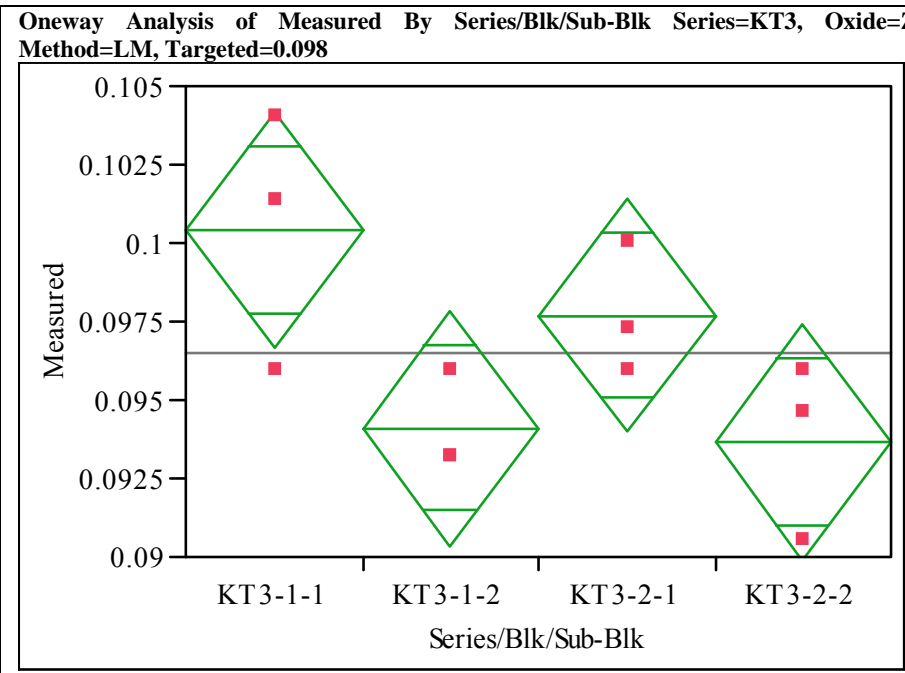

\section{Oneway Anova}

Summary of Fit

$\begin{array}{ll}\text { Rsquare } & 0.591757 \\ \text { Adj Rsquare } & 0.438665\end{array}$

0.438665
Root Mean Square Error $\quad 0.002812$

Root Mean Square Error $\quad 0.002812$
Mean of Response

Observations (or Sum Wgts)

Analysis of Variance

Source DF Sum of Squares Mean Square F Ratio Prob $>$ F

$\begin{array}{lrrrrr} & & & & \\ \text { Series/Blk/Sub-Blk } & 3 & 0.00009169 & 0.000031 & 3.8654 & 0.0560\end{array}$

$\begin{array}{lrrr}\text { Error } & 8 & 0.00006325 & 7.907 \mathrm{e}-6\end{array}$

$\begin{array}{lrr}\text { C. Total } & 11 & 0.00015494\end{array}$

Means for Oneway Anova

Level Number Mean Std Error Lower 95\% Upper 95\%

\begin{tabular}{lrrrrr} 
Level & Number & Mean & Std Error & Lower $95 \%$ & Upper $95 \%$ \\
KT3-1-1 & 3 & 0.100409 & 0.00162 & 0.09667 & 0.10415 \\
\hline
\end{tabular}

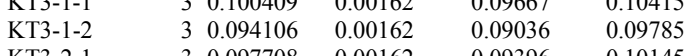

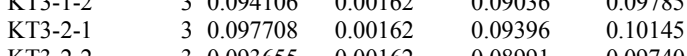

$\begin{array}{llllll}\text { KT3-2-2 } & 3 & 0.093655 & 0.00162 & 0.08991 & 0.09740\end{array}$

Std Error uses a pooled estimate of error variance 
Exhibit B-4. Plots of Measured versus Targeted Concentrations by Glass ID by Oxide for the KT03-Series.

Oxide=Al2O3 (wt \%)

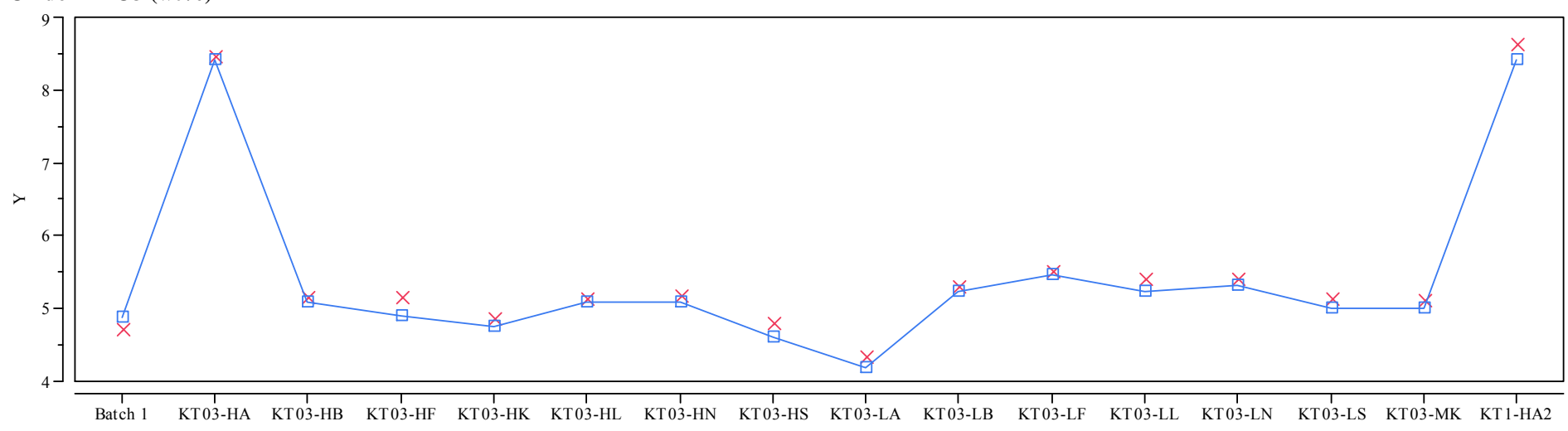

Glass ID

Oxide=B2O3 (wt\%)

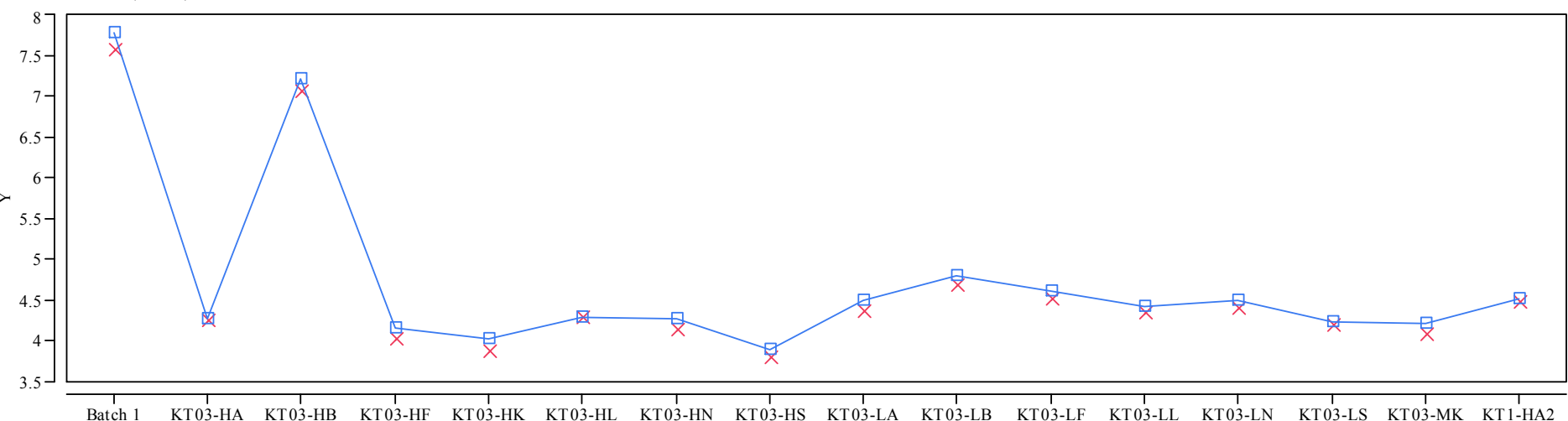

Glass ID

$\mathrm{Y} \times$ Measured $\quad \square-$ Targeted 
Exhibit B-4. Plots of Measured versus Targeted Concentrations by Glass ID by Oxide for the KT03-Series. (continued) Oxide $=\mathbf{B a O}(w t \%)$

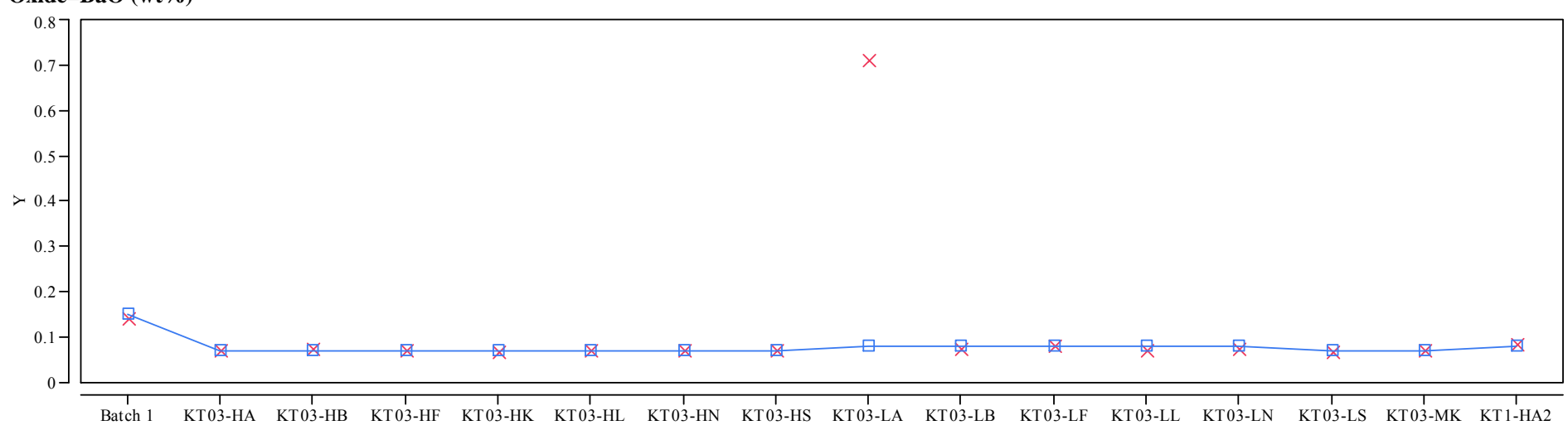

Glass ID

Oxide $=\mathrm{CaO}(\mathrm{wt} \%)$

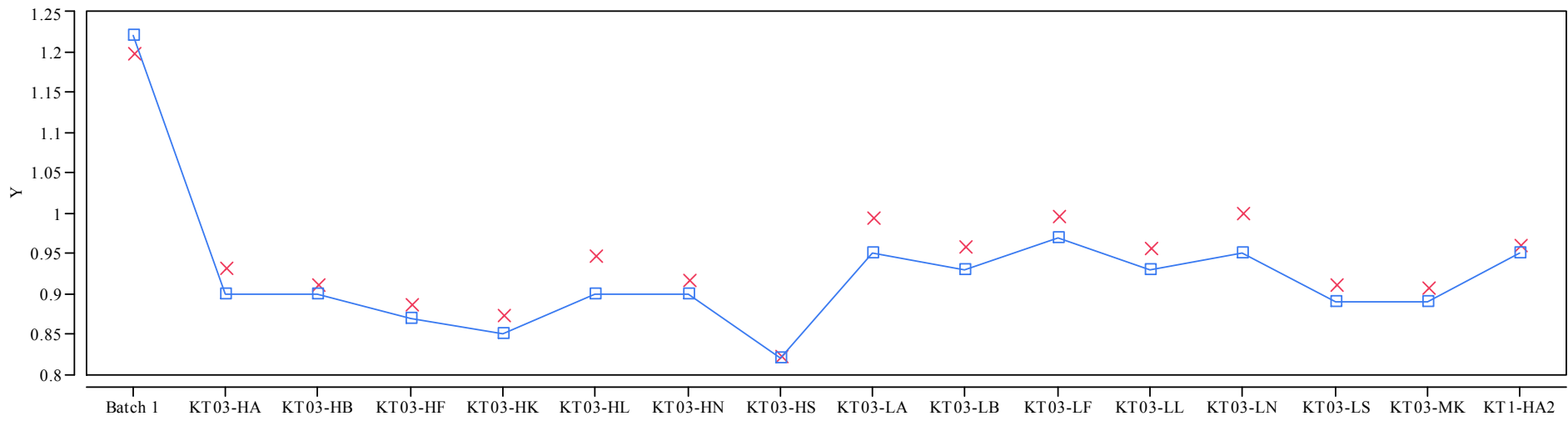

Glass ID

$\mathrm{Y} \times$ Measured $\square-$ Targeted 
Exhibit B-4. Plots of Measured versus Targeted Concentrations by Glass ID by Oxide for the KT03-Series. (continued) Oxide $=\mathrm{Ce} 2 \mathrm{O} 3(\mathrm{wt} \%)$

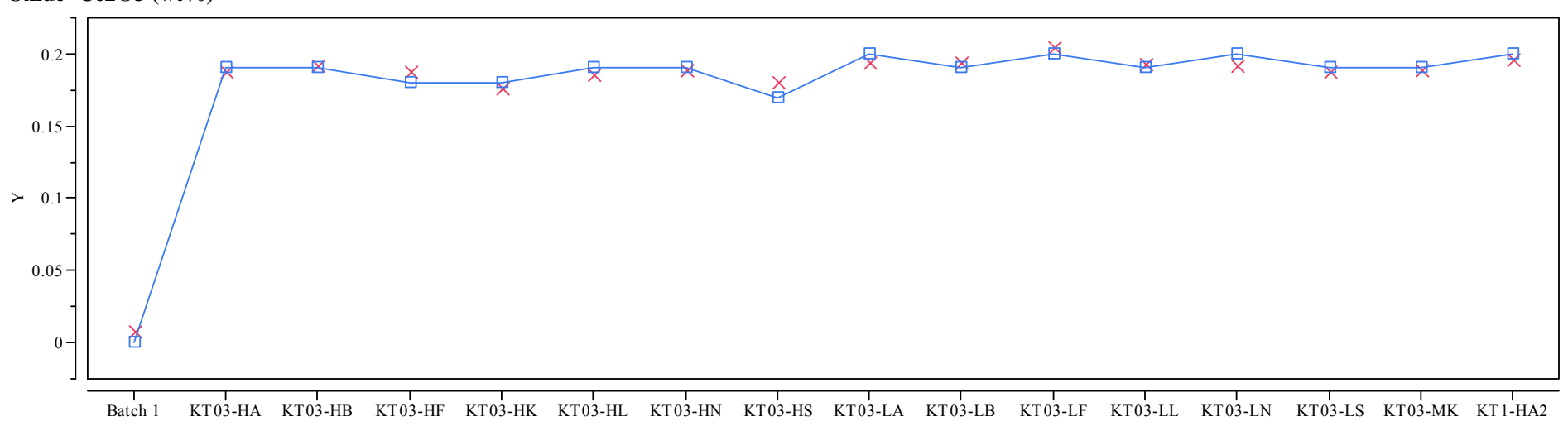

Glass ID

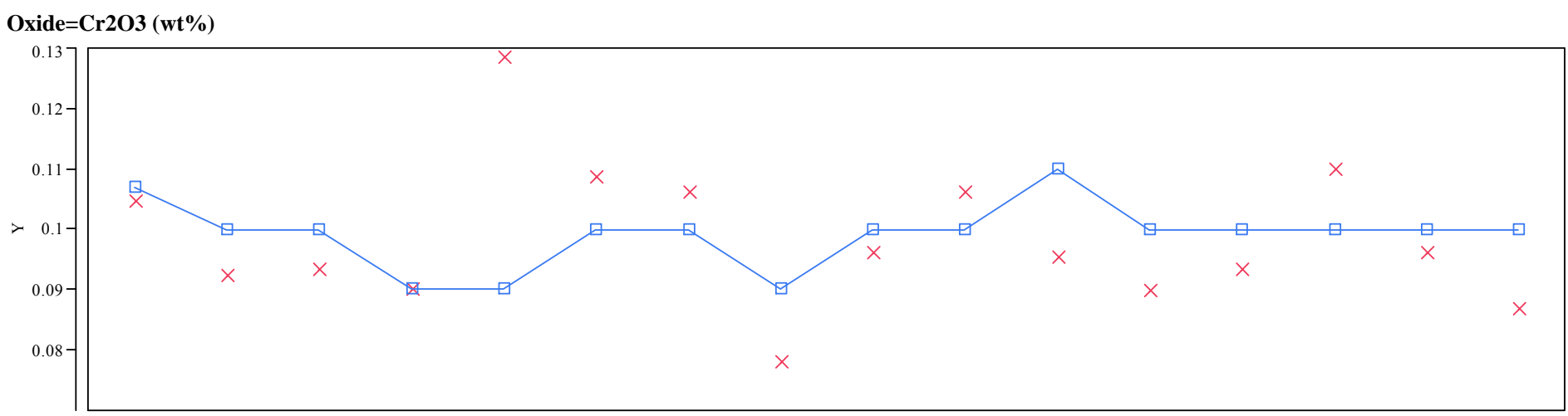

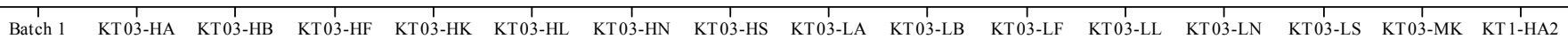

$$
\text { Glass ID }
$$

$\mathrm{Y} \times$ Measured $\quad \square-$ Targeted 
Exhibit B-4. Plots of Measured versus Targeted Concentrations by Glass ID by Oxide for the KT03-Series. (continued) Oxide $=\mathrm{CuO}(\mathrm{wt} \%)$

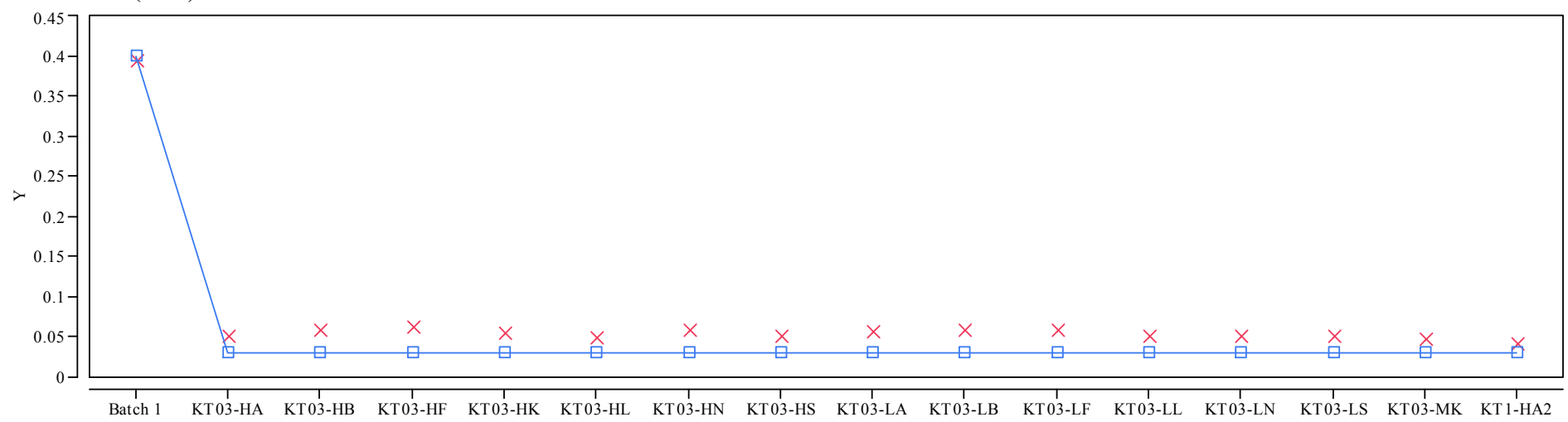

Glass ID

Oxide=Fe2O3 (wt $\%)$

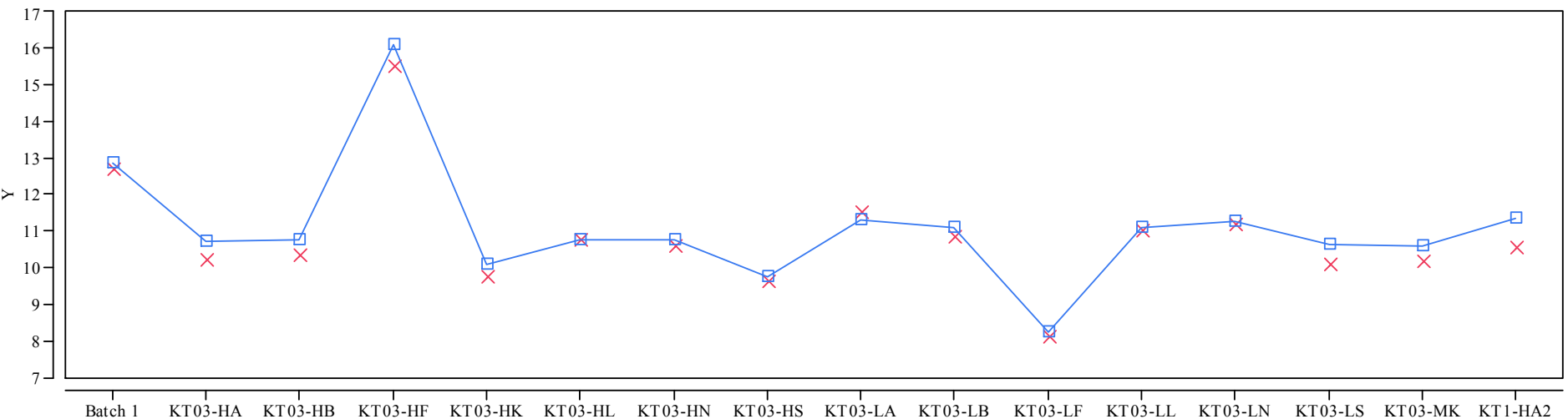

Glass ID

$\mathrm{Y} \times$ Measured $\quad \square-$ Targeted 
Exhibit B-4. Plots of Measured versus Targeted Concentrations by Glass ID by Oxide for the KT03-Series. (continued) Oxide=K2O (wt\%)

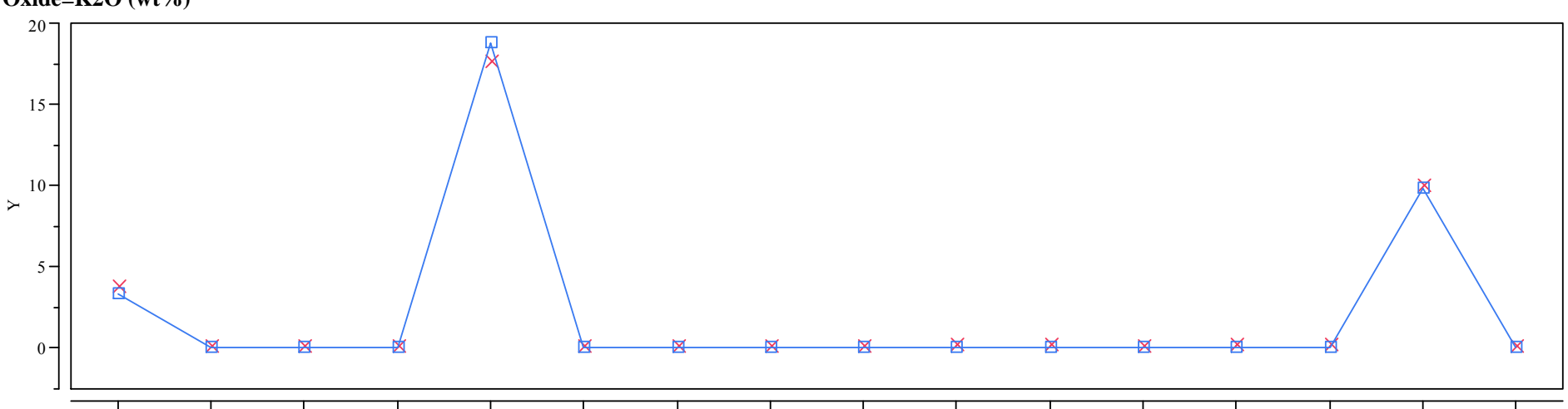

Batch 1 KT03-HA

Glass ID

Oxide=La2O3 (wt \%)

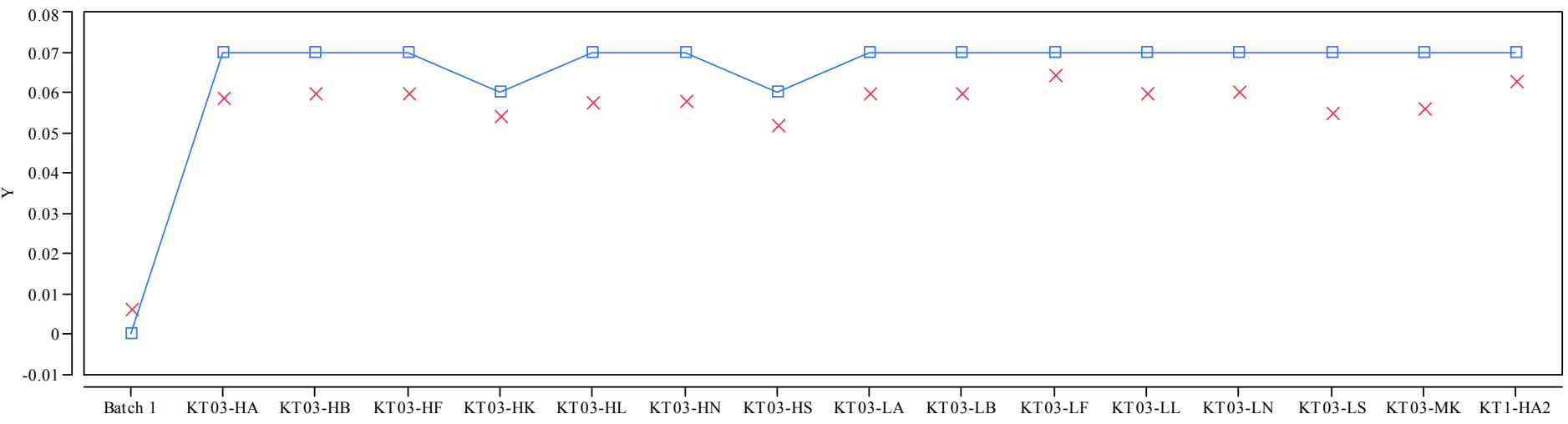

Glass ID

$\mathrm{Y} \times$ Measured $\square-$ Targeted 
Exhibit B-4. Plots of Measured versus Targeted Concentrations by Glass ID by Oxide for the KT03-Series. (continued) Oxide=Li2O (wt\%)

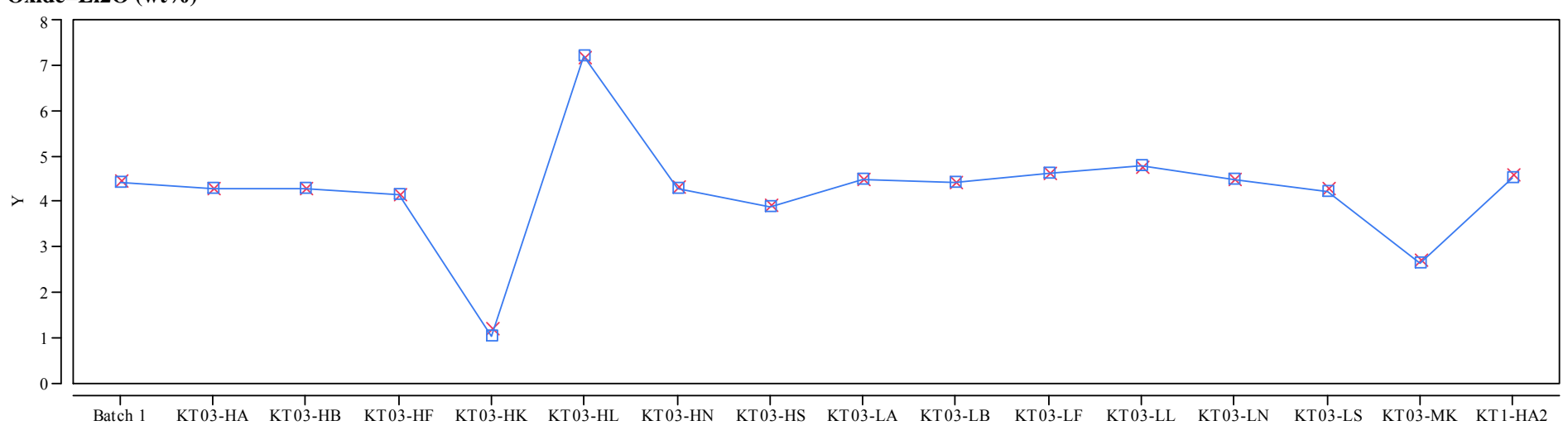

Glass ID

Oxide=MgO (wt \%)

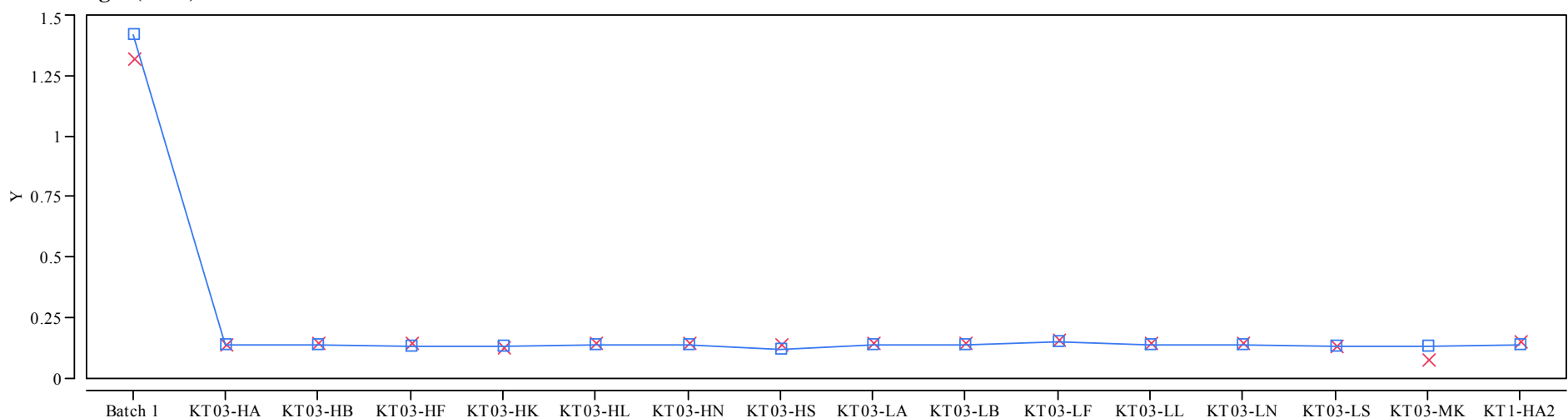

Glass ID

$\mathrm{Y} \times$ Measured $\quad \square-$ Targeted 
Exhibit B-4. Plots of Measured versus Targeted Concentrations by Glass ID by Oxide for the KT03-Series. (continued) Oxide $=\mathrm{MnO}(\mathrm{wt} \%)$

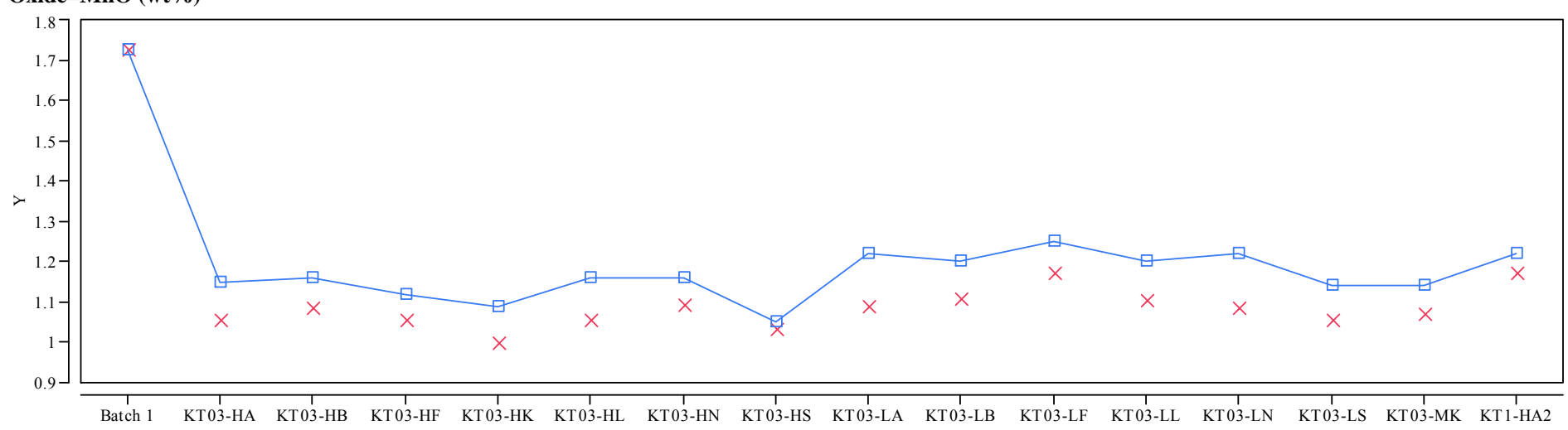

Glass ID

Oxide=Na2O $(w t \%)$

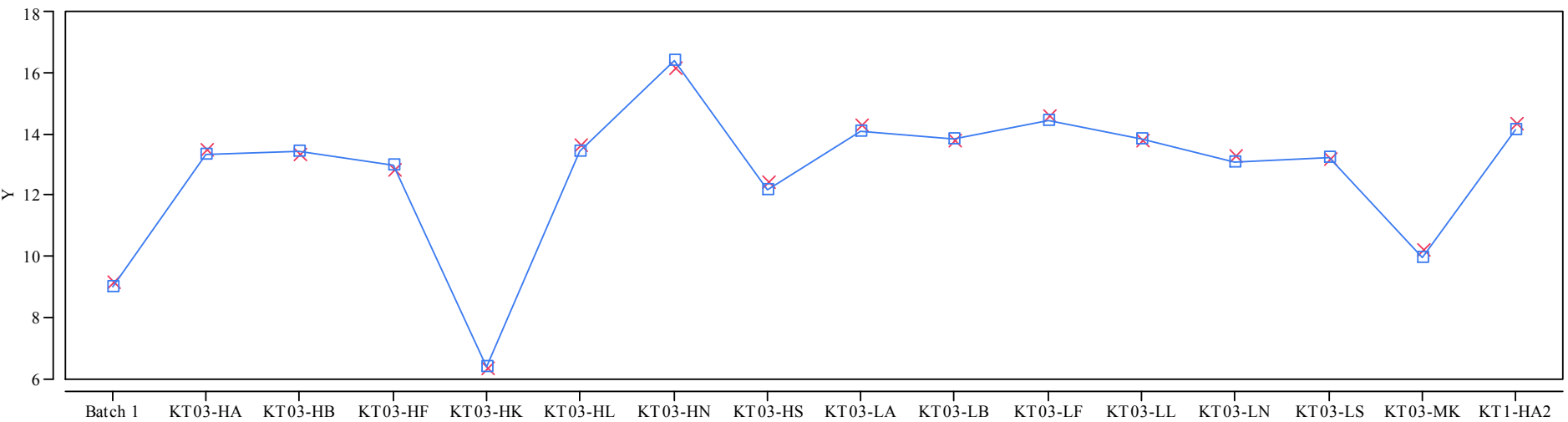

Glass ID

$\mathrm{Y} \times$ Measured $\quad \square-$ Targeted 
Exhibit B-4. Plots of Measured versus Targeted Concentrations by Glass ID by Oxide for the KT03-Series. (continued) Oxide $=\mathrm{Nb} 2 \mathrm{O} 5(\mathrm{wt} \%)$

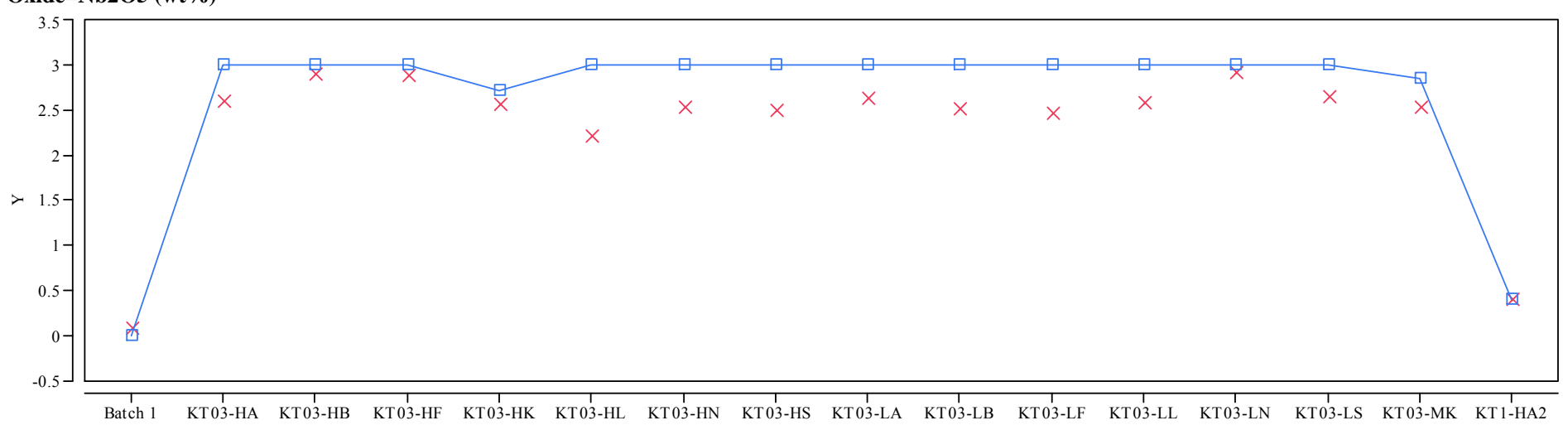

Glass ID

Oxide $=\mathrm{NiO}(\mathrm{wt} \%)$

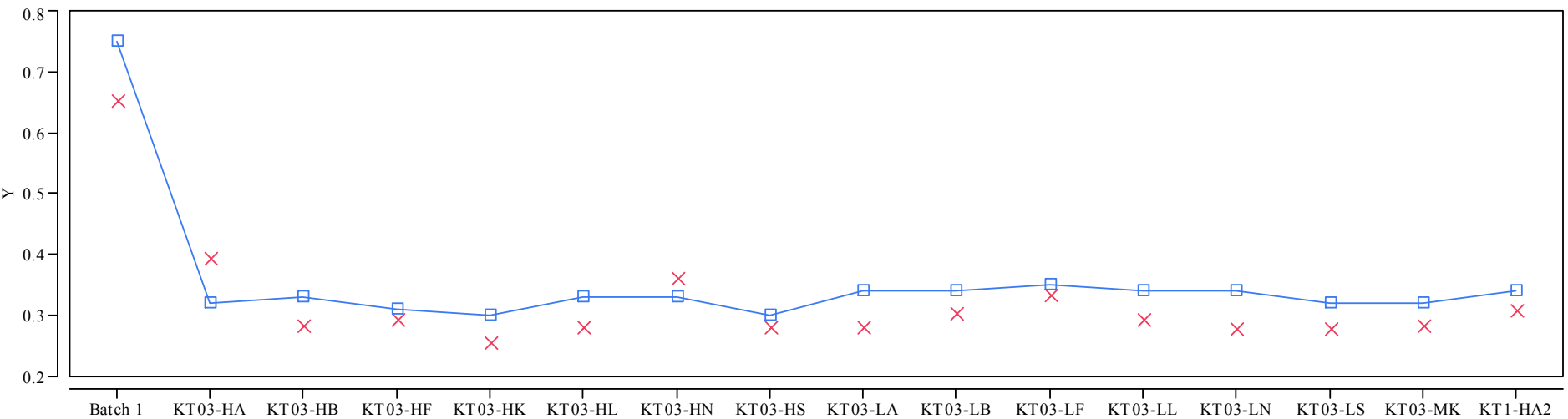

Glass ID

$\mathrm{Y} \times$ Measured $\square-$ Targeted 
Exhibit B-4. Plots of Measured versus Targeted Concentrations by Glass ID by Oxide for the KT03-Series. (continued) Oxide=PbO (wt\%)

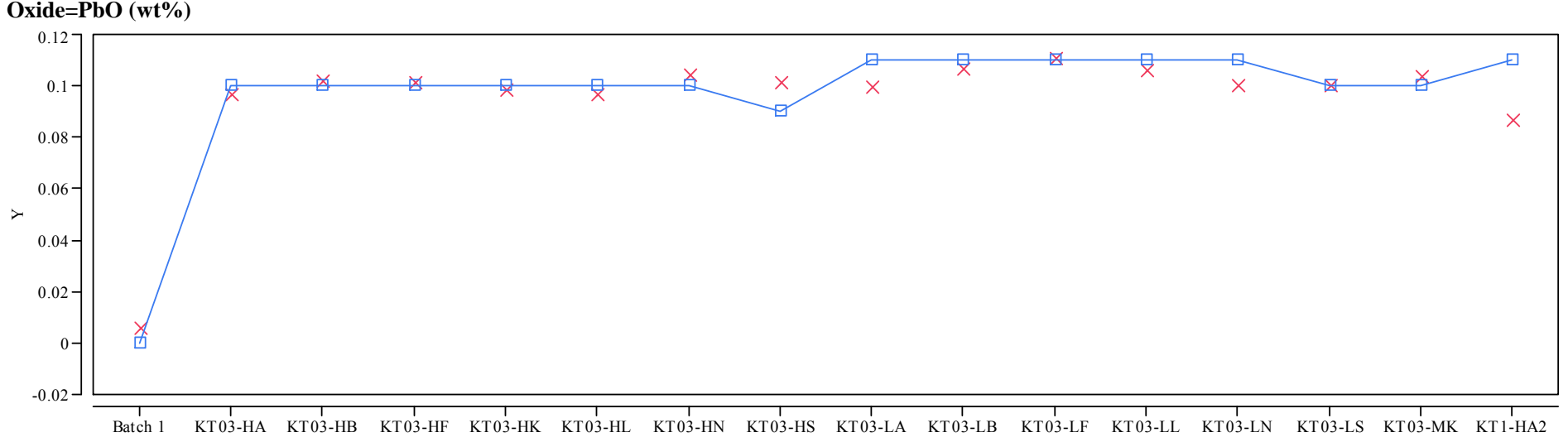

Glass ID

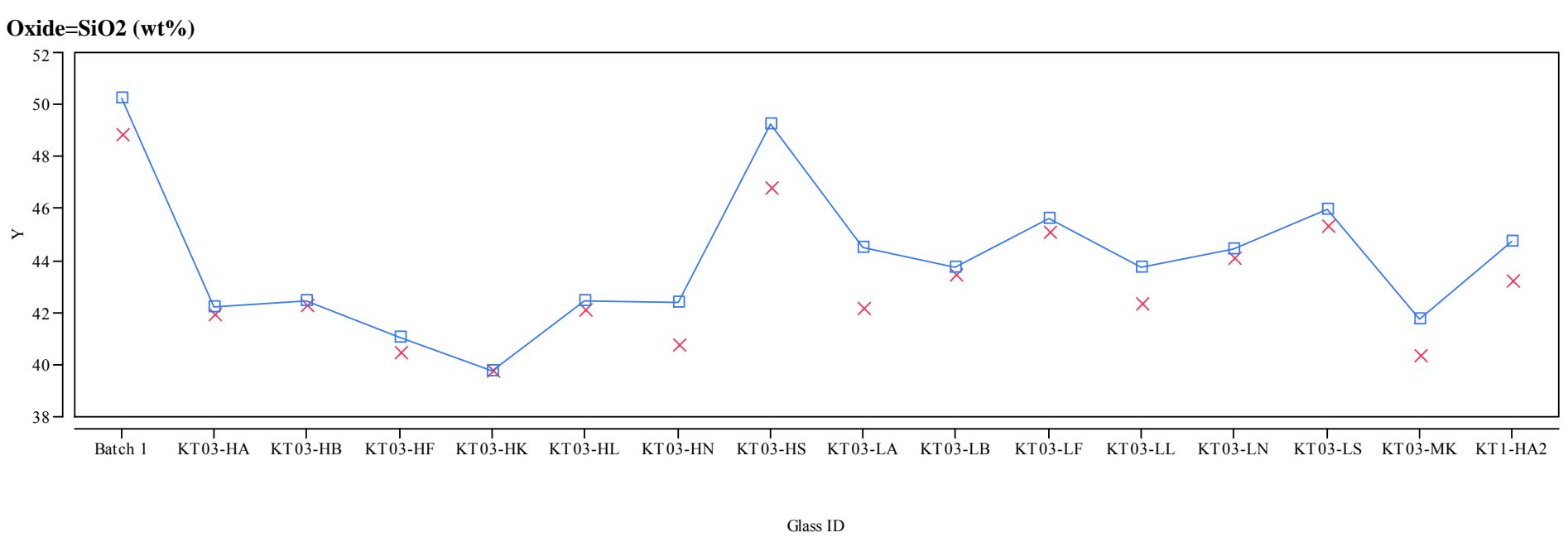

Y $\times$ Measured $\quad \square-$ Targeted 
Exhibit B-4. Plots of Measured versus Targeted Concentrations by Glass ID by Oxide for the KT03-Series. (continued) Oxide $=\mathrm{SO} 4(\mathrm{wt} \%)$

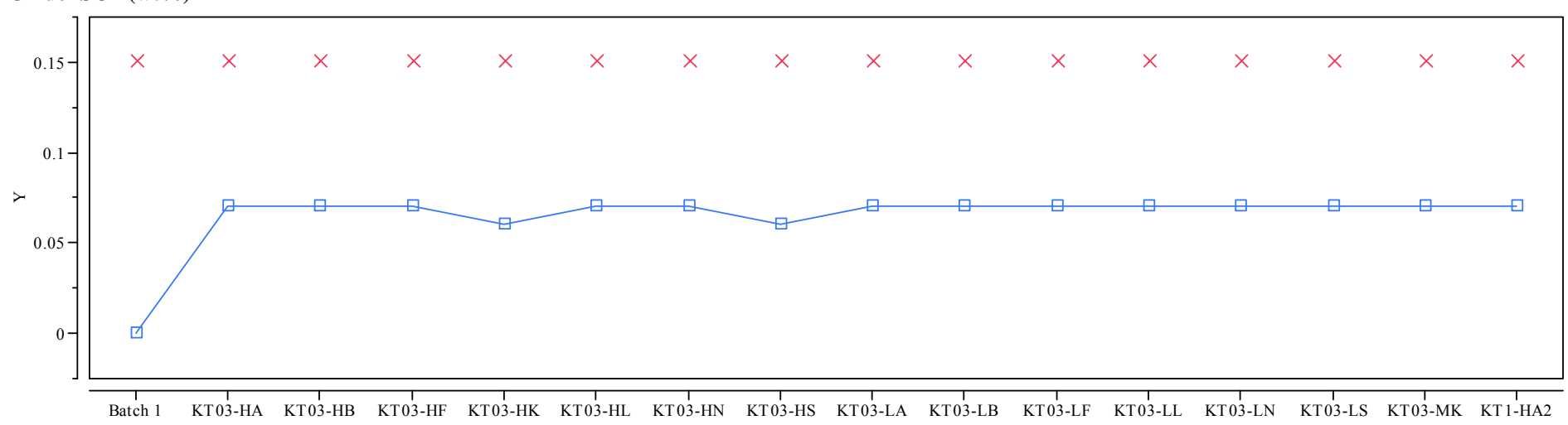

Glass ID

Oxide=TiO2 $($ wt $\%)$

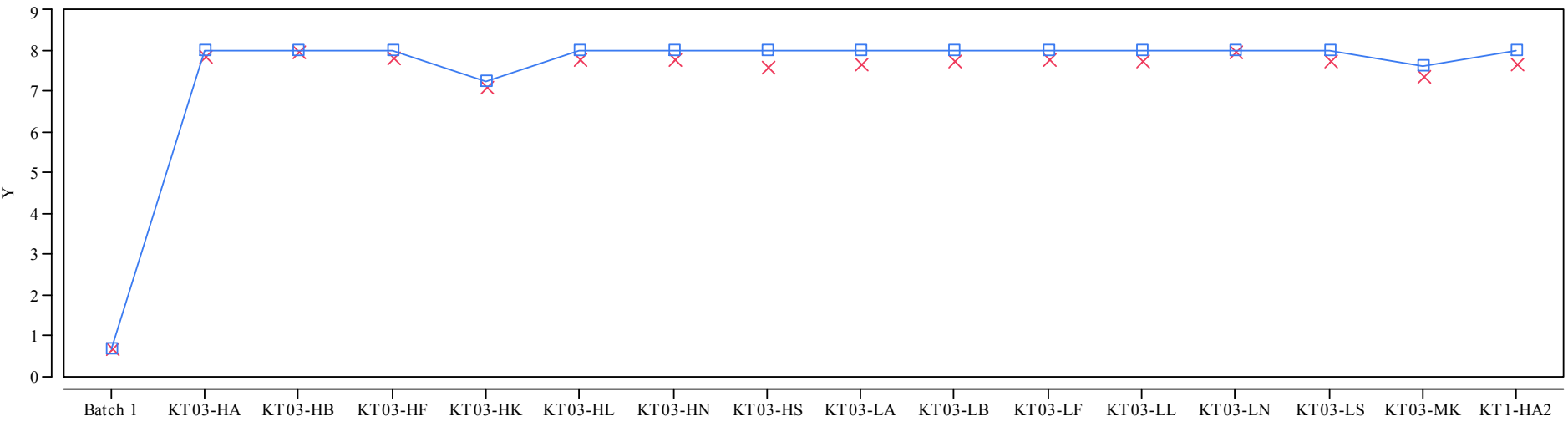

Glass ID

$\mathrm{Y} \times$ Measured $\quad \square-$ Targeted 
Exhibit B-4. Plots of Measured versus Targeted Concentrations by Glass ID by Oxide for the KT03-Series. (continued) Oxide $=\mathrm{ZnO}(\mathrm{wt} \%)$

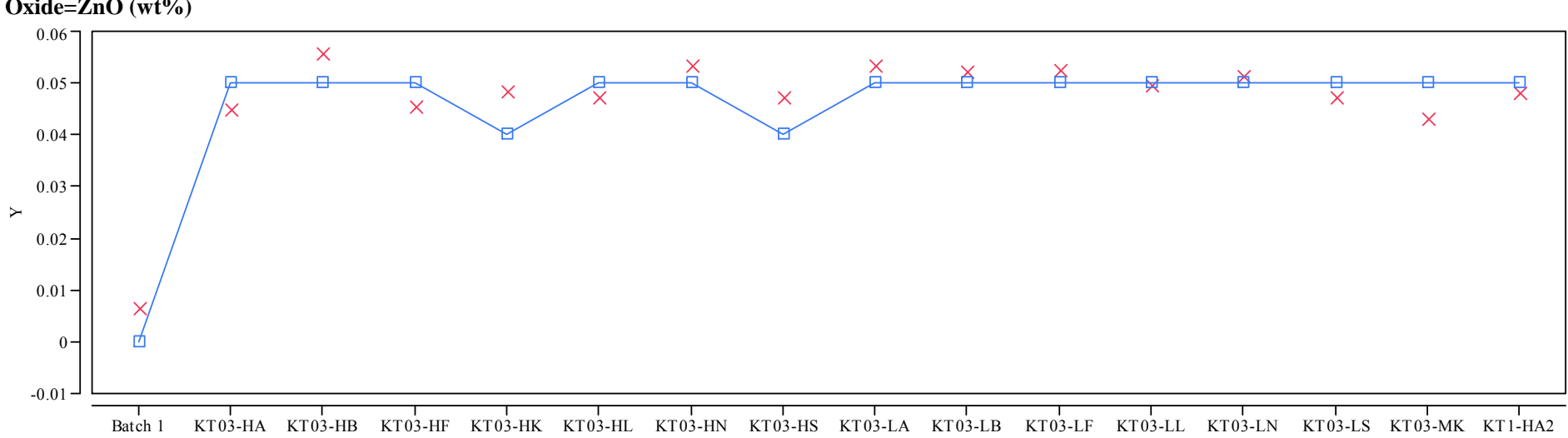

Glass ID

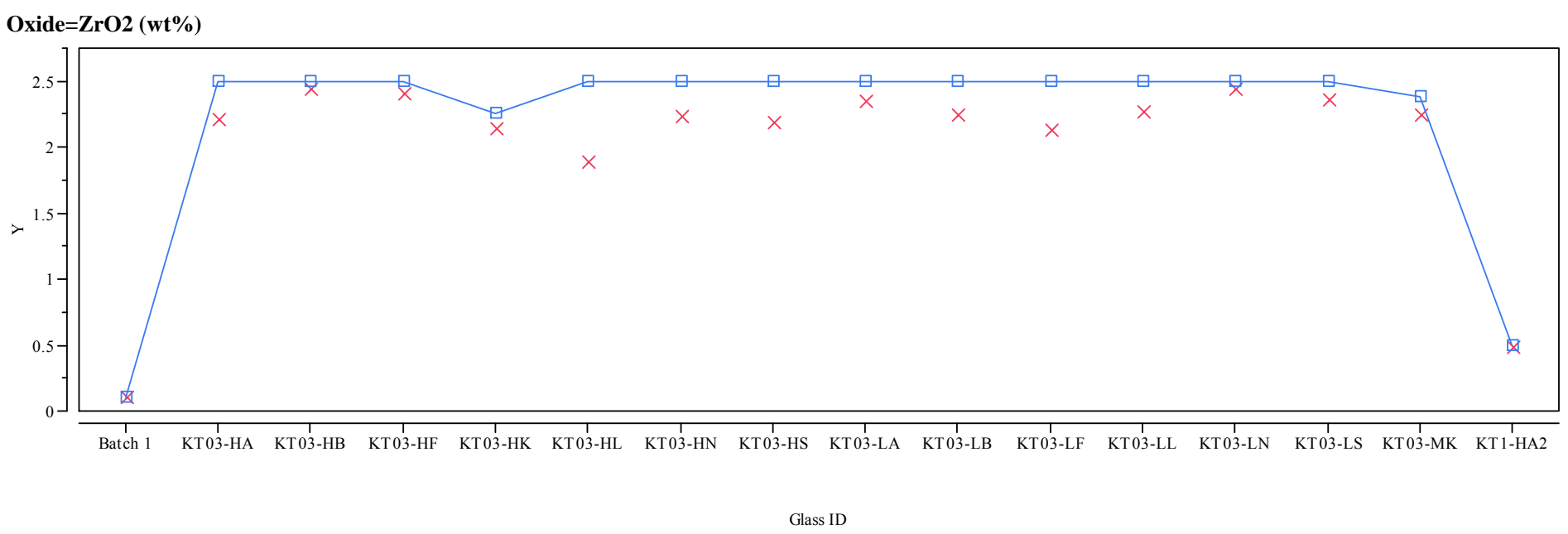

$\mathrm{Y} \times$ Measured $\quad \square-$ Targeted 
Exhibit B-4. Plots of Measured versus Targeted Concentrations by Glass ID by Oxide for the KT03-Series. (continued) Oxide=Sum of Oxides

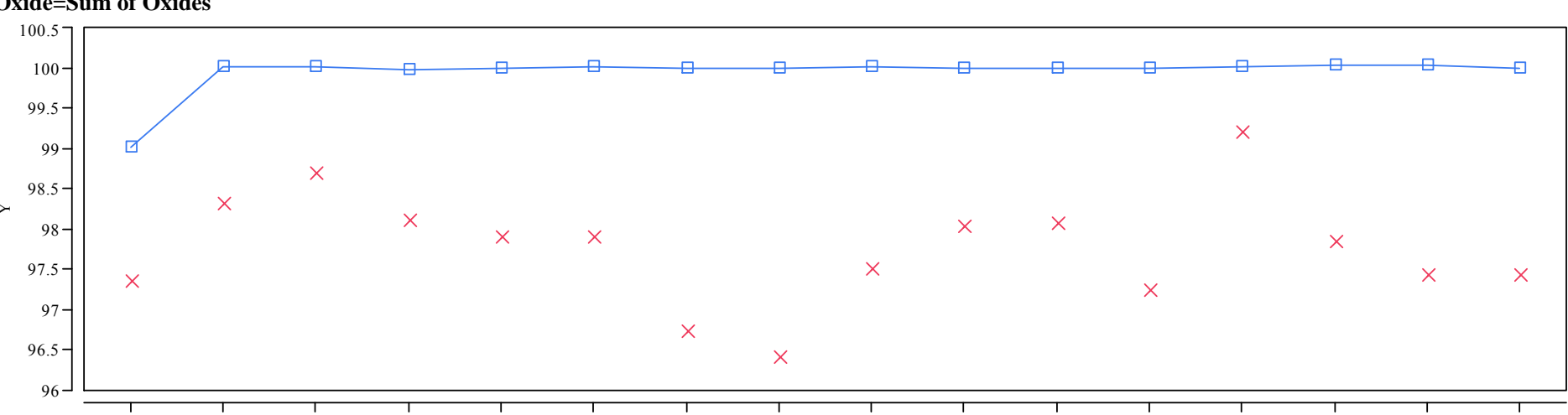

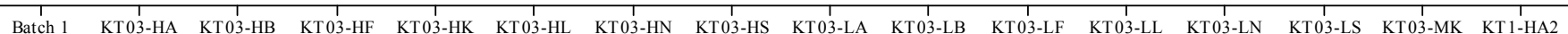

$$
\text { Glass ID }
$$

Y $\times$ Measured

$$
\square \text { - Targeted }
$$


SRNL-STI-2010-00566

Revision 0

Appendix C. Data Supporting the Chemical Composition Measurements of the KT04-Series Glasses 
SRNL-STI-2010-00566

Revision 0

Table C-1. PSAL Chemical Composition Measurements of the KT04-Series of Glasses Using LM Preparation Method (Part 1).

\begin{tabular}{|c|c|c|c|c|c|c|c|c|c|c|c|c|c|c|}
\hline Glass ID & Block & $\begin{array}{c}\text { Sub- } \\
\text { Blk }\end{array}$ & Seq & Lab ID & $\begin{array}{c}\text { Al } \\
(w t \%)\end{array}$ & $\begin{array}{c}\text { Ba } \\
(w t \%)\end{array}$ & $\begin{array}{c}\text { Ca } \\
(w t \%)\end{array}$ & $\begin{array}{c}\text { Ce } \\
\text { (wt\%) }\end{array}$ & $\begin{array}{c}\mathrm{Cr} \\
(\mathrm{wt} \%)\end{array}$ & $\begin{array}{c}\mathrm{Cu} \\
(\mathrm{wt} \%)\end{array}$ & $\begin{array}{c}\text { Fe } \\
(w t \%)\end{array}$ & $\begin{array}{c}\mathbf{K} \\
(\mathrm{wt} \%)\end{array}$ & $\begin{array}{c}\text { La } \\
(w t \%)\end{array}$ & $\underset{(w t \%)}{M g}$ \\
\hline Batch 1 & 1 & 1 & 1 & BCHPF111 & 2.53 & 0.126 & 0.862 & $<0.010$ & 0.076 & 0.312 & 8.78 & 2.29 & $<0.010$ & 0.822 \\
\hline KT04-09 & 1 & 1 & 2 & A10LM21 & 3.08 & 0.059 & 0.730 & 0.157 & 0.046 & 0.069 & 8.45 & 0.089 & 0.058 & 0.064 \\
\hline KT04-07 & 1 & 1 & 3 & A04LM11 & 3.08 & 0.062 & 0.652 & 0.066 & 0.076 & 0.034 & 6.47 & 0.072 & 0.029 & 0.067 \\
\hline KT04-08 & 1 & 1 & 4 & A02LM11 & 2.78 & 0.063 & 0.603 & 0.069 & 0.072 & 0.044 & 6.42 & 0.077 & 0.030 & 0.066 \\
\hline KT04-03 & 1 & 1 & 5 & A03LM11 & 2.49 & 0.055 & 0.726 & 0.199 & 0.054 & 0.037 & 7.81 & 0.039 & 0.053 & 0.086 \\
\hline KT04-06 & 1 & 1 & 6 & A06LM21 & 3.94 & 0.055 & 0.735 & 0.085 & 0.059 & 0.051 & 6.13 & 0.112 & 0.023 & 0.058 \\
\hline Batch 1 & 1 & 1 & 7 & BCHPF112 & 2.50 & 0.127 & 0.855 & $<0.010$ & 0.076 & 0.311 & 8.80 & 2.27 & $<0.010$ & 0.816 \\
\hline KT04-07 & 1 & 1 & 8 & A04LM21 & 3.09 & 0.063 & 0.651 & 0.066 & 0.076 & 0.036 & 6.40 & 0.074 & 0.028 & 0.066 \\
\hline KT04-06 & 1 & 1 & 9 & A06LM11 & 3.94 & 0.056 & 0.738 & 0.087 & 0.060 & 0.056 & 6.12 & 0.115 & 0.024 & 0.058 \\
\hline KT04-08 & 1 & 1 & 10 & A02LM21 & 2.76 & 0.064 & 0.604 & 0.068 & 0.073 & 0.039 & 6.30 & 0.081 & 0.029 & 0.064 \\
\hline KT04-09 & 1 & 1 & 11 & A10LM11 & 3.06 & 0.060 & 0.727 & 0.159 & 0.049 & 0.046 & 8.42 & 0.090 & 0.058 & 0.064 \\
\hline KT04-03 & 1 & 1 & 12 & A03LM21 & 2.44 & 0.057 & 0.708 & 0.206 & 0.057 & 0.037 & 8.13 & 0.034 & 0.054 & 0.090 \\
\hline Batch 1 & 1 & 1 & 13 & BCHPF113 & 2.52 & 0.127 & 0.850 & $<0.010$ & 0.077 & 0.310 & 8.82 & 2.26 & $<0.010$ & 0.820 \\
\hline Batch 1 & 1 & 2 & 1 & BCHPF121 & 2.54 & 0.123 & 0.859 & $<0.010$ & 0.075 & 0.310 & 8.70 & 2.25 & $<0.010$ & 0.816 \\
\hline KT04-06 & 1 & 2 & 2 & A06LM22 & 4.03 & 0.054 & 0.728 & 0.087 & 0.059 & 0.050 & 6.08 & 0.111 & 0.022 & 0.057 \\
\hline KT04-06 & 1 & 2 & 3 & A06LM12 & 4.01 & 0.054 & 0.742 & 0.086 & 0.058 & 0.055 & 6.15 & 0.115 & 0.022 & 0.056 \\
\hline KT04-03 & 1 & 2 & 4 & A03LM12 & 2.52 & 0.054 & 0.734 & 0.199 & 0.054 & 0.037 & 7.73 & 0.040 & 0.051 & 0.083 \\
\hline KT04-07 & 1 & 2 & 5 & A04LM12 & 3.12 & 0.060 & 0.655 & 0.067 & 0.075 & 0.034 & 6.37 & 0.074 & 0.027 & 0.064 \\
\hline KT04-08 & 1 & 2 & 6 & A02LM12 & 2.84 & 0.061 & 0.611 & 0.070 & 0.071 & 0.045 & 6.28 & 0.079 & 0.028 & 0.063 \\
\hline Batch 1 & 1 & 2 & 7 & BCHPF122 & 2.57 & 0.120 & 0.869 & $<0.010$ & 0.073 & 0.313 & 8.58 & 2.29 & $<0.010$ & 0.792 \\
\hline KT04-08 & 1 & 2 & 8 & A02LM22 & 2.82 & 0.061 & 0.616 & 0.068 & 0.071 & 0.039 & 6.19 & 0.083 & 0.027 & 0.061 \\
\hline KT04-09 & 1 & 2 & 9 & A10LM22 & 3.11 & 0.058 & 0.736 & 0.159 & 0.047 & 0.070 & 8.40 & 0.091 & 0.057 & 0.061 \\
\hline KT04-03 & 1 & 2 & 10 & A03LM22 & 2.52 & 0.057 & 0.719 & 0.208 & 0.056 & 0.037 & 7.99 & 0.036 & 0.053 & 0.086 \\
\hline KT04-09 & 1 & 2 & 11 & A10LM12 & 3.15 & 0.059 & 0.738 & 0.161 & 0.048 & 0.047 & 8.41 & 0.093 & 0.057 & 0.061 \\
\hline KT04-07 & 1 & 2 & 12 & A04LM22 & 3.12 & 0.062 & 0.649 & 0.067 & 0.075 & 0.036 & 6.40 & 0.074 & 0.027 & 0.064 \\
\hline Batch 1 & 1 & 2 & 13 & BCHPF123 & 2.57 & 0.127 & 0.853 & $<0.010$ & 0.076 & 0.314 & 8.86 & 2.25 & $<0.010$ & 0.818 \\
\hline Batch 1 & 2 & 1 & 1 & BCHPF211 & 2.50 & 0.121 & 0.854 & $<0.010$ & 0.073 & 0.304 & 8.68 & 2.31 & $<0.010$ & 0.803 \\
\hline KT04-05 & 2 & 1 & 2 & A01LM21 & 3.69 & 0.054 & 0.687 & 0.118 & 0.101 & 0.056 & 6.55 & 0.070 & 0.049 & 0.086 \\
\hline KT04-10 & 2 & 1 & 3 & A05LM21 & 3.02 & 0.068 & 0.711 & 0.213 & 0.054 & 0.044 & 9.28 & 0.037 & 0.064 & 0.066 \\
\hline KT04-10 & 2 & 1 & 4 & A05LM11 & 3.03 & 0.067 & 0.719 & 0.210 & 0.054 & 0.043 & 9.31 & 0.040 & 0.063 & 0.065 \\
\hline KT04-02 & 2 & 1 & 5 & A07LM21 & 2.79 & 0.060 & 0.696 & 0.242 & 0.055 & 0.040 & 7.86 & 0.032 & 0.054 & 0.087 \\
\hline KT04-01 & 2 & 1 & 6 & A08LM11 & 3.13 & 0.054 & 0.698 & 0.230 & 0.050 & 0.047 & 8.19 & 0.035 & 0.076 & 0.083 \\
\hline Batch 1 & 2 & 1 & 7 & BCHPF212 & 2.48 & 0.122 & 0.861 & $<0.010$ & 0.073 & 0.310 & 8.76 & 2.32 & $<0.010$ & 0.783 \\
\hline KT04-01 & 2 & 1 & 8 & A08LM21 & 3.07 & 0.054 & 0.688 & 0.232 & 0.050 & 0.043 & 8.24 & 0.033 & 0.077 & 0.083 \\
\hline KT04-05 & 2 & 1 & 9 & A01LM11 & 3.69 & 0.052 & 0.681 & 0.116 & 0.098 & 0.046 & 6.62 & 0.067 & 0.049 & 0.086 \\
\hline KT04-04 & 2 & 1 & 10 & A09LM11 & 2.67 & 0.051 & 0.655 & 0.168 & 0.053 & 0.042 & 8.25 & 0.040 & 0.048 & 0.082 \\
\hline KT04-02 & 2 & 1 & 11 & A07LM11 & 2.78 & 0.057 & 0.709 & 0.233 & 0.052 & 0.035 & 7.96 & 0.034 & 0.052 & 0.084 \\
\hline KT04-04 & 2 & 1 & 12 & A09LM21 & 2.65 & 0.050 & 0.659 & 0.164 & 0.052 & 0.056 & 8.27 & 0.043 & 0.047 & 0.080 \\
\hline Batch 1 & 2 & 1 & 13 & BCHPF213 & 2.55 & 0.117 & 0.879 & $<0.010$ & 0.072 & 0.311 & 9.46 & 2.36 & $<0.010$ & 0.754 \\
\hline Batch 1 & 2 & 2 & 1 & BCHPF221 & 2.52 & 0.128 & 0.843 & $<0.010$ & 0.079 & 0.317 & 9.06 & 2.28 & $<0.010$ & 0.811 \\
\hline KT04-01 & 2 & 2 & 2 & A08LM22 & 3.11 & 0.059 & 0.674 & 0.235 & 0.054 & 0.041 & 8.12 & 0.027 & 0.079 & 0.088 \\
\hline KT04-05 & 2 & 2 & 3 & A01LM22 & 3.74 & 0.058 & 0.671 & 0.118 & 0.106 & 0.045 & 6.51 & 0.063 & 0.051 & 0.090 \\
\hline KT04-01 & 2 & 2 & 4 & A08LM12 & 3.17 & 0.058 & 0.694 & 0.231 & 0.053 & 0.045 & 8.03 & 0.029 & 0.078 & 0.087 \\
\hline KT04-10 & 2 & 2 & 5 & A05LM12 & 3.10 & 0.071 & 0.704 & 0.209 & 0.057 & 0.040 & 9.24 & 0.034 & 0.065 & 0.069 \\
\hline KT04-02 & 2 & 2 & 6 & A07LM22 & 2.85 & 0.063 & 0.683 & 0.242 & 0.057 & 0.037 & 7.83 & 0.026 & 0.055 & 0.091 \\
\hline Batch 1 & 2 & 2 & 7 & BCHPF222 & 2.52 & 0.125 & 0.851 & $<0.010$ & 0.077 & 0.309 & 8.75 & 2.28 & $<0.010$ & 0.803 \\
\hline KT04-04 & 2 & 2 & 8 & A09LM12 & 2.75 & 0.055 & 0.640 & 0.168 & 0.057 & 0.040 & 8.07 & 0.033 & 0.050 & 0.088 \\
\hline KT04-05 & 2 & 2 & 9 & A01LM12 & 3.77 & 0.056 & 0.666 & 0.117 & 0.104 & 0.043 & 6.56 & 0.059 & 0.051 & 0.092 \\
\hline KT04-04 & 2 & 2 & 10 & A09LM22 & 2.72 & 0.054 & 0.640 & 0.164 & 0.055 & 0.053 & 8.10 & 0.036 & 0.049 & 0.085 \\
\hline KT04-10 & 2 & 2 & 11 & A05LM22 & 3.12 & 0.070 & 0.692 & 0.208 & 0.056 & 0.040 & 9.18 & 0.030 & 0.065 & 0.071 \\
\hline KT04-02 & 2 & 2 & 12 & A07LM12 & 2.89 & 0.061 & 0.694 & 0.233 & 0.057 & 0.032 & 7.88 & 0.028 & 0.054 & 0.090 \\
\hline Batch 1 & 2 & 2 & 13 & BCHPF223 & 2.57 & 0.125 & 0.847 & $<0.010$ & 0.076 & 0.307 & 8.85 & 2.27 & $<0.010$ & 0.804 \\
\hline
\end{tabular}


Table C-2. PSAL Chemical Composition Measurements of the KT04-Series of Glasses Using LM Preparation Method (Part 2).

\begin{tabular}{|c|c|c|c|c|c|c|c|c|c|c|c|c|c|}
\hline Glass ID & Block & Sub-Blk & Seq & Lab ID & Mn (wt\%) & Na (wt\%) & Nb (wt\%) & Ni (wt\%) & Pb (wt\%) & Si (wt \%) & Ti (wt\%) & Zn (wt \%) & Zr (wt\%) \\
\hline Batch 1 & 1 & 1 & 1 & BCHPF111 & 1.32 & 6.63 & $<0.100$ & 0.535 & $<0.010$ & 23.2 & 0.393 & $<0.010$ & 0.068 \\
\hline \begin{tabular}{|l|} 
KT04-09 \\
\end{tabular} & 1 & 1 & 2 & A10LM21 & 0.425 & 10.5 & 0.571 & 0.349 & 0.110 & 22.6 & 2.92 & 0.037 & 0.547 \\
\hline \begin{tabular}{|l|} 
KT04-07 \\
\end{tabular} & 1 & 1 & 3 & A04LM11 & 0.559 & 10.6 & 0.782 & 0.445 & 0.061 & 22.7 & 2.94 & 0.064 & 0.726 \\
\hline \begin{tabular}{|l|} 
KT04-08 \\
\end{tabular} & 1 & 1 & 4 & A02LM11 & 0.747 & 11.6 & 0.891 & 0.427 & 0.063 & 23.2 & 3.01 & 0.034 & 0.786 \\
\hline \begin{tabular}{|l|} 
KT04-03 \\
\end{tabular} & 1 & 1 & 5 & A03LM11 & 1.41 & 10.7 & 0.734 & 0.204 & 0.096 & 22.8 & 2.63 & 0.029 & 0.676 \\
\hline KT04-06 & 1 & 1 & 6 & A06LM21 & 0.907 & 10.2 & 0.657 & 0.106 & 0.051 & 22.9 & 2.63 & 0.065 & 0.646 \\
\hline Batch 1 & 1 & 1 & 7 & BCHPF112 & 1.31 & 6.63 & $<0.100$ & 0.536 & $<0.010$ & 23.3 & 0.392 & $<0.010$ & 0.069 \\
\hline \begin{tabular}{|l|} 
KT04-07 \\
\end{tabular} & 1 & 1 & 8 & A04LM21 & 0.548 & 10.7 & 0.800 & 0.441 & 0.061 & 23.0 & 2.93 & 0.065 & 0.741 \\
\hline \begin{tabular}{|l|} 
KT04-06 \\
\end{tabular} & 1 & 1 & 9 & A06LM11 & 0.907 & 10.3 & 0.675 & 0.105 & 0.054 & 23.0 & 2.62 & 0.068 & 0.658 \\
\hline \begin{tabular}{|l|} 
KT04-08 \\
\end{tabular} & 1 & 1 & 10 & A02LM21 & 0.731 & 11.3 & 0.878 & 0.410 & 0.061 & 23.0 & 2.97 & 0.035 & 0.778 \\
\hline \begin{tabular}{|l|} 
KT04-09 \\
\end{tabular} & 1 & 1 & 11 & A10LM11 & 0.427 & 10.7 & 0.575 & 0.337 & 0.111 & 22.6 & 2.94 & 0.039 & 0.550 \\
\hline \begin{tabular}{|l|} 
KT04-03 \\
\end{tabular} & 1 & 1 & 12 & A03LM21 & 1.40 & 11.1 & 0.720 & 0.215 & 0.104 & 22.9 & 2.53 & 0.030 & 0.695 \\
\hline \begin{tabular}{|l|} 
Batch 1 \\
\end{tabular} & 1 & 1 & 13 & BCHPF113 & 1.32 & 6.95 & $<0.100$ & 0.534 & $<0.010$ & 23.7 & 0.394 & $<0.010$ & 0.068 \\
\hline Batch 1 & 1 & 2 & 1 & BCHPF121 & 1.31 & 6.73 & $<0.100$ & 0.531 & $<0.010$ & 23.0 & 0.395 & $<0.010$ & 0.067 \\
\hline KT04-06 & 1 & 2 & 2 & A06LM22 & 0.901 & 10.4 & 0.657 & 0.105 & 0.050 & 22.6 & 2.62 & 0.063 & 0.646 \\
\hline \begin{tabular}{|l|} 
KT04-06 \\
\end{tabular} & 1 & 2 & 3 & A06LM12 & 0.898 & 10.5 & 0.669 & 0.103 & 0.052 & 22.8 & 2.61 & 0.065 & 0.653 \\
\hline \begin{tabular}{|l|} 
KT04-03 \\
\end{tabular} & 1 & 2 & 4 & A03LM12 & 1.40 & 10.8 & 0.728 & 0.200 & 0.093 & 22.5 & 2.62 & 0.027 & 0.670 \\
\hline \begin{tabular}{|l|} 
KT04-07 \\
\end{tabular} & 1 & 2 & 5 & A04LM12 & 0.537 & 10.8 & 0.775 & 0.438 & 0.059 & 22.4 & 2.92 & 0.061 & 0.721 \\
\hline \begin{tabular}{|l|} 
KT04-08 \\
\end{tabular} & 1 & 2 & 6 & A02LM12 & 0.724 & 12.0 & 0.883 & 0.419 & 0.059 & 22.9 & 2.98 & 0.032 & 0.784 \\
\hline \begin{tabular}{|l|} 
Batch 1 \\
\end{tabular} & 1 & 2 & 7 & BCHPF122 & 1.30 & 6.80 & $<0.100$ & 0.518 & $<0.010$ & 22.9 & 0.388 & $<0.010$ & 0.067 \\
\hline \begin{tabular}{|l|} 
KT04-08 \\
\end{tabular} & 1 & 2 & 8 & A02LM22 & 0.719 & 11.8 & 0.878 & 0.402 & 0.057 & 22.6 & 2.95 & 0.033 & 0.767 \\
\hline \begin{tabular}{|l|} 
KT04-09 \\
\end{tabular} & 1 & 2 & 9 & A10LM22 & 0.410 & 10.7 & 0.563 & 0.342 & 0.108 & 22.0 & 2.90 & 0.036 & 0.547 \\
\hline \begin{tabular}{|l|} 
KT04-03 \\
\end{tabular} & 1 & 2 & 10 & A03LM22 & 1.40 & 11.1 & 0.722 & 0.211 & 0.104 & 22.1 & 2.60 & 0.029 & 0.698 \\
\hline \begin{tabular}{|l|} 
KT04-09 \\
\end{tabular} & 1 & 2 & 11 & A10LM12 & 0.416 & 11.1 & 0.576 & 0.332 & 0.109 & 22.0 & 2.92 & 0.037 & 0.551 \\
\hline \begin{tabular}{|l|} 
KT04-07 \\
\end{tabular} & 1 & 2 & 12 & A04LM22 & 0.535 & 11.1 & 0.785 & 0.433 & 0.059 & 22.9 & 2.83 & 0.063 & 0.738 \\
\hline Batch 1 & 1 & 2 & 13 & BCHPF123 & 1.33 & 7.12 & $<0.100$ & 0.530 & $<0.010$ & 23.0 & 0.397 & $<0.010$ & 0.068 \\
\hline \begin{tabular}{|l|} 
Batch 1 \\
\end{tabular} & 2 & 1 & 1 & BCHPF211 & 1.30 & 6.70 & $<0.100$ & 0.537 & $<0.010$ & 23.3 & 0.392 & $<0.010$ & 0.067 \\
\hline KT04-05 & 2 & 1 & 2 & A01LM21 & 0.768 & 10.0 & 0.702 & 0.081 & 0.051 & 23.5 & 2.64 & 0.030 & 0.693 \\
\hline \begin{tabular}{|l|} 
KT04-10 \\
\end{tabular} & 2 & 1 & 3 & A05LM21 & 0.268 & 9.62 & 0.534 & 0.333 & 0.119 & 22.5 & 2.73 & 0.032 & 0.545 \\
\hline \begin{tabular}{|l|} 
KT04-10 \\
\end{tabular} & 2 & 1 & 4 & A05LM11 & 0.266 & 9.98 & 0.533 & 0.331 & 0.117 & 22.6 & 2.74 & 0.033 & 0.540 \\
\hline KT04-02 & 2 & 1 & 5 & A07LM21 & 1.33 & 10.4 & 0.733 & 0.134 & 0.103 & 22.9 & 2.67 & 0.029 & 0.708 \\
\hline \begin{tabular}{|l|} 
KT04-01 \\
\end{tabular} & 2 & 1 & 6 & A08LM11 & 1.50 & 9.58 & 0.643 & 0.232 & 0.116 & 22.5 & 2.59 & $<0.010$ & 0.608 \\
\hline Batch 1 & 2 & 1 & 7 & BCHPF212 & 1.31 & 6.59 & $<0.100$ & 0.522 & $<0.010$ & 23.4 & 0.384 & $<0.010$ & 0.067 \\
\hline \begin{tabular}{|l|} 
KT04-01 \\
\end{tabular} & 2 & 1 & 8 & A08LM21 & 1.49 & 9.54 & 0.669 & 0.233 & 0.120 & 22.5 & 2.58 & $<0.010$ & 0.623 \\
\hline \begin{tabular}{|l|} 
KT04-05 \\
\end{tabular} & 2 & 1 & 9 & A01LM11 & 0.768 & 9.85 & 0.708 & 0.081 & 0.051 & 23.5 & 2.65 & 0.028 & 0.690 \\
\hline \begin{tabular}{|l|} 
KT04-04 \\
\end{tabular} & 2 & 1 & 10 & A09LM11 & 0.803 & 9.81 & 0.674 & 0.100 & 0.071 & 23.2 & 2.62 & 0.056 & 0.620 \\
\hline \begin{tabular}{|l|} 
KT04-02 \\
\end{tabular} & 2 & 1 & 11 & A07LM11 & 1.34 & 10.3 & 0.717 & 0.132 & 0.096 & 22.9 & 2.67 & 0.026 & 0.682 \\
\hline KT04-04 & 2 & 1 & 12 & A09LM21 & 0.799 & 10.2 & 0.663 & 0.110 & 0.068 & 23.4 & 2.63 & 0.054 & 0.605 \\
\hline \begin{tabular}{|l|} 
Batch 1 \\
\end{tabular} & 2 & 1 & 13 & BCHPF213 & 1.41 & 6.96 & $<0.100$ & 0.512 & $<0.010$ & 24.5 & 0.387 & $<0.010$ & 0.067 \\
\hline Batch 1 & 2 & 2 & 1 & BCHPF221 & 1.33 & 6.72 & $<0.100$ & 0.537 & $<0.010$ & 23.1 & 0.385 & $<0.010$ & 0.066 \\
\hline \begin{tabular}{|l|} 
KT04-01 \\
\end{tabular} & 2 & 2 & 2 & A08LM22 & 1.49 & 9.96 & 0.681 & 0.239 & 0.126 & 22.1 & 2.56 & $<0.010$ & 0.624 \\
\hline \begin{tabular}{|l|} 
KT04-05 \\
\end{tabular} & 2 & 2 & 3 & A01LM22 & 0.772 & 10.2 & 0.712 & 0.083 & 0.054 & 23.1 & 2.63 & 0.031 & 0.695 \\
\hline \begin{tabular}{|l|} 
KT04-01 \\
\end{tabular} & 2 & 2 & 4 & A08LM12 & 1.49 & 10.0 & 0.652 & 0.236 & 0.121 & 22.1 & 2.56 & $<0.010$ & 0.609 \\
\hline \begin{tabular}{|l|} 
KT04-10 \\
\end{tabular} & 2 & 2 & 5 & A05LM12 & 0.281 & 10.3 & 0.533 & 0.338 & 0.121 & 22.2 & 2.72 & 0.035 & 0.538 \\
\hline \begin{tabular}{|l|} 
KT04-02 \\
\end{tabular} & 2 & 2 & 6 & A07LM22 & 1.34 & 10.6 & 0.743 & 0.137 & 0.106 & 22.5 & 2.63 & 0.031 & 0.704 \\
\hline Batch 1 & 2 & 2 & 7 & BCHPF222 & 1.32 & 6.64 & $<0.100$ & 0.531 & $<0.010$ & 23.1 & 0.394 & $<0.010$ & 0.066 \\
\hline \begin{tabular}{|l|} 
KT04-04 \\
\end{tabular} & 2 & 2 & 8 & A09LM12 & 0.800 & 10.3 & 0.687 & 0.105 & 0.075 & 22.8 & 2.59 & 0.060 & 0.624 \\
\hline \begin{tabular}{|l|} 
KT04-05 \\
\end{tabular} & 2 & 2 & 9 & A01LM12 & 0.778 & 10.2 & 0.721 & 0.086 & 0.054 & 23.0 & 2.60 & 0.031 & 0.693 \\
\hline \begin{tabular}{|l|} 
KT04-04 \\
\end{tabular} & 2 & 2 & 10 & A09LM22 & 0.800 & 10.8 & 0.676 & 0.115 & 0.071 & 22.8 & 2.56 & 0.058 & 0.606 \\
\hline KT04-10 & 2 & 2 & 11 & A05LM22 & 0.280 & 10.2 & 0.546 & 0.343 & 0.121 & 22.0 & 2.69 & 0.034 & 0.538 \\
\hline \begin{tabular}{|l|} 
KT04-02 \\
\end{tabular} & 2 & 2 & 12 & A07LM12 & 1.35 & 10.9 & 0.743 & 0.136 & 0.100 & 22.3 & 2.62 & 0.028 & 0.679 \\
\hline Batch 1 & 2 & 2 & 13 & BCHPF223 & 1.34 & 6.89 & $<0.100$ & 0.532 & $<0.010$ & 22.9 & 0.389 & $<0.010$ & 0.066 \\
\hline
\end{tabular}


Table C-3. PSAL Chemical Composition Measurements of the KT04-Series of Glasses Using PF Preparation Method.

\begin{tabular}{|c|c|c|c|c|c|c|}
\hline Glass ID & Block & Sub-Blk & Sequence & Lab ID & B (wt \%) & Li (wt\%) \\
\hline Batch 1 & 1 & 1 & 1 & BCHPF111 & 2.47 & 2.08 \\
\hline KT04-10 & 1 & 1 & 2 & A05PF21 & 1.88 & 1.68 \\
\hline KT04-01 & 1 & 1 & 3 & A08PF21 & 1.86 & 1.68 \\
\hline KT04-02 & 1 & 1 & 4 & A07PF21 & 1.81 & 1.70 \\
\hline KT04-02 & 1 & 1 & 5 & A07PF11 & 1.84 & 1.70 \\
\hline KT04-04 & 1 & 1 & 6 & A09PF21 & 1.82 & 1.68 \\
\hline Batch 1 & 1 & 1 & 7 & BCHPF112 & 2.41 & 2.12 \\
\hline KT04-10 & 1 & 1 & 8 & A05PF11 & 1.84 & 1.67 \\
\hline KT04-01 & 1 & 1 & 9 & A08PF11 & 1.87 & 1.67 \\
\hline KT04-03 & 1 & 1 & 10 & A03PF11 & 1.86 & 1.69 \\
\hline KT04-03 & 1 & 1 & 11 & A03PF21 & 1.84 & 1.71 \\
\hline KT04-04 & 1 & 1 & 12 & A09PF11 & 1.85 & 1.69 \\
\hline Batch 1 & 1 & 1 & 13 & BCHPF113 & 2.39 & 2.11 \\
\hline Batch 1 & 1 & 2 & 1 & BCHPF121 & 2.45 & 2.09 \\
\hline KT04-03 & 1 & 2 & 2 & A03PF12 & 1.83 & 1.68 \\
\hline KT04-10 & 1 & 2 & 3 & A05PF12 & 1.80 & 1.69 \\
\hline KT04-04 & 1 & 2 & 4 & A09PF22 & 1.82 & 1.67 \\
\hline KT04-04 & 1 & 2 & 5 & A09PF12 & 1.77 & 1.67 \\
\hline KT04-01 & 1 & 2 & 6 & A08PF22 & 1.80 & 1.68 \\
\hline Batch 1 & 1 & 2 & 7 & BCHPF122 & 2.34 & 2.11 \\
\hline KT04-02 & 1 & 2 & 8 & A07PF22 & 1.82 & 1.70 \\
\hline KT04-03 & 1 & 2 & 9 & A03PF22 & 1.81 & 1.67 \\
\hline KT04-02 & 1 & 2 & 10 & A07PF12 & 1.79 & 1.69 \\
\hline KT04-10 & 1 & 2 & 11 & A05PF22 & 1.79 & 1.67 \\
\hline KT04-01 & 1 & 2 & 12 & A08PF12 & 1.78 & 1.66 \\
\hline Batch 1 & 1 & 2 & 13 & BCHPF123 & 2.37 & 2.11 \\
\hline Batch 1 & 2 & 1 & 1 & BCHPF211 & 2.42 & 2.11 \\
\hline KT04-09 & 2 & 1 & 2 & A10PF21 & 1.79 & 1.68 \\
\hline KT04-05 & 2 & 1 & 3 & A01PF21 & 1.79 & 1.67 \\
\hline KT04-06 & 2 & 1 & 4 & A06PF11 & 1.79 & 1.68 \\
\hline KT04-07 & 2 & 1 & 5 & A04PF21 & 1.76 & 1.69 \\
\hline KT04-07 & 2 & 1 & 6 & A04PF11 & 1.76 & 1.68 \\
\hline Batch 1 & 2 & 1 & 7 & BCHPF212 & 2.28 & 2.10 \\
\hline KT04-08 & 2 & 1 & 8 & A02PF11 & 1.77 & 1.66 \\
\hline KT04-05 & 2 & 1 & 9 & A01PF11 & 1.79 & 1.66 \\
\hline KT04-08 & 2 & 1 & 10 & A02PF21 & 1.77 & 1.68 \\
\hline KT04-09 & 2 & 1 & 11 & A10PF11 & 1.78 & 1.66 \\
\hline KT04-06 & 2 & 1 & 12 & A06PF21 & 1.77 & 1.68 \\
\hline Batch 1 & 2 & 1 & 13 & BCHPF213 & 2.31 & 2.09 \\
\hline Batch 1 & 2 & 2 & 1 & BCHPF221 & 2.43 & 2.16 \\
\hline KT04-08 & 2 & 2 & 2 & A02PF12 & 1.86 & 1.72 \\
\hline KT04-05 & 2 & 2 & 3 & A01PF12 & 1.87 & 1.73 \\
\hline KT04-09 & 2 & 2 & 4 & A10PF22 & 1.86 & 1.71 \\
\hline KT04-07 & 2 & 2 & 5 & A04PF12 & 1.87 & 1.72 \\
\hline KT04-06 & 2 & 2 & 6 & A06PF12 & 1.88 & 1.71 \\
\hline Batch 1 & 2 & 2 & 7 & BCHPF222 & 2.39 & 2.13 \\
\hline KT04-05 & 2 & 2 & 8 & A01PF22 & 1.89 & 1.73 \\
\hline KT04-08 & 2 & 2 & 9 & A02PF22 & 1.88 & 1.74 \\
\hline KT04-09 & 2 & 2 & 10 & A10PF12 & 1.89 & 1.72 \\
\hline KT04-07 & 2 & 2 & 11 & A04PF22 & 1.86 & 1.73 \\
\hline KT04-06 & 2 & 2 & 12 & A06PF22 & 1.88 & 1.74 \\
\hline Batch 1 & 2 & 2 & 13 & BCHPF223 & 2.43 & 2.17 \\
\hline
\end{tabular}


Table C-4. Comparison of Measured versus Targeted Composition for KT04 Glasses.

\begin{tabular}{|c|c|c|c|c|c|}
\hline Glass ID & Oxide & $\begin{array}{l}\text { Measured } \\
\text { (wt\%) }\end{array}$ & $\begin{array}{c}\text { Targeted } \\
(\mathbf{w t} \%)\end{array}$ & $\begin{array}{c}\text { Difference of Measured } \\
\text { versus Targeted }\end{array}$ & $\begin{array}{c}\text { \% Difference of } \\
\text { Measured versus } \\
\text { Targeted }\end{array}$ \\
\hline Batch 1 & $\mathrm{Al}_{2} \mathrm{O}_{3}$ & 4.7820 & 4.8770 & -0.0950 & $-1.9 \%$ \\
\hline Batch 1 & $\mathrm{~B}_{2} \mathrm{O}_{3}$ & 7.6982 & 7.7770 & -0.0788 & $-1.0 \%$ \\
\hline Batch 1 & $\mathrm{BaO}$ & 0.1384 & 0.1510 & -0.0126 & $-8.3 \%$ \\
\hline Batch 1 & $\mathrm{CaO}$ & 1.1990 & 1.2200 & -0.0210 & $-1.7 \%$ \\
\hline Batch 1 & $\mathrm{Ce}_{2} \mathrm{O}_{3}$ & 0.0059 & 0.0000 & 0.0059 & \\
\hline Batch 1 & $\mathrm{Cr}_{2} \mathrm{O}_{3}$ & 0.1100 & 0.1070 & 0.0030 & $2.8 \%$ \\
\hline Batch 1 & $\mathrm{CuO}$ & 0.3889 & 0.3990 & -0.0101 & $-2.5 \%$ \\
\hline Batch 1 & $\mathrm{Fe}_{2} \mathrm{O}_{3}$ & 12.6409 & 12.8390 & -0.1981 & $-1.5 \%$ \\
\hline Batch 1 & $\mathrm{~K}_{2} \mathrm{O}$ & 2.7535 & 3.3270 & -0.5735 & $-17.2 \%$ \\
\hline Batch 1 & $\mathrm{La}_{2} \mathrm{O}_{3}$ & 0.0059 & 0.0000 & 0.0059 & \\
\hline Batch 1 & $\mathrm{Li}_{2} \mathrm{O}$ & 4.5534 & 4.4290 & 0.1244 & $2.8 \%$ \\
\hline Batch 1 & $\mathrm{MgO}$ & 1.3324 & 1.4190 & -0.0866 & $-6.1 \%$ \\
\hline Batch 1 & $\mathrm{MnO}$ & 1.7108 & 1.7260 & -0.0152 & $-0.9 \%$ \\
\hline Batch 1 & $\mathrm{Na}_{2} \mathrm{O}$ & 9.1394 & 9.0030 & 0.1364 & $1.5 \%$ \\
\hline Batch 1 & $\mathrm{Nb}_{2} \mathrm{O}_{5}$ & 0.0715 & 0.0000 & 0.0715 & \\
\hline Batch 1 & $\mathrm{NiO}$ & 0.6739 & 0.7510 & -0.0771 & $-10.3 \%$ \\
\hline Batch 1 & $\mathrm{PbO}$ & 0.0054 & 0.0000 & 0.0054 & \\
\hline Batch 1 & $\mathrm{SiO}_{2}$ & 49.8100 & 50.2200 & -0.4100 & $-0.8 \%$ \\
\hline Batch 1 & $\mathrm{TiO}_{2}$ & 0.6519 & 0.6770 & -0.0251 & $-3.7 \%$ \\
\hline Batch 1 & $\mathrm{ZnO}$ & 0.0062 & 0.0000 & 0.0062 & \\
\hline Batch 1 & $\mathrm{ZrO}_{2}$ & 0.0907 & 0.0980 & -0.0073 & $-7.4 \%$ \\
\hline Batch 1 & Sum & 97.7686 & 99.0200 & -1.2514 & $-1.3 \%$ \\
\hline KT04-01 & $\mathrm{Al}_{2} \mathrm{O}_{3}$ & 5.8952 & 5.8000 & 0.0952 & $1.6 \%$ \\
\hline KT04-01 & $\mathrm{B}_{2} \mathrm{O}_{3}$ & 5.8844 & 6.0000 & -0.1156 & $-1.9 \%$ \\
\hline KT04-01 & $\mathrm{BaO}$ & 0.0628 & 0.0700 & -0.0072 & $-10.3 \%$ \\
\hline KT04-01 & $\mathrm{CaO}$ & 0.9633 & 0.9400 & 0.0233 & $2.5 \%$ \\
\hline KT04-01 & $\mathrm{Ce}_{2} \mathrm{O}_{3}$ & 0.2717 & 0.2900 & -0.0183 & $-6.3 \%$ \\
\hline KT04-01 & $\mathrm{Cr}_{2} \mathrm{O}_{3}$ & 0.0756 & 0.0900 & -0.0144 & $-16.0 \%$ \\
\hline KT04-01 & $\mathrm{CuO}$ & 0.0551 & 0.0400 & 0.0151 & $37.7 \%$ \\
\hline KT04-01 & $\mathrm{Fe}_{2} \mathrm{O}_{3}$ & 11.6449 & 12.1100 & -0.4651 & $-3.8 \%$ \\
\hline KT04-01 & $\mathrm{K}_{2} \mathrm{O}$ & 0.0373 & 0.0400 & -0.0027 & $-6.6 \%$ \\
\hline KT04-01 & $\mathrm{La}_{2} \mathrm{O}_{3}$ & 0.0909 & 0.1100 & -0.0191 & $-17.4 \%$ \\
\hline KT04-01 & $\mathrm{Li}_{2} \mathrm{O}$ & 3.6007 & 3.6000 & 0.0007 & $0.0 \%$ \\
\hline KT04-01 & $\mathrm{MgO}$ & 0.1414 & 0.1500 & -0.0086 & $-5.8 \%$ \\
\hline KT04-01 & $\mathrm{MnO}$ & 1.9271 & 1.9300 & -0.0029 & $-0.1 \%$ \\
\hline KT04-01 & $\mathrm{Na}_{2} \mathrm{O}$ & 13.1700 & 13.2200 & -0.0500 & $-0.4 \%$ \\
\hline KT04-01 & $\mathrm{Nb}_{2} \mathrm{O}_{5}$ & 0.9459 & 1.0400 & -0.0941 & $-9.0 \%$ \\
\hline KT04-01 & $\mathrm{NiO}$ & 0.2990 & 0.3500 & -0.0510 & $-14.6 \%$ \\
\hline KT04-01 & $\mathrm{PbO}$ & 0.1301 & 0.1600 & -0.0299 & $-18.7 \%$ \\
\hline KT04-01 & $\mathrm{SiO}_{2}$ & 47.7064 & 48.8000 & -1.0936 & $-2.2 \%$ \\
\hline KT04-01 & $\mathrm{TiO}_{2}$ & 4.2909 & 4.3500 & -0.0591 & $-1.4 \%$ \\
\hline KT04-01 & $\mathrm{ZnO}$ & 0.0062 & 0.0000 & 0.0062 & \\
\hline KT04-01 & $\mathrm{ZrO}_{2}$ & 0.8321 & 0.9200 & -0.0879 & $-9.6 \%$ \\
\hline KT04-01 & Sum & 98.0312 & 100.0100 & -1.9788 & $-2.0 \%$ \\
\hline KT04-02 & $\mathrm{Al}_{2} \mathrm{O}_{3}$ & 5.3426 & 5.1900 & 0.1526 & $2.9 \%$ \\
\hline KT04-02 & $\mathrm{B}_{2} \mathrm{O}_{3}$ & 5.8441 & 6.0000 & -0.1559 & $-2.6 \%$ \\
\hline KT04-02 & $\mathrm{BaO}$ & 0.0673 & 0.0700 & -0.0027 & $-3.9 \%$ \\
\hline KT04-02 & $\mathrm{CaO}$ & 0.9731 & 0.9500 & 0.0231 & $2.4 \%$ \\
\hline KT04-02 & $\mathrm{Ce}_{2} \mathrm{O}_{3}$ & 0.2782 & 0.2900 & -0.0118 & $-4.1 \%$ \\
\hline KT04-02 & $\mathrm{Cr}_{2} \mathrm{O}_{3}$ & 0.0808 & 0.0900 & -0.0092 & $-10.3 \%$ \\
\hline KT04-02 & $\mathrm{CuO}$ & 0.0451 & 0.0400 & 0.0051 & $12.7 \%$ \\
\hline KT04-02 & $\mathrm{Fe}_{2} \mathrm{O}_{3}$ & 11.2696 & 11.5600 & -0.2904 & $-2.5 \%$ \\
\hline KT04-02 & $\mathrm{K}_{2} \mathrm{O}$ & 0.0361 & 0.0400 & -0.0039 & $-9.7 \%$ \\
\hline KT04-02 & $\mathrm{La}_{2} \mathrm{O}_{3}$ & 0.0630 & 0.0700 & -0.0070 & $-9.9 \%$ \\
\hline KT04-02 & $\mathrm{Li}_{2} \mathrm{O}$ & 3.6545 & 3.6000 & 0.0545 & $1.5 \%$ \\
\hline KT04-02 & $\mathrm{MgO}$ & 0.1459 & 0.1500 & -0.0041 & $-2.7 \%$ \\
\hline KT04-02 & $\mathrm{MnO}$ & 1.7302 & 1.7100 & 0.0202 & $1.2 \%$ \\
\hline KT04-02 & $\mathrm{Na}_{2} \mathrm{O}$ & 14.2214 & 14.0800 & 0.1414 & $1.0 \%$ \\
\hline KT04-02 & $\mathrm{Nb}_{2} \mathrm{O}_{5}$ & 1.0500 & 1.1000 & -0.0500 & $-4.5 \%$ \\
\hline
\end{tabular}


Table C-4. Comparison of Measured versus Targeted Composition for KT04 Glasses. (continued)

\begin{tabular}{|c|c|c|c|c|c|}
\hline Glass ID & Oxide & $\begin{array}{l}\text { Measured } \\
(\mathbf{w t} \%)\end{array}$ & $\begin{array}{c}\text { Targeted } \\
\text { (wt\%) }\end{array}$ & $\begin{array}{c}\text { Difference of Measured } \\
\text { versus Targeted }\end{array}$ & $\begin{array}{c}\text { \% Difference of } \\
\text { Measured versus } \\
\text { Targeted } \\
\end{array}$ \\
\hline KT04-02 & $\mathrm{NiO}$ & 0.1715 & 0.2000 & -0.0285 & $-14.3 \%$ \\
\hline KT04-02 & $\mathrm{PbO}$ & 0.1091 & 0.1300 & -0.0209 & $-16.1 \%$ \\
\hline KT04-02 & $\mathrm{SiO}_{2}$ & 48.4551 & 49.3200 & -0.8649 & $-1.8 \%$ \\
\hline KT04-02 & $\mathrm{TiO}_{2}$ & 4.4160 & 4.3800 & 0.0360 & $0.8 \%$ \\
\hline KT04-02 & $\mathrm{ZnO}$ & 0.0355 & 0.0400 & -0.0045 & $-11.3 \%$ \\
\hline KT04-02 & $\mathrm{ZrO}_{2}$ & 0.9364 & 1.0100 & -0.0736 & $-7.3 \%$ \\
\hline KT04-02 & Sum & 98.9256 & 100.0200 & -1.0944 & $-1.1 \%$ \\
\hline KT04-03 & $\mathrm{Al}_{2} \mathrm{O}_{3}$ & 4.7096 & 4.4900 & 0.2196 & $4.9 \%$ \\
\hline KT04-03 & $\mathrm{B}_{2} \mathrm{O}_{3}$ & 5.9085 & 6.0000 & -0.0915 & $-1.5 \%$ \\
\hline KT04-03 & $\mathrm{BaO}$ & 0.0622 & 0.0700 & -0.0078 & $-11.1 \%$ \\
\hline KT04-03 & $\mathrm{CaO}$ & 1.0099 & 0.9600 & 0.0499 & $5.2 \%$ \\
\hline KT04-03 & $\mathrm{Ce}_{2} \mathrm{O}_{3}$ & 0.2378 & 0.2600 & -0.0222 & $-8.5 \%$ \\
\hline KT04-03 & $\mathrm{Cr}_{2} \mathrm{O}_{3}$ & 0.0808 & 0.0900 & -0.0092 & $-10.3 \%$ \\
\hline KT04-03 & $\mathrm{CuO}$ & 0.0463 & 0.0400 & 0.0063 & $15.8 \%$ \\
\hline KT04-03 & $\mathrm{Fe}_{2} \mathrm{O}_{3}$ & 11.3161 & 11.5300 & -0.2139 & $-1.9 \%$ \\
\hline KT04-03 & $\mathrm{K}_{2} \mathrm{O}$ & 0.0449 & 0.0400 & 0.0049 & $12.2 \%$ \\
\hline KT04-03 & $\mathrm{La}_{2} \mathrm{O}_{3}$ & 0.0619 & 0.0700 & -0.0081 & $-11.6 \%$ \\
\hline KT04-03 & $\mathrm{Li}_{2} \mathrm{O}$ & 3.6330 & 3.6000 & 0.0330 & $0.9 \%$ \\
\hline KT04-03 & $\mathrm{MgO}$ & 0.1430 & 0.1500 & -0.0070 & $-4.6 \%$ \\
\hline KT04-03 & $\mathrm{MnO}$ & 1.8109 & 1.8100 & 0.0009 & $0.1 \%$ \\
\hline KT04-03 & $\mathrm{Na}_{2} \mathrm{O}$ & 14.7269 & 14.3600 & 0.3669 & $2.6 \%$ \\
\hline KT04-03 & $\mathrm{Nb}_{2} \mathrm{O}_{5}$ & 1.0385 & 1.1000 & -0.0615 & $-5.6 \%$ \\
\hline KT04-03 & $\mathrm{NiO}$ & 0.2640 & 0.3200 & -0.0560 & $-17.5 \%$ \\
\hline KT04-03 & $\mathrm{PbO}$ & 0.1069 & 0.1300 & -0.0231 & $-17.8 \%$ \\
\hline KT04-03 & $\mathrm{SiO}_{2}$ & 48.2947 & 49.5300 & -1.2353 & $-2.5 \%$ \\
\hline KT04-03 & $\mathrm{TiO}_{2}$ & 4.3285 & 4.4000 & -0.0715 & $-1.6 \%$ \\
\hline KT04-03 & $\mathrm{ZnO}$ & 0.0358 & 0.0400 & -0.0042 & $-10.5 \%$ \\
\hline KT04-03 & $\mathrm{ZrO}_{2}$ & 0.9250 & 1.0200 & -0.0950 & $-9.3 \%$ \\
\hline KT04-03 & Sum & 98.7851 & 100.0100 & -1.2249 & $-1.2 \%$ \\
\hline KT04-04 & $\mathrm{Al}_{2} \mathrm{O}_{3}$ & 5.0969 & 4.9700 & 0.1269 & $2.6 \%$ \\
\hline KT04-04 & $\mathrm{B}_{2} \mathrm{O}_{3}$ & 5.8441 & 6.0000 & -0.1559 & $-2.6 \%$ \\
\hline KT04-04 & $\mathrm{BaO}$ & 0.0586 & 0.0700 & -0.0114 & $-16.3 \%$ \\
\hline KT04-04 & $\mathrm{CaO}$ & 0.9074 & 0.8600 & 0.0474 & $5.5 \%$ \\
\hline KT04-04 & $\mathrm{Ce}_{2} \mathrm{O}_{3}$ & 0.1944 & 0.2200 & -0.0256 & $-11.6 \%$ \\
\hline KT04-04 & $\mathrm{Cr}_{2} \mathrm{O}_{3}$ & 0.0793 & 0.0900 & -0.0107 & $-11.9 \%$ \\
\hline KT04-04 & $\mathrm{CuO}$ & 0.0598 & 0.0400 & 0.0198 & $49.4 \%$ \\
\hline KT04-04 & $\mathrm{Fe}_{2} \mathrm{O}_{3}$ & 11.6842 & 12.1700 & -0.4858 & $-4.0 \%$ \\
\hline KT04-04 & $\mathrm{K}_{2} \mathrm{O}$ & 0.0458 & 0.0400 & 0.0058 & $14.4 \%$ \\
\hline KT04-04 & $\mathrm{La}_{2} \mathrm{O}_{3}$ & 0.0569 & 0.0700 & -0.0131 & $-18.7 \%$ \\
\hline KT04-04 & $\mathrm{Li}_{2} \mathrm{O}$ & 3.6115 & 3.6000 & 0.0115 & $0.3 \%$ \\
\hline KT04-04 & $\mathrm{MgO}$ & 0.1389 & 0.1500 & -0.0111 & $-7.4 \%$ \\
\hline KT04-04 & $\mathrm{MnO}$ & 1.0336 & 1.0700 & -0.0364 & $-3.4 \%$ \\
\hline KT04-04 & $\mathrm{Na}_{2} \mathrm{O}$ & 13.8541 & 13.8000 & 0.0541 & $0.4 \%$ \\
\hline KT04-04 & $\mathrm{Nb}_{2} \mathrm{O}_{5}$ & 0.9656 & 1.0600 & -0.0944 & $-8.9 \%$ \\
\hline KT04-04 & $\mathrm{NiO}$ & 0.1368 & 0.1600 & -0.0232 & $-14.5 \%$ \\
\hline KT04-04 & $\mathrm{PbO}$ & 0.0768 & 0.1000 & -0.0232 & $-23.2 \%$ \\
\hline KT04-04 & $\mathrm{SiO}_{2}$ & 49.3109 & 50.1300 & -0.8191 & $-1.6 \%$ \\
\hline KT04-04 & $\mathrm{TiO}_{2}$ & 4.3368 & 4.4100 & -0.0732 & $-1.7 \%$ \\
\hline KT04-04 & $\mathrm{ZnO}$ & 0.0710 & 0.0800 & -0.0090 & $-11.3 \%$ \\
\hline KT04-04 & $\mathrm{ZrO}_{2}$ & 0.8291 & 0.9400 & -0.1109 & $-11.8 \%$ \\
\hline KT04-04 & Sum & 98.3923 & 100.0300 & -1.6377 & $-1.6 \%$ \\
\hline KT04-05 & $\mathrm{Al}_{2} \mathrm{O}_{3}$ & 7.0337 & 6.8900 & 0.1437 & $2.1 \%$ \\
\hline KT04-05 & $\mathrm{B}_{2} \mathrm{O}_{3}$ & 5.9085 & 6.0000 & -0.0915 & $-1.5 \%$ \\
\hline KT04-05 & $\mathrm{BaO}$ & 0.0614 & 0.0700 & -0.0086 & $-12.3 \%$ \\
\hline KT04-05 & $\mathrm{CaO}$ & 0.9462 & 0.9400 & 0.0062 & $0.7 \%$ \\
\hline KT04-05 & $\mathrm{Ce}_{2} \mathrm{O}_{3}$ & 0.1373 & 0.1400 & -0.0027 & $-1.9 \%$ \\
\hline KT04-05 & $\mathrm{Cr}_{2} \mathrm{O}_{3}$ & 0.1494 & 0.1300 & 0.0194 & $15.0 \%$ \\
\hline KT04-05 & $\mathrm{CuO}$ & 0.0595 & 0.0400 & 0.0195 & $48.7 \%$ \\
\hline
\end{tabular}


Table C-4. Comparison of Measured versus Targeted Composition for KT04 Glasses. (continued)

\begin{tabular}{|c|c|c|c|c|c|}
\hline Glass ID & Oxide & $\begin{array}{l}\text { Measured } \\
(\mathbf{w t} \%)\end{array}$ & $\begin{array}{c}\text { Targeted } \\
\text { (wt\%) }\end{array}$ & $\begin{array}{c}\text { Difference of Measured } \\
\text { versus Targeted }\end{array}$ & $\begin{array}{c}\text { \% Difference of } \\
\text { Measured versus } \\
\text { Targeted }\end{array}$ \\
\hline KT04-05 & $\mathrm{Fe}_{2} \mathrm{O}_{3}$ & 9.3788 & 9.6700 & -0.2912 & $-3.0 \%$ \\
\hline KT04-05 & $\mathrm{K}_{2} \mathrm{O}$ & 0.0780 & 0.0700 & 0.0080 & $11.4 \%$ \\
\hline KT04-05 & $\mathrm{La}_{2} \mathrm{O}_{3}$ & 0.0586 & 0.0700 & -0.0114 & $-16.2 \%$ \\
\hline KT04-05 & $\mathrm{Li}_{2} \mathrm{O}$ & 3.6545 & 3.6000 & 0.0545 & $1.5 \%$ \\
\hline KT04-05 & $\mathrm{MgO}$ & 0.1468 & 0.1500 & -0.0032 & $-2.2 \%$ \\
\hline KT04-05 & $\mathrm{MnO}$ & 0.9962 & 1.0300 & -0.0338 & $-3.3 \%$ \\
\hline KT04-05 & $\mathrm{Na}_{2} \mathrm{O}$ & 13.5643 & 13.7600 & -0.1958 & $-1.4 \%$ \\
\hline KT04-05 & $\mathrm{Nb}_{2} \mathrm{O}_{5}$ & 1.0167 & 1.0800 & -0.0633 & $-5.9 \%$ \\
\hline KT04-05 & $\mathrm{NiO}$ & 0.1053 & 0.1200 & -0.0147 & $-12.3 \%$ \\
\hline KT04-05 & $\mathrm{PbO}$ & 0.0566 & 0.0700 & -0.0134 & $-19.2 \%$ \\
\hline KT04-05 & $\mathrm{SiO}_{2}$ & 49.7922 & 50.6700 & -0.8778 & $-1.7 \%$ \\
\hline KT04-05 & $\mathrm{TiO}_{2}$ & 4.3868 & 4.4700 & -0.0832 & $-1.9 \%$ \\
\hline KT04-05 & $\mathrm{ZnO}$ & 0.0373 & 0.0400 & -0.0027 & $-6.6 \%$ \\
\hline KT04-05 & $\mathrm{ZrO}_{2}$ & 0.9358 & 1.0000 & -0.0642 & $-6.4 \%$ \\
\hline KT04-05 & Sum & 98.5040 & 100.0100 & -1.5060 & $-1.5 \%$ \\
\hline KT04-06 & $\mathrm{Al}_{2} \mathrm{O}_{3}$ & 7.5202 & 7.3100 & 0.2102 & $2.9 \%$ \\
\hline KT04-06 & $\mathrm{B}_{2} \mathrm{O}_{3}$ & 5.8924 & 6.0000 & -0.1076 & $-1.8 \%$ \\
\hline KT04-06 & $\mathrm{BaO}$ & 0.0611 & 0.0700 & -0.0089 & $-12.7 \%$ \\
\hline KT04-06 & $\mathrm{CaO}$ & 1.0295 & 1.0000 & 0.0295 & $2.9 \%$ \\
\hline KT04-06 & $\mathrm{Ce}_{2} \mathrm{O}_{3}$ & 0.1010 & 0.1100 & -0.0090 & $-8.2 \%$ \\
\hline KT04-06 & $\mathrm{Cr}_{2} \mathrm{O}_{3}$ & 0.0862 & 0.1400 & -0.0538 & $-38.4 \%$ \\
\hline KT04-06 & $\mathrm{CuO}$ & 0.0663 & 0.0400 & 0.0263 & $65.9 \%$ \\
\hline KT04-06 & $\mathrm{Fe}_{2} \mathrm{O}_{3}$ & 8.7498 & 9.1200 & -0.3702 & $-4.1 \%$ \\
\hline KT04-06 & $\mathrm{K}_{2} \mathrm{O}$ & 0.1364 & 0.1100 & 0.0264 & $24.0 \%$ \\
\hline KT04-06 & $\mathrm{La}_{2} \mathrm{O}_{3}$ & 0.0267 & 0.0400 & -0.0133 & $-33.3 \%$ \\
\hline KT04-06 & $\mathrm{Li}_{2} \mathrm{O}$ & 3.6653 & 3.6000 & 0.0653 & $1.8 \%$ \\
\hline KT04-06 & $\mathrm{MgO}$ & 0.0949 & 0.1000 & -0.0051 & $-5.1 \%$ \\
\hline KT04-06 & $\mathrm{MnO}$ & 1.1663 & 1.2000 & -0.0337 & $-2.8 \%$ \\
\hline KT04-06 & $\mathrm{Na}_{2} \mathrm{O}$ & 13.9518 & 13.7700 & 0.1818 & $1.3 \%$ \\
\hline KT04-06 & $\mathrm{Nb}_{2} \mathrm{O}_{5}$ & 0.9506 & 1.0300 & -0.0794 & $-7.7 \%$ \\
\hline KT04-06 & $\mathrm{NiO}$ & 0.1333 & 0.1600 & -0.0267 & $-16.7 \%$ \\
\hline KT04-06 & $\mathrm{PbO}$ & 0.0557 & 0.0700 & -0.0143 & $-20.4 \%$ \\
\hline KT04-06 & $\mathrm{SiO}_{2}$ & 48.8295 & 50.6600 & -1.8305 & $-3.6 \%$ \\
\hline KT04-06 & $\mathrm{TiO}_{2}$ & 4.3702 & 4.4200 & -0.0498 & $-1.1 \%$ \\
\hline KT04-06 & $\mathrm{ZnO}$ & 0.0812 & 0.0800 & 0.0012 & $1.5 \%$ \\
\hline KT04-06 & $\mathrm{ZrO}_{2}$ & 0.8790 & 0.9700 & -0.0910 & $-9.4 \%$ \\
\hline KT04-06 & Sum & 97.8476 & 100.0000 & -2.1524 & $-2.2 \%$ \\
\hline KT04-07 & $\mathrm{Al}_{2} \mathrm{O}_{3}$ & 5.8622 & 5.6900 & 0.1722 & $3.0 \%$ \\
\hline KT04-07 & $\mathrm{B}_{2} \mathrm{O}_{3}$ & 5.8361 & 6.0000 & -0.1639 & $-2.7 \%$ \\
\hline KT04-07 & $\mathrm{BaO}$ & 0.0689 & 0.0800 & -0.0111 & $-13.8 \%$ \\
\hline KT04-07 & $\mathrm{CaO}$ & 0.9119 & 0.9000 & 0.0119 & $1.3 \%$ \\
\hline KT04-07 & $\mathrm{Ce}_{2} \mathrm{O}_{3}$ & 0.0779 & 0.0800 & -0.0021 & $-2.6 \%$ \\
\hline KT04-07 & $\mathrm{Cr}_{2} \mathrm{O}_{3}$ & 0.1104 & 0.1500 & -0.0396 & $-26.4 \%$ \\
\hline KT04-07 & $\mathrm{CuO}$ & 0.0438 & 0.0400 & 0.0038 & $9.5 \%$ \\
\hline KT04-07 & $\mathrm{Fe}_{2} \mathrm{O}_{3}$ & 9.1644 & 9.4900 & -0.3256 & $-3.4 \%$ \\
\hline KT04-07 & $\mathrm{K}_{2} \mathrm{O}$ & 0.0885 & 0.0800 & 0.0085 & $10.7 \%$ \\
\hline KT04-07 & $\mathrm{La}_{2} \mathrm{O}_{3}$ & 0.0325 & 0.0400 & -0.0075 & $-18.6 \%$ \\
\hline KT04-07 & $\mathrm{Li}_{2} \mathrm{O}$ & 3.6707 & 3.6000 & 0.0707 & $2.0 \%$ \\
\hline KT04-07 & $\mathrm{MgO}$ & 0.1082 & 0.1100 & -0.0018 & $-1.6 \%$ \\
\hline KT04-07 & $\mathrm{MnO}$ & 0.7034 & 0.7400 & -0.0366 & $-4.9 \%$ \\
\hline KT04-07 & $\mathrm{Na}_{2} \mathrm{O}$ & 14.5584 & 14.6400 & -0.0816 & $-0.6 \%$ \\
\hline KT04-07 & $\mathrm{Nb}_{2} \mathrm{O}_{5}$ & 1.1237 & 1.2100 & -0.0863 & $-7.1 \%$ \\
\hline KT04-07 & $\mathrm{NiO}$ & 0.5589 & 0.6500 & -0.0911 & $-14.0 \%$ \\
\hline KT04-07 & $\mathrm{PbO}$ & 0.0646 & 0.0700 & -0.0054 & $-7.7 \%$ \\
\hline KT04-07 & $\mathrm{SiO}_{2}$ & 48.6691 & 50.3800 & -1.7109 & $-3.4 \%$ \\
\hline KT04-07 & $\mathrm{TiO}_{2}$ & 4.8455 & 4.9000 & -0.0545 & $-1.1 \%$ \\
\hline KT04-07 & $\mathrm{ZnO}$ & 0.0787 & 0.0800 & -0.0013 & $-1.6 \%$ \\
\hline KT04-07 & $\mathrm{ZrO}_{2}$ & 0.9881 & 1.0700 & -0.0819 & $-7.7 \%$ \\
\hline
\end{tabular}


Table C-4. Comparison of Measured versus Targeted Composition for KT04 Glasses. (continued)

\begin{tabular}{|c|c|c|c|c|c|}
\hline Glass ID & Oxide & $\begin{array}{l}\text { Measured } \\
\text { (wt\%) }\end{array}$ & $\begin{array}{l}\text { Targeted } \\
(\mathrm{wt} \%)\end{array}$ & $\begin{array}{c}\text { Difference of Measured } \\
\text { versus Targeted }\end{array}$ & $\begin{array}{c}\text { \% Difference of } \\
\text { Measured versu } \\
\text { Targeted }\end{array}$ \\
\hline KT04-07 & Sum & 97.5660 & 100.0000 & -2.4340 & $-2.4 \%$ \\
\hline KT04-08 & $\mathrm{Al}_{2} \mathrm{O}_{3}$ & 5.2906 & 5.0700 & 0.2206 & $4.4 \%$ \\
\hline KT04-08 & $\mathrm{B}_{2} \mathrm{O}_{3}$ & 5.8602 & 6.0000 & -0.1398 & $-2.3 \%$ \\
\hline KT04-08 & $\mathrm{BaO}$ & 0.0695 & 0.0800 & -0.0105 & $-13.1 \%$ \\
\hline KT04-08 & $\mathrm{CaO}$ & 0.8514 & 0.8100 & 0.0414 & $5.1 \%$ \\
\hline KT04-08 & $\mathrm{Ce}_{2} \mathrm{O}_{3}$ & 0.0805 & 0.0800 & 0.0005 & $0.7 \%$ \\
\hline KT04-08 & $\mathrm{Cr}_{2} \mathrm{O}_{3}$ & 0.1049 & 0.1000 & 0.0049 & $4.9 \%$ \\
\hline KT04-08 & $\mathrm{CuO}$ & 0.0523 & 0.0400 & 0.0123 & $30.7 \%$ \\
\hline KT04-08 & $\mathrm{Fe}_{2} \mathrm{O}_{3}$ & 9.0035 & 9.2400 & -0.2365 & $-2.6 \%$ \\
\hline KT04-08 & $\mathrm{K}_{2} \mathrm{O}$ & 0.0964 & 0.0800 & 0.0164 & $20.5 \%$ \\
\hline KT04-08 & $\mathrm{La}_{2} \mathrm{O}_{3}$ & 0.0334 & 0.0400 & -0.0066 & $-16.4 \%$ \\
\hline KT04-08 & $\mathrm{Li}_{2} \mathrm{O}$ & 3.6599 & 3.6000 & 0.0599 & $1.7 \%$ \\
\hline KT04-08 & $\mathrm{MgO}$ & 0.1053 & 0.1100 & -0.0047 & $-4.3 \%$ \\
\hline KT04-08 & $\mathrm{MnO}$ & 0.9429 & 0.9700 & -0.0271 & $-2.8 \%$ \\
\hline KT04-08 & $\mathrm{Na}_{2} \mathrm{O}$ & 15.7379 & 15.5600 & 0.1779 & $1.1 \%$ \\
\hline KT04-08 & $\mathrm{Nb}_{2} \mathrm{O}_{5}$ & 1.2624 & 1.3100 & -0.0476 & $-3.6 \%$ \\
\hline KT04-08 & $\mathrm{NiO}$ & 0.5275 & 0.6100 & -0.0825 & $-13.5 \%$ \\
\hline KT04-08 & $\mathrm{PbO}$ & 0.0646 & 0.0700 & -0.0054 & $-7.7 \%$ \\
\hline KT04-08 & $\mathrm{SiO}_{2}$ & 49.0435 & 50.0400 & -0.9965 & $-2.0 \%$ \\
\hline KT04-08 & $\mathrm{TiO}_{2}$ & 4.9665 & 4.9900 & -0.0235 & $-0.5 \%$ \\
\hline KT04-08 & $\mathrm{ZnO}$ & 0.0417 & 0.0400 & 0.0017 & $4.3 \%$ \\
\hline KT04-08 & $\mathrm{ZrO}_{2}$ & 1.0519 & 1.1400 & -0.0881 & $-7.7 \%$ \\
\hline KT04-08 & Sum & 98.8468 & 99.9800 & -1.1332 & $-1.1 \%$ \\
\hline KT04-09 & $\mathrm{Al}_{2} \mathrm{O}_{3}$ & 5.8575 & 5.5300 & 0.3275 & $5.9 \%$ \\
\hline KT04-09 & $\mathrm{B}_{2} \mathrm{O}_{3}$ & 5.8924 & 6.0000 & -0.1076 & $-1.8 \%$ \\
\hline KT04-09 & $\mathrm{BaO}$ & 0.0659 & 0.0800 & -0.0141 & $-17.7 \%$ \\
\hline KT04-09 & $\mathrm{CaO}$ & 1.0253 & 0.9600 & 0.0653 & $6.8 \%$ \\
\hline KT04-09 & $\mathrm{Ce}_{2} \mathrm{O}_{3}$ & 0.1862 & 0.2000 & -0.0138 & $-6.9 \%$ \\
\hline KT04-09 & $\mathrm{Cr}_{2} \mathrm{O}_{3}$ & 0.0694 & 0.1000 & -0.0306 & $-30.6 \%$ \\
\hline KT04-09 & $\mathrm{CuO}$ & 0.0726 & 0.0400 & 0.0326 & $81.5 \%$ \\
\hline KT04-09 & $\mathrm{Fe}_{2} \mathrm{O}_{3}$ & 12.0381 & 12.4300 & -0.3919 & $-3.2 \%$ \\
\hline KT04-09 & $\mathrm{K}_{2} \mathrm{O}$ & 0.1093 & 0.0800 & 0.0293 & $36.6 \%$ \\
\hline KT04-09 & $\mathrm{La}_{2} \mathrm{O}_{3}$ & 0.0674 & 0.0800 & -0.0126 & $-15.7 \%$ \\
\hline KT04-09 & $\mathrm{Li}_{2} \mathrm{O}$ & 3.6438 & 3.6000 & 0.0438 & $1.2 \%$ \\
\hline KT04-09 & $\mathrm{MgO}$ & 0.1036 & 0.1100 & -0.0064 & $-5.8 \%$ \\
\hline KT04-09 & $\mathrm{MnO}$ & 0.5417 & 0.5800 & -0.0383 & $-6.6 \%$ \\
\hline KT04-09 & $\mathrm{Na}_{2} \mathrm{O}$ & 14.4910 & 14.1500 & 0.3410 & $2.4 \%$ \\
\hline KT04-09 & $\mathrm{Nb}_{2} \mathrm{O}_{5}$ & 0.8172 & 0.8500 & -0.0328 & $-3.9 \%$ \\
\hline KT04-09 & $\mathrm{NiO}$ & 0.4327 & 0.5200 & -0.0874 & $-16.8 \%$ \\
\hline KT04-09 & $\mathrm{PbO}$ & 0.1180 & 0.1500 & -0.0320 & $-21.4 \%$ \\
\hline KT04-09 & $\mathrm{SiO}_{2}$ & 47.7064 & 48.7900 & -1.0836 & $-2.2 \%$ \\
\hline KT04-09 & $\mathrm{TiO}_{2}$ & 4.8706 & 4.8800 & -0.0094 & $-0.2 \%$ \\
\hline KT04-09 & $\mathrm{ZnO}$ & 0.0464 & 0.0400 & 0.0064 & $15.9 \%$ \\
\hline KT04-09 & $\mathrm{ZrO}_{2}$ & 0.7413 & 0.8100 & -0.0687 & $-8.5 \%$ \\
\hline KT04-09 & Sum & 98.8965 & 99.9800 & -1.0835 & $-1.1 \%$ \\
\hline KT04-10 & $\mathrm{Al}_{2} \mathrm{O}_{3}$ & 5.7960 & 5.6800 & 0.1160 & $2.0 \%$ \\
\hline KT04-10 & $\mathrm{B}_{2} \mathrm{O}_{3}$ & 5.8844 & 6.0000 & -0.1156 & $-1.9 \%$ \\
\hline KT04-10 & $\mathrm{BaO}$ & 0.0770 & 0.0800 & -0.0030 & $-3.7 \%$ \\
\hline KT04-10 & $\mathrm{CaO}$ & 0.9885 & 0.9800 & 0.0085 & $0.9 \%$ \\
\hline KT04-10 & $\mathrm{Ce}_{2} \mathrm{O}_{3}$ & 0.2460 & 0.2500 & -0.0040 & $-1.6 \%$ \\
\hline KT04-10 & $\mathrm{Cr}_{2} \mathrm{O}_{3}$ & 0.0808 & 0.1000 & -0.0192 & $-19.2 \%$ \\
\hline KT04-10 & $\mathrm{CuO}$ & 0.0523 & 0.0400 & 0.0123 & $30.7 \%$ \\
\hline KT04-10 & $\mathrm{Fe}_{2} \mathrm{O}_{3}$ & 13.2283 & 13.7400 & -0.5117 & $-3.7 \%$ \\
\hline KT04-10 & $\mathrm{K}_{2} \mathrm{O}$ & 0.0425 & 0.0400 & 0.0025 & $6.2 \%$ \\
\hline KT04-10 & $\mathrm{La}_{2} \mathrm{O}_{3}$ & 0.0754 & 0.0800 & -0.0046 & $-5.8 \%$ \\
\hline KT04-10 & $\mathrm{Li}_{2} \mathrm{O}$ & 3.6115 & 3.6000 & 0.0115 & $0.3 \%$ \\
\hline KT04-10 & $\mathrm{MgO}$ & 0.1123 & 0.1200 & -0.0077 & $-6.4 \%$ \\
\hline KT04-10 & $\mathrm{MnO}$ & 0.3535 & 0.4100 & -0.0565 & $-13.8 \%$ \\
\hline
\end{tabular}


SRNL-STI-2010-00566

Revision 0

Table C-4. Comparison of Measured versus Targeted Composition for KT04 Glasses. (continued)

\begin{tabular}{|c|c|c|c|c|c|}
\hline Glass ID & Oxide & $\begin{array}{c}\text { Measured } \\
\mathbf{( w t \% )}\end{array}$ & $\begin{array}{c}\text { Targeted } \\
\mathbf{( w t \% )}\end{array}$ & $\begin{array}{c}\text { Difference of Measured } \\
\text { versus Targeted }\end{array}$ & $\begin{array}{c}\text { \% Difference of } \\
\text { Measured versus } \\
\text { Targeted }\end{array}$ \\
\hline $\mathrm{KT04-10}$ & $\mathrm{Na}_{2} \mathrm{O}$ & 13.5137 & 13.6300 & -0.1163 & $-0.9 \%$ \\
\hline $\mathrm{KT} 04-10$ & $\mathrm{Nb}_{2} \mathrm{O}_{5}$ & 0.7675 & 0.7900 & -0.0225 & $-2.9 \%$ \\
\hline $\mathrm{KT} 04-10$ & $\mathrm{NiO}$ & 0.4279 & 0.4900 & -0.0621 & $-12.7 \%$ \\
\hline $\mathrm{KT} 04-10$ & $\mathrm{PbO}$ & 0.1287 & 0.1500 & -0.0213 & $-14.2 \%$ \\
\hline $\mathrm{KT} 04-10$ & $\mathrm{SiO}_{2}$ & 47.7599 & 48.4500 & -0.6901 & $-1.4 \%$ \\
\hline $\mathrm{KT} 04-10$ & $\mathrm{TiO}_{2}$ & 4.5370 & 4.5600 & -0.0230 & $-0.5 \%$ \\
\hline $\mathrm{KT} 04-10$ & $\mathrm{ZnO}$ & 0.0417 & 0.0400 & 0.0017 & $4.3 \%$ \\
\hline $\mathrm{KT} 04-10$ & $\mathrm{ZrO}_{2}$ & 0.7298 & 0.7700 & -0.0402 & $-5.2 \%$ \\
\hline $\mathrm{KT} 04-10$ & $\mathrm{Sum}$ & 98.4545 & 100.0000 & -1.5455 & $-1.5 \%$ \\
\hline
\end{tabular}


Exhibit C-1 Measurements in Analytical Sequence for KT04-Series by Preparation Method by Oxide.

Oxide $=\mathrm{Al} 2 \mathrm{O} 3$ (wt \%), Prep Method $=$ LM

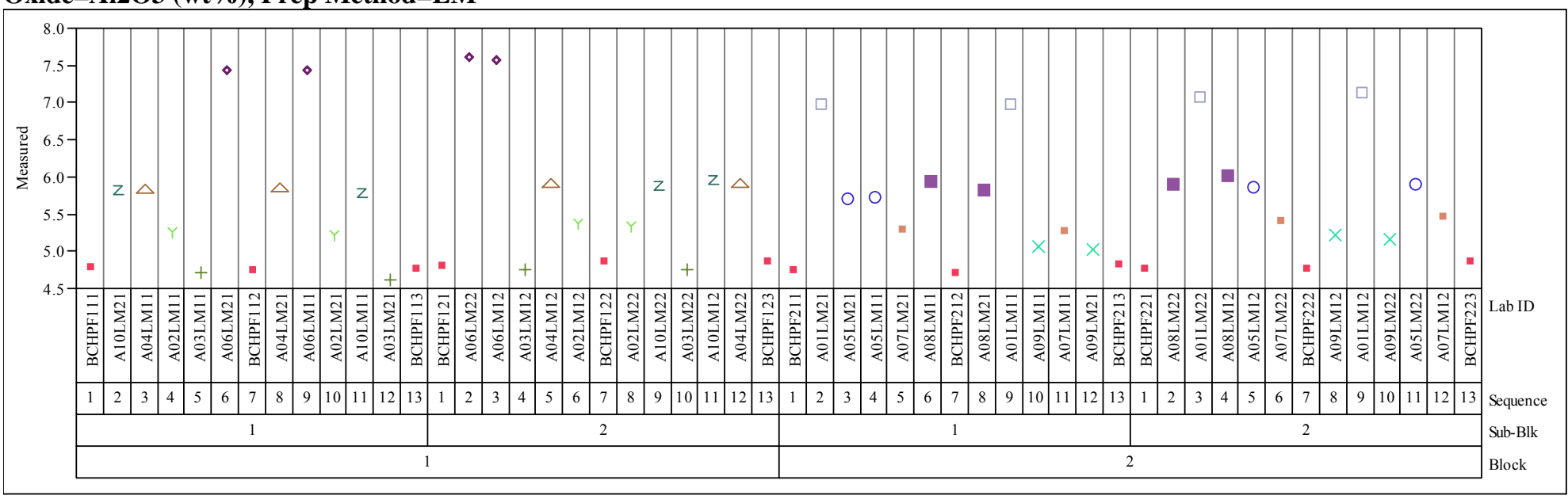

Oxide=B2O3 (wt\%), Prep Method=PF

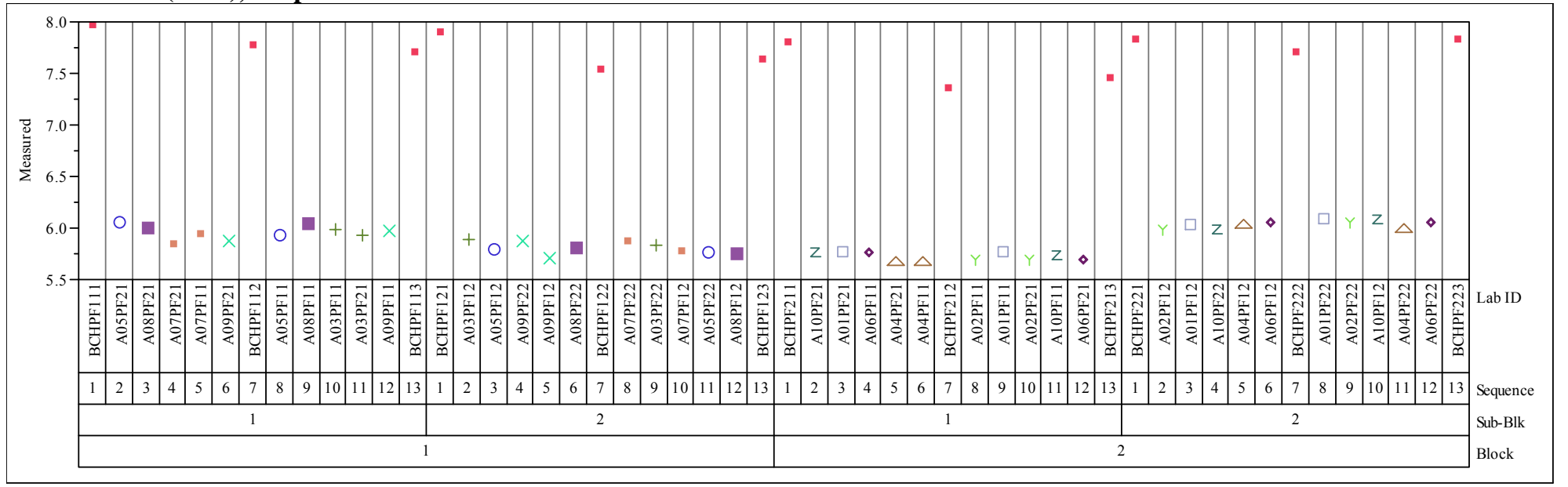


Exhibit C-1 Measurements in Analytical Sequence for KT04-Series by Preparation Method by Oxide. (continued) Oxide=BaO (wt \%), Prep Method=LM

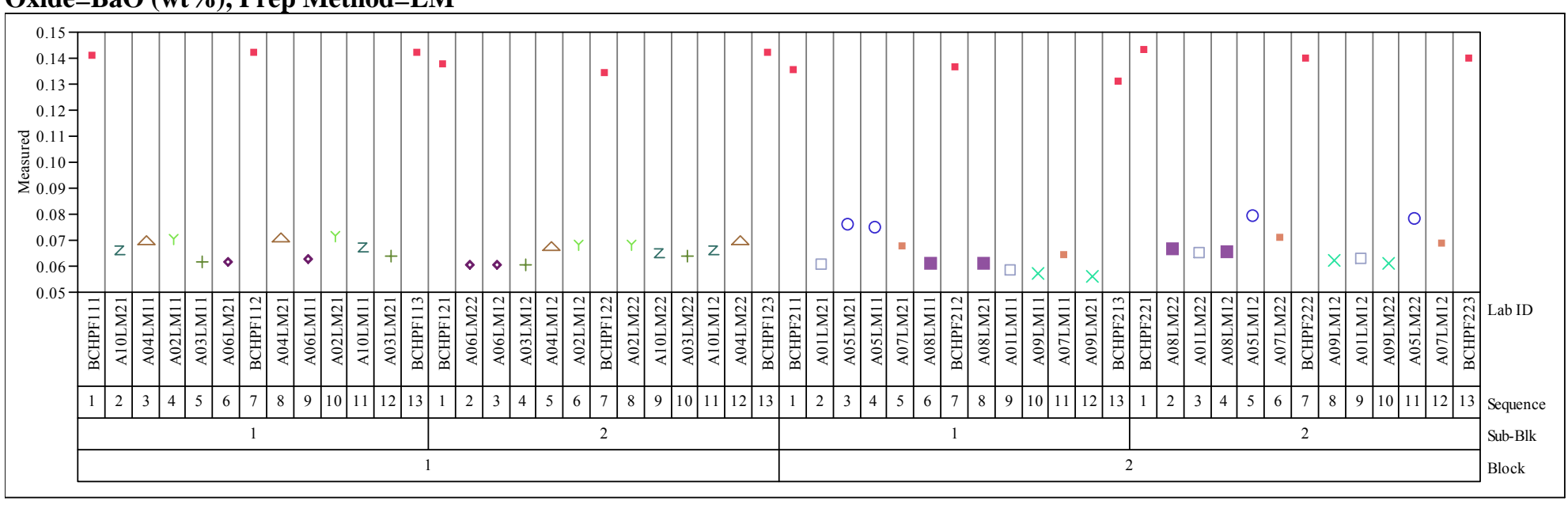

\section{Oxide $=\mathrm{CaO}$ (wt \%), Prep Method $=\mathrm{LM}$}

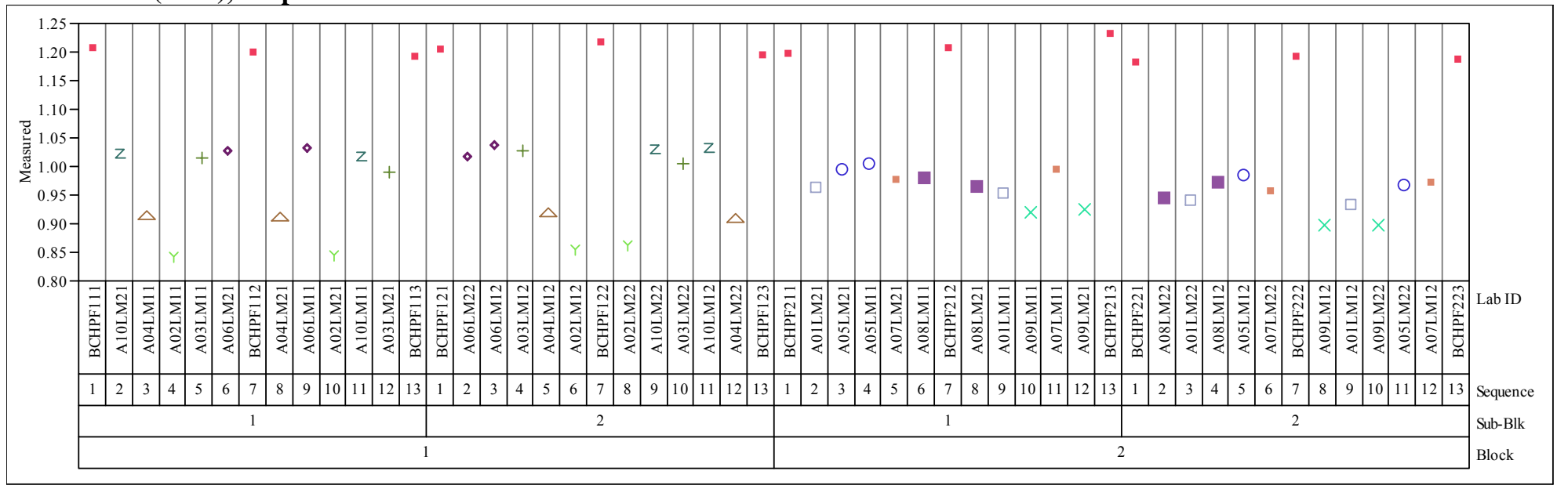


Exhibit C-1 Measurements in Analytical Sequence for KT04-Series by Preparation Method by Oxide. (continued) Oxide $=\mathrm{Ce} 2 \mathrm{O} 3$ (wt\%), Prep Method=LM

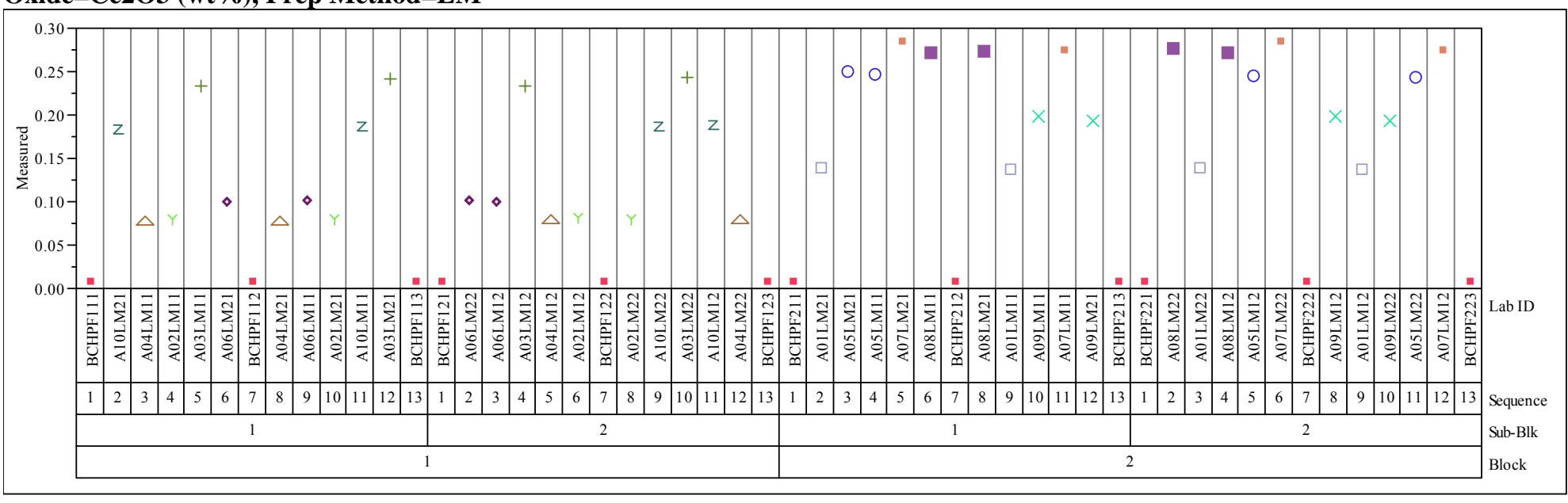

\section{Oxide $=$ Cr2O3 (wt\%), Prep Method $=$ LM}

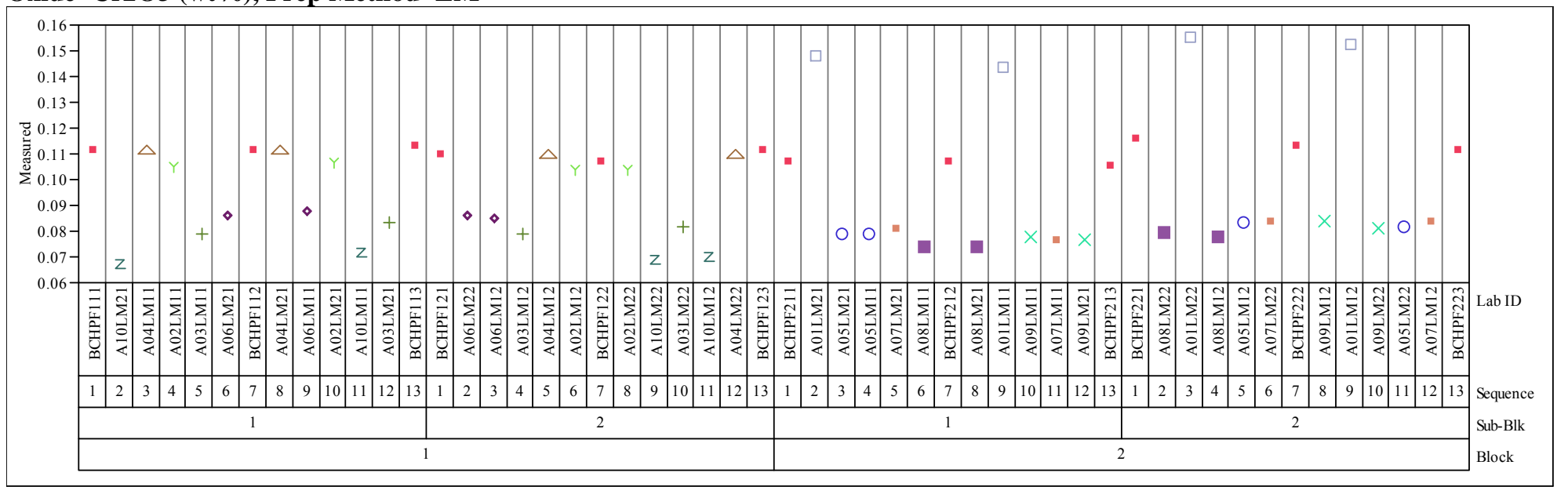


Exhibit C-1 Measurements in Analytical Sequence for KT04-Series by Preparation Method by Oxide. (continued) Oxide $=\mathrm{CuO}($ wt \%), Prep Method $=$ LM

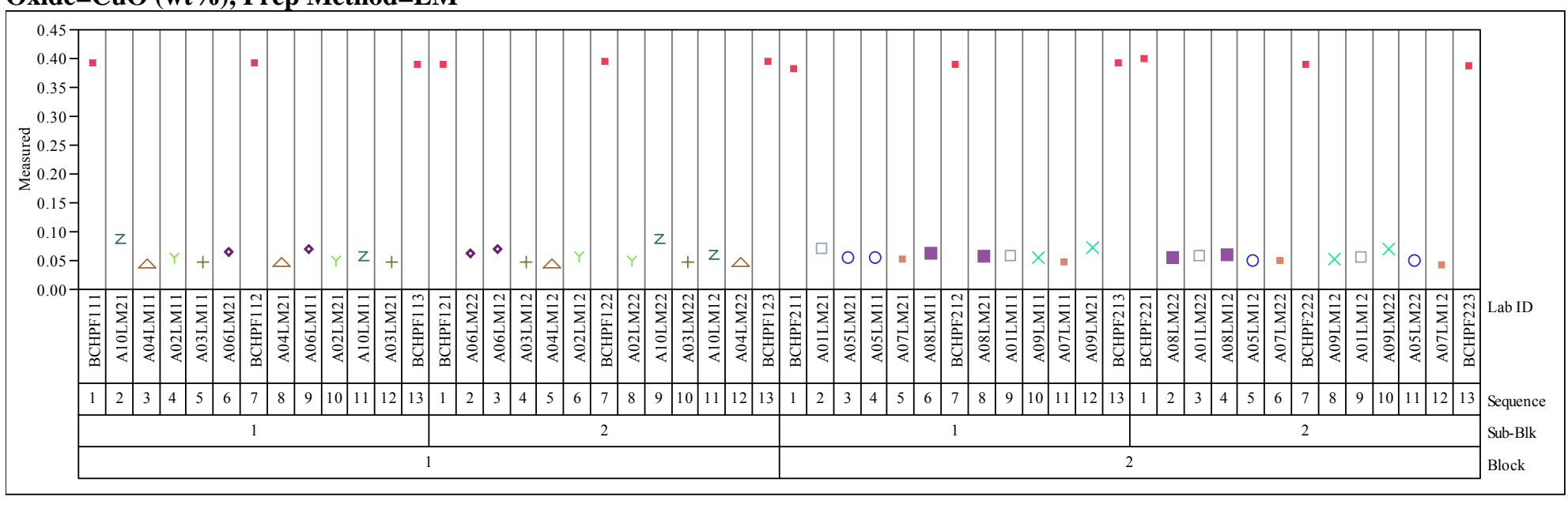

Oxide=Fe2O3 (wt\%), Prep Method=LM

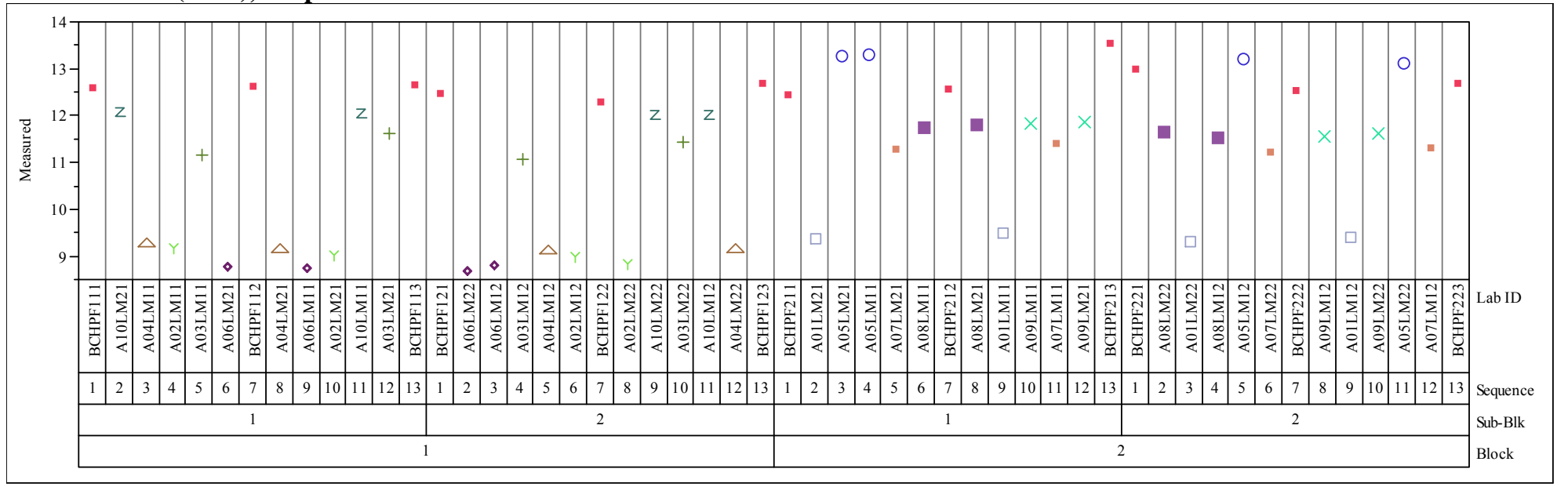


Exhibit C-1 Measurements in Analytical Sequence for KT04-Series by Preparation Method by Oxide. (continued) Oxide=K2O (wt\%), Prep Method=LM

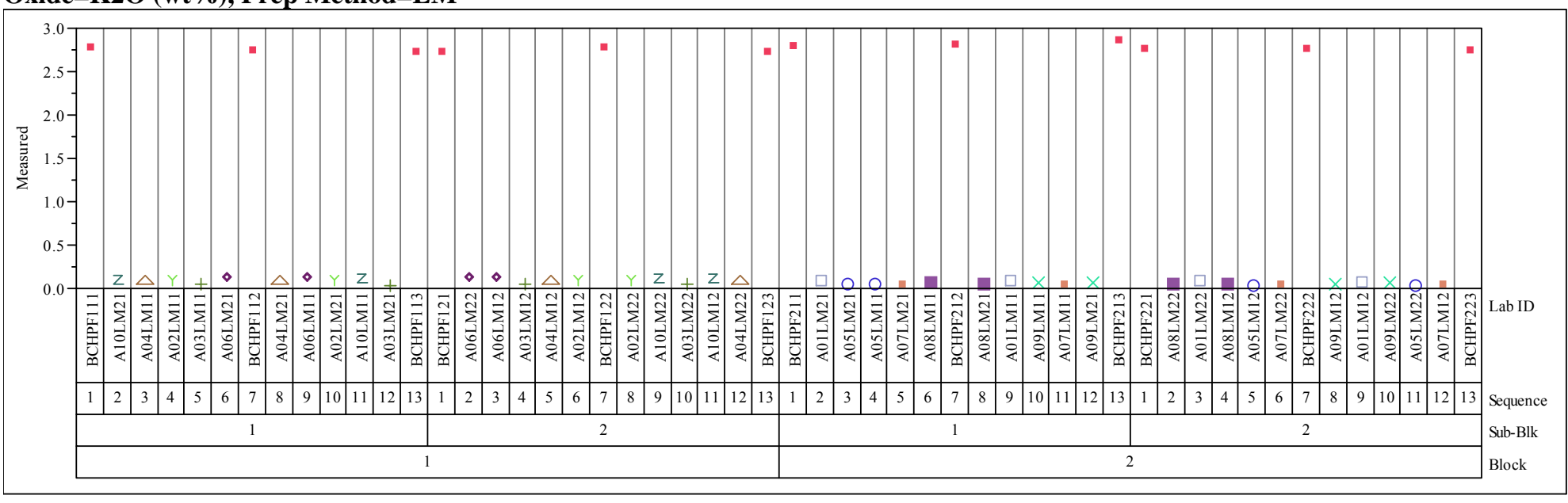

Oxide=La2O3 (wt\%), Prep Method=LM

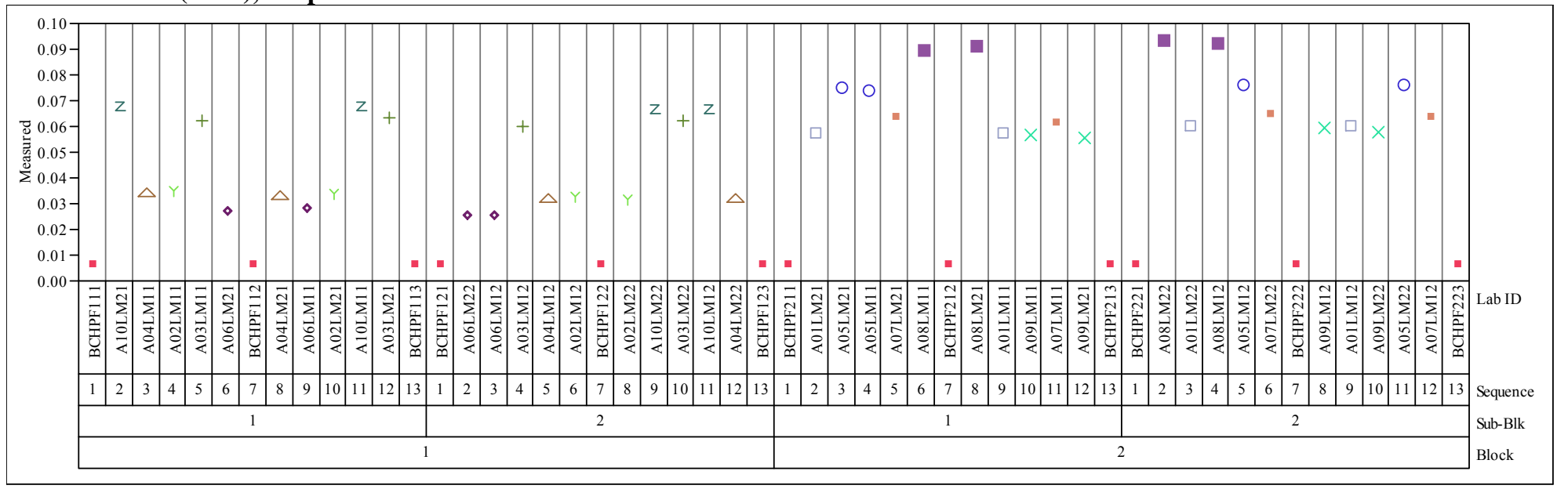


Exhibit C-1 Measurements in Analytical Sequence for KT04-Series by Preparation Method by Oxide. (continued) Oxide=Li2O (wt\%), Prep Method=PF

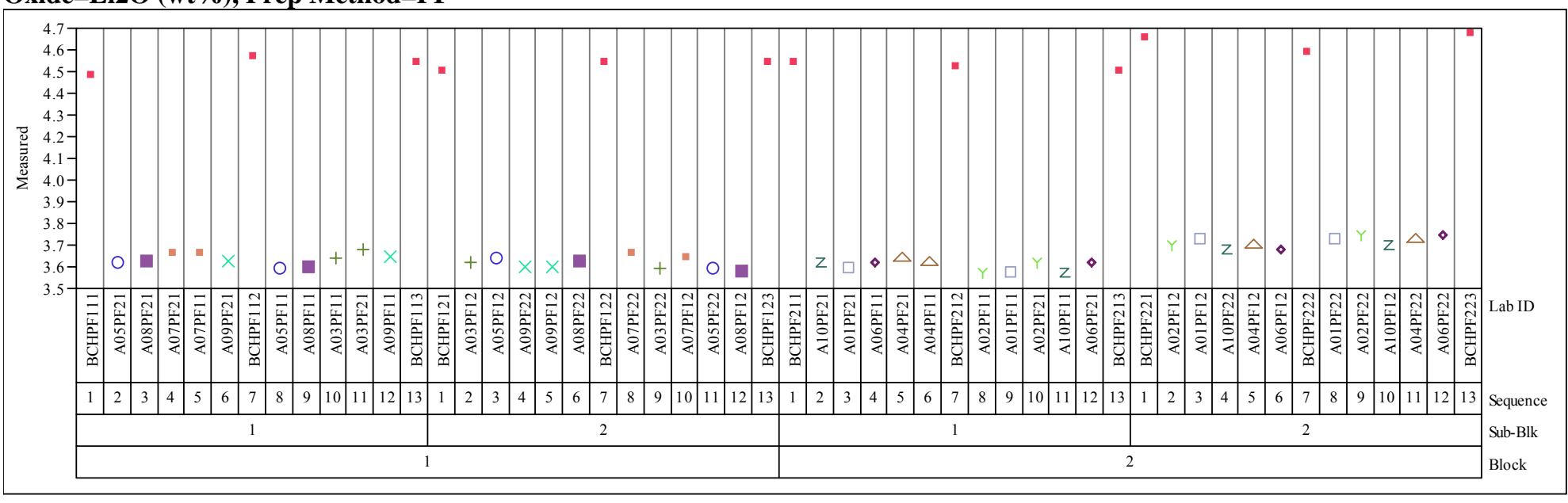

Oxide=MgO (wt\%), Prep Method=LM

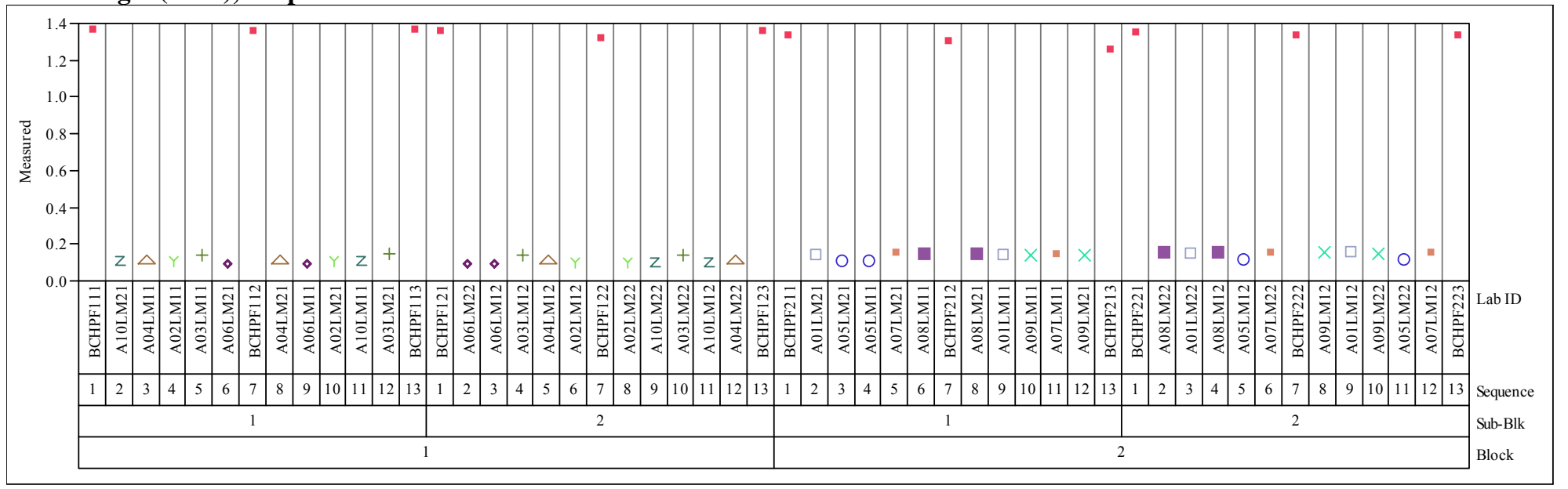


Exhibit C-1 Measurements in Analytical Sequence for KT04-Series by Preparation Method by Oxide. (continued) Oxide=MnO (wt\%), Prep Method=LM

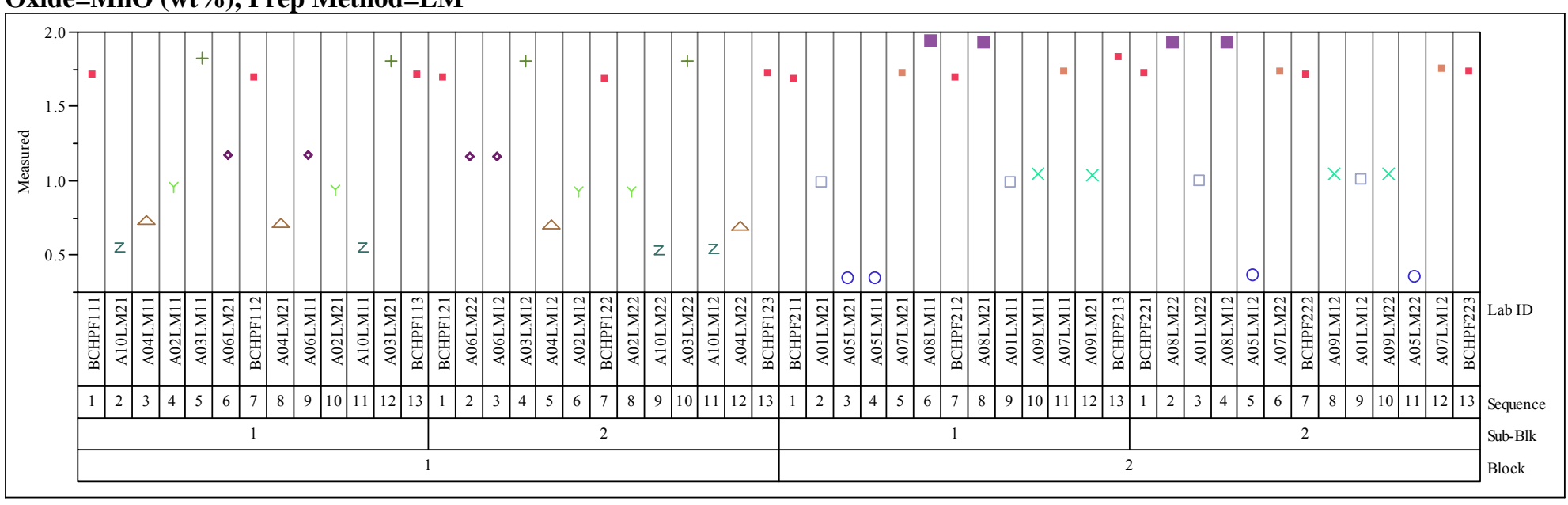

Oxide=Na2O (wt\%), Prep Method $=$ LM

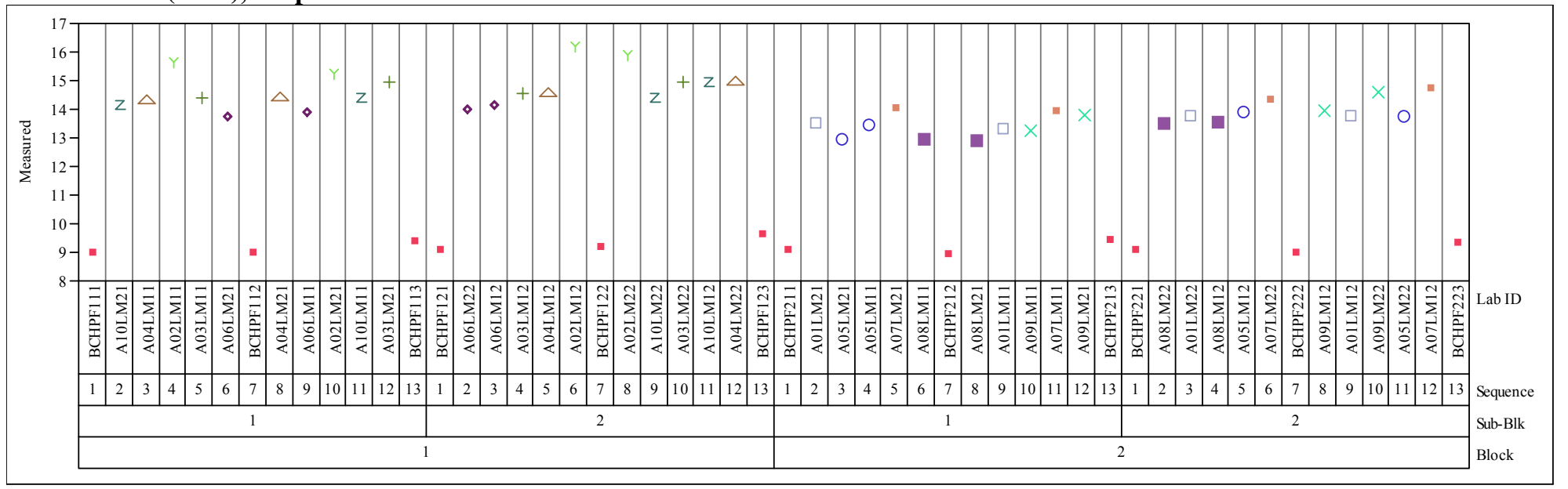


Exhibit C-1 Measurements in Analytical Sequence for KT04-Series by Preparation Method by Oxide. (continued) Oxide=Nb2O5 (wt\%), Prep Method=LM

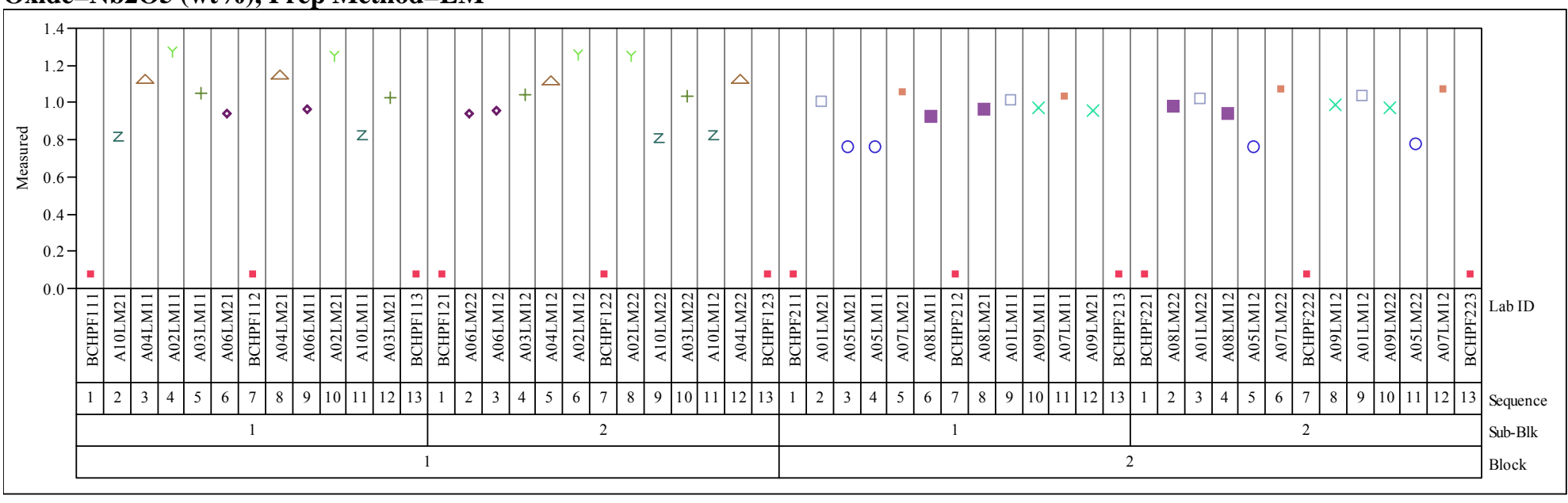

Oxide=NiO (wt\%), Prep Method=LM

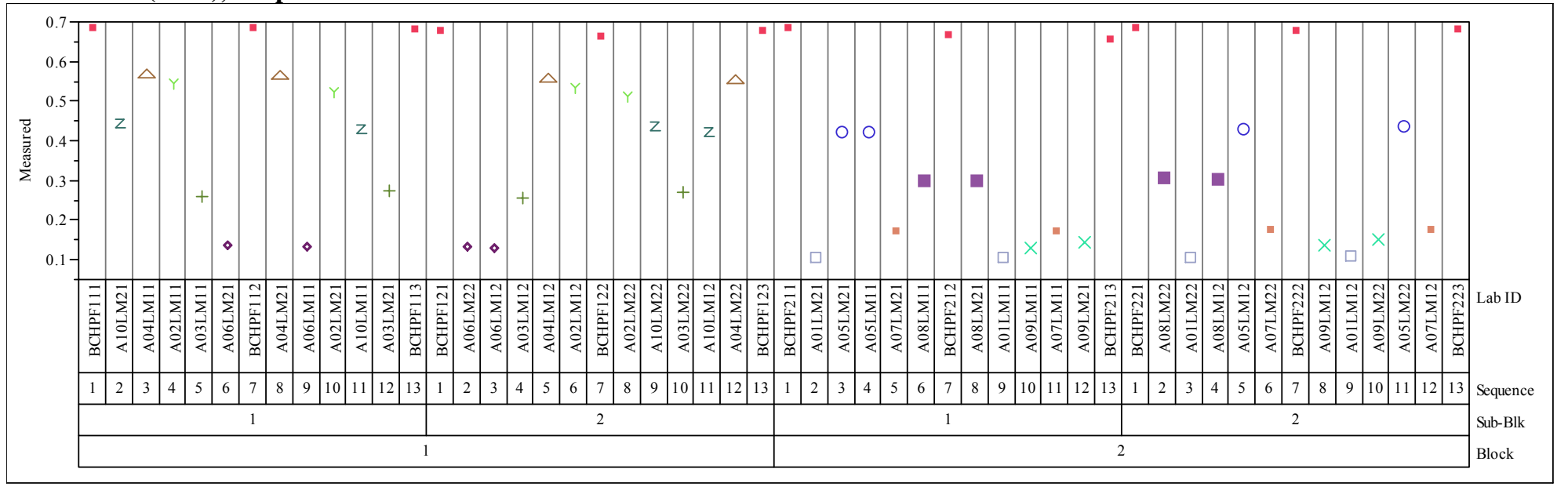


Exhibit C-1 Measurements in Analytical Sequence for KT04-Series by Preparation Method by Oxide. (continued) Oxide=PbO (wt \%), Prep Method=LM

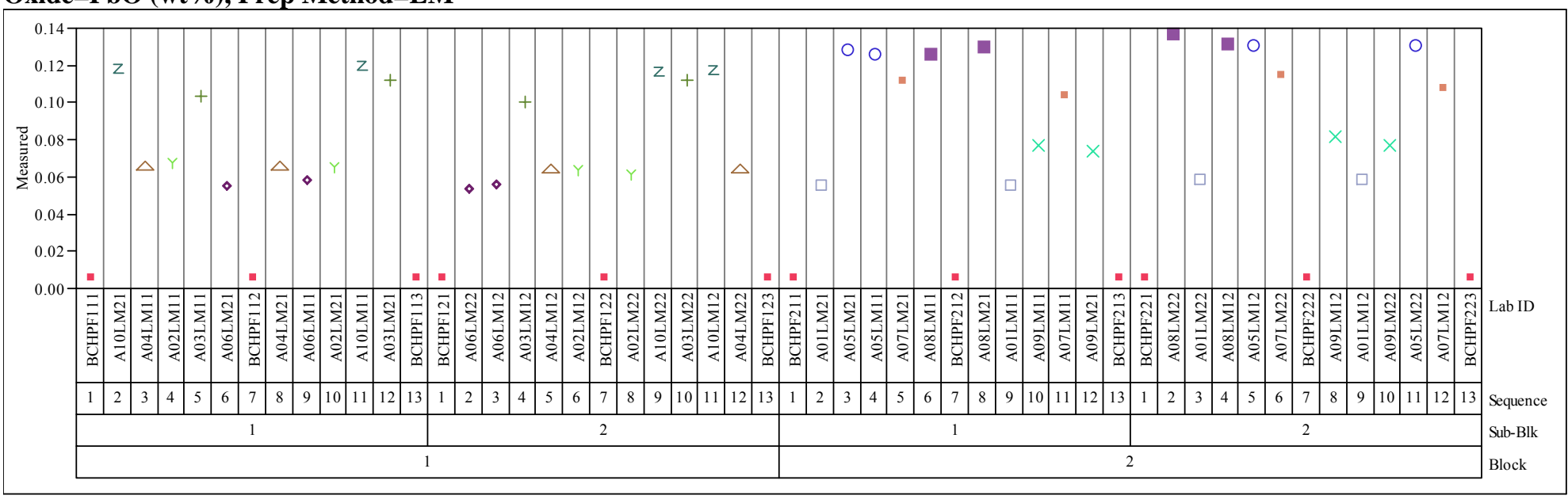

Oxide=SiO2 (wt\%), Prep Method=LM

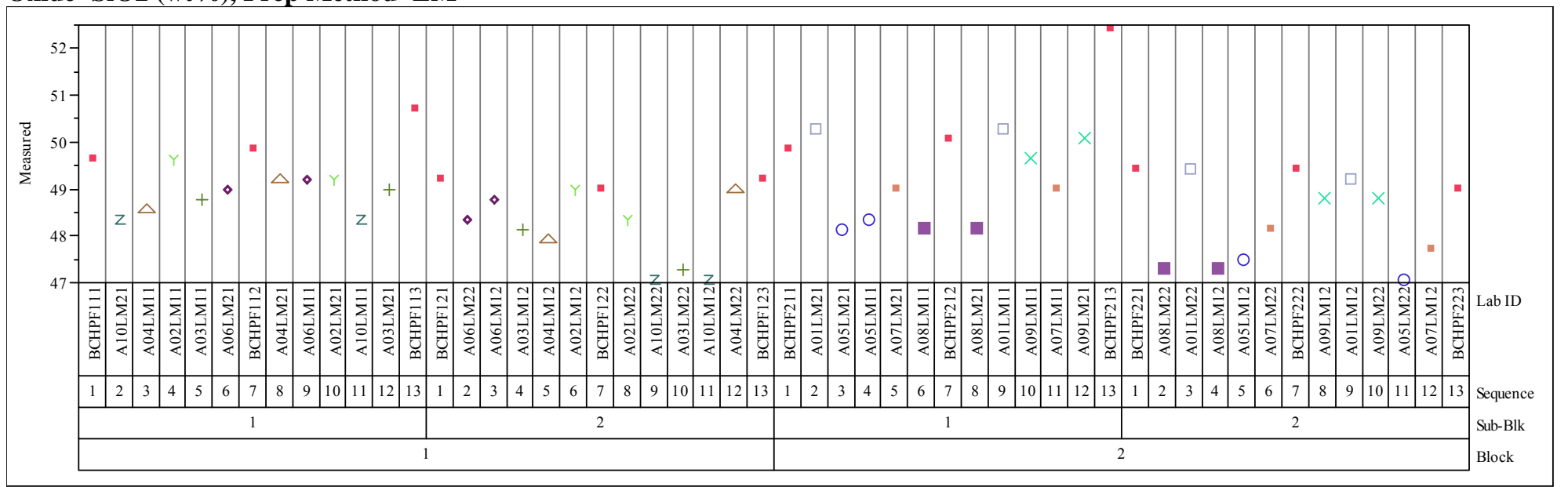


Exhibit C-1 Measurements in Analytical Sequence for KT04-Series by Preparation Method by Oxide. (continued) Oxide=TiO2 (wt\%), Prep Method=LM

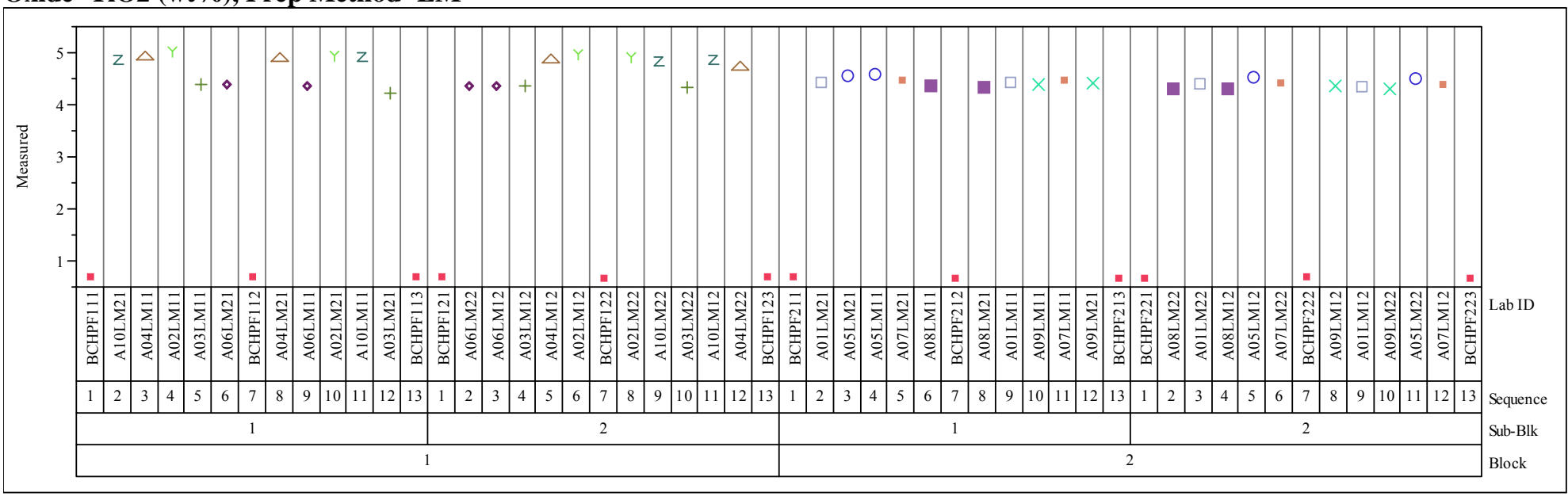

\section{Oxide=ZnO (wt\%), Prep Method=LM}

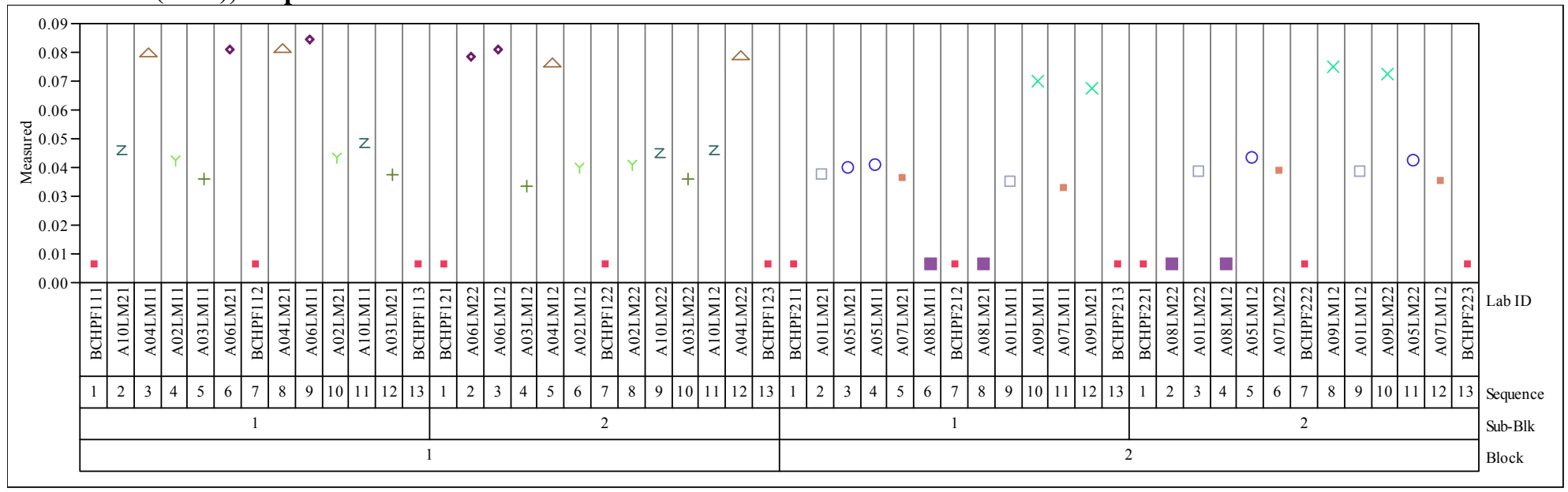


Exhibit C-1 Measurements in Analytical Sequence for KT04-Series by Preparation Method by Oxide. (continued) Oxide=ZrO2 (wt\%), Prep Method=LM

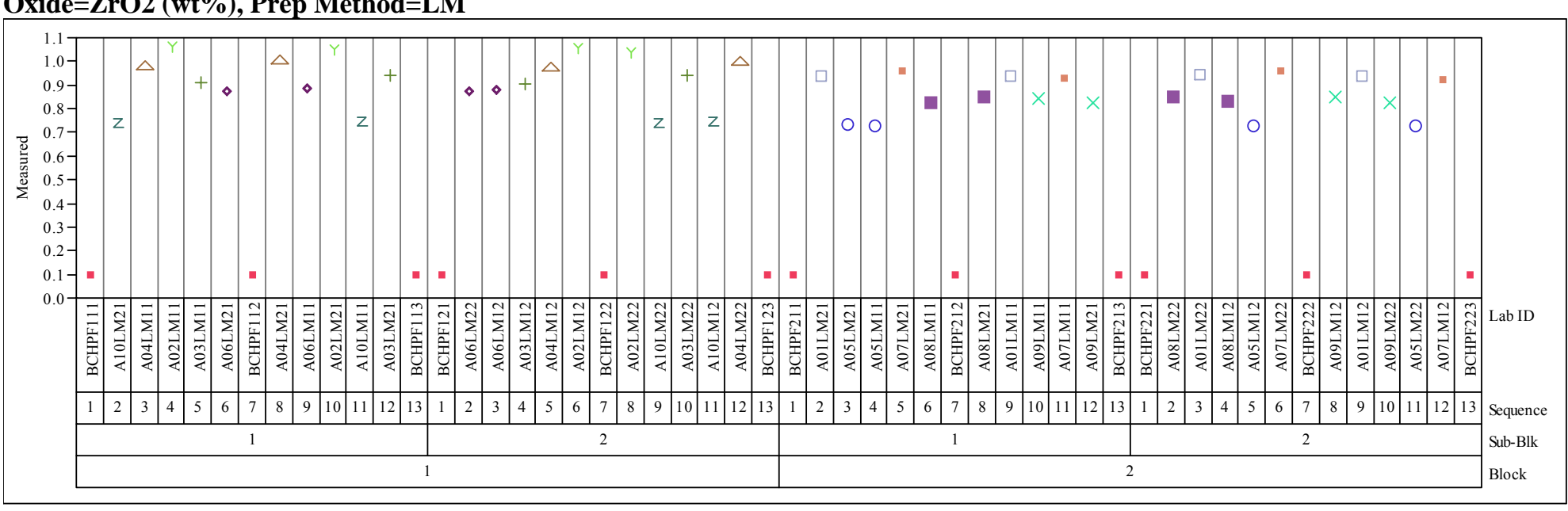


Exhibit C-2. Measurements for Each KT01-Series Glass ID by Preparation Method by Oxide.

Oxide=Al2O3 (wt \%), Prep Method $=$ LM

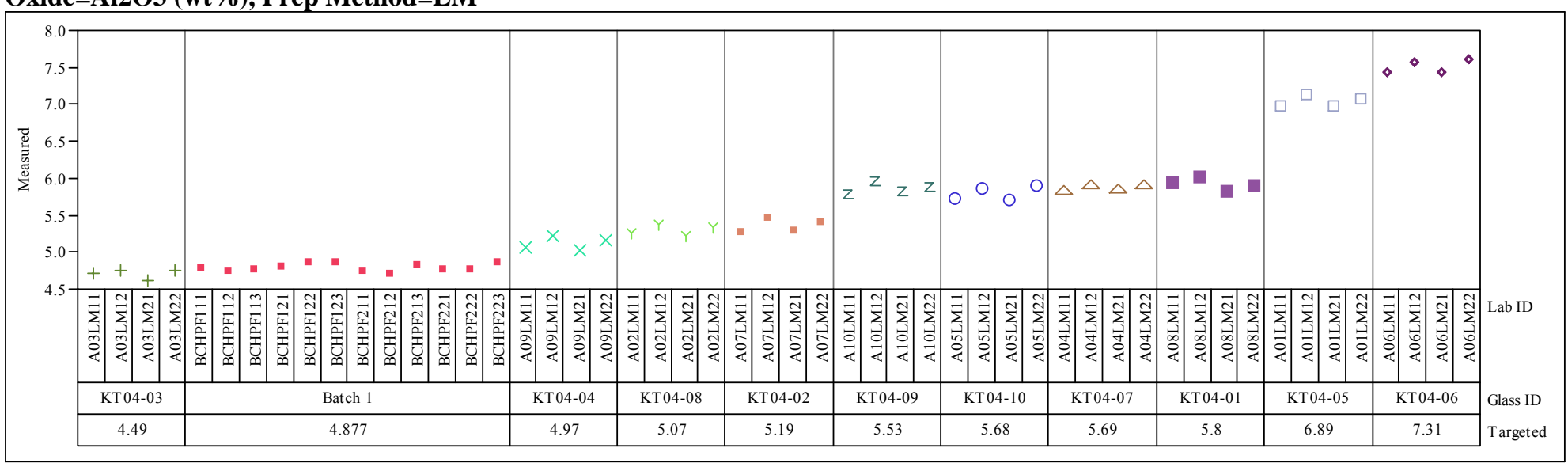

Oxide=B2O3 (wt\%), Prep Method=PF

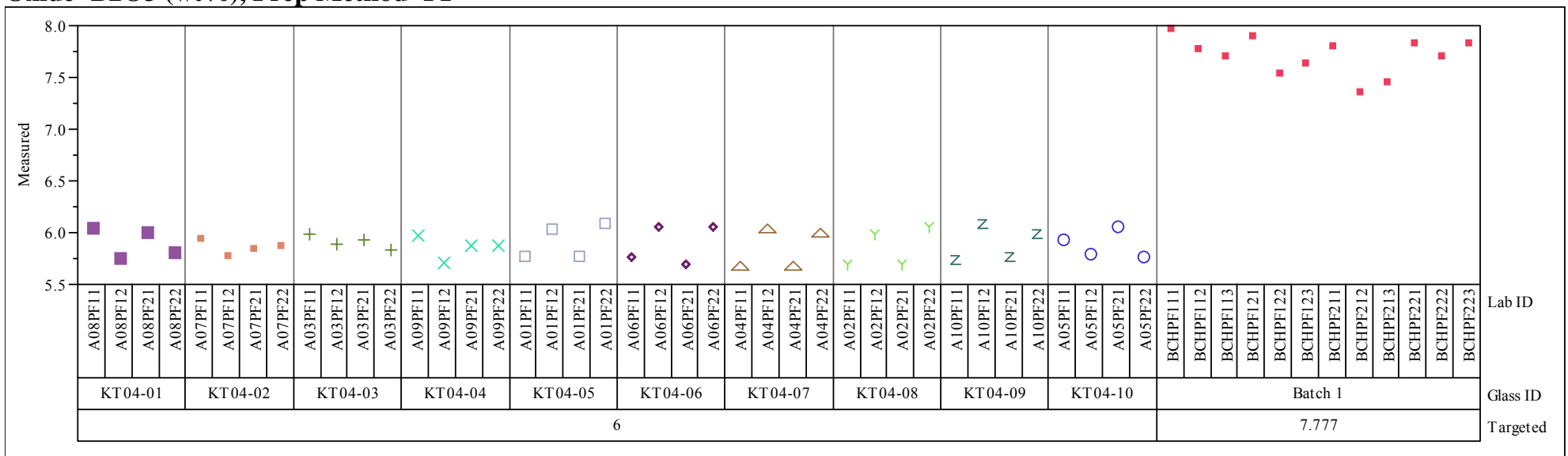




\section{Exhibit C-2. Measurements for Each KT01-Series Glass ID by Preparation Method by Oxide. (continued)}

Oxide=BaO (wt \%), Prep Method=LM

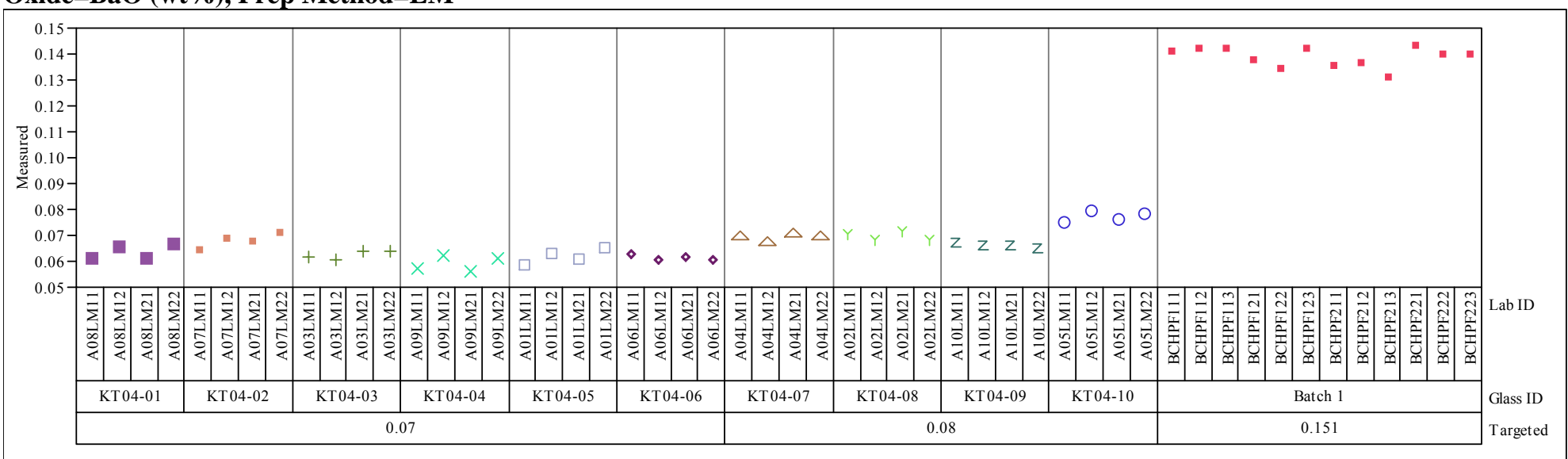

Oxide $=\mathrm{CaO}$ (wt\%), Prep Method $=$ LM

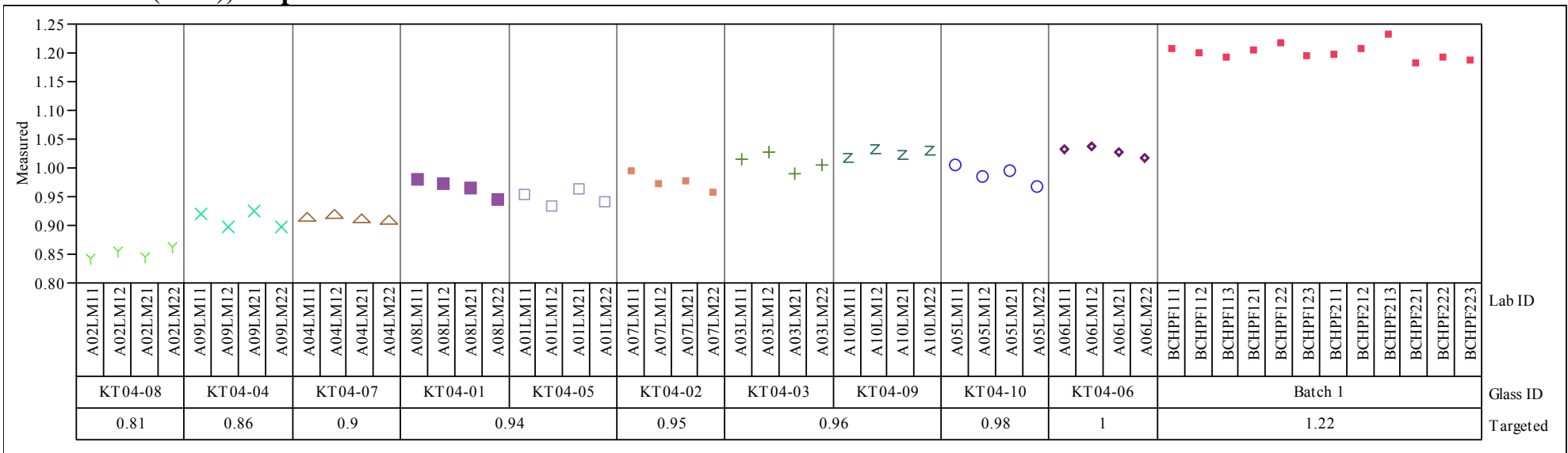




\section{Exhibit C-2. Measurements for Each KT01-Series Glass ID by Preparation Method by Oxide. (continued)}

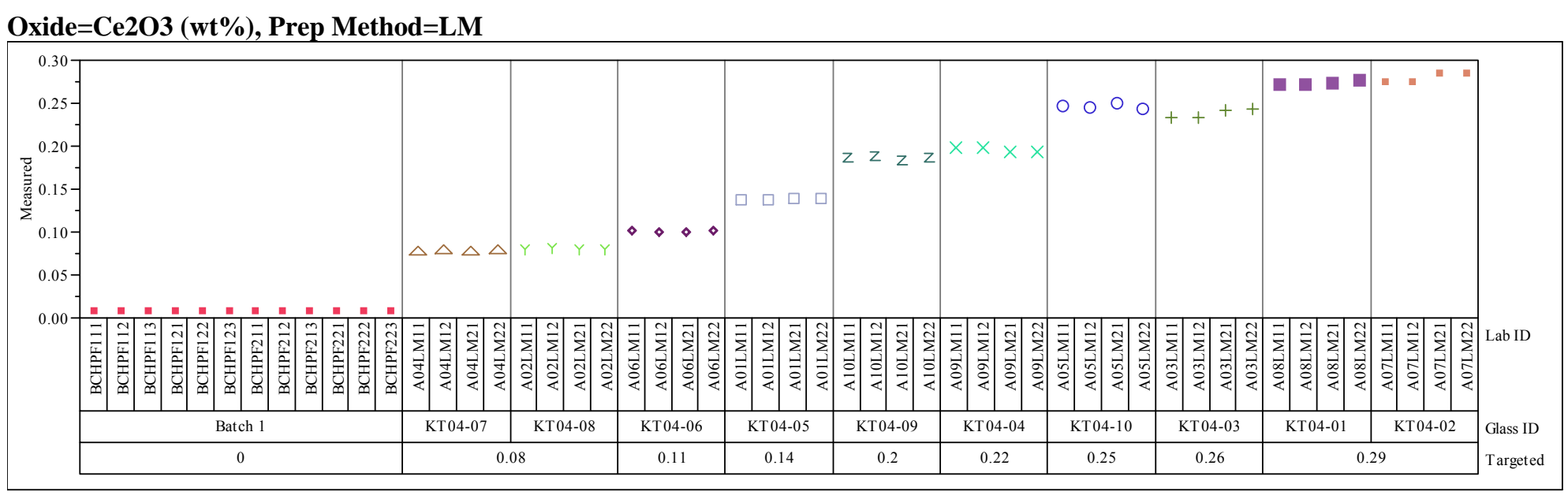

\section{Oxide $=\mathrm{Cr} 2 \mathrm{O} 3$ (wt\%), Prep Method $=$ LM}

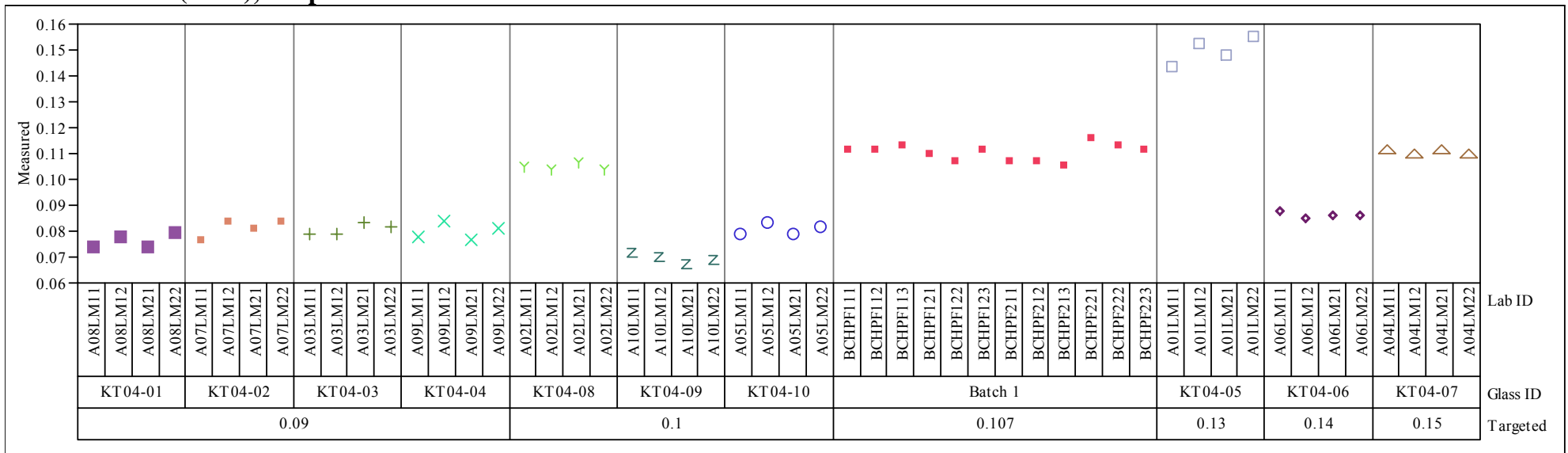




\section{Exhibit C-2. Measurements for Each KT01-Series Glass ID by Preparation Method by Oxide. (continued)}

\section{Oxide $=\mathrm{CuO}$ (wt\%), Prep Method=LM}

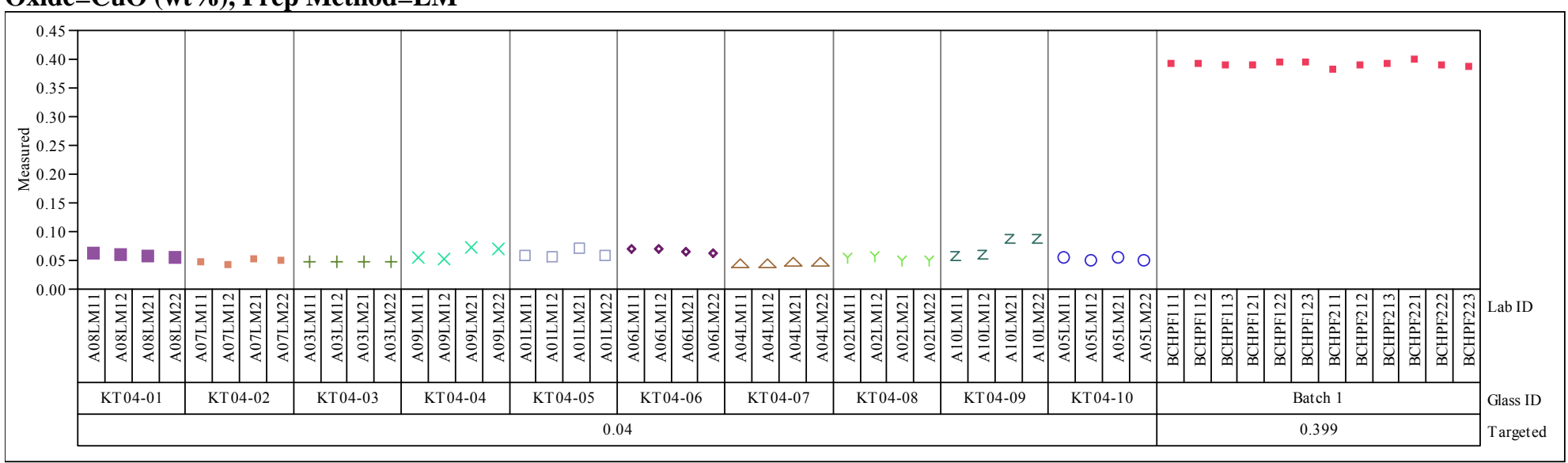

Oxide=Fe2O3 (wt\%), Prep Method=LM

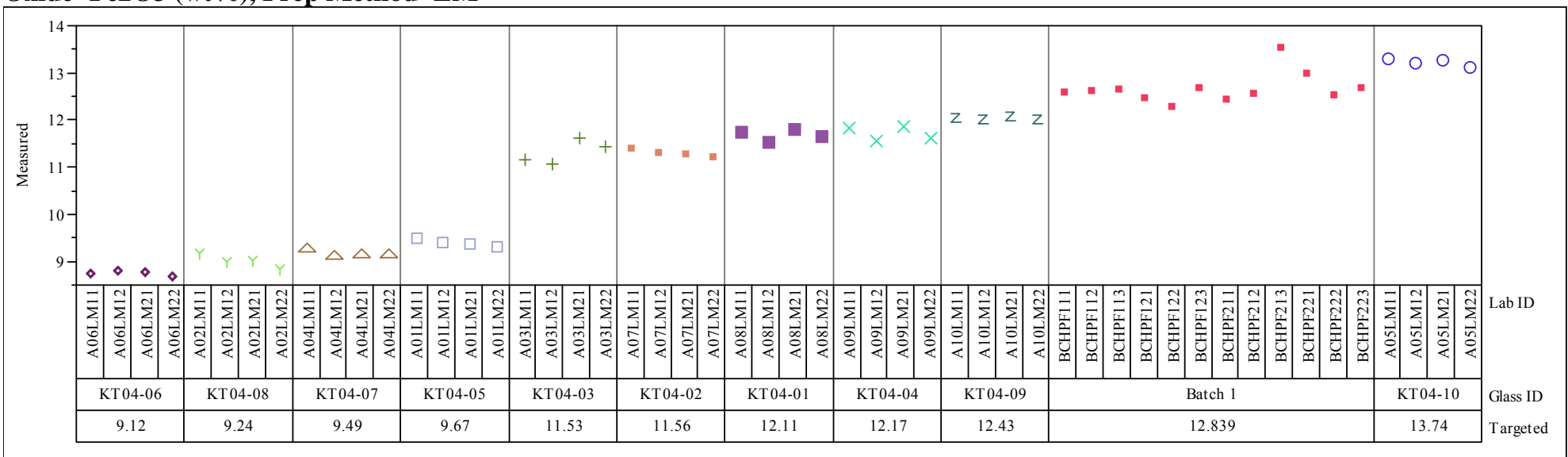




\section{Exhibit C-2. Measurements for Each KT01-Series Glass ID by Preparation Method by Oxide. (continued)}

\section{Oxide=K2O (wt\%), Prep Method=LM}

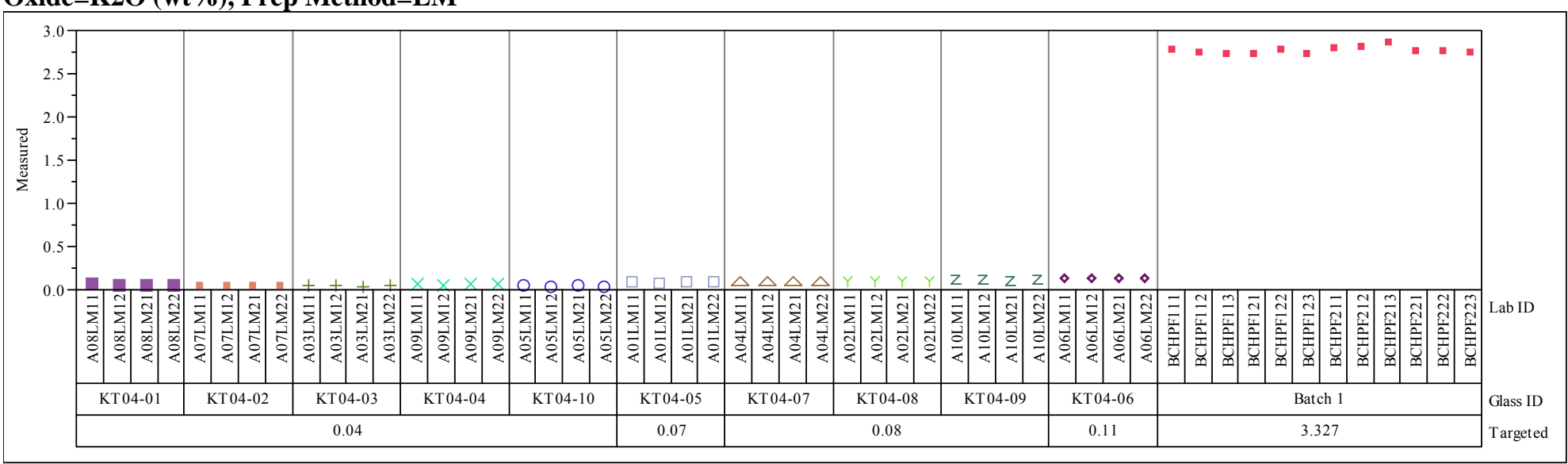

\section{Oxide=La2O3 (wt\%), Prep Method=LM}

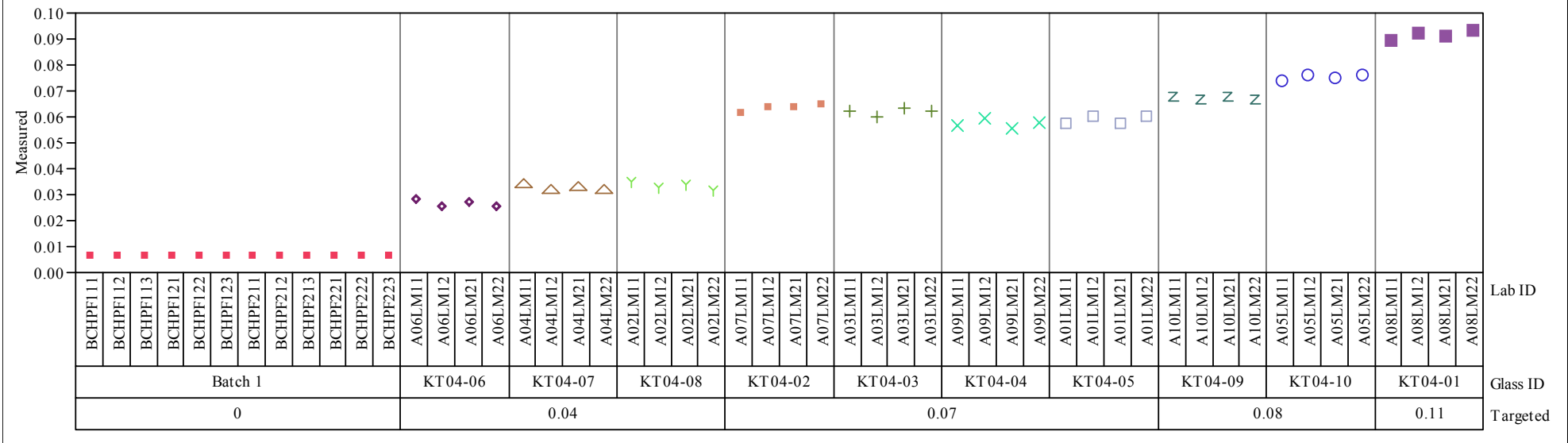




\section{Exhibit C-2. Measurements for Each KT01-Series Glass ID by Preparation Method by Oxide. (continued)}

Oxide=Li2O (wt\%), Prep Method=PF

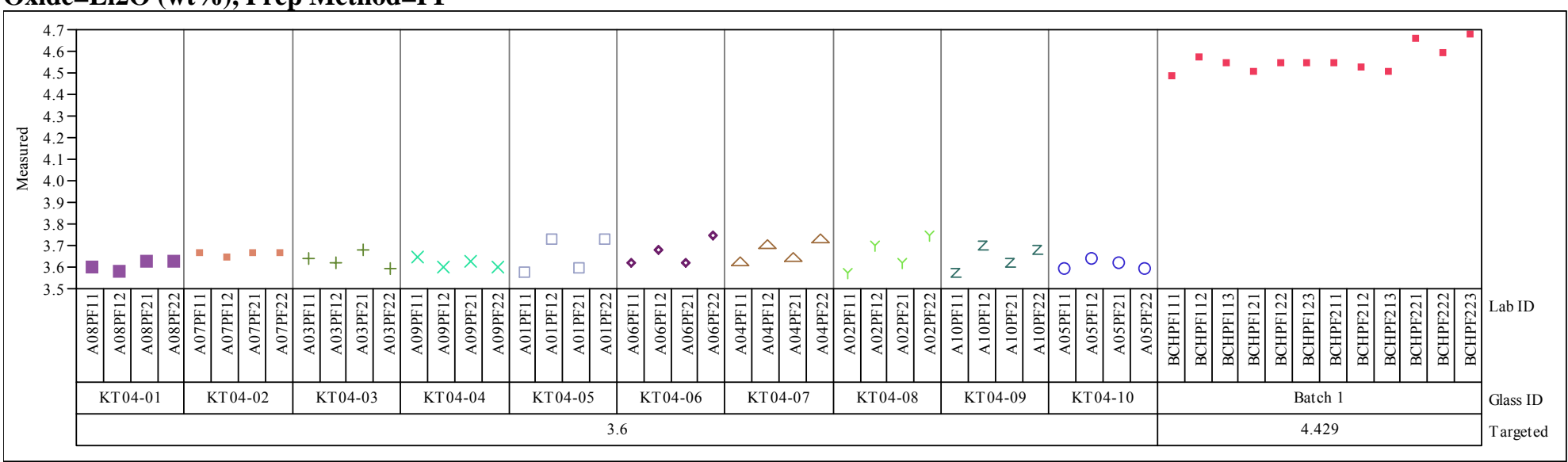

Oxide=MgO (wt\%), Prep Method=LM

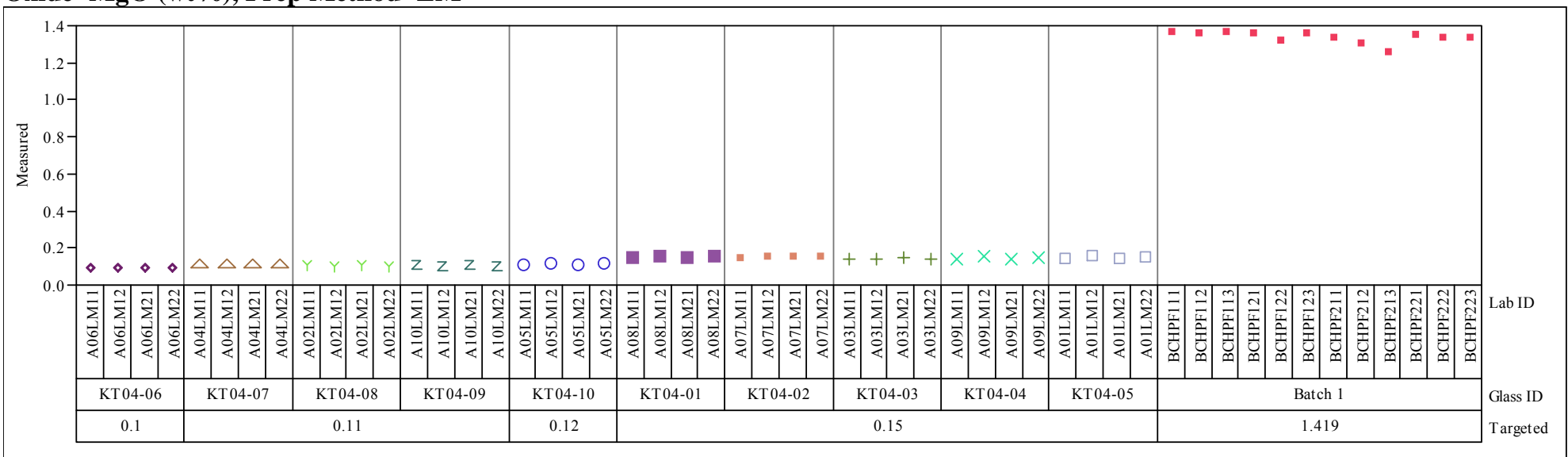




\section{Exhibit C-2. Measurements for Each KT01-Series Glass ID by Preparation Method by Oxide. (continued)}

\section{Oxide=MnO (wt \%), Prep Method=LM}

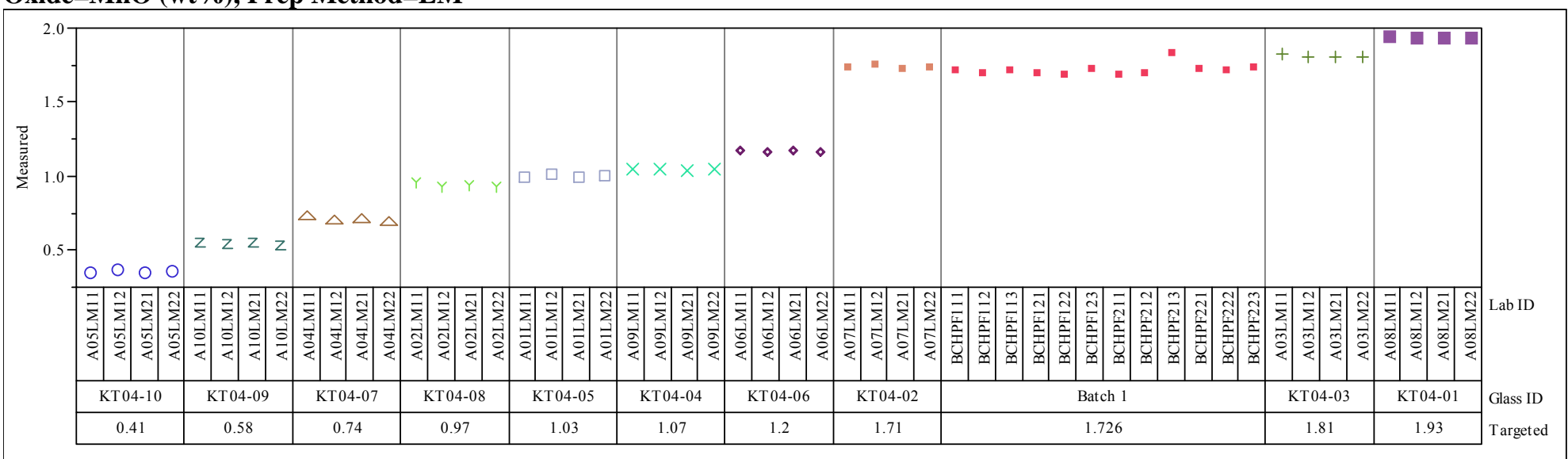

Oxide $=\mathrm{Na2O}$ (wt\%), Prep Method $=$ LM

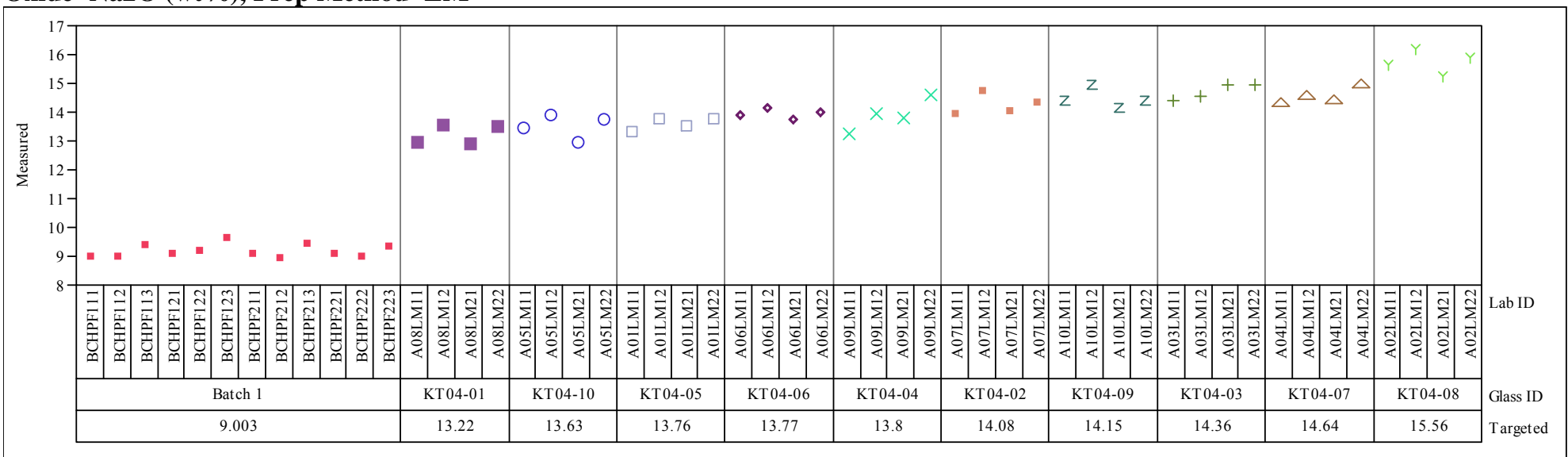


Exhibit C-2. Measurements for Each KT01-Series Glass ID by Preparation Method by Oxide. (continued) Oxide=Nb2O5 (wt\%), Prep Method=LM

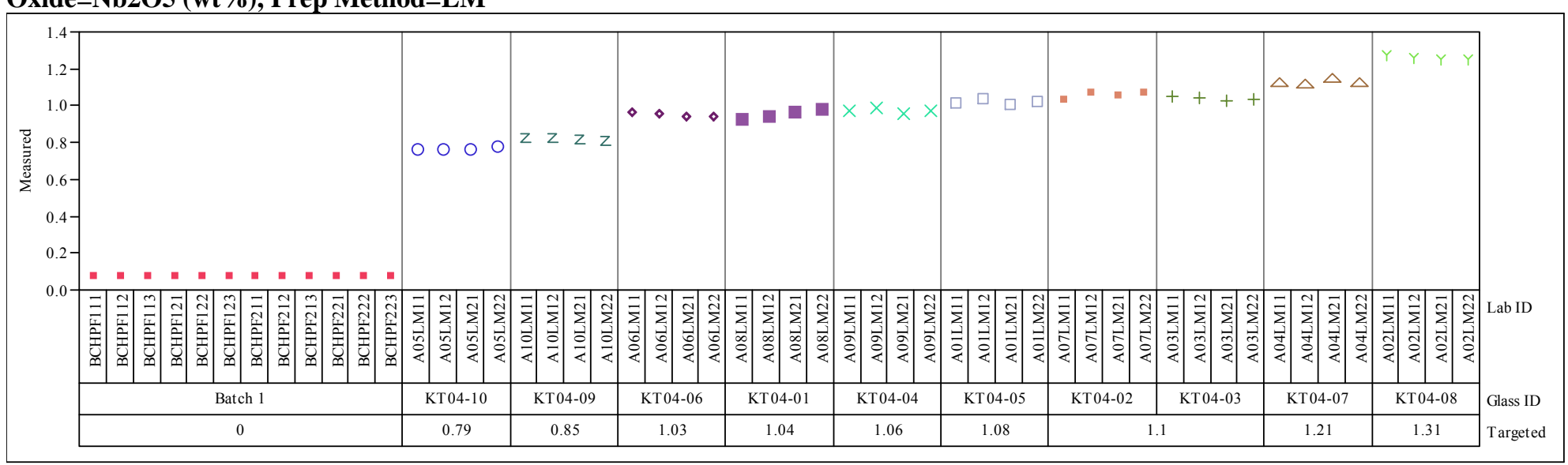

Oxide=NiO (wt\%), Prep Method=LM

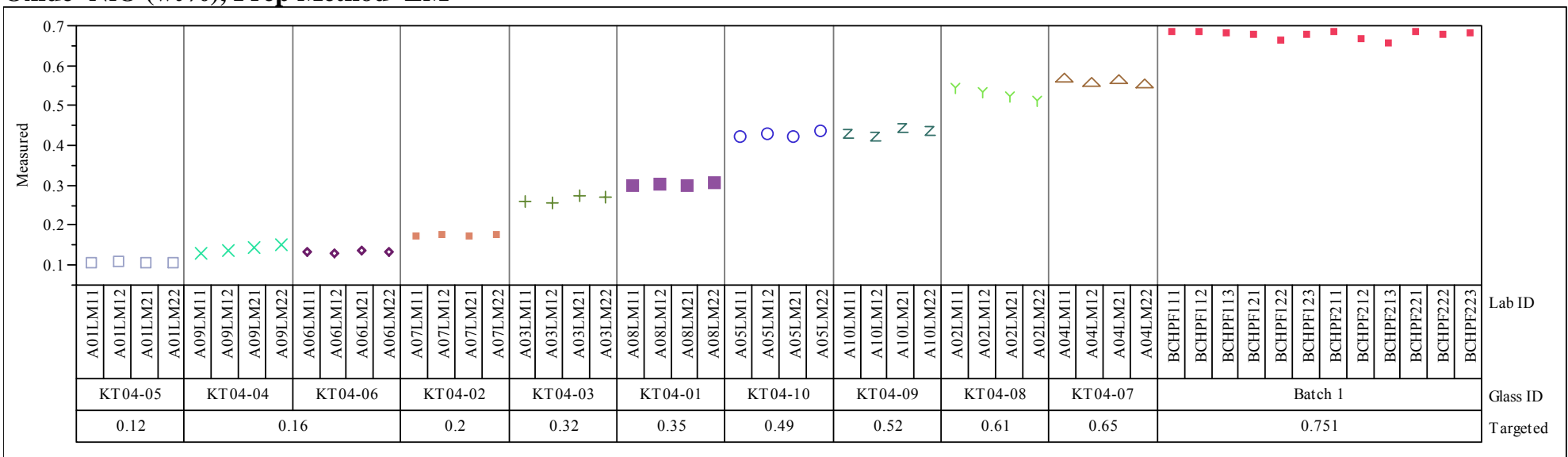




\section{Exhibit C-2. Measurements for Each KT01-Series Glass ID by Preparation Method by Oxide. (continued)}

\section{Oxide=PbO (wt \%), Prep Method $=$ LM}

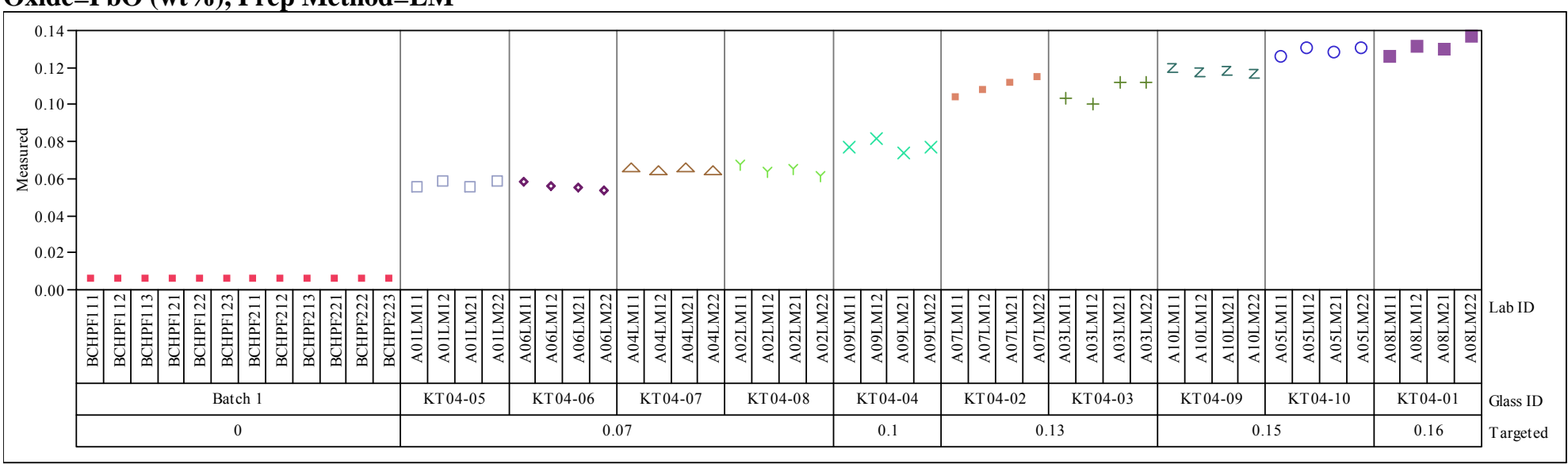

Oxide=SiO2 (wt\%), Prep Method=LM

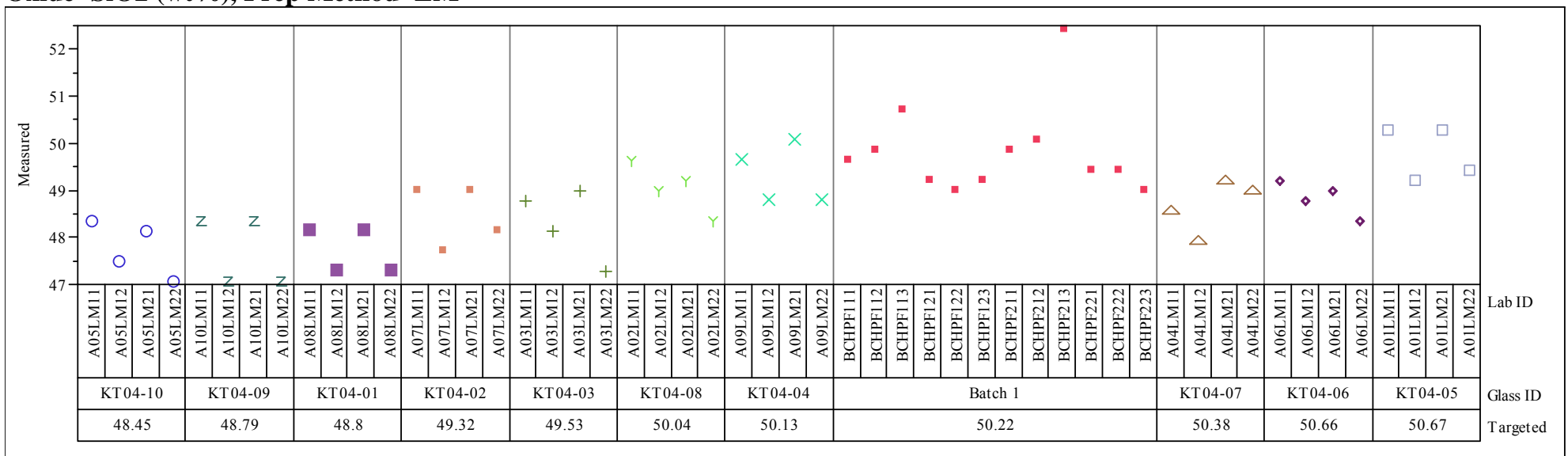


Exhibit C-2. Measurements for Each KT01-Series Glass ID by Preparation Method by Oxide. (continued) Oxide=TiO2 (wt\%), Prep Method=LM

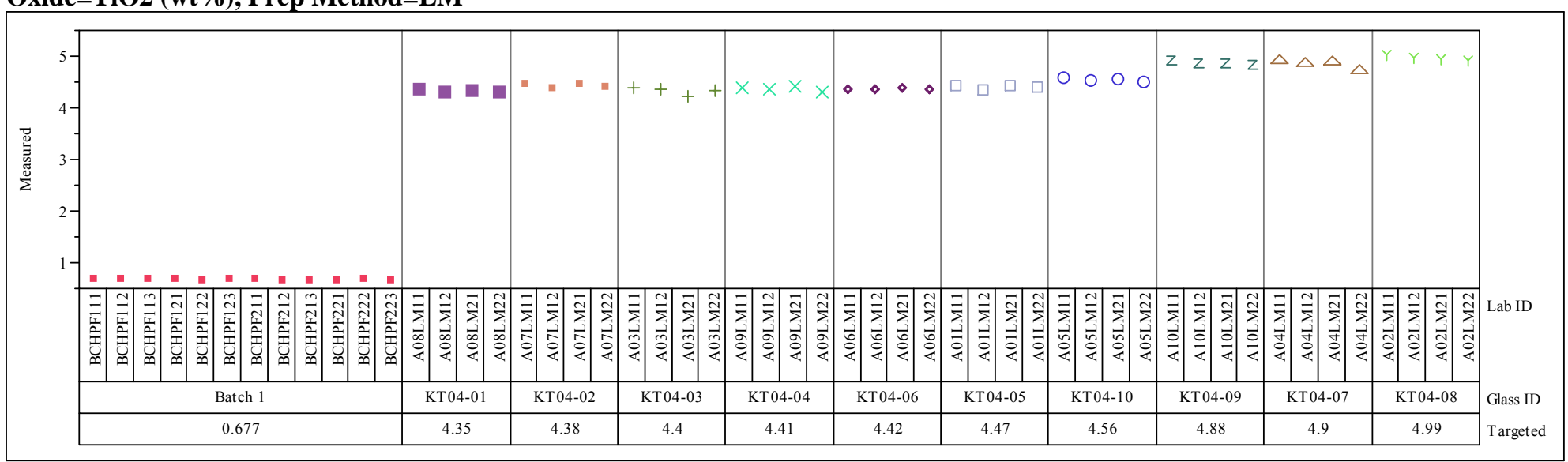

Oxide $=\mathrm{ZnO}$ (wt\%), Prep Method=LM

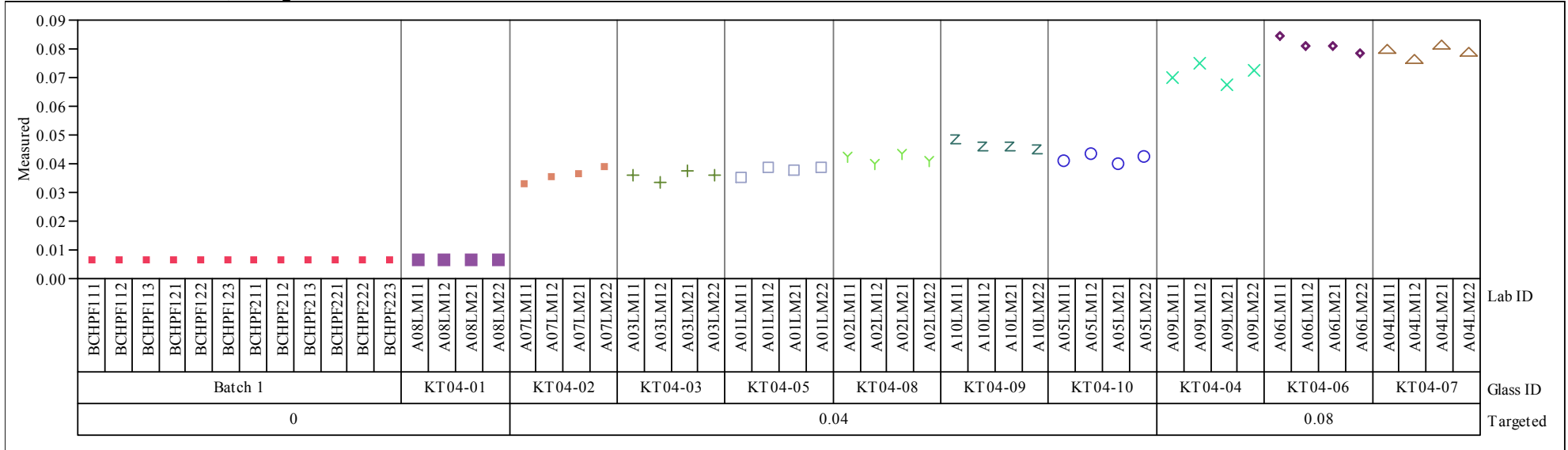


Exhibit C-2. Measurements for Each KT01-Series Glass ID by Preparation Method by Oxide. (continued) Oxide $=\mathrm{ZrO2}$ (wt\%), Prep Method=LM

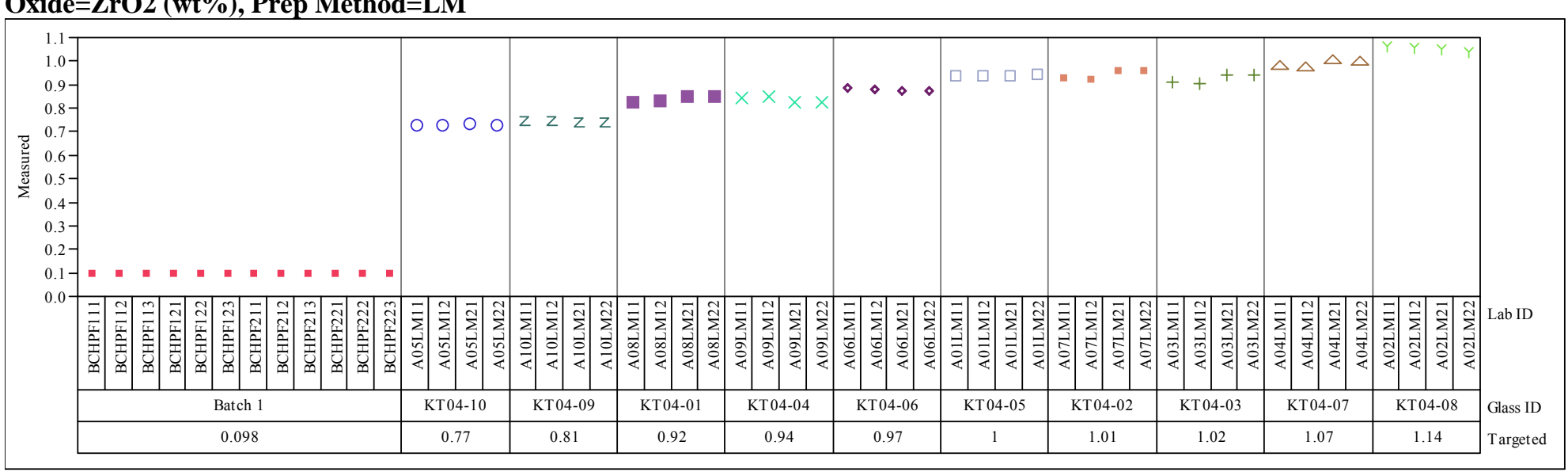


Exhibit C-3. Statistical Evaluation of the ICP-AES Calibration Effects from the KT04 Batch 1 Results by Oxide.

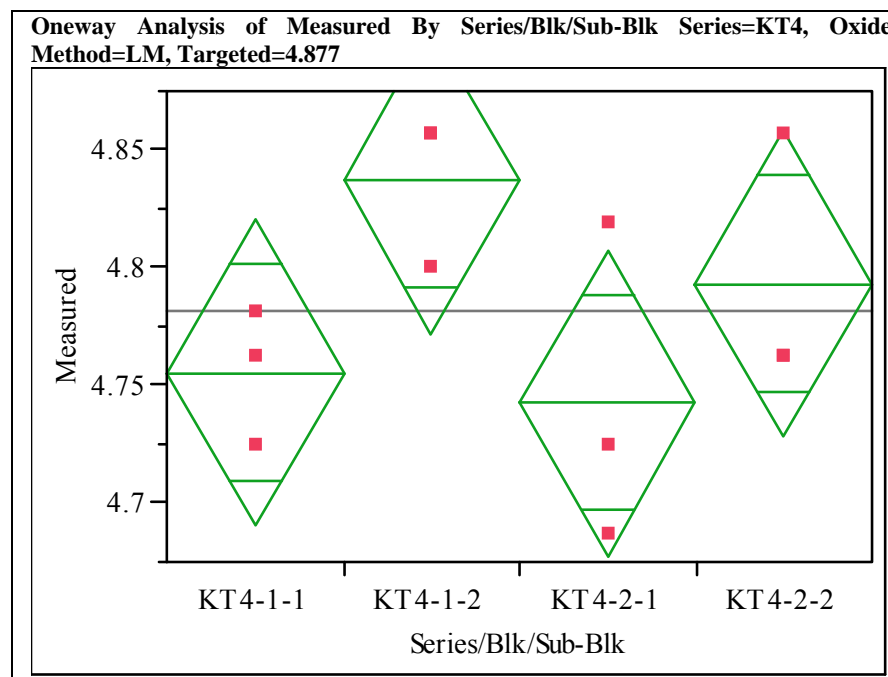

\section{Oneway Anova}

Summary o

$\begin{array}{ll}\text { Rsquare } & 0.460826 \\ & 0.258635\end{array}$

$\begin{array}{ll}\text { Adj Rsquare } & 0.258635 \\ \text { Root Mean Square Error } & 0.048787\end{array}$

Root Mean Square Error $\quad 0.048787$

Mean of Response 4.78201

Analysis of Variance

Source DF Sum of Squares Mean Square F Ratio Prob $>$ F

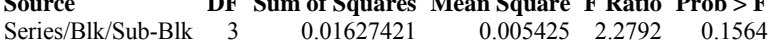

$\begin{array}{lrrr}\text { Series/Blk/Sub-Blk } & 3 & 0.01627421 & 0.005425 \\ \text { Error } & 8 & 0.01904112 & 0.002380 \\ \text { C. } & 11 & 0.03531533 & \end{array}$

110.03531533

Means for Oneway Anova

Level Number Mean Std Error Lower 95\% Upper 95\%

$\begin{array}{lrrrrr}\text { LTevel } & \text { Number } & \text { Mean } & & & \\ \text { KTd Error } & \text { Lower } 95 \% & \text { Upper 95\% } \\ \text { K } & 3 & 4.75524 & 0.02817 & 4.6903 & 4.8202\end{array}$

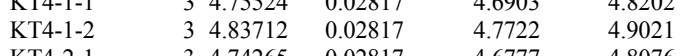

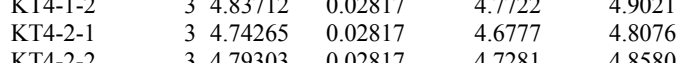

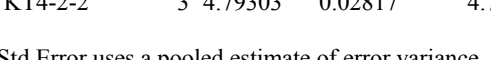

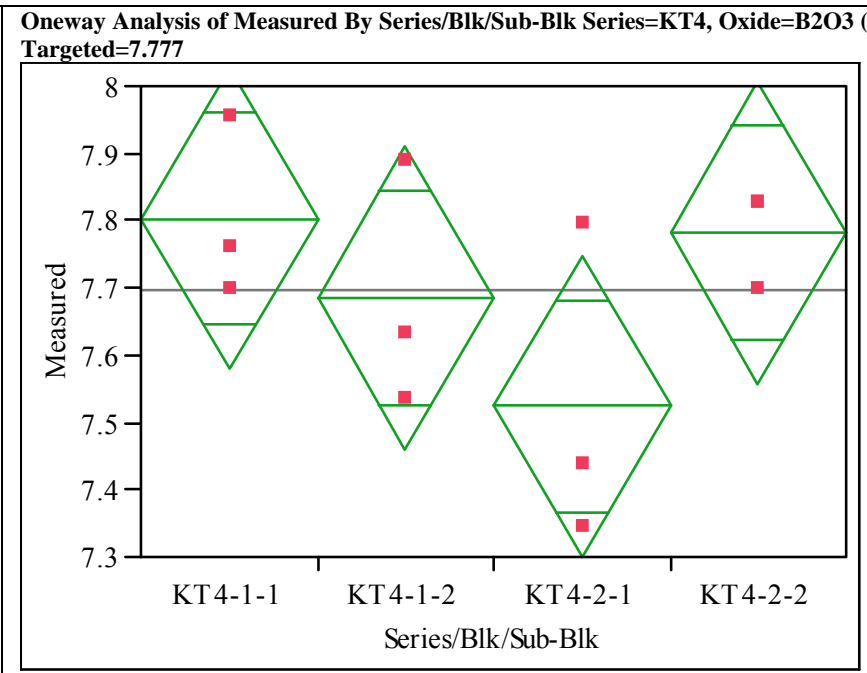

\section{Oneway Anova}

Summary of Fit

$\begin{array}{ll}\text { Rsquare } & 0.390759 \\ \text { Adj Rsquare } & 0.162294 \\ \text { Root Mean Square Error } & 0.168341 \\ \text { Mean of Response } & 7.698244\end{array}$

Observations (or Sum Wgts)

Analysis of Variance

Source DF Sum of Squares Mean Square F Ratio Prob $>$ F

$\begin{array}{lrrrrr} & & & & \\ \text { Series/Blk/Sub-Blk } & 3 & 0.14540778 & 0.048469 & 1.7104 & 0.2417\end{array}$

$\begin{array}{lrrr}\text { Error } & 8 & 0.22670826 & 0.028339 \\ \text { C. Total } & 11 & 0.37211604 & \end{array}$

C. Tota

Means for Oneway Anova

Level Number Mean Std Error Lower 95\% Upper 95\%

\begin{tabular}{lrrrrr}
\hline KT4-1-1 & 3 & 7.80289 & 0.09719 & 7.5788 & 8.0270
\end{tabular}

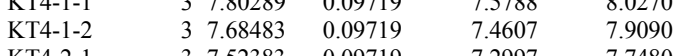

\begin{tabular}{llllll} 
KT4-1-2 & 3 & 7.68483 & 0.09719 & 7.4607 & 7.9090 \\
KT4-2-1 & 3 & 7.52383 & 0.09719 & 7.2997 & 7.7480 \\
\hline
\end{tabular}

KT4-2-2

Std Error uses a pooled estimate of error variance 
Exhibit C-3. Statistical Evaluation of the ICP-AES Calibration Effects from the KT04 Batch 1 Results by Oxide. (continued)

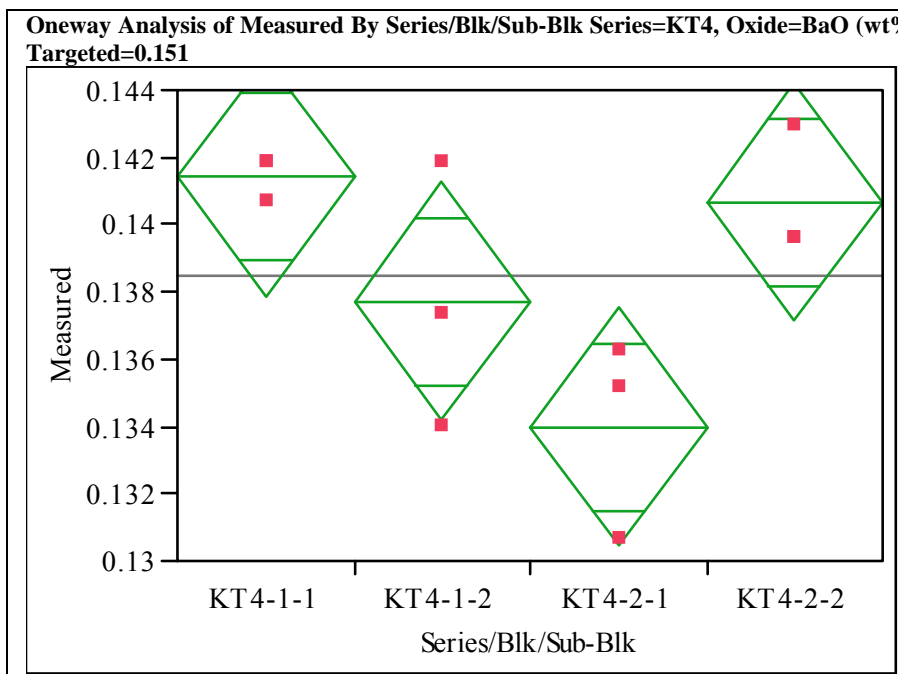

\section{Oneway Anova}

Summary of Fit

Rsquare $\quad 0.645833$

\begin{tabular}{ll} 
Adj Rsquare & 0.513021 \\
Root Mean Square Error & 0.002658 \\
\hline
\end{tabular}

$\begin{array}{ll}\text { Root Mean Square Error } & 0.002658 \\ \text { Mean of Response } & 0.138446\end{array}$

$\begin{array}{lr}\text { Mean of Response } & 0.138446 \\ \text { Observations (or Sum Wgts) } & 12\end{array}$

Analysis of Variance

Source DF Sum of Squares Mean Square F Ratio Prob $>$ F

$\begin{array}{lrrrrr} & \text { Dource } & & & & \\ \text { Series/Blk/Sub-Blk } & 3 & 0.00010305 & 0.000034 & 4.8627 & 0.0328\end{array}$

$\begin{array}{lrrr}\text { Series/Blk/Sub-Blk } & 3 & 0.00010305 & 0.000034 \\ \text { Error } & 8 & 0.00005651 & 7.064 \mathrm{e}-6 \\ \text { C. Total } & 11 & 0.00015956 & \end{array}$

Means for Oneway Anova

Level Number Mean Std Error Lower 95\% Upper 95\%

$\begin{array}{lrrrrr}\text { KT4-1-1 } & 3 & 0.141423 & 0.00153 & 0.13788 & 0.14496\end{array}$

$\begin{array}{llllll}\text { KT4-1-1 } & 3 & 0.141423 & 0.00153 & 0.13788 & 0.14496 \\ \text { KT-1-2 } & 3 & 0.137702 & 0.00153 & 0.13416 & 0.14124\end{array}$

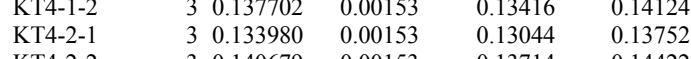

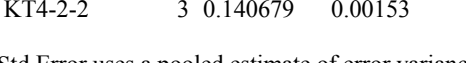

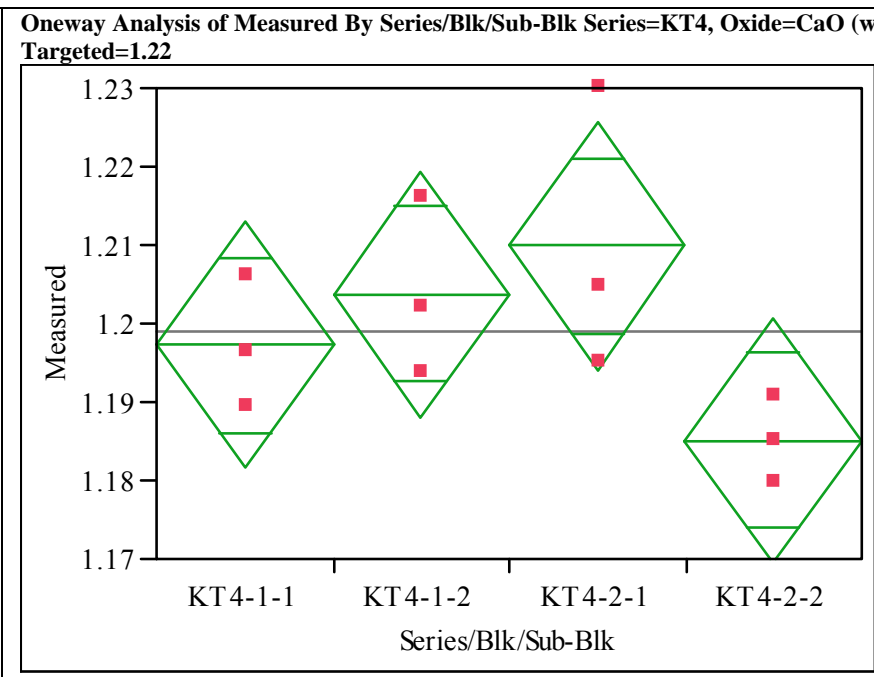

\section{Oneway Anova}

Summary of Fit

Rsquare $\quad 0.475491$

$\begin{array}{lr}\text { Adj Rsquare } & 0.2788 \\ \text { Root Mean Square Error } & 0.01179\end{array}$

Root Mean Square Error $\quad 0.01179$
Mean of Response

Observations (or Sum Wgts)

Analysis of Variance

Source DF Sum of Squares Mean Square F Ratio Prob $>$ F

$\begin{array}{lrrrrr} & & & \\ \text { Series/Blk/Sub-Blk } & 3 & 0.00100808 & 0.000336 & 2.4174 & 0.1415\end{array}$

$\begin{array}{lrrr}\text { Error } & 8 & 0.00111201 & 0.000139 \\ \text { C. Total } & 11 & 0.00212009 & \end{array}$

Means for Oneway Anova

Level Number Mean Std Error Lower 95\% Upper 95\%

\begin{tabular}{lrrrrr} 
KT4-1-1 & 3 & 1.19725 & 0.00681 & 1.1816 & 1.2129 \\
\hline
\end{tabular}

$\begin{array}{llllll}\text { KT4-1-1 } & 3 & 1.19725 & 0.00681 & 1.1816 & 1.2129 \\ \text { KT4-1-2 } & 3 & 1.20378 & 0.00681 & 1.1881 & 1.2195 \\ \text { KT4-2-1 } & 3 & 1.20984 & 0.00681 & 1.1941 & 1.2255\end{array}$

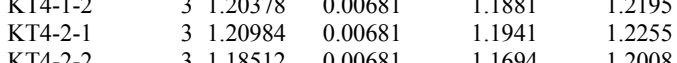

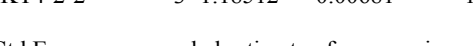

Std Error uses a pooled estimate of error variance 
Exhibit C-3. Statistical Evaluation of the ICP-AES Calibration Effects from the KT04 Batch 1 Results by Oxide. (continued)

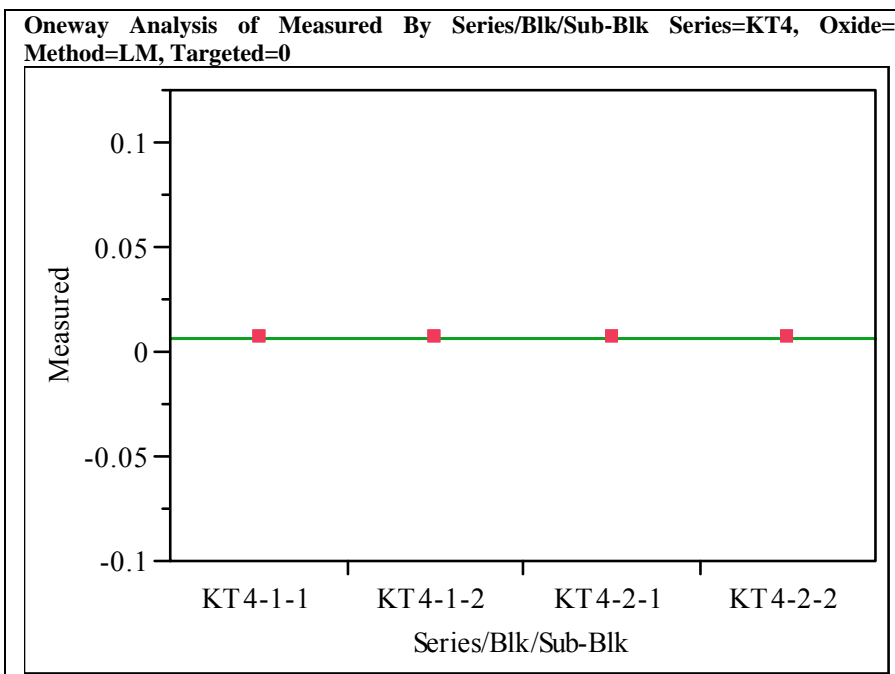

\section{Oneway Anova}

Summary of Fit

Rsquare

Adj Rsquare

$\begin{array}{lr}\text { Root Mean Square Error } & 0 \\ \text { Mean of Response } & 0.005857\end{array}$

Analysis of Variance

Source DF Sum of Squares Mean Square F Ratio Prob $>$ F

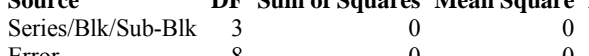

$\begin{array}{ll}\text { Series/Blk/Sub-Blk } & 3 \\ \text { Error } & 8\end{array}$

$\begin{array}{rl}8 & 0 \\ 11 & 0\end{array}$

0

Means for Oneway Anova

Level Number Mean Std Error Lower 95\% Upper 95\%

$\begin{array}{lrrrrr}\text { KT4-1-1 } & 3 & 0.005857 & 0 & 0.00586 & 0.00586\end{array}$

$\begin{array}{llllll}\text { KT }-1-1 & 3 & 0.005857 & 0 & 0.00586 & 0.00586 \\ \text { KT4-1-2 } & 3 & 0.005857 & 0 & 0.00586 & 0.00586\end{array}$

$\begin{array}{llllll}\text { KT4-1-2 } & 3 & 0.005857 & 0 & 0.00586 & 0.00586 \\ \text { KT4-2-1 } & 3 & 0.005857 & 0 & 0.00586 & 0.00586 \\ \text { KT4-2-2 } & 3 & 0.005857 & 0 & 0.00586 & 0.00586\end{array}$

Std Error uses a pooled estimate of error variance

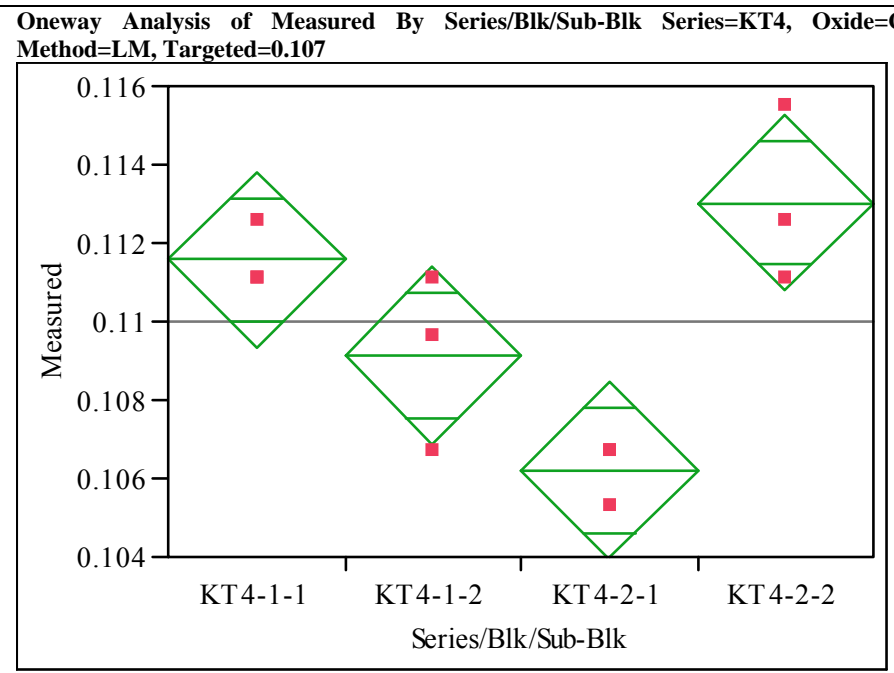

\section{Oneway Anova}

Summary of Fit

$\begin{array}{ll}\text { Rsquare } & 0.778929 \\ \text { Adj Rsquare } & 0.696028 \\ \text { Root Mean Square Error } & 0.001688 \\ \text { Mean of Response } & 0.109985\end{array}$

Observations (or Sum Wgts)

Analysis of Variance

Source DF Sum of Squares Mean Square F Ratio Prob $>$ F

$\begin{array}{lrrrrr} & \text { DF } & \text { Sum of Squares } & \text { Mean Square } & & \\ \text { Series/Blk/Sub-Blk } & 3 & 0.00008029 & 0.000027 & 9.3958 & 0.0053\end{array}$

$\begin{array}{lrrr}\text { Error } & 8 & 0.00002279 & 2.848 \mathrm{e}-6 \\ \text { C. Total } & 11 & 0.00010308 & \end{array}$

Means for Oneway Anova

Level Number Mean Std Error Lower 95\% Upper 95\%

\begin{tabular}{lrrrrr} 
LTevel & Number & Mean & Std Error & Lower $95 \%$ & Upper $95 \%$ \\
KT4-1 & 3 & 0.111569 & 0.00097 & 0.10932 & 0.11382 \\
\hline
\end{tabular}

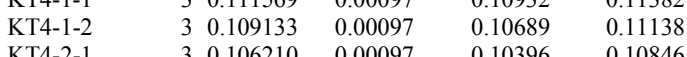

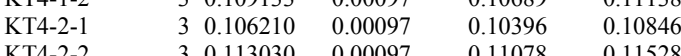

Std Error uses a pooled estimate of error variance 
Exhibit C-3. Statistical Evaluation of the ICP-AES Calibration Effects from the KT04 Batch 1 Results by Oxide. (continued)

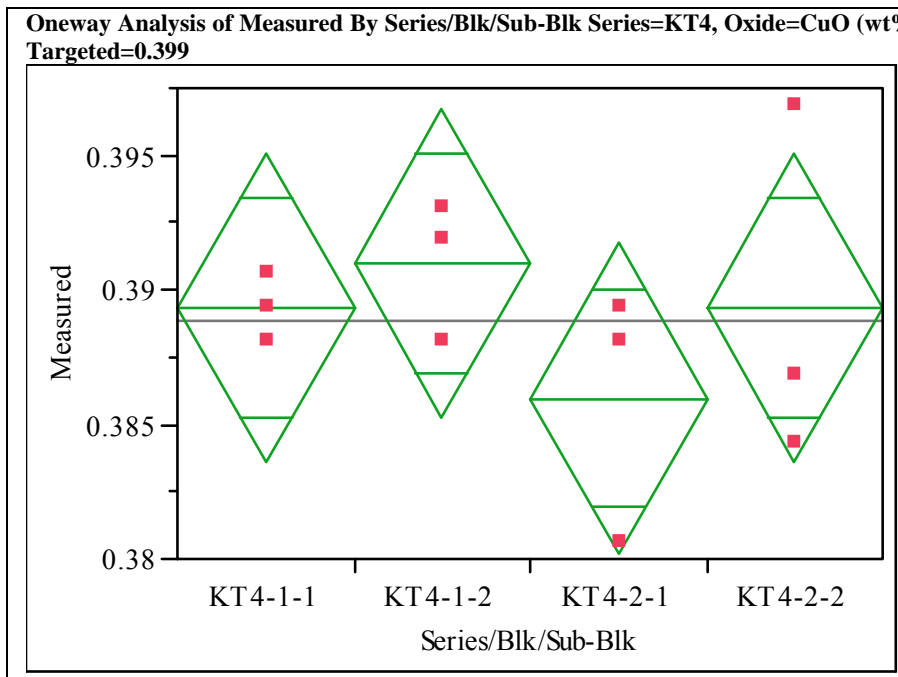

\section{Oneway Anova}

Summary of Fit

Rsquare $\quad 0.209945$

$\begin{array}{ll}\text { Adj Rsquare } & -0.08633 \\ \text { Root Mean Square Error } & 0.00432\end{array}$

$\begin{array}{ll}\text { Root Mean Square Error } & 0.004321 \\ \text { Mean of Response } & 0.388893\end{array}$

$\begin{array}{lr}\text { Mean of Response } & 0.388893 \\ \text { Observations (or Sum Wgts) } & 12\end{array}$

Analysis of Variance

Source DF Sum of Squares Mean Square F Ratio Prob $>$ F

Series/Blk/Sub-Blk $3 \quad 0.00003970 \quad 0.000013 \quad 0.7086 \quad 0.5734$

$\begin{array}{lll}\text { Error } & 8 & 0.00014939\end{array}$

$\begin{array}{lll}0.000013 & 0.7086 & 0.5734 \\ 0.000019 & & \end{array}$

C. Total

$\begin{array}{rr}8 & 0.00014939 \\ 11 & 0.00018909\end{array}$

Means for Oneway Anova

Level Number Mean Std Error Lower 95\% Upper 95\%

$\begin{array}{llllll}\text { KT4-1-1 } & 3 & 0.389310 & 0.00249 & 0.38356 & 0.39506\end{array}$

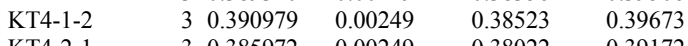

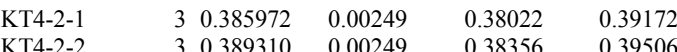

Std Error uses a pooled estimate of error variance

Oneway Analysis of Measured By Series/Blk/Sub-Blk Series=KT4, Oxide=Fe2O3 (wt\%), Prep Method $=$ LM, Targeted $=12.839$

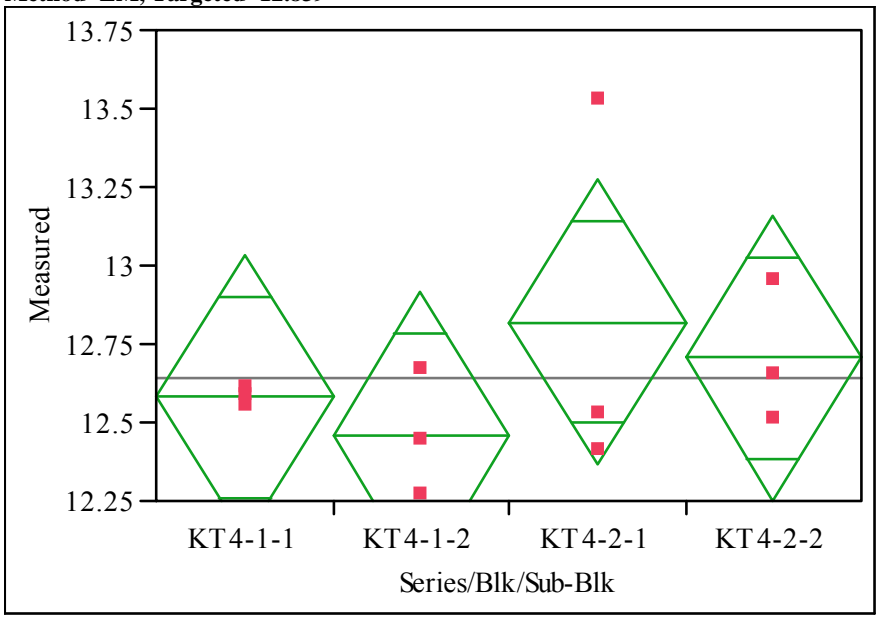

\section{Oneway Anova}

Summary of Fit

Rsquare $\quad 0.189991$

$\begin{array}{ll} & -0.11376 \\ \text { Adj Rsquare } & 0.342308\end{array}$

Root Mean Square Error $\quad 0.342308$

Observations (or Sum Wgts)

Analysis of Variance

Source DF Sum of Squares Mean Square F Ratio Prob $>$ F

$\begin{array}{lrrrrr} & \text { DF } & \text { Sum of Squares } & \text { Mean Square } & & \\ \text { Series/Blk/Sub-Blk } & 3 & 0.2198708 & 0.073290 & 0.6255 & 0.6184\end{array}$

$\begin{array}{lrrr}\text { Error } & 8 & 0.9373977 & 0.117175 \\ \text { C. Total } & 11 & 1.1572685 & \end{array}$

Means for Oneway Anova

Level Number Mean Std Error Lower 95\% Upper 95\%

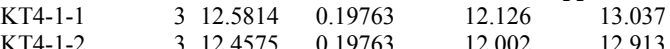

$\begin{array}{llllll}\text { KT4-1-2 } & 3 & 12.4575 & 0.19763 & 12.002 & 12.913 \\ \text { KT4-2-1 } & 3 & 12.8196 & 0.19763 & 12.364 & 13.275\end{array}$

$\begin{array}{llllll}\text { KT4-2-1 } & 3 & 12.8196 & 0.19763 & 12.364 & 13.275 \\ \text { KT4-2-2 } & 3 & 12.7053 & 0.19763 & 12.250 & 13.161\end{array}$

Std Error uses a pooled estimate of error variance 
Exhibit C-3. Statistical Evaluation of the ICP-AES Calibration Effects from the KT04 Batch 1 Results by Oxide. (continued)

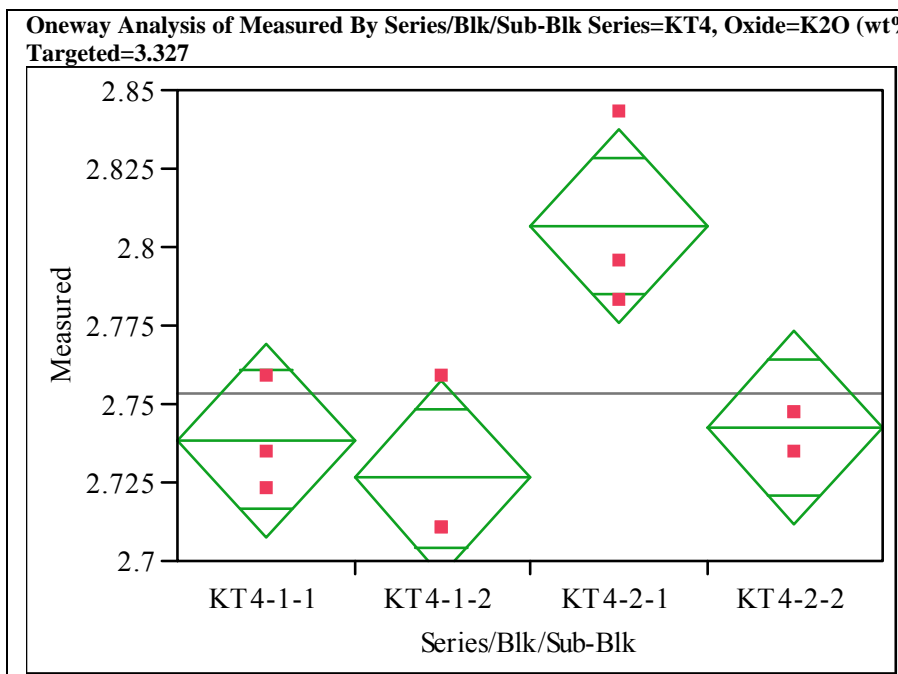

\section{Oneway Anova}

Summary of Fit

Rsquare $\quad 0.729527$

$\begin{array}{ll}\text { Adj Rsquare } & 0.628099 \\ \text { Root Mean Square Error } & 0.023327\end{array}$

Root Mean Square Error $\quad 0.023327$

$\begin{array}{lr}\text { Mean of Response } & 2.753515 \\ \text { Observations (or Sum Wgts) } & 12\end{array}$

Analysis of Variance

$\begin{array}{lrrrrr}\text { Source } & \text { DF } & \text { Sum of Squares } & \text { Mean Square } & \text { F Ratio } & \text { Prob }>\text { F } \\ \text { Series/Blk/Sub-Blk } & 3 & 0.01174150 & 0.003914 & 7.1926 & 0.0117\end{array}$

$\begin{array}{lrrr}\text { Series/Blk/Sub-Blk } & 3 & 0.01174150 & 0.003914 \\ \text { Error } & 8 & 0.00435318 & 0.000544\end{array}$

$\begin{array}{rr}8 & 0.00435318 \\ 11 & 0.01609469\end{array}$

Means for Oneway Anova

Level Number Mean Std Error Lower 95\% Upper 95\%

\begin{tabular}{lrrrrr} 
KT4-1-1 & 3 & 2.73846 & 0.01347 & 2.7074 & 2.7695 \\
\hline
\end{tabular}

$\begin{array}{llllll}\text { KT4-1-1 } & 3 & 2.73846 & 0.01347 & 2.7074 & 2.7695 \\ \text { KT4-1-2 } & 3 & 2.72641 & 0.01347 & 2.6954 & 2.7575\end{array}$

$\begin{array}{llllll}\text { KT4-1-2 } & 3 & 2.72641 & 0.01347 & 2.6954 & 2.7575 \\ \text { KT4-2-1 } & 3 & 2.80672 & 0.01347 & 2.7757 & 2.8378\end{array}$

$\begin{array}{llll}\text { KT4-2-2 } & 32.74247 & 0.01347 & 2.711\end{array}$

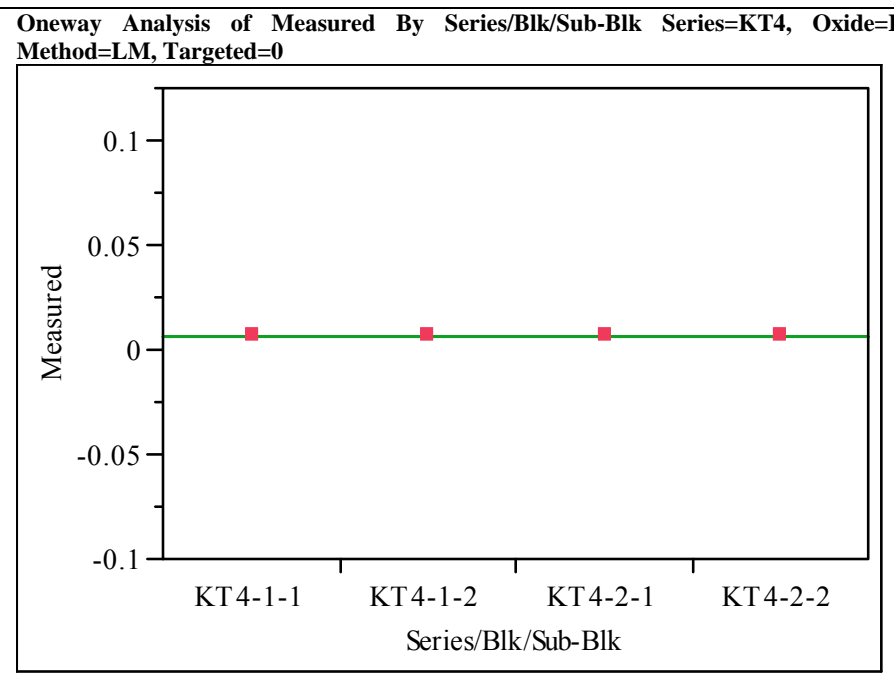

\section{Oneway Anova}

Summary of Fit

\section{Rsquare}

Adj Rsquare

$\begin{array}{lr}\text { Root Mean Square Error } & 0 \\ \text { Mean of Response } & 0.005864\end{array}$

Observations (or Sum Wgts)

Analysis of Variance

Source DF Sum of Squares Mean Square F Ratio Prob $>$ F

$\begin{array}{lrrr}\text { Source } & \text { DF } & \text { Sum of Squares } & \text { Mean Square } \\ \text { Series/Blk/Sub-Blk } & 3 & 0 & 0 \\ \text { Error } & 8 & 0 & 0 \\ \text { C. Total } & 11 & 0 & \\ & & & \end{array}$

Means for Oneway Anova

Level Number Mean Std Error Lower 95\% Upper 95\%

$\begin{array}{lrrrrr}\text { KT4-1-1 } & 3 & 0.005864 & 0 & 0.00586 & 0.00586 \\ \text { KT } & 3 & 0.005864 & 0 & 0.00586 & 0.00586\end{array}$

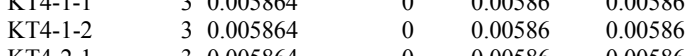

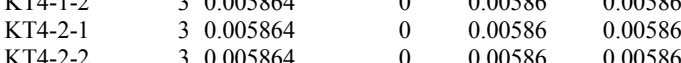

Std Error uses a pooled estimate of error variance 
Exhibit C-3. Statistical Evaluation of the ICP-AES Calibration Effects from the KT04 Batch 1 Results by Oxide. (continued)

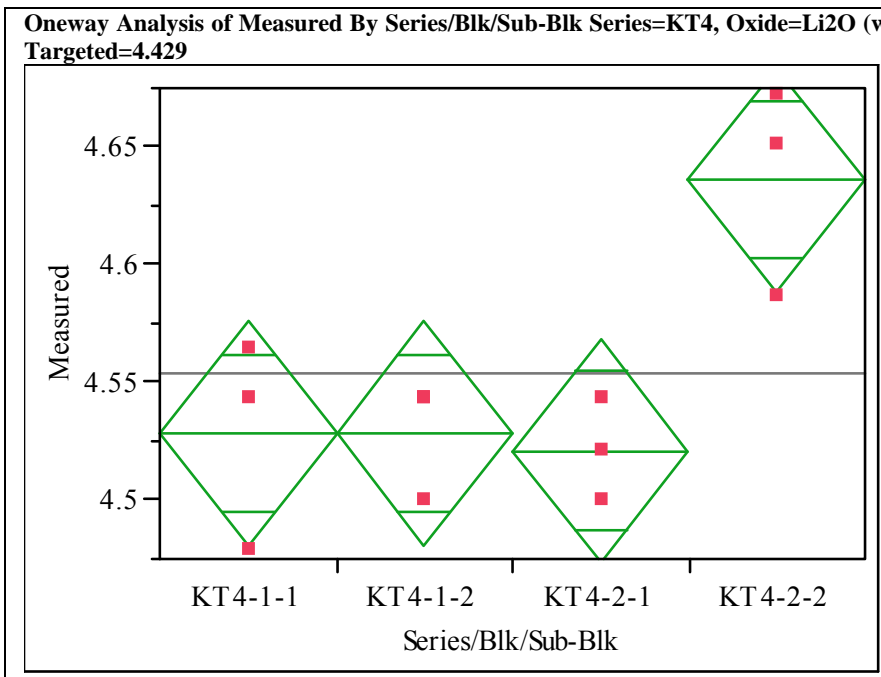

\section{Oneway Anova}

Summary of Fit

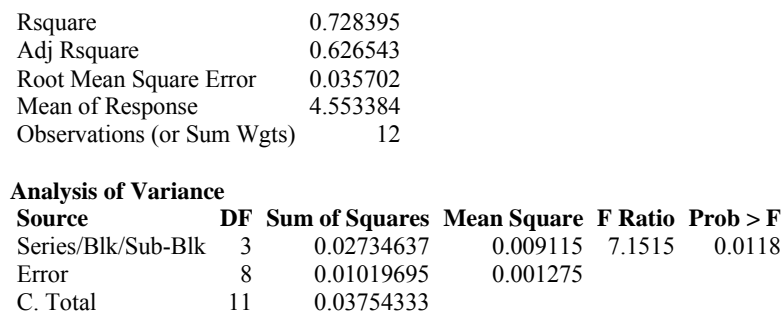

$8 \quad 0.02734637$

C. Total

0.03754333

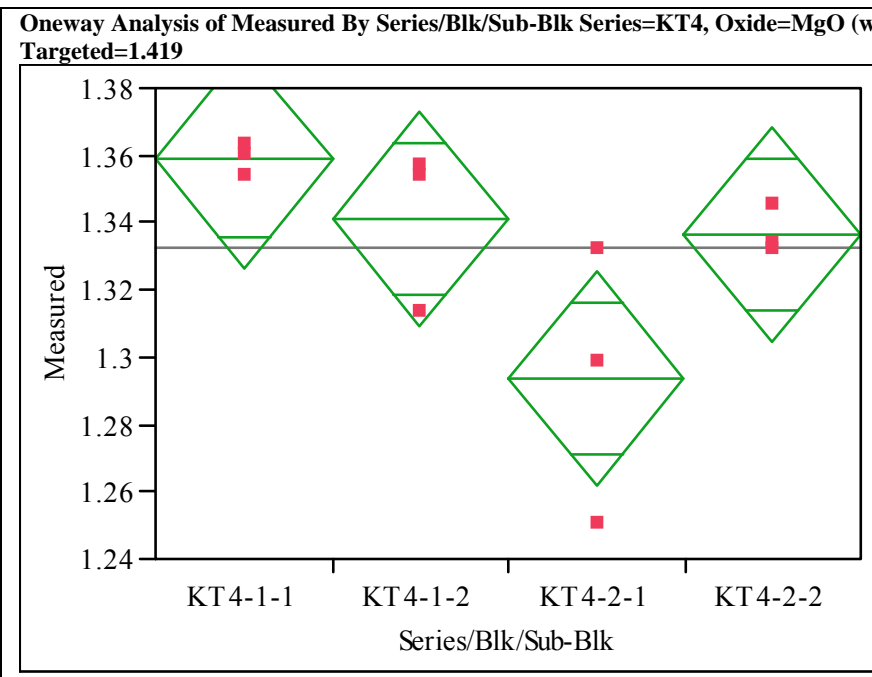

\section{Oneway Anova}

\section{ummary of Fit}

$\begin{array}{lr}\text { Rsquare } & 0.59749 \\ \text { Adj Rsquare } & 0.446549 \\ \text { Root Mean Square Error } & 0.024098 \\ \text { Mean of Response } & 1.332444\end{array}$

Observations (or Sum Wgts)

Analysis of Variance

Source DF Sum of Squares Mean Square F Ratio Prob $>$ F

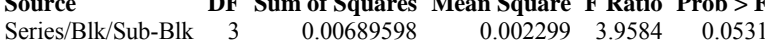

$\begin{array}{lrrr}\text { Error } & 8 & 0.00464560 & 0.000581\end{array}$

$\begin{array}{lrr}\text { C. Total } & 11 & 0.01154158\end{array}$

Means for Oneway Anova

Level Number Mean Std Error Lower 95\% Upper 95\%

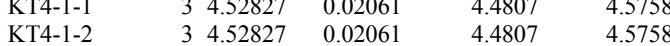

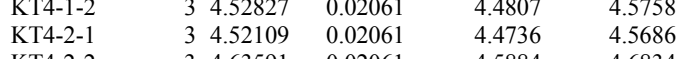

Means for Oneway Anova

Level Number Mean Std Error Lower 95\% Upper 95\%

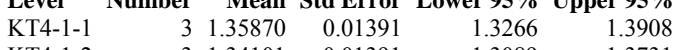

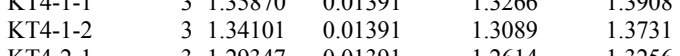

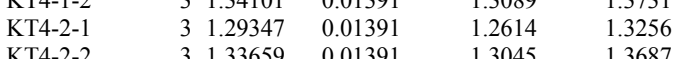

Std Error uses a pooled estimate of error variance

Std Error uses a pooled estimate of error variance 
Exhibit C-3. Statistical Evaluation of the ICP-AES Calibration Effects from the KT04 Batch 1 Results by Oxide. (continued)

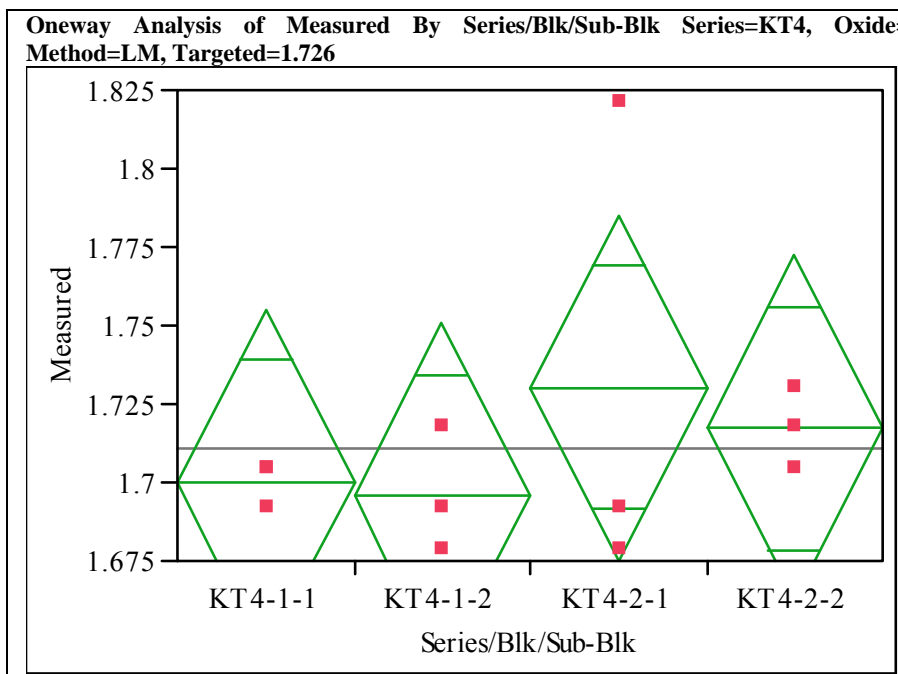

\section{Oneway Anova}

Summary of Fit

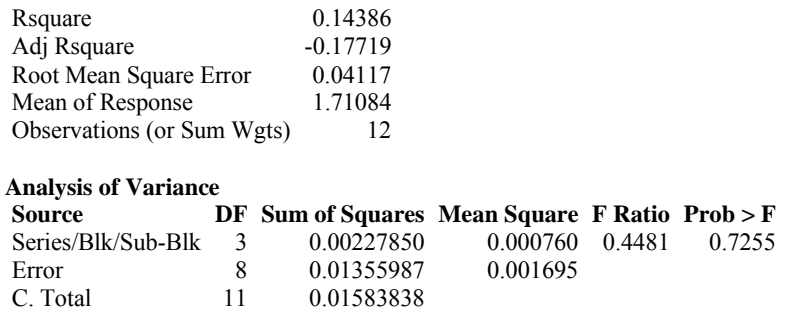

Std Error uses a pooled estimate of error variance

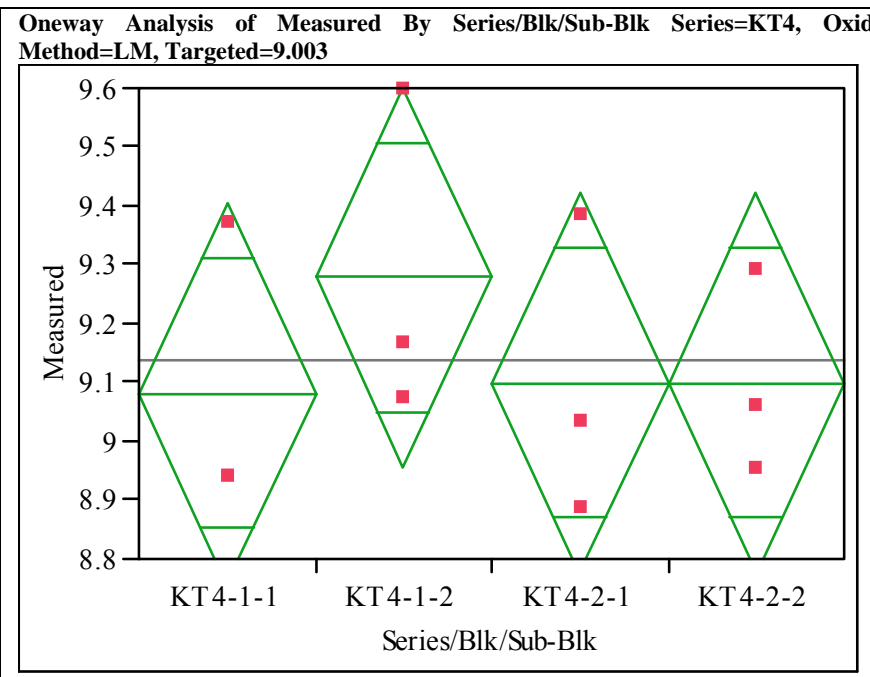

\section{Oneway Anova}

Summary of Fit
0.142322
Adj Rsquare
Root Mean Square Error $\quad \begin{array}{r}-0.17931 \\ 0.242796\end{array}$
Mean of Response $\quad \begin{array}{r}0.242796 \\ 9.13944\end{array}$

Observations (or Sum Wgts) 9.13944

Analysis of Variance

Source DF Sum of Squares Mean Square F Ratio Prob $>$ F

$\begin{array}{lrrrrr} & & & & \\ \text { Series/Blk/Sub-Blk } & 3 & 0.07825661 & 0.026086 & 0.4425 & 0.7291\end{array}$

$\begin{array}{lrrr}\text { Error } & 8 & 0.47159906 & 0.058950 \\ \text { C. Total } & 11 & 0.54985567 & \end{array}$

Means for Oneway Anova

Level Number Mean Std Error Lower 95\% Upper 95\%

$\begin{array}{llllll}\text { KT4-1-1 } & 3 & 9.08103 & 0.14018 & 8.7578 & 9.4043\end{array}$

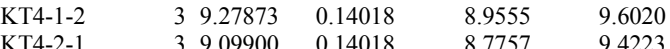

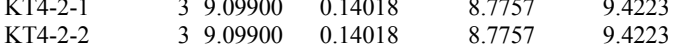

Std Error uses a pooled estimate of error variance 
Exhibit C-3. Statistical Evaluation of the ICP-AES Calibration Effects from the KT04 Batch 1 Results by Oxide. (continued)

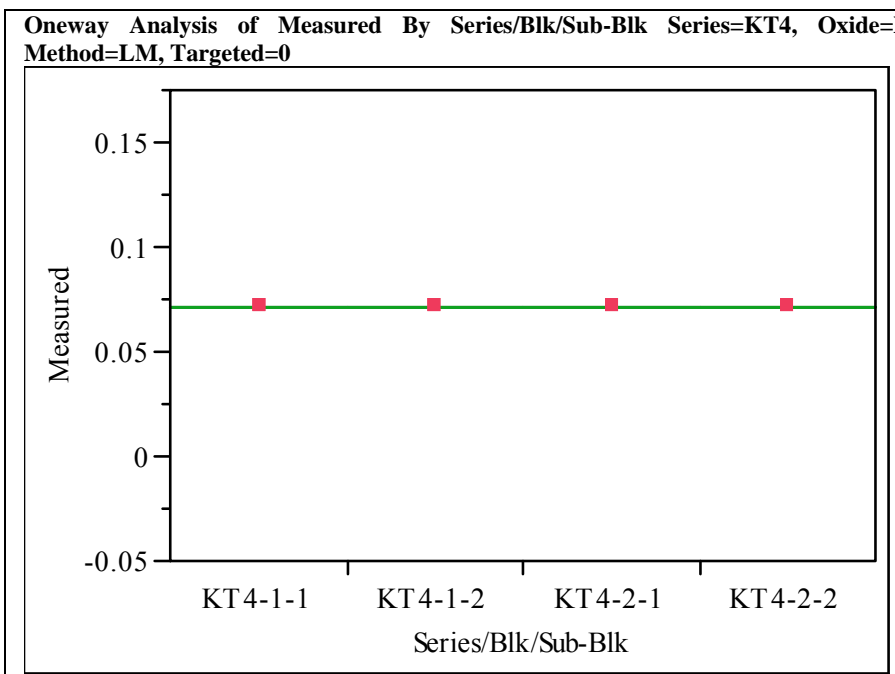

\section{Oneway Anova}

Summary of Fit

Rsquare

Adj Rsquare

$\begin{array}{lr}\text { Root Mean Square Error } & 0 \\ \text { Mean of Response } & 0.071525\end{array}$

Mean of Response 0.071525

Analysis of Variance

Source DF Sum of Squares Mean Square F Ratio Prob $>$ F

$\begin{array}{lrrr}\text { Source } & \text { DF } & \text { Sum of Squares } \\ \text { Series/Blk/Sub-Blk } & 3 & 0 & 0 \\ \text { Error } & 8 & 0 & 0\end{array}$

Error

0

Means for Oneway Anova

Level Number Mean Std Error Lower 95\% Upper 95\%

$\begin{array}{lrrrrr}\text { KT4-1-1 } & 3 & 0.071525 & 0 & 0.07153 & 0.07153 \\ \text { KT4-1-2 } & 3 & 0.071525 & 0 & 0.07153 & 0.07153\end{array}$

$\begin{array}{llllll}\text { KT4-1-2 } & 3 & 0.071525 & 0 & 0.07153 & 0.07153 \\ \text { KT4-2-1 } & 3 & 0.071525 & 0 & 0.07153 & 0.07153\end{array}$

$\begin{array}{llllll}\text { KT4-1-2 } & 3 & 0.071525 & 0 & 0.07153 & 0.07153 \\ \text { KT4-2-1 } & 3 & 0.071525 & 0 & 0.07153 & 0.07153 \\ \text { KT4-2-2 } & 3 & 0.071525 & 0 & 0.07153 & 0.07153\end{array}$

Std Error uses a pooled estimate of error variance

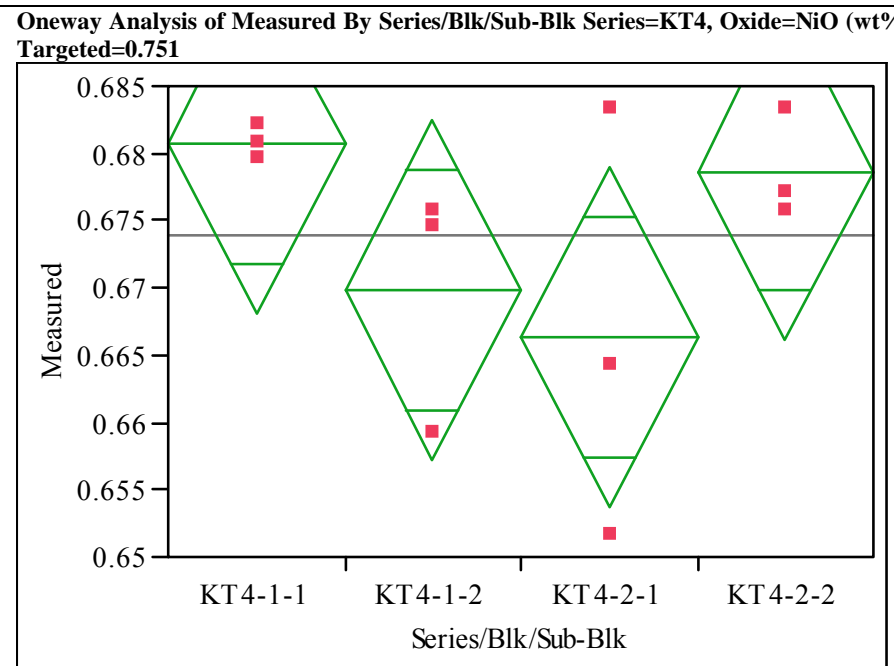

\section{Oneway Anova}

Summary of Fit

$\begin{array}{lr}\text { Rsquare } & 0.375454 \\ \text { Adj Rsquare } & 0.14125\end{array}$

0.14125
Adj Rsquare

Root Mean Square Error $\quad 0.00948$

Observations (or Sum Wgts) 0.673895

Analysis of Variance

Source DF Sum of Squares Mean Square F Ratio Prob $>$ F

$\begin{array}{lrrrrr} & & & & & \\ \text { Souries/Blk/Sub-Blk } & 3 & 0.00043221 & 0.000144 & 1.6031 & 0.2636\end{array}$

$\begin{array}{lrrr}\text { Error } & 8 & 0.00071895 & 0.000090\end{array}$

$\begin{array}{lrr}\text { C. Total } & 11 & 0.00115116\end{array}$

Means for Oneway Anova

Level Number Mean Std Error Lower 95\% Upper 95\%

\begin{tabular}{lrrrrr}
\hline KT4-1-1 & 3 & 0.680788 & 0.00547 & 0.66817 & 0.69341
\end{tabular}

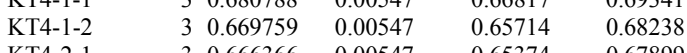

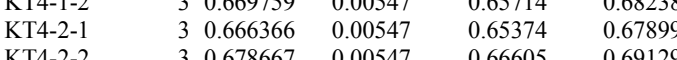

Std Error uses a pooled estimate of error variance 
Exhibit C-3. Statistical Evaluation of the ICP-AES Calibration Effects from the KT04 Batch 1 Results by Oxide. (continued)

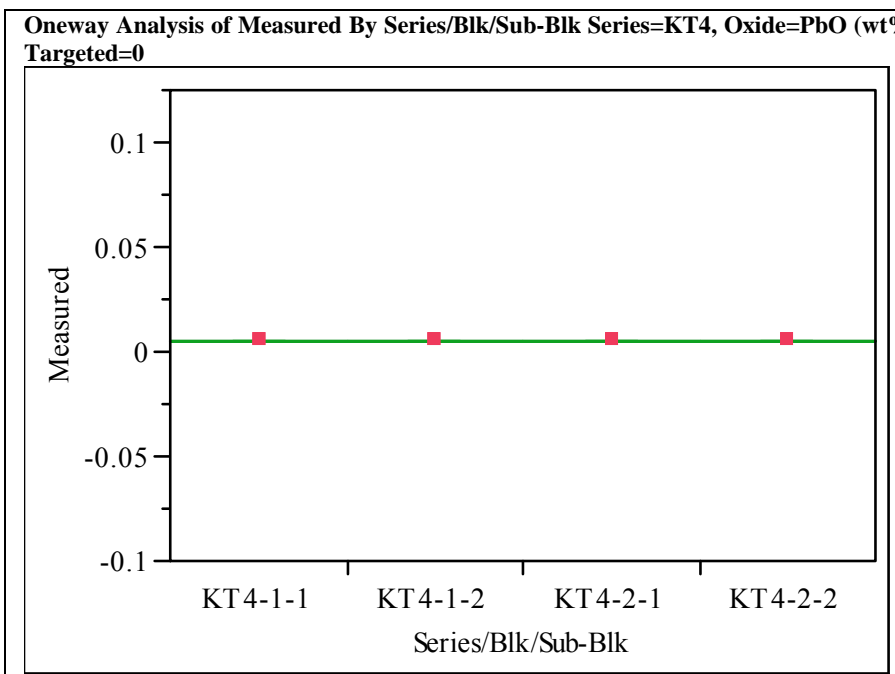

\section{Oneway Anova}

Summary of Fit

Rsquare

$\begin{array}{lr} & 0 \\ \text { Adj Rsquare } & -0.375\end{array}$

Root Mean Square Error $\quad \begin{array}{r}-0.375 \\ 1.06 \mathrm{e}-18\end{array}$

Mean of Response $\quad 0.005386$

Observations (or Sum Wgts) 0.005386

Observations (or Sum wh

Source DF Sum of Squares Mean Square F Ratio Prob $>$ F

$\begin{array}{lrrr}\text { Series/Blk/Sub-Blk } & 3 & 0 & 0 \\ \text { Error } & 8 & 9.0278 \mathrm{e}-36 & 1.128 \mathrm{e}-36\end{array}$

Error

Means for Oneway Anova

Level Number Mean Std Error Lower 95\% Upper 95\%

\begin{tabular}{lrrrrr}
\hline KT4-1-1 & 3 & 0.005386 & $6.133 \mathrm{e}-19$ & 0.00539 & 0.00539
\end{tabular}

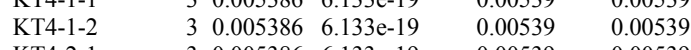

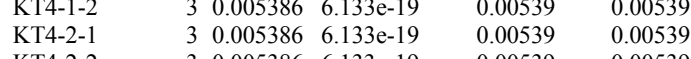

$\begin{array}{llllll}\text { KT4-2-2 } & 3 & 0.005386 & 6.133 \mathrm{e}-19 & 0.00539 & 0.00539\end{array}$

Std Error uses a pooled estimate of error variance

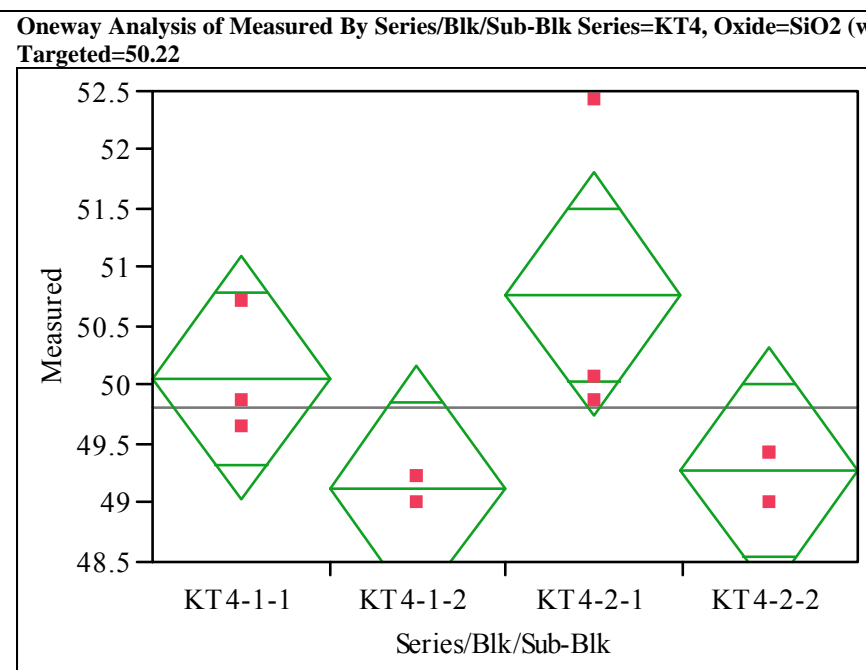

\section{Oneway Anova}

Summary of Fit

$\begin{array}{ll}\text { Rsquare } & 0.517451 \\ \text { Adj Rsquare } & 0.336495\end{array}$

0.336495
Root Mean Square Error $\quad 0.778717$

Root Mean Square Error $\quad 0.778717$

\begin{tabular}{lr} 
Observations (or Sum Wgts) & 49.81004 \\
\hline
\end{tabular}

Analysis of Variance

Source DF Sum of Squares Mean Square F Ratio Prob $>$ F

$\begin{array}{lrrrrr} & & & & & \\ \text { Series/Blk/Sub-Blk } & 3 & 5.202074 & 1.73402 & 2.8595 & 0.1043\end{array}$

$\begin{array}{lrrr}\text { Error } & 8 & 4.851201 & 0.60640\end{array}$

$\begin{array}{lrr}\text { C. Total } & 11 & 10.053275\end{array}$

Means for Oneway Anova

Level Number Mean Std Error Lower 95\% Upper 95\%

\begin{tabular}{lrrrrr} 
Level & Number & Mean & Std Error & Lower 95\% & Uper 95\% \\
KT4-1-1 & 3 & 50.0596 & 0.44959 & 49.023 & 51.096 \\
KT4-1-2 & 3 & 49.1326 & 0.44959 & 48.096 & 50.169 \\
\hline
\end{tabular}

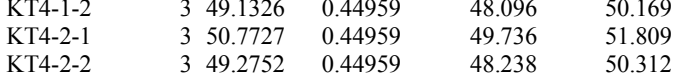

Std Error uses a pooled estimate of error variance 
Exhibit C-3. Statistical Evaluation of the ICP-AES Calibration Effects from the KT04 Batch 1 Results by Oxide. (continued)

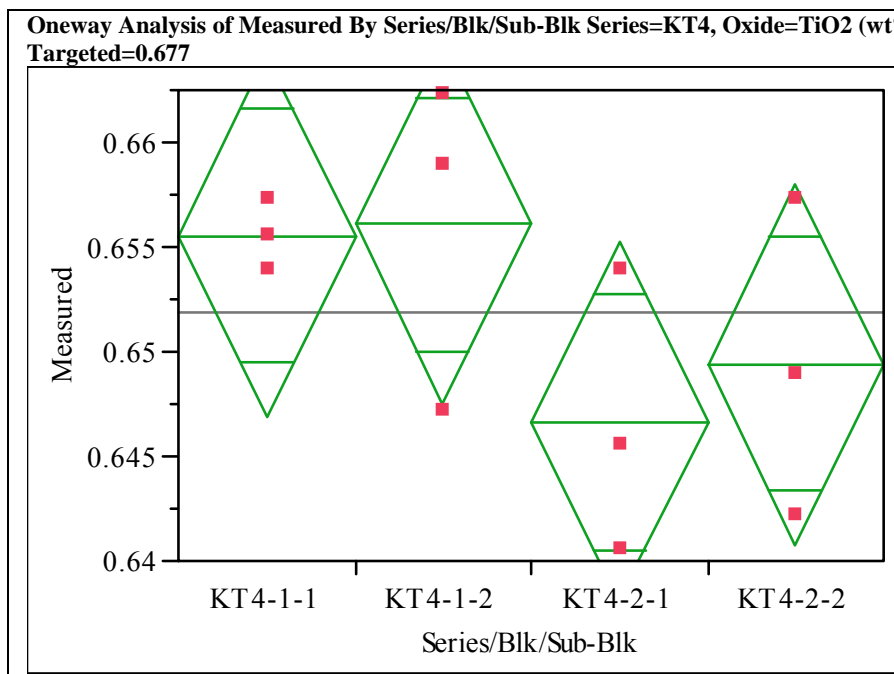

\section{Oneway Anova}

Summary of Fit

$\begin{array}{ll}\text { Rsquare } & 0.367311 \\ \text { Adj Rsquare } & 0.130053\end{array}$

$\begin{array}{lr}\text { Adj Rsquare } & 0.130053 \\ \text { Root Mean Square Error } \quad 0.00646\end{array}$

Root Mean Square Error $\quad 0.00646$
Mean of Response

Mean of Response 0.6519

Analysis of Variance

Source DF Sum of Squares Mean Square F Ratio Prob $>$ F

Series/Blk/Sub-Blk $3 \quad 0.00019383 \quad 0.000065 \quad 1.5481 \quad 0.2757$

$\begin{array}{lrrr}\text { Series/Blk/Sub-Blk } & 3 & 0.00019383 & 0.000065 \\ \text { Error } & 8 & 0.00033387 & 0.000042 \\ \text { C. Total } & 11 & 0.00052770 & \end{array}$

Means for Oneway Anova

Level Number Mean Std Error Lower 95\% Upper 95\%

$\begin{array}{llllll}\text { KT4-1-1 } & 3 & 0.655524 & 0.00373 & 0.64692 & 0.66412\end{array}$

$\begin{array}{llllll}\text { KT4-1-2 } & 3 & 0.656080 & 0.00373 & 0.64748 & 0.66468\end{array}$

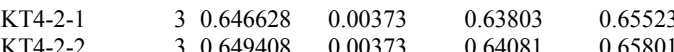

Std Error uses a pooled estimate of error variance

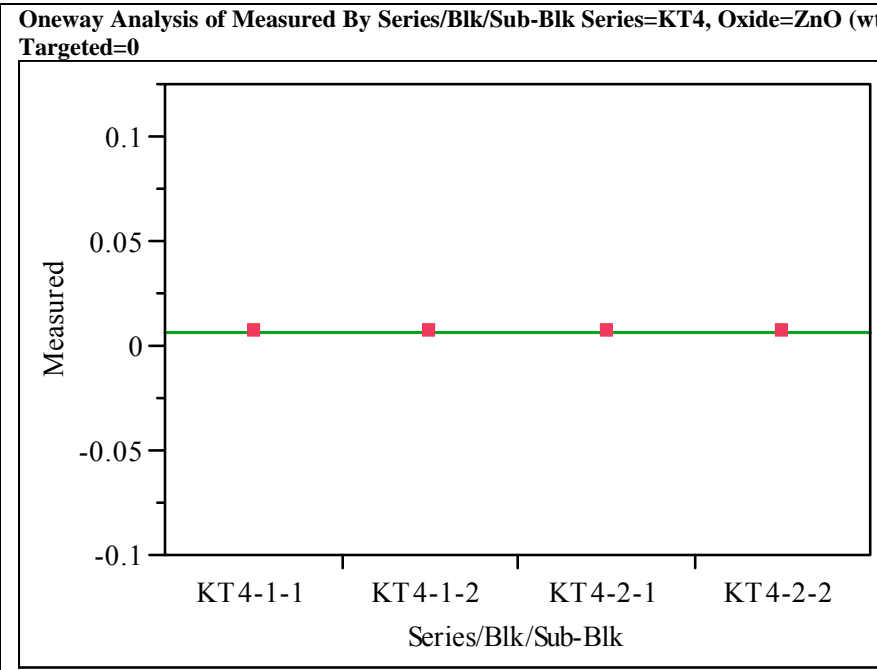

\section{Oneway Anova}

Summary of Fit

Rsquare

Adj Rsquare

Root Mean Square Error $\quad 0$

0.006224

Observations (or Sum Wgts)

Analysis of Variance

Source DF Sum of Squares Mean Square F Ratio Prob $>$ F

$\begin{array}{lccc}\text { Serres/Blk/Sub-Blk } & 3 & 0 & 0 \\ \text { Error } & 8 & 0 & 0\end{array}$

$\begin{array}{lrr}\text { Error } & 8 & 0 \\ \text { C. Total } & 11 & 0\end{array}$

Means for Oneway Anova

Level Number Mean Std Error Lower 95\% Upper 95\%

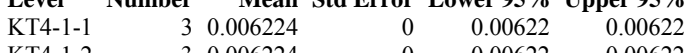

$\begin{array}{llllll}\text { KT4-1-1 } & 3 & 0.006224 & 0 & 0.0622 & 0.00622 \\ \text { KT4-1-2 } & 3 & 0.006224 & 0 & 0.00622 & 0.00622\end{array}$

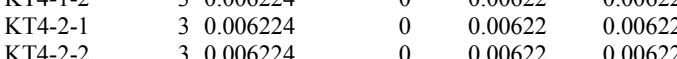

Std Error uses a pooled estimate of error variance 


\section{Exhibit C-3. Statistical Evaluation of the ICP-AES Calibration Effects from the KT04 Batch 1 Results by Oxide. (continued)}

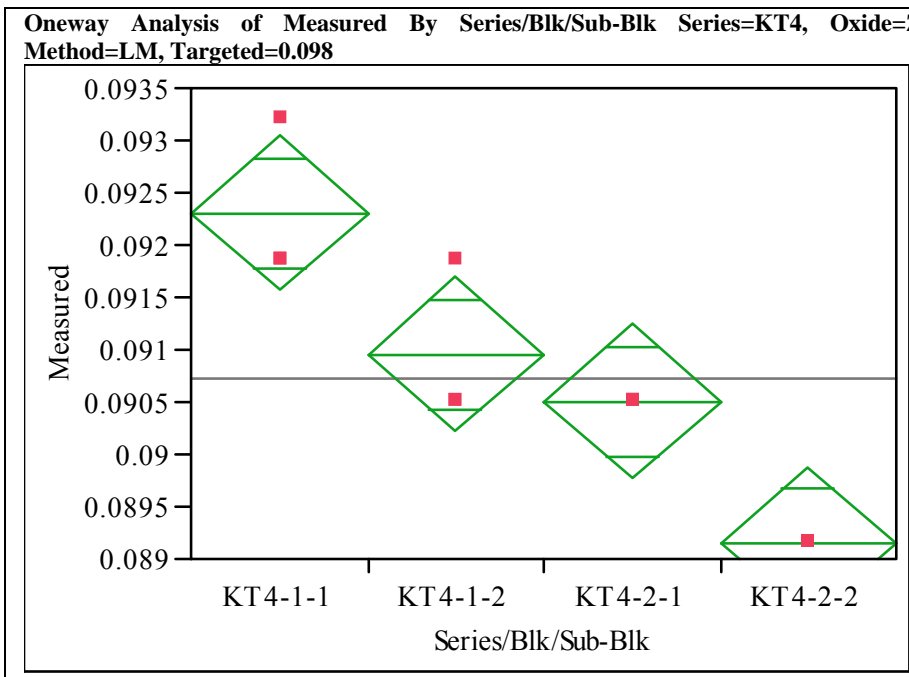

Oneway Anova

Summary of Fit

$\begin{array}{ll}\text { Rsquare } & 0.862069\end{array}$

$\begin{array}{ll}\text { Adj Rsquare } & 0.810345 \\ \text { Root Mean Square Error } & 0.00055\end{array}$

Root Mean Square Error $\quad 0.00055$

Mean of Response 0.090729

Analysis of Variance

Source DF Sum of Squares Mean Square F Ratio Prob $>$ F

$\begin{array}{lrrrrr}\text { Series/Blk/Sub-Blk } & 3 & 0.00001521 & 5.0685 \mathrm{e}-6 & 16.6667 & 0.0008 \\ \text { Error } & 8 & 0.00000243 & 3.0411 \mathrm{e}-7 & & \end{array}$

Error

C. Total

Means for Oneway Anova

Level Number Mean Std Error Lower 95\% Upper 95\%

$\begin{array}{llllll}\text { KT4-1-1 } & 3 & 0.092305 & 0.00032 & 0.09157 & 0.09304 \\ \text { KT4-1-2 } & 3 & 0.090954 & 0.00032 & 0.09022 & 0.09169\end{array}$

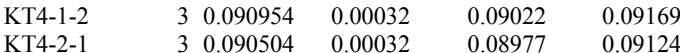

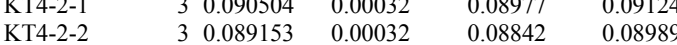

Std Error uses a pooled estimate of error variance 
Exhibit C-4. Plots of Measured versus Targeted Concentrations by Glass ID by Oxide for the KT04-Series.

Oxide=Al2O3 (wt \%)

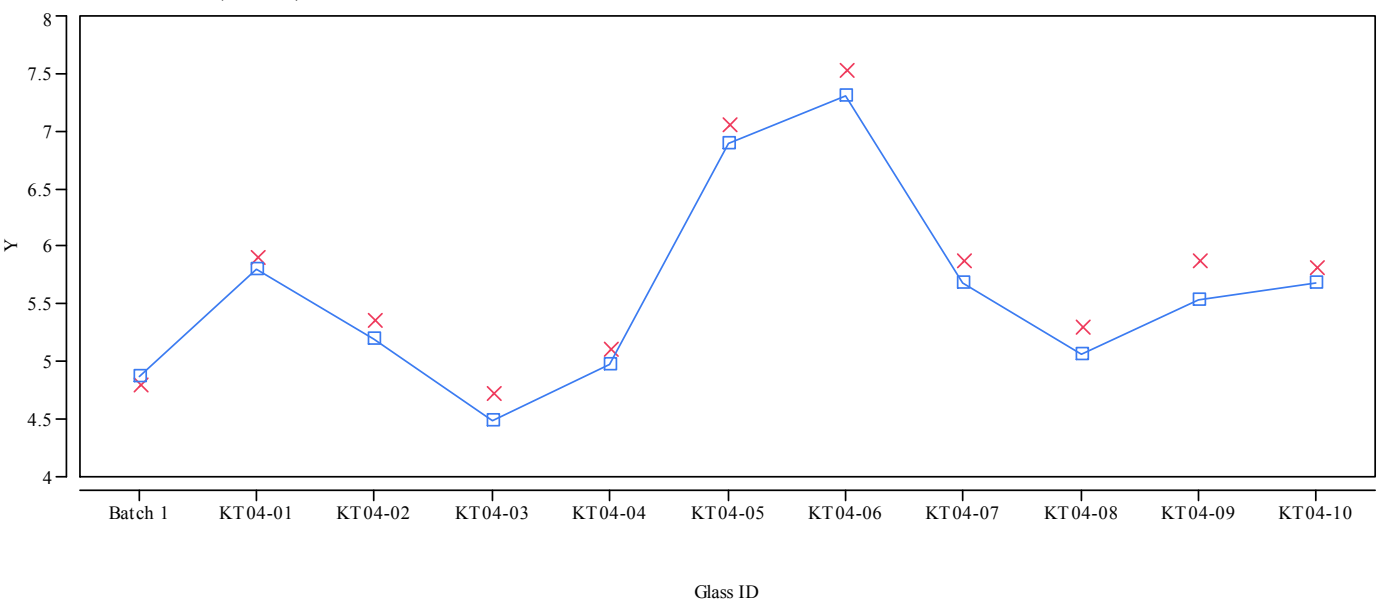

$\mathrm{Y} \times$ Measured $\square-$ Targeted

Oxide=B2O3 (wt \%)

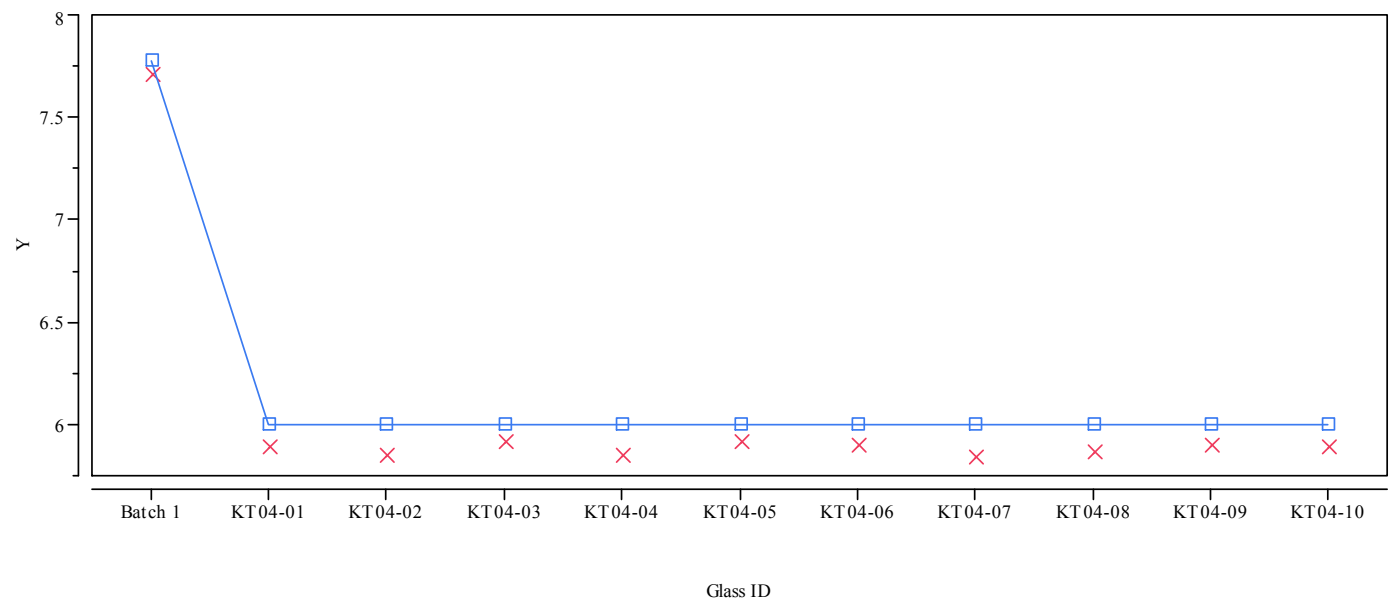

Y $\times$ Measured $\square-$ Targeted 
Exhibit C-4. Plots of Measured versus Targeted Concentrations by Glass ID by Oxide for the KT04-Series. (continued)

\section{Oxide $=\mathrm{BaO}(\mathrm{wt} \%)$}

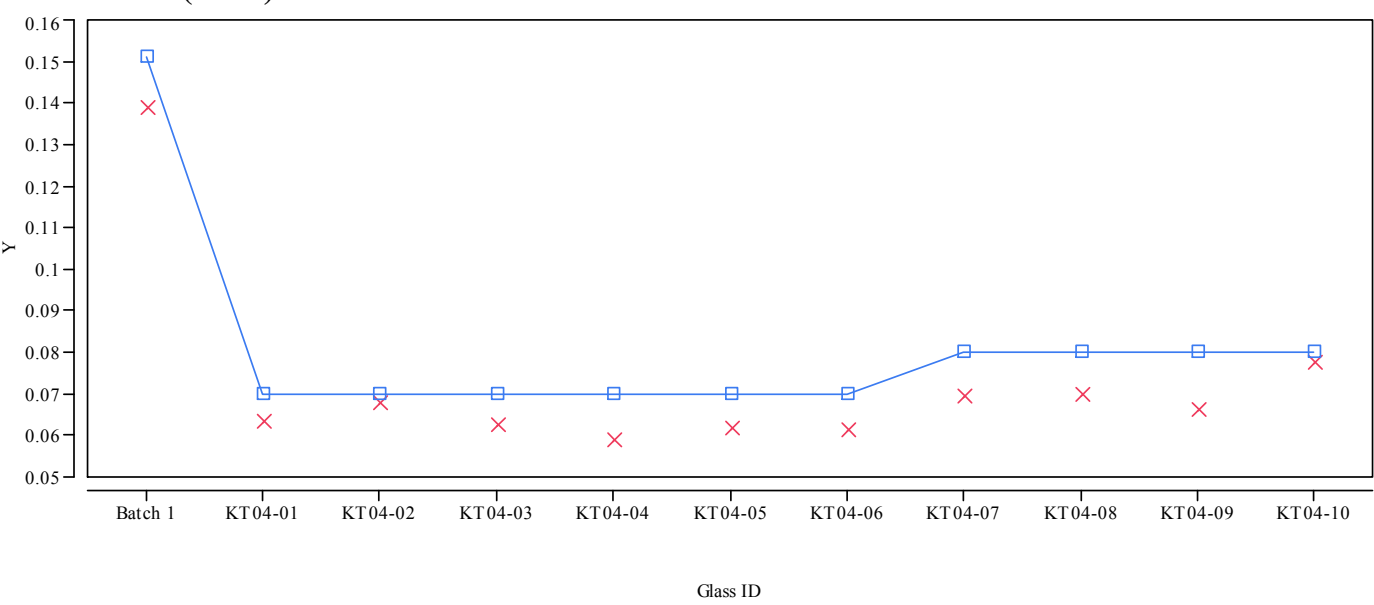

$\mathrm{Y} \times$ Measured $\square$ T Targeted

\section{Oxide= $\mathrm{CaO}(\mathrm{wt} \%)$}

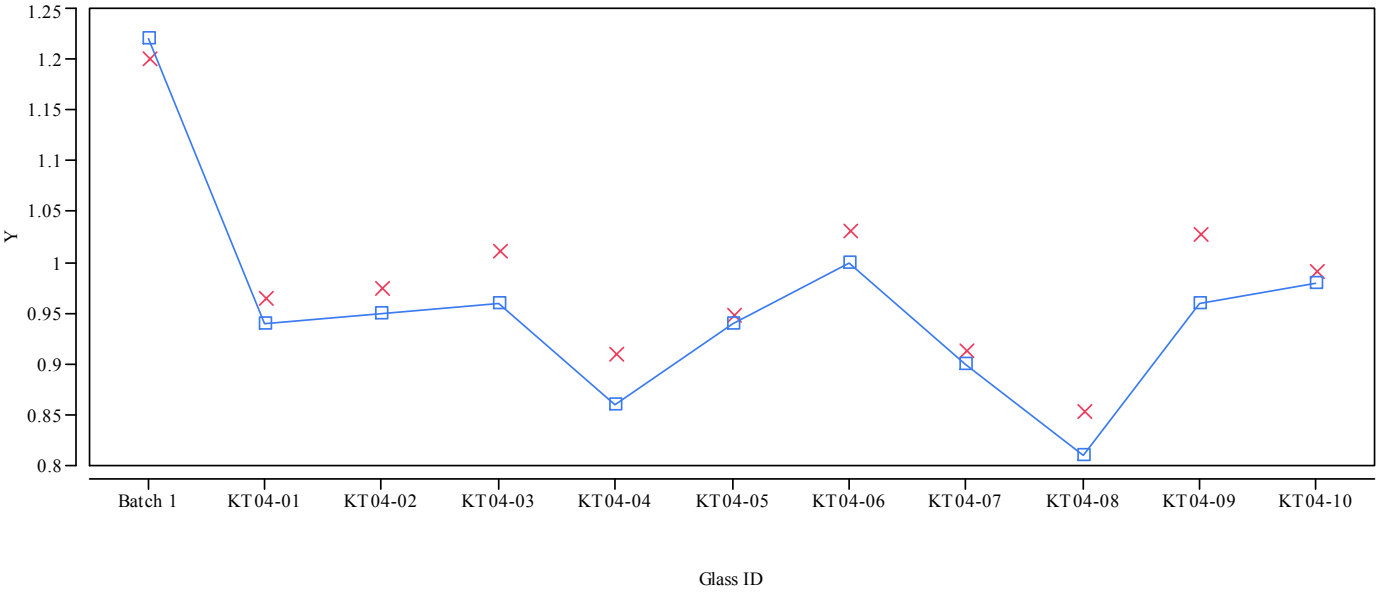

Y $\times$ Measured $\square \longrightarrow$ Targeted 
Exhibit C-4. Plots of Measured versus Targeted Concentrations by Glass ID by Oxide for the KT04-Series. (continued)

Oxide=Ce2O3 (wt \%)

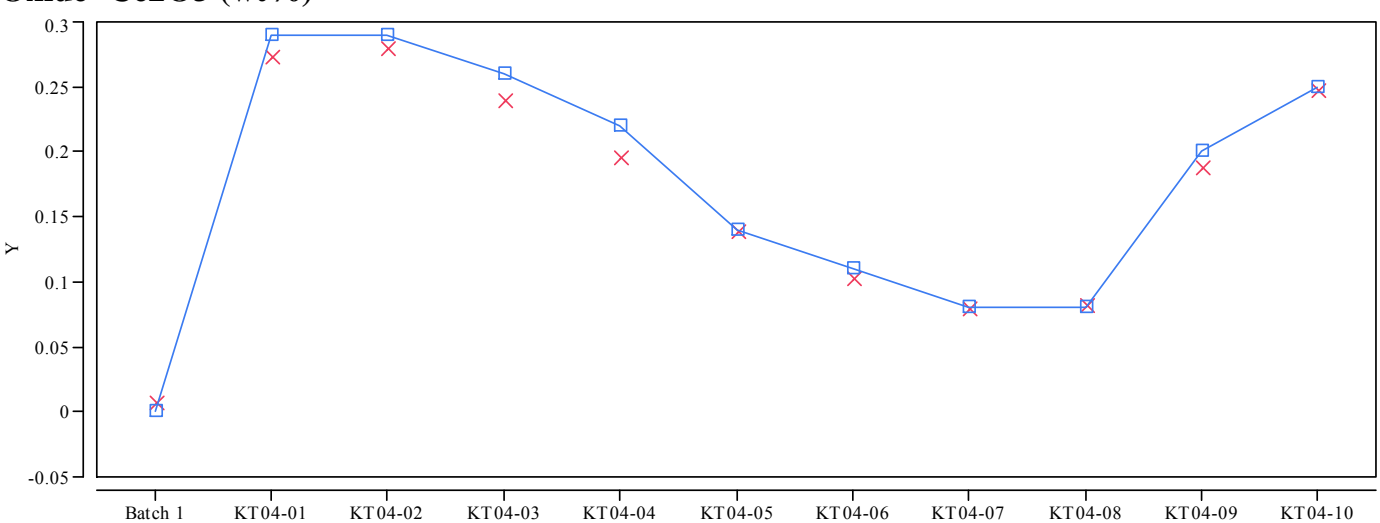

Glass ID

Y $\times$ Measured $\square$ Targeted

Oxide $=\mathrm{Cr} 2 \mathrm{O} 3(\mathrm{wt} \%)$

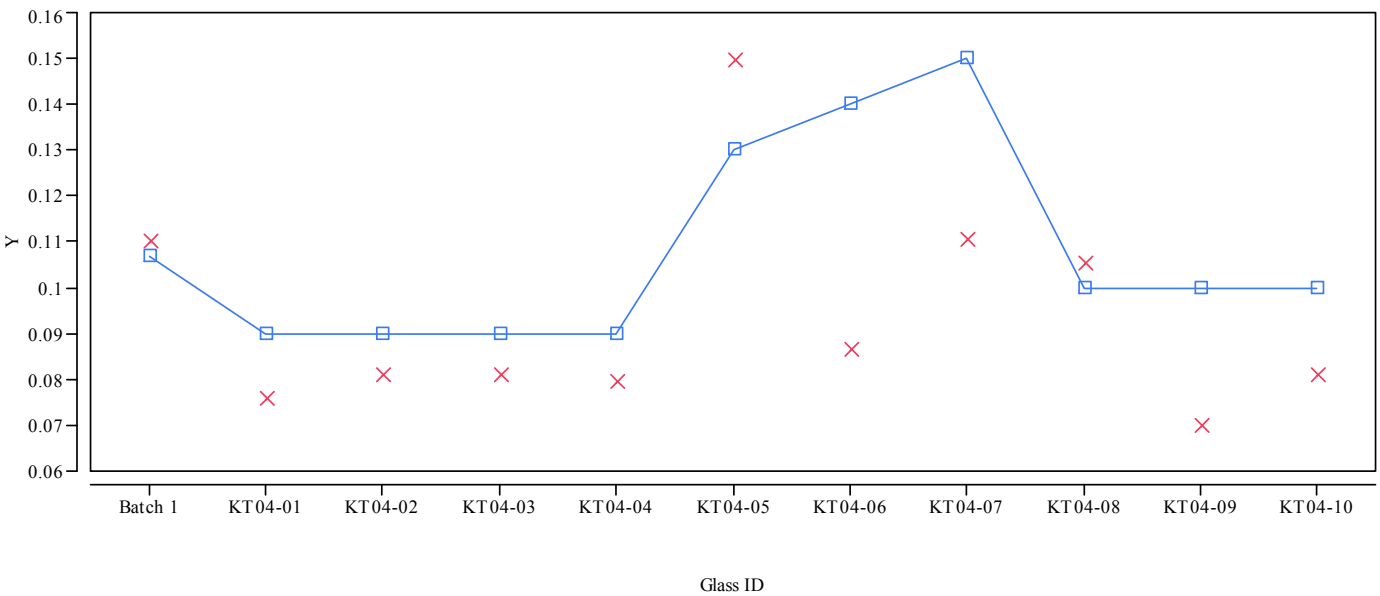

Y $\times$ Measured $\square-$ Targeted 
Exhibit C-4. Plots of Measured versus Targeted Concentrations by Glass ID by Oxide for the KT04-Series. (continued)

\section{Oxide $=\mathrm{CuO}(\mathrm{wt} \%)$}

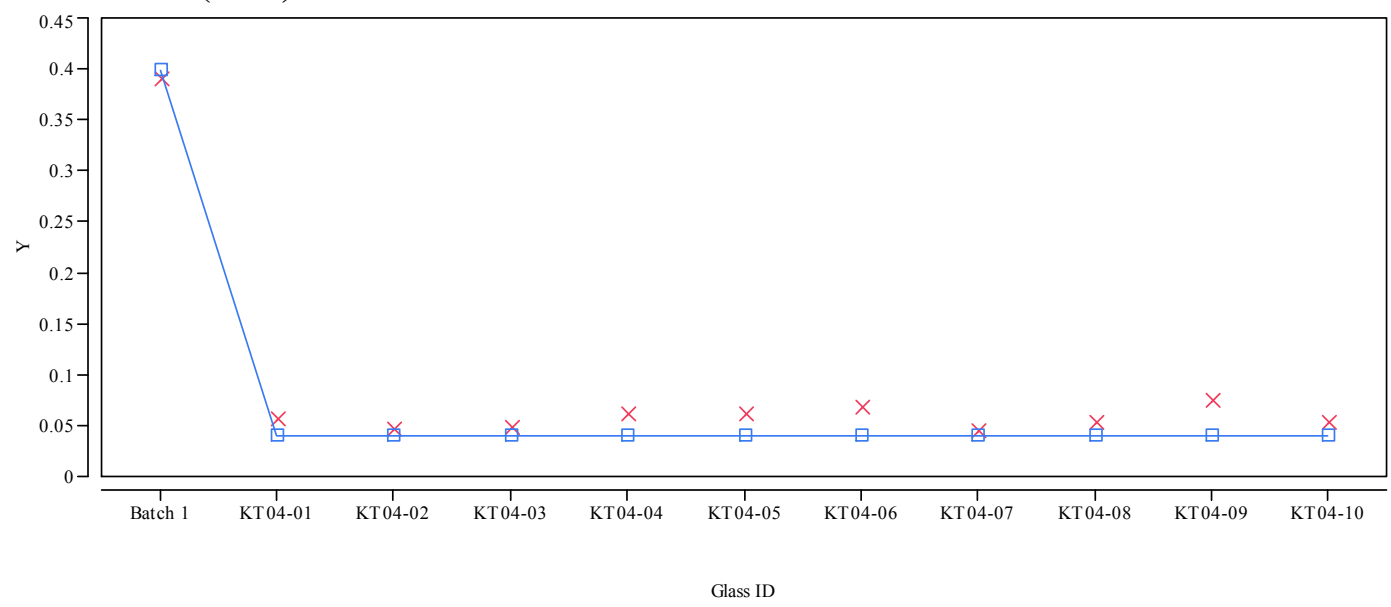

$\mathrm{Y} \times$ Measured $\square$ - Targeted

Oxide=Fe2O3 (wt \%)

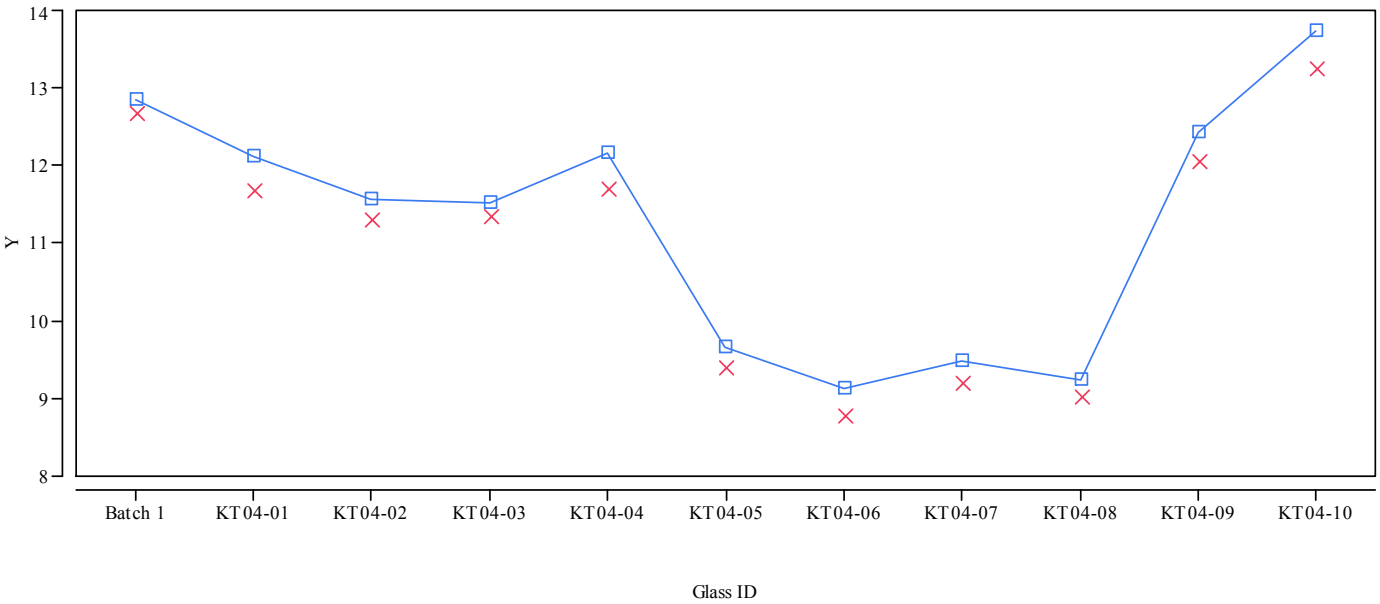

Y $\times$ Measured $\square$ Targeted 
Exhibit C-4. Plots of Measured versus Targeted Concentrations by Glass ID by Oxide for the KT04-Series. (continued)

\section{Oxide=K2O (wt\%)}

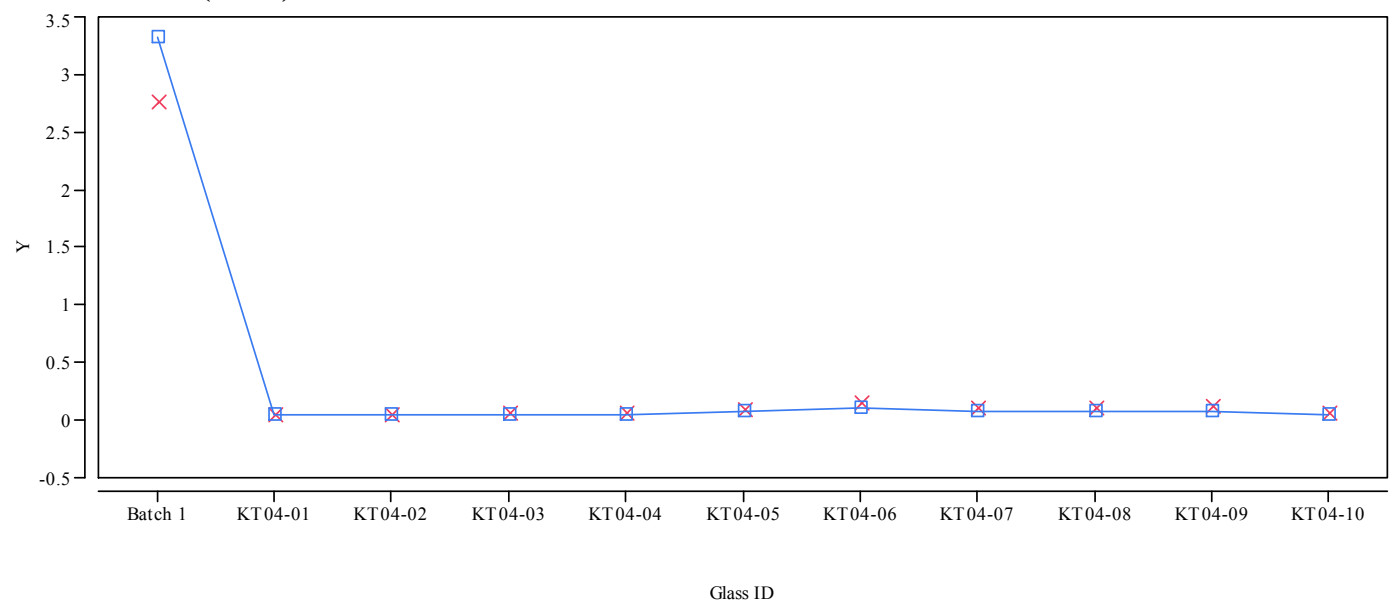

$\mathrm{Y} \times$ Measured $\square$ - Targeted

Oxide=La2O3 (wt\%)

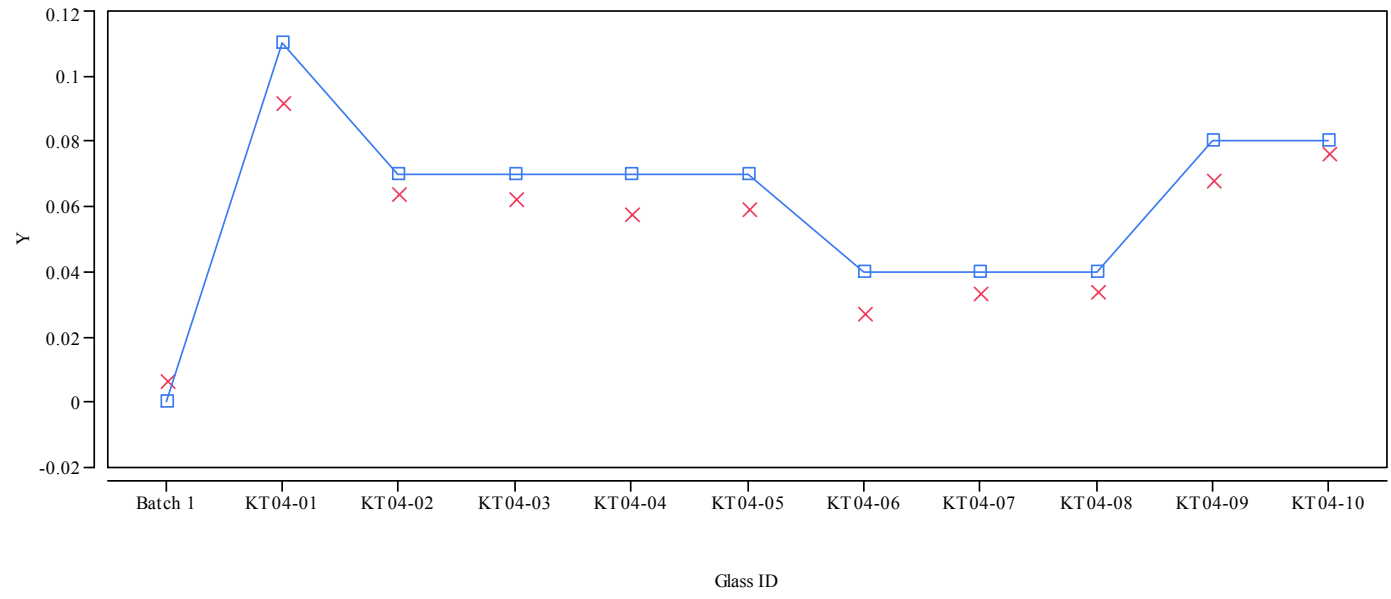

Y $\times$ Measured $\square$ - Targeted 
Exhibit C-4. Plots of Measured versus Targeted Concentrations by Glass ID by Oxide for the KT04-Series. (continued)

\section{Oxide $=\mathrm{Li2O}(\mathrm{wt} \%)$}

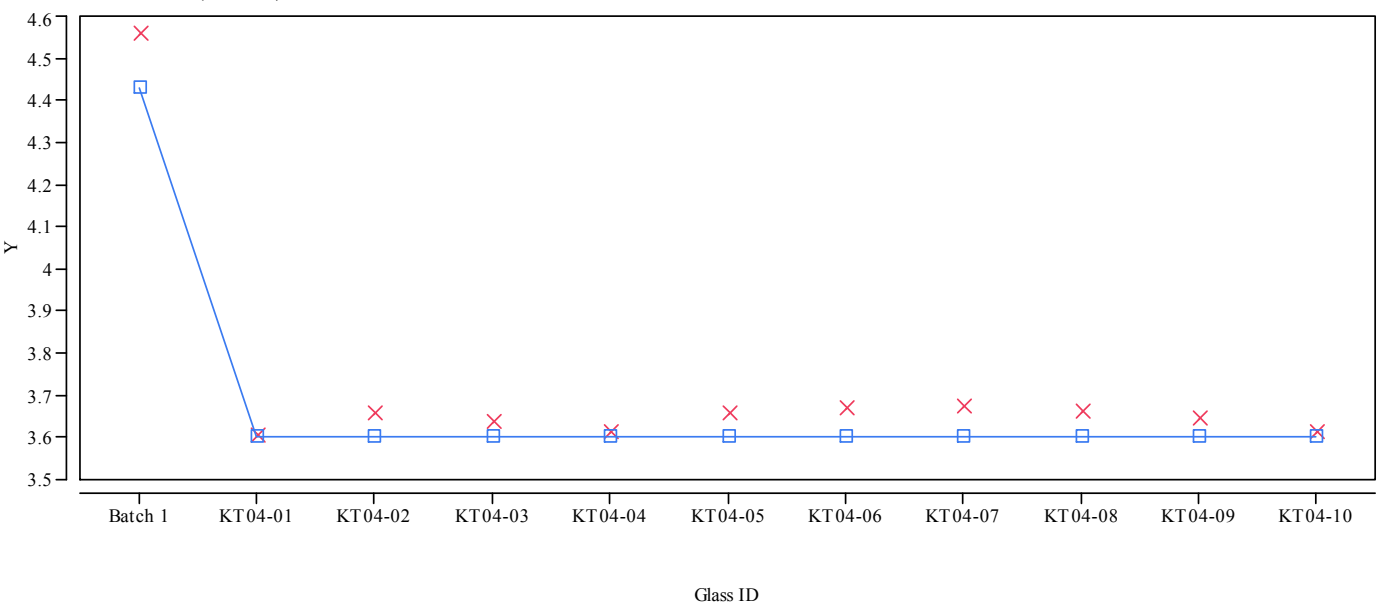

Y $\times$ Measured $\square \longrightarrow$ Targeted

\section{Oxide $=\mathrm{MgO}(\mathrm{wt} \%)$}

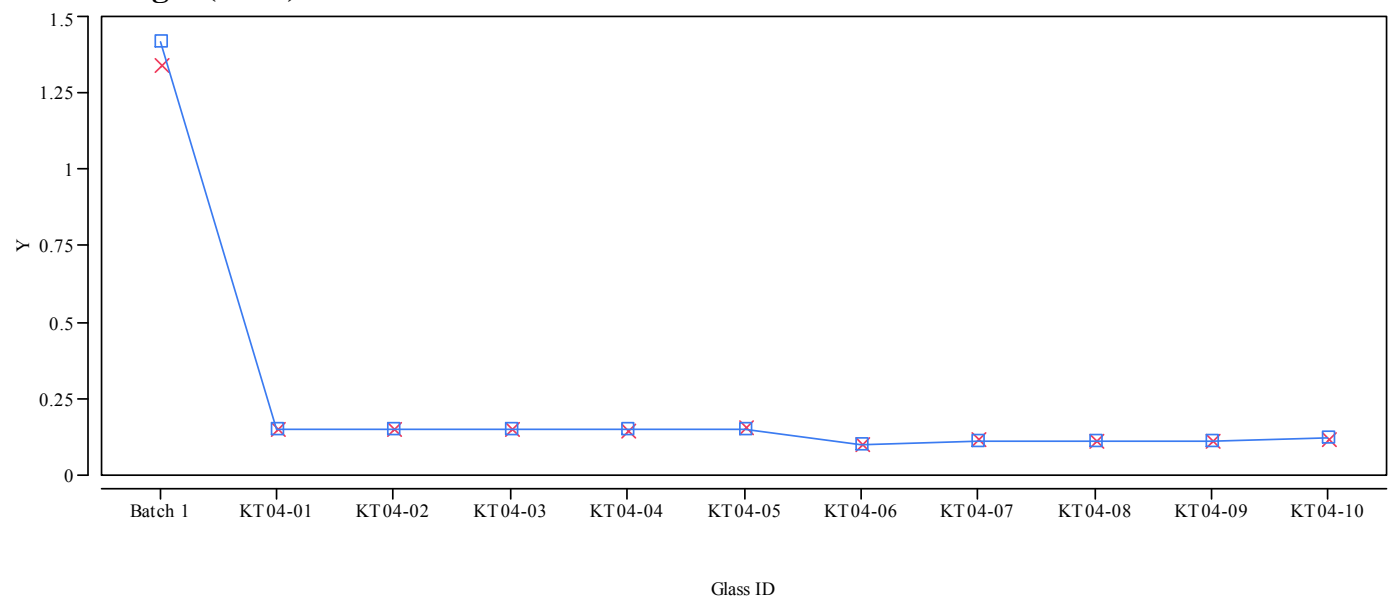

$\mathrm{Y} \times$ Measured $\square$ T argeted 
Exhibit C-4. Plots of Measured versus Targeted Concentrations by Glass ID by Oxide for the KT04-Series. (continued)

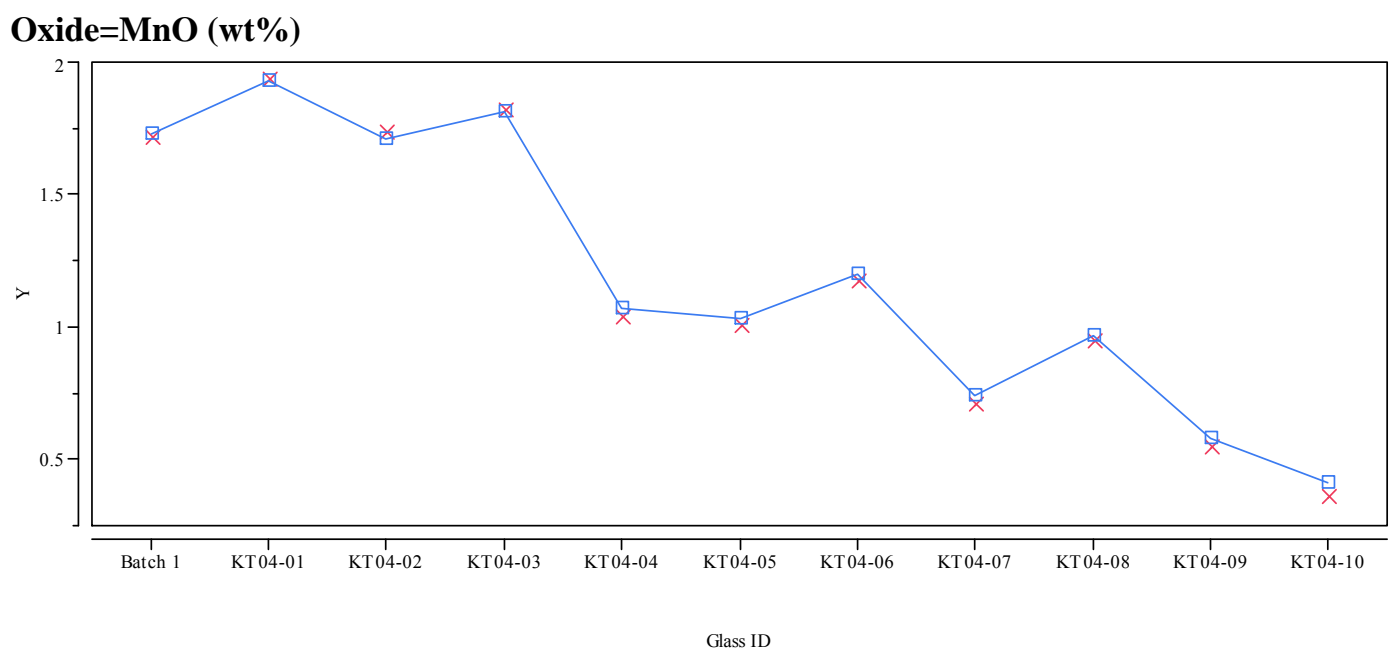

$\mathrm{Y} \times$ Measured $\square$ Targeted

\section{Oxide $=\mathrm{Na} 2 \mathrm{O}(\mathrm{wt} \%)$}

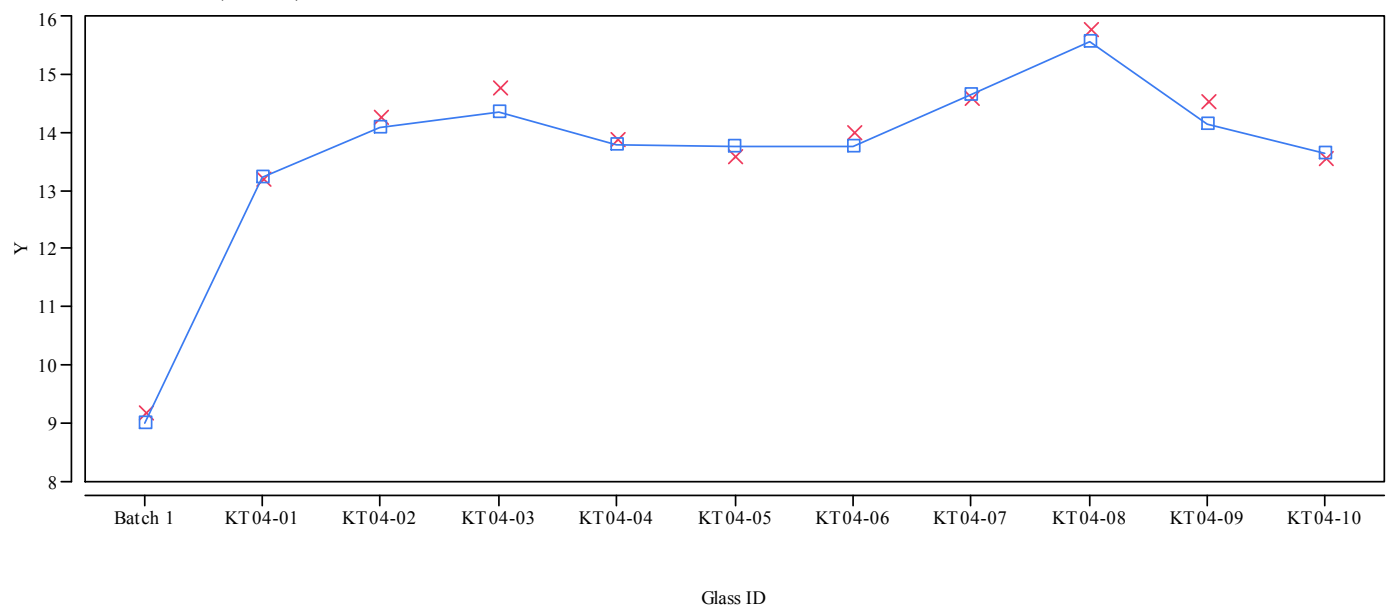

Y $\times$ Measured $\square-$ Targeted 
Exhibit C-4. Plots of Measured versus Targeted Concentrations by Glass ID by Oxide for the KT04-Series. (continued)

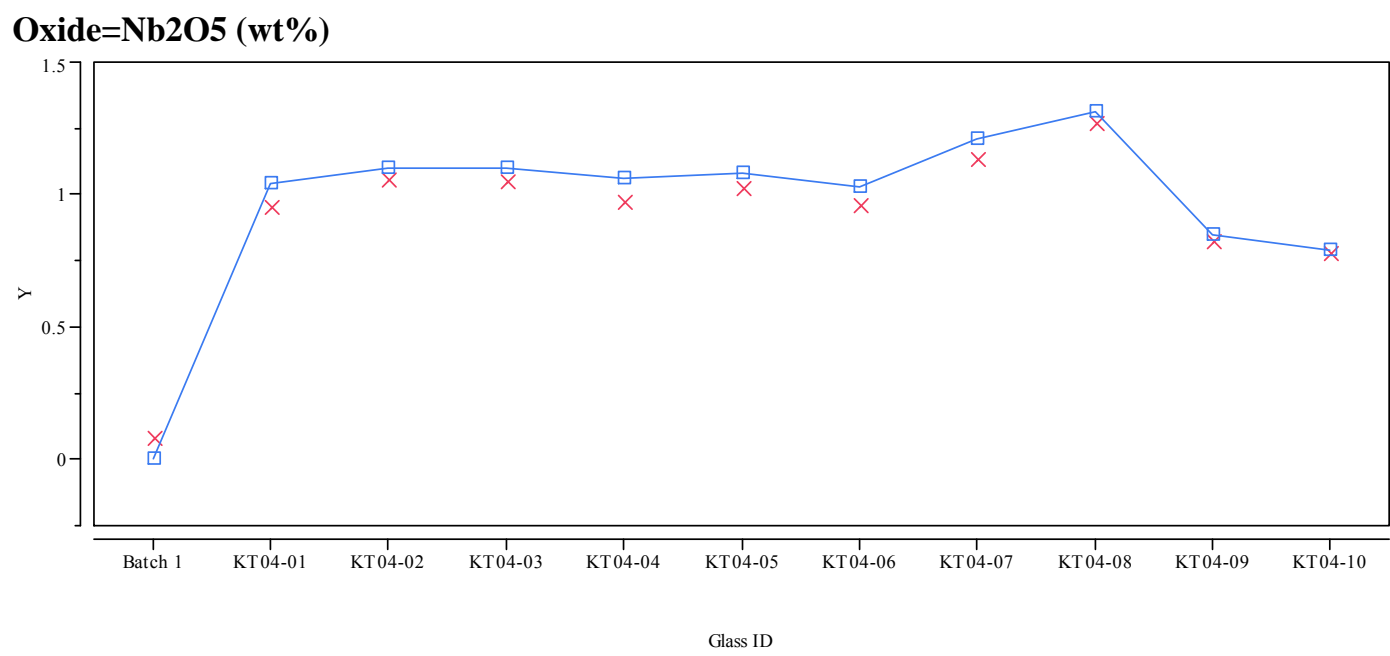

$\mathrm{Y} \times$ Measured $\square$ Targeted

\section{Oxide $=\mathrm{NiO}(\mathrm{wt} \%)$}

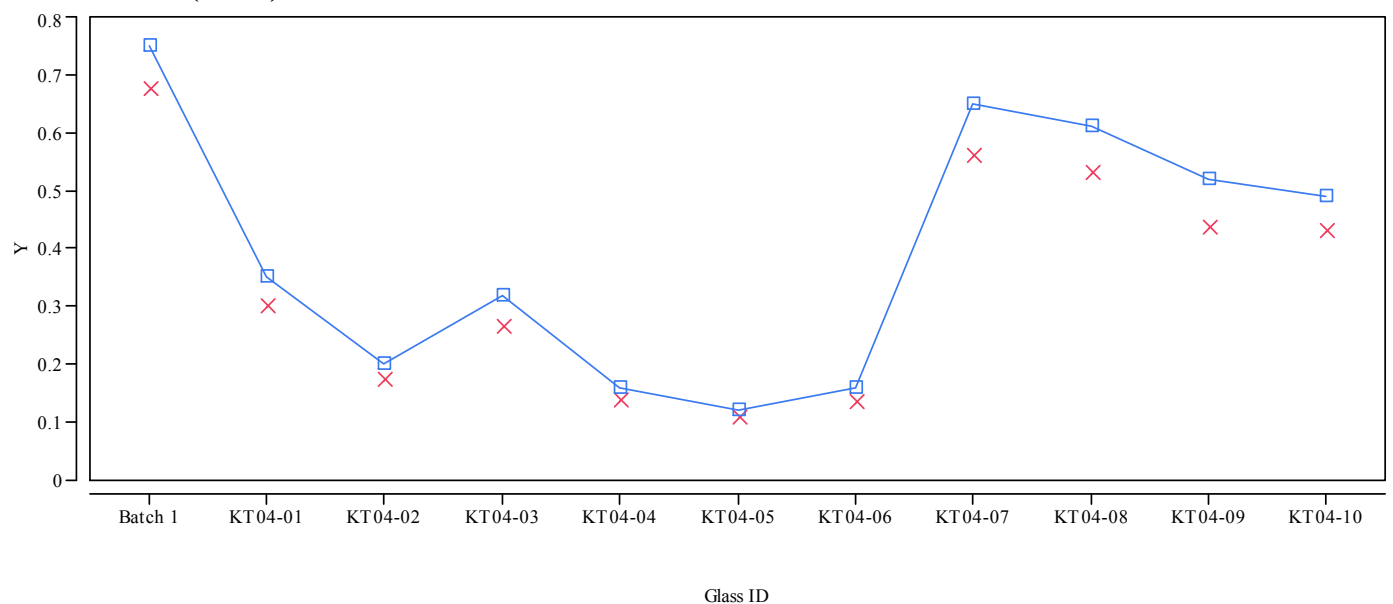

$\mathrm{Y} \times$ Measured $\square$ Targeted 
Exhibit C-4. Plots of Measured versus Targeted Concentrations by Glass ID by Oxide for the KT04-Series. (continued)

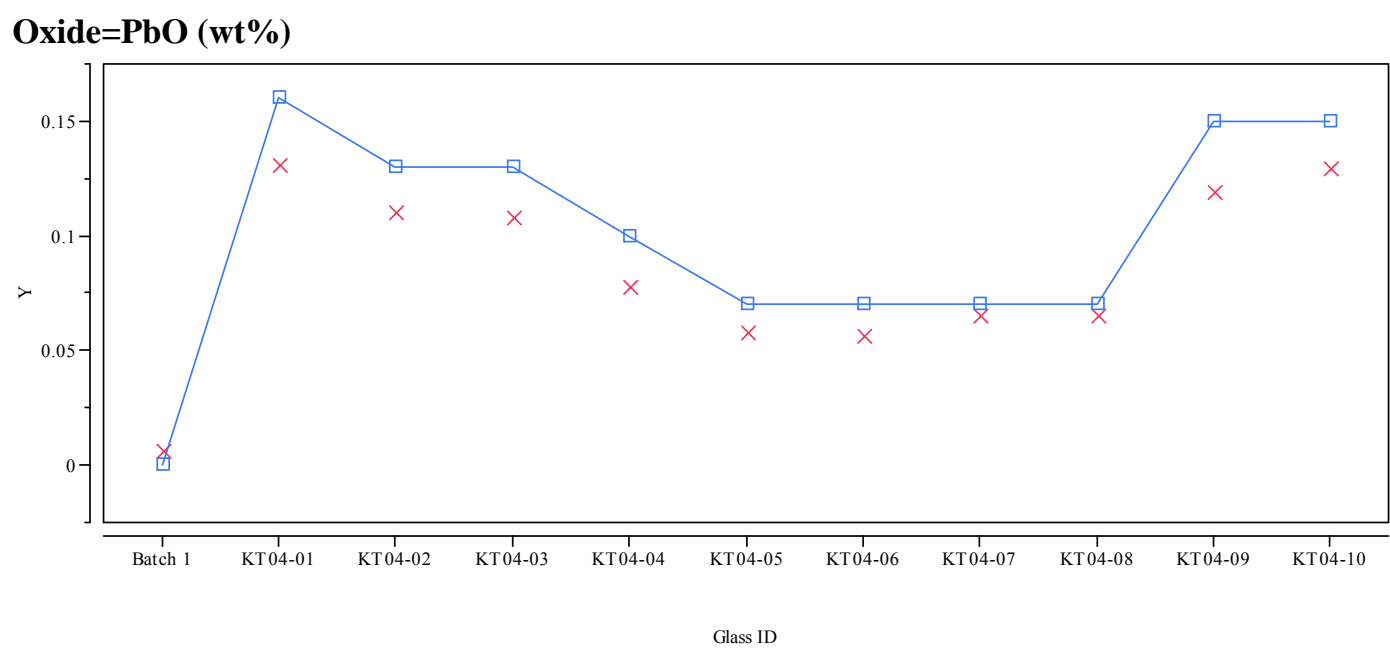

Y $\times$ Measured $\square-$ Targeted

Oxide $=\mathrm{SiO} 2(\mathrm{wt} \%)$

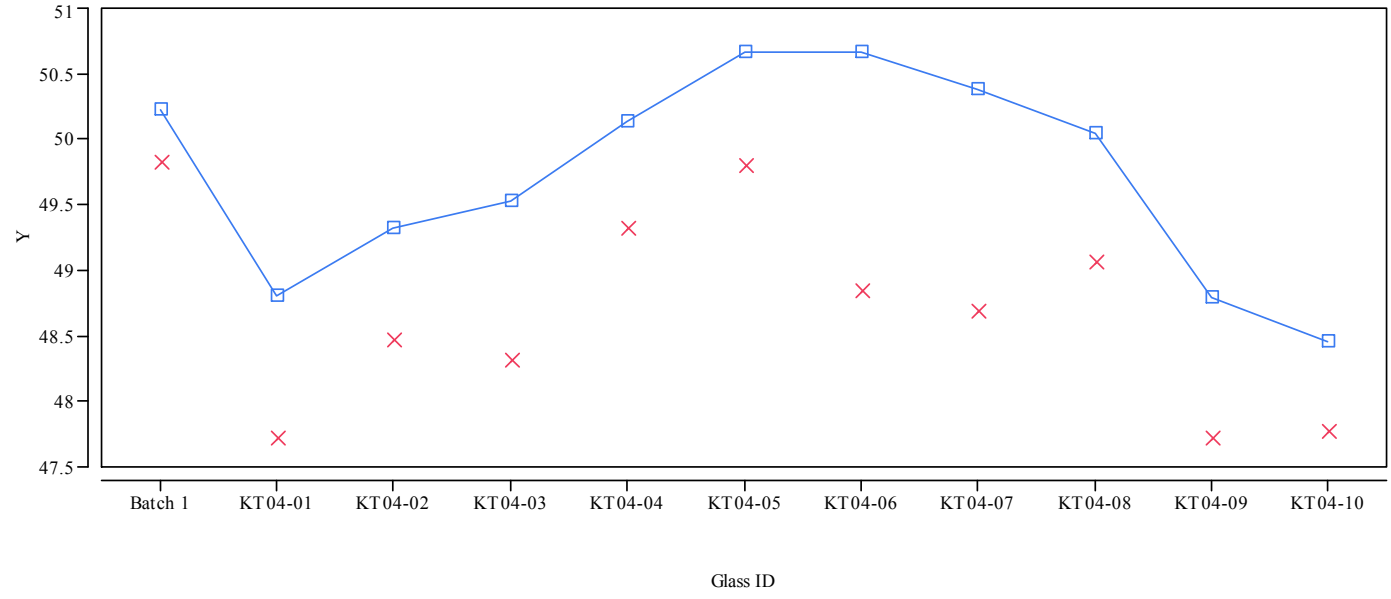

Y $\times$ Measured $\square$ Targeted 
Exhibit C-4. Plots of Measured versus Targeted Concentrations by Glass ID by Oxide for the KT04-Series. (continued)

Oxide=TiO2 (wt\%)

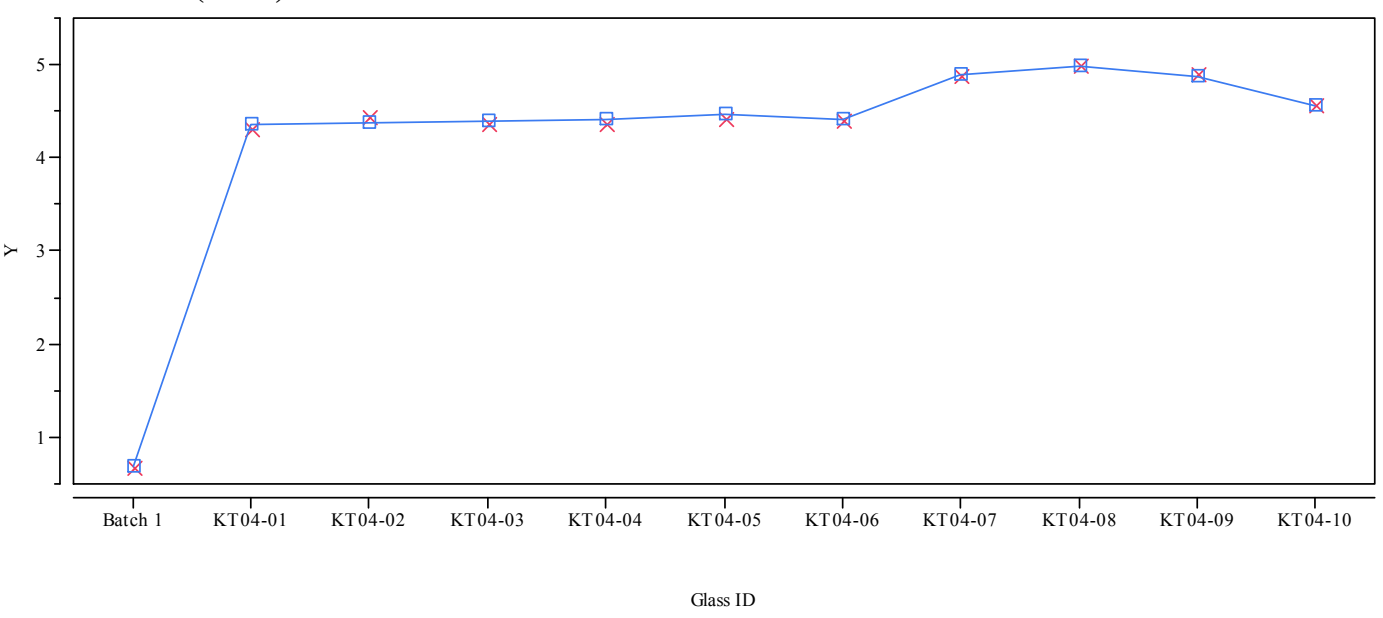

$\mathrm{Y} \times$ Measured $\square$ - Targeted

\section{Oxide $=\mathrm{ZnO}$ (wt \%)}

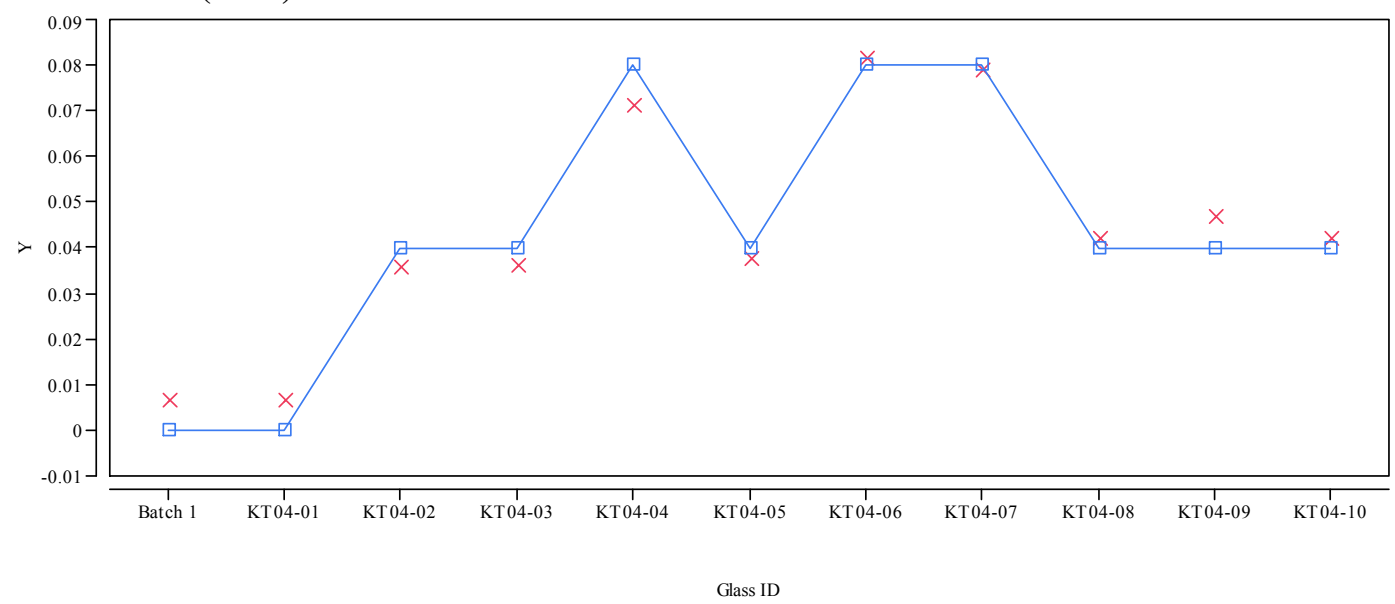

$\mathrm{Y} \times$ Measured $\square$ - Targeted 
Exhibit C-4. Plots of Measured versus Targeted Concentrations by Glass ID by Oxide for the KT04-Series. (continued)

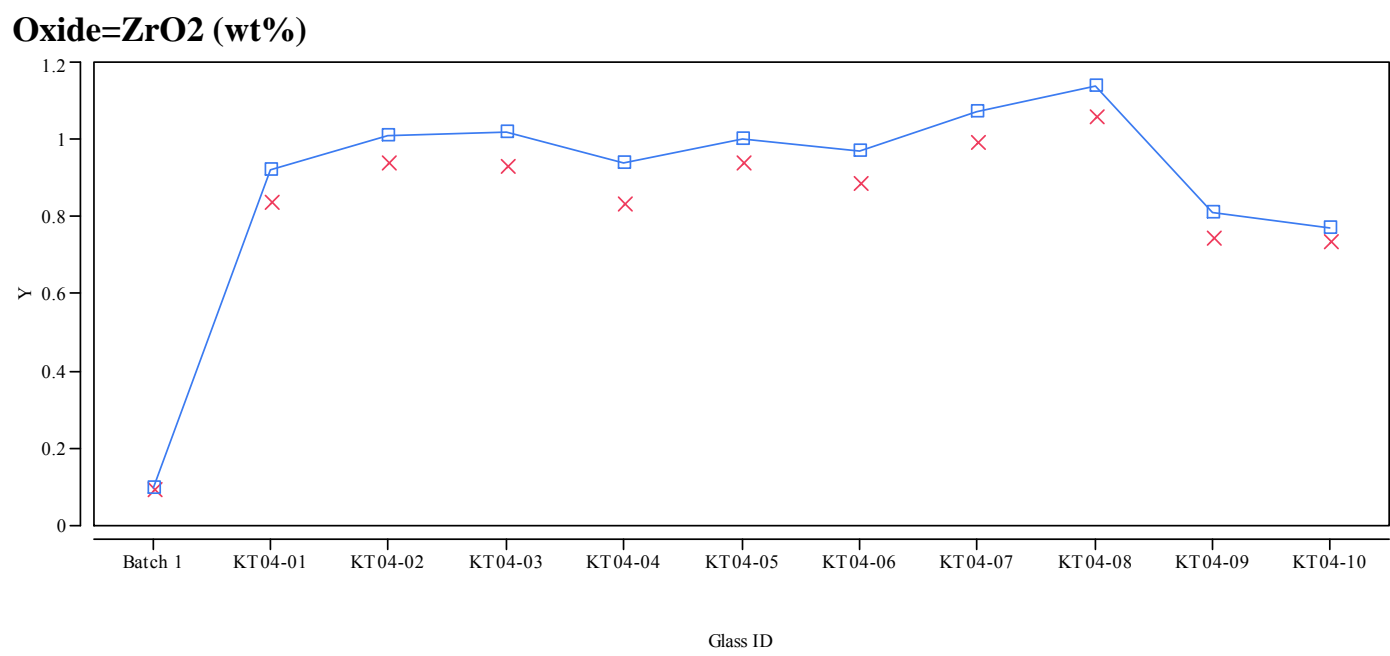

$\mathrm{Y} \times$ Measured $\square$ Targeted

Oxide $=$ Sum of Oxides

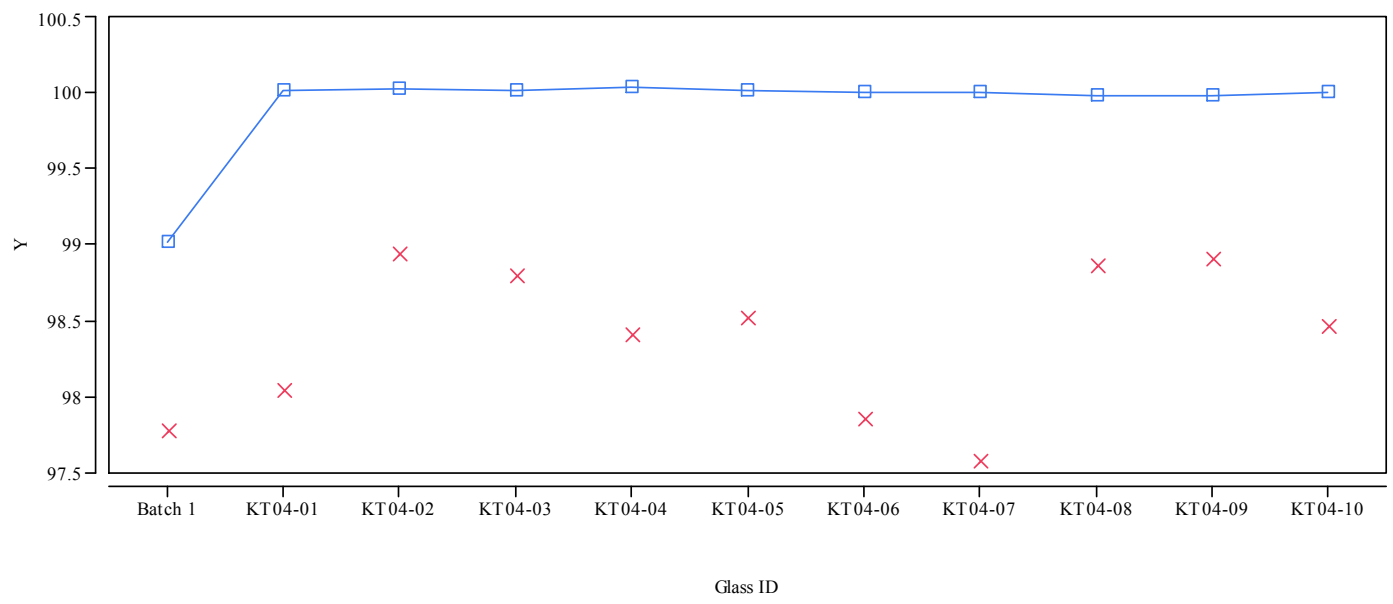

Y $\times$ Measured $\square-$ Targeted 
SRNL-STI-2010-00566

Revision 0

Appendix D. Data Supporting the PCT Measurements of the KT01-Series Glasses 
Table D-1. Measurements of the PCT Solutions for the KT01-Series Glasses As-Received (ar) and After Appropriate Adjustments (ppm).

\begin{tabular}{|c|c|c|c|c|c|c|c|c|c|c|c|c|}
\hline Glass ID & $\begin{array}{c}\text { Heat } \\
\text { Treatment }\end{array}$ & Block & Seq & $\begin{array}{c}\text { Lab } \\
\text { ID }\end{array}$ & $\begin{array}{l}\text { B } \\
\text { ar }\end{array}$ & $\begin{array}{l}\mathrm{Li} \\
\mathrm{ar}\end{array}$ & $\begin{array}{l}\mathrm{Na} \\
\mathrm{ar}\end{array}$ & $\begin{array}{l}\mathrm{Si} \\
\mathrm{ar}\end{array}$ & $\begin{array}{c}\text { B } \\
(\mathrm{ppm})\end{array}$ & $\begin{array}{c}\mathbf{L i} \\
(\mathbf{p p m})\end{array}$ & $\begin{array}{c}\mathrm{Na} \\
(\mathrm{ppm})\end{array}$ & $\begin{array}{c}\mathrm{Si} \\
(\mathbf{p p m})\end{array}$ \\
\hline Soln Std & ref & 1 & 1 & std-11-1 & 21.2 & 9.90 & 82.1 & 47.2 & 21.20 & 9.90 & 82.10 & 47.20 \\
\hline KT1-HS & $\mathrm{ccc}$ & 1 & 2 & S76 & 5.83 & 9.98 & 47.9 & 71.2 & 9.72 & 16.63 & 79.83 & 118.67 \\
\hline EA & ref & 1 & 3 & S70 & 40.3 & 12.2 & 107 & 52.9 & 671.67 & 203.33 & 1783.34 & 881.67 \\
\hline ARM-1 & ref & 1 & 4 & S85 & 11.0 & 8.30 & 21.5 & 34.4 & 18.33 & 13.83 & 35.83 & 57.33 \\
\hline KT1-HN & quenched & 1 & 5 & S72 & 8.09 & 11.8 & 91.3 & 81.4 & 13.48 & 19.67 & 152.17 & 135.67 \\
\hline KT1-HL & $\mathrm{ccc}$ & 1 & 6 & S78 & 12.9 & 57.7 & 105 & 173.6 & 21.50 & 96.17 & 175.00 & 289.34 \\
\hline blank & ref & 1 & 7 & S47 & 0.422 & $<1.00$ & $<0.100$ & $<0.100$ & 0.70 & 0.83 & 0.08 & 0.08 \\
\hline KT1-HA & $\mathrm{ccc}$ & 1 & 8 & S16 & 6.16 & 10.3 & 59.8 & 65.8 & 10.27 & 17.17 & 99.67 & 109.67 \\
\hline KT1-LL & quenched & 1 & 9 & S68 & 7.03 & 11.3 & 63.7 & 72.4 & 11.72 & 18.83 & 106.17 & 120.67 \\
\hline KT1-LS & quenched & 1 & 10 & S37 & 6.71 & 10.7 & 61.3 & 69.5 & 11.18 & 17.83 & 102.17 & 115.84 \\
\hline KT1-HN & $\mathrm{ccc}$ & 1 & 11 & S67 & 7.79 & 12.8 & 85.2 & 82.4 & 12.98 & 21.33 & 142.00 & 137.34 \\
\hline KT1-HB & quenched & 1 & 12 & S56 & 9.54 & 9.58 & 52.1 & 60.9 & 15.90 & 15.97 & 86.84 & 101.50 \\
\hline KT1-LA & $\mathrm{ccc}$ & 1 & 13 & S77 & 7.88 & 12.7 & 66.7 & 79.4 & 13.13 & 21.17 & 111.17 & 132.34 \\
\hline KT1-LF & $\mathrm{ccc}$ & 1 & 14 & S59 & 6.38 & 11.3 & 62.4 & 72.6 & 10.63 & 18.83 & 104.00 & 121.00 \\
\hline KT1-LN & quenched & 1 & 15 & S63 & 6.02 & 10.1 & 43.8 & 63.1 & 10.03 & 16.83 & 73.00 & 105.17 \\
\hline KT1-LN & $\mathrm{ccc}$ & 1 & 16 & S07 & 5.94 & 10.2 & 40.9 & 63.1 & 9.90 & 17.00 & 68.17 & 105.17 \\
\hline Soln Std & ref & 1 & 17 & std-11-2 & 20.6 & 10.0 & 83.2 & 47.3 & 20.60 & 10.00 & 83.20 & 47.30 \\
\hline KT1-HB & $\mathrm{ccc}$ & 1 & 18 & S15 & 9.68 & 9.55 & 47.2 & 57.7 & 16.13 & 15.92 & 78.67 & 96.17 \\
\hline KT1-LL & $\mathrm{ccc}$ & 1 & 19 & S45 & 6.62 & 11.0 & 54.9 & 67.0 & 11.03 & 18.33 & 91.50 & 111.67 \\
\hline KT1-LS & $\mathrm{ccc}$ & 1 & 20 & S86 & 6.84 & 11.2 & 57.1 & 69.1 & 11.40 & 18.67 & 95.17 & 115.17 \\
\hline KT1-HK & $\mathrm{ccc}$ & 1 & 21 & S46 & 4.98 & 5.00 & 41.8 & 52.7 & 8.30 & 8.33 & 69.67 & 87.84 \\
\hline KT1-LA & quenched & 1 & 22 & S19 & 8.13 & 12.8 & 74.4 & 83.1 & 13.55 & 21.33 & 124.00 & 138.50 \\
\hline KT1-HF & $\mathrm{ccc}$ & 1 & 23 & S01 & 6.27 & 10.3 & 59.3 & 70.9 & 10.45 & 17.17 & 98.84 & 118.17 \\
\hline KT1-LB & $\mathrm{ccc}$ & 1 & 24 & S62 & 7.14 & 11.8 & 60.4 & 72.4 & 11.90 & 19.67 & 100.67 & 120.67 \\
\hline KT1-HF & quenched & 1 & 25 & $\mathrm{~S} 22$ & 7.08 & 11.2 & 59.1 & 68.0 & 11.80 & 18.67 & 98.50 & 113.34 \\
\hline KT1-HS & quenched & 1 & 26 & S38 & 5.88 & 9.91 & 51.2 & 71.0 & 9.80 & 16.52 & 85.34 & 118.34 \\
\hline KT1-LF & quenched & 1 & 27 & S64 & 6.22 & 10.8 & 67.2 & 73.7 & 10.37 & 18.00 & 112.00 & 122.84 \\
\hline KT1-LB & quenched & 1 & 28 & S52 & 6.72 & 10.6 & 62.4 & 68.1 & 11.20 & 17.67 & 104.00 & 113.50 \\
\hline KT1-HA & quenched & 1 & 29 & S55 & 5.92 & 9.50 & 59.7 & 63.8 & 9.87 & 15.83 & 99.50 & 106.34 \\
\hline KT1-HL & quenched & 1 & 30 & S13 & 10.2 & 26.8 & 94.6 & 105 & 17.00 & 44.67 & 157.67 & 175.00 \\
\hline KT1-HK & quenched & 1 & 31 & S39 & 5.39 & 5.49 & 48.4 & 48.1 & 8.98 & 9.15 & 80.67 & 80.17 \\
\hline Soln Std & ref & 1 & 32 & std-11-3 & 19.6 & 9.68 & 79.4 & 45.7 & 19.60 & 9.68 & 79.40 & 45.70 \\
\hline Soln Std & ref & 2 & 1 & \begin{tabular}{|l|} 
std-12-1 \\
\end{tabular} & 21.3 & 10.2 & 83.5 & 48.4 & 21.30 & 10.20 & 83.50 & 48.40 \\
\hline KT1-HB & quenched & 2 & 2 & $\mathrm{~S} 23$ & 9.49 & 9.31 & 48.9 & 58.3 & 15.82 & 15.52 & 81.50 & 97.17 \\
\hline KT1-HB & $\mathrm{ccc}$ & 2 & 3 & S05 & 9.87 & 10.3 & 51.4 & 62.4 & 16.45 & 17.17 & 85.67 & 104.00 \\
\hline KT1-LA & $\mathrm{ccc}$ & 2 & 4 & S34 & 7.87 & 13.2 & 68.2 & 80.7 & 13.12 & 22.00 & 113.67 & 134.50 \\
\hline KT1-LF & quenched & 2 & 5 & S82 & 6.32 & 11.1 & 68.8 & 74.4 & 10.53 & 18.50 & 114.67 & 124.00 \\
\hline KT1-LF & $\mathrm{ccc}$ & 2 & 6 & S09 & 6.12 & 11.4 & 62.3 & 72.1 & 10.20 & 19.00 & 103.84 & 120.17 \\
\hline KT1-LL & $\mathrm{ccc}$ & 2 & 7 & S18 & 6.16 & 11.3 & 56.4 & 68.0 & 10.27 & 18.83 & 94.00 & 113.34 \\
\hline ARM-1 & ref & 2 & 8 & $\mathrm{~S} 20$ & 9.37 & 7.77 & 20.1 & 31.7 & 15.62 & 12.95 & 33.50 & 52.83 \\
\hline KT1-HK & $\mathrm{ccc}$ & 2 & 9 & S74 & 4.94 & 5.53 & 47.5 & 58.3 & 8.23 & 9.22 & 79.17 & 97.17 \\
\hline KT1-HN & quenched & 2 & 10 & S69 & 7.22 & 11.9 & 90.2 & 80.4 & 12.03 & 19.83 & 150.34 & 134.00 \\
\hline KT1-HL & $\mathrm{ccc}$ & 2 & 11 & S03 & 12.4 & 58.1 & 109 & 222 & 20.67 & 96.84 & 181.67 & 370.01 \\
\hline KT1-HN & $\mathrm{ccc}$ & 2 & 12 & S35 & 7.26 & 12.7 & 83.6 & 80.1 & 12.10 & 21.17 & 139.34 & 133.50 \\
\hline KT1-HF & $\mathrm{ccc}$ & 2 & 13 & S60 & 5.44 & 9.77 & 58.0 & 67.4 & 9.07 & 16.28 & 96.67 & 112.34 \\
\hline KT1-HF & quenched & 2 & 14 & S26 & 6.61 & 11.2 & 58.3 & 67.8 & 11.02 & 18.67 & 97.17 & 113.00 \\
\hline KT1-LL & quenched & 2 & 15 & $\mathrm{~S} 12$ & 6.30 & 11.3 & 64.1 & 71.3 & 10.50 & 18.83 & 106.84 & 118.84 \\
\hline KT1-LB & $\mathrm{ccc}$ & 2 & 16 & S36 & 6.52 & 11.9 & 61.2 & 71.7 & 10.87 & 19.83 & 102.00 & 119.50 \\
\hline Soln Std & ref & 2 & 17 & std-12-2 & 20.2 & 10.1 & 83.4 & 48.1 & 20.20 & 10.10 & 83.40 & 48.10 \\
\hline KT1-LB & quenched & 2 & 18 & S04 & 7.14 & 11.5 & 65.1 & 72.8 & 11.90 & 19.17 & 108.50 & 121.34 \\
\hline KT1-LN & quenched & 2 & 19 & S57 & 5.80 & 10.4 & 44.1 & 69.3 & 9.67 & 17.33 & 73.50 & 115.50 \\
\hline EA & ref & 2 & 20 & S06 & 39.2 & 12.0 & 105 & 51.5 & 653.33 & 200.00 & 1750.00 & 858.34 \\
\hline KT1-LS & quenched & 2 & 21 & S30 & 6.48 & 10.8 & 62.2 & 68.8 & 10.80 & 18.00 & 103.67 & 114.67 \\
\hline KT1-HS & $\mathrm{ccc}$ & 2 & 22 & S54 & 5.21 & 9.98 & 47.9 & 69.7 & 8.68 & 16.63 & 79.83 & 116.17 \\
\hline KT1-LA & quenched & 2 & 23 & S42 & 7.37 & 12.8 & 74.6 & 82.1 & 12.28 & 21.33 & 124.34 & 136.84 \\
\hline KT1-LS & $\mathrm{ccc}$ & 2 & 24 & S50 & 6.10 & 11.5 & 58.7 & 69.0 & 10.17 & 19.17 & 97.84 & 115.00 \\
\hline KT1-LN & $\mathrm{ccc}$ & 2 & 25 & S80 & 5.42 & 10.5 & 42.1 & 62.4 & 9.03 & 17.50 & 70.17 & 104.00 \\
\hline KT1-HA & quenched & 2 & 26 & S71 & 5.54 & 9.88 & 63.4 & 64.0 & 9.23 & 16.47 & 105.67 & 106.67 \\
\hline KT1-HA & $\mathrm{ccc}$ & 2 & 27 & S84 & 5.33 & 10.2 & 57.7 & 63.4 & 8.88 & 17.00 & 96.17 & 105.67 \\
\hline KT1-HK & quenched & 2 & 28 & S79 & 5.42 & 5.91 & 52.4 & 52.7 & 9.03 & 9.85 & 87.34 & 87.84 \\
\hline KT1-HL & quenched & 2 & 29 & S27 & 10.7 & 30.1 & 105 & 117 & 17.83 & 50.17 & 175.00 & 195.00 \\
\hline KT1-HS & quenched & 2 & 30 & S75 & 4.94 & 9.85 & 50.8 & 69.8 & 8.23 & 16.42 & 84.67 & 116.34 \\
\hline Soln Std & ref & 2 & 31 & std-12-3 & 20.0 & 10.2 & 84.3 & 47.9 & 20.00 & 10.20 & 84.30 & 47.90 \\
\hline Soln Std & ref & 3 & 1 & std-13-1 & 21.1 & 10.0 & 82.3 & 48.4 & 21.10 & 10.00 & 82.30 & 48.40 \\
\hline KT1-HF & quenched & 3 & $\frac{1}{2}$ & S51 & 7.50 & 11.1 & 57.2 & 67.8 & 12.50 & 18.50 & 95.34 & 113.00 \\
\hline KT1-HA & $\mathrm{ccc}$ & 3 & 3 & S14 & 5.99 & 10.0 & 55.5 & 62.8 & 9.98 & 16.67 & 92.50 & 104.67 \\
\hline KT1-HL & $\mathrm{ccc}$ & 3 & 4 & S32 & 12.8 & 58.0 & 106 & 170 & 21.33 & 96.67 & 176.67 & 283.34 \\
\hline
\end{tabular}


Table D-1. Measurements of the PCT Solutions for the KT01-Series Glasses As-Received (ar) and After Appropriate Adjustments (ppm). (continued)

\begin{tabular}{|c|c|c|c|c|c|c|c|c|c|c|c|c|}
\hline Glass ID & $\begin{array}{c}\text { Heat } \\
\text { Treatment }\end{array}$ & Block & Seq & $\begin{array}{c}\text { Lab } \\
\text { ID }\end{array}$ & $\begin{array}{l}\text { B } \\
\text { ar }\end{array}$ & $\begin{array}{l}\mathrm{Li} \\
\text { ar }\end{array}$ & $\begin{array}{l}\mathrm{Na} \\
\text { ar }\end{array}$ & $\begin{array}{l}\text { Si } \\
\text { ar }\end{array}$ & $\begin{array}{c}\text { B } \\
(\mathrm{ppm})\end{array}$ & $\begin{array}{c}\mathbf{L i} \\
(\mathrm{ppm})\end{array}$ & $\begin{array}{c}\mathrm{Na} \\
(\mathrm{ppm})\end{array}$ & $\begin{array}{c}\mathrm{Si} \\
\text { (ppm) }\end{array}$ \\
\hline ARM-1 & ref & 3 & 5 & S21 & 9.83 & 7.92 & 20.4 & 32.5 & 16.38 & 13.20 & 34.00 & 54.17 \\
\hline blank & ref & 3 & 6 & S83 & $<0.100$ & $<1.00$ & 0.144 & $<0.100$ & 0.08 & 0.83 & 0.24 & 0.08 \\
\hline KT1-LN & $\mathrm{ccc}$ & 3 & 7 & S28 & 5.82 & 10.5 & 41.9 & 63.4 & 9.70 & 17.50 & 69.83 & 105.67 \\
\hline KT1-HB & quenched & 3 & 8 & S10 & 8.78 & 9.23 & 49.1 & 58.2 & 14.63 & 15.38 & 81.83 & 97.00 \\
\hline KT1-HK & quenched & 3 & 9 & S25 & 5.76 & 5.91 & 52.2 & 53.4 & 9.60 & 9.85 & 87.00 & 89.00 \\
\hline KT1-LF & $\mathrm{ccc}$ & 3 & 10 & S66 & 6.00 & 11.5 & 62.1 & 73.9 & 10.00 & 19.17 & 103.50 & 123.17 \\
\hline KT1-HS & quenched & 3 & 11 & S44 & 5.28 & 9.87 & 50.4 & 70.6 & 8.80 & 16.45 & 84.00 & 117.67 \\
\hline KT1-HS & ccc & 3 & 12 & S81 & 5.23 & 10.0 & 47.7 & 70.4 & 8.72 & 16.67 & 79.50 & 117.34 \\
\hline KT1-LA & quenched & 3 & 13 & S53 & 7.56 & 12.6 & 72.6 & 81.5 & 12.60 & 21.00 & 121.00 & 135.84 \\
\hline KT1-LF & quenched & 3 & 14 & S33 & 5.83 & 10.7 & 66.3 & 71.8 & 9.72 & 17.83 & 110.50 & 119.67 \\
\hline KT1-LN & quenched & 3 & 15 & S61 & 5.71 & 10.4 & 45.5 & 64.3 & 9.52 & 17.33 & 75.83 & 107.17 \\
\hline KT1-HK & $\mathrm{ccc}$ & 3 & 16 & S24 & 5.09 & 5.70 & 48.4 & 60.1 & 8.48 & 9.50 & 80.67 & 100.17 \\
\hline Soln Std & ref & 3 & 17 & std-13-2 & 20.3 & 10.3 & 84.3 & 49.0 & 20.30 & 10.30 & 84.30 & 49.00 \\
\hline KT1-HA & quenched & 3 & 18 & S49 & 6.19 & 9.88 & 63.5 & 64.6 & 10.32 & 16.47 & 105.84 & 107.67 \\
\hline KT1-HB & ccc & 3 & 19 & S58 & 9.64 & 10.5 & 51.0 & 62.8 & 16.07 & 17.50 & 85.00 & 104.67 \\
\hline KT1-LL & $\mathrm{ccc}$ & 3 & 20 & S02 & 6.32 & 11.5 & 58.2 & 69.7 & 10.53 & 19.17 & 97.00 & 116.17 \\
\hline EA & ref & 3 & 21 & S08 & 39.4 & 12.2 & 106 & 52.8 & 656.67 & 203.33 & 1766.67 & 880.00 \\
\hline KT1-LS & quenched & 3 & 22 & S41 & 6.56 & 10.8 & 61.2 & 68.8 & 10.93 & 18.00 & 102.00 & 114.67 \\
\hline KT1-HF & $\mathrm{ccc}$ & 3 & 23 & S65 & 5.71 & 9.71 & 56.4 & 67.8 & 9.52 & 16.18 & 94.00 & 113.00 \\
\hline KT1-LB & $\mathrm{ccc}$ & 3 & 24 & S48 & 6.81 & 11.8 & 59.6 & 71.7 & 11.35 & 19.67 & 99.34 & 119.50 \\
\hline KT1-HN & quenched & 3 & 25 & S31 & 7.31 & 11.8 & 89.4 & 80.4 & 12.18 & 19.67 & 149.00 & 134.00 \\
\hline KT1-LA & $\mathrm{ccc}$ & 3 & 26 & S40 & 7.38 & 12.8 & 67.0 & 79.3 & 12.30 & 21.33 & 111.67 & 132.17 \\
\hline KT1-HN & $\mathrm{ccc}$ & 3 & 27 & S11 & 7.21 & 12.7 & 83.1 & 80.6 & 12.02 & 21.17 & 138.50 & 134.34 \\
\hline KT1-LL & quenched & 3 & 28 & S29 & 6.24 & 11.1 & 61.4 & 70.7 & 10.40 & 18.50 & 102.34 & 117.84 \\
\hline KT1-HL & quenched & 3 & 29 & S17 & 9.87 & 27.5 & 96.4 & 107 & 16.45 & 45.83 & 160.67 & 178.34 \\
\hline KT1-LS & $\mathrm{ccc}$ & 3 & 30 & S43 & 6.20 & 11.4 & 57.5 & 68.9 & 10.33 & 19.00 & 95.84 & 114.84 \\
\hline KT1-LB & quenched & 3 & 31 & S73 & 6.58 & 11.2 & 63.6 & 71.6 & 10.97 & 18.67 & 106.00 & 119.34 \\
\hline Soln Std & ref & 3 & 32 & std-13-3 & 20.4 & 10.3 & 85.4 & 48.9 & 20.40 & 10.30 & 85.40 & 48.90 \\
\hline
\end{tabular}


Table D-2. Normalized PCT Results for the KT01-Series Glasses by Heat Treatment by Compositional View.

\begin{tabular}{|c|c|c|c|c|c|c|c|c|c|c|}
\hline $\begin{array}{c}\text { Glass } \\
\text { ID }\end{array}$ & $\begin{array}{c}\text { Heat } \\
\text { Treatment }\end{array}$ & $\begin{array}{l}\text { Comp } \\
\text { View }\end{array}$ & $\begin{array}{c}\log N L[B \\
(g / L)]\end{array}$ & $\begin{array}{c}\log N L[L i \\
(g / L)]\end{array}$ & $\begin{array}{c}\log \mathrm{NL}[\mathrm{Na} \\
(\mathrm{g} / \mathrm{L})]\end{array}$ & $\begin{array}{c}\log N L[S i \\
(g / L)]\end{array}$ & $\begin{array}{c}\mathrm{NL} \\
\mathrm{B}(\mathrm{g} / \mathrm{L})\end{array}$ & $\begin{array}{c}\mathrm{NL} \\
\mathrm{Li}(\mathrm{g} / \mathrm{L})\end{array}$ & $\begin{array}{c}\mathrm{NL} \\
\mathrm{Na}(\mathrm{g} / \mathrm{L})\end{array}$ & $\begin{array}{c}\mathrm{NL} \\
\mathrm{Si}(\mathrm{g} / \mathrm{L})\end{array}$ \\
\hline ARM & ref & reference & -0.3215 & -0.2482 & -0.3188 & -0.5988 & 0.477 & 0.565 & 0.480 & 0.252 \\
\hline EA & ref & reference & 1.2746 & 1.0094 & 1.1515 & 0.5836 & 18.821 & 10.220 & 14.175 & 3.834 \\
\hline KT1-HA & $\mathrm{ccc}$ & measured & -0.2102 & -0.1222 & -0.0666 & -0.3238 & 0.616 & 0.755 & 0.858 & 0.475 \\
\hline KT1-HB & ccc & measured & -0.1384 & -0.0852 & -0.0903 & -0.3083 & 0.727 & 0.822 & 0.812 & 0.492 \\
\hline KT1-HF & $\mathrm{ccc}$ & measured & -0.1588 & -0.0831 & -0.0108 & -0.2499 & 0.694 & 0.826 & 0.976 & 0.563 \\
\hline KT1-HK & $\mathrm{ccc}$ & measured & -0.2125 & 0.1465 & 0.1585 & -0.3088 & 0.613 & 1.401 & 1.440 & 0.491 \\
\hline KT1-HL & ccc & measured & 0.1657 & 0.4669 & 0.2391 & 0.1791 & 1.465 & 2.930 & 1.734 & 1.510 \\
\hline KT1-HN & $\mathrm{ccc}$ & measured & -0.0694 & 0.0098 & 0.0750 & -0.1891 & 0.852 & 1.023 & 1.188 & 0.647 \\
\hline KT1-HS & $\mathrm{ccc}$ & measured & -0.1875 & -0.0830 & -0.0928 & -0.2874 & 0.649 & 0.826 & 0.808 & 0.516 \\
\hline KT1-LA & $\mathrm{ccc}$ & measured & -0.0534 & 0.0050 & 0.0085 & -0.2101 & 0.884 & 1.012 & 1.020 & 0.616 \\
\hline KT1-LB & $\mathrm{ccc}$ & measured & -0.1256 & -0.0339 & -0.0235 & -0.2526 & 0.749 & 0.925 & 0.947 & 0.559 \\
\hline KT1-LF & $\mathrm{ccc}$ & measured & -0.1779 & -0.0734 & -0.0253 & -0.2678 & 0.664 & 0.844 & 0.943 & 0.540 \\
\hline KT1-LL & ccc & measured & -0.1498 & -0.0669 & -0.0474 & -0.2705 & 0.708 & 0.857 & 0.897 & 0.536 \\
\hline KT1-LN & ccc & measured & -0.1959 & -0.0971 & -0.1301 & -0.3195 & 0.637 & 0.800 & 0.741 & 0.479 \\
\hline KT1-LS & $\mathrm{ccc}$ & measured & -0.1405 & -0.0524 & -0.0479 & -0.2667 & 0.724 & 0.886 & 0.896 & 0.541 \\
\hline KT1-HA & quenched & measured & -0.2056 & -0.1403 & -0.0338 & -0.3228 & 0.623 & 0.724 & 0.925 & 0.476 \\
\hline KT1-HB & quenched & measured & -0.1597 & -0.1181 & -0.0887 & -0.3213 & 0.692 & 0.762 & 0.815 & 0.477 \\
\hline KT1-HF & quenched & measured & -0.0735 & -0.0318 & -0.0085 & -0.2551 & 0.844 & 0.929 & 0.981 & 0.556 \\
\hline KT1-HK & quenched & measured & -0.1697 & 0.1749 & 0.2049 & -0.3538 & 0.677 & 1.496 & 1.603 & 0.443 \\
\hline KT1-HL & quenched & measured & 0.0728 & 0.1527 & 0.2049 & -0.0535 & 1.182 & 1.421 & 1.603 & 0.884 \\
\hline KT1-HN & quenched & measured & -0.0627 & -0.0221 & 0.1066 & -0.1907 & 0.866 & 0.950 & 1.278 & 0.645 \\
\hline KT1-HS & quenched & measured & -0.1926 & -0.0878 & -0.0667 & -0.2872 & 0.642 & 0.817 & 0.858 & 0.516 \\
\hline KT1-LA & quenched & measured & -0.0549 & -0.0006 & 0.0489 & -0.1971 & 0.881 & 0.999 & 1.119 & 0.635 \\
\hline KT1-LB & quenched & measured & -0.1262 & -0.0620 & -0.0004 & -0.2595 & 0.748 & 0.867 & 0.999 & 0.550 \\
\hline KT1-LF & quenched & measured & -0.1811 & -0.0943 & 0.0093 & -0.2653 & 0.659 & 0.805 & 1.022 & 0.543 \\
\hline KT1-LL & quenched & measured & -0.1397 & -0.0681 & 0.0004 & -0.2504 & 0.725 & 0.855 & 1.001 & 0.562 \\
\hline KT1-LN & quenched & measured & -0.1870 & -0.1013 & -0.1016 & -0.3023 & 0.650 & 0.792 & 0.791 & 0.499 \\
\hline KT1-LS & quenched & measured & -0.1263 & -0.0760 & -0.0202 & -0.2665 & 0.748 & 0.840 & 0.955 & 0.541 \\
\hline KT1-HA & $\mathrm{ccc}$ & targeted & -0.1609 & -0.0931 & -0.0382 & -0.2923 & 0.690 & 0.807 & 0.916 & 0.510 \\
\hline KT1-HB & $\mathrm{ccc}$ & targeted & -0.1395 & -0.0975 & -0.1033 & -0.3155 & 0.725 & 0.799 & 0.788 & 0.484 \\
\hline KT1-HF & $\mathrm{ccc}$ & targeted & -0.1526 & -0.0939 & -0.0270 & -0.2520 & 0.704 & 0.806 & 0.940 & 0.560 \\
\hline KT1-HK & $\mathrm{ccc}$ & targeted & -0.1943 & 0.1802 & 0.1618 & -0.3111 & 0.639 & 1.514 & 1.451 & 0.489 \\
\hline KT1-HL & $\mathrm{ccc}$ & targeted & 0.1764 & 0.4605 & 0.2272 & 0.1718 & 1.501 & 2.887 & 1.687 & 1.485 \\
\hline KT1-HN & $\mathrm{ccc}$ & targeted & -0.0601 & -0.0001 & 0.0605 & -0.1943 & 0.871 & 1.000 & 1.150 & 0.639 \\
\hline KT1-HS & $\mathrm{ccc}$ & targeted & -0.1771 & -0.0862 & -0.1045 & -0.2924 & 0.665 & 0.820 & 0.786 & 0.510 \\
\hline KT1-LA & $\mathrm{ccc}$ & targeted & -0.0601 & -0.0113 & 0.0075 & -0.2178 & 0.871 & 0.974 & 1.017 & 0.606 \\
\hline KT1-LB & $\mathrm{ccc}$ & targeted & -0.1178 & -0.0413 & -0.0321 & -0.2555 & 0.762 & 0.909 & 0.929 & 0.555 \\
\hline KT1-LF & $\mathrm{ccc}$ & targeted & -0.1688 & -0.0766 & -0.0378 & -0.2690 & 0.678 & 0.838 & 0.917 & 0.538 \\
\hline KT1-LL & $\mathrm{ccc}$ & targeted & -0.1359 & -0.0746 & -0.0612 & -0.2785 & 0.731 & 0.842 & 0.869 & 0.527 \\
\hline KT1-LN & $\mathrm{ccc}$ & targeted & -0.1913 & -0.1066 & -0.1453 & -0.3226 & 0.644 & 0.782 & 0.716 & 0.476 \\
\hline KT1-LS & ccc & targeted & -0.1381 & -0.0616 & -0.0550 & -0.2714 & 0.728 & 0.868 & 0.881 & 0.535 \\
\hline KT1-HA & quenched & targeted & -0.1563 & -0.1112 & -0.0053 & -0.2913 & 0.698 & 0.774 & 0.988 & 0.511 \\
\hline KT1-HB & quenched & targeted & -0.1609 & -0.1303 & -0.1017 & -0.3286 & 0.690 & 0.741 & 0.791 & 0.469 \\
\hline KT1-HF & quenched & targeted & -0.0673 & -0.0426 & -0.0247 & -0.2572 & 0.856 & 0.907 & 0.945 & 0.553 \\
\hline KT1-HK & quenched & targeted & -0.1516 & 0.2086 & 0.2082 & -0.3561 & 0.705 & 1.617 & 1.615 & 0.441 \\
\hline KT1-HL & quenched & targeted & 0.0834 & 0.1462 & 0.1930 & -0.0607 & 1.212 & 1.400 & 1.559 & 0.870 \\
\hline KT1-HN & quenched & targeted & -0.0534 & -0.0319 & 0.0921 & -0.1959 & 0.884 & 0.929 & 1.236 & 0.637 \\
\hline KT1-HS & quenched & targeted & -0.1822 & -0.0910 & -0.0784 & -0.2922 & 0.657 & 0.811 & 0.835 & 0.510 \\
\hline KT1-LA & quenched & targeted & -0.0616 & -0.0169 & 0.0479 & -0.2048 & 0.868 & 0.962 & 1.117 & 0.624 \\
\hline KT1-LB & quenched & targeted & -0.1184 & -0.0694 & -0.0090 & -0.2624 & 0.761 & 0.852 & 0.979 & 0.547 \\
\hline KT1-LF & quenched & targeted & -0.1720 & -0.0975 & -0.0032 & -0.2665 & 0.673 & 0.799 & 0.993 & 0.541 \\
\hline KT1-LL & quenched & targeted & -0.1258 & -0.0759 & -0.0134 & -0.2583 & 0.749 & 0.840 & 0.970 & 0.552 \\
\hline KT1-LN & quenched & targeted & -0.1823 & -0.1108 & -0.1168 & -0.3054 & 0.657 & 0.775 & 0.764 & 0.495 \\
\hline KT1-LS & quenched & targeted & -0.1240 & -0.0851 & -0.0273 & -0.2712 & 0.752 & 0.822 & 0.939 & 0.536 \\
\hline
\end{tabular}


Exhibit D-1. KT01 PCT Measurements (ppm values) in Analytical Sequence by Element.

Variability Chart for B (ppm)

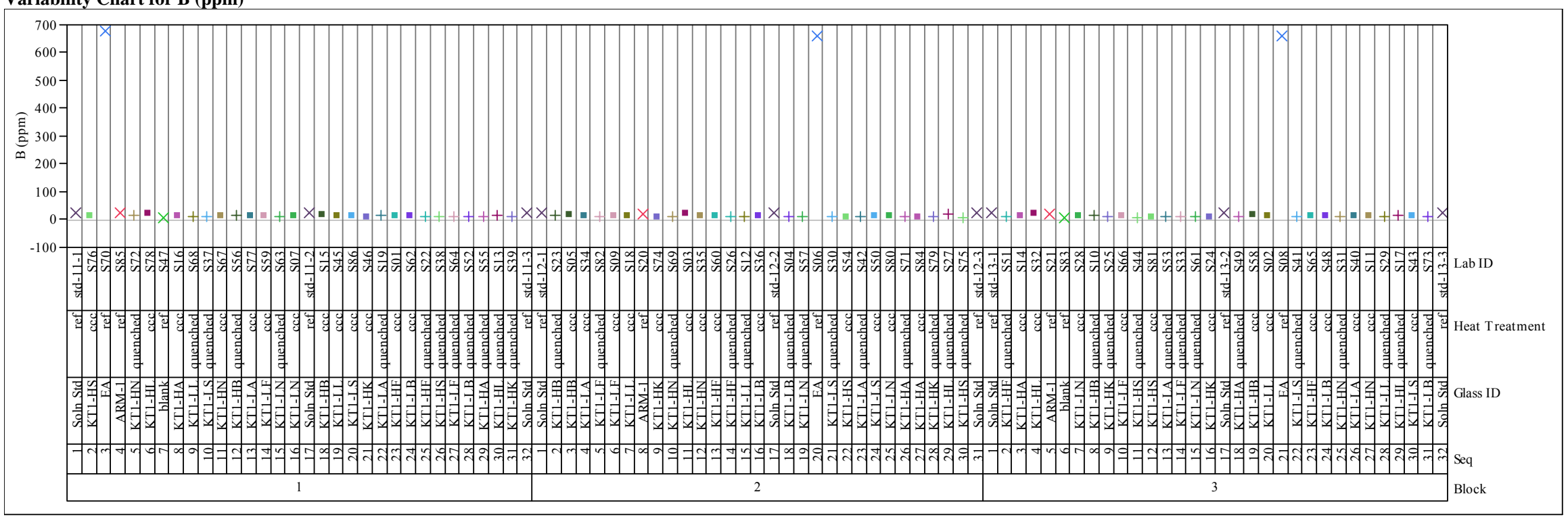


Exhibit D-1. KT01 PCT Measurements (ppm values) in Analytical Sequence by Element. (continued)

Variability Chart for Li (ppm)

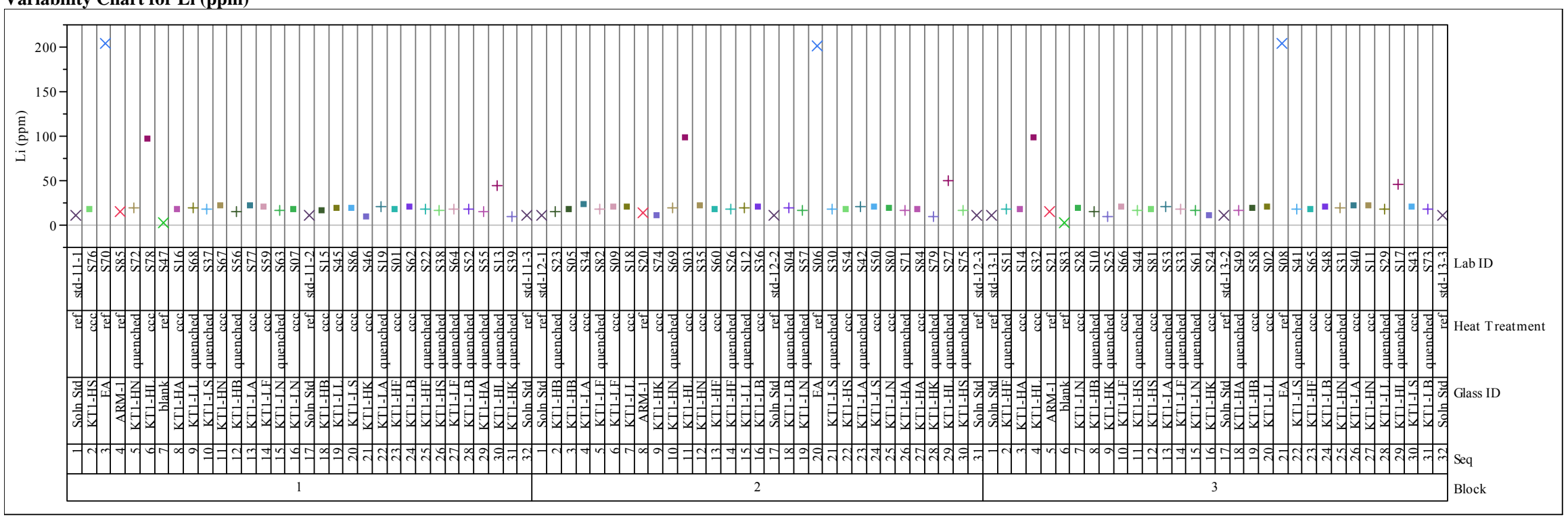


Exhibit D-1. KT01 PCT Measurements (ppm values) in Analytical Sequence by Element. (continued)

Variability Chart for Na (ppm)

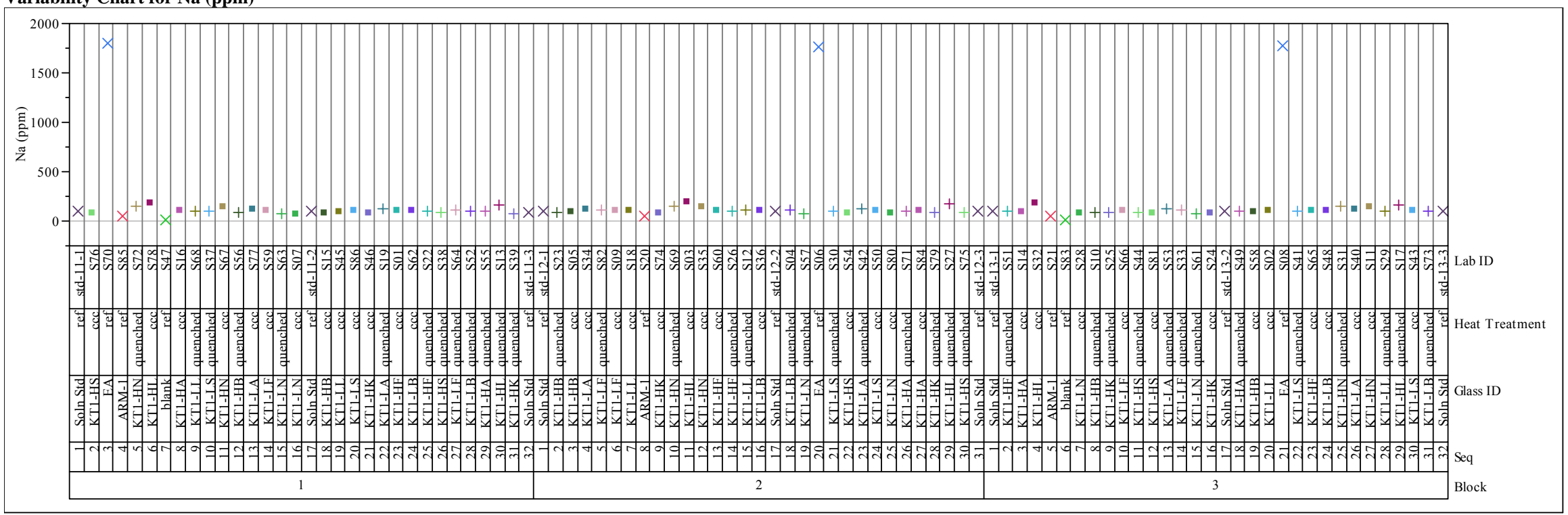




\section{Exhibit D-1. KT01 PCT Measurements (ppm values) in Analytical Sequence by Element. (continued)}

Variability Chart for Si (ppm)

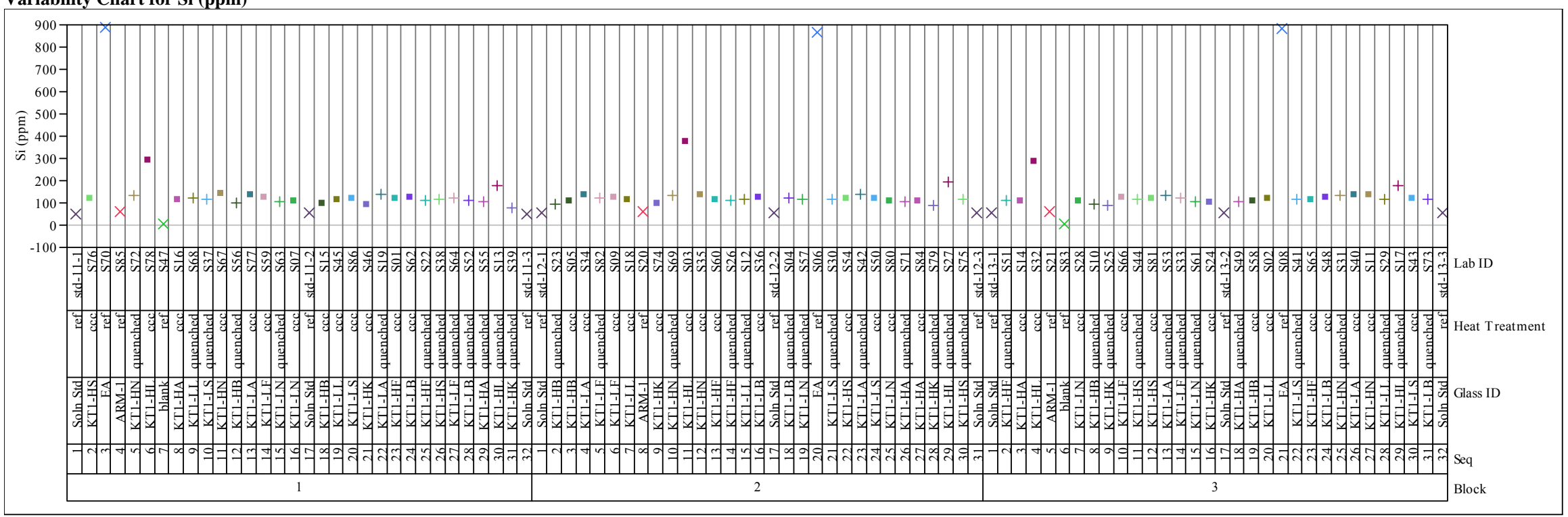


Exhibit D-2. Statistical Evaluation of the ICP-AES Calibration Effects from the KT01 Multi-Element Standard Solution Results by Oxide.

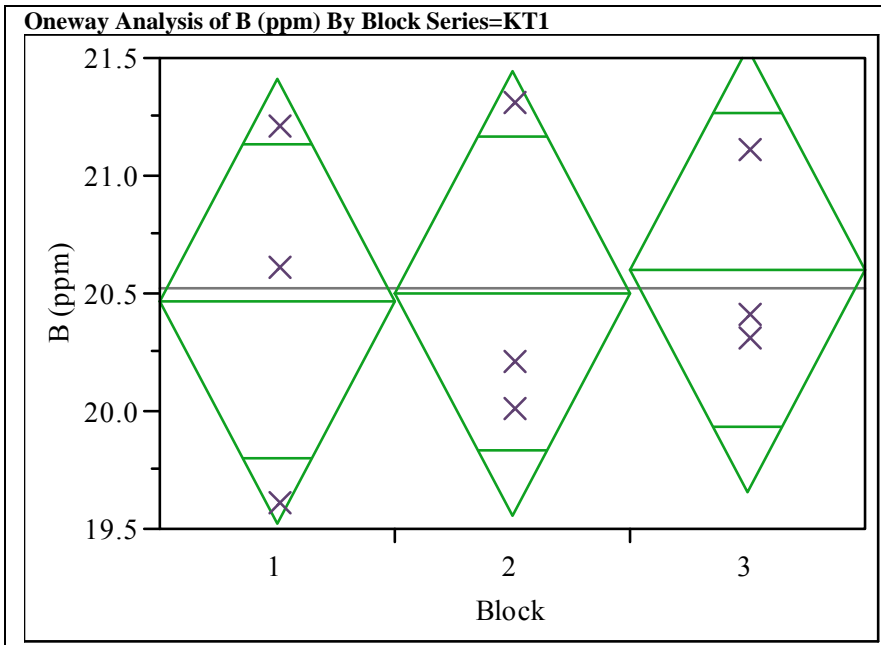

\section{Oneway Anova}

Summary of Fit

$$
\begin{array}{lr}
\text { Rsquare } & 0.010717 \\
\text { Adj Rsquare } & -0.31904 \\
\text { Root Mean Square Error } & 0.666667 \\
\text { Mean of Response } & 20.52222 \\
\text { Observations (or Sum Wgts) } & 9 \\
& \\
\text { Analysis of Variance } &
\end{array}
$$

Source DF Sum of Squares Mean Square F Ratio Prob $>$ F

$\begin{array}{lrrrrr}\text { Block } & 2 & 0.0288889 & 0.014444 & 0.0325 & 0.9682\end{array}$

$\begin{array}{llll}\text { Elock } & 2 & 0.0288889 & 0.014444 \\ \text { Error } & 6 & 2.6666667 & 0.444444\end{array}$

C. Total $8 \quad 2.6955556$

Means for Oneway Anova

Level Number Mean Std Error Lower 95\% Upper 95\%

$\begin{array}{lrrrrr}1 & 3 & 20.4667 & 0.38490 & 19.525 & 21.408 \\ 2 & 3 & 20.5000 & 0.38490 & 19.558 & 21.442\end{array}$

$\begin{array}{llllll}1 & 3 & 20.4667 & 0.38490 & 19.525 & 21.408 \\ 2 & 3 & 20.5000 & 0.38490 & 19.558 & 21.442 \\ 3 & 3 & 20.6000 & 0.38490 & 19.658 & 21.542\end{array}$

Std Error uses a pooled estimate of error variance

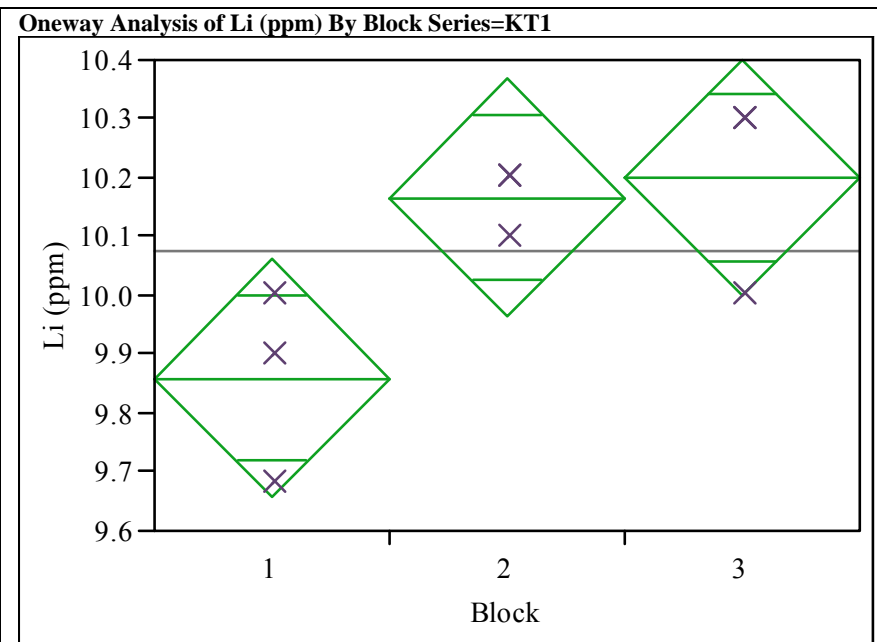

\section{Oneway Anova}

Summary of Fit

$\begin{array}{ll}\text { Rsquare } & 0.636681 \\ \text { Adj Rsquare } & 0.515575\end{array}$

0.536815
Adj Rsquare
Root Mean Square Error $\quad 0.141578$

Root Mean Square Error $\quad 0.141578$

Observations (or Sum Wgts) 10.07556

Analysis of Variance

Source DF Sum of Squares Mean Square F Ratio Prob $>$ F

$\begin{array}{lrrrrr}\text { Block } & 2 & 0.21075556 & 0.105378 & 5.2572 & 0.0480\end{array}$

$\begin{array}{llll}\text { Error } & 6 & 0.12026667 & 0.020044\end{array}$

$\begin{array}{lll}\text { C. Total } & 8 & 0.33102222\end{array}$

Means for Oneway Anova

Level Number Mean Std Error Lower 95\% Upper 95\%

$\begin{array}{lrrrrr} & & & & & \\ 1 & 3 & 9.8600 & 0.08174 & 9.660 & 10.060 \\ 3 & 3 & 10.1667 & 0.08174 & 9.967 & 10.367\end{array}$

$\begin{array}{rrrrrr}2 & 3 & 10.1667 & 0.08174 & 9.967 & 10.367 \\ 3 & 3 & 10.2000 & 0.08174 & 10.000 & 10.400\end{array}$

Std Error uses a pooled estimate of error variance 
Exhibit D-2. Statistical Evaluation of the ICP-AES Calibration Effects from the KT01 Multi-Element Standard Solution Results by Oxide. (continued)

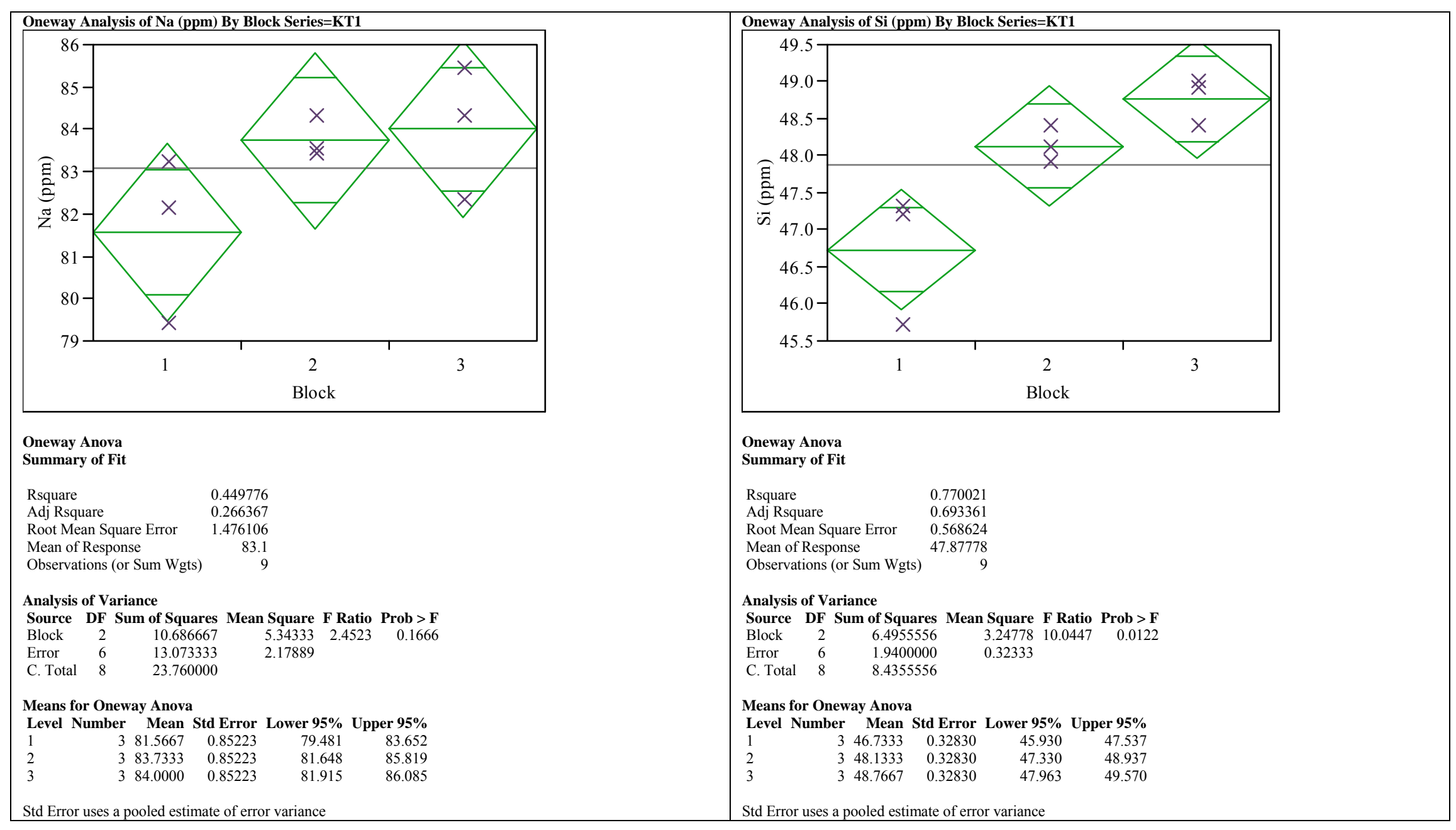




\section{Exhibit D-3. KT01 PCT Results (as common logarithms) Grouped by Glass ID and Heat Treatment.}

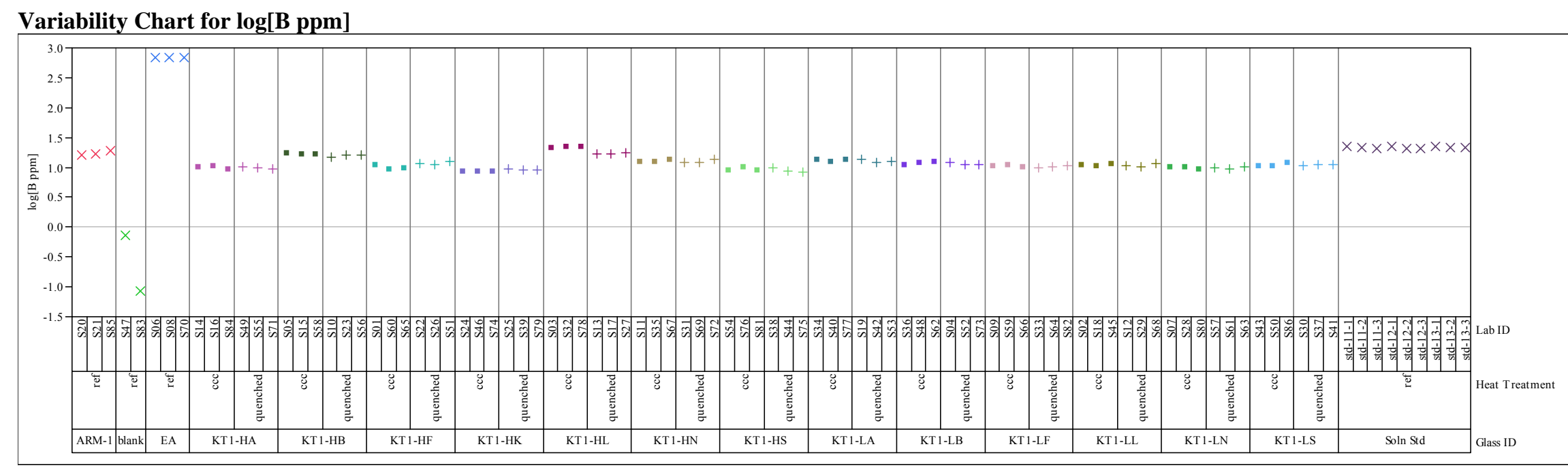

\section{Variability Chart for log[Li ppm]}

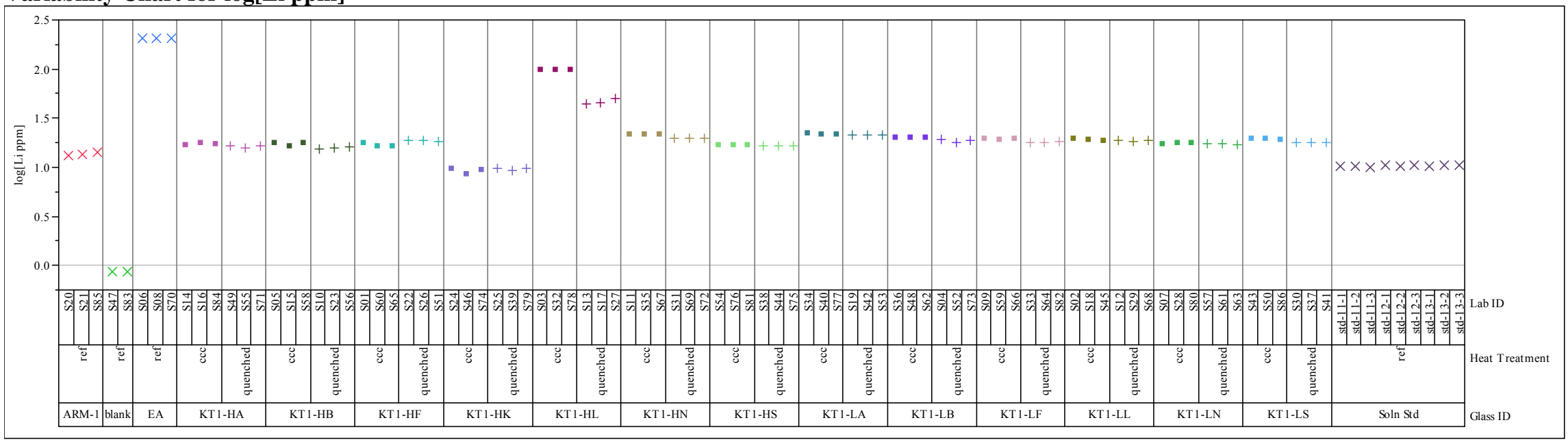


Exhibit D-3. KT01 PCT Results (as common logarithms) Grouped by Glass ID and Heat Treatment. (continued) Variability Chart for log[Na ppm]

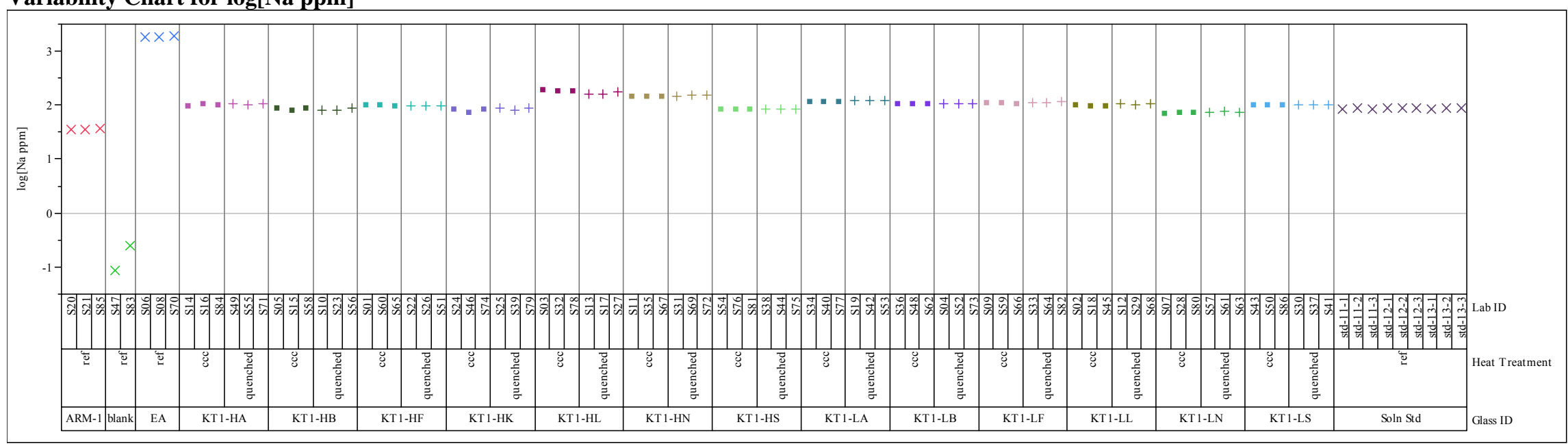

\section{Variability Chart for log[Si ppm]}

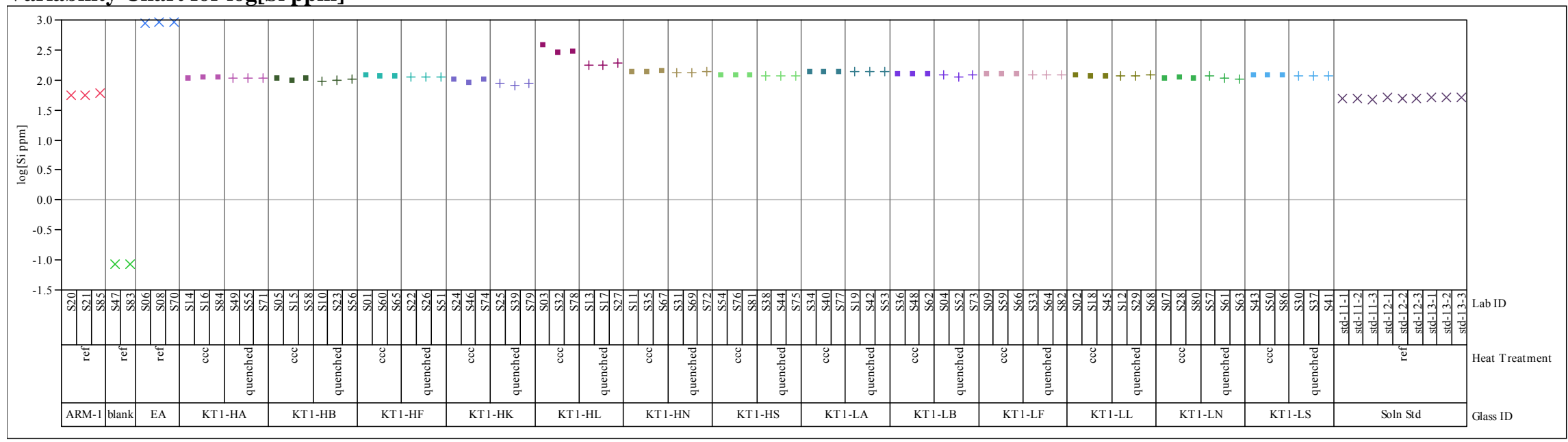


Exhibit D-4. Correlations and Scatter Plots of the KT01 Normalized PCTs Over All Compositional Views and Heat Treatments.

\section{Multivariate Correlations}

$\begin{array}{ccccc} & \log \text { NL[B (g/L)] } & \log \text { NL[Li(g/L)] } & \log \text { NL[Na (g/L)] } & \log \text { NL[Si (g/L)] } \\ \log \text { NL[B (g/L)] } & 1.0000 & 0.8503 & 0.8862 & 0.9149 \\ \log \text { NL[Li(g/L)] } & 0.8503 & 1.0000 & 0.9259 & 0.8632 \\ \log \text { NL[Na (g/L)] } & 0.8862 & 0.9259 & 1.0000 & 0.8247 \\ \log \text { NL[Si (g/L)] } & 0.9149 & 0.8632 & 0.8247 & 1.0000\end{array}$

\section{Scatterplot Matrix}

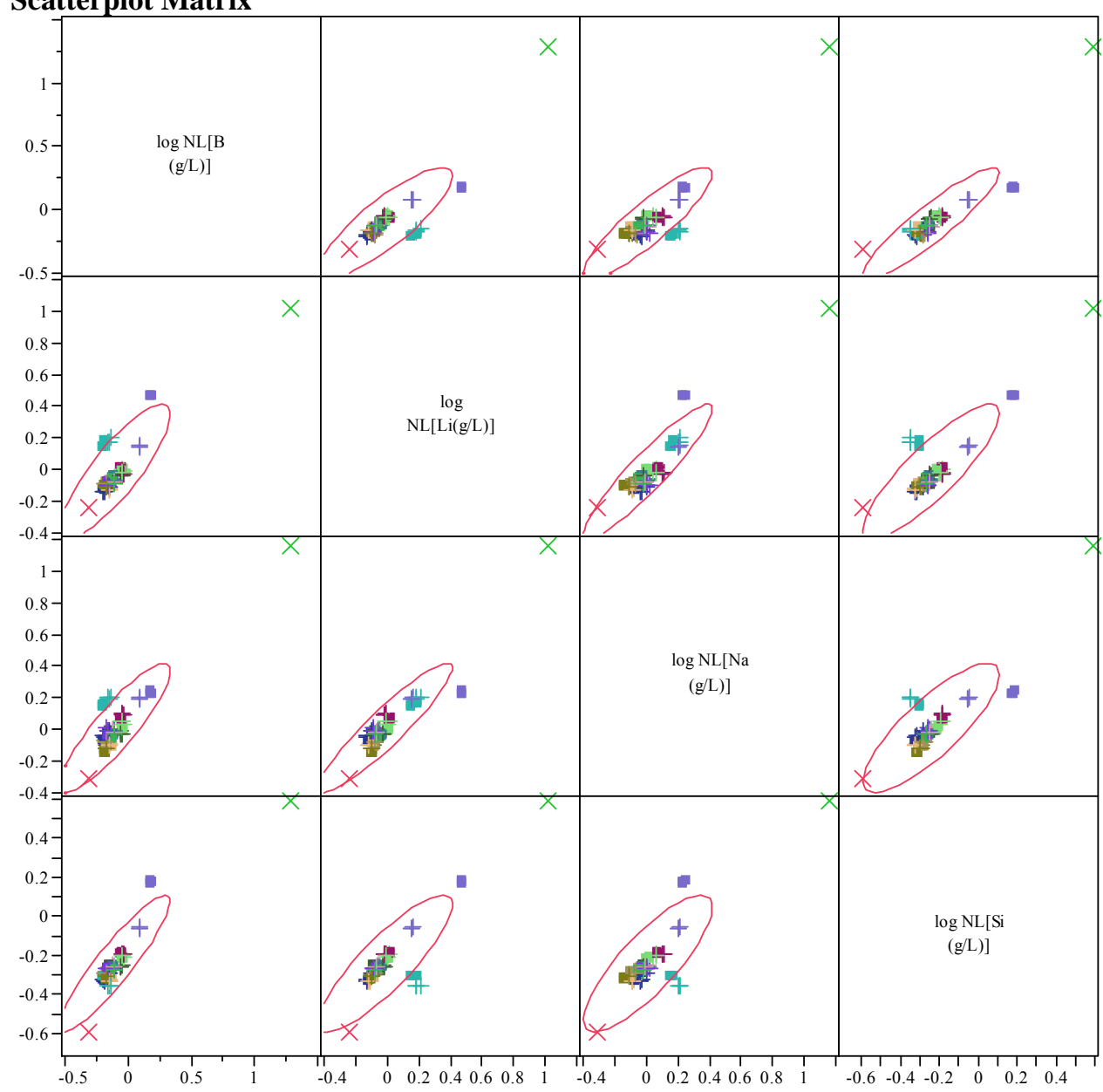


Exhibit D-5. Effects of Heat Treatment for the KT01 Glasses by Compositional View.

Variability Chart for $\log$ NL[B $(\mathrm{g} / \mathrm{L})]$

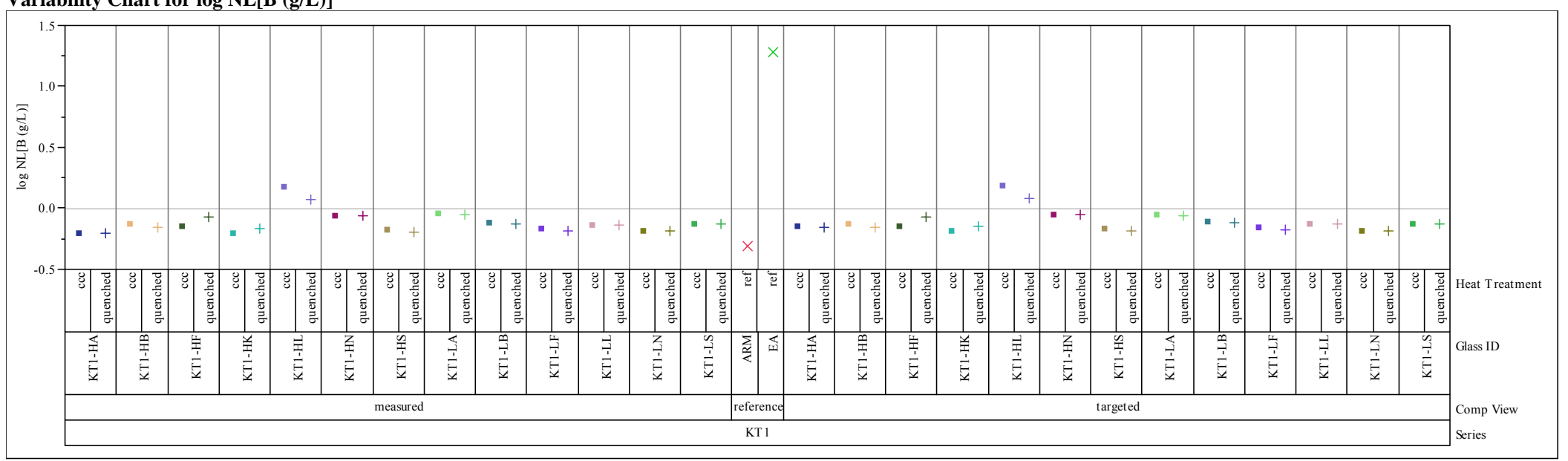

Variability Chart for $\log N L[L i(g / L)]$

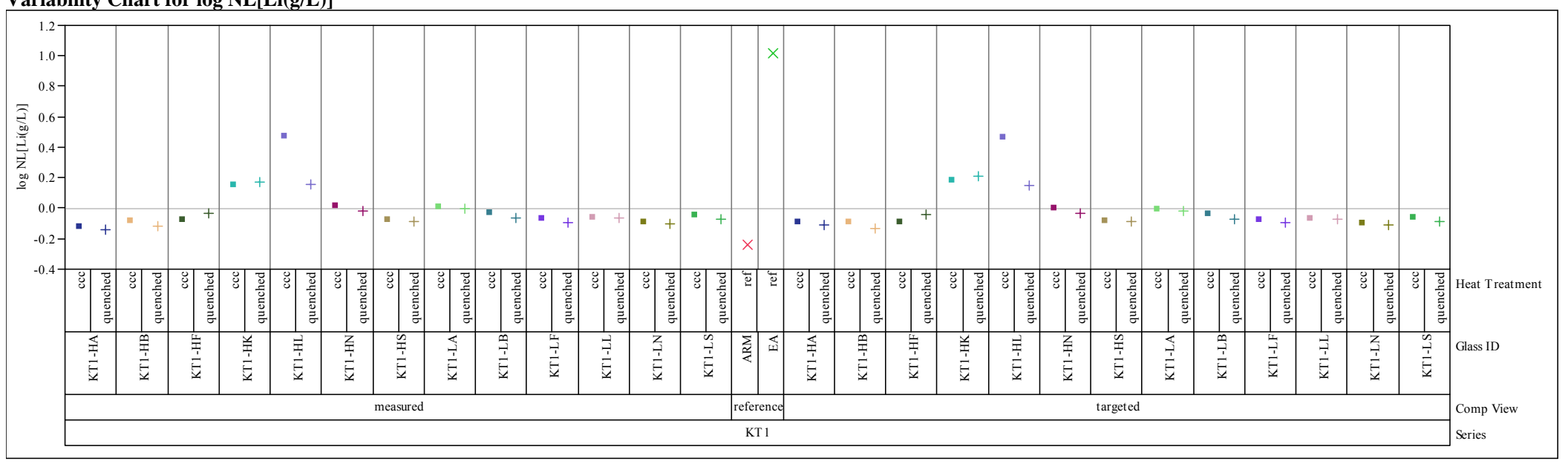




\section{Exhibit D-5. Effects of Heat Treatment for the KT01 Glasses by Compositional View. (continued)}

Variability Chart for log NL[Na (g/L)]

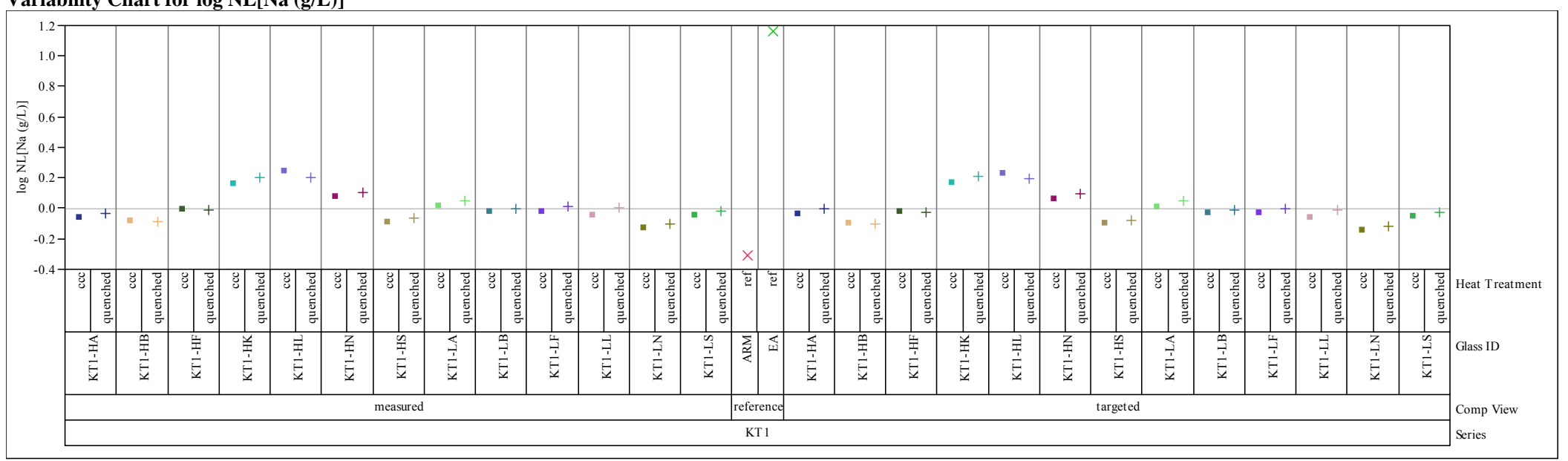

\section{Variability Chart for $\log \mathrm{NL}[\mathrm{Si}(\mathrm{g} / \mathrm{L})]$}

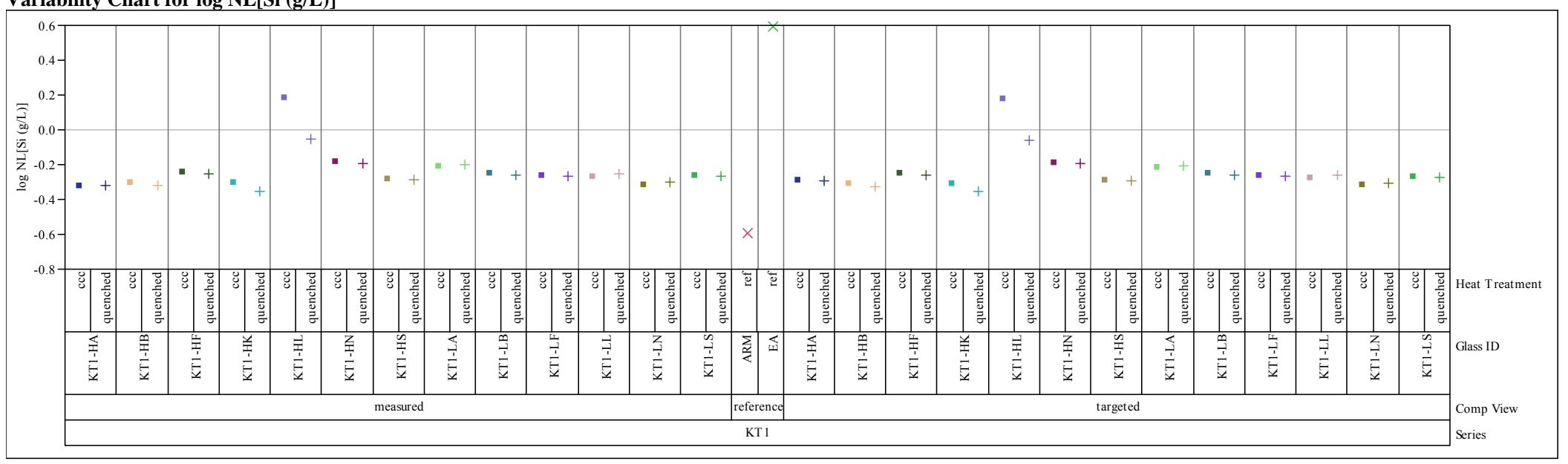


Exhibit D-6. KT01-Series PCT Measurements versus Durability Model Predictions.
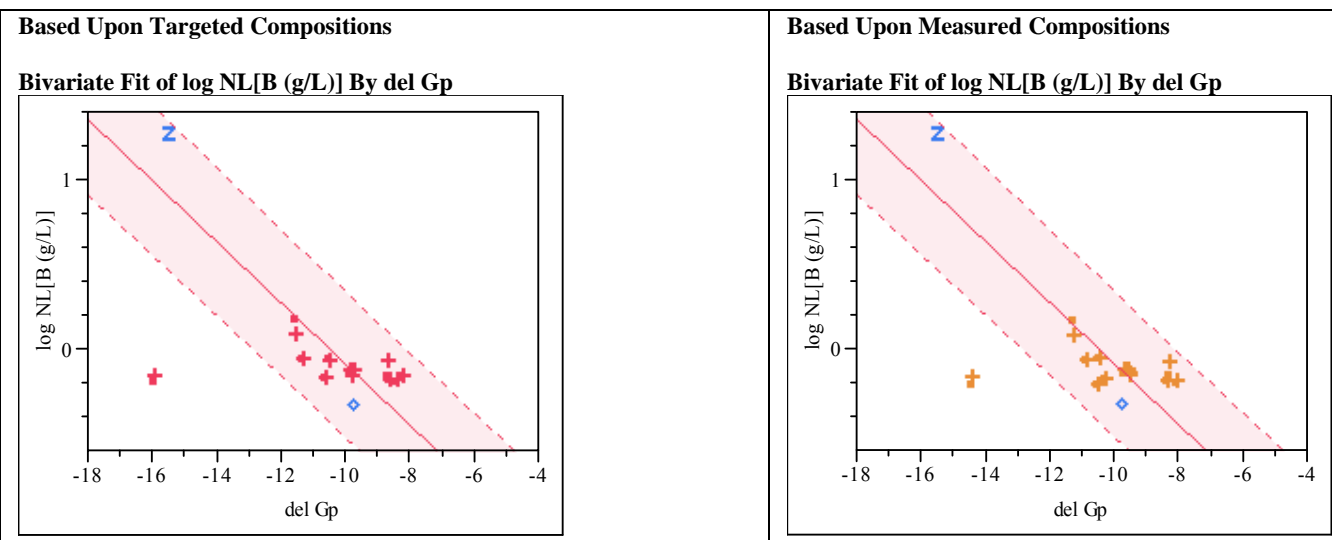

Bivariate Fit of $\log \mathrm{NL}[\mathrm{Li}$ (g/L)] By del Gp

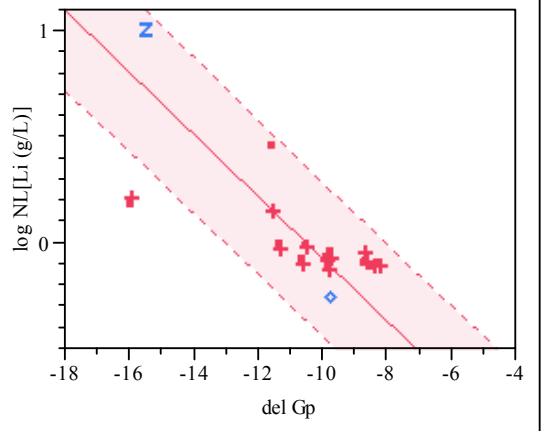

Bivariate Fit of log NL[Li (g/L)] By del Gp

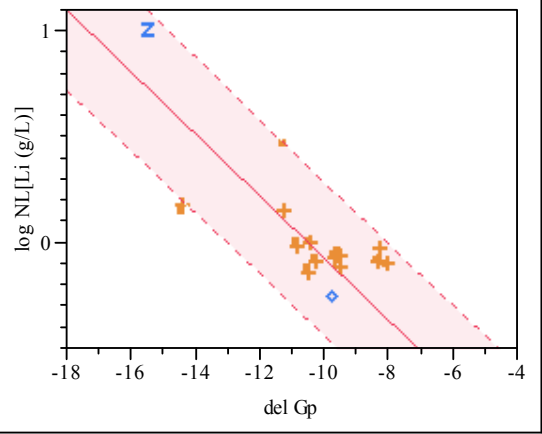

Bivariate Fit of $\log \mathrm{NL}[\mathrm{Na}(\mathrm{g} / \mathrm{L})]$ By del Gp

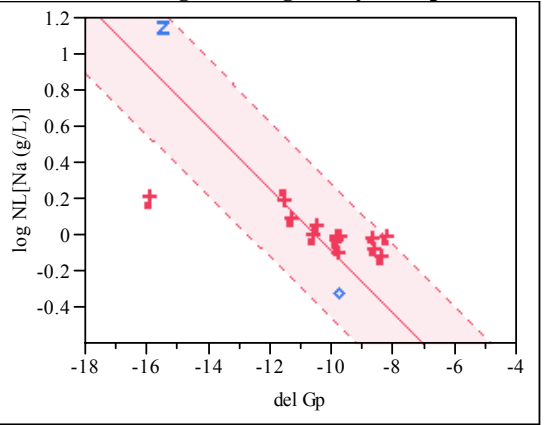

Bivariate Fit of $\log$ NL[Na (g/L)] By del Gp

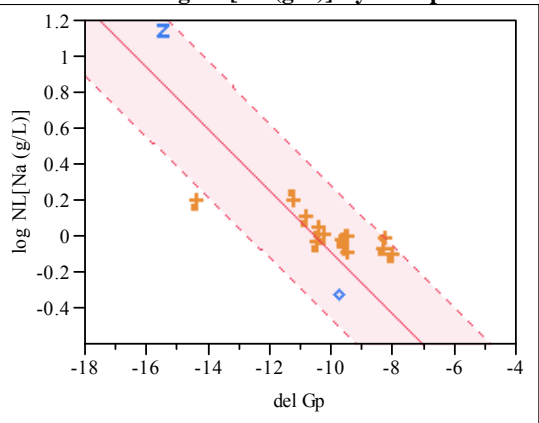

Bivariate Fit of log NL[Si (g/L)] By del Gp

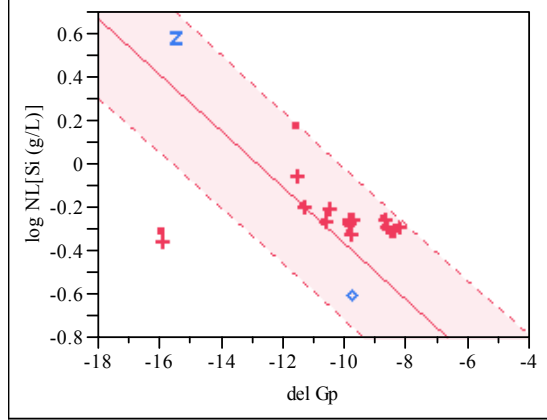

Bivariate Fit of $\log$ NL[Si (g/L)] By del Gp

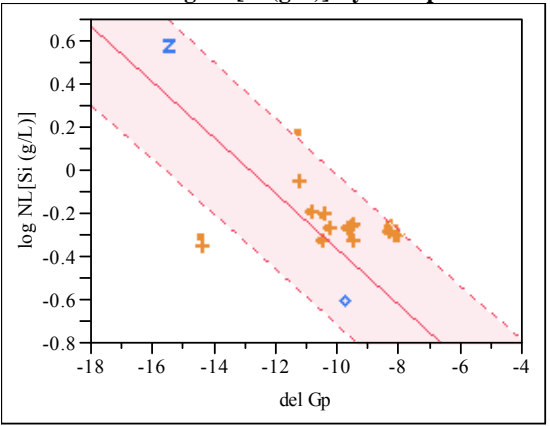

z Environmental Assessment Benchmark Glass

+ + Quenched Study Glasses

$\diamond$ Approved Reference Material Glass

- Slowly Cooled Study Glasses 
SRNL-STI-2010-00566

Revision 0

Appendix E. Data Supporting the PCT Measurements of the KT03-Series Glasses 
Table E-1. Measurements of the PCT Solutions for the KT03-Series Glasses As-Received (ar) and After Appropriate Adjustments (ppm).

\begin{tabular}{|c|c|c|c|c|c|c|c|c|c|c|c|c|}
\hline ID & \begin{tabular}{|c|} 
Heat \\
Treatment
\end{tabular} & Block & Seq & D & B ar & Li ar & Na ar & Si ar & pm) & pm) & pm) & Si (ppm) \\
\hline Soln Std & ref & 1 & 1 & STD-11 & 20.4 & 10.2 & 85.0 & 50.3 & 20.40 & 10.20 & 85.00 & 50.30 \\
\hline KT03-HS & $\mathrm{ccc}$ & 1 & 2 & V76 & 3.32 & 7.48 & 29.2 & 54.7 & 5.53 & 12.47 & 48.67 & 91.17 \\
\hline EA & ref & 1 & 3 & 70 & 35.0 & 11.4 & 93.7 & 54.0 & 583.33 & 190.00 & 1561.67 & 900.00 \\
\hline KT03-HN & quenched & 1 & 4 & V72 & 6.06 & \begin{tabular}{|l|}
9.48 \\
\end{tabular} & 78.4 & 66.7 & 10.10 & 15.80 & 130.67 & 111.17 \\
\hline KT03-HL & $\mathrm{ccc}$ & 1 & 5 & V78 & 6.98 & \begin{tabular}{|l|}
48.9 \\
\end{tabular} & 76.9 & 146 & 11.63 & 1.50 & 128.17 & 243.34 \\
\hline blank & ref & 1 & 6 & V47 & $<0.100$ & $<1.00$ & $<0.100$ & $<0.100$ & 0.08 & 0.83 & 0.08 & 0.08 \\
\hline KT1-HA2 & $\mathrm{ccc}$ & 1 & 7 & V16 & 5.03 & \begin{tabular}{|l|}
9.39 \\
\end{tabular} & $\begin{array}{l}51.1 \\
\end{array}$ & 63.6 & .38 & 15.65 & 5.17 & 106.00 \\
\hline KT03-LL & uenched & 1 & 8 & V68 & 76 & 11.4 & 54.7 & 64.4 & .60 & 9.00 & .17 & 107.34 \\
\hline KT03-LS & quenched & 1 & 9 & V37 & 50 & 8.44 & 39.69 & 55.7 & 7.50 & 4.07 & 66.15 & 92.84 \\
\hline KT03-HN & $\mathrm{ccc}$ & 1 & 10 & V67 & 95 & 10.3 & 71.3 & 66.4 & 9.92 & 7.17 & 118.84 & 110.67 \\
\hline -HB & denched & 1 & 11 & V56 & 93 & \begin{tabular}{|l|}
9.43 \\
\end{tabular} & 43.9 & 55.6 & 14.88 & 5.72 & 17 & 92.67 \\
\hline LA & $\mathrm{ccc}$ & 1 & 12 & V77 & 13 & \begin{tabular}{|l|}
10.8 \\
\end{tabular} & 51.4 & 64.9 & 10.22 & 3.00 & .67 & 108.17 \\
\hline LF & ccc & 1 & 13 & V59 & 46 & \begin{tabular}{|l|}
10.8 \\
\end{tabular} & 52.9 & 65.2 & 9.10 & .00 & .17 & 108.67 \\
\hline $\mathrm{LN}$ & enches & 1 & 14 & V63 & 18 & \begin{tabular}{|l|}
10.6 \\
\end{tabular} & 46.7 & 62.6 & 10.30 & .67 & 33 & 4.34 \\
\hline $\mathrm{LN}$ & $\mathrm{ccc}$ & 1 & 15 & V07 & 12 & \begin{tabular}{|l|}
10.2 \\
\end{tabular} & 42.0 & 59.1 & 8.53 & .00 & 0 & .50 \\
\hline MK & ccc & 1 & 16 & V87 & 05 & \begin{tabular}{|l|}
6.64 \\
\end{tabular} & 34.0 & 46.7 & 5 & 07 & & 7.83 \\
\hline $\mathrm{HA}$ & ienchec & 1 & 17 & V93 & 16 & 22 & 38.3 & 48.7 & 3 & .70 & 33 & .17 \\
\hline Std & ref & 1 & 18 & STD-12 & 19.9 & 9.90 & 78.6 & 49.5 & 9.90 & 90 & 50 & 9.50 \\
\hline -HB & $\mathrm{ccc}$ & 1 & 19 & V15 & 88 & \begin{tabular}{|l|}
9.40 \\
\end{tabular} & 41.0 & 54.6 & 14.80 & 5.67 & & 1.00 \\
\hline MK & enchec & 1 & 20 & V88 & 90 & 74 & 34.9 & 44.3 & & 23 & & .83 \\
\hline A-1 & & 1 & 21 & V92 & 70 & \begin{tabular}{|l|}
7.99 \\
\end{tabular} & 19.9 & 35.5 & 16.17 & .32 & 17 & 9.17 \\
\hline $\mathrm{LL}$ & $\mathrm{coc}-\mathrm{c}^{2}$ & 1 & 22 & V45 & 37 & \begin{tabular}{|l|}
10.8 \\
\end{tabular} & 46.9 & 59.3 & 8.95 & 3.00 & & 8.84 \\
\hline $\mathrm{LS}$ & $\mathrm{cc}$ & 1 & 23 & V86 & 54 & 5 & 38.9 & 57.7 & 7 & 4.92 & & 6.17 \\
\hline $\mathrm{HK}$ & $\mathrm{ccc}$ & 1 & 24 & V46 & 41 & 6 & 41.5 & 49.6 & 5 & 60 & 17 & 82.67 \\
\hline LA & uenche & 1 & 25 & V19 & 94 & \begin{tabular}{|l|}
10.4 \\
\end{tabular} & 53.8 & 64.9 & .90 & 7.33 & 67 & 108.17 \\
\hline $\mathrm{HF}$ & & 1 & 26 & V01 & 78 & \begin{tabular}{|l|}
9.38 \\
\end{tabular} & 45.8 & 58.8 & 7.97 & 63 & & 98.00 \\
\hline LB & ccc & 1 & 27 & V62 & 32 & \begin{tabular}{|l|}
10.4 \\
\end{tabular} & 48.0 & 61.3 & 0 & 7.33 & 0 & 102.17 \\
\hline KT03-HF & quenche & 1 & 28 & V22 & 56 & 9.26 & 43.5 & 53.4 & .27 & 5.43 & 0 & 89.00 \\
\hline $\mathrm{HS}$ & quench & 1 & 29 & T. & 3 & \begin{tabular}{|l|}
7.92 \\
\end{tabular} & 31.1 & 57.0 & 5 & 20 & & 5.00 \\
\hline $\mathrm{LF}$ & quench & 1 & 30 & V64 & 2 & 29 & 44.9 & 53.8 & 7 & 3.82 & 33 & 89.67 \\
\hline KT03-HA & $\mathrm{ccc}$ & 1 & 31 & V98 & 15 & 9.55 & 52.5 & 61.1 & 8 & 5.92 & 0 & 101.84 \\
\hline LB & quench & 1 & 32 & 15 & 7 & 0 & 49.0 & 59.1 & .45 & 17 & & 98.50 \\
\hline KT1-HA2 & quenc & 1 & 33 & $\mathrm{~V}$ & 77 & \begin{tabular}{|l|}
9.75 \\
\end{tabular} & 53.0 & 64.6 & 8 & .25 & & 107.67 \\
\hline KT03-HL & quenched & 1 & 34 & V13 & 65 & 23.0 & 71.9 & 82.8 & 12.75 & 8.33 & .84 & 138.00 \\
\hline $\mathrm{HK}$ & quenched & 1 & 35 & V39 & 3 & 8 & 55.4 & 29.8 & .17 & 97 & & 49.67 \\
\hline Soln Std & $\operatorname{ref}$ & 1 & 36 & STD-13 & .0 & \begin{tabular}{|l|}
10.1 \\
\end{tabular} & .3 & 49.4 & .00 & .10 & 0 & 9.40 \\
\hline Std & ref & 2 & 1 & STD-21 & 20.3 & \begin{tabular}{|l|}
10.1 \\
\end{tabular} & 82.2 & 50.3 & .30 & .10 & .20 & 50.30 \\
\hline KT03-HB & quenche & 2 & 2 & $\mathrm{~V} 23$ & 86 & \begin{tabular}{|l|}
9.19 \\
\end{tabular} & 45.4 & 55.0 & 4.77 & 32 & 5.67 & 91.67 \\
\hline KT03-HB & $\mathrm{ccc}$ & 2 & 3 & V05 & 43 & \begin{tabular}{|l|}
8.97 \\
\end{tabular} & 43.0 & 51.4 & 14.05 & 4.95 & .67 & 85.67 \\
\hline KT03-LA & $\operatorname{ccc}$ & 2 & 4 & V34 & 24 & 11.6 & 56.0 & 67.7 & 10.40 & 9.33 & 34 & 112.84 \\
\hline KT03-LF & quenche & 2 & 5 & V82 & 5.05 & 9.66 & 54.7 & 61.0 & 8.42 & 6.10 & 1.17 & 101.67 \\
\hline KT03-LF & $\mathrm{ccc}$ & 2 & 6 & V09 & 32 & \begin{tabular}{|l|}
10.8 \\
\end{tabular} & 54.5 & 64.4 & 87 & 8.00 & 0.84 & 107.34 \\
\hline KT1-HA2 & $\operatorname{ccc}$ & 2 & 7 & V18 & 92 & 9.54 & 54.4 & 63.1 & 8.20 & 5.90 & 0.67 & 105.17 \\
\hline ARM-1 & ref & 2 & 8 & V20 & 9.43 & \begin{tabular}{|l|}
7.88 \\
\end{tabular} & 22.4 & 34.9 & 15.72 & 3.13 & 37.33 & 58.17 \\
\hline KT03-HK & $\operatorname{ccc}$ & 2 & 9 & V74 & 4.29 & \begin{tabular}{|l|}
4.58 \\
\end{tabular} & 42.0 & 49.7 & 7.15 & 7.63 & 70.00 & 82.83 \\
\hline KT03-HN & quenched & 2 & 10 & V69 & 5.99 & \begin{tabular}{|l|}
9.77 \\
\end{tabular} & 81.6 & 68.3 & 9.98 & 6.28 & 136.00 & 113.84 \\
\hline KT03-LL & quenched & 2 & 11 & V94 & 5.62 & \begin{tabular}{|l|}
11.4 \\
\end{tabular} & 56.3 & 63.9 & 9.37 & 9.00 & 93.84 & 106.50 \\
\hline KT03-HL & $\mathrm{ccc}$ & 2 & 12 & V03 & 6.98 & \begin{tabular}{|l|}
48.9 \\
\end{tabular} & 79.4 & 142.4 & 11.63 & 1.50 & 132.34 & 237.34 \\
\hline KT03-HN & $\operatorname{ccc}$ & 2 & 13 & V35 & 5.78 & 10.4 & 73.9 & 66.0 & 9.63 & 7.33 & 123.17 & 110.00 \\
\hline KT03-HF & $\mathrm{ccc}$ & 2 & 14 & V60 & 4.74 & 9.98 & 50.2 & 60.3 & 7.90 & 6.63 & 83.67 & 100.50 \\
\hline KT03-HF & quenched & 2 & 15 & V26 & 5.70 & \begin{tabular}{|l|}
9.79 \\
\end{tabular} & 47.5 & 55.9 & 9.50 & 6.32 & 9.17 & 93.17 \\
\hline KT1-HA2 & quenched & 2 & 16 & V12 & 5.15 & 10.0 & 53.4 & 65.5 & 8.58 & 16.67 & 89.00 & 109.17 \\
\hline KT03-LB & $\mathrm{ccc}$ & 2 & 17 & V36 & 5.82 & 10.8 & 52.9 & 62.1 & 9.70 & 8.00 & 8.17 & 103.50 \\
\hline Soln Std & ref & 2 & 18 & STD-22 & 19.7 & \begin{tabular}{|l|}
10.2 \\
\end{tabular} & 83.2 & 49.4 & 19.70 & 10.20 & 83.20 & 49.40 \\
\hline KT03-MK & quenched & 2 & 19 & V89 & 3.96 & 6.95 & 37.4 & 44.9 & 6.60 & 11.58 & 62.33 & 74.83 \\
\hline KT03-LB & quenched & 2 & 20 & V04 & 5.88 & \begin{tabular}{|l|}
0.1 \\
\end{tabular} & 54.2 & 61.0 & 9.80 & 16.83 & 90.34 & 101.67 \\
\hline EA & ref & 2 & 21 & V90 & 34.8 & 11.6 & 97.9 & 54.1 & 580.00 & 193.33 & 1631.67 & 901.67 \\
\hline KT03-LL & $\mathrm{ccc}$ & 2 & 22 & V97 & 5.63 & \begin{tabular}{|l|}
11.3 \\
\end{tabular} & 50.5 & 60.7 & 9.38 & 18.83 & 84.17 & 101.17 \\
\hline
\end{tabular}


Table E-1. Measurements of the PCT Solutions for the KT03-Series Glasses As-Received (ar) and After Appropriate Adjustments (ppm). (continued)

\begin{tabular}{|c|c|c|c|c|c|c|c|c|c|c|c|c|}
\hline Glass ID & $\begin{array}{c}\text { Heat } \\
\text { Treatment }\end{array}$ & Block & Seq & Lab ID & B ar & Li ar & Na ar & Si ar & B (ppm) & Li (ppm) & Na (ppm) & Si (ppm) \\
\hline KT03-LN & quenched & 2 & 23 & V57 & 5.75 & 10.2 & 46.3 & 59.6 & 9.58 & 17.00 & 77.17 & 99.34 \\
\hline KT03-MK & $\mathrm{ccc}$ & 2 & 24 & V06 & 3.53 & 6.21 & 32.0 & 42.8 & 5.88 & 10.35 & 53.33 & 71.33 \\
\hline KT03-LS & quenched & 2 & 25 & V30 & 4.39 & 8.49 & 41.1 & 55.3 & 7.32 & 14.15 & 68.50 & 92.17 \\
\hline KT03-HS & ccc & 2 & 26 & V54 & 3.45 & 7.91 & 30.8 & 55.7 & 5.75 & 13.18 & 51.33 & 92.84 \\
\hline KT03-LA & quenched & 2 & 27 & V42 & 5.92 & 10.7 & 57.6 & 65.1 & 9.87 & 17.83 & 96.00 & 108.50 \\
\hline KT03-LS & $\mathrm{ccc}$ & 2 & 28 & V50 & 4.98 & 10.3 & 48.5 & 65.5 & 8.30 & 17.17 & 80.83 & 109.17 \\
\hline KT03-LN & $\mathrm{ccc}$ & 2 & 29 & V80 & 4.99 & 10.3 & 43.5 & 59.2 & 8.32 & 17.17 & 72.50 & 98.67 \\
\hline KT03-HA & quenched & 2 & 30 & V71 & 4.26 & 8.57 & 41.1 & 50.4 & 7.10 & 14.28 & 68.50 & 84.00 \\
\hline KT03-HA & $\mathrm{ccc}$ & 2 & 31 & V84 & 3.99 & 8.36 & 46.0 & 53.3 & 6.65 & 13.93 & 76.67 & 88.84 \\
\hline KT03-HK & quenched & 2 & 32 & V79 & 10.2 & 5.34 & 56.6 & 29.2 & 17.00 & 8.90 & 94.34 & 48.67 \\
\hline KT03-HL & quenched & 2 & 33 & V27 & 7.51 & 23.0 & 71.9 & 81.3 & 12.52 & 38.33 & 119.84 & 135.50 \\
\hline KT03-HS & quenched & 2 & 34 & V75 & 3.55 & 8.10 & 32.2 & 57.4 & 5.92 & 13.50 & 53.67 & 95.67 \\
\hline Soln Std & ref & 2 & 35 & STD-23 & 19.8 & 10.1 & 82.5 & 49.6 & 19.80 & 10.10 & 82.50 & 49.60 \\
\hline Soln Std & ref & 3 & 1 & STD-31 & 20.4 & 10.2 & 84.1 & 49.4 & 20.40 & 10.20 & 84.10 & 49.40 \\
\hline KT03-HF & quenched & 3 & 2 & V51 & 5.95 & 9.60 & 45.8 & 54.3 & 9.92 & 16.00 & 76.33 & 90.50 \\
\hline KT03-HA & $\mathrm{ccc}$ & 3 & 3 & V14 & 4.28 & 8.31 & 45.9 & 53.1 & 7.13 & 13.85 & 76.50 & 88.50 \\
\hline KT03-HL & ccc & 3 & 4 & V32 & 6.86 & 48.8 & 77.2 & 140.9 & 11.43 & 81.33 & 128.67 & 234.84 \\
\hline KT03-MK & quenched & 3 & 5 & V21 & 3.93 & 7.09 & 37.6 & 45.1 & 6.55 & 11.82 & 62.67 & 75.17 \\
\hline KT03-LS & quenched & 3 & 6 & V95 & 4.52 & 8.55 & 41.4 & 55.5 & 7.53 & 14.25 & 69.00 & 92.50 \\
\hline blank & ref & 3 & 7 & V83 & $<0.100$ & $<1.00$ & $<0.100$ & $<0.100$ & 0.08 & 0.83 & 0.08 & 0.08 \\
\hline KT03-LN & $\mathrm{ccc}$ & 3 & 8 & V28 & 4.83 & 9.66 & 42.3 & 55.8 & 8.05 & 16.10 & 70.50 & 93.00 \\
\hline KT03-HB & quenched & 3 & 9 & V10 & 8.49 & 9.18 & 44.7 & 53.2 & 14.15 & 15.30 & 74.50 & 88.67 \\
\hline KT03-HK & quenched & 3 & 10 & V25 & 10.1 & 5.33 & 54.9 & 29.2 & 16.83 & 8.88 & 91.50 & 48.67 \\
\hline KT03-LF & $\mathrm{ccc}$ & 3 & 11 & V66 & 5.59 & 11.4 & 60.2 & 67.0 & 9.32 & 19.00 & 100.34 & 111.67 \\
\hline KT03-HS & quenched & 3 & 12 & V44 & 3.65 & 8.04 & 33.6 & 56.3 & 6.08 & 13.40 & 56.00 & 93.84 \\
\hline KT03-HS & ccc & 3 & 13 & V81 & 3.56 & 8.04 & 33.3 & 56.2 & 5.93 & 13.40 & 55.50 & 93.67 \\
\hline KT03-LA & quenched & 3 & 14 & V53 & 5.83 & 10.5 & 55.8 & 63.7 & 9.72 & 17.50 & 93.00 & 106.17 \\
\hline KT03-LF & quenched & 3 & 15 & V33 & 5.29 & 10.3 & 57.6 & 63.6 & 8.82 & 17.17 & 96.00 & 106.00 \\
\hline KT03-LN & quenched & 3 & 16 & V61 & 5.67 & 10.2 & 46.2 & 58.6 & 9.45 & 17.00 & 77.00 & 97.67 \\
\hline KT03-HK & $\mathrm{ccc}$ & 3 & 17 & V24 & 4.37 & 4.74 & 44.3 & 50.0 & 7.28 & 7.90 & 73.83 & 83.34 \\
\hline Soln Std & ref & 3 & 18 & STD-32 & 19.6 & 10.2 & 82.5 & 48.9 & 19.60 & 10.20 & 82.50 & 48.90 \\
\hline KT03-HA & quenched & 3 & 19 & V49 & 4.58 & 8.82 & 42.2 & 50.5 & 7.63 & 14.70 & 70.33 & 84.17 \\
\hline KT03-MK & $\mathrm{ccc}$ & 3 & 20 & V91 & 3.68 & 6.19 & 31.7 & 41.1 & 6.13 & 10.32 & 52.83 & 68.50 \\
\hline ARM-1 & ref & 3 & 21 & V85 & 9.34 & 8.05 & 21.0 & 34.3 & 15.57 & 13.42 & 35.00 & 57.17 \\
\hline KT03-HB & $\mathrm{ccc}$ & 3 & 22 & V58 & 8.41 & 9.13 & 41.0 & 50.9 & 14.02 & 15.22 & 68.33 & 84.84 \\
\hline KT03-LL & $\mathrm{ccc}$ & 3 & 23 & V02 & 5.60 & 11.8 & 52.0 & 62.5 & 9.33 & 19.67 & 86.67 & 104.17 \\
\hline EA & ref & 3 & 24 & V08 & 34.3 & 11.3 & 95.4 & 52.2 & 571.67 & 188.33 & 1590.00 & 870.00 \\
\hline KT1-HA2 & quenched & 3 & 25 & V41 & 5.46 & 9.98 & 53.6 & 65.2 & 9.10 & 16.63 & 89.34 & 108.67 \\
\hline KT03-HF & $\mathrm{ccc}$ & 3 & 26 & V65 & 4.78 & 9.70 & 48.8 & 58.7 & 7.97 & 16.17 & 81.33 & 97.84 \\
\hline KT03-LB & $\mathrm{ccc}$ & 3 & 27 & V48 & 5.74 & 10.5 & 49.9 & 60.3 & 9.57 & 17.50 & 83.17 & 100.50 \\
\hline KT03-HN & quenched & 3 & 28 & V31 & 5.98 & 9.82 & 82.4 & 66.2 & 9.97 & 16.37 & 137.34 & 110.34 \\
\hline KT03-LA & $\mathrm{ccc}$ & 3 & 29 & V40 & 6.10 & 11.7 & 56.9 & 66.0 & 10.17 & 19.50 & 94.84 & 110.00 \\
\hline KT03-HN & $\mathrm{ccc}$ & 3 & 30 & V11 & 5.99 & 10.7 & 76.1 & 66.5 & 9.98 & 17.83 & 126.84 & 110.84 \\
\hline KT03-LL & quenched & 3 & 31 & V29 & 5.72 & 11.6 & 56.7 & 63.4 & 9.53 & 19.33 & 94.50 & 105.67 \\
\hline KT03-LS & $\mathrm{ccc}$ & 3 & 32 & V96 & 4.40 & 9.31 & 41.5 & 57.4 & 7.33 & 15.52 & 69.17 & 95.67 \\
\hline KT03-HL & quenched & 3 & 33 & V17 & 7.52 & 23.3 & 74.3 & 81.3 & 12.53 & 38.83 & 123.84 & 135.50 \\
\hline KT1-HA2 & $\mathrm{ccc}$ & 3 & 34 & V43 & 4.89 & 9.58 & 52.5 & 63.0 & 8.15 & 15.97 & 87.50 & 105.00 \\
\hline KT03-LB & quenched & 3 & 35 & V73 & 5.72 & 10.1 & 51.5 & 59.9 & 9.53 & 16.83 & 85.84 & 99.84 \\
\hline Soln Std & ref & 3 & 36 & STD-33 & 19.5 & 10.2 & 82.8 & 48.8 & 19.50 & 10.20 & 82.80 & 48.80 \\
\hline
\end{tabular}


Table E-2. Normalized PCT Results for the KT03-Series Glasses and KT01-HA2 by Heat Treatment by Compositional View.

\begin{tabular}{|c|c|c|c|c|c|c|c|c|c|c|}
\hline $\begin{array}{c}\text { Glass } \\
\text { ID }\end{array}$ & Heat Treatment & $\begin{array}{l}\text { Comp } \\
\text { View }\end{array}$ & $\begin{array}{c}\log \mathrm{NL}[\mathrm{B} \\
(\mathrm{g} / \mathrm{L})]\end{array}$ & $\begin{array}{c}\log N L[L i \\
(g / L)]\end{array}$ & $\begin{array}{c}\log \mathrm{NL}[\mathrm{Na} \\
(\mathrm{g} / \mathrm{L})]\end{array}$ & $\begin{array}{c}\log N L[S i \\
(g / L)]\end{array}$ & $\begin{array}{c}\mathrm{NL} \\
\mathrm{B}(\mathrm{g} / \mathrm{L})\end{array}$ & $\begin{array}{c}\mathrm{NL} \\
\mathrm{Li}(\mathrm{g} / \mathrm{L})\end{array}$ & $\begin{array}{c}\mathrm{NL} \\
\mathrm{Na}(\mathrm{g} / \mathrm{L})\end{array}$ & $\begin{array}{c}\text { NL } \\
\text { Si (g/L) }\end{array}$ \\
\hline EA & ref & reference & 1.2169 & 0.9836 & 1.1069 & 0.5921 & 16.479 & 9.630 & 12.791 & 3.909 \\
\hline KT03-HA & $\mathrm{ccc}$ & targeted & -0.2527 & -0.1349 & -0.0924 & -0.3275 & 0.559 & 0.733 & 0.808 & 0.470 \\
\hline KT03-HA & quenched & measured & -0.2614 & -0.1415 & -0.1695 & -0.3721 & 0.548 & 0.722 & 0.677 & 0.425 \\
\hline KT03-HA & quenched & targeted & -0.2643 & -0.1444 & -0.1665 & -0.3757 & 0.544 & 0.717 & 0.682 & 0.421 \\
\hline KT03-HB & quenched & measured & -0.1752 & -0.1068 & -0.1224 & -0.3360 & 0.668 & 0.782 & 0.754 & 0.461 \\
\hline KT03-HB & quenched & targeted & -0.1852 & -0.1107 & -0.1266 & -0.3387 & 0.653 & 0.775 & 0.747 & 0.458 \\
\hline KT03-HF & $\mathrm{ccc}$ & measured & -0.1960 & -0.0743 & -0.0716 & -0.2813 & 0.637 & 0.843 & 0.848 & 0.523 \\
\hline KT03-HF & $\mathrm{ccc}$ & targeted & -0.2101 & -0.0771 & -0.0784 & -0.2885 & 0.616 & 0.837 & 0.835 & 0.515 \\
\hline KT03-HF & quenched & measured & -0.1157 & -0.0804 & -0.0963 & -0.3174 & 0.766 & 0.831 & 0.801 & 0.481 \\
\hline KT03-HF & quenched & targeted & -0.1299 & -0.0833 & -0.1030 & -0.3247 & 0.742 & 0.826 & 0.789 & 0.473 \\
\hline KT03-HL & $\mathrm{ccc}$ & measured & -0.0598 & 0.3917 & 0.1096 & 0.0841 & 0.871 & 2.464 & 1.287 & 1.214 \\
\hline KT03-HL & $\mathrm{ccc}$ & targeted & -0.0614 & 0.3866 & 0.1146 & 0.0797 & 0.868 & 2.435 & 1.302 & 1.202 \\
\hline KT03-HL & quenched & measured & -0.0227 & 0.0663 & 0.0800 & -0.1588 & 0.949 & 1.165 & 1.202 & 0.694 \\
\hline KT03-HL & quenched & targeted & -0.0243 & 0.0612 & 0.0850 & -0.1631 & 0.946 & 1.151 & 1.216 & 0.687 \\
\hline KT03-HN & $\mathrm{ccc}$ & measured & -0.1141 & -0.0567 & 0.0122 & -0.2360 & 0.769 & 0.878 & 1.028 & 0.581 \\
\hline KT03-HN & $\mathrm{ccc}$ & targeted & -0.1305 & -0.0568 & 0.0041 & -0.2538 & 0.741 & 0.877 & 1.010 & 0.557 \\
\hline KT03-HN & quenched & measured & -0.1065 & -0.0902 & 0.0518 & -0.2310 & 0.783 & 0.812 & 1.127 & 0.587 \\
\hline KT03-HN & quenched & targeted & -0.1229 & -0.0903 & 0.0437 & -0.2489 & 0.754 & 0.812 & 1.106 & 0.564 \\
\hline KT03-HS & ccc & measured & -0.3123 & -0.1428 & -0.2493 & -0.3731 & 0.487 & 0.720 & 0.563 & 0.424 \\
\hline KT03-HS & $\mathrm{ccc}$ & targeted & -0.3234 & -0.1426 & -0.2413 & -0.3957 & 0.475 & 0.720 & 0.574 & 0.402 \\
\hline KT03-HS & quenched & measured & -0.2916 & -0.1311 & -0.2325 & -0.3625 & 0.511 & 0.739 & 0.586 & 0.434 \\
\hline KT03-LB & quenched & targeted & -0.1914 & -0.0921 & -0.0773 & -0.3106 & 0.644 & 0.809 & 0.837 & 0.489 \\
\hline KT03-LF & $\mathrm{ccc}$ & measured & -0.1859 & -0.0643 & -0.0651 & -0.2850 & 0.652 & 0.862 & 0.861 & 0.519 \\
\hline KT03-LF & $\mathrm{ccc}$ & targeted & -0.1972 & -0.0676 & -0.0612 & -0.2907 & 0.635 & 0.856 & 0.868 & 0.512 \\
\hline KT03-LF & quenched & measured & -0.2422 & -0.1334 & -0.0947 & -0.3282 & 0.573 & 0.736 & 0.804 & 0.470 \\
\hline KT03-LF & quenched & targeted & -0.2535 & -0.1367 & -0.0908 & -0.3339 & 0.558 & 0.730 & 0.811 & 0.464 \\
\hline KT03-LL & $\mathrm{ccc}$ & measured & -0.1632 & -0.0658 & -0.0899 & -0.2897 & 0.687 & 0.859 & 0.813 & 0.513 \\
\hline KT03-LL & $\mathrm{ccc}$ & targeted & -0.1728 & -0.0736 & -0.0925 & -0.3047 & 0.672 & 0.844 & 0.808 & 0.496 \\
\hline KT03-LL & quenched & measured & -0.1502 & -0.0592 & -0.0394 & -0.2682 & 0.708 & 0.873 & 0.913 & 0.539 \\
\hline KT03-LL & quenched & targeted & -0.1599 & -0.0669 & -0.0419 & -0.2833 & 0.692 & 0.857 & 0.908 & 0.521 \\
\hline KT03-LN & $\mathrm{ccc}$ & measured & -0.2146 & -0.0909 & -0.1400 & -0.3280 & 0.610 & 0.811 & 0.724 & 0.470 \\
\hline KT03-LN & $\mathrm{ccc}$ & targeted & -0.2254 & -0.0952 & -0.1354 & -0.3321 & 0.595 & 0.803 & 0.732 & 0.465 \\
\hline KT03-LN & quenched & measured & -0.1436 & -0.0789 & -0.1029 & -0.3116 & 0.718 & 0.834 & 0.789 & 0.488 \\
\hline KT03-LN & quenched & targeted & -0.1545 & -0.0832 & -0.0982 & -0.3157 & 0.701 & 0.826 & 0.798 & 0.483 \\
\hline KT03-LS & $\mathrm{ccc}$ & measured & -0.2245 & -0.0942 & -0.1351 & -0.3247 & 0.596 & 0.805 & 0.733 & 0.474 \\
\hline KT03-LS & $\mathrm{ccc}$ & targeted & -0.2307 & -0.0936 & -0.1391 & -0.3315 & 0.588 & 0.806 & 0.726 & 0.466 \\
\hline KT03-LS & quenched & measured & -0.2401 & -0.1430 & -0.1565 & -0.3592 & 0.575 & 0.719 & 0.697 & 0.437 \\
\hline KT03-LS & quenched & targeted & -0.2464 & -0.1424 & -0.1605 & -0.3660 & 0.567 & 0.720 & 0.691 & 0.431 \\
\hline KT03-MK & $\mathrm{ccc}$ & measured & -0.3057 & -0.0684 & -0.1427 & -0.4147 & 0.495 & 0.854 & 0.720 & 0.385 \\
\hline KT03-MK & $\mathrm{ccc}$ & targeted & -0.3219 & -0.0660 & -0.1341 & -0.4304 & 0.477 & 0.859 & 0.734 & 0.371 \\
\hline KT03-MK & quenched & measured & -0.2850 & -0.0303 & -0.0916 & -0.4019 & 0.519 & 0.933 & 0.810 & 0.396 \\
\hline KT03-MK & quenched & targeted & -0.3012 & -0.0279 & -0.0831 & -0.4177 & 0.500 & 0.938 & 0.826 & 0.382 \\
\hline KT1-HA2 & $\mathrm{ccc}$ & measured & -0.2253 & -0.1246 & -0.0821 & -0.2820 & 0.595 & 0.751 & 0.828 & 0.522 \\
\hline KT1-HA2 & $\mathrm{ccc}$ & targeted & -0.2312 & -0.1224 & -0.0775 & -0.2974 & 0.587 & 0.754 & 0.837 & 0.504 \\
\hline KT1-HA2 & quenched & measured & -0.1960 & -0.1064 & -0.0765 & -0.2694 & 0.637 & 0.783 & 0.839 & 0.538 \\
\hline
\end{tabular}


Exhibit E-1. KT03 PCT Measurements (ppm values) in Analytical Sequence by Element.

Variability Chart for B (ppm)

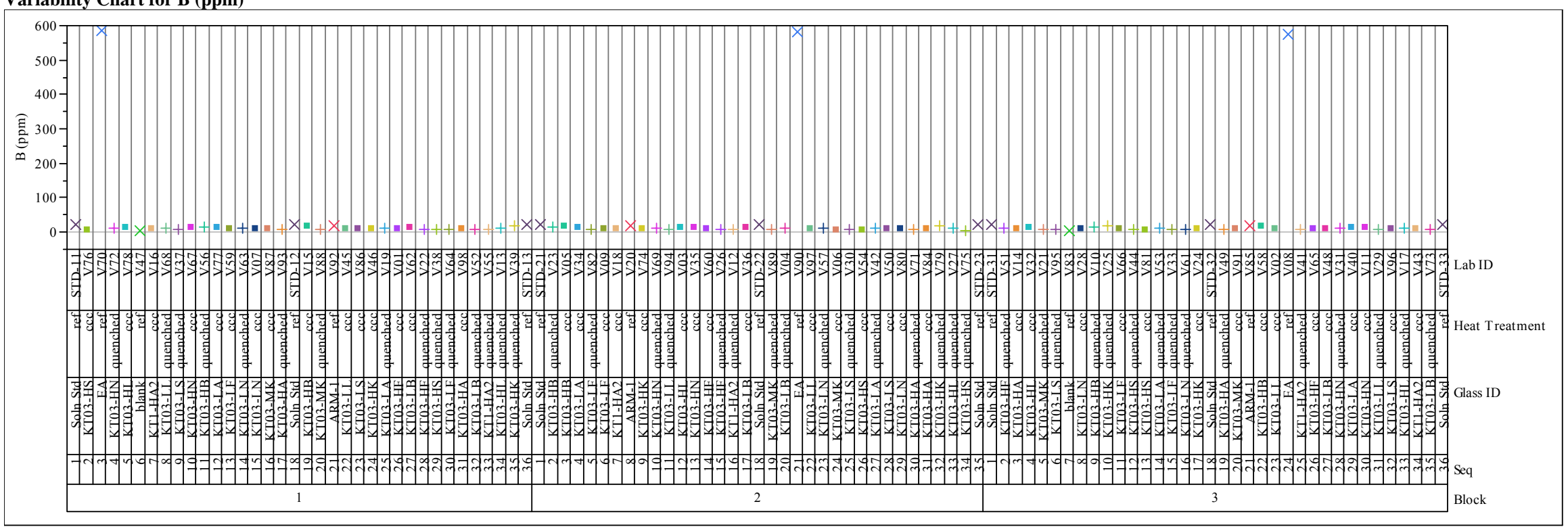


Exhibit E-1. KT03 PCT Measurements (ppm values) in Analytical Sequence by Element. (continued)

Variability Chart for Li (ppm)

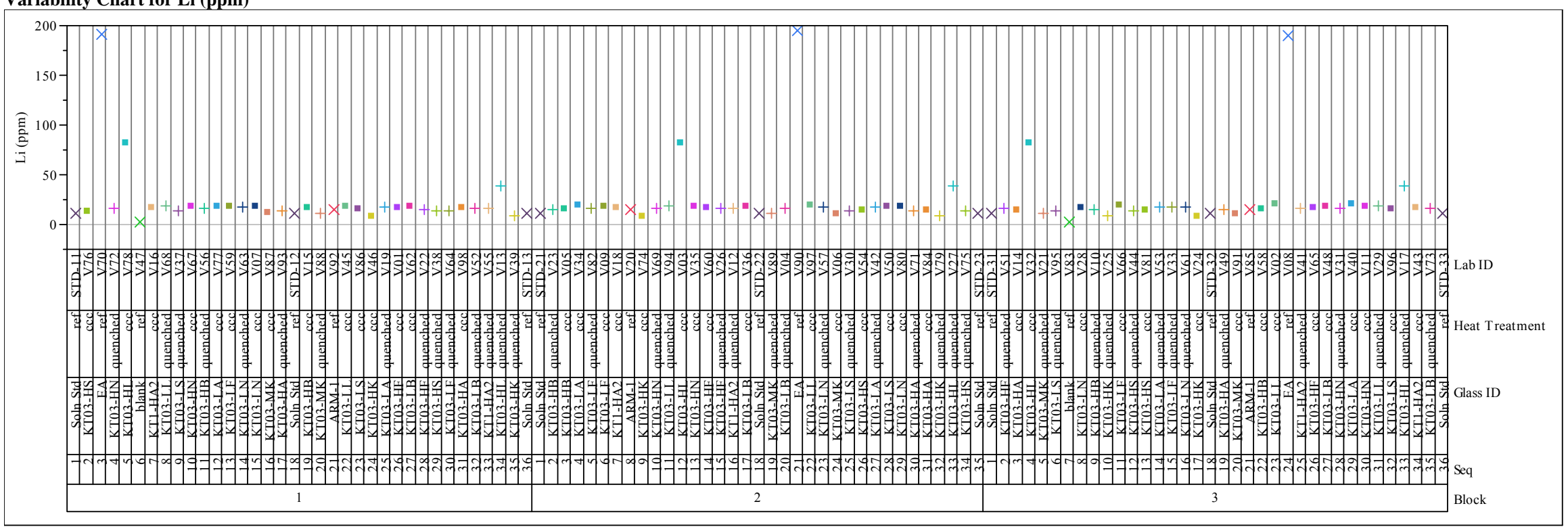


Exhibit E-1. KT03 PCT Measurements (ppm values) in Analytical Sequence by Element. (continued)

Variability Chart for Na (ppm)

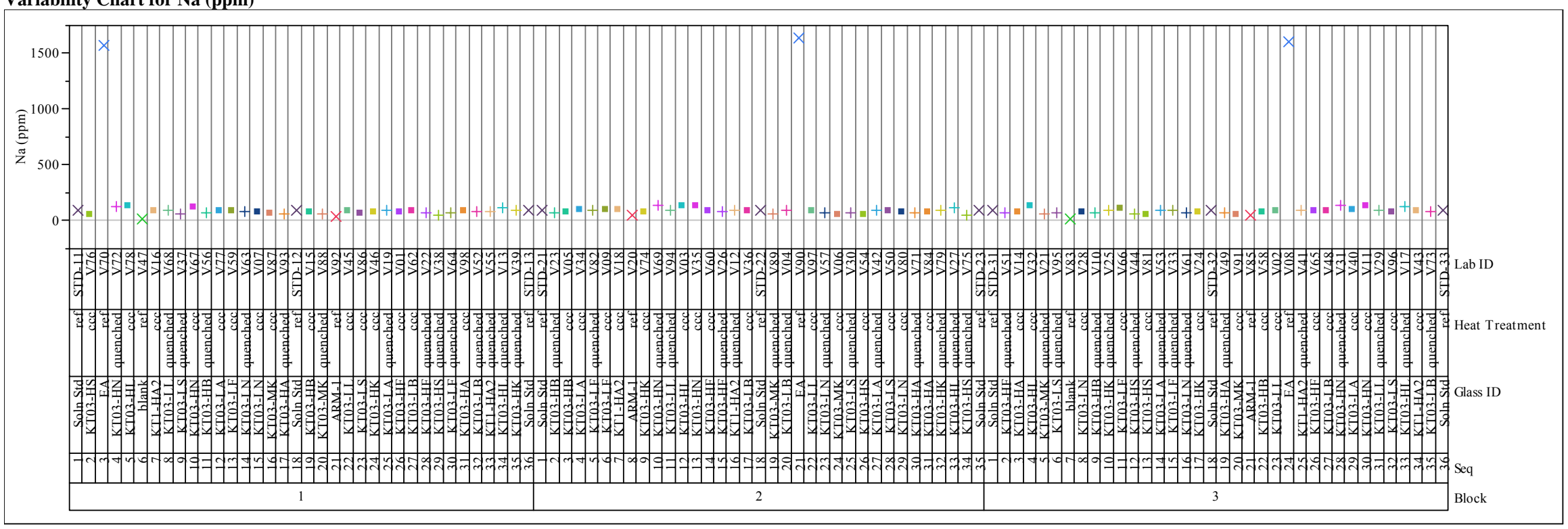


Exhibit E-1. KT03 PCT Measurements (ppm values) in Analytical Sequence by Element. (continued)

Variability Chart for Si (ppm)

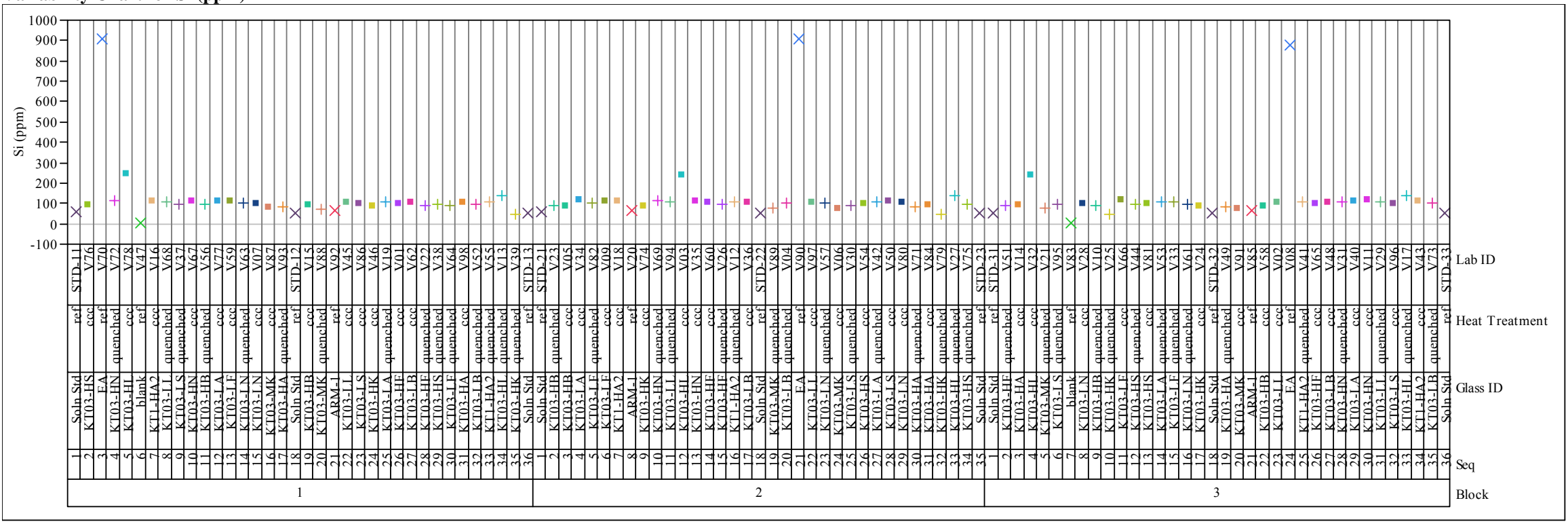


Exhibit E-2. Statistical Evaluation of the ICP-AES Calibration Effects from the KT03 Multi-Element Standard Solution Results by Oxide.

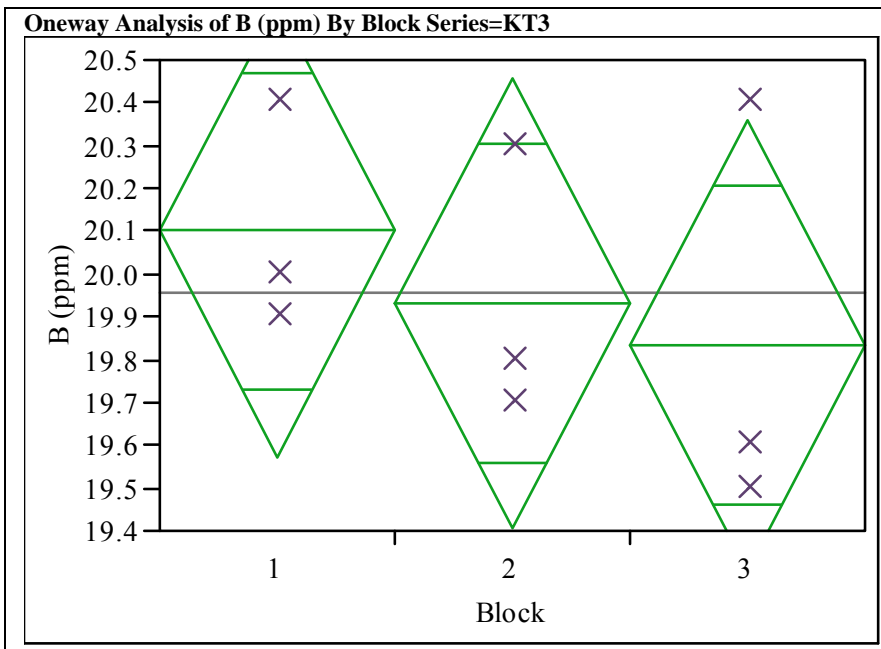

\section{Oneway Anova}

Summary of Fit

$\begin{array}{ll}\text { Rsquare } & 0.115566 \\ \text { Adj Rsquare } & -0.17925\end{array}$

-0.17925
Rdj Rsquare
Root Mean Square Error $\quad 0.372678$

$\begin{array}{ll}\text { Root Mean Square Error } \quad 0.372678 \\ \text { Mean of Response } & 19.95556\end{array}$

Mean of Response 19.95556

Analysis of Variance

Source DF Sum of Squares Mean Square F Ratio Prob $>$ F

$\begin{array}{lrrrrr}\text { Block } & 2 & 0.10888889 & 0.054444 & 0.3920 & 0.6918\end{array}$

$\begin{array}{llll}\text { Elock } & 2 & 0.10888889 & 0.054444 \\ \text { Error } & 6 & 0.83333333 & 0.138889\end{array}$

$\begin{array}{lll}\text { C. Total } & 8 & 0.94222222\end{array}$

Means for Oneway Anova

Means for Oneway Anova
Level Number Mean Std Error Lower 95\% Upper 95\%

$\begin{array}{llrrrr}1 & 3 & 20.1000 & 0.21517 & 19.574 & 20.626 \\ 2 & 3 & 19.9333 & 0.21517 & 19.407 & 20.460\end{array}$

$\begin{array}{llllll}2 & 3 & 19.9333 & 0.21517 & 19.407 & 20.460 \\ 3 & 3 & 19.8333 & 0.21517 & 19.307 & 20.360\end{array}$

Std Error uses a pooled estimate of error variance

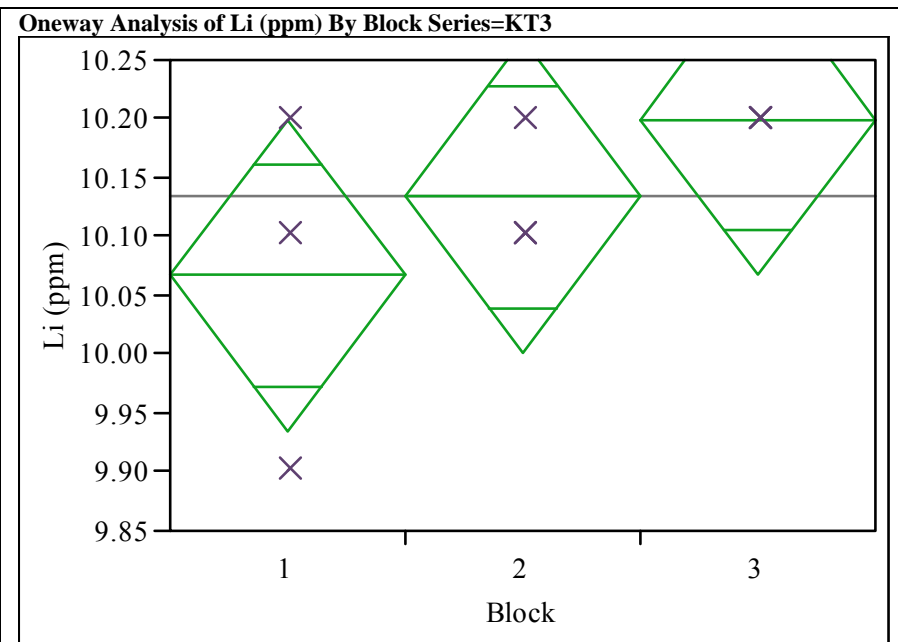

\section{Oneway Anova}

Summary of Fit

$\begin{array}{ll}\text { Rsquare } & 0.333333 \\ \text { Adj Rsquare } & 0.11111\end{array}$

$\begin{array}{ll}\text { Adj Rsquare } & 0.111111 \\ \text { Root Mean Square Error } & 0.094281\end{array}$

$\begin{array}{ll}\text { Root Mean Square Error } \quad 0.094281 \\ \text { Mean of Response } & 10.13333\end{array}$

Observations (or Sum Wgts)

Analysis of Variance

Source DF Sum of Squares Mean Square F Ratio Prob > F

$\begin{array}{lrrrrr}\text { Block } & 2 & 0.02666667 & 0.013333 & 1.5000 & 0.2963\end{array}$

$\begin{array}{llll}\text { Error } & 6 & 0.05333333 & 0.008889\end{array}$

$\begin{array}{lll}\text { C. Total } & 8 & 0.08000000\end{array}$

\section{Means for Oneway Anova}

Level Number Mean Std Error Lower 95\% Upper 95\%

$\begin{array}{rrrrrr}1 & 3 & 10.0667 & 0.05443 & 9.933 & 10.200 \\ 1 & 3 & 10.1333 & 0.0543 & 10.000 & 10.267\end{array}$

$\begin{array}{llllrr}2 & 3 & 10.1333 & 0.05443 & 10.000 & 10.267 \\ 3 & 3 & 10.2000 & 0.05443 & 10.067 & 10.333\end{array}$

Std Error uses a pooled estimate of error variance 
Exhibit E-2. Statistical Evaluation of the ICP-AES Calibration Effects from the KT03 Multi-Element Standard Solution Results by Oxide. (continued)

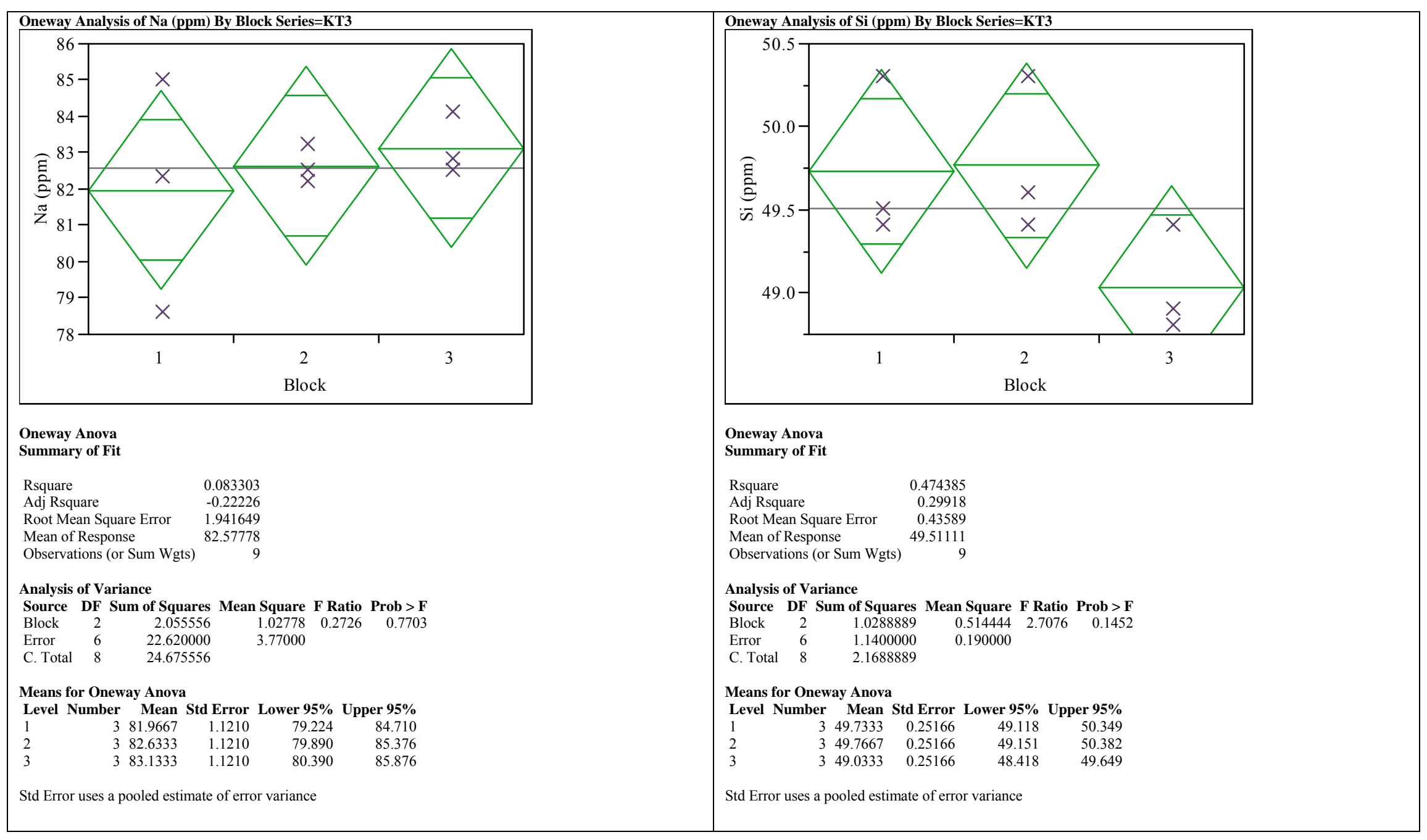




\section{Exhibit E-3. KT03 PCT Results (as common logarithms) Grouped by Glass ID and Heat Treatment.}

Variability Chart for log[B ppm]
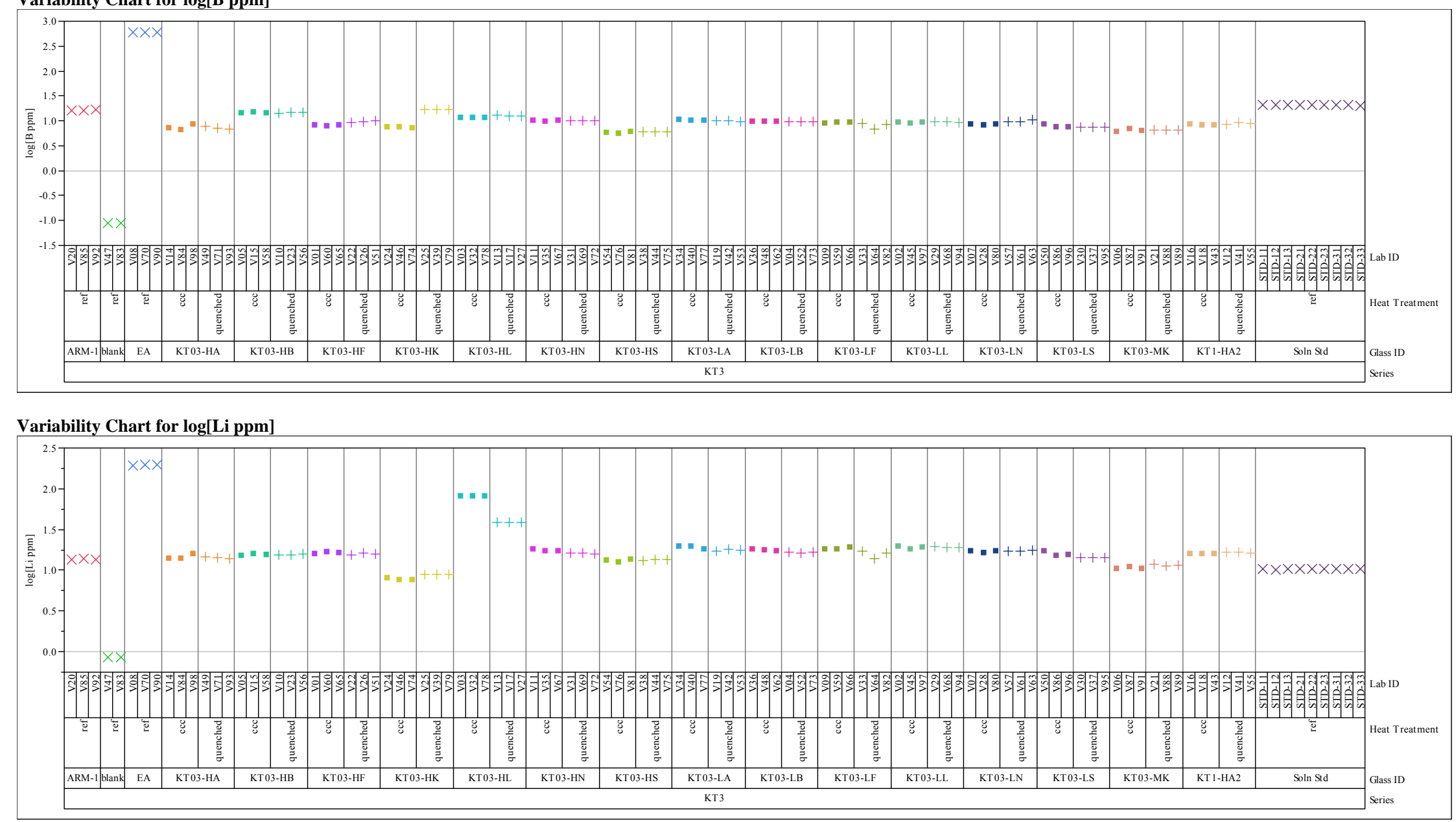
Exhibit E-3. KT03 PCT Results (as common logarithms) Grouped by Glass ID and Heat Treatment. (continued) Variability Chart for log[Na ppm]
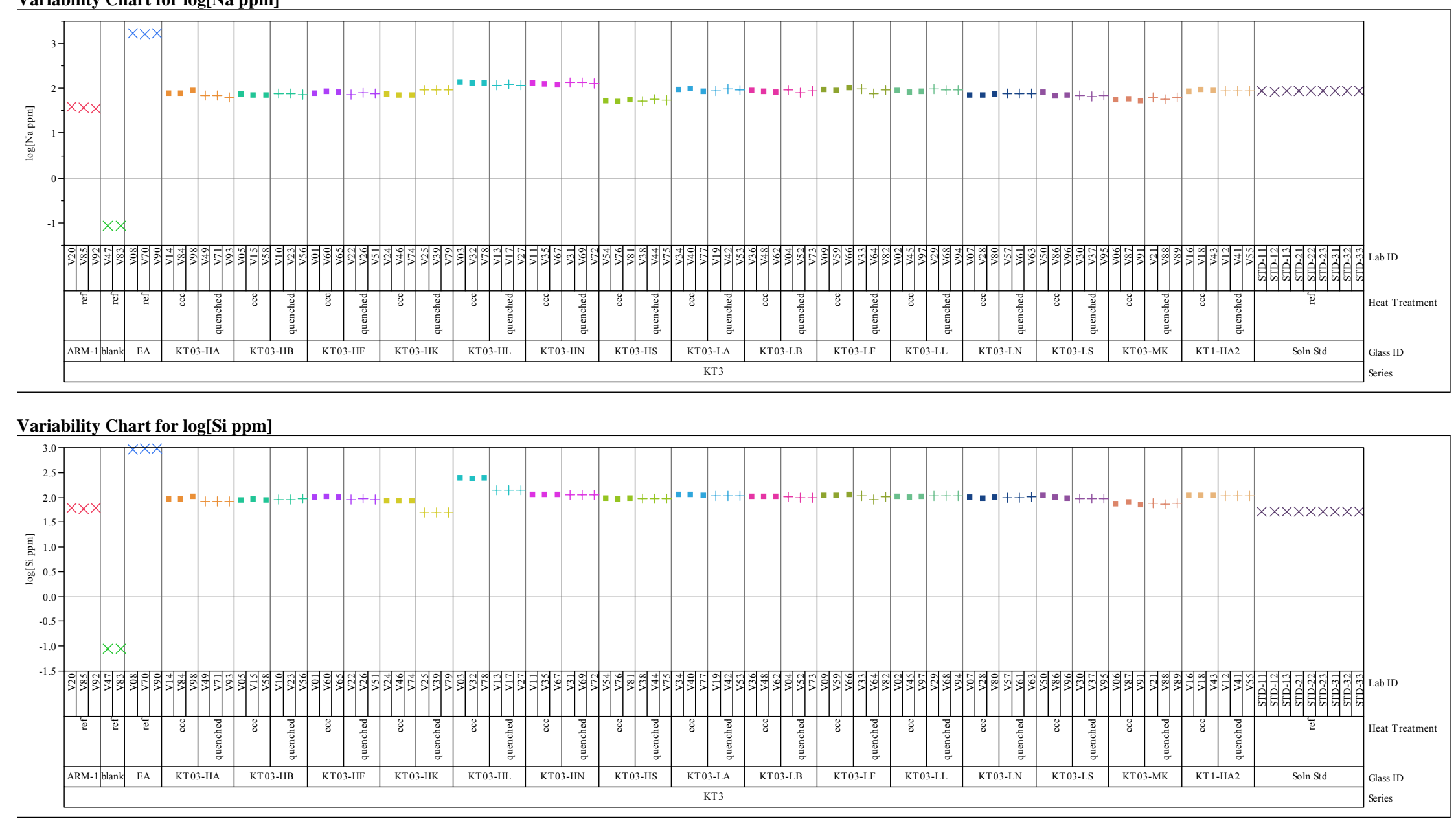
Exhibit E-4. Correlations and Scatter Plots of the KT03 Normalized PCTs Over All Compositional Views and Heat Treatments.

\section{Multivariate Correlations}

$\begin{array}{ccccc} & \log \text { NL[B (g/L)] } & \log \text { NL[Li(g/L)] } & \log \text { NL[Na (g/L)] } & \log \text { NL[Si (g/L)] } \\ \log \text { NL[B (g/L)] } & 1.0000 & 0.8565 & 0.9279 & 0.7278 \\ \log \text { NL[Li(g/L)] } & 0.8565 & 1.0000 & 0.9260 & 0.7214 \\ \log \text { NL[Na (g/L)] } & 0.9279 & 0.9260 & 1.0000 & 0.6776 \\ \log \text { NL[Si (g/L)] } & 0.7278 & 0.7214 & 0.6776 & 1.0000\end{array}$

\section{Scatterplot Matrix}

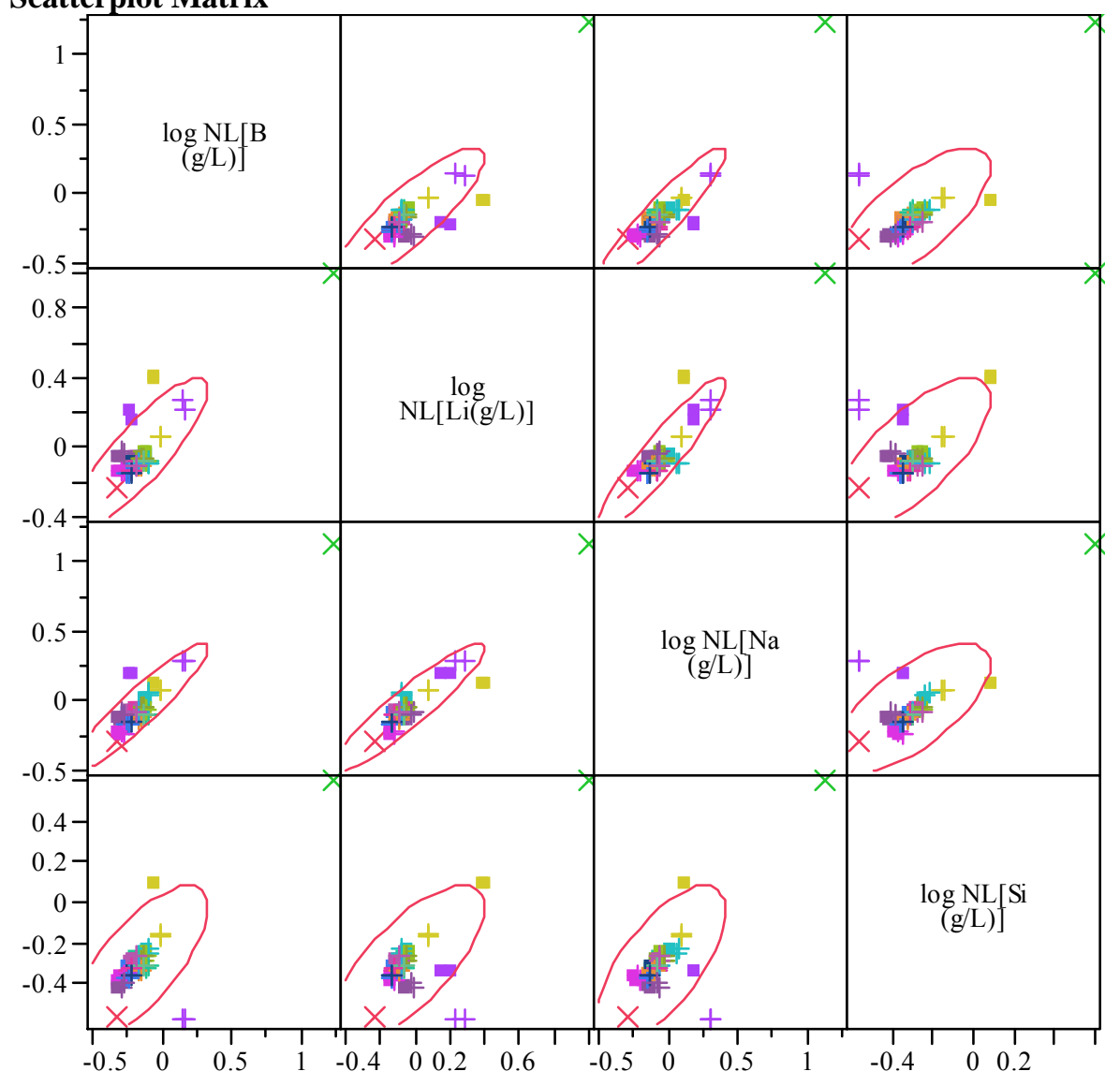




\section{Exhibit E-5. Effects of Heat Treatment for the KT03 Glasses by Compositional View.}
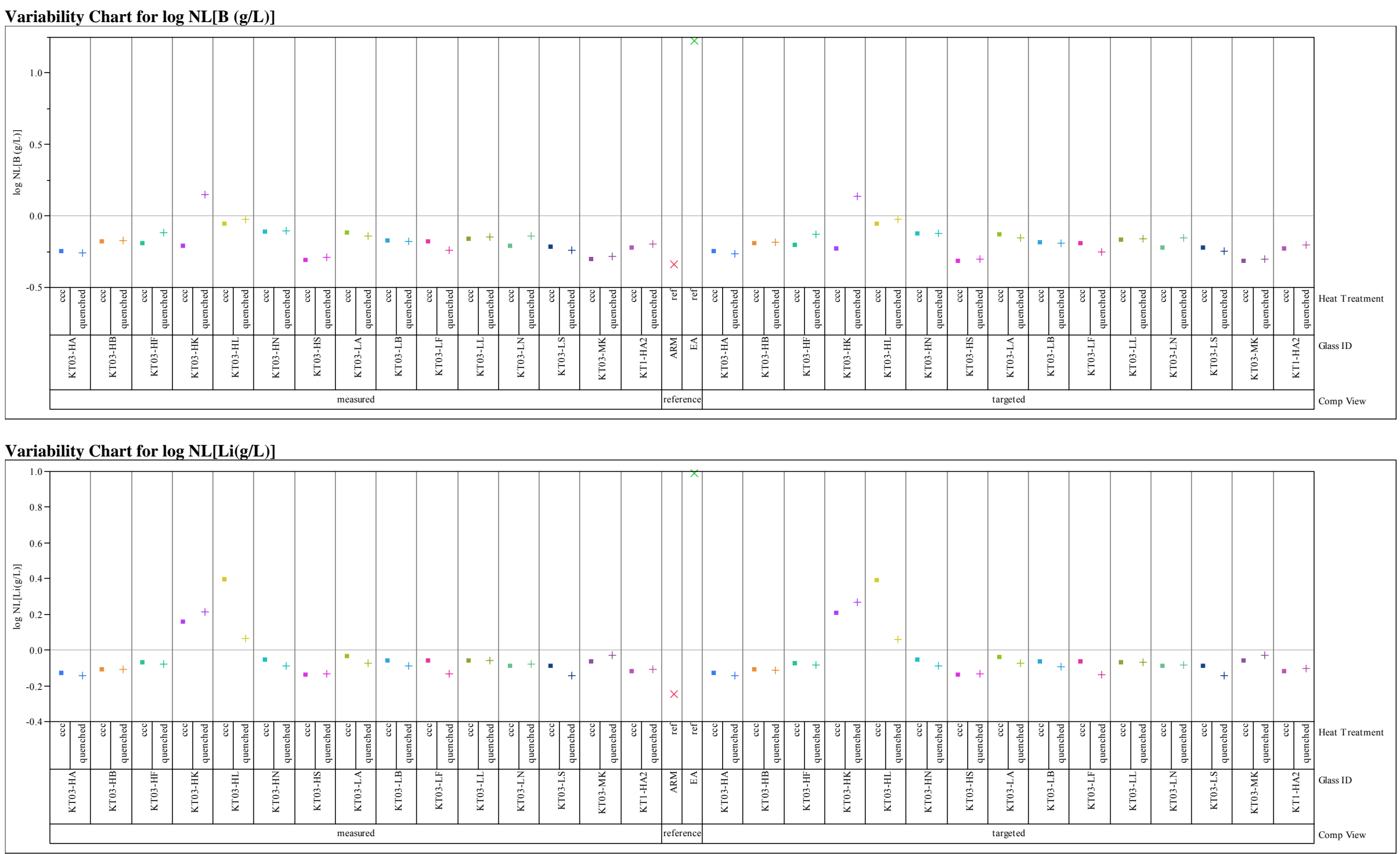


\section{Exhibit E-5. Effects of Heat Treatment for the KT03 Glasses by Compositional View. (continued)}

Variability Chart for log NL[Na (g/L)]

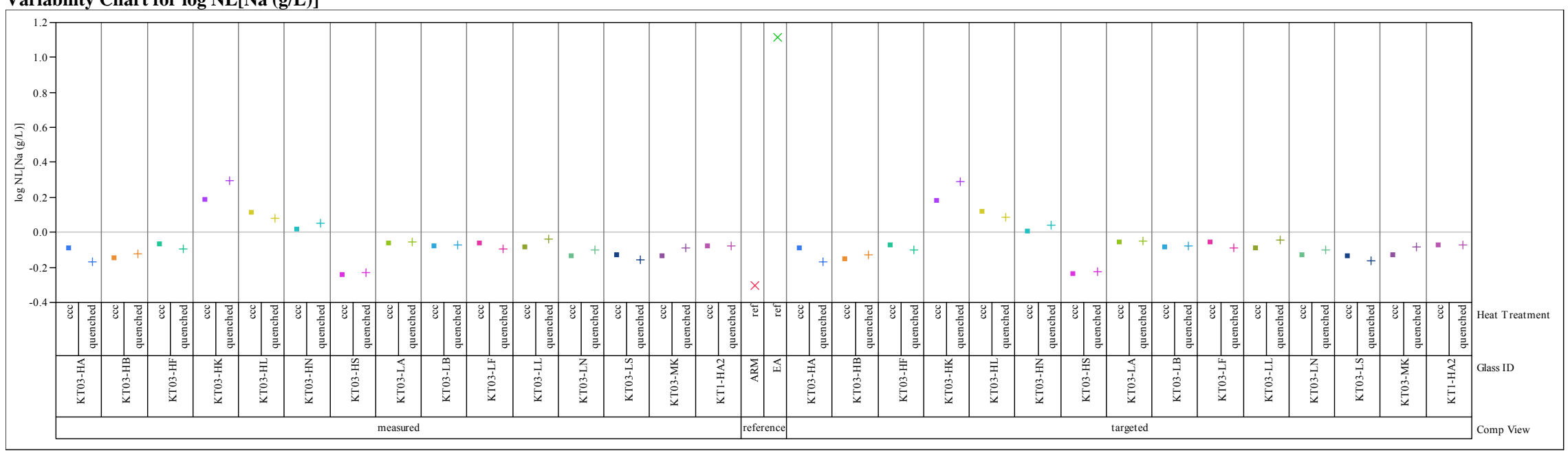

\section{Variability Chart for $\log \mathrm{NL}[\mathrm{Si}(\mathrm{g} / \mathrm{L})]$}

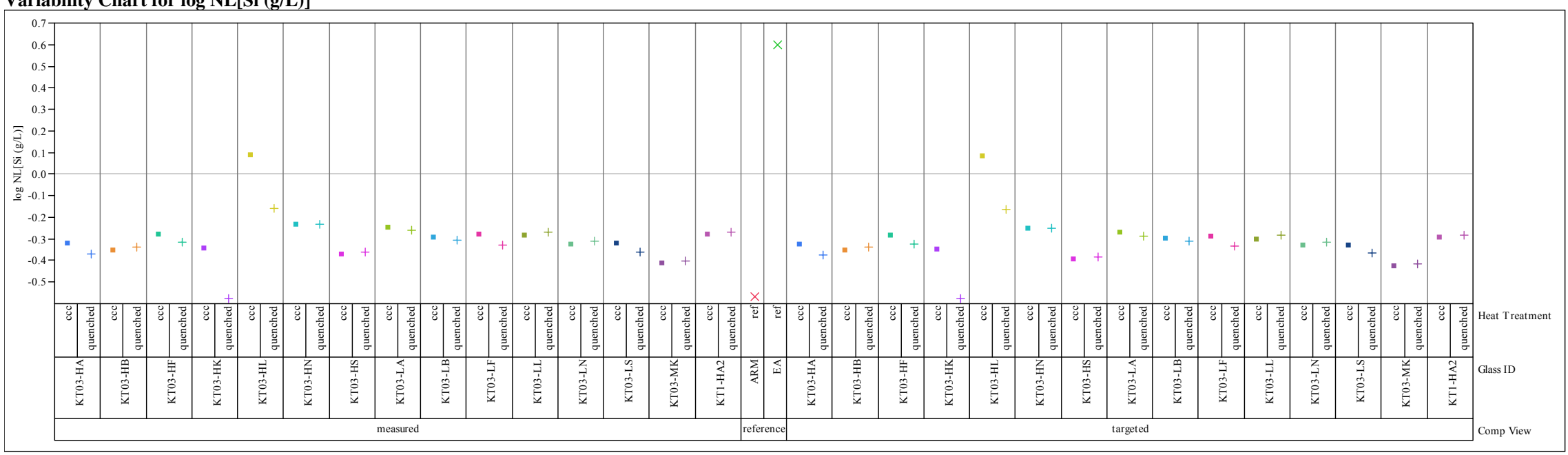


Exhibit E-6. KT03-Series PCT Measurements versus Durability Model Predictions.

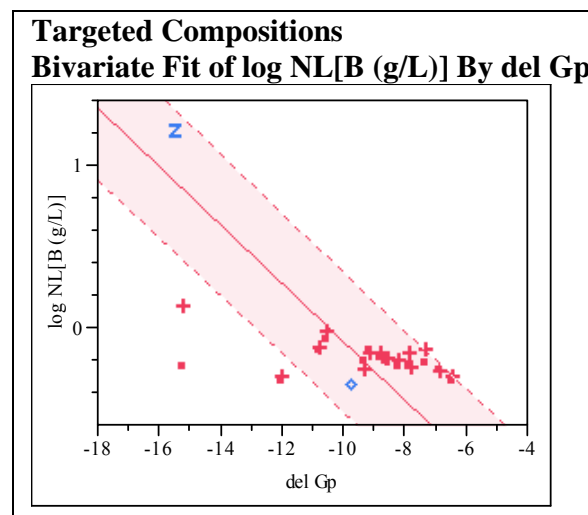

Bivariate Fit of log NL[Li (g/L)] By del Gp

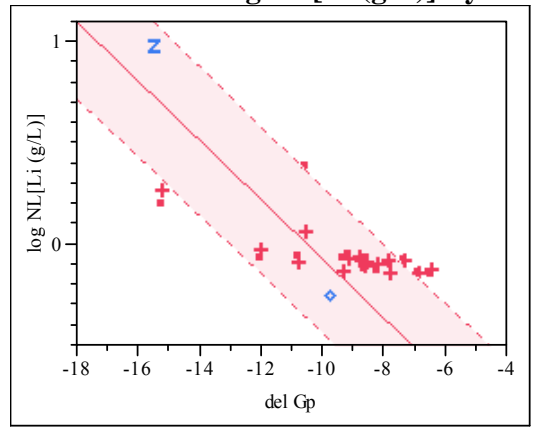

Bivariate Fit of $\log$ NL[Na (g/L)] By del Gp

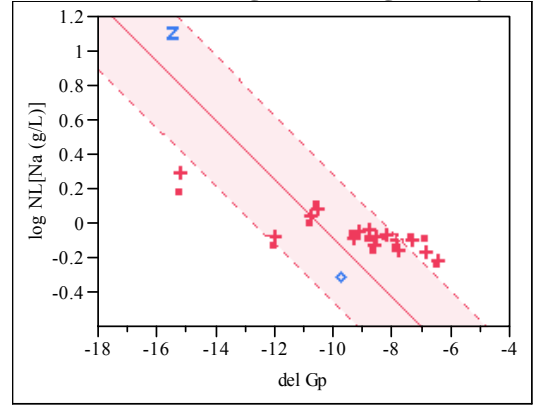

Bivariate Fit of $\log \mathrm{NL}[\mathrm{Si}(\mathrm{g} / \mathrm{L})]$ By del Gp

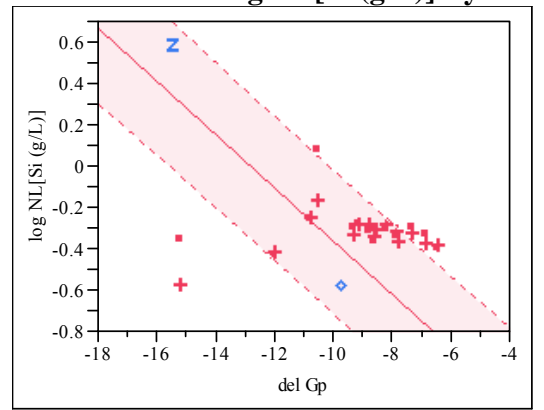

\section{Measured Compositions}

Bivariate Fit of $\log \mathrm{NL}[\mathrm{B}(\mathrm{g} / \mathrm{L})]$ By del Gp

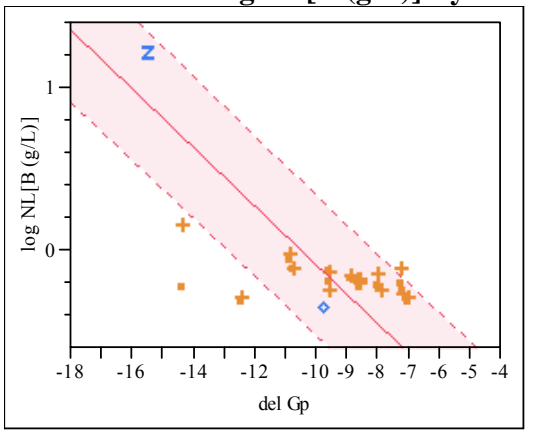

Bivariate Fit of $\log \mathrm{NL}[\mathrm{Li}(\mathrm{g} / \mathrm{L})]$ By del Gp

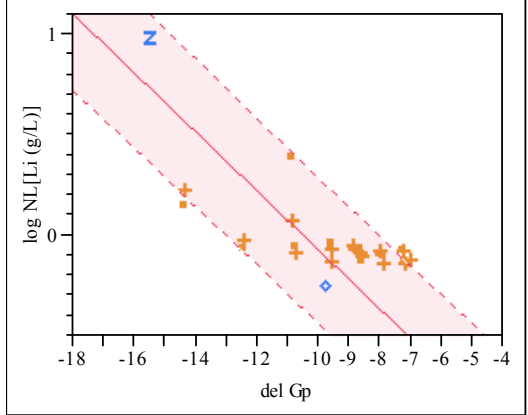

Bivariate Fit of $\log$ NL[Na (g/L)] By del Gp

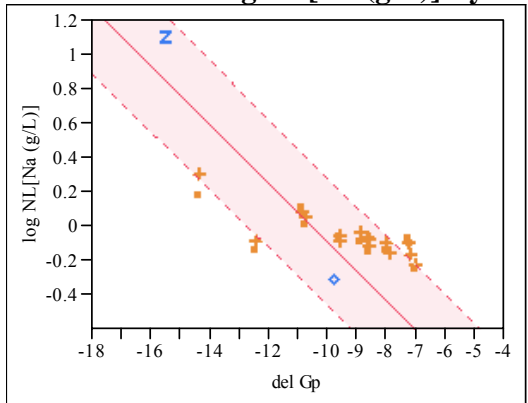

Bivariate Fit of log NL[Si (g/L)] By del Gp

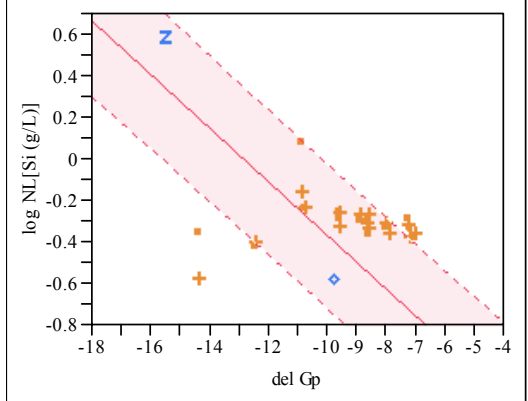

z Environmental Assessment Benchmark Glass

$\diamond$ Approved Reference Material Glass
++ Quenched Study Glasses

- Slowly Cooled Study Glasses 
SRNL-STI-2010-00566

Revision 0

Appendix F. Data Supporting the PCT Measurements of the KT04-Series Glasses 
Table F-1. Measurements of the PCT Solutions for the KT04-Series Glasses As-Received (ar) and After Appropriate Adjustments (ppm).

\begin{tabular}{|c|c|c|c|c|c|c|c|c|c|c|c|c|}
\hline Glass ID & Heat Treatment & Block & Seq & Lab ID & B ar & Li ar & Na ar & Si ar & B (ppm) & Li (ppm) & Na (ppm) & Si (ppm) \\
\hline Soln Std & ref & 1 & 1 & STD-11 & 20.7 & 10.1 & 82.3 & 49.9 & 20.70 & 10.10 & 82.30 & 49.90 \\
\hline KT04-01 & quenched & 1 & 2 & B33 & 6.08 & 6.60 & 41.3 & 55.2 & 10.13 & 11.00 & 68.83 & 92.00 \\
\hline KT04-03 & $\mathrm{ccc}$ & 1 & 3 & $\mathrm{~B} 10$ & 6.91 & 7.33 & 44.3 & 61.0 & 11.52 & 12.22 & 73.83 & 101.67 \\
\hline KT04-05 & $\mathrm{ccc}$ & 1 & 4 & B39 & 5.23 & 6.35 & 35.1 & 56.9 & 8.72 & 10.58 & 8.50 & 94.84 \\
\hline KT04-04 & $\mathrm{ccc}$ & 1 & 5 & B06 & 6.14 & 7.11 & 38.0 & 61.0 & 10.23 & 11.85 & 63.33 & 101.67 \\
\hline KT04-03 & quenched & 1 & 6 & B08 & 6.85 & 7.21 & 49.0 & 63.6 & 11.42 & 12.02 & 81.67 & 106.00 \\
\hline KT04-08 & quenched & 1 & 7 & B16 & 6.35 & 6.65 & 56.7 & 64.6 & 10.58 & 11.08 & 94.50 & 107.67 \\
\hline KT04-09 & $\mathrm{ccc}$ & 1 & 8 & B36 & 6.22 & 6.92 & 42.3 & 59.7 & 10.37 & 1.53 & 70.50 & 99.50 \\
\hline KT04-10 & $\mathrm{ccc}$ & 1 & 9 & B02 & 6.37 & 7.15 & 38.9 & 59.0 & 10.62 & 11.92 & 64.83 & 98.34 \\
\hline EA & ref & 1 & 10 & B52 & 39.4 & 11.8 & 108 & 54.8 & 656.67 & 196.67 & 1800.00 & 913.34 \\
\hline KT04-06 & $\mathrm{ccc}$ & 1 & 11 & B42 & 5.21 & 6.22 & 33.4 & 55.0 & 8.68 & 10.37 & 55.67 & 91.67 \\
\hline KT04-07 & quenched & 1 & 12 & B49 & 5.69 & 6.36 & 44.1 & 59.6 & 9.48 & 0.60 & 3.50 & 99.34 \\
\hline KT04-04 & quenched & 1 & 13 & B13 & 6.44 & 7.32 & 43.0 & 64.0 & 10.73 & 20 & 1.67 & 106.67 \\
\hline Soln Std & ref & 1 & 14 & STD-12 & 20.0 & 10.1 & 82.9 & 50.0 & 20.00 & .10 & 2.90 & 50.00 \\
\hline KT04-09 & uenched & 1 & 15 & B60 & 6.56 & 6.98 & 43.9 & 59.9 & 10.93 & .63 & 3.17 & 9.84 \\
\hline ARM-1 & ref & 1 & 16 & B01 & 10.08 & 8.34 & 20.8 & 35.7 & 16.80 & .90 & .67 & 9.50 \\
\hline KT04-06 & uenched & 1 & 17 & B57 & 4.91 & 6.05 & 34.6 & 53.4 & 8.18 & .08 & 7.67 & 9.00 \\
\hline KT04-02 & quenc & 1 & 18 & B47 & 6.21 & 6.91 & 43.2 & 58.5 & .35 & .52 & 2.00 & 7.50 \\
\hline KT04-10 & quencl & 1 & 19 & B14 & 6.06 & 6.90 & 39.1 & 57.4 & 10.10 & .50 & 5.17 & 5.67 \\
\hline KT04-07 & $\mathrm{ccc}$ & 1 & 20 & B66 & 5.49 & 6.49 & 41.4 & 58.8 & 9.15 & 0.82 & 99.00 & 98.00 \\
\hline blank & ref & 1 & 21 & B31 & $<0.100$ & $<1.00$ & $<0.100$ & $<0.100$ & .08 & 0.83 & 08 & 0.08 \\
\hline KT04-05 & uenched & 1 & 22 & B62 & 5.59 & 7.05 & 41.0 & 63.2 & 9.32 & 1.75 & 8.33 & 105.34 \\
\hline KT04-08 & & 1 & 23 & B20 & 6.01 & 7.00 & 51.0 & 63.4 & 10.02 & 1.67 & 5.00 & 105.67 \\
\hline 4-02 & $\mathrm{cc}$ & 1 & 24 & B28 & 6.01 & 6.95 & 40.6 & 56.9 & 10.02 & .58 & 7.67 & 94.84 \\
\hline KT04-01 & $\mathrm{cc}$ & 1 & 25 & B18 & .02 & 6.82 & 34.6 & 54.4 & 10.03 & .37 & 7.67 & 0.67 \\
\hline Soln Std & ref & 1 & 26 & STD-12 & 19.68 & 10.00 & 81.0 & 49.0 & 68 & 0.00 & 1.00 & 49.00 \\
\hline Std & ref & 2 & 1 & STD-21 & 20.7 & 10.0 & 83.3 & 50.0 & 20.70 & 0.00 & .30 & 50.00 \\
\hline KT04-06 & uenche & 2 & 2 & B03 & 4.88 & 6.05 & 5.1 & 53.9 & 8.13 & 0.08 & 8.50 & 89.84 \\
\hline KT04-06 & $\mathrm{ccc}$ & 2 & 3 & B26 & 1.83 & 6.27 & 3.8 & 55.4 & .05 & .45 & 6.33 & 92.34 \\
\hline KT04-08 & quenched & 2 & 4 & B43 & .79 & 6.42 & 3.8 & 61.7 & 9.65 & .70 & 9.67 & 102.84 \\
\hline KT04-02 & quenched & 2 & 5 & B61 & .50 & 7.36 & 47.4 & 63.4 & 10.83 & .27 & 9.00 & 105.67 \\
\hline KT04-03 & $\mathrm{ccc}$ & 2 & 6 & B68 & 40 & 7.28 & 45.2 & 60.2 & 10.67 & 2.13 & 5.33 & 100.34 \\
\hline KT04-01 & $\mathrm{ccc}$ & 2 & 7 & B58 & 87 & 6.78 & 5.0 & 54.4 & 9.78 & .30 & 33 & 90.67 \\
\hline KT04-08 & $\mathrm{ccc}$ & 2 & 8 & B41 & 5.64 & 6.72 & 9.3 & 62.1 & .40 & .20 & 2.17 & 103.50 \\
\hline KT04-04 & uenche & 2 & 9 & B53 & 5.55 & 6.68 & 2.2 & 57.8 & 9.25 & .13 & 0.33 & 96.34 \\
\hline KT04-05 & $\mathrm{ccc}$ & 2 & 10 & B23 & 83 & 6.40 & 7.1 & 57.1 & .05 & 0.67 & 1.83 & 95.17 \\
\hline KT04-05 & ench & 2 & 11 & B59 & 79 & 6.44 & 8.4 & 57.2 & 7.98 & 0.73 & 4.00 & 95.34 \\
\hline KT04-07 & nched & 2 & 12 & B30 & 4.87 & 6.05 & 42.5 & 56.6 & 8.12 & 0.08 & .83 & 4.34 \\
\hline KT04-01 & nched & 2 & 13 & B64 & 5.46 & 6.60 & 5.2 & 53.3 & 9.10 & 1.00 & 0.33 & 88.84 \\
\hline Soln Std & ref & 2 & 14 & STD-22 & 19.8 & 10.1 & 81.7 & 49.4 & 19.80 & 0.10 & 1.70 & 49.40 \\
\hline KT04-04 & $\operatorname{ccc}$ & 2 & 15 & B67 & 6.39 & 7.05 & 39.8 & 60.5 & 10.65 & 75 & 5.33 & 100.84 \\
\hline KT04-09 & denched & 2 & 16 & B17 & 6.79 & 7.35 & 49.5 & 64.2 & 11.32 & 2.25 & 82.50 & 107.00 \\
\hline KT04-10 & quenched & 2 & 17 & B05 & 6.06 & \begin{tabular}{|l|}
6.99 \\
\end{tabular} & 42.0 & 58.6 & 10.10 & 11.65 & 70.00 & 97.67 \\
\hline KT04-02 & $\mathrm{ccc}$ & 2 & 18 & B54 & 5.97 & \begin{tabular}{|l|}
7.01 \\
\end{tabular} & 42.0 & 57.7 & 9.95 & 11.68 & 70.00 & 96.17 \\
\hline EA & ref & 2 & 19 & B09 & 31.8 & \begin{tabular}{|l|}
9.60 \\
\end{tabular} & 142 & 53.0 & 530.00 & 160.00 & 2366.67 & 883.34 \\
\hline KT04-09 & $\mathrm{ccc}$ & 2 & 20 & B48 & 5.95 & \begin{tabular}{|l|}
6.61 \\
\end{tabular} & 41.1 & 57.2 & 9.92 & 11.02 & 68.50 & 95.34 \\
\hline KT04-07 & $\mathrm{ccc}$ & 2 & 21 & B44 & 5.32 & 6.41 & 43.9 & 58.2 & 8.87 & 10.68 & 3.17 & 97.00 \\
\hline ARM-1 & ref & 2 & 22 & B22 & 9.75 & 8.20 & 21.8 & 35.6 & 16.25 & 3.67 & 36.33 & 59.33 \\
\hline KT04-10 & $\mathrm{ccc}$ & 2 & 23 & B27 & 6.15 & 7.15 & 40.5 & 59.0 & 10.25 & 11.92 & 77.50 & 98.34 \\
\hline KT04-03 & uenche & 2 & 24 & B34 & 6.49 & 7.21 & 50.4 & 61.4 & 10.82 & 2.02 & 84.00 & 102.34 \\
\hline Soln Std & ref & 2 & 25 & STD-23 & 19.9 & \begin{tabular}{|l|}
10.1 \\
\end{tabular} & 82.9 & 49.7 & 19.90 & 10.10 & 82.90 & 49.70 \\
\hline Soln Std & ref & 3 & 1 & STD-31 & 20.6 & 10.0 & 82.7 & 49.0 & 20.60 & 10.00 & 82.70 & 49.00 \\
\hline KT04-01 & quenched & 3 & 2 & B32 & 6.59 & 6.86 & 37.9 & 55.7 & 10.98 & 11.43 & 63.17 & 92.84 \\
\hline KT04-04 & quenched & 3 & 3 & B29 & 6.20 & 6.62 & 43.6 & 57.9 & 10.33 & 11.03 & 72.67 & 96.50 \\
\hline KT04-09 & quenched & 3 & 4 & B24 & 6.58 & 6.89 & 45.8 & 59.4 & 10.97 & 11.48 & 76.33 & 99.00 \\
\hline KT04-02 & $\mathrm{ccc}$ & 3 & 5 & B15 & 6.30 & 6.76 & 41.6 & 55.7 & 10.50 & 11.27 & 69.33 & 92.84 \\
\hline KT04-02 & quenched & 3 & 6 & B19 & 6.22 & 6.65 & 44.0 & 56.7 & 10.37 & 11.08 & 73.33 & 94.50 \\
\hline KT04-06 & quenched & 3 & 7 & $\mathrm{~B} 63$ & 5.19 & 6.24 & 40.4 & 55.5 & 8.65 & 10.40 & 67.33 & 92.50 \\
\hline KT04-01 & $\mathrm{ccc}$ & 3 & 8 & B35 & 6.07 & 6.57 & 35.9 & 53.2 & 10.12 & 10.95 & 59.83 & 88.67 \\
\hline
\end{tabular}


Table F-1. Measurements of the PCT Solutions for the KT04-Series Glasses As-Received (ar) and After Appropriate Adjustments (ppm). (continued)

\begin{tabular}{|c|c|c|c|c|c|c|c|c|c|c|c|c|}
\hline Glass ID & Heat Treatment & Block & Seq & Lab ID & B ar & Li ar & Na ar & Si ar & B (ppm) & Li (ppm) & Na (ppm) & Si (ppm) \\
\hline KT04-08 & quenched & 3 & 9 & B46 & 5.98 & 6.41 & 53.9 & 61.6 & 9.97 & 10.68 & 89.84 & 102.67 \\
\hline EA & ref & 3 & 10 & B40 & 37.5 & 11.3 & 102 & 52.4 & 625.00 & 188.33 & 1700.00 & 873.34 \\
\hline blank & ref & 3 & 11 & B25 & $<0.100$ & $<1.00$ & $<0.100$ & $<0.100$ & 0.08 & 0.83 & 0.08 & 0.08 \\
\hline KT04-03 & quenched & 3 & 12 & B51 & 6.92 & 7.20 & 49.7 & 60.9 & 11.53 & 12.00 & 82.83 & 101.50 \\
\hline ARM-1 & ref & 3 & 13 & B38 & 10.7 & 8.44 & 24.4 & 36.3 & 17.83 & 14.07 & 40.67 & 60.50 \\
\hline Soln Std & ref & 3 & 14 & STD-32 & 20.7 & 10.2 & 84.7 & 50.0 & 20.70 & 10.20 & 84.70 & 50.00 \\
\hline KT04-03 & ccc & 3 & 15 & B56 & 7.34 & 7.33 & 49.6 & 59.9 & 12.23 & 12.22 & 82.67 & 99.84 \\
\hline KT04-08 & ccc & 3 & 16 & B21 & 6.41 & 7.03 & 50.9 & 63.1 & 10.68 & 11.72 & 84.84 & 105.17 \\
\hline KT04-09 & ccc & 3 & 17 & B65 & 6.27 & 6.69 & 43.2 & 57.2 & 10.45 & 11.15 & 72.00 & 95.34 \\
\hline KT04-10 & quenched & 3 & 18 & B45 & 6.26 & 6.89 & 40.9 & 56.9 & 10.43 & 11.48 & 68.17 & 94.84 \\
\hline KT04-05 & quenched & 3 & 19 & B55 & 5.22 & 6.35 & 37.8 & 55.7 & 8.70 & 10.58 & 63.00 & 92.84 \\
\hline KT04-10 & ccc & 3 & 20 & B37 & 6.28 & 7.01 & 39.1 & 57.2 & 10.47 & 11.68 & 65.17 & 95.34 \\
\hline KT04-06 & ccc & 3 & 21 & B50 & 5.01 & 6.21 & 34.6 & 54.5 & 8.35 & 10.35 & 57.67 & 90.84 \\
\hline KT04-07 & quenched & 3 & 22 & B11 & 5.60 & 6.29 & 45.1 & 58.5 & 9.33 & 10.48 & 75.17 & 97.50 \\
\hline KT04-07 & ccc & 3 & 23 & B04 & 5.66 & 6.41 & 44.1 & 58.8 & 9.43 & 10.68 & 73.50 & 98.00 \\
\hline KT04-04 & ccc & 3 & 24 & B07 & 6.11 & 6.87 & 44.9 & 59.1 & 10.18 & 11.45 & 74.83 & 98.50 \\
\hline KT04-05 & ccc & 3 & 25 & B12 & 5.86 & 6.96 & 41.5 & 62.2 & 9.77 & 11.60 & 69.17 & 103.67 \\
\hline Soln Std & ref & 3 & 26 & STD-33 & 20.5 & 10.0 & 83.0 & 49.2 & 20.50 & 10.00 & 83.00 & 49.20 \\
\hline
\end{tabular}


Table F-2. Normalized PCT Results for the KT04-Series Glasses by Heat Treatment by Compositional View.

\begin{tabular}{|c|c|c|c|c|c|c|c|c|c|c|}
\hline $\begin{array}{c}\text { Glass } \\
\text { ID }\end{array}$ & Heat Treatment & $\begin{array}{l}\text { Comp } \\
\text { View }\end{array}$ & $\begin{array}{c}\log N L[B \\
(g / L)]\end{array}$ & $\begin{array}{c}\log N L[L i \\
(g / L)]\end{array}$ & $\begin{array}{c}\log \text { NL[Na } \\
(\mathrm{g} / \mathrm{L})]\end{array}$ & $\begin{array}{c}\log N L[S i \\
(g / L)]\end{array}$ & $\begin{array}{c}\mathrm{NL} \\
\mathrm{B}(\mathrm{g} / \mathrm{L})\end{array}$ & $\begin{array}{c}\mathrm{NL} \\
\mathrm{Li}(\mathrm{g} / \mathrm{L})\end{array}$ & $\begin{array}{c}\text { NL } \\
\mathrm{Na}(\mathrm{g} / \mathrm{L})\end{array}$ & $\begin{array}{c}\text { NL } \\
\text { Si (g/L) }\end{array}$ \\
\hline EA & ref & reference & 1.2339 & 0.9612 & 1.1910 & 0.5918 & 17.137 & 9.146 & 15.524 & 3.906 \\
\hline KT04-01 & $\mathrm{ccc}$ & targeted & -0.2713 & -0.1739 & -0.2236 & -0.4039 & 0.535 & 0.670 & 0.598 & 0.395 \\
\hline KT04-01 & quenched & measured & -0.2600 & -0.1764 & -0.1836 & -0.3883 & 0.550 & 0.666 & 0.655 & 0.409 \\
\hline KT04-01 & quenched & targeted & -0.2685 & -0.1763 & -0.1853 & -0.3981 & 0.539 & 0.666 & 0.653 & 0.400 \\
\hline KT04-02 & quenched & measured & -0.2371 & -0.1649 & -0.1498 & -0.3589 & 0.579 & 0.684 & 0.708 & 0.438 \\
\hline KT04-02 & quenched & targeted & -0.2485 & -0.1584 & -0.1455 & -0.3666 & 0.564 & 0.694 & 0.715 & 0.430 \\
\hline KT04-03 & $\mathrm{ccc}$ & measured & -0.2047 & -0.1413 & -0.1509 & -0.3510 & 0.624 & 0.722 & 0.706 & 0.446 \\
\hline KT04-03 & $\mathrm{ccc}$ & targeted & -0.2113 & -0.1373 & -0.1399 & -0.3620 & 0.615 & 0.729 & 0.725 & 0.435 \\
\hline KT04-03 & quenched & measured & -0.2124 & -0.1477 & -0.1202 & -0.3397 & 0.613 & 0.712 & 0.758 & 0.457 \\
\hline KT04-03 & quenched & targeted & -0.2191 & -0.1437 & -0.1093 & -0.3507 & 0.604 & 0.718 & 0.778 & 0.446 \\
\hline KT04-05 & $\mathrm{ccc}$ & measured & -0.3183 & -0.1908 & -0.2033 & -0.3765 & 0.480 & 0.645 & 0.626 & 0.420 \\
\hline KT04-05 & $\mathrm{ccc}$ & targeted & -0.3250 & -0.1842 & -0.2095 & -0.3841 & 0.473 & 0.654 & 0.617 & 0.413 \\
\hline KT04-05 & quenched & measured & -0.3266 & -0.1880 & -0.1893 & -0.3770 & 0.471 & 0.649 & 0.647 & 0.420 \\
\hline KT04-05 & quenched & targeted & -0.3333 & -0.1815 & -0.1955 & -0.3846 & 0.464 & 0.658 & 0.637 & 0.412 \\
\hline KT04-06 & ccc & measured & -0.3404 & -0.2145 & -0.2625 & -0.3965 & 0.457 & 0.610 & 0.546 & 0.401 \\
\hline KT04-06 & $\mathrm{ccc}$ & targeted & -0.3482 & -0.2067 & -0.2568 & -0.4124 & 0.448 & 0.621 & 0.554 & 0.387 \\
\hline KT04-06 & quenched & measured & -0.3424 & -0.2230 & -0.2295 & -0.4021 & 0.455 & 0.598 & 0.590 & 0.396 \\
\hline KT04-06 & quenched & targeted & -0.3502 & -0.2152 & -0.2238 & -0.4181 & 0.446 & 0.609 & 0.597 & 0.382 \\
\hline KT04-07 & $\mathrm{ccc}$ & measured & -0.2970 & -0.2012 & -0.1769 & -0.3672 & 0.505 & 0.629 & 0.665 & 0.429 \\
\hline KT04-07 & $\mathrm{ccc}$ & targeted & -0.3090 & -0.1928 & -0.1794 & -0.3822 & 0.491 & 0.642 & 0.662 & 0.415 \\
\hline KT04-07 & quenched & measured & -0.3061 & -0.2152 & -0.1692 & -0.3701 & 0.494 & 0.609 & 0.677 & 0.427 \\
\hline KT04-09 & quenched & targeted & -0.2261 & -0.1520 & -0.1332 & -0.3500 & 0.594 & 0.705 & 0.736 & 0.447 \\
\hline KT04-10 & $\mathrm{ccc}$ & measured & -0.2430 & -0.1514 & -0.1827 & -0.3606 & 0.571 & 0.706 & 0.657 & 0.436 \\
\hline KT04-10 & $\mathrm{ccc}$ & targeted & -0.2515 & -0.1500 & -0.1864 & -0.3668 & 0.560 & 0.708 & 0.651 & 0.430 \\
\hline KT04-10 & quenched & measured & -0.2528 & -0.1623 & -0.1702 & -0.3663 & 0.559 & 0.688 & 0.676 & 0.430 \\
\hline KT04-10 & quenched & targeted & -0.2613 & -0.1609 & -0.1739 & -0.3725 & 0.548 & 0.690 & 0.670 & 0.424 \\
\hline
\end{tabular}




\section{Exhibit F-1. KT04 PCT Measurements (ppm values) in Analytical Sequence by Element.}

Variability Chart for B (ppm)

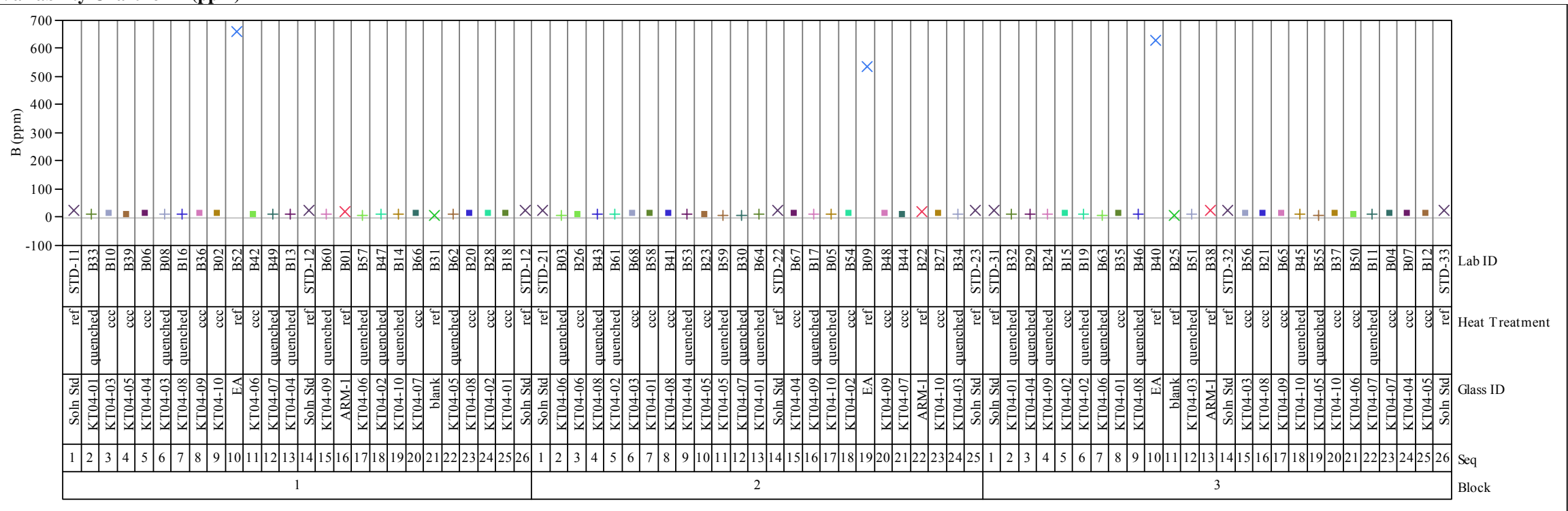




\section{Exhibit F-1. KT04 PCT Measurements (ppm values) in Analytical Sequence by Element. (continued)}

Variability Chart for Li (ppm)

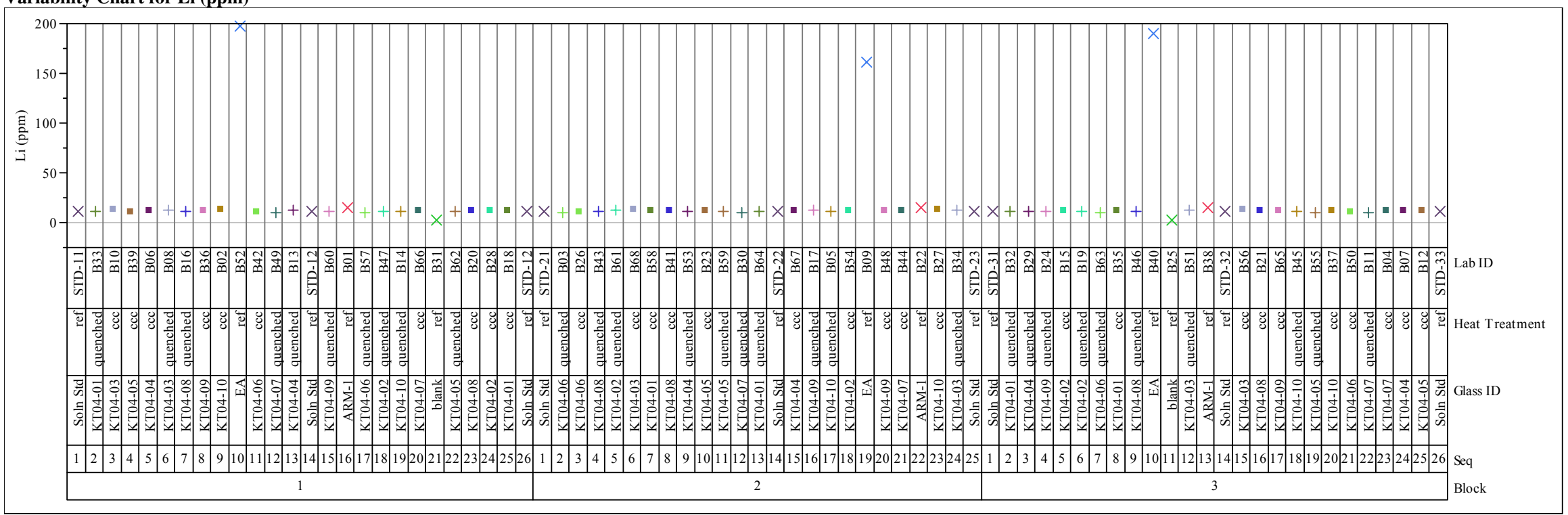




\section{Exhibit F-1. KT04 PCT Measurements (ppm values) in Analytical Sequence by Element. (continued)}

Variability Chart for Na (ppm)

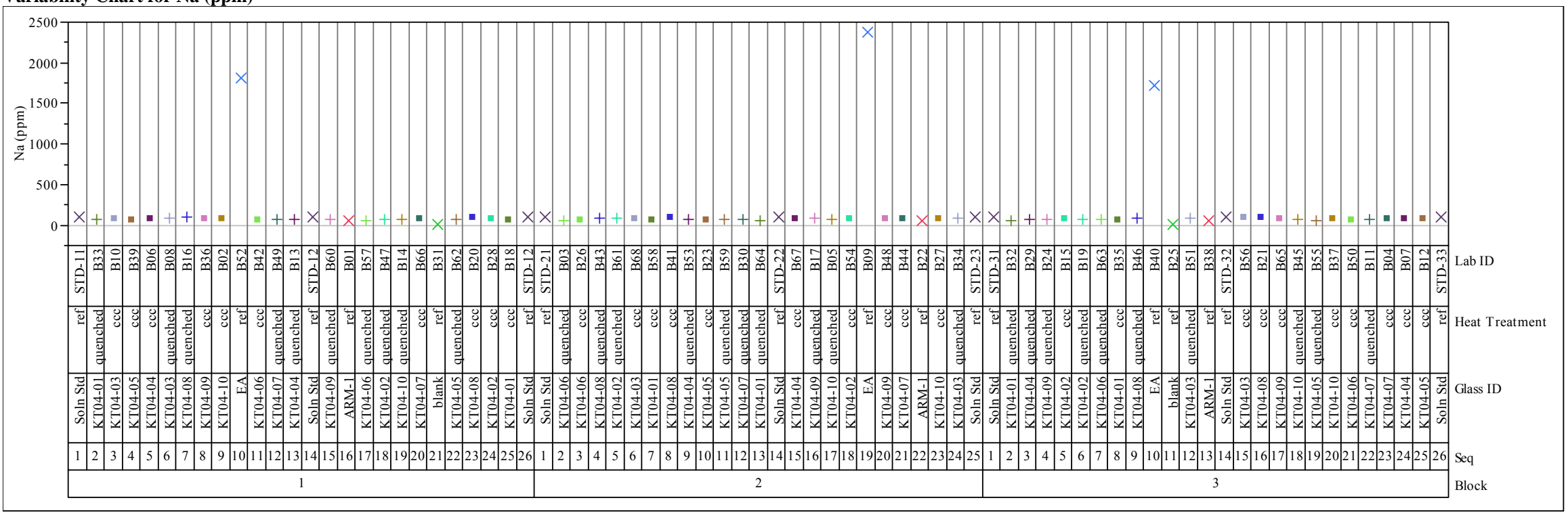




\section{Exhibit F-1. KT04 PCT Measurements (ppm values) in Analytical Sequence by Element. (continued)}

Variability Chart for Si (ppm)

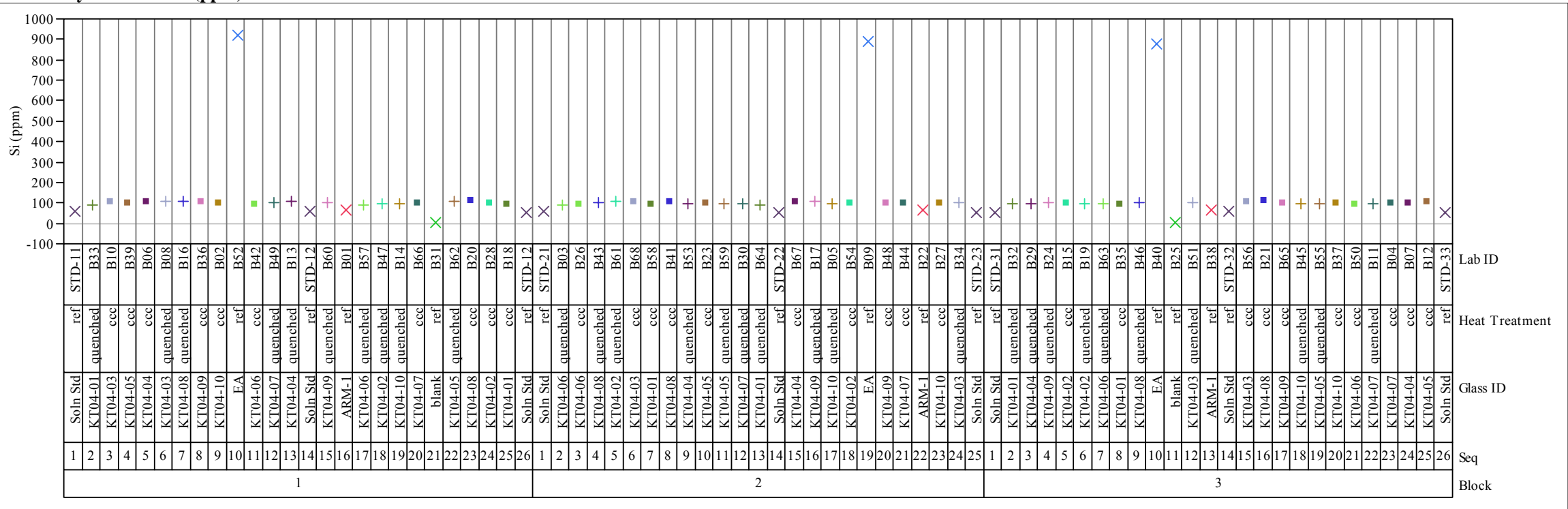


Exhibit F-2. Statistical Evaluation of the ICP-AES Calibration Effects from the KT04 Multi-Element Standard Solution Results by Oxide.

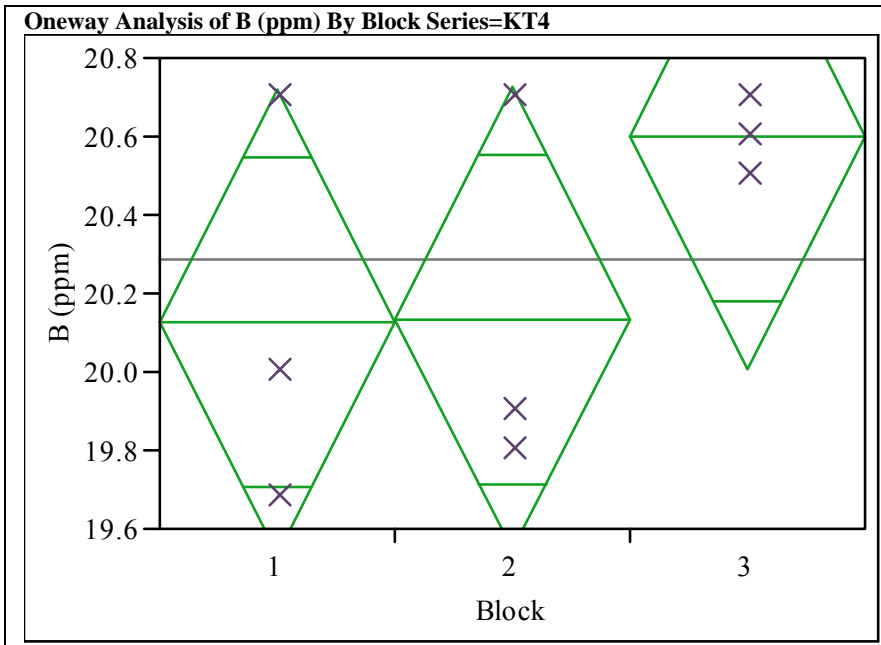

\section{Oneway Anova}

Summary

$$
\begin{array}{lr}
\text { Rsquare } & 0.295999 \\
\text { Adj Rsquare } & 0.061331 \\
\text { Root Mean Square Error } & 0.418516 \\
\text { Mean of Response } & 20.28667 \\
\text { Observations (or Sum Wgts) } & 9 \\
\multicolumn{2}{l}{\text { Analysis of Variance }}
\end{array}
$$

Source DF Sum of Squares Mean Square F Ratio Prob $>$ F

$\begin{array}{lrrrrr}\text { Block } & 2 & 0.4418667 & 0.220933 & 1.2614 & 0.3489\end{array}$

$\begin{array}{llll}\text { Error } & 6 & 1.0509333 & 0.175156\end{array}$

C. Total $8 \quad 1.4928000$

$$
0.175156
$$

Means for Oneway Anova

Level Number Mean Std Error Lower 95\% Upper 95\%

$\begin{array}{llllrr} & 3 & 20.1267 & 0.24163 & 19.535 & 20.718 \\ 1 & 3 & 20.1333 & 0.24163 & 19.542 & 20.725\end{array}$

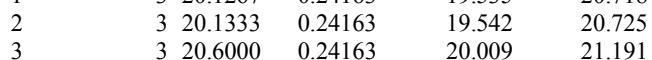

Std Error uses a pooled estimate of error variance

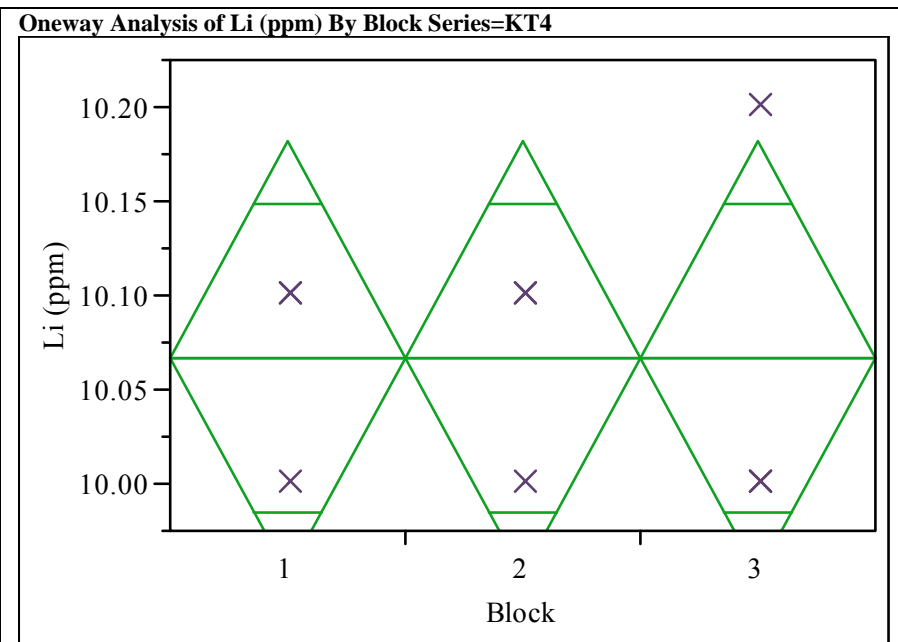

\section{Oneway Anova}

Summary of Fit

Rsquare
Adj Rsquare

$\begin{array}{lr}0.33333 \\ \text { Adj Rsquare } & -0.08165\end{array}$

Root Mean Square Error $\quad 0.08165$

Observations (or Sum Wgts)

Analysis of Variance

Source DF Sum of Squares Mean Square F Ratio Prob $>$ F

$\begin{array}{llllll}\text { Block } & 2 & 1.8933 \mathrm{e}-29 & 9.47 \mathrm{e}-30 & 0.0000 & 1.0000\end{array}$

$\begin{array}{lll}\text { Block } & 2 & 1.8933 \mathrm{e}-29 \\ \text { Error } & 6 & 0.04000000\end{array}$

C. Total $8 \quad 0.04000000$

\section{Means for Oneway Anova}

Level Number Mean Std Error Lower 95\% Upper 95\%

$\begin{array}{rrrrrr}1 & 3 & 10.0667 & 0.04714 & 9.9513 & 10.182 \\ 1 & 3 & 10.0667 & 0.04714 & 9.9513 & 10.182\end{array}$

$\begin{array}{llllll}2 & 3 & 10.0667 & 0.04714 & 9.9513 & 10.182 \\ 3 & 3 & 10.0667 & 0.04714 & 9.9513 & 10.182\end{array}$

Std Error uses a pooled estimate of error variance 
Exhibit F-2. Statistical Evaluation of the ICP-AES Calibration Effects from the KT04 Multi-Element Standard Solution Results by Oxide. (continued)

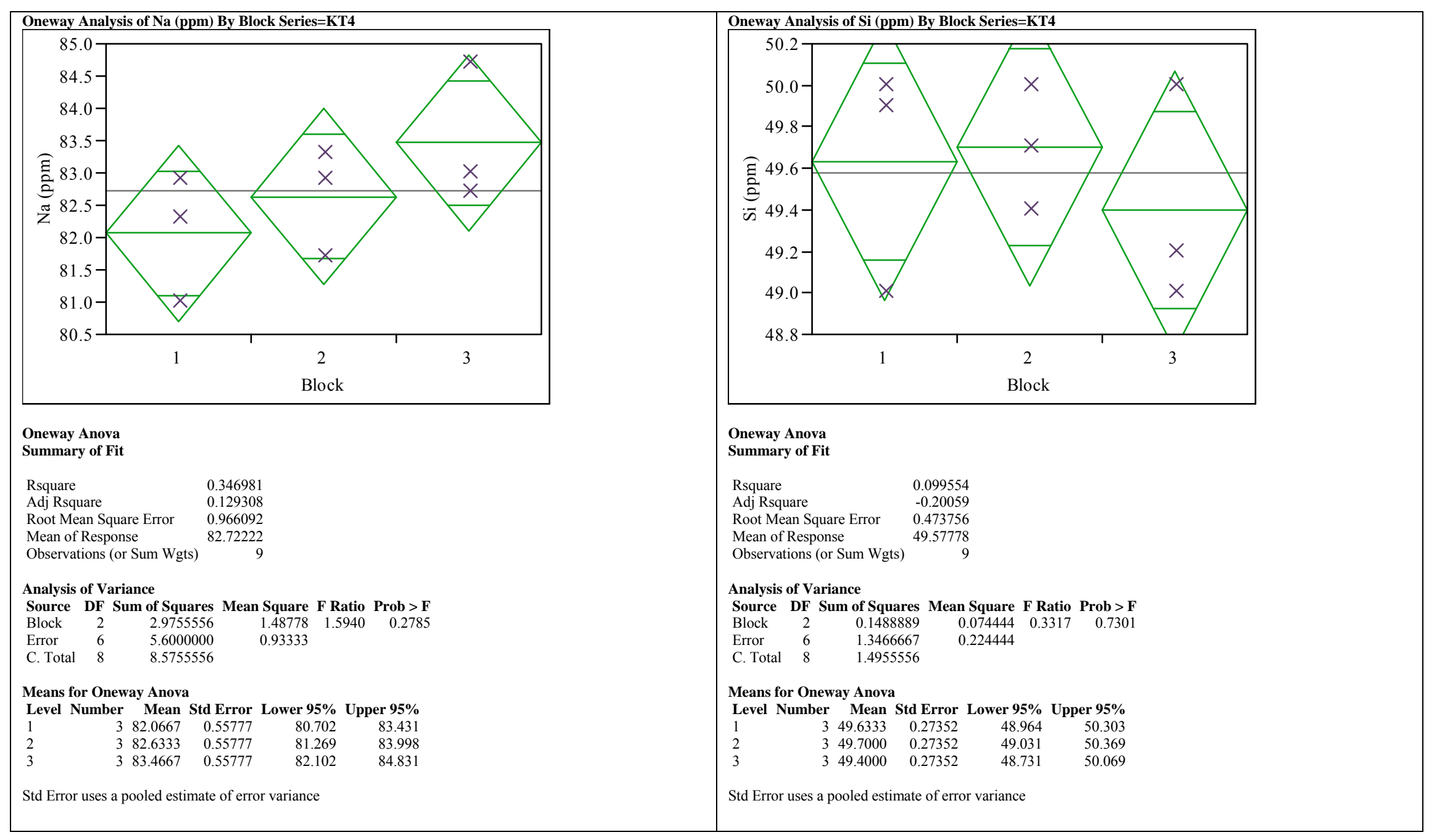




\section{Exhibit F-3. KT04 PCT Results (as common logarithms) Grouped by Glass ID and Heat Treatment.}

Variability Chart for log[B ppm]

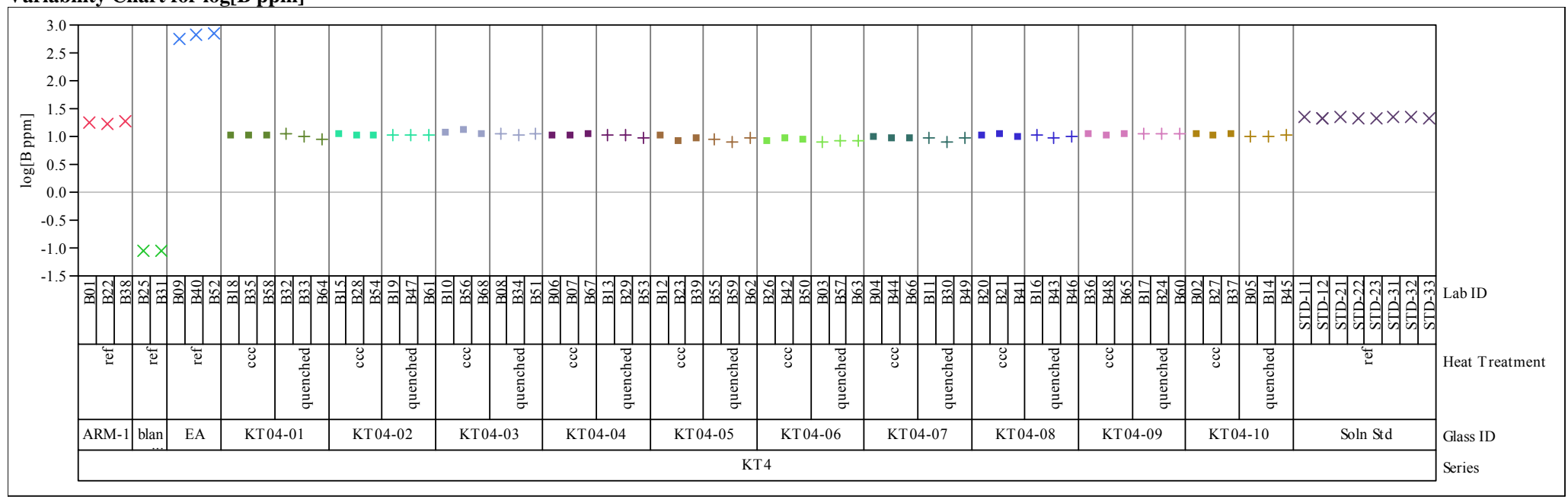

Variability Chart for $\log [\mathrm{Li}$ ppm]

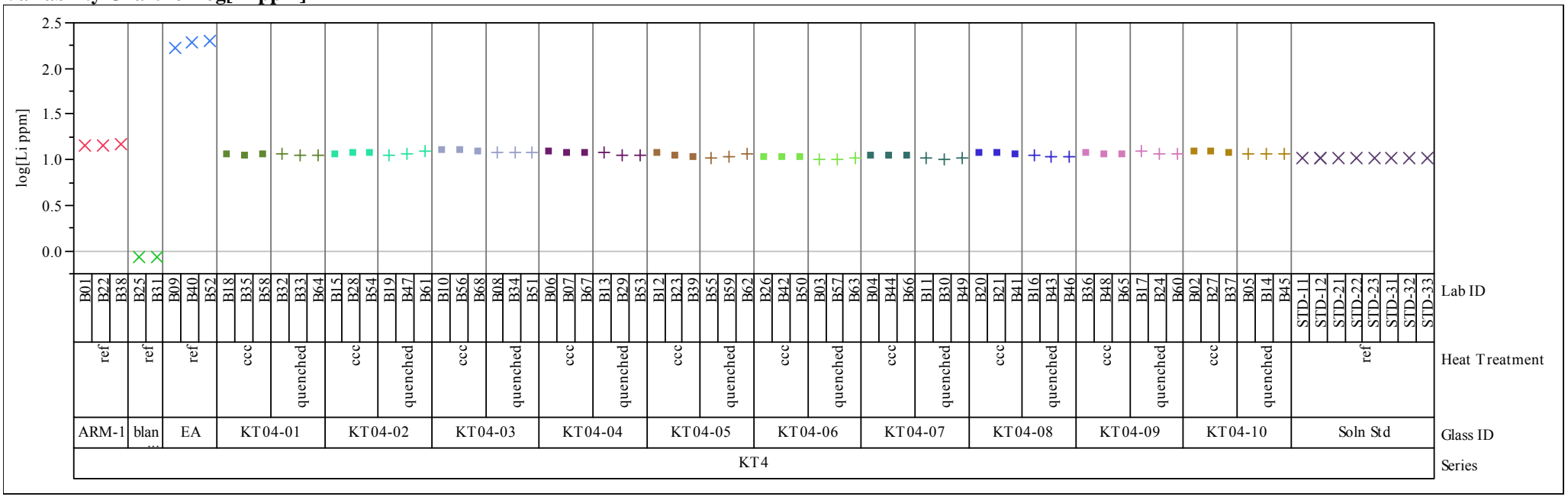


Exhibit F-3. KT04 PCT Results (as common logarithms) Grouped by Glass ID and Heat Treatment. (continued) Variability Chart for log[Na ppm]

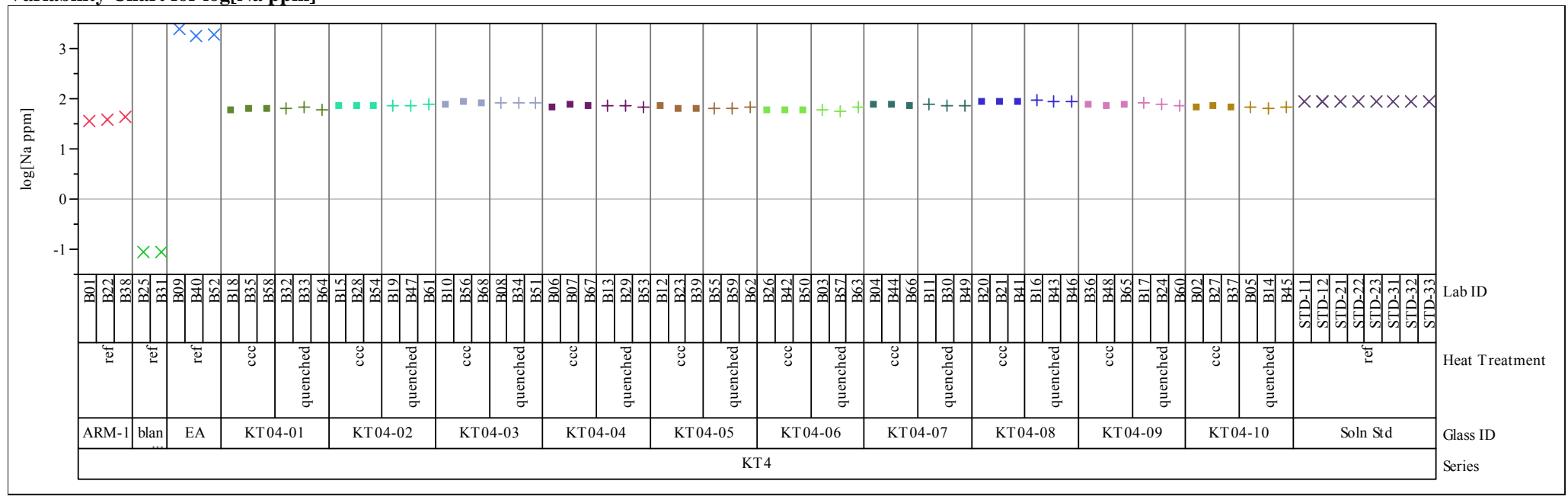

Variability Chart for log[Si ppm]

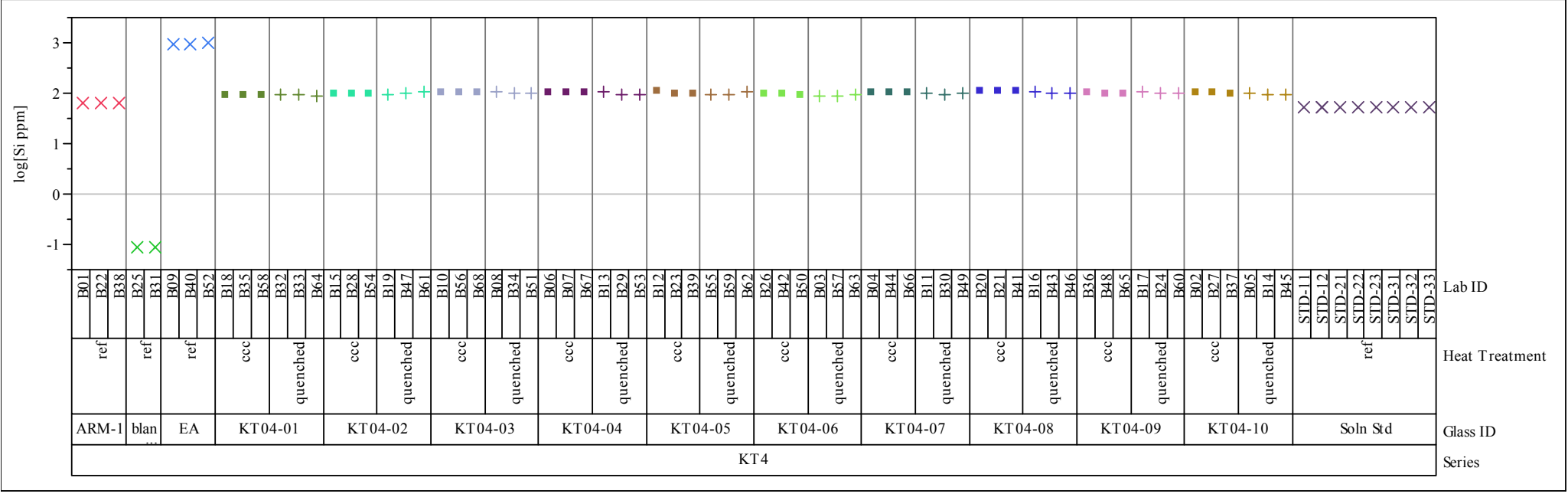


Exhibit F-4. Correlations and Scatter Plots of the KT01 Normalized PCTs Over All Compositional Views and Heat Treatments.

\section{Multivariate Correlations}

$\begin{array}{ccccc} & \log \text { NL[B (g/L)] } & \log \text { NL[Li(g/L)] } & \log \text { NL[Na (g/L)] } & \log \text { NL[Si (g/L)] } \\ \log \text { NL[B (g/L)] } & 1.0000 & 0.9962 & 0.9901 & 0.9802 \\ \log \text { NL[Li(g/L)] } & 0.9962 & 1.0000 & 0.9887 & 0.9833 \\ \log \text { NL[Na (g/L)] } & 0.9901 & 0.9887 & 1.0000 & 0.9902 \\ \log \text { NL[Si (g/L)] } & 0.9802 & 0.9833 & 0.9902 & 1.0000\end{array}$

\section{Scatterplot Matrix}

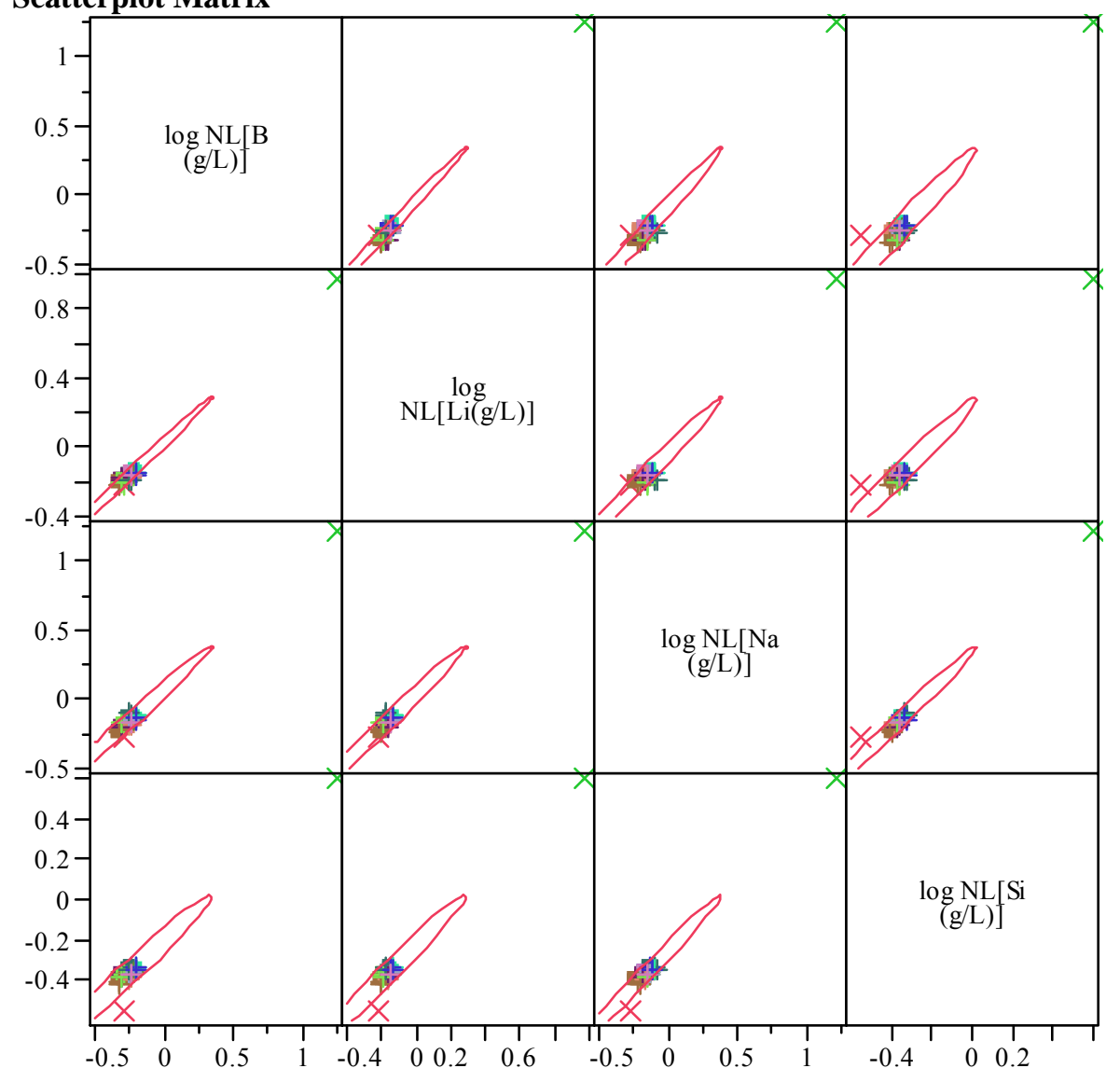


Exhibit F-5. Effects of Heat Treatment for the KT04 Glasses by Compositional View.
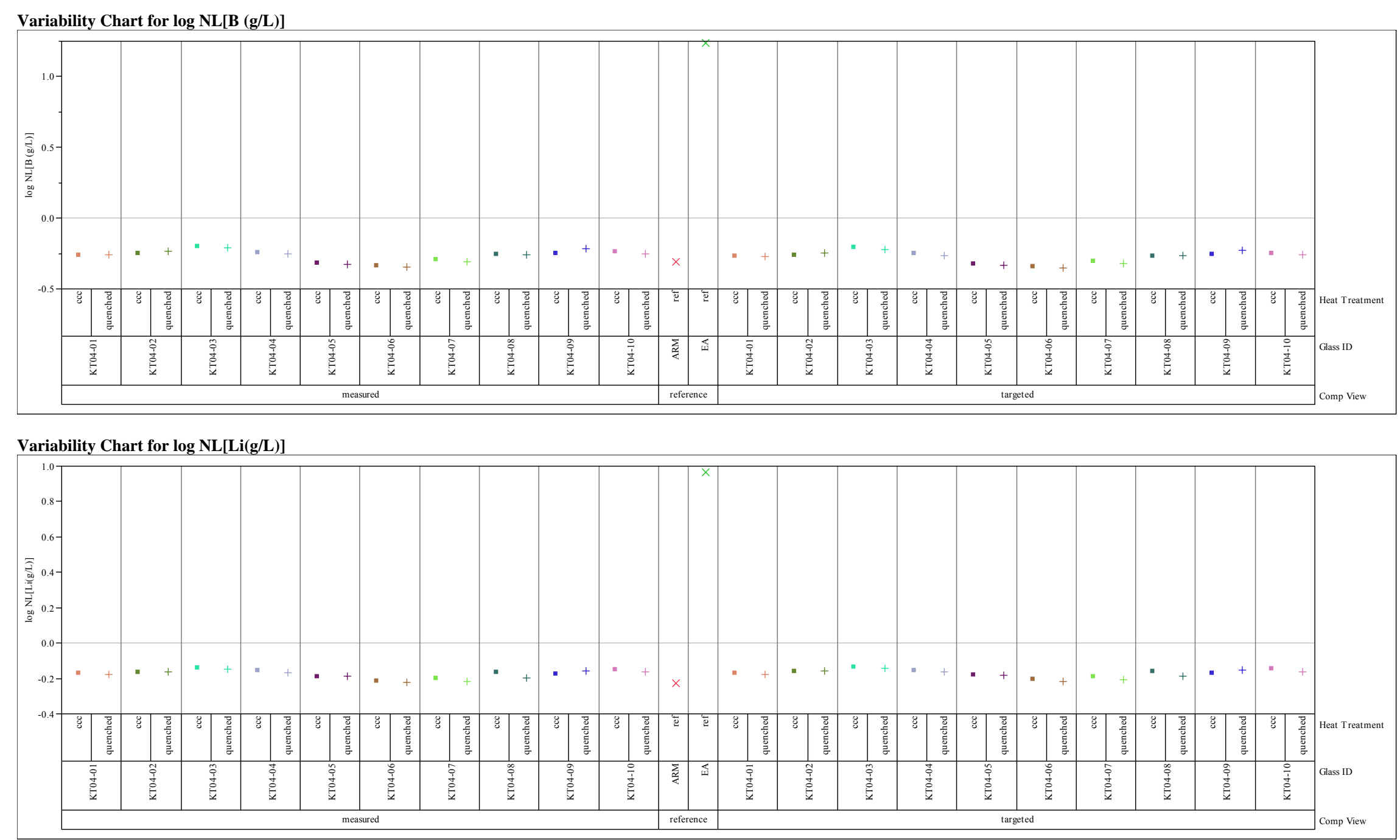


\section{Exhibit F-5. Effects of Heat Treatment for the KT04 Glasses by Compositional View. (continued)}

Variability Chart for log NL[Na (g/L)]

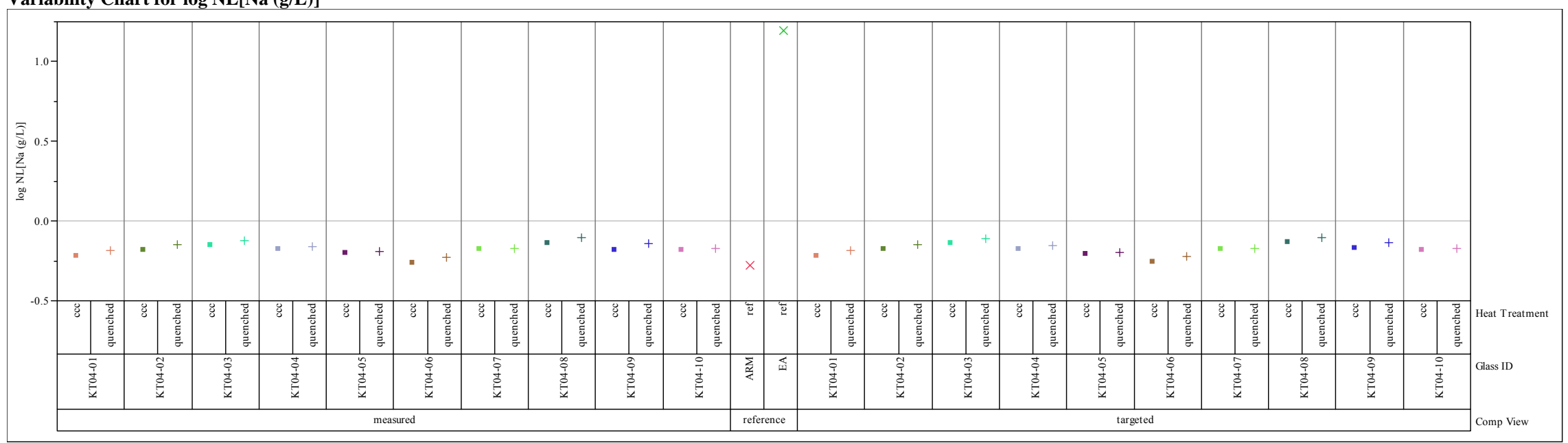

\section{Variability Chart for $\log \mathrm{NL}[\mathrm{Si}(\mathrm{g} / \mathrm{L})]$}

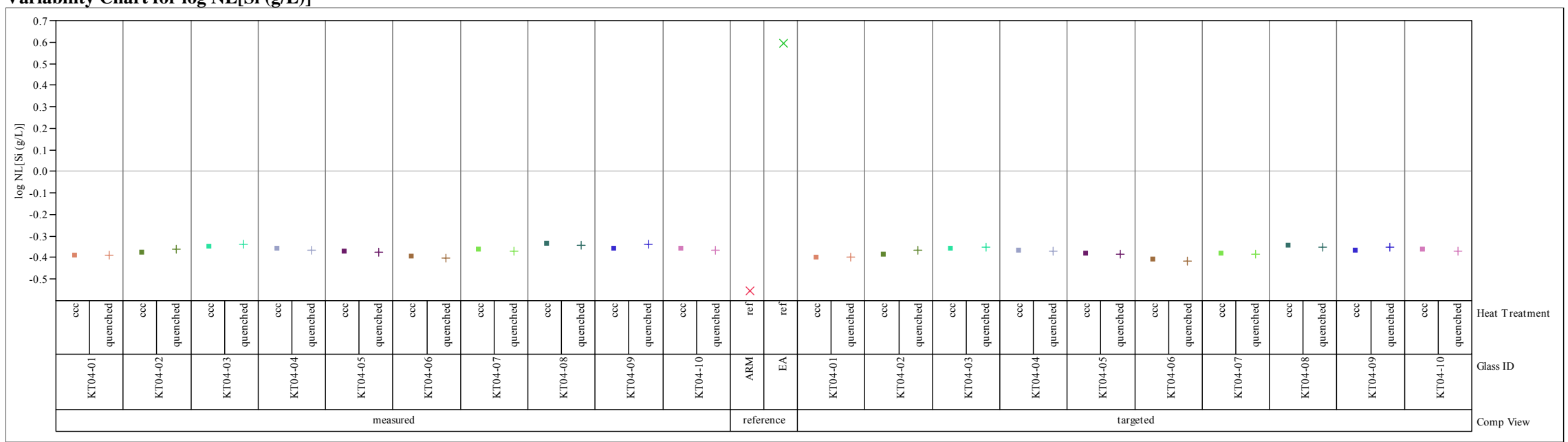


Exhibit F-6. KT04-Series PCT Measurements versus Durability Model Predictions.

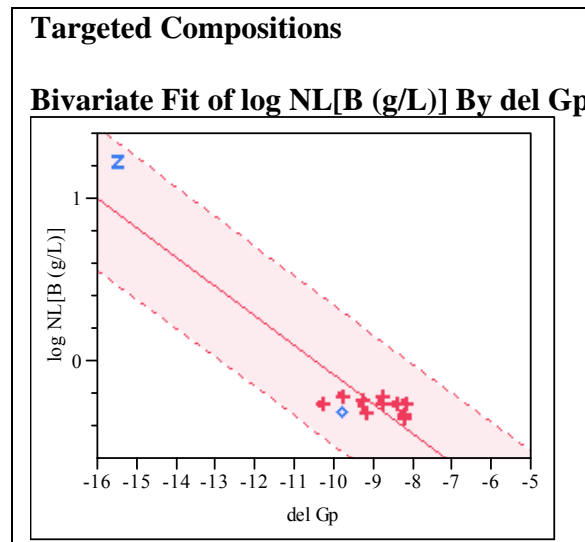

Bivariate Fit of $\log \mathrm{NL}[\mathrm{Li}(\mathrm{g} / \mathrm{L})]$ By del Gp

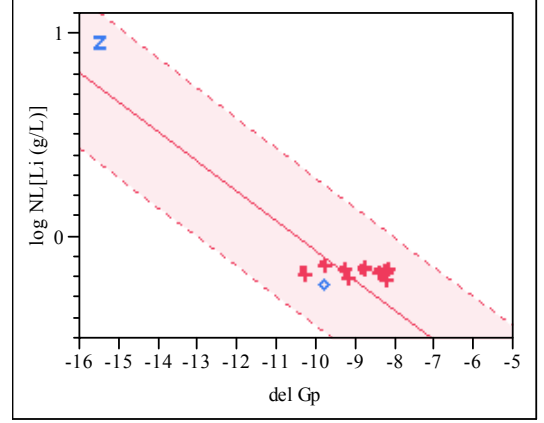

Bivariate Fit of $\log \mathrm{NL}[\mathrm{Na}(\mathrm{g} / \mathrm{L})]$ By del Gp

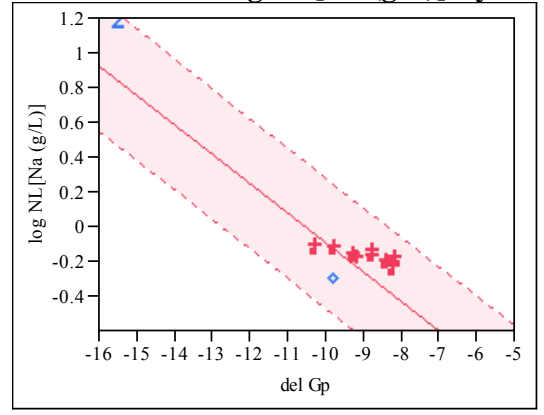

Bivariate Fit of $\log \mathrm{NL}[\mathrm{Si}(\mathrm{g} / \mathrm{L})]$ By del Gp

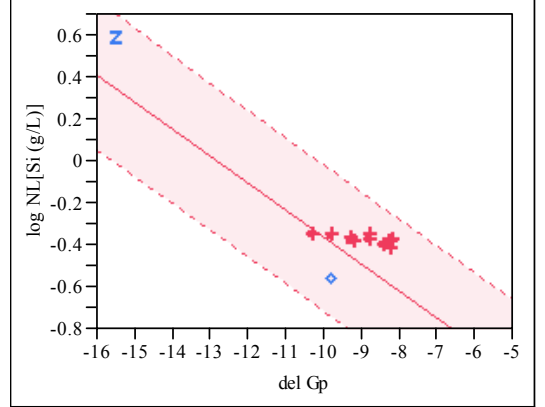

\section{Measured Compositions}

Bivariate Fit of $\log \mathrm{NL}[\mathrm{B}(\mathrm{g} / \mathrm{L})]$ By del Gp

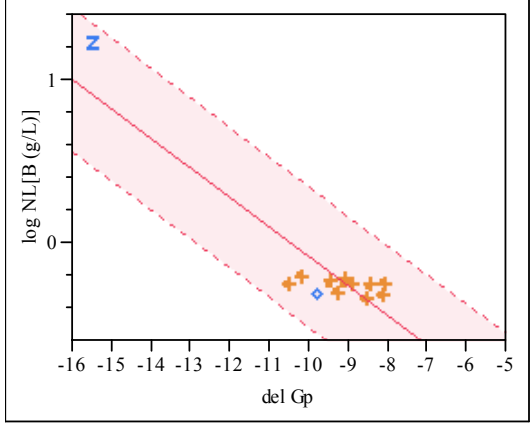

Bivariate Fit of $\log \mathrm{NL}[\mathrm{Li}(\mathrm{g} / \mathrm{L})]$ By del Gp

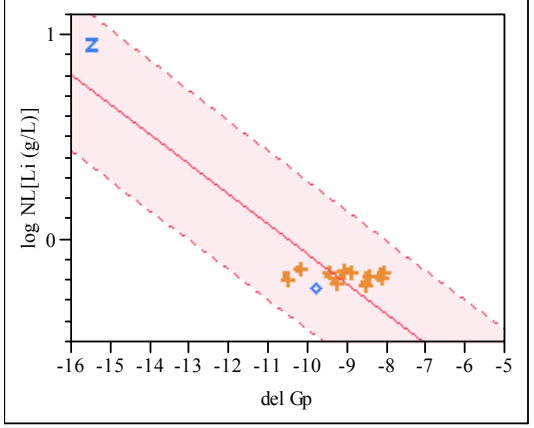

Bivariate Fit of $\log$ NL[Na $(\mathrm{g} / \mathrm{L})]$ By del Gp

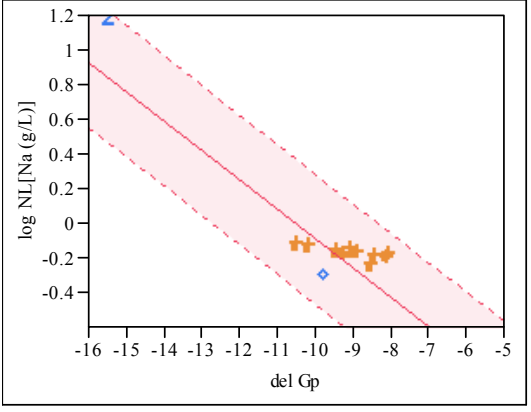

Bivariate Fit of $\log \mathrm{NL}[\mathrm{Si}(\mathrm{g} / \mathrm{L})]$ By del Gp

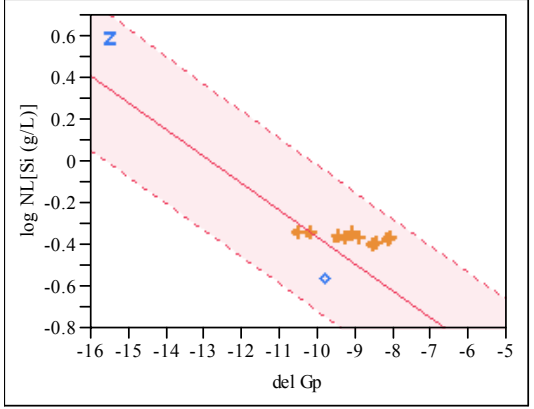

z Environmental Assessment Benchmark Glass

$\diamond$ Approved Reference Material Glass
++ Quenched Study Glasses

- Slowly Cooled Study Glasses 
SRNL-STI-2010-00566

Revision 0

Appendix G. Results from Fitting Fulcher Equations to the Viscosity Measurements of the KT01-Series Glasses 


\section{Exhibit G-1. Results from Fitting Fulcher Equations to the Viscosity Measurements of the} KT01-Series Glasses.

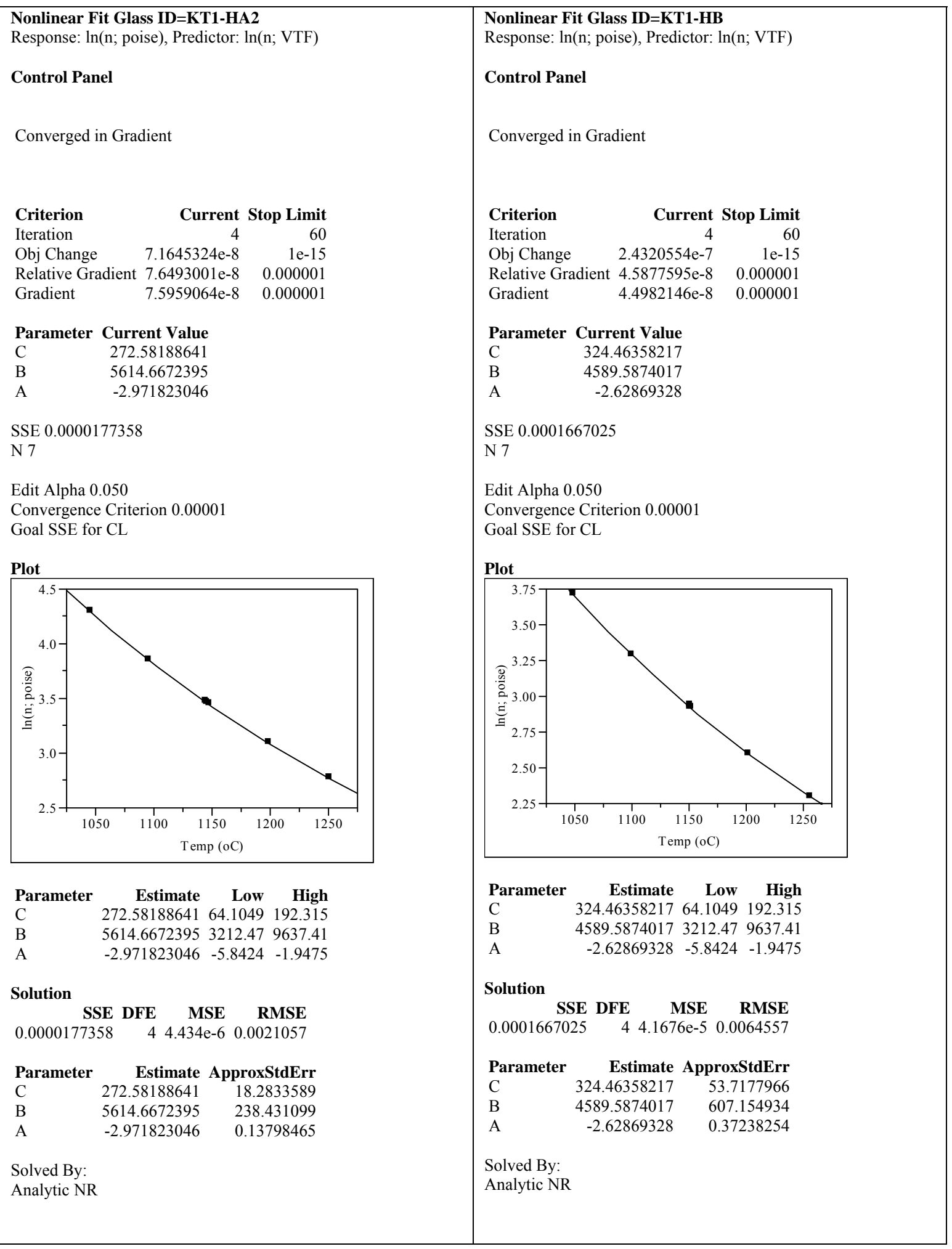




\section{Exhibit G-1. Results from Fitting Fulcher Equations to the Viscosity Measurements of the} KT01-Series Glasses. (continued)

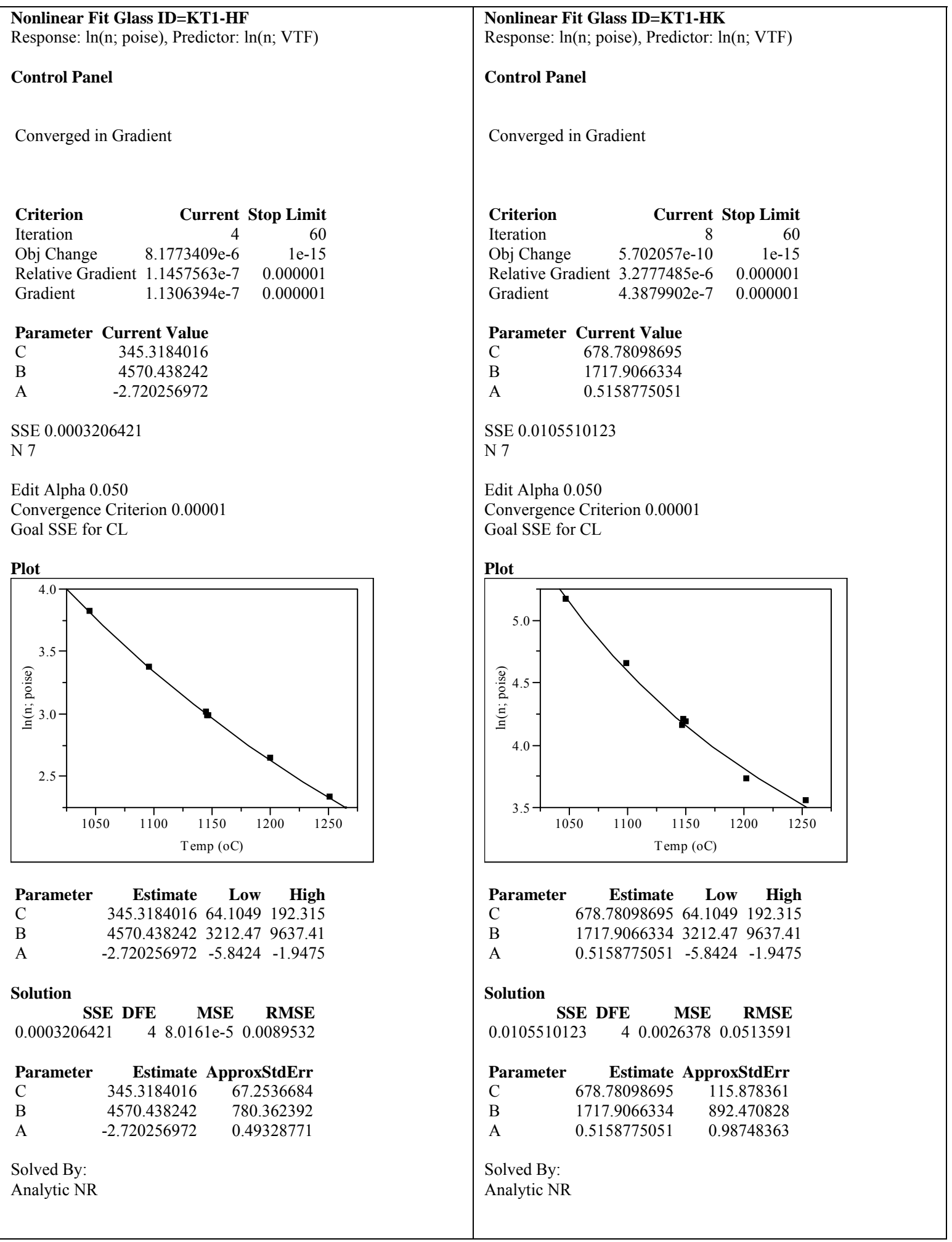




\section{Exhibit G-1. Results from Fitting Fulcher Equations to the Viscosity Measurements of the} KT01-Series Glasses. (continued)

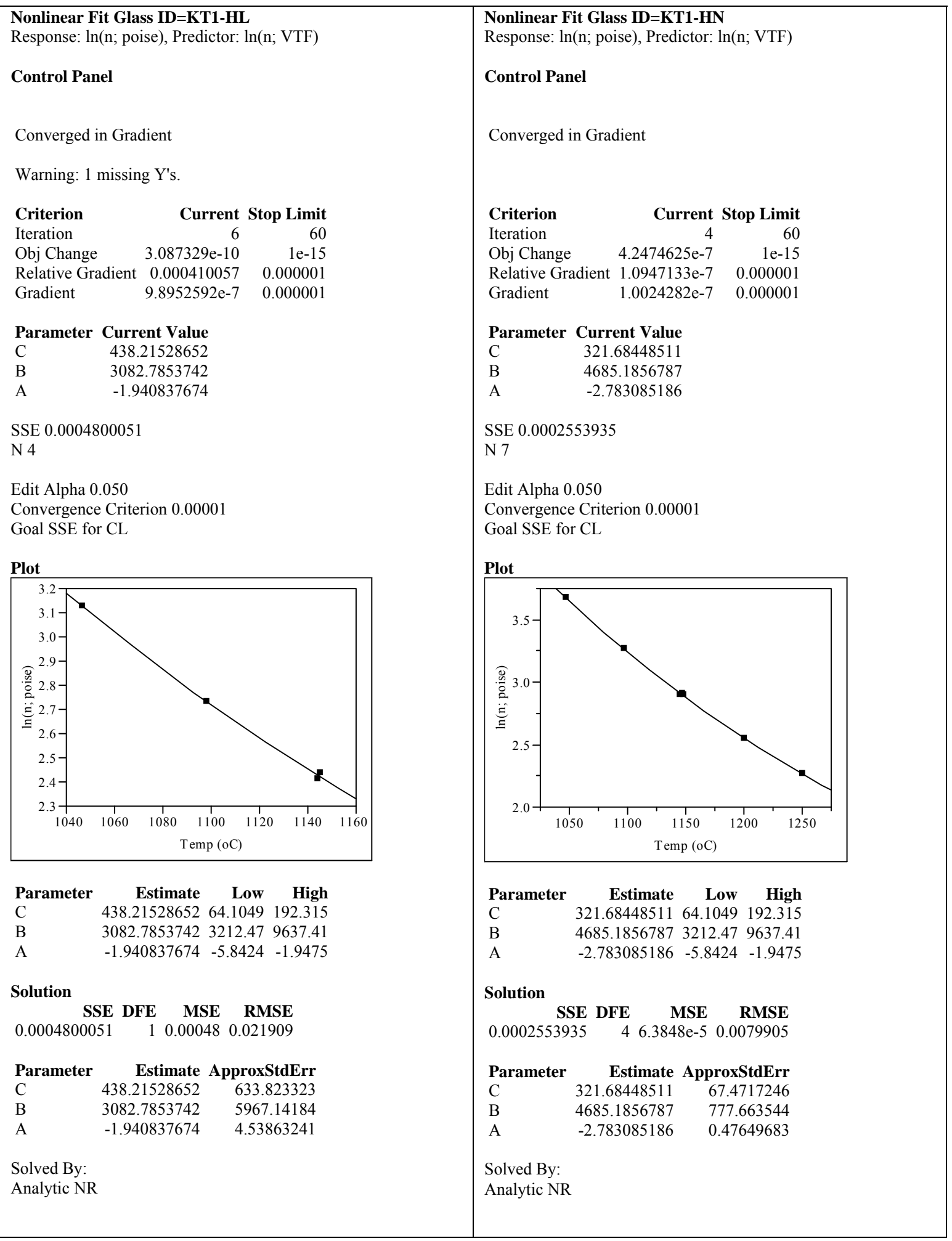




\section{Exhibit G-1. Results from Fitting Fulcher Equations to the Viscosity Measurements of the} KT01-Series Glasses. (continued)

\begin{tabular}{|c|c|}
\hline $\begin{array}{l}\text { Nonlinear Fit Glass ID=KT1-HS } \\
\text { Response: } \ln (n ; \text { poise }) \text {, Predictor: } \ln (n ; \text { VTF })\end{array}$ & $\begin{array}{l}\text { Nonlinear Fit Glass ID=KT1-LA } \\
\text { Response: } \ln (\mathrm{n} \text {; poise), Predictor: } \ln (\mathrm{n} ; \mathrm{VTF})\end{array}$ \\
\hline Control Panel & Control Panel \\
\hline Converged in Gradient & Converged in Gradient \\
\hline $\begin{array}{lrr}\text { Criterion } & \text { Current } & \text { Stop Limit } \\
\text { Iteration } & 4 & 60 \\
\text { Obj Change } & 7.341071 \mathrm{e}-11 & 1 \mathrm{e}-15 \\
\text { Relative Gradient } & 2.0745692 \mathrm{e}-9 & 0.000001 \\
\text { Gradient } & 1.1252127 \mathrm{e}-9 & 0.000001\end{array}$ & $\begin{array}{lrr}\text { Criterion } & \text { Current } & \text { Stop Limit } \\
\text { Iteration } & 4 & 60 \\
\text { Obj Change } & 6.107442 \mathrm{e}-7 & 1 \mathrm{e}-15 \\
\text { Relative Gradient } & 4.4833869 \mathrm{e}-8 & 0.000001 \\
\text { Gradient } & 4.4270611 \mathrm{e}-8 & 0.000001\end{array}$ \\
\hline $\begin{array}{lr}\text { Parameter } & \text { Current Value } \\
\text { C } & 207.43634541 \\
\text { B } & 6676.2470589 \\
\text { A } & -3.591204552\end{array}$ & $\begin{array}{lr}\text { Parameter } & \text { Current Value } \\
\text { C } & 318.75966405 \\
\text { B } & 4779.9084894 \\
\text { A } & -2.763798524\end{array}$ \\
\hline $\begin{array}{l}\text { SSE } 0.0000426649 \\
\text { N } 6\end{array}$ & $\begin{array}{l}\text { SSE } 0.0006001086 \\
\text { N } 7\end{array}$ \\
\hline $\begin{array}{l}\text { Edit Alpha } 0.050 \\
\text { Convergence Criterion } 0.00001 \\
\text { Goal SSE for CL }\end{array}$ & $\begin{array}{l}\text { Edit Alpha } 0.050 \\
\text { Convergence Criterion } 0.00001 \\
\text { Goal SSE for CL }\end{array}$ \\
\hline$\underbrace{1}_{1050}{ }_{1100}^{1}$ & Plot \\
\hline $\begin{array}{lrrr}\text { Parameter } & \text { Estimate } & \text { Low } & \text { High } \\
\text { C } & 207.43634541 & 64.1049 & 192.315 \\
\text { B } & 6676.2470589 & 3212.47 & 9637.41 \\
\text { A } & -3.591204552 & -5.8424 & -1.9475\end{array}$ & $\begin{array}{lrrr}\text { Parameter } & \text { Estimate } & \text { Low } & \text { High } \\
\text { C } & 318.75966405 & 64.1049 & 192.315 \\
\text { B } & 4779.9084894 & 3212.47 & 9637.41 \\
\text { A } & -2.763798524 & -5.8424 & -1.9475\end{array}$ \\
\hline 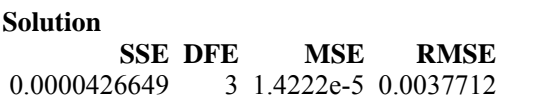 & $\begin{array}{rrrrr}\text { Solution } & & & & \\
\text { SSE } & \text { DFE } & \text { MSE } & \text { RMSE } \\
0.0006001086 & 4 & 0.00015 & 0.0122486\end{array}$ \\
\hline $\begin{array}{lrr}\text { Parameter } & \text { Estimate } & \text { ApproxStdErr } \\
\text { C } & 207.43634541 & 37.6916267 \\
\text { B } & 6676.2470589 & 541.779711 \\
\text { A } & -3.591204552 & 0.2904694\end{array}$ & $\begin{array}{lrr}\text { Parameter } & \text { Estimate } & \text { ApproxStdErr } \\
\mathrm{C} & 318.75966405 & 99.8128742 \\
\mathrm{~B} & 4779.9084894 & 1169.39902 \\
\mathrm{~A} & -2.763798524 & 0.71383863\end{array}$ \\
\hline $\begin{array}{l}\text { Solved By: } \\
\text { Analytic NR }\end{array}$ & $\begin{array}{l}\text { Solved By: } \\
\text { Analytic NR }\end{array}$ \\
\hline
\end{tabular}




\section{Exhibit G-1. Results from Fitting Fulcher Equations to the Viscosity Measurements of the} KT01-Series Glasses. (continued)

\begin{tabular}{|c|c|}
\hline $\begin{array}{l}\text { Nonlinear Fit Glass ID=KT1-LB } \\
\text { Response: } \ln (n \text {; poise), Predictor: } \ln (n \text {; VTF) }\end{array}$ & $\begin{array}{l}\text { Nonlinear Fit Glass ID=KT1-LF } \\
\text { Response: } \ln (\mathrm{n} \text {; poise), Predictor: } \ln (\mathrm{n} ; \mathrm{VTF})\end{array}$ \\
\hline Control Panel & Control Panel \\
\hline Converged in Gradient & Converged in Gradient \\
\hline Current Stop Limit & Current Stop Limit \\
\hline Iteration & $4 \quad 60$ \\
\hline $2.8131587 \mathrm{e}-8$ & $2.850766 \mathrm{e}-10$ \\
\hline Relative Gradient $6.149598 \mathrm{e}-10 \quad 0.000001$ & Relative Gradient $3.059204 \mathrm{e}-10 \quad 0.000001$ \\
\hline Gradient $\quad 4.860766 \mathrm{e}-10 \quad 0.000001$ & Gradient $\quad 1.282965 \mathrm{e}-10 \quad 0.000001$ \\
\hline Parameter Current Value & Parameter Current Value \\
\hline C $\quad 275.98447951$ & $\begin{array}{ll}\mathrm{C} & 228.74616791\end{array}$ \\
\hline 5370.8378721 & 6086.9880788 \\
\hline-3.018664365 & -3.359017457 \\
\hline SSE & \\
\hline $\begin{array}{l}0.0001054217 \mathrm{~N} \\
7\end{array}$ & $\begin{array}{l}\text { SSE } 0.0000934483 \\
\text { N } 7\end{array}$ \\
\hline $\begin{array}{l}\text { Edit Alpha } 0.050 \\
\text { Convergence Criterion } 0.00001 \\
\text { Goal SSE for CL }\end{array}$ & $\begin{array}{l}\text { Edit Alpha } 0.050 \\
\text { Convergence Criterion } 0.00001 \\
\text { Goal SSE for CL }\end{array}$ \\
\hline Plot & Plot \\
\hline $\begin{array}{ccccc}1 & 1 & 1 & 1 & 1 \\
1050 & 1100 & 1150 & 1200 & 1250 \\
& & \end{array}$ & $\begin{array}{cccc}1050 & 1100 & 1150 & 1200 \\
& & 1150 & \\
\text { Temp }(\mathrm{oC}) & \end{array}$ \\
\hline Estimate Low High & Estimate Low \\
\hline $275.98447951 \quad 64.1049 \quad 192.315$ & $228.74616791 \quad 64.1049 \quad 192.315$ \\
\hline $\begin{array}{llll}5370.8378721 & 3212.47 & 9637.41\end{array}$ & $6086.9880788 \quad 3212.479637 .41$ \\
\hline $\begin{array}{lll}-3.018664365 & -5.8424 & -1.9475\end{array}$ & $\begin{array}{lll}-3.359017457 & -5.8424 & -1.9475\end{array}$ \\
\hline Solution & Solution \\
\hline $\begin{array}{rrrr}\text { SSE } & \text { DFE } & \text { MSE } & \text { RMSE } \\
0.0001054217 & 4 & 2.6355 \mathrm{e}-5 & 0.0051338\end{array}$ & $\begin{array}{rrrr}\text { SSE } & \text { DFE } & \text { MSE } & \text { RMSE } \\
0.0000934483 & 4 & 2.3362 \mathrm{e}-5 & 0.0048334\end{array}$ \\
\hline Estimate ApproxStdErr & Estimate ApproxStdErr \\
\hline 275.98447951 & 228.74616791 \\
\hline 5370.8378721 & 6086.9880788 \\
\hline-3.018664365 & -3.359017457 \\
\hline $\begin{array}{l}\text { Solved By: } \\
\text { Analytic NR }\end{array}$ & $\begin{array}{l}\text { Solved By: } \\
\text { Analytic NR }\end{array}$ \\
\hline
\end{tabular}




\section{Exhibit G-1. Results from Fitting Fulcher Equations to the Viscosity Measurements of the} KT01-Series Glasses. (continued)

\begin{tabular}{|c|c|}
\hline \multirow{2}{*}{$\begin{array}{l}\text { Nonlinear Fit Glass ID=KT1-LL } \\
\text { Response: } \ln (\mathrm{n} \text {; poise), Predictor: } \ln (\mathrm{n} \text {; VTF) }\end{array}$} & \multirow{2}{*}{$\begin{array}{l}\text { Nonlinear Fit Glass ID=KT1-LN } \\
\text { Response: } \ln (n \text {; poise), Predictor: } \ln (n ; \text { VTF) }\end{array}$} \\
\hline & \\
\hline Control Panel & Control Panel \\
\hline Converged in Gradient & Converged in Gradient \\
\hline Current Stop Limit & Current Stop Limit \\
\hline Iteration & Iteration \\
\hline $4.4618791 \mathrm{e}-8$ & $2.426674 \mathrm{e}-12$ \\
\hline Relative Gradient $7.9156147 \mathrm{e}-9 \quad 0.000001$ & Relative Gradient $1.6302144 \mathrm{e}-9 \quad 0.000001$ \\
\hline Gradient $\quad 4.0694908 \mathrm{e}-9 \quad 0.000001$ & Gradient $\quad 2.950439 \mathrm{e}-10 \quad 0.000001$ \\
\hline Parameter Current Value & Parameter Current Value \\
\hline 277.95408035 & 188.5707596 \\
\hline 5404.9672434 & 6788.5163104 \\
\hline-3.150404726 & -3.729581521 \\
\hline $\begin{array}{l}\text { SSE } 0.0003785986 \\
\text { N } 7\end{array}$ & $\begin{array}{l}\text { SSE } 0.0001099648 \\
\text { N } 7\end{array}$ \\
\hline $\begin{array}{l}\text { Edit Alpha } 0.050 \\
\text { Convergence Criterion } 0.00001 \\
\text { Goal SSE for CL }\end{array}$ & $\begin{array}{l}\text { Edit Alpha } 0.050 \\
\text { Convergence Criterion } 0.00001 \\
\text { Goal SSE for CL }\end{array}$ \\
\hline Plot & Plot \\
\hline $\begin{array}{ccccc}1 & \mid & \mid & \mid & \mid \\
1050 & 1100 & 1150 & 1200 & 1250\end{array}$ & $\begin{array}{llll}1050 & 1100 & 1150 & 1200\end{array}$ \\
\hline Estimate Low High & Parameter \\
\hline $277.95408035 \quad 64.1049 \quad 192.315$ & $188.5707596 \quad 64.1049 \quad 192.315$ \\
\hline $5404.9672434 \quad 3212.479637 .41$ & $6788.5163104 \quad 3212.479637 .41$ \\
\hline $\begin{array}{lll}-3.150404726 & -5.8424 & -1.9475\end{array}$ & $\begin{array}{lll}-3.729581521 & -5.8424 & -1.9475\end{array}$ \\
\hline Solution & Solution \\
\hline $\begin{array}{rrrr}\text { SSE } & \text { DFE } & \text { MSE } & \text { RMSE } \\
0.0003785986 & 4 & 9.465 \mathrm{e}-5 & 0.0097288\end{array}$ & $\begin{array}{rrrr}\text { SSE } & \text { DFE } & \text { MSE } & \text { RMSE } \\
0.0001099648 & 4 & 0.0000275 & 0.0052432\end{array}$ \\
\hline Estimate ApproxStdErr & Estimate ApproxStdErr \\
\hline $\begin{array}{lll}\mathrm{C} & 277.95408035 & 87.5110799\end{array}$ & $\begin{array}{lll}\mathrm{C} & 188.5707596 & 55.8320281\end{array}$ \\
\hline $5404.9672434 \quad 1098.17012$ & 6788.5163104 \\
\hline-3.150404726 & -3.729581521 \\
\hline $\begin{array}{l}\text { Solved By: } \\
\text { Analytic NR }\end{array}$ & $\begin{array}{l}\text { Solved By: } \\
\text { Analytic NR }\end{array}$ \\
\hline
\end{tabular}


Exhibit G-1. Results from Fitting Fulcher Equations to the Viscosity Measurements of the KT01-Series Glasses. (continued)

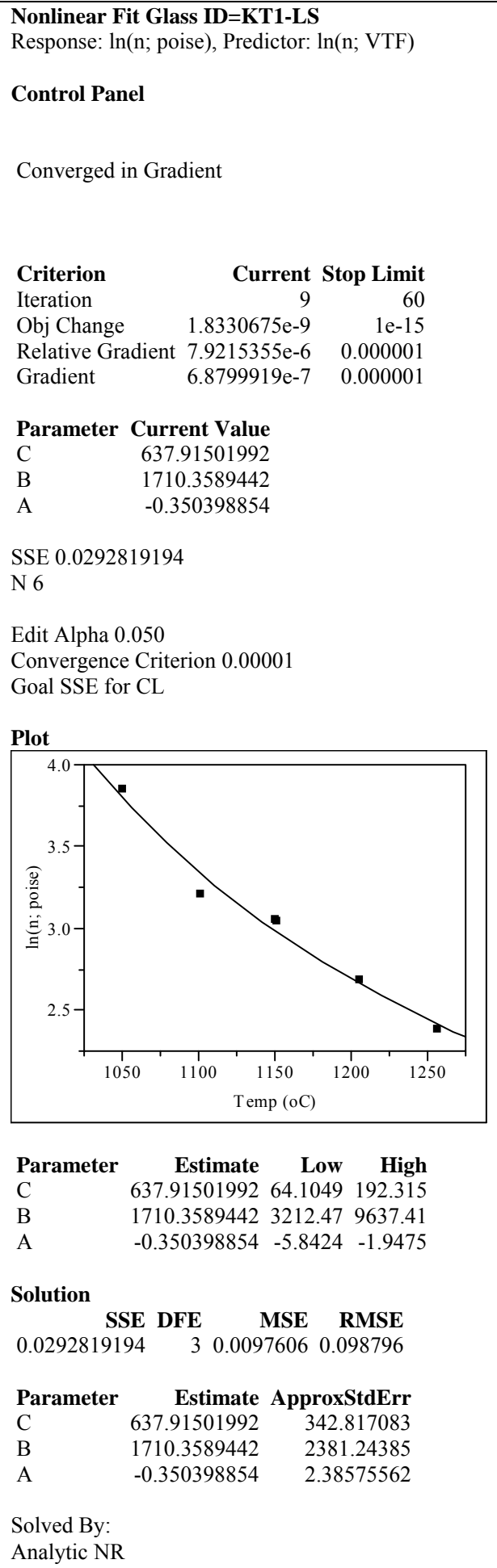


SRNL-STI-2010-00566

Revision 0

Appendix H. Results from Fitting Fulcher Equations to the Viscosity Measurements for KT03-Series Glasses 
Exhibit H-1. Results from Fitting Fulcher Equations to the Viscosity Measurements of the KT03-Series Glasses.

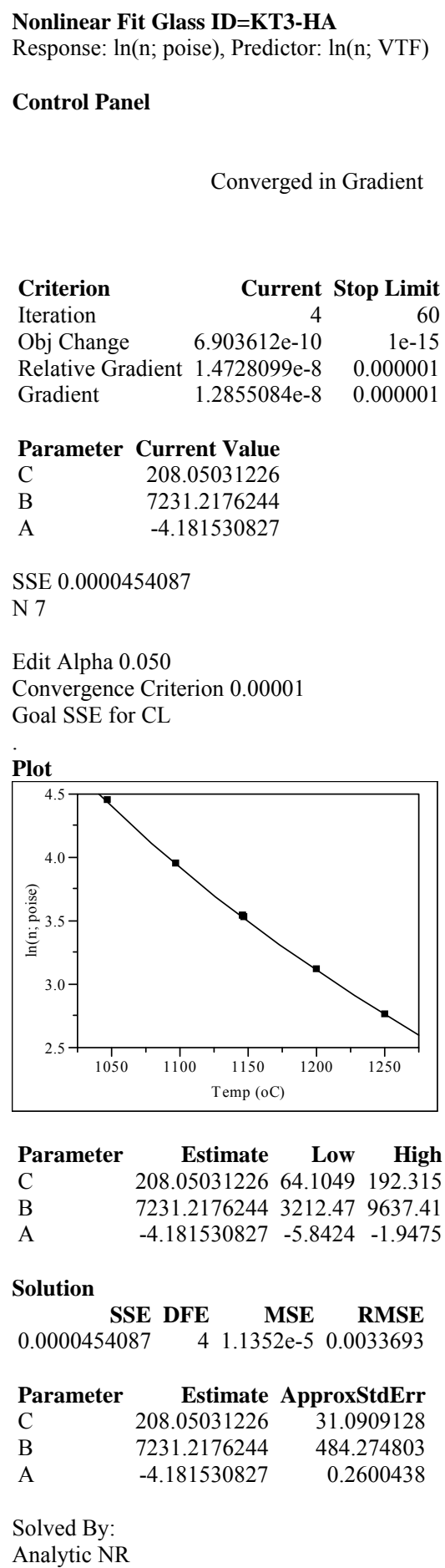

Nonlinear Fit Glass ID=KT3-HB

Response: $\ln (\mathrm{n}$; poise), Predictor: $\ln (\mathrm{n}$; VTF)

Control Panel

Converged in Gradient

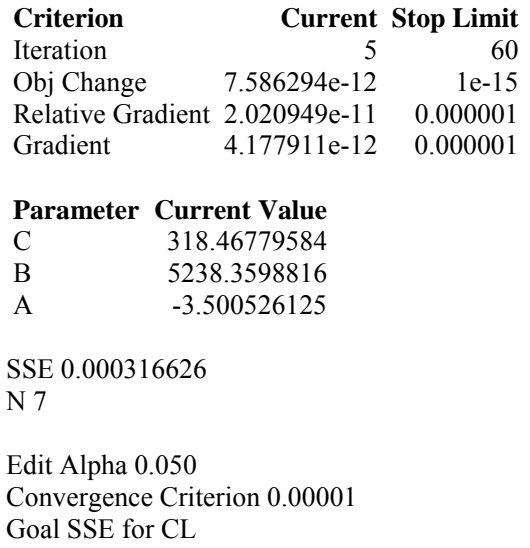

Plot

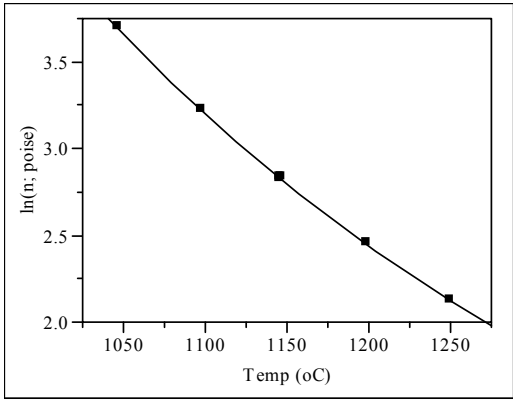

Parameter Estimate Low High

$\begin{array}{llll}\text { C } & 318.46779584 & 64.1049 & 192.315\end{array}$

B $\quad 5238.3598816 \quad 3212.479637 .41$

$\begin{array}{lllll}\text { A } & -3.500526125 & -5.8424 & -1.9475\end{array}$

Solution

SSE DFE MSE RMSE

$\begin{array}{llll}0.000316626 & 4 & 7.9157 \mathrm{e}-5 & 0.008897\end{array}$

Parameter Estimate ApproxStdErr

$\begin{array}{lll}\text { C } & 318.46779584 & 68.22062\end{array}$

$\begin{array}{lll}\text { B } & 5238.3598816 & 876.872918\end{array}$

$\begin{array}{lll}\text { A } & -3.500526125 & 0.53595608\end{array}$

Solved By:

Analytic NR 
Exhibit H-1. Results from Fitting Fulcher Equations to the Viscosity Measurements of the KT03-Series Glasses. (continued)

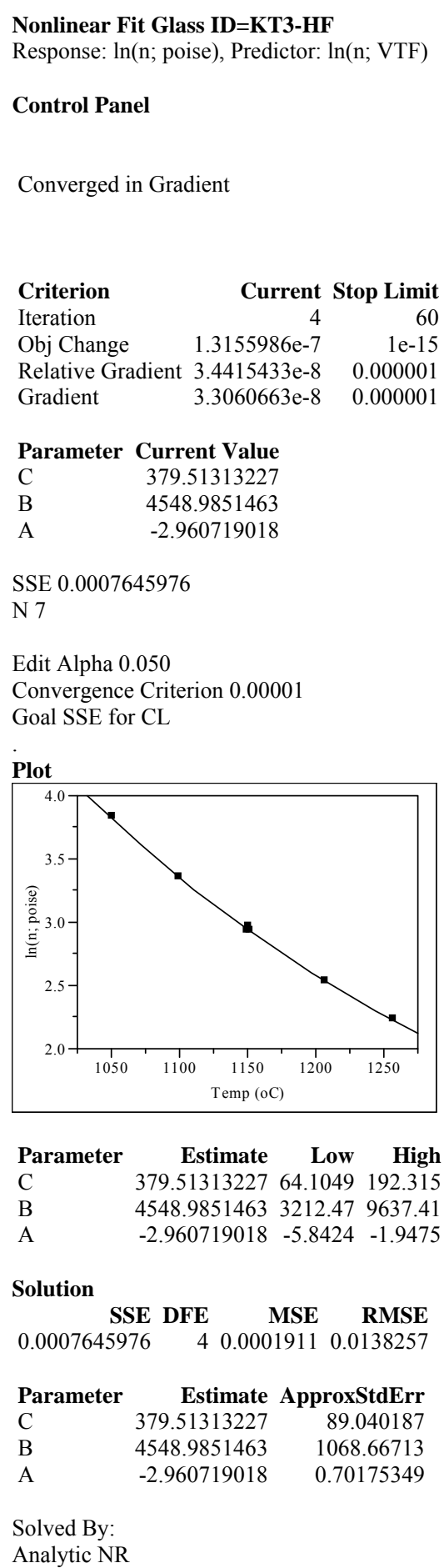

\section{Nonlinear Fit Glass ID=KT3-HK}

Response: $\ln (\mathrm{n}$; poise), Predictor: $\ln (\mathrm{n}$; VTF)

Control Panel

Converged in Gradient

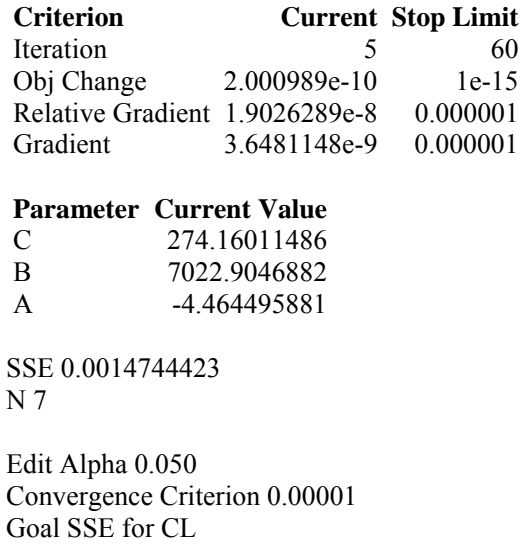

Plot

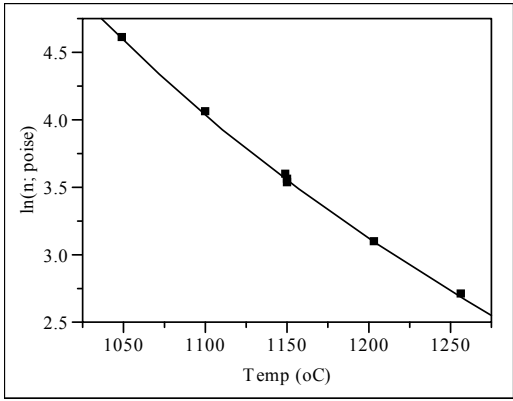

Parameter Estimate Low High

$\begin{array}{llll}\text { C } & 274.16011486 & 64.1049 & 192.315\end{array}$

$\begin{array}{lllll}\text { B } & 7022.9046882 & 3212.47 & 9637.41\end{array}$

$\begin{array}{llll}\text { A } & -4.464495881 & -5.8424 & -1.9475\end{array}$

Solution

SSE DFE MSE RMSE $\begin{array}{llll}0.0014744423 \quad 4 & 0.0003686 & 0.0191992\end{array}$

Parameter Estimate ApproxStdErr

$\begin{array}{llr}\text { C } & 274.16011486 & 132.96427\end{array}$

$\begin{array}{lll}\text { B } & 7022.9046882 & 2160.50683\end{array}$

$\begin{array}{lll}\text { A } & -4.464495881 & 1.24546739\end{array}$

Solved By:

Analytic NR 
Exhibit H-1. Results from Fitting Fulcher Equations to the Viscosity Measurements of the KT03-Series Glasses. (continued)

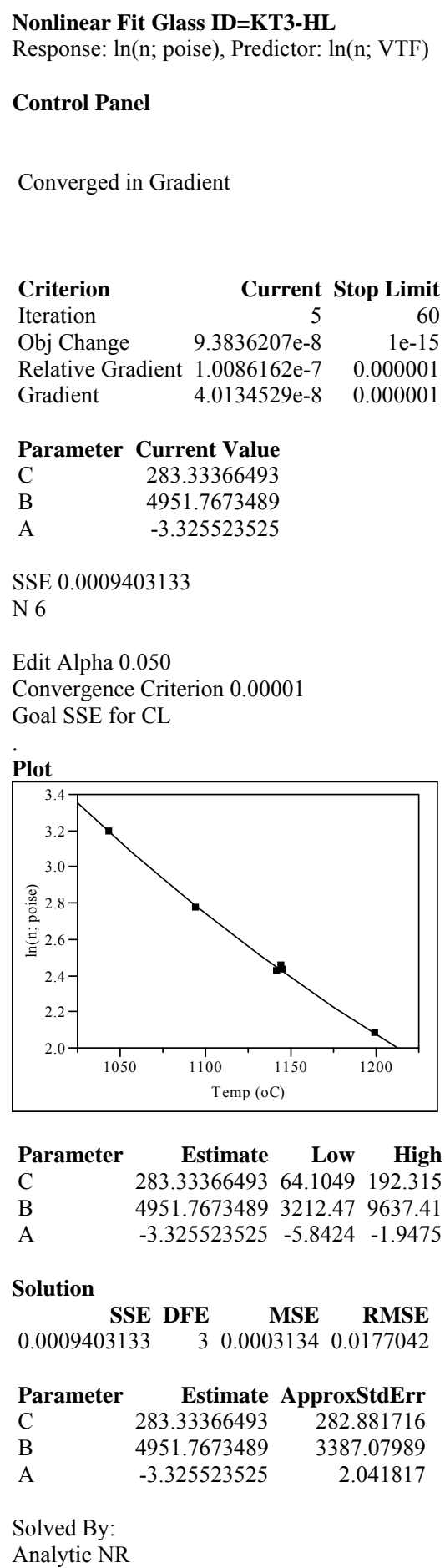

\section{Nonlinear Fit Glass ID=KT3-HN}

Response: $\ln (n$; poise), Predictor: $\ln (n$; VTF)

Control Panel

Converged in Gradient

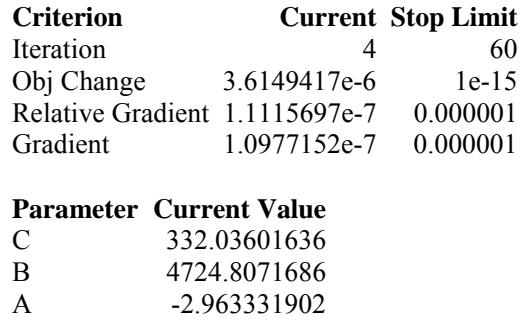

Plot

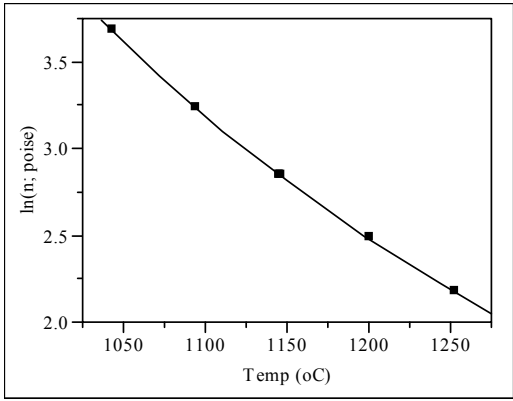

Parameter Estimate Low High

$\begin{array}{llll}\text { C } & 332.03601636 & 64.1049 & 192.315\end{array}$

B $\quad 4724.8071686 \quad 3212.479637 .41$

$\begin{array}{llll}\text { A } & -2.963331902 & -5.8424 & -1.9475\end{array}$

Solution

SSE DFE MSE RMSE

$\begin{array}{llll}0.0000814272 & 4 & 2.0357 \mathrm{e}-5 & 0.0045119\end{array}$

Parameter Estimate ApproxStdErr

$\begin{array}{llr}\text { C } & 332.03601636 & 33.8667161\end{array}$

$\begin{array}{lll}\text { B } & 4724.8071686 & 399.808301\end{array}$

$\begin{array}{lll}\text { A } & -2.963331902 & 0.24873021\end{array}$

Solved By:

Analytic NR 
Exhibit H-1. Results from Fitting Fulcher Equations to the Viscosity Measurements of the KT03-Series Glasses. (continued)

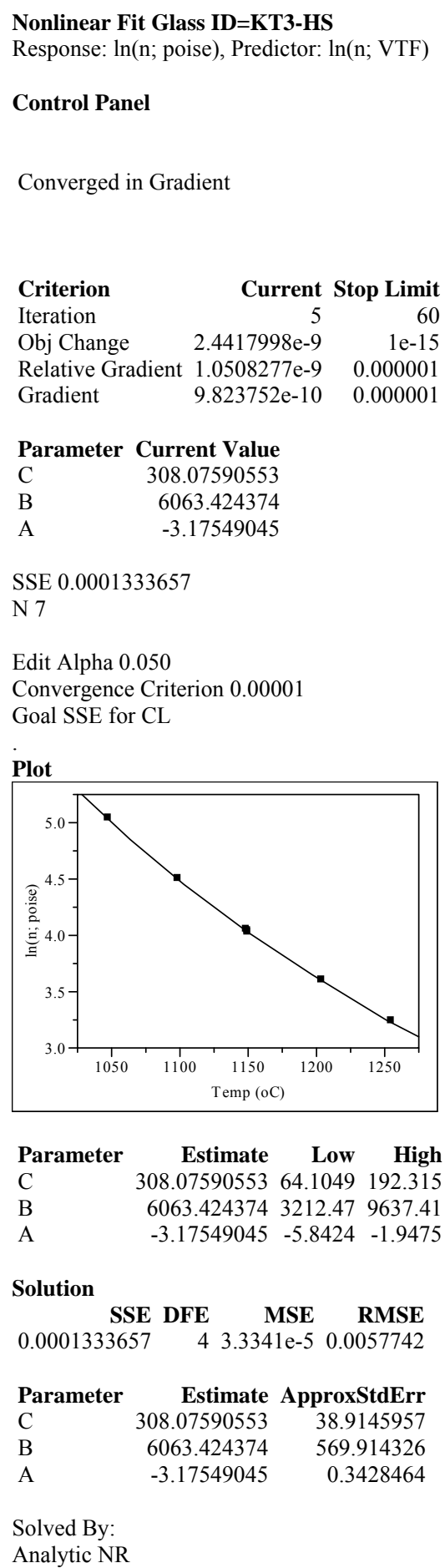

Nonlinear Fit Glass ID=KT3-LA

Response: $\ln (\mathrm{n}$; poise), Predictor: $\ln (\mathrm{n}$; VTF)

Control Panel

Converged in Gradient

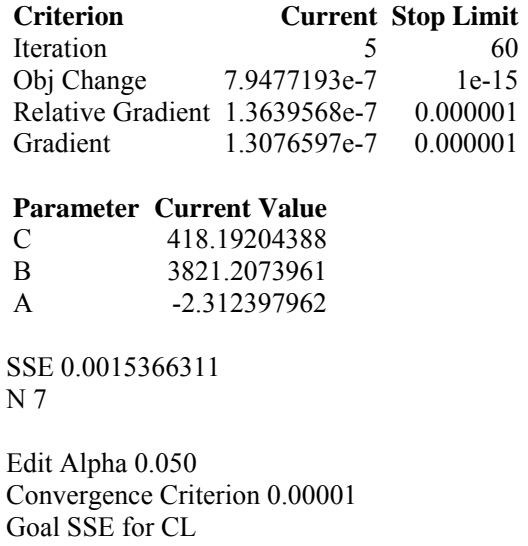

Plot

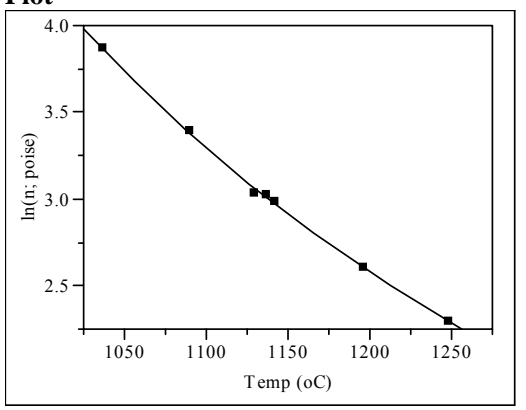

Parameter Estimate Low High

$\begin{array}{llll}\text { C } & 418.19204388 & 64.1049 & 192.315\end{array}$

$\begin{array}{llll}\text { B } & 3821.2073961 & 3212.47 & 9637.41\end{array}$

$\begin{array}{llll}\text { A } & -2.312397962 & -5.8424 & -1.9475\end{array}$

Solution

SSE DFE MSE RMSE

$\begin{array}{llll}0.0015366311 & 4 & 0.0003842 & 0.0195999\end{array}$

Parameter Estimate ApproxStdErr

$\begin{array}{llr}\text { C } & 418.19204388 & 108.882141\end{array}$

$\begin{array}{lll}\text { B } & 3821.2073961 & 1175.78498\end{array}$

$\begin{array}{lll}\text { A } & -2.312397962 & 0.82643608\end{array}$

Solved By:

Analytic NR 
Exhibit H-1. Results from Fitting Fulcher Equations to the Viscosity Measurements of the KT03-Series Glasses. (continued)

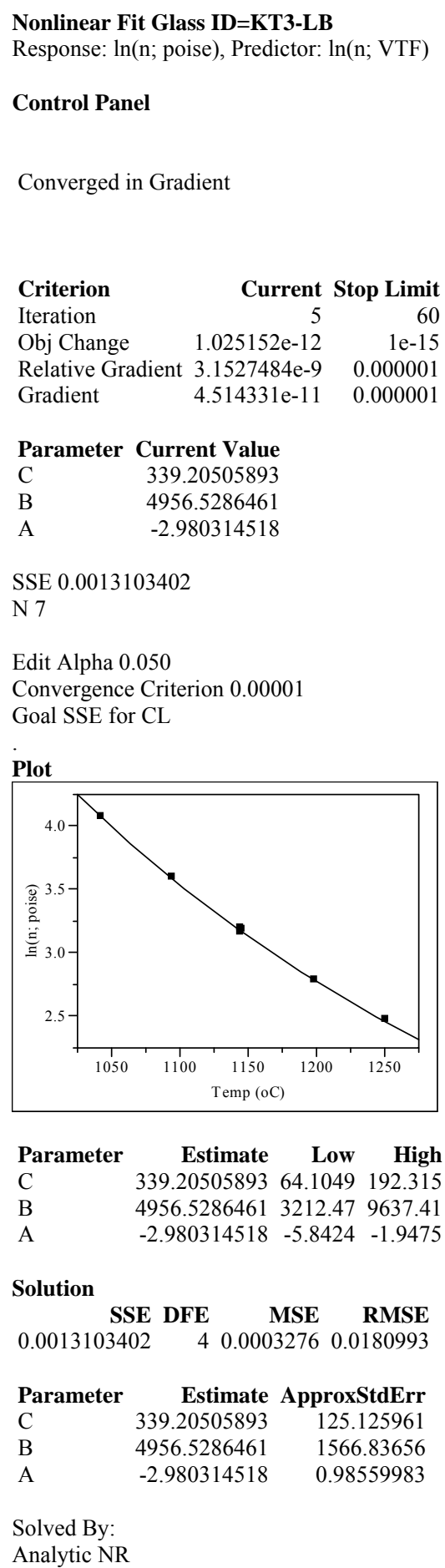

\section{Nonlinear Fit Glass ID=KT3-LF}

Response: $\ln (\mathrm{n}$; poise), Predictor: $\ln (\mathrm{n}$; VTF)

Control Panel

Converged in Gradient

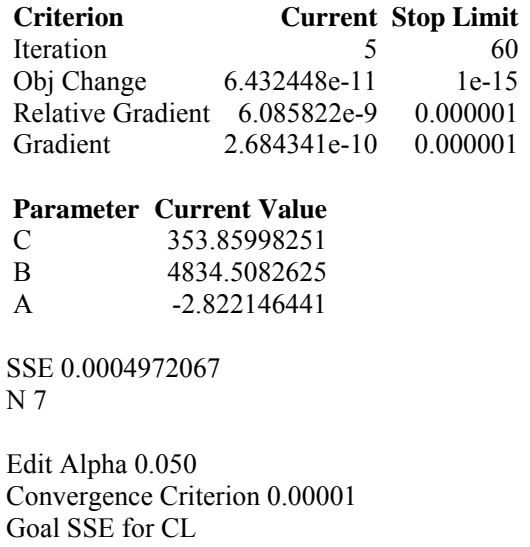

Plot

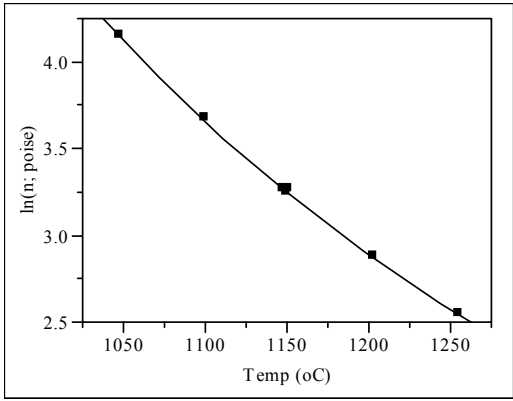

Parameter Estimate Low High

C $\quad 353.8599825164 .1049 \quad 192.315$

B $\quad 4834.5082625 \quad 3212.479637 .41$

$\begin{array}{llll}\text { A } & -2.822146441 & -5.8424 & -1.9475\end{array}$

Solution

SSE DFE MSE RMSE $\begin{array}{llll}0.0004972067 & 4 & 0.0001243 & 0.0111491\end{array}$

$\begin{array}{lrr}\text { Parameter } & \text { Estimate } & \text { ApproxStdErr } \\ \text { C } & 353.85998251 & 75.7489401 \\ \text { B } & 4834.5082625 & 937.175834 \\ \text { A } & -2.822146441 & 0.59713183\end{array}$

Solved By:

Analytic NR 
Exhibit H-1. Results from Fitting Fulcher Equations to the Viscosity Measurements of the KT03-Series Glasses. (continued)

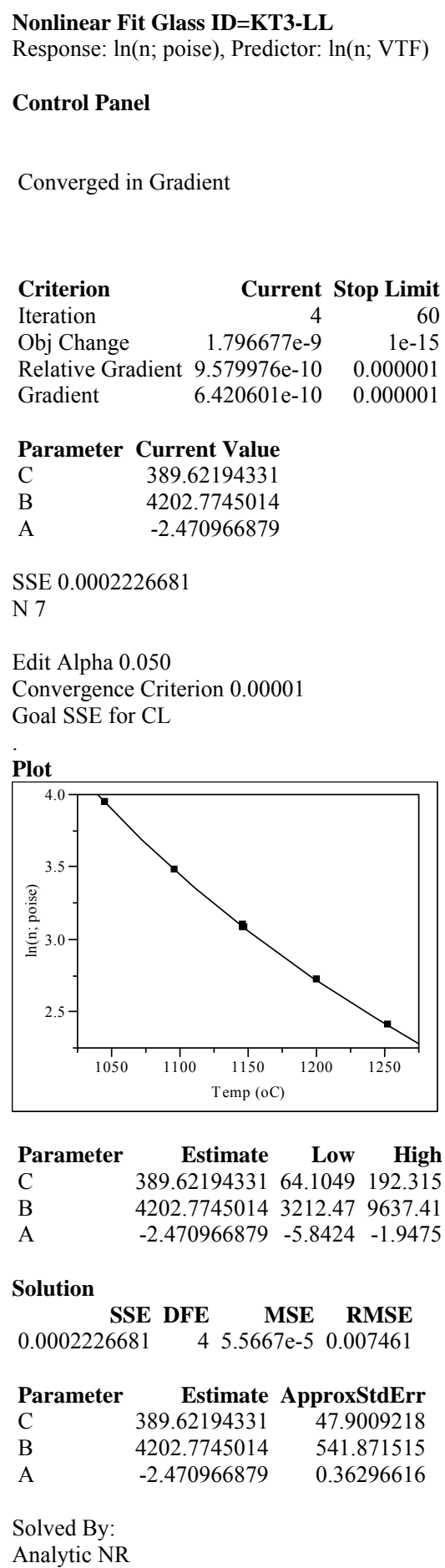

Nonlinear Fit Glass ID=KT3-LN

Response: $\ln (\mathrm{n}$; poise), Predictor: $\ln (\mathrm{n}$; VTF)

Control Panel

Converged in Gradient

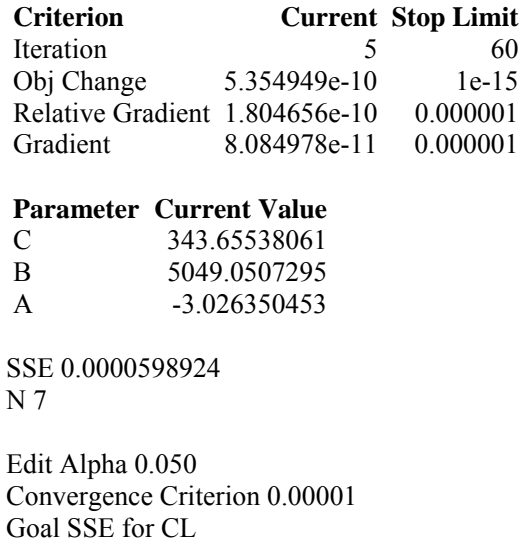

Plot

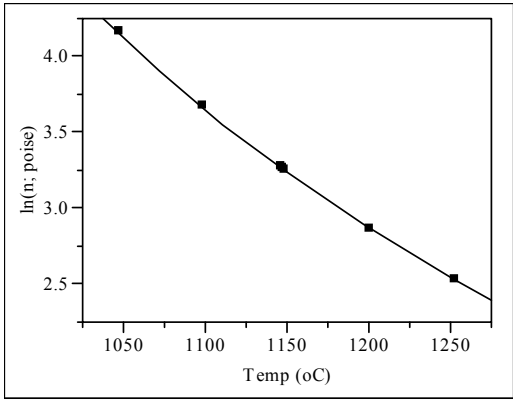

Parameter Estimate Low High

C $\quad 343.6553806164 .1049 \quad 192.315$

B $\quad 5049.05072953212 .479637 .41$

$\begin{array}{llll}\text { A } & -3.026350453 & -5.8424 & -1.9475\end{array}$

Solution SSE DFE MSE RMSE $\begin{array}{llll}0.0000598924 & 4 & 1.4973 \mathrm{e}-5 & 0.0038695\end{array}$

Parameter Estimate ApproxStdErr $\begin{array}{llr}\text { C } & 343.65538061 & 26.6404698\end{array}$

$\begin{array}{lll}\text { B } & 5049.0507295 & 340.078139\end{array}$

$\begin{array}{lll}\text { A } & -3.026350453 & 0.21410428\end{array}$

Solved By:

Analytic NR 
Exhibit H-1. Results from Fitting Fulcher Equations to the Viscosity Measurements of the KT03-Series Glasses. (continued)

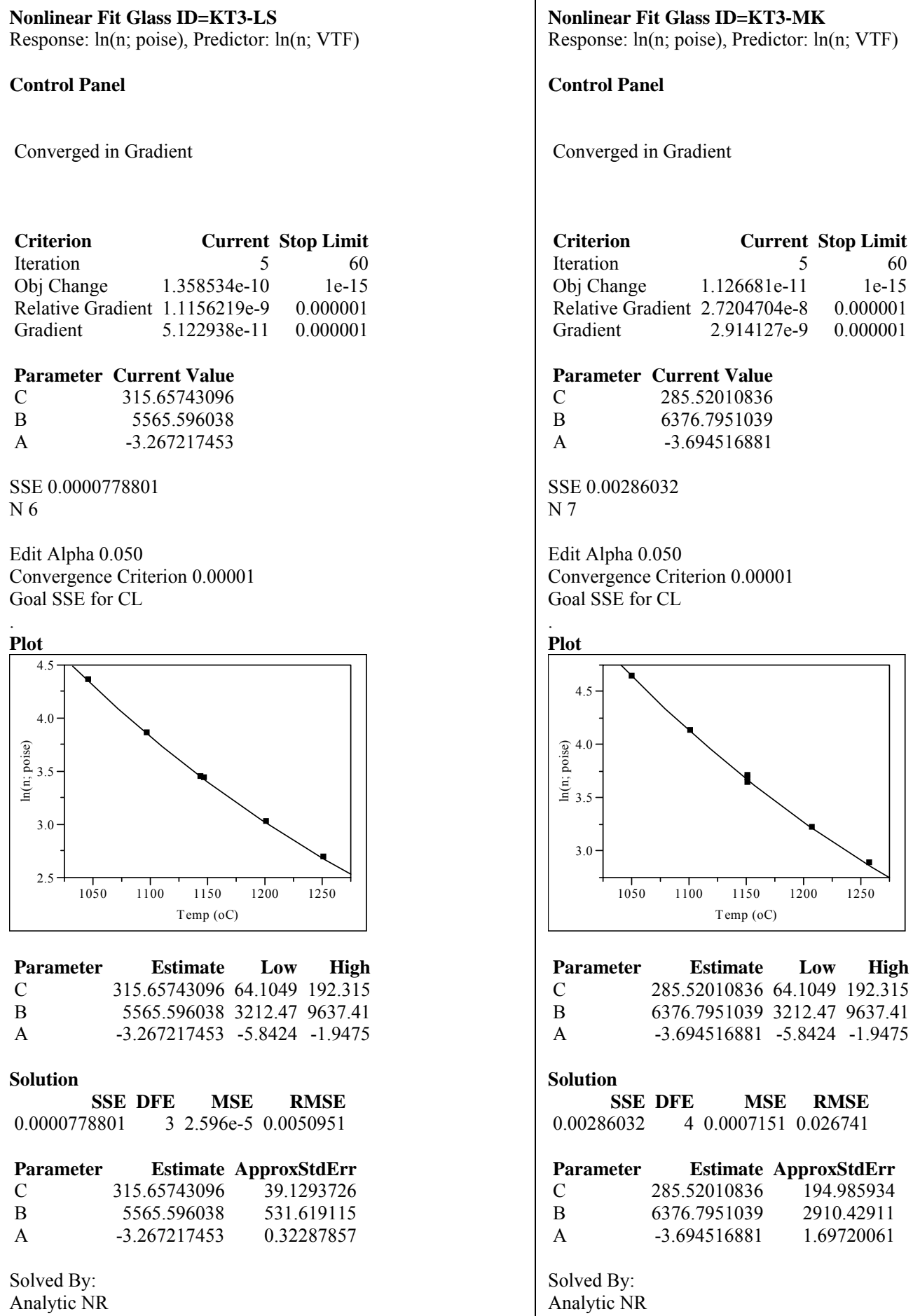

Nonlinear Fit Glass ID=KT3-MK

Response: $\ln (\mathrm{n}$; poise), Predictor: $\ln (\mathrm{n} ; \mathrm{VTF})$

Control Panel

Converged in Gradient

Plot

Parameter Estimate Low High

C $\quad 285.5201083664 .1049192 .315$

B $\quad 6376.79510393212 .479637 .41$

A $\quad-3.694516881 \quad-5.8424 \quad-1.9475$

Solution SSE DFE MSE RMSE $\begin{array}{llllll}0.00286032 & 4 & 0.0007151 & 0.026741\end{array}$

Parameter Estimate ApproxStdErr $\begin{array}{lll}\text { C } & 285.52010836 & 194.985934\end{array}$

$\begin{array}{lll}\text { B } & 6376.7951039 & 2910.42911\end{array}$

A $\quad-3.694516881 \quad 1.69720061$

Solved By:

Analytic NR 
SRNL-STI-2010-00566

Revision 0

Appendix I. Results from Fitting Fulcher Equations

to the Viscosity Measurements of the KT04-Series Glasses 
Exhibit I-1. Results from Fitting Fulcher Equations to the Viscosity Measurements of the KT04-Series Glasses.

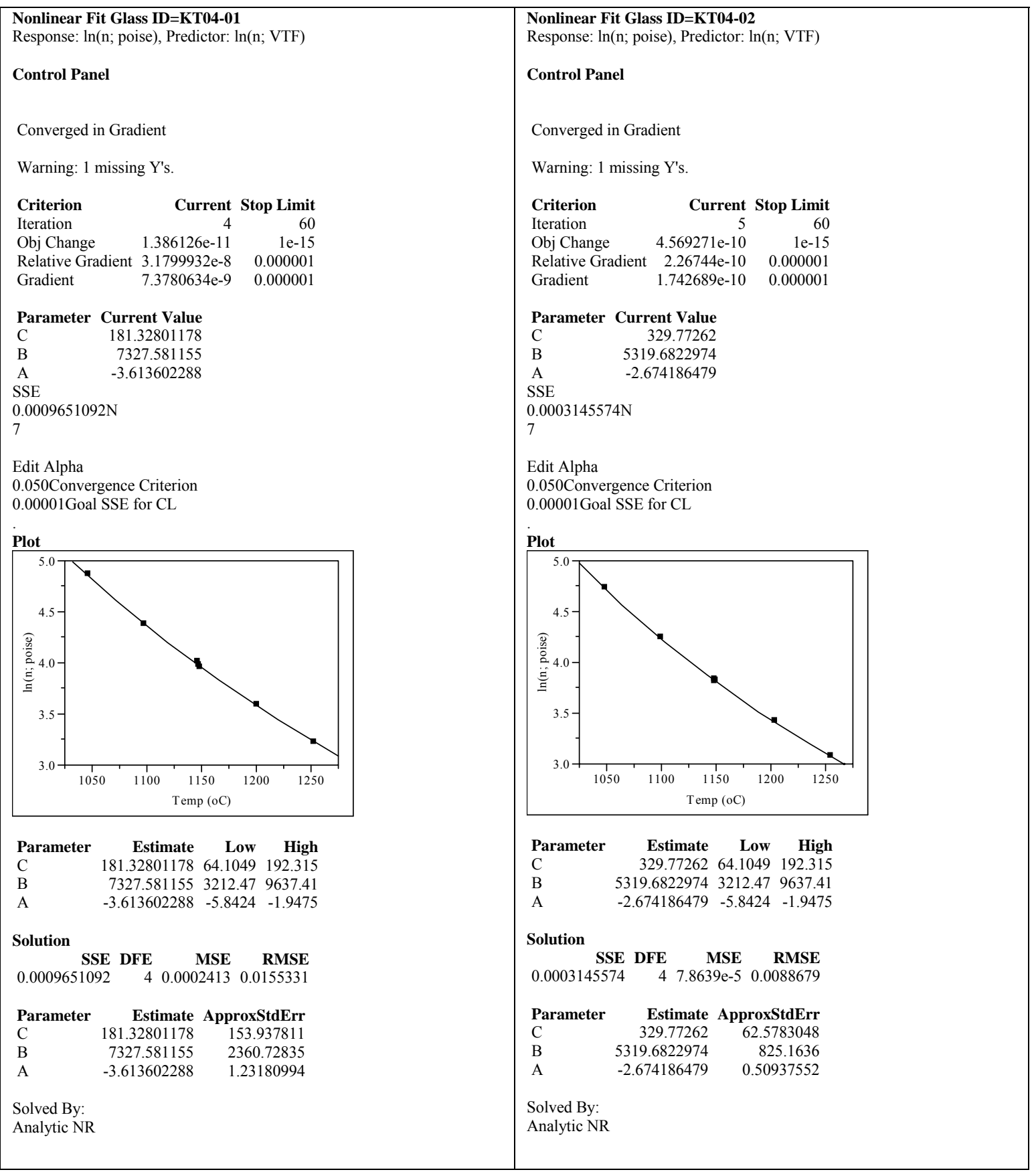


Exhibit I-1. Results from Fitting Fulcher Equations to the Viscosity Measurements of the KT04-Series Glasses. (continued)

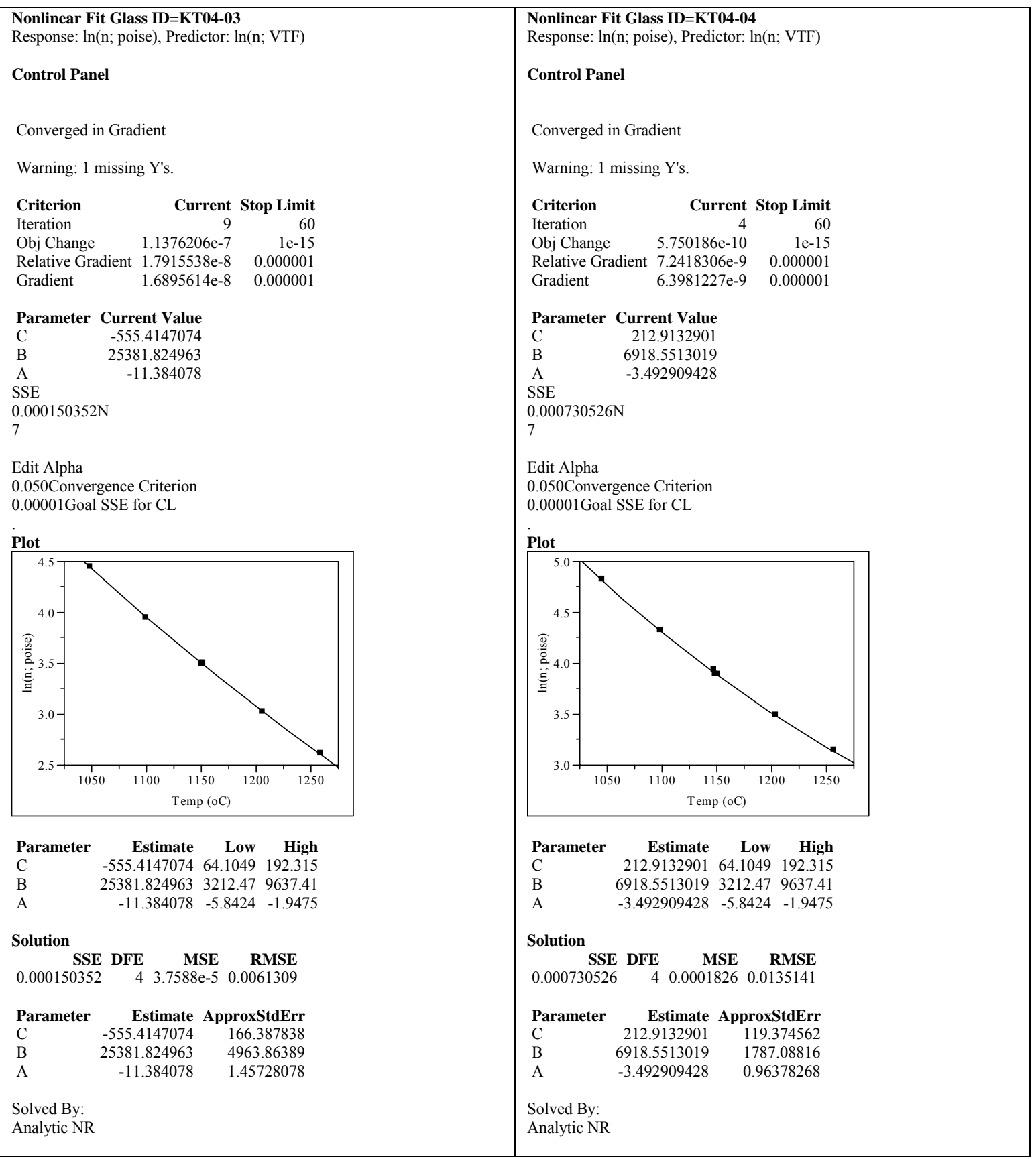


Exhibit I-1. Results from Fitting Fulcher Equations to the Viscosity Measurements of the KT04-Series Glasses. (continued)

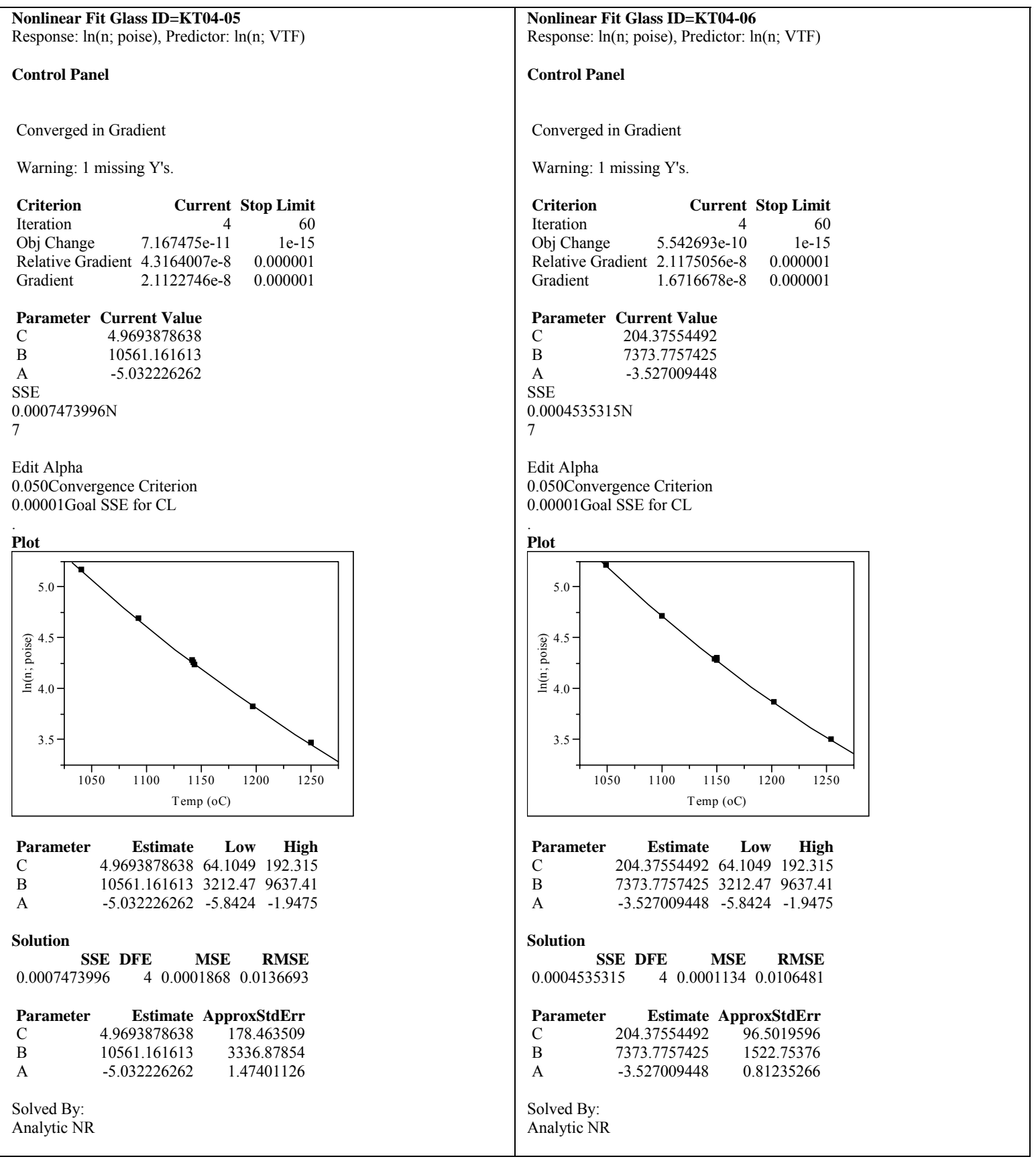


Exhibit I-1. Results from Fitting Fulcher Equations to the Viscosity Measurements of the KT04-Series Glasses. (continued)

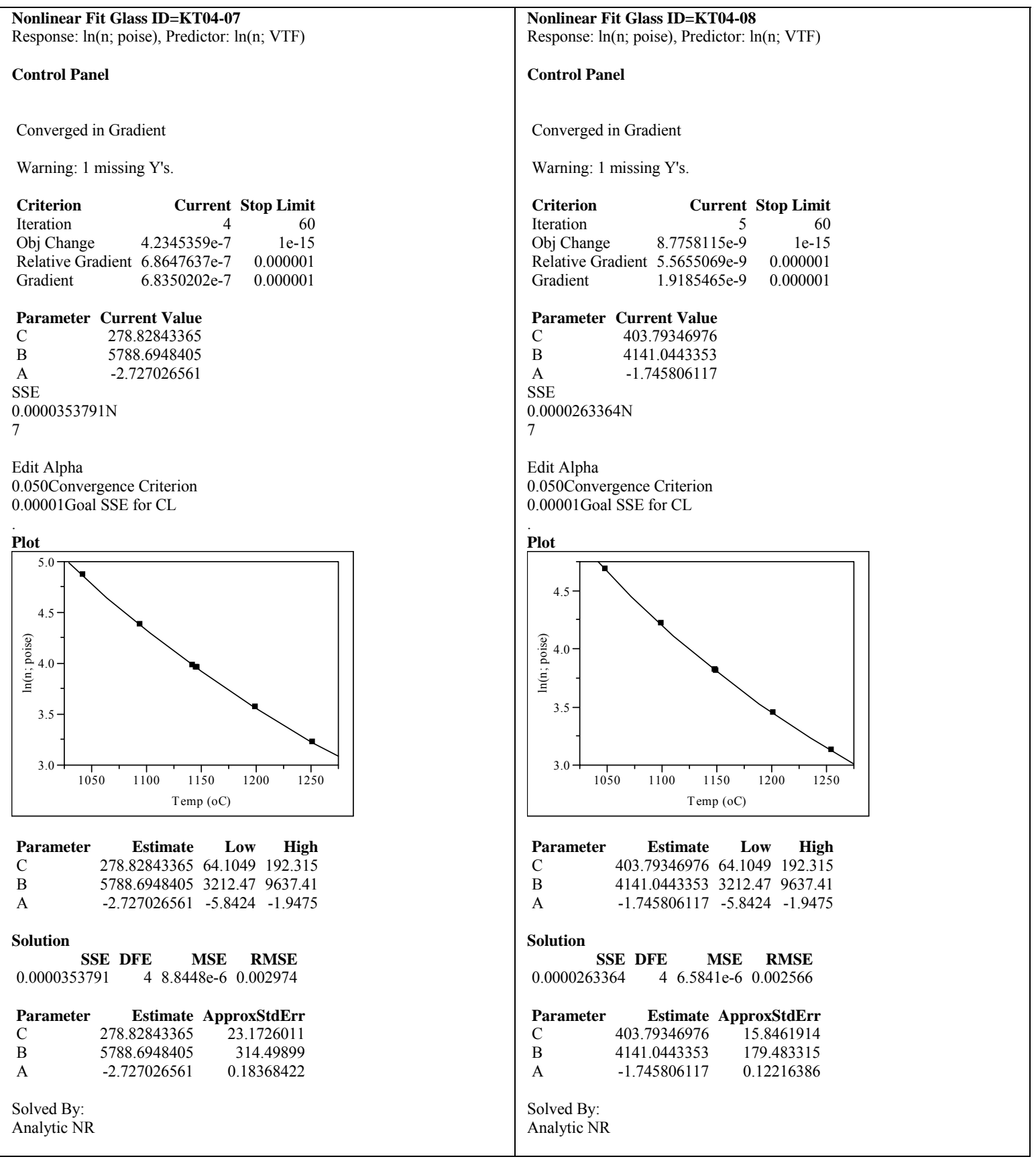


Exhibit I-1. Results from Fitting Fulcher Equations to the Viscosity Measurements of the KT04-Series Glasses. (continued)

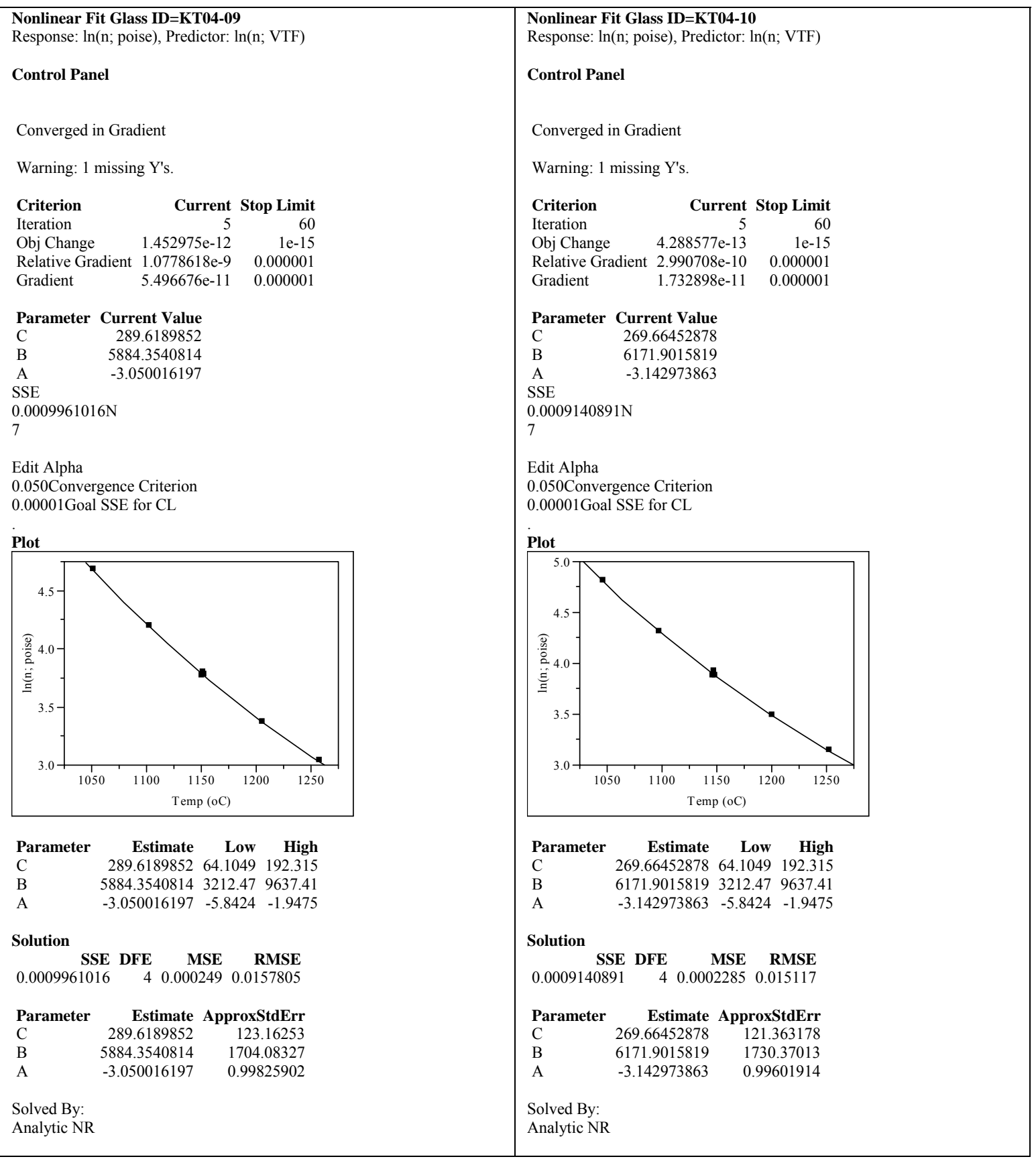


SRNL-STI-2010-00566

Revision 0

Appendix J. Summarized Viscosity Data for the KT01, KT03, and KT04-Series Glasses 
Table J-1. Model Predicted Viscosities with Confidence Intervals (CI) and Measured Viscosities (Fulcher Fits) at $1150{ }^{\circ} \mathrm{C}$ for the KT01, KT03 and KT04-Series Glasses by Compositional View.

\begin{tabular}{|c|c|c|c|c|c|c|}
\hline $\begin{array}{c}\text { Compositional } \\
\text { View }\end{array}$ & Glass ID & $\begin{array}{c}\text { Viscosity } \\
\text { Prediction (P) }\end{array}$ & $\begin{array}{l}\text { Lower CI for } \\
\text { Prediction (P) }\end{array}$ & $\begin{array}{l}\text { Upper CI for } \\
\text { Prediction (P) }\end{array}$ & $\begin{array}{l}\text { Meas. Visc. } \\
\text { (Fulcher) (P) }\end{array}$ & $\begin{array}{c}\text { Model } \\
\text { Predictable? }\end{array}$ \\
\hline measured & KT1-HA2 & 30.63 & 20.86 & 44.96 & 30.79 & Yes \\
\hline measured & KT1-HB & 21.65 & 14.72 & 31.84 & 18.74 & Yes \\
\hline measured & KT1-HF & 20.09 & 13.66 & 29.56 & 19.29 & Yes \\
\hline measured & KT1-HK & 29.59 & 20.15 & 43.44 & 64.17 & No \\
\hline measured & KT1-HL & 9.96 & 6.73 & 14.74 & 10.92 & Yes \\
\hline measured & KT1-HN & 18.78 & 12.76 & 27.64 & 17.69 & Yes \\
\hline measured & KT1-HS & 44.25 & 30.18 & 64.88 & 32.85 & Yes \\
\hline measured & KT1-LA & 21.75 & 14.79 & 31.99 & 19.82 & Yes \\
\hline measured & KT1-LB & 26.29 & 17.89 & 38.62 & 22.79 & Yes \\
\hline measured & KT1-LF & 34.40 & 23.44 & 50.47 & 25.75 & Yes \\
\hline measured & KT1-LL & 24.54 & 16.70 & 36.06 & 21.07 & Yes \\
\hline measured & KT1-LN & 36.79 & 25.08 & 53.98 & 27.97 & Yes \\
\hline measured & KT1-LS & 24.49 & 16.67 & 35.99 & 19.88 & Yes \\
\hline targeted & KT1-HA2 & 34.01 & 23.18 & 49.91 & 30.79 & Yes \\
\hline targeted & KT1-HB & 20.37 & 13.85 & 29.97 & 18.74 & Yes \\
\hline targeted & KT1-HF & 17.78 & 12.08 & 26.19 & 19.29 & Yes \\
\hline targeted & KT1-HK & 25.09 & 17.08 & 36.87 & 64.17 & No \\
\hline targeted & KT1-HL & 9.84 & 6.65 & 14.56 & 10.92 & Yes \\
\hline targeted & KT1-HN & 17.41 & 11.82 & 25.64 & 17.69 & Yes \\
\hline targeted & KT1-HS & 43.03 & 29.35 & 63.10 & 32.85 & Yes \\
\hline targeted & KT1-LA & 21.56 & 14.66 & 31.70 & 19.82 & Yes \\
\hline targeted & KT1-LB & 24.56 & 16.71 & 36.10 & 22.79 & Yes \\
\hline targeted & KT1-LF & 31.64 & 21.56 & 46.45 & 25.75 & Yes \\
\hline targeted & KT1-LL & 23.73 & 16.14 & 34.87 & 21.07 & Yes \\
\hline targeted & KT1-LN & 33.39 & 22.76 & 49.01 & 27.97 & Yes \\
\hline targeted & KT1-LS & 23.43 & 15.94 & 34.45 & 19.88 & Yes \\
\hline measured & KT03-HA & 35.69 & 24.33 & 52.36 & 32.96 & Yes \\
\hline measured & KT03-HB & 20.91 & 14.22 & 30.76 & 16.43 & Yes \\
\hline measured & KT03-HF & 17.32 & 11.76 & 25.51 & 18.98 & Yes \\
\hline measured & KT03-HK & 29.25 & 19.92 & 42.94 & 34.95 & Yes \\
\hline measured & KT03-HL & 8.12 & 5.48 & 12.04 & 10.89 & Yes \\
\hline measured & KT03-HN & 12.96 & 8.78 & 19.13 & 16.66 & Yes \\
\hline measured & KT03-HS & 55.00 & 37.52 & 80.61 & 56.06 & Yes \\
\hline measured & KT03-LA & 16.86 & 11.45 & 24.83 & 18.34 & Yes \\
\hline measured & KT03-LB & 24.77 & 16.86 & 36.40 & 22.94 & Yes \\
\hline measured & KT03-LF & 29.35 & 19.99 & 43.10 & 25.8 & Yes \\
\hline measured & KT03-LL & 19.85 & 13.49 & 29.20 & 21.25 & Yes \\
\hline measured & KT03-LN & 28.48 & 19.39 & 41.82 & 25.41 & Yes \\
\hline measured & KT03-LS & 37.04 & 25.25 & 54.33 & 30.07 & Yes \\
\hline measured & KT03-MK & 21.47 & 14.60 & 31.57 & 39.72 & No \\
\hline targeted & KT03-HA & 35.76 & 24.38 & 52.47 & 32.96 & Yes \\
\hline targeted & KT03-HB & 19.71 & 13.39 & 29.00 & 16.43 & Yes \\
\hline targeted & KT03-HF & 16.58 & 11.26 & 24.43 & 18.98 & Yes \\
\hline targeted & KT03-HK & 25.15 & 17.12 & 36.96 & 34.95 & Yes \\
\hline targeted & KT03-HL & 8.49 & 5.73 & 12.57 & 10.89 & Yes \\
\hline targeted & KT03-HN & 14.60 & 9.90 & 21.52 & 16.66 & Yes \\
\hline targeted & KT03-HS & 66.53 & 45.40 & 97.50 & 56.06 & Yes \\
\hline targeted & KT03-LA & 22.07 & 15.01 & 32.45 & 18.34 & Yes \\
\hline targeted & KT03-LB & 24.14 & 16.42 & 35.48 & 22.94 & Yes \\
\hline targeted & KT03-LF & 30.77 & 20.96 & 45.16 & 25.8 & Yes \\
\hline targeted & KT03-LL & 21.69 & 14.75 & 31.90 & 21.25 & Yes \\
\hline targeted & KT03-LN & 29.36 & 19.99 & 43.10 & 25.41 & Yes \\
\hline targeted & KT03-LS & 37.09 & 25.28 & 54.41 & 30.07 & Yes \\
\hline targeted & KT03-MK & 25.13 & 17.10 & 36.93 & 39.72 & No \\
\hline measured & KT04-01 & 48.92 & 33.37 & 71.72 & 52.94 & Yes \\
\hline measured & KT04-02 & 42.17 & 28.75 & 61.83 & 41.91 & Yes \\
\hline measured & KT04-03 & 36.17 & 24.65 & 53.06 & 30.66 & Yes \\
\hline measured & KT04-04 & 46.17 & 31.49 & 67.69 & 45.34 & Yes \\
\hline
\end{tabular}


Table J-1. Model Predicted Viscosities with Confidence Intervals (CI) and Measured Viscosities (Fulcher Fits) at $1150{ }^{\circ} \mathrm{C}$ for the KT01, KT03 and KT04-Series Glasses by Compositional View. (continued)

\begin{tabular}{|c|c|c|c|c|c|c|}
\hline $\begin{array}{c}\text { Compositional } \\
\text { View }\end{array}$ & Glass ID & $\begin{array}{c}\text { Viscosity } \\
\text { Prediction (P) }\end{array}$ & $\begin{array}{l}\text { Lower CI for } \\
\text { Prediction (P) }\end{array}$ & $\begin{array}{l}\text { Upper CI for } \\
\text { Prediction (P) }\end{array}$ & $\begin{array}{c}\text { Meas. Visc. } \\
\text { (Fulcher) (P) }\end{array}$ & $\begin{array}{c}\text { Model } \\
\text { Predictable? }\end{array}$ \\
\hline measured & KT04-05 & 67.12 & 45.80 & 98.36 & 67.32 & Yes \\
\hline measured & KT04-06 & 63.61 & 43.40 & 93.22 & 66.34 & Yes \\
\hline measured & KT04-07 & 48.06 & 32.78 & 70.46 & 51.21 & Yes \\
\hline measured & KT04-08 & 39.55 & 26.97 & 58.01 & 45.69 & Yes \\
\hline measured & KT04-09 & 37.76 & 25.74 & 55.39 & 40.99 & Yes \\
\hline measured & KT04-10 & 41.67 & 28.42 & 61.11 & 44.34 & Yes \\
\hline targeted & KT04-01 & 50.58 & 34.51 & 74.15 & 52.94 & Yes \\
\hline targeted & KT04-02 & 45.06 & 30.73 & 66.06 & 41.91 & Yes \\
\hline targeted & KT04-03 & 41.22 & 28.10 & 60.45 & 30.66 & Yes \\
\hline targeted & KT04-04 & 47.35 & 32.30 & 69.42 & 45.34 & Yes \\
\hline targeted & KT04-05 & 68.04 & 46.43 & 99.71 & 67.32 & Yes \\
\hline targeted & KT04-06 & 72.52 & 49.48 & 106.27 & 66.34 & Yes \\
\hline targeted & KT04-07 & 52.90 & 36.09 & 77.53 & 51.21 & Yes \\
\hline targeted & KT04-08 & 43.03 & 29.34 & 63.10 & 45.69 & Yes \\
\hline targeted & KT04-09 & 41.69 & 28.43 & 61.14 & 40.99 & Yes \\
\hline targeted & KT04-10 & 41.36 & 28.20 & 60.66 & 44.34 & Yes \\
\hline
\end{tabular}


Exhibit J-1. Plots of Measured Versus Predicted Viscosity Values by Series Based on Measured and Target Compositions.

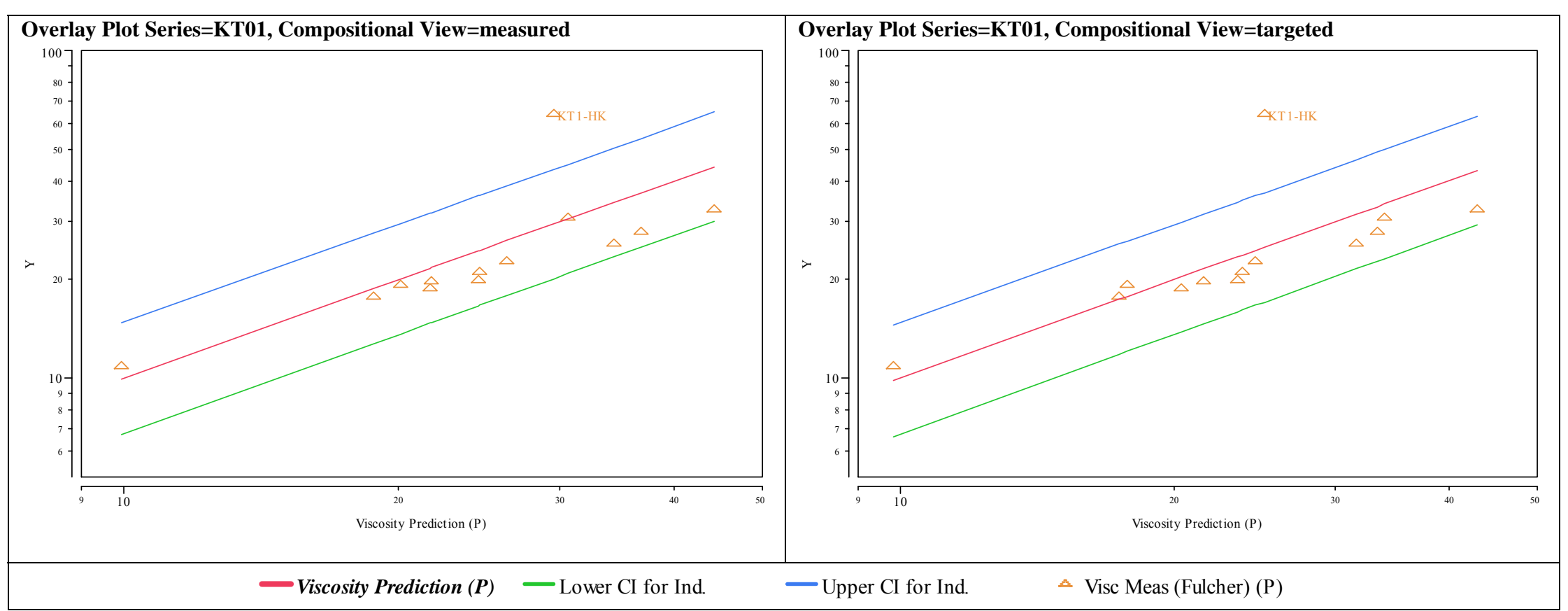


Exhibit J-1. Plots of Measured Versus Predicted Viscosity Values by Series Based on Measured and Target Compositions. (continued)

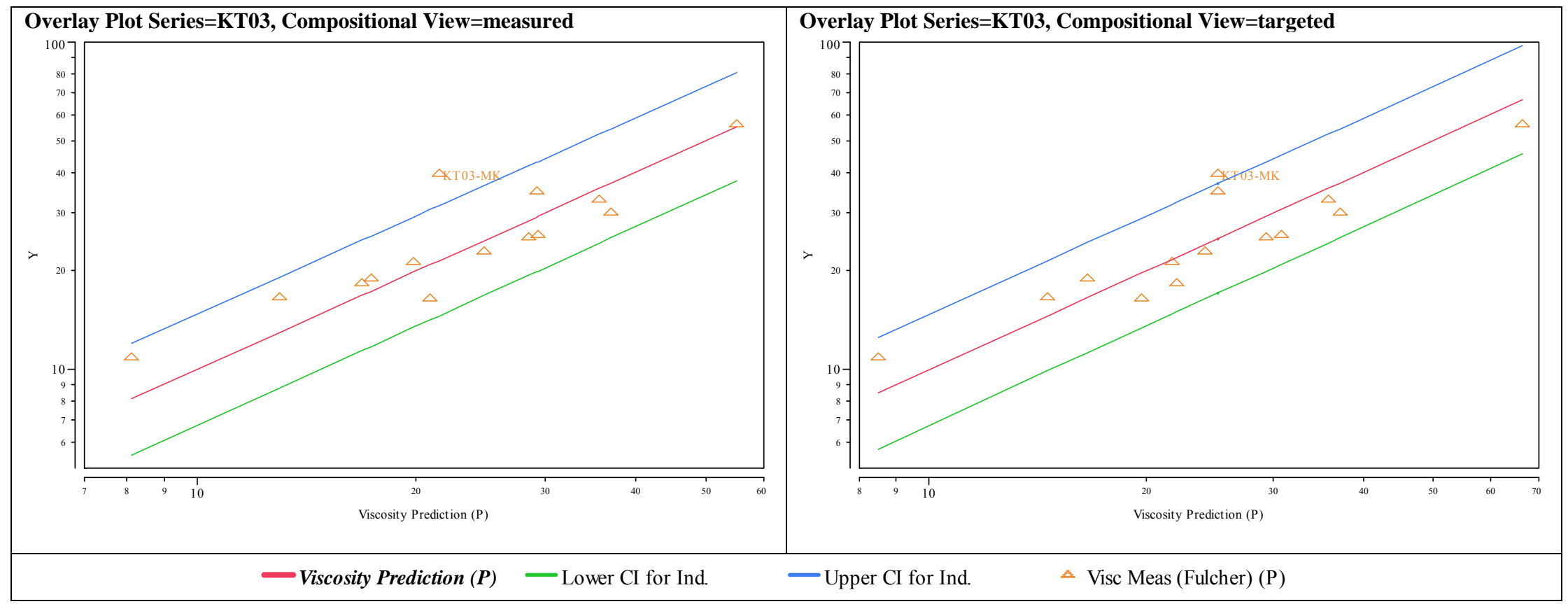


Exhibit J-1. Plots of Measured Versus Predicted Viscosity Values by Series Based on Measured and Target Compositions. (continued)

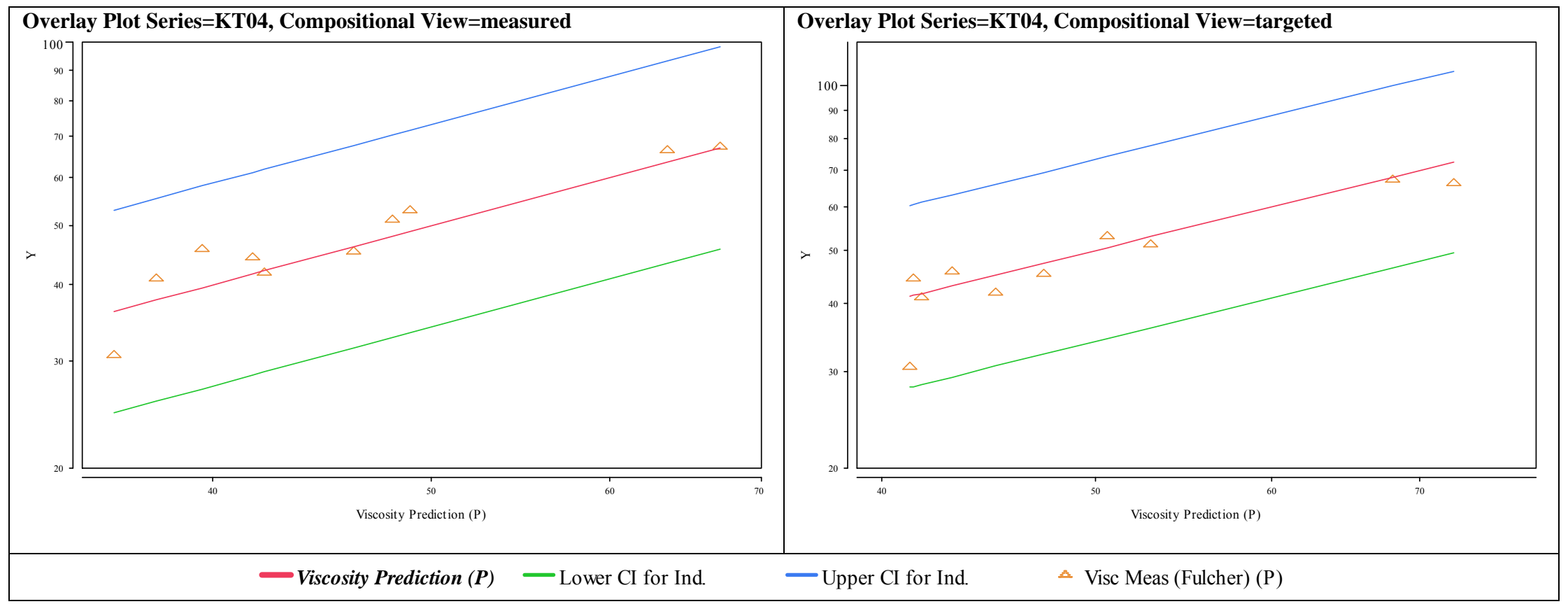


Exhibit J-2. Plots of Measured Versus Predicted Viscosity Values for all of the KT01, KT03 and KT04-Series Glasses, Based on Measured and Target Compositions.

Overlay Plot Compositional View=measured

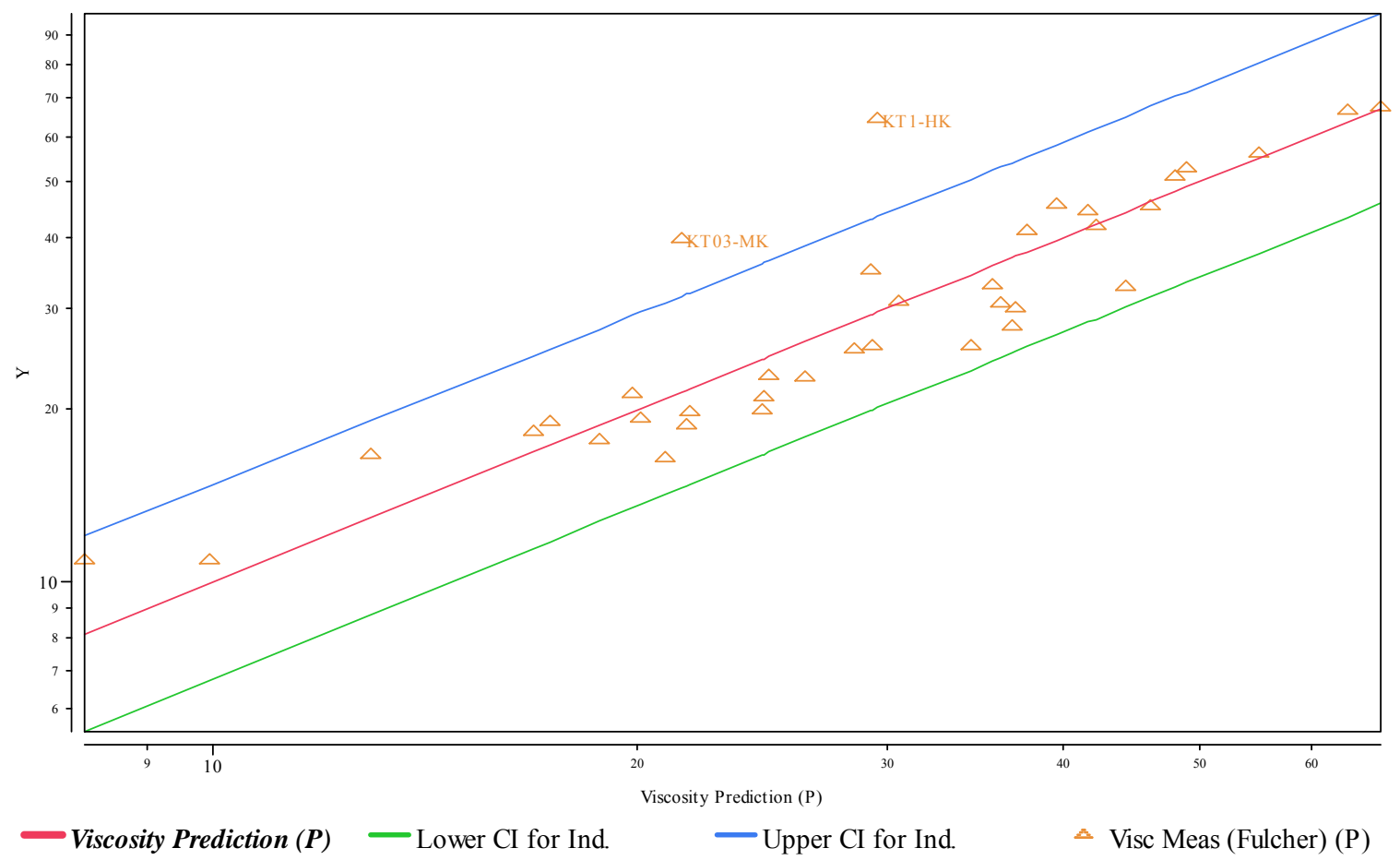

Overlay Plot Compositional View=targeted

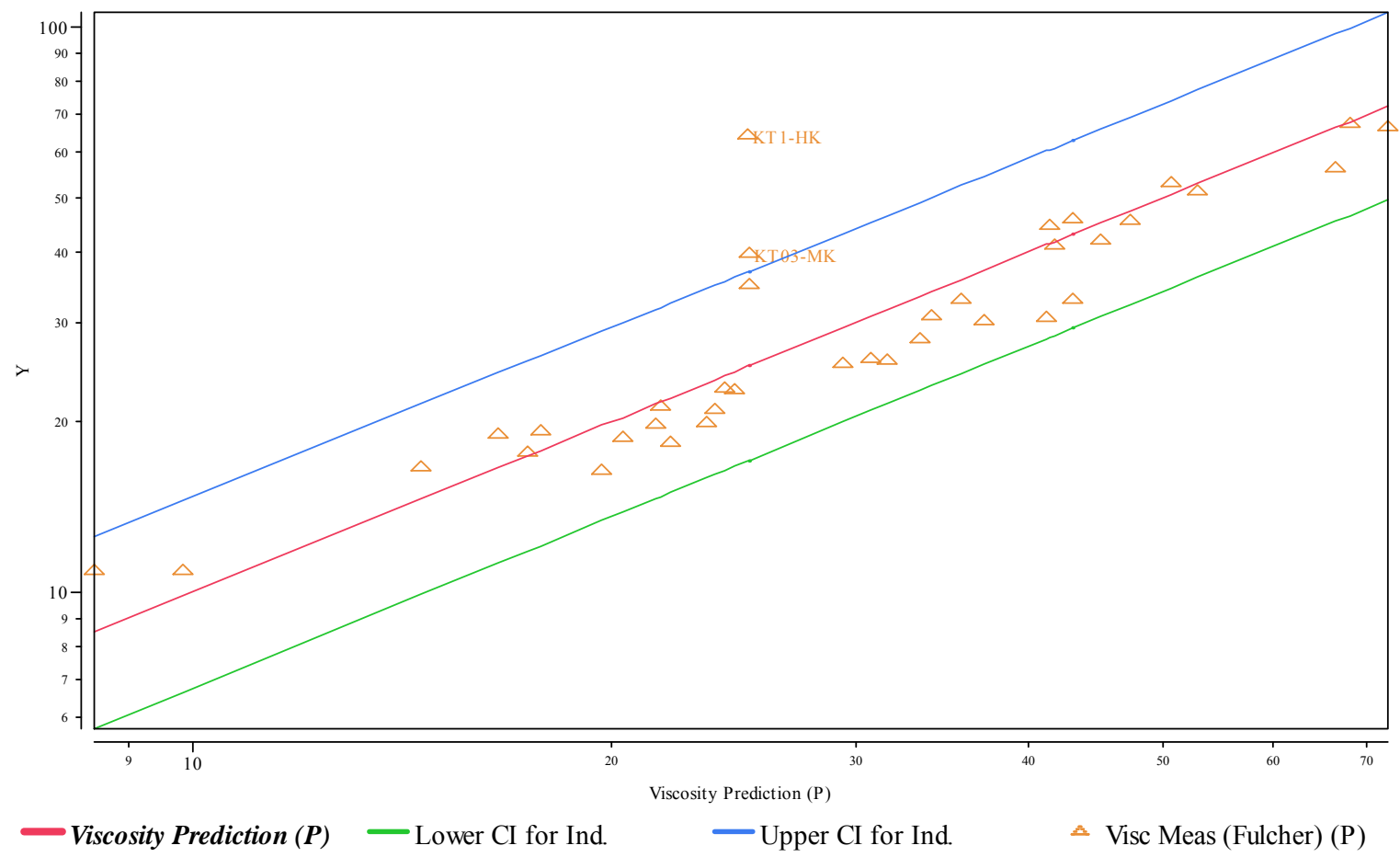




\section{Distribution:}

J. W. Amoroso, 999-W

C. J. Bannochie, 773-42A

A. B. Barnes, 999-W

A. L. Billings, 999-W

J. M. Bricker, 704-27S

M. A. Broome, 704-29S

C. L. Crawford, 773-42A

D. A. Crowley, 773-43A

R. E. Edwards, 766-H

T. B. Edwards, 999-W

T. L. Fellinger, 704-26S

S. D. Fink, 773-A

K. M. Fox, 999-W

B. J. Giddings, 786-5A

J. M. Gillam, 766-H

B. A. Hamm, 766-H

C. C. Herman, 999-W

D. T. Herman, 735-11A

R. N. Hinds, 704-S

E. W. Holtzscheiter, 704-15S
T. H. Huff, 766-H

J. F. Iaukea, 704-30S

C. M. Jantzen, 773-A

F. C. Johnson, 999-W

D. C. Koopman, 999-W

D. D. Larsen, 766-H

P. L. Lee, 703-41A

S. L. Marra, 773-A

D. H. Miller, 999-W

J. E. Occhipinti, 704-S

D. K. Peeler, 999-W

F. M. Pennebaker, 773-42A

J. W. Ray, 704-S

M. A. Rios-Armstrong, 766-H

H. B. Shah, 766-H

D. C. Sherburne, 704-S

A. V. Staub, 704-27S

M. E. Stone, 999-W

J. P. Vaughan, 773-41A 